YTS-1435

FINAL

\title{
CALENDAR YEAR 1995 GROUNDWATER QUALITY REPORT FOR THE CHESTNUT RIDGE HYDROGEOLOGIC REGIME, OAK RIDGE Y-12 PLANT, OAK RIDGE, TENNESSEE
}

\author{
1995 Groundwater Quality Data and Calculated Rate \\ of Contaminant Migration
}

February 1996

Prepared by

Envirommental Maragement Departuent

Health, Safety, Environment, and Accountability Organization

Oak Ridge Y-12 Plant

Oak Ridge, Tennessee 37831

Managed by

LOCKHEED MARTIN ENERGY SYSTEMS, INC.

for the

U.S. DEPARTMENT OF ENERGY

under

Contract DE-AC05-840R21400

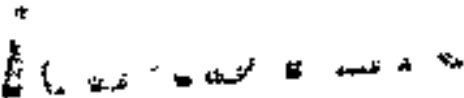




\section{DISCLANMER}

Portions of this document may be nlevible in electronic image products. Images are produced from the best avallable original document. 


\section{TABLE OF CONTENTS}

Section

Pape

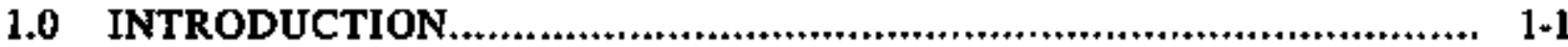

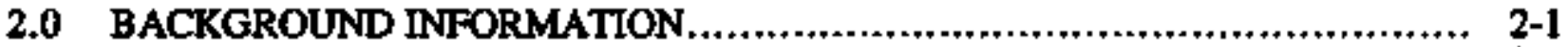

2.1 Regulatory Framework......................................................... 2-1

2.2 CERCLA Operable U'nits...................................................... 2-3

2.2.1 Chestnat Ridge Security Pits.............................................. 2-3

2.2.2 Ash Disposal Basin .................................................. 2-4

2.2.3 United Naclear Corponation Site ....................................... 2-5

2.2.4 Rogers Quarty ...................................................... $2-6$

2.3 RCRA Treatment, Storage, or Disposal Facilities................................... 2-6

2.3.1 Chestnut Ridge Sediment Disposal Basin ........................ 2-6

2.3.2 Kerr Hollow Quarry .............................................. 2-7

2.3.3 East Chestnut Ridge Waste Pile ................................... 2-8

2.4 Solid Waste Disposal Facilities ................................................... 2-8

2.4.1 Industrial Landifll II................................................... 2-9

2.4.2 Industrial Landfill IV....................................................... $2-9$

2.4.3 Constructiond Demolition Landfill VI.................................... 2-9

2.4.4 Industrial Landfill $V$ and Constructiondemolition Landfill VII......... 2-10

2.5 Chestnut Ridge Borrow Area Waste Pile ............................................. 2-10

3.0 CY 1995 GROUNDWATER PROTECTION PROGRAM............................ 3-1

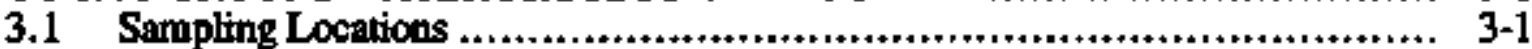

3.1.1 RCRA Interim Status Assessment Monitoring .............................. 3-1

3.1.2 RCRA Interim Status Detection Monitoning................................ 3-2

3.1.3 SWDF Detection/Background Monitoring ............................... 3-2

3.1.4 CERCLA Record of Decision Monitoring................................ 3-2

3.1.5 RCRA Post-Closure Detection Monitoring................................. 3-2

3.1.6 Best Management Practice Monitoring..................................... 3-3

3.2 Sampling Frequency............................................................ 3-3

3.3 Sample Collection................................................................. 3-4

3.4 Quality Assurance/Quality Control Sampling ..................................... 3-4

3.5 Laboratory Analysis ................................................................. 3-5

3.6 Data Maragement................................................................ 3-6

3.7 Analytical Results.................................................................. 3-7

4.0 HYDROGEOLOGIC FRAMEWORK ................................................. 4-1

4.1 Geology ......................................................................... 4-1

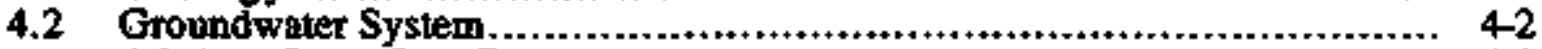

4.2.1 Stormflow Zone .............................................................. 4 4.3

4.2.2 Vadose Zone .................................................................. 4.3

4.2.3 Groundwater Zone ................................................. 4-4

4.2.4 Aquiclude.............................................................. 4.6

4.3 Groundwater Flow Directions..................................................... 4-6

4.4 Rate of Groundwater Flow and Contaminant Migration ..................... 4-7

4.4.1 Residuum............................................................. 4-8

4.4.2 Bedrock .................................................................... 4.8

4.5 Surface Water System .......................................................... 4.9 
TABLE OF CONTENTS (cont'd)

Section

5.0 REFERENCES

Anpendiges
A Figures
B Tables
C Monitoring Well Construction Details
D Sampling Sequences
E Gronndwater Quality Data
F Field Dupticate Data
G Radiological Chemistry Data Summary
H Groundwater Elevations, 1995
I Ion-Charge Balance Calculations
J Trace Metal Concentrations that Exceed Maximum Contaminant Levels
K Nitrate Concentrations
L QAVC Data Summaries
M Validated Resnles of Volatile Organic Compound Analyses 


\section{LIST OF FIGURES}

\section{Eipune}

1 Regional Location of the Y-12 Pant

2 Hydrogeologic Regimes at the Y-12 Pant

A-2

3 Waste-Management Sites and CERCLA Operable Units in the

Chestnut Ridge Hydrogeologic Regine

A-3

4 Sampling Locations for Groundwater and Surface Water Monitocing During 1995

5 Topography and Bedrock Geology in the Chestnut Ridge Hydrogeologic Regime.

6 Schematic Profile of Hydrostratigraphic Units in the Chestmut Ridge Hydrogeologic Regime.

7 Groundwater Elevations in the Chestnut Ridge Hydrogeologic Regime

8 Hydrographs for Selected Sites in the Chestnot Ridge Regime A- 8 


\section{LIST OF TABLES}

Table

Page

1 Waste-Management Sites and CERCLA Operable Units in the Chestnut Ridge Hydrogeologic Regime .......................................... B-1

2 CY 1995 Monitoring Program Summary ........................................ B-2

3 General Construction Information for Monitoring Wells Sampled During 1995.... B-14 


\section{ACRONYMS AND ABBREVIATIONS}

\begin{tabular}{|c|c|}
\hline $\begin{array}{l}\text { Of } \\
\text { Of } \\
\text { O } \\
\text { PO } \\
\text { Q } \\
\text { RO } \\
\text { RI } \\
\text { RF } \\
\text { RO } \\
\text { SA } \\
\text { SE } \\
\text { SV } \\
\text { SV } \\
\text { II } \\
\text { TI }\end{array}$ & 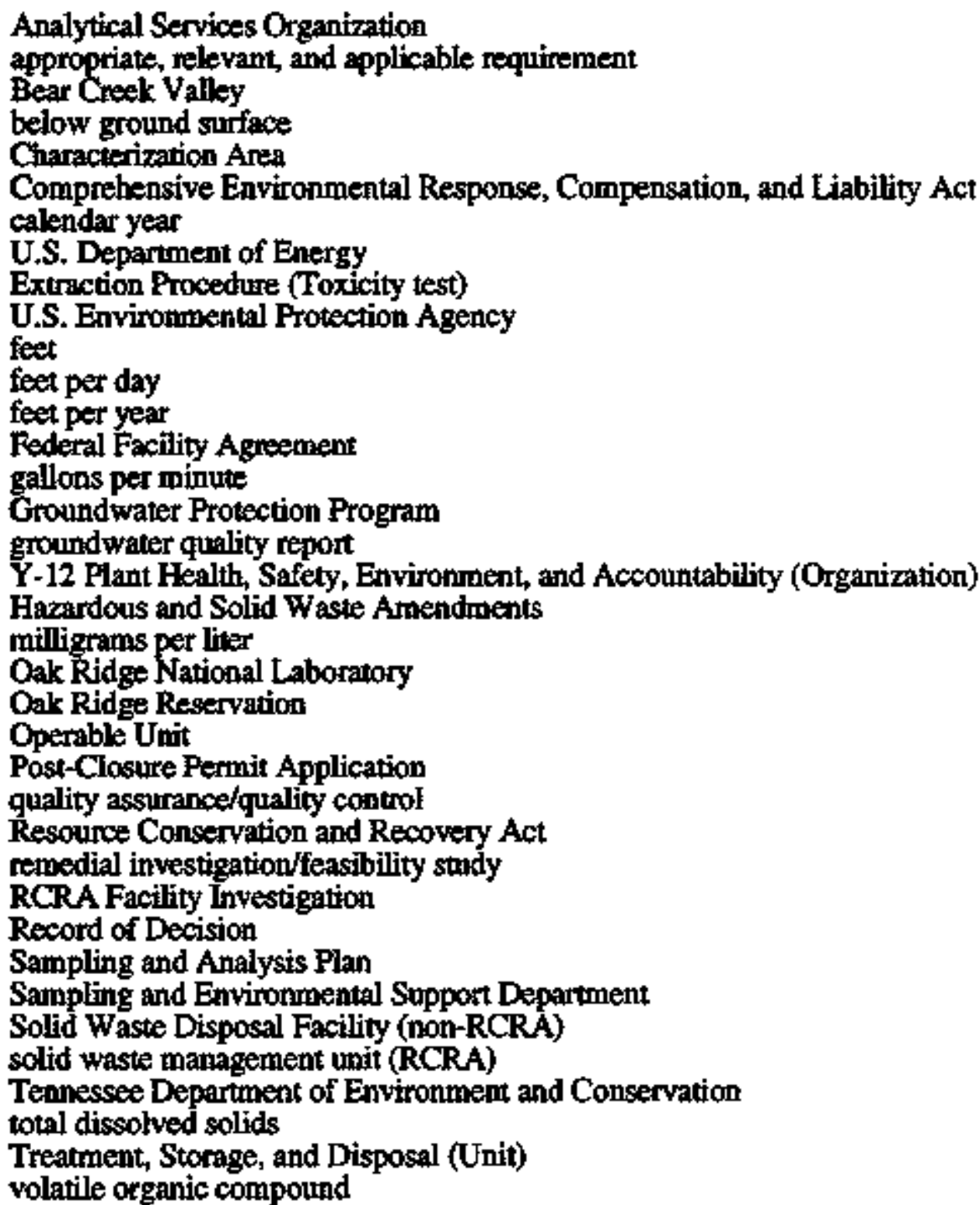 \\
\hline
\end{tabular}




\subsection{INTRODUCTION}

This annual groundwater quality report (GWQR) contains groundwater quality data obtained during the 1995 calendar year (CY) at several hazardous and nonhazartous waste management facilities associated with the U.S. Department of Energy (DOE) Y-12 Plant located on the DOE Oak Ridge Reservation (ORR) sontheast of Oak Ridge, Tennessee (Figure 1). These sites are located south of the Y-12 Plant in the Chestnut Ridge Hydrogeologic Regime (Chestnut Ridge Regime), which is one of three regimes defined for the purposes of groundwater quality monitoring at the Y-12 Plant. The Environmental Management Department of the Y-12 Plant Health, Safety, Environment, and Accountability (HSEA) Organization manages the groundwater monitoring activities in each regime as part of the Y-12 Plant Groundwater Protection Program (GWPP). The U.S. Environmental Protection Agency (EPA) identification number for the Y-12 Plant is TN3 890090001.

The GWQR for the Chestnut Ridge Regime is completed in two-parts: Part 1 (this report) containing the gromdwater quality data and Part 2 containing a detailed evaluation of the data. The primary purpose of this report is to serve as a reference containing the groundwater quality data obtained each year under the lead of the Y-12 Plant GWPP. Because it contains information needed to comply with Resource Conservation and Recovery Act (RCRA) interim status assessment monitoring and post-closure reporting requirements, this report is submitted to the Tennessee Department of Environment and Conservation (TDEC) by the RCRA reporting deadline (March 1 of the following CY). Part 2 of the annual groundwater report, to be issued mid-year, will contain a regime-wide evaluation of groundwater quality, present the findings and status of ongoing hydrogeologic studies, describe changes in monitoring priorities, and present planned modifications to the groundwater sampling and analysis activities. 


\subsection{BACKGROUND INFORMATION}

The Chestnut Ridge Regime is located south of the Y-12 Plant, and is flanked to the notth by Bear Creek Valley (BCV) and to the south by Bethel Valley. The regime encompasses the portion of Chestnut Ridge extending from Scarboro Road east of the Y-12 Plant to an umnamed drainage basin on the ridge located southwest of the western end of the Y-12 Plant (Figare 2). Groundwater quality monitoring has been conducted at sites within the Chestnut Ridge Regirne since the late 1970s. Monitoring programs have evolved in response to changing regulatory requirements and evaluation of monitoring data.

Groundwater quality monitoring during CY 1995 in the Chestnut Ridge Regime was performed at three classes of waste management facilities:

- sites subject to regulation under the Comprehensive Environmental Response, Compensation, and Liability Act (CERCIA);

- hazardous waste treatment, storage, and disposal (TSD) units subject to regulation under RCRA as well as CERCLA; and

- nonhazardous solid waste disposal facilities (SWDFs).

In addition, groundwater monitoring was conducted as a best management practice at one temporary waste storage facility that does not fall under any of the three regulatory classes. This facility (Chestnut Ridge Borrow Area Waste Pile) is classified as a Strdy Area for which future characterization and disposition under CERCL_A are required. A brief description of each of the sites in the regime, inclnding waste inventories and disposal histories, and a discussion of the regulatory status and groundwater monitoring history of each site are provided in the following sections.

\subsection{Regulatory Framework}

In 1984, the EPA and the TDEC determined that a number of the waste management units at the Y-12 Plant were either hazardous waste TSD units or solid waste management units (SWMUs) subject to regulation under Section 3004(u) of RCRA (Welch ef al. 1987). The RCRA TSDs were grinted RCRA interim status until operating or post-closure permits were obtained by the DOE. Accordingly interim stanus assessment monitoring or detection monitoring was initiated beginning in CY 1986. The process to prepare RCRA post-closure permit applications (PCPAs) for these sites was initiated. The DOE began submittal of PCPAs to TDEC in CY 1987. At designated SWMUs, RCRA Facility Investigations (RFIs) were initiated, as requined under the 
Hazardous and Solid Waste Amendments (HSWA) permit issued for the ORR by the EPA in CY 1986.

In November 1989, the ORR was added to the National Priority List. In January 1992, the DOE, the EPA, and the TDEC negotiated a Federal Facility Agreement (FFA) regarding environmental restoration at the ORR under CERCLA. This agreement superseded the HSWA permit and CERCLA became the primary regalatory driver for several RCRA TSD units and RCRA-regulated SWMUs at the Y-12 Plant. As such, the DOE appealed the applicability of RCRA post-closure care, corrective action, and groundwater monitoring nequirements to CERCLA Operable Units (OUs).

In April 1993, the DOE, the TDEC, and Lockheed Martin Energy Systems, Inc. (formerly Martin Marietra Energy Systerts, Inc.) signed an Agreed Order for the S-3 Site RCRA post-closure permit, thereby resolving the appeal and formally agreeing to proceed with CERCLA as a lead regulatory program with RCRA as an appropriate, relevant, and applicable requirement (ARAR). Under this agreement, RCRA will be applied as an ARAR to the extent that post-closure maintenance and care of former TSD facilities will be conducted in compliance with the terms of RCRA post-closure pennits. Groundwater monitoring is to be integrated with the CERCLA remedial investigation/feasibility study (RUFS) programs with RCRA conective action deferred to CERCLA. Groundwater monitoring data reporting will comply with RCRA post-closure permit conditions as well as RUFS requirenents. Per agrement with TDEC, three RCRA post-closure permits will be issued for the Y-12 Plant encompassing each of the hydrogeologic regimes. Specific requirements for interim status sites within each regime will be incorporated into the pernits as separate modifications.

Efforts were initiated early in CY 1994 by DOE and Energy Systems to modify the FFA strategy for RI/FS implementation. The FFA originally defined two types of CERCLA OUs for which an RV/FS would be performed: (1) source control OUs conprised of individual or groups of waste management sites, and (2) integrator OUs that encompass groundwater and surface water, independent of the source control OUs that may contribute to the contarnination of these media (U.S. Department of Energy 1994a). However, as the RIs for OUs within BCV progressed, it became increasingly evident that separation of source control and integrator OU's was not the most rechnically feasible approach to implementation of the RI/FS process. An agreement was reached among regulatory agencies and the DOE to proceed, where feasible, with an integrated RIFS approach. In the integrated approach, source and integrator OUs are addressed concurrently in a Characterization Area (CA) defined by watershed boundaries, and specific sites or locations of highest risk or concem are targeted for focused studies. Additionally, efforts were initiated to develop a conceptual model of groundwater flow and contaminant migration that is tailored to the specific characteristics of the groundwater flow system in $\mathrm{BCV}$.

95.069051020795 
Because there is no historical evidence of intermingling plumes from Chestnut Ridge Regine sites, no integrating $C A$ bas been established. Groundwater and surface water actions ane included in the RIFS for each source OU. Four source OUs are currently listed in the FFA (Table 1): one former RCRA TSD unit (the Chestnut Ridge Security Pits [Security Pits]) and three former RCRA SWMUs (Ash Disposal Basin, United Nuclear Corporation Site, and Rogers Quarry). Two additional fomer RCRA TSD units (Kerr Hollow Quarry and the Chestnut Ridge Sediment Disposal Basin [Sediment Disposal Basin]) are listed as lower priority study areas because they have remained in interim status detection monitoring since 1986 and no releases of contaminants to the environment have been confirmed.

The RCRA PCPAs for all TSD units in the Chestnut Ridge Regime have been subrmitted to the TDEC. The post-closure permit for the Sediment Disposal Basin was issued Septernber 18, 1995 and specified continued detection monitoring. The RCRA PCPAs for the Security Pits and the Ker Hollow Quarry proposed corrective action monitoring and continued detection monitoring, respectively. Kerr Hollow Quarry and the Sediment Disposal Basin will be managed primarily under the terms of the RCRA post-closure permits because they are not OUs. In addition, a CERCLA Record of Decision (ROD) was issued for Kerr Hollow Quarry in September 1995 that defined administrative controls for the site following waste removal actions. As specified under the RCRA post-closure permits, if detection monitoring at these two facilities confirms a futore release of contamination, the priority of the site will be re-evaluated under CERCLA and potentially elevated to OU status and an RI/FS initiated.

In addition to CERCLARCRA regulated units, five nonhazardous SWDFs (landfills) are currently subject to groundwater monitoring under provisions of TDEC Rule 1200-1-07. Baseline groundwater quality monitoring has been completed for all of these facilities and all are currently under semiannual detection monitoring in accordance with each facility's operating class and permits. Detnils reganding the monitoring histories for these facilities are included with the facility descriptions.

\subsection{Cercla Operable Units}

\subsubsection{Chestnut Ridge Security Pits}

The 1992 FFA designated the Security Pits as Chesnut Ridge OU 01. The site is also regulated as a TSD under RCRA. An RI work plan for this OU was prepared and was submitted for review by the EPA (Region IV) and the TDEC in CY 1993 (U.S. Department of Energy 1993a). Located on the crest of Chestnat Ridge, south of the central portion of the Y-12 Plant (Figure 3), the Security Pits were operated between 1973 and 1988. When in operation, the site 
was a series of trenches used for the disposal of hazardous and nonhazardous wastes. The disposal trenches were about 8 to 10 feet (ft) wide, 10 to $18 \mathrm{ft}$ deep, and 700 to $800 \mathrm{ft}$ long. Typically, several trenches were operated simultaneously to allow for the segregation of wastes in separate cells. Particularly reactive materials were disposed in six anger holes, each about $2 \mathrm{ft}$ in diameter and $10 \mathrm{ft}$ deep, located at the eastern end of the site (Butz and Stoner 1983).

The Security Pits contain an estimated 4,639 tons of waste materials. Detailed waste inventories are classified, but an unclassified inventory listed ten major wastes types that included acids, fiberglass, beryllium, biological material, debris, heavy metals, inorganics, organics, thorium, and uranium (Martin Marietta Energy Systems, Inc. 1989a).

Disposal of hazatdous waste in the Security Pits ceased in December 1984 and disposal of nonhazardous waste ceased on November 8, 1988. A low-permeability cap was constructed over the disposal trenches in 1989 in accordance with a RCRA closure plan (Martin Marietta Energy Systems, Inc. 1988a). Also in 1989, a RCRA PCPA for the Security Pits was initially submitted to the TDEC. Pertinent aspects of the PCPA for the Security Pits were modified in response to the Aptil 1993 Agreed Order and a revised PCPA was re-submitted to the TDEC in November 1994 (Martin Marietta Energy Systems, Inc. 1994a).

Groundwater monitoring at the Security Pits began with one well (well 1080) in CY 1982. Additional monitoring wells were installed at the site in CY 1985 and were periodically sampled as part of the Y-12 Plant historical and characterization monitoring programs. The site was granted RCRA interim status in November 1985 and RCRA detection monitoring was initiated in January 1986 (Bailey, personal communication 1989). The detection and characterization monitoring programs confirmed the presence of groundwater contamination at the site. Assessment monitoring in accordance with RCRA regulations for interim stans TSD facilities began in January 1988 and has continued to the present. In addition to tnonitoring in support of the RI, assessment monitoring at the Security Pits will continue pending final administrative disposition of the PCPA for the site, whereupon permit-specified monitoting requirements will be implemented.

\subsubsection{Ash Disposol Basin}

The Ash Disposal Basin, also known as the Filled Coal Ash Pond, is located on the southerri flank of Chestnut Ridge about one-half mile south of the Y-12 Plant (Figure 3). The basin was created in 1955 by the construction of an earthen dam across a northem tributary of McCoy Branch. Fly-ash slurry from the Y-12 Steam Plant was pumped into the Ash Disposal Basin until it was filled in July 1967 (Battelle Columbus Division 1988). Since then, the fly-ash slurry by-passed the Ash Disposal Basin through an emergency spillway on the eastern abutment. 
and discharged directly into McCoy Branch, which was diverted to direct the slurry into Rogers Quarry (King et al. 1989). Ash disposal ceased in July 1993 per agreement with the TDEC.

Groundwater monitoring at the Ash Disposal Basin began in 1986 with the installation of several monitoring wells that were sampled as part of the Y-12 Plant characterization monitoting program. In 1987, the Ash Disposal Basin was identified as a RCRA SWMU (Welch et al 1987), and wells at the site were subsequently sampled for the purposes of a site-specific RFI. In CY 1990, RFI monitoring activities at the site were incorporated into the Security Pits quarterly RCRA assessment monitoring schedule, as recommended in the Comprehensive Groundwater Monitoring Plan for the Deparment of Energy Y-12 Plant Oak Ridge, Tennessee (Geraghty \& Miller, Inc. 1990a).

The FFA designated the Ash Disposal Basin as Chestnut Ridge OU2. The RI work plan for this OU (U.S. Department of Energy 1993b) was approved in early 1993 by the EPA and TDEC. Field activities for the RI were completed in June 1993 and included one round of groundwater sampling from wells at the site. An RI report was issued in August 1994 (U.S. Department of Energy 1994b), followed by an FS in January 1995 (U.S. Department of Energy 1995). Preparation of a proposed plan for public review and comment is in progress.

\subsubsection{United Nuclear Corporation Site}

The United Nuclear Corporation Site (Chestnut Ridge OU3) lies on the crest of Chestnut Ridge southeast of the west end of the Y-12 Plant (Figure 3). The site was excavated to a depth of about $32 \mathrm{ft}$ and was used to landfill 11,000 dnums (55-gallon) of sludge fixed in cement, 18,000 dnums of contaninated soil, and 288 boxes of contaminated process and demolition materials (U.S. Department of Energy 1993c). The site ceased operations in 1984 (Grutzeck 1987) and was capped and closed in CY 1992 in accordance with an approved CERCLA ROD and a RCRA closure plan (U.S. Department of Energy 1991; Martin Marietta Energy Systems, Inc. 1989b). During site closure (in spring of 1992), approximately 7,000 cubic yards of low-level radiationcontaminated soil and concrete from the Elza Gate Site were placed in the United Nuclear Corporation Site as substitute fill material. The TDEC granted formal approval for the disposal of these materials on January 2, 1992.

Characterization monitoring at the site startes in 1985 and continued until 1988, when monitoring in support of an RFI was initiated. In CY 1990, monitoring wells at the site were incorporated into the ongoing quarterly assessment monitoring program for the Security Pits. Semiannual monitoring at the site in accordance with the ROD has continued since closure of the site under the management of the Y-12 Plant Environmental Restoration Surveillance and Maintenance Program (Martin Marietta Energy Systems, Inc. 1992). 


\subsubsection{Rogers Quarry}

Rogers Quarry is located in the southwest portion of the Chestnut Ridge Regime about 3 miles west of Kert Hollow Quary (Figure 3). The quarry was used from the 1940s through the late-1950s as a source of stone-construction material, and was abandoned in the early 1960s when it filled with water. Until July 1993, the site received fly-ash shturry from the Y-12 Stearn Plant that was discharged into McCoy Branch (King et al. 1989); disposal of fly ash in Rogers Quarry ceased per agreement with the TDEC.

Wells were installed at the site in 1985 and sampled as part of the Y-12 Plant characterization monitoring program. Although jdentified as a RCRA SWMU in 1987 (Weich et al. 1987), an RFI was not initiated at Rogers Quany. Groundwater monitoring since 1987 has continued as a best management practice of the Y-12 Plant GWPP. Monitoring results have not indicated that groundwater contamination is present at the site.

The FFA designated Rogers Quanty as Chestnut Ridge OU4 (Table 1). An RI work plan for this OU was submitted for review by the EPA and TDEC in CY 1993 (U.S. Department of Energy 1993d).

\subsection{RCRA Treatment, Storage, or Disposal Facilities}

There are three RCRA-regulated hazardous waste TSD facilities in the Chestnut Ridge Regime that are not designated as CERCLA OUs (Table 1): the Sediment Disposal Basin, Kerr Hollow Quarry, and the East Chestnut Ridge Waste Pile. The Seciment Disposal Basin and Kert Hollow Quarry are closed facilities granted RCRA interim status in 1986. The East Chestnut Ridge Waste Pile is a lined waste pile facility built and operated in accordance with a RCRA operating permit issued by the TDEC in 1987.

\subsubsection{Chestnut Ridge Sediment Disposal Basin}

The Sediment Disposal Basin is southeast of the east end of the Y-12 Plant (Figure 3). Beginning in 1973, the site received soil and sediment that was periodically dredged from New Hope Pond, a closed surface impoundnent located at the east end of the Y-12 Plant. These soils and sediments contained polychlorinated biphenyls, mercury, and uranium. Between November 1987 and April 1988, the Sediment Disposal Basin also received mercury-contaminated soils from several locations in the Y-12 Plant. Results of Extraction Procedure (EP) Toxicity analyses showed that the soils did not exhibit the characteristics of a hazardous waste (Saunders 1983; 95-069M55020796 
Kimbrough and McMahon 1988a and 1988b). In 1989, the Sediment Disposal Basin was closed in accordance with a TDEC-approved RCRA closure plan. A revised RCRA PCPA was prepaned for the site and submitted to the TDEC in September 1994 (Martin Marietta Energy Systens, Inc. 1994b). A final RCRA post-closure permit was issued by the TDEC on September 18, 1995.

Defection monitoring in accordance with RCRA interim status requirements was conducted at the Sediment Disposal Basin between 1987 and October 1995. No statistical evidence of groundwater contamination has been determined at the site. Upon issuance of the RCRA post-closure permit by TDEC, semiannual post-closure detection monitoring was initiated in accordance with permit provisions. Under post-closure, analyses and statistical evaluations of specific indicator parameters, characteristic of wastes emplaced at the site, will be conducted.

\subsubsection{Kerr Hollow Quarry}

The Kerr Hollow Quarry is located near the south-central portion of the Chestnut Ridge Regime (Figure 3) and was a source of stone construction material in the 1940 s until it filled with water and was abandoned. From the early-1950s until Novenber 1988, Kert Hollow Quary was nsed for the disposal of reactive materials from the Y-12 Plant and the Oak Ridge National Laboratory (ORNL).

Between CYs 1990 and 1993, waste materials were removed from the quarry in an effort to obtain certified clean-closure status from the TDEC. These efforts were discontinued in CY 1993 and the site was closed with some of the wastes in place. Because clean closure of the site was not achieved, a RCRA PCPA for the site was prepared and submitted to the TDEC in Jone 1995. When issued by the TDEC, the post-closure penmit for the site is expected to require groundwater monitoring under a RCRA detection monitoring or modified corrective action program.

Detection monitoring in accordance with RCRA interim status requirements has been in progress at the Kerr Hollow Quarry since CY 1988. Required statistical evaluations of the monitoring data have not indicated that groundwater contamination is present at the site. However, estimated low concentrations of tetrachloroethene, carton tetrachloride, and chloroform have been sporadically detected in samples collected from wells GW-142 and GW-144. All volatile organic compound (VOC) levels have remained below EPA primary drinking water standards and laboratory reporting limits. These VOCs were first detected in samples from the wells in CY 1990. The frequency of occurrence of VOCs increased during CY 1992, then decreased through CY 1993. No VOCs have been detected in well GW-142 since the third guarter of CY 1993. During CY 1994, the only VOC reported for samples from well GW-144 was carbon tetrachioride, detected only in the second quarter sample. In CY 1995, first quarter samples from GW-144 contained low levels of carbon tetrachloride and tetrachloroethene and second quarter samples 
contained carbon tetrachloride only. This trend in the VOC detection frequency over time suggests a potential association with corrective action (debris removal and container shredding) at the site that was performed from August 1990 until October 1993. Continued monitoring and trending of these VOC concentrations was proposed in the RCRA PCPA.

Total elemental uranium and/or strontium concentrations reported for unfiltered samples collected from wells GW-142, GW-145, and GW-146 at the site have consistently exceeded concentrations typically reported for other wells in the regime. The lack of associated elevated alpha or beta activity may indicate that the elevated uraniom and strontium concentrations reflect natural geochemical variation. Alternately, the elevated concentrations of these metals may reflect potential impacts of waste disposal at the site. Isotopic uranium and strontium analysis conducted in CY 1995 indicates that the elemental uraniurn and strontium are not radiogenic. Continued isotopic analyses are planned for further evaluation of treads.

\subsubsection{East Chestnut Ridge Waste Pile}

The East Chestnut Ridge Waste Pile is a lined, hazardous waste management facility constructed in CY 1987 as a storage site for contaminated soils from the Y-12 Plant. The site is located in the eastem portion of the Chestmut Ridge Regime near the Sediment Disposal Basin (Figure 3).

As a lined facility, the East Chestnut Ridge Waste Pile is exempt from groundwater monitoring requirements under RCRA. Nevertheless; groundwater quality monitoring has been performed at the site since CY 1987 as a best management practice of the Y-12 Plant GWPP. Monitoring results have not indicated that groundwater contamination is present at the site.

\subsection{Solid Waste Dispasal Facilities}

There ane five permitted nonhazardous waste landfills in the Chestnut Ridge Regime (Table 1): Industrial Landfills II, IV, and V, and Construction/Demolition Landfills VI and VII. These five permitted landfills are classified as either Class II or Class IV facilities, as defined in the most recent (December 1993) Rules of the Tennessee Department of Environment and Conservation, Division of Solid Waste Management, Chapter 1200-1-7, Solid Waste Processing and Dispasal.

Groundwater monitoring at each of the SWDFs in the Chestuut Ridge Regime is performed in accordance with the requirements for detection monitoring contained in paragraph 1200-1-7 .02(7) of the TDEC solid waste regulations and each facility operating pernit. Detection monitoring at some of the permitted SWDFs includes site-specific requirements (i.e., additional 95-069MSN2070796 
analytical parameters) speciffed in the operating permit for the site, as requested by the TDEC, or are performed as a best management practice of the Y-12 Plant GWPP.

Detection monitoring was initiated at Indestrial Landfill II in CY 1982 and at Industrial Landfill IV in CY 1987. Construction/Demolition Landfills VI and VII and Industrial Landfill V are newly permitted facilities. As required under the TDEC solid waste regulations and permit requirements, quarterly sampling to establish background water quality conditions for detection monitoring purposes was initiated in May 1993 at Construction/Demolition Landfills VI and VI, and Industrial Landfill V. Background monitoring protocols were modified in early 1994 to comply with amendments to TDEC Rule 1200-1-7-.04(7), effective January 1, 1994. All backgmound monitoring was completed and a semiannual detection monitoring program instituted at the end of the first quarter of CY 1995.

\subsubsection{Industrial Landfill II}

Industrial Landfill II, also known as the Y-12 Plant Centralized Sanitary Landfill II, is a Class II SWDF located near the western end of the Chestnut Ridge Regime (Figure 3). The landfill is used for disposal of combustible and decomposable solid waste and construction spoil material from the Y-12 Plant, the Oak Ridge K-25 Site, ORNL, and DOE prime contractors in Oak Ridge. These wastes include scrap metal, glass, paper products, plastics, wood, organic garbage, textile products, asphalt noofing materiałs, and special wastes such as asbestos and beryllium oxide. The landfill is near capacity and partial closure was initiated in CY 1994.

\subsubsection{Industrial Landfil IV}

Industrial Landfill IV is a Class II SWDF located near the west end of the Chestnut Ridge Regime (Figure 3). This site has been operated since CY 1989 for disposal of nonhazardous, nonradioactive industrial wastes. Wastes disposed at the site include cardboard, plastics, nubber, scrap metal, wood, paper, and special wastes. Based on current waste volumes, the site is expected to receive approximately 12,000 cubic feet of waste per year.

\subsubsection{Construction/Demolition Landfill VI}

Construction/Demolition Landfill VI (formerly the Industrial Landfill II Expansion) is a new Class IV SWDF constnucted in CY 1993. Lacated adjacent to Industrial Landfill II (Figure 3), the TDEC issued an operating permit for the site on October 26, 1993. Waste disposal at the site began in April 1994. 


\subsubsection{Industrial Landfil $V$ and Construction/Demolition Landfill VII}

Industrial Landfiti V and Construction/Demolition Landfill VII ane new Class II and Class IV SWDFs, respectively, located adjacent to each other in the south-central part of the Chestnut Ridge Regime (Figure 3). Both sites are also collectively known as the Steam Ptant Ash Disposal Landfill. Permits for esch site were issued by the TDEC on April 26, 1993. Construction of Industrial Landfill V was completed in April 1994 and the site is currently in service. Construction of Construction/Demolition Landfill VII was completed December 1994 and the TDEC granted approval to operate the facility in January 1995. Operation of the facility will not commence immediately in consideration of available space in Construction/Deroolition Landfill VI.

\subsection{Chestnut Ridge Borrow Area Waste Pile}

The Chestnat Ridge Borrow Area Waste Pile, also known as the Civic Center Spoil Pile, is located near the eastem end of the Chestnut Ridge Regime (Figure 3). The site was built as a temporary storage area for contaminated soils nemoved from the Oak Ridge Civic Center properties and the Oak Ridge Sewer Line Beltway. Soils in both areas contained merenry and other metals (and passibly some organic compounds) believed to have originated from the Y-12 Plant Results of EP Toxicity analyses indicated that the soils do not exhibit the toxicity characteristic of a hazardous waste. The site is curently listed as a Study Area to be investigated under CERCLA. A. soil sampling plan designed to determine if the soils are toxic hazardous wastes based on results of the Toxicity Characteristic Leaching Procedure was submitted to the TDEC for review in September 1992 (Science Applications Intemational Corporation 1992).

The Chestnut Ridge Bortow Area Waste Pile is approximately $70 \mathrm{ft}$ wide, 10 to $12 \mathrm{ft}$ deep, and $200 \mathrm{ft}$ long. The botton and walls of the pit are lined with polyvinyl chloride and the bottorn is sloped to facilitate drainage. Perimeter channels divert surface runoff and a plastic liner on top of the pile restricts infiltration. Approximately 3,000 cubic yards of soil are now stored at the facility. Groundwater monitoring at the site is conducted as best management practice of the Y-12 Plant GWPP. 


\subsection{CY 1995 GROUNDWATER PROTECTION PROGRAM}

The following sections describe the groundwater sampling activities performed during CY 1995 in the Chestnut Ridge Regine under the lead of the Y-12 Plant GWPP. Included are details regarding the groundwater sampling locations, frequency, and procedures, analytical parameters, quality assurance/quality control (QA/QC) sampling, and data management.

\subsection{Sampling Locations}

Table 2 provides a detailed listing of CY 1995 monitoring activities by programmatic driver. The table depicts monitoring locations, sampling frequency, and parameters as originally specified in the CY 1995 Sampling and Analysis Plan (SAP) (HSW Environmental Consultants, Inc. 1994). Changes to the SAP during the course of CY 1995 are documented as addenda. Addenda to the SAP are necessary for various reasons, such as changing regulatory requirements at sites or the need to add sites/modify parameters for special projects. Sorne addenda are issued to document administrative decisions or provide clatification on techuical issues. Table 2 provides a summary listing of addenda that modified the CY 1995 monitoring progfam and the changes specified by each one.

Grondwater monitoring during CY 1995 involved the collection of groundwater samples frow 73 monitoring wells and two springs (Table 2). Locations of these sampling points are shown on Figure 4. Data regarding the elevation of the screened or open hole interval for the monitoring wells are summarized in Table 3; detailed well construction information is contained in Appendix C.

Samples were collected to comply with six monitoring programs: (1) RCRA interim status assessment monitoring, (2) RCRA interim status detection monitoring, (3) SWDF detection monitoring, (4) post-closure monitoring in accordance with a CERCLA ROD, (5) RCRA postclosure detection monitoring, and (6) best management practice monitoring. Descriptions of the monitoring well networks used for each program are provided in the following sections.

\subsubsection{RCRA Intertm Status Assessment Momitoring}

Assessment monitoring during CY 1995 involved 10 monitoring wells at the Security Pits (Table 2). Assessment monitoring at the Security Pits has been in progress since CY 1988 and will be discontinned when a RCRA post-closure permit is issued for the site by the TDEC. 


\subsubsection{RCRA Interim Status Detection Monitoring}

Detection monitoring in accordance with the RCRA interim status regulations was performed during CY 1995 at the Sediment Disposal Basin and Kerr Hollow Quarry, and included a total of 15 monitoring wells (Table 2). Four of the eight interim status detection monitoring wells at the Chesmut Ridge Sediruent Disposal Basin were dropped during the fourth quarter of CY 1995 upon issuance of the RCRA post-closure permit for the site. As required for interim status detection monitoring, results of selected indicator parameters (e.g., pH and specific conductance) were analyzed to identify any statistically significant changes in groundwater quality. Results of these statistical analyses were submitted to the TDEC in July 1995 (Lockheed Martin Energy Systems, Inc. 1995a and 1995b) and February 1996 (Lockheed Martin Energy Systems, Inc. 1996a and 1996b). The anmal RCRA detection monitoring report for these facilities also was submitted in February 1996 (Lockheed Martin Energy Systems, Inc. 1996c).

\subsubsection{SWDF Detection/Background Monitoring}

As noted in Section 2.4, groundwater monitoring in accordance with the TDEC solid waste management regolations is performed at all of the SWDFs in the regime. In CY 1995, 24 monitoring wells and one spring at the SWDFs were sampled for detection or background monitoring purposes (Table 2).

\subsubsection{CERCLA Record of Decision Monitoring}

Six monitoring wells were sampled on a semiannual basis in accordance with an approved CERCLA ROD for the United Nuclear Corporation Site. These data are obtained as part of the specified post-closure surveillance and mainsenance activities and reported to the Y-12 Plant Environmental Restoration Surveillance and Mainterance Program.

\subsubsection{RCRA Past-Closure Detection Monitoring}

Detection monitoring was conducted during the fourth quarter of CY 1995 at the Sediment Disposal Basin in accordance with the final RCRA post-closure permit issued Seplember 18, 1995. Four of the previous interim status detection monitoring wells comprise the formal post-closure detection monitoring network (Table 2). Statistical evaluations of indicator parameters were conducted to identify any statistically sigaificant changes in groundwater quality indicative of a release of contamination to groundwater. Results of these evaluations along with other 95-069155020796 
permit-reguired data were submitted to the TDEC in February 1996 (Lockheed Martin Energy Systems, Inc. 1996b and 1996c).

\subsubsection{Best Maniagement Practice Monitoring}

Groundwater samples were collected from 18 monitoring wells and one spring (SCR2.2SP) during CY 1995 as a best management practice of the Y 12 Plant GWPP (Table 2). Best management practice monitoring was performed at five sites where regulation-driven monitoring is not specified. For example, groundwater quality monitoring at the East Chestmut Ridge Waste Pile has been performed since 1987 even though this hazardous waste TSD unit is a lined facility exempt from RCRA groundwater monitoring requirements.

\subsection{Sampling Frequency}

Groundwater samples were collected during each quarter of CY 1994. First through fourth quarter sampling events were performed January 4 - March 20, April 4 - May 16, Joly 10 August 14, and $O$ ctober 6 - November 17, respectively.

As sumnarized in the following table, 25 of the wells inclnded in the previously described monitoring programs were sampled at a guarterly frequency, and 48 wells and sping CBS-1 were sampled semiannually Station SCR2.2SP was sampled once during the second quarter.

\begin{tabular}{|c|c|c|}
\hline \multirow[t]{2}{*}{ Site } & \multicolumn{2}{|c|}{$\begin{array}{l}\text { Sampling Frequency } \\
\text { (No. of Wells) }\end{array}$} \\
\hline & Quarteriy & Seniannugly \\
\hline $\begin{array}{l}\text { Chestnut Ridge Secunity Pits } \\
\text { Chestnot Ridge Sediment Disposal Basin }\end{array}$ & $\begin{array}{c}10 \\
8\end{array}$ & + \\
\hline Kerr Hollow Quarry & 7 & \\
\hline Industrial Landfill II & $=$ & 3 \\
\hline Industrial Landfill IV & . & 5 \\
\hline Industrial Landfill V & 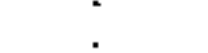 & 5 \\
\hline Consuruction/Demolition Landfill vi & r & 7 \\
\hline Constraction/Demolition Landfill VII & . & 4 \\
\hline Ash Disposal Basin & . & 4 \\
\hline Chestrut Ridge Bortow Area Waste Pile & . & 6 \\
\hline East Chestnat Ridge Waste Pile & . & 4 \\
\hline Rogers Quarry & & 4 \\
\hline United Nuclear Corporation Site & & 6 \\
\hline tal & 25 & 48 \\
\hline
\end{tabular}

Monitoring wells sampled on a semianual basis are located at sites for which a quarterly monitoring frequency is not a regulatory, DOE, or best management practice requirement. 
Beginning in Aptil 1994, in response to changing requirements, semiannual sampling frequencies for selected wells were phased into the ongoing quarterly monitoring programs. This process continued in CY 1995. Consequently, some wells scheduled for semiannual sampling actially were sampled three times during CY 1995. Semiannual sampling of spring CBS-1 was performed to obtain samples during high and low flow conditions.

\subsection{Sample Collection}

Groundwater samples were collected by personnel from the Oal Ridge K-25 Site Sampling and Environmental Support Department (SESD). Samples from the spring were collected by personnel from the HSEA Organization. Pre-defined sampling sequences were followed to minimize the potential for cross-contamination (Appendix D). Sampling sequences were constructed to sample from the least contaminated wells to the most contaminated wells at a site or in a sampling group (a series of monitoring wells grouped for sampling and data-tracking purposes). At sites where no groundwater contamination is present, samples were collected from the farthest upgradient wells first

Groundwater sampling was perfomed in accordance with a technical procedure for groundwater sampling (SESD-TP-8204) approved by the Y-12 Plant GWPP Manager. Descriptions of the field methods and procedures used to collect the groundwater samples are contained in Environmental Surveillance Quality Control Program, Martin Marietta Energy Systems, Inc. (Martin Marietta Energy Systems, Inc. 1988b). The EPA (Region IV) and the TDEC have approved these methods and procedures (U.S. Environmental Protection Agency and Tennessee Department of Environment and Conservation 1988).

\subsection{Quality Assurance/Quality Control Sampling}

The QA/QC samples included laboratory blanks, tip blanks, exuipment tinsates, field blanks, and duplicate groundwater samples. A total of 165 laboratory blanks, 121 trip blanks, 36 equipment rinsate samples, 4 field blanks, and 34 duplicate samples were analyzed doring CY 1995. The total number of each $Q A / Q C$ sample type analyzed during each quarter fluctuated according to sampling schedules; the ranges are as follows: 22 to 60 laboratory blanks, 20 to 41 trip blanks, 6 to 14 equipment rinsates, 1 field blank, and 5 to 14 duplicate samples.

Laboratory blanks were prepared using deionized water and analyzed along with the groundwater samples. Trip blanks were prepared in the laboratory using deionized water and transported anopened in coolers containing groundwater samples to be analyzed for VOCs. Equipment rinsates were obtained frorn the deionized water used to decontarninate the groundwater 95-109945020796 
sampling equipment. Equipment rinsate samples were collected after a sampling team had completed sampling at a site or finished a sampling group (typically no more than 10 wells grouped for sampling and data tracking purposes). If more than one sampling team collected samples at a site or within a sampling group, each team collected an equipment rinsate sample. In addition, if a sampling team used more than one pump at a site or sampling group, an equipment rinsate was collected for each pump. Field blanks were prepared in the field using deionized water and were collected at a frequency of about 10 percent of the sampling groups. Duplicate groundwater samples were collected at a frequency of at least one per sampling group.

\subsection{Laboratory Analysis}

Groundwater samples collected from each monitoring well and spring were analyzed for the parameters and constituents specified on Table 2. As shown, samples from all of the wells were analyzed for a standard suite of analytes that included:

- principal cations and anions;

- trace metals (a term used to differentiate metals that are typically minor groundwater constituents, such as cobalt and nickel, from metals that are principal ions in groundwater, sach as magnesium and sodium);

- VOCs;

- gross alpha activity and grass beta activity;

- total suspended solids, total dissolved solids (TDS), and turbidity;

- field and laboratory determinations of pH and specific conductance; and

- field determinations of temperature, dissolved oxygen, and oxidation-reduction potential.

In addition to the standard suite of analyses, groundwater samples collected frotn wells at some sites in the Chestnut Ridge Regime were analyzed for other compounds or parameters as required by specific regulations, operating pernits, post-closure requirements, or best management practices (Table 2). For example, samples collected from wells at Construction/Demolition Landfill VI were analyzed for additional organic compounds and other required parameters specified by the TDEC solid waste management regulations and operating permit Samples from other wells, such as those at Kerr Hollow Quarry, were analyzed for isotopic uraniun and strontium- $89 / 90$ because of elevated elemental uranium and strontium values observed in some wells. 
All of the laboratory blanks, trip blanks, field blanks, and equipment rinsate samples were analyzed for the suite of VOCs listed on Table 2. Selected equipment rinsate samples also were analyzed for trace metals (Indrectively Coupled Plasma analyses), organic compounds, gross alpha and gross beta activity, and radionuclides. Duplicate groundwater samples were analyzed for the constibuents and parameters specified for the well from which the duplicate sample was collected.

Analyses of the groundwater and QA/QC samples were performed by two different laboratories: the K-25 Analytical Services Organization (ASO) and the ORNL ASO. The bulk of the analyses was performed by the K-25 ASO. Only selected radiochemical analyses were performed by the ORNL ASO.

\subsection{Dota Management}

Analytical results obtained by the analytical laboratories were verified and entered into the Y-12 Plant GWPP Groundwater Monitoring Data Base. All monitoring data are subjected to a three-step verification, validation, and evaluation process. Brief descriptions of the data verification/validation/evaluation procedures are as follows; details are provided in the Y-12 Plant Groundwater Protection Program Groundwater Monitoring Program Data Management Plan (Martin Marietta Energy Systems, Inc. 1993).

The majority of analytical results obtained from each quarterly sampling event was downloaded directly into the data base from data files provided by the analytical laboratory. A few data required mannal input from hardcopy docmentation (taboratory reports and freld data sheets). For manually input data, a dopble-entry procedure was followed whereby the hardcopy data were input twice into separate data files. A computer program then compared the two input data files and identified discrepancies, which were then accounted for and corrected.

When all data for each sampling event were in the data base, a computer program then verified that all results were received, the parameter names were correct, correct units were reported, and that the results reported for each parameter were within an expected range or were valid numeric values. As a final step in the data verification process, printouts of the data were visually checked against the laboratory reports and field data sheets for completeness and obvious data outliers. The appropriate laboratory was contacted to resolve incomplete data transfers, discrepancies between electronic and hardcopy versions of the data, and questions regarding data outliers.

Data validation consists of calculating ion-charge balances for each sample, comparisons of sample data to corresponding laboratory and field QAVC data, and validation of VOCs using EPA validation methods. The tabulation of the results of these data processing steps has historically been presented as informational appendices in the Part 1 GWQRs, but not discussed in detail 95-16920150207\%5 
because of time constraints imposed by the March 1 reporting deadline. These results will be evaluated fully in the Part 2 GWQRs issued in mid-year.

Data evaluation consists of trending of environmental data and laboratory perforraance. redefining plume configurations where required, and detailed review of particular contaminants or sites of concerm. The results of data evaluation have historically been presented in the Part 2 GWQRs; the results of evaluation of CY 1995 data will again be presented in these reports.

\subsection{Analyticaj Results}

Groundwater quaity data obtained during CY 1995 are contained in Appendices E, F, and G. Appendix E contains the analytical results for each groundwater sample and Appendix $F$ contains the results for the field duplicate samples. Radiochemical data are presented in Appendix $\mathrm{G}$. The data presented in these appendices were generated from the following quarterly data sets: first quarter - Q1_95.SD2, second quarter - Q2_95R1.SD2, third quarter Q3_95R1_SD2, and fourth quarter - Q4_95.SD2 (H\&R Tecbnical Associates, Inc. 1995a, 1995b, 1996a, and 1996b). Water level elevations obtained during comprehensive semiamnual measurement events are presented in Appendix $\mathrm{H}$, which form the data base for potentiometric surface maps discussed in Section 4.0.

Informational appendices that highlight selected data or present results of datd verification and evaluation processes include ion-charge balances (Appendix I), comparison of trace metals to EPA primary driaking water standards (Appendix $\mathrm{J}$ ), calculation of antual average nitrate concentrations (Appendix K), tabulation of QAVC data (Appendix L), and results of VOC validation (Appendix M). 


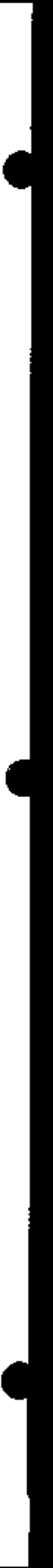




\subsection{HYDROGEOLOGIC FRAMEWORK}

This section contains a general description of the complex hydrogeologic system in the Chestrut Ridge Regime. It provides sufficient descriptive detail needed for RCRA assessment monitoring reporting requirements regarding evaluation of groundwater flow directions. In past GWQRs, the bydrogeologic overview was based primarily on the conceptual framework and associated nomenclature presented in: Status Report - A Hydrologic Framework for the Oak Ridge Reservation (Solomon ef al. 1992). However, the overview for this report has been revised because flow in the Maynardville Limestone, a key component of the groundwater flow system in BCV, is not specifically addressed in Solomon th al. (1992), and some components of the ORR hydrogeologic framework (e.g., the stormflow zone) appear to be of lesser hydrologic significance at the Y-12 Plant than indicated for other areas on the ORR. In general, the revised description of the hydrogeologic system incorporates: (1) applicable aspects of the conceptral framework described in Solomon et al (1992), (2) hydrologic characteristics evaluated by Moore (1988 and 1989), and (3) findings of the RI for the BCV CA (Science Applications International Corporation 1995).

\subsection{Geelogy}

The geology on the ORR is generally characterized by thrust-faulted sequences of southeast-dipping, clastic (primarily shale and siltstone) and carbonate (limestone and dolostone) strata of Lower Cambrian to Lower Ordovician age. In the Y-12 Plant area, the primarily folostone strata of the Knox Group form Chestnut Ridge, interbedded limestone and shale formations of the Conasauga Group underlie BCV to the north, and shale and siltstone beds of the Rome Formation form Pine Ridge (Figure 5). Strike and dip of bedding in the Chestnut Ridge Regime is generally N $55^{\circ} \mathrm{E}$ and $45^{\circ} \mathrm{SE}$, respectively (not referenced to Y-12 Plant grid system).

With the exception of Kerr Hollow Quarry and Rogers Quarty, the sites in the Chestnut Ridge Regime are directly anderlain by reddish-brown to yellow-orange residuum overlying the Knox Group. The residuum is characteristically slightly acidic, predominantly composed of clays and iron sesquioxides (Jennings 1985), and contains semi-continuous, relict beds of fractured chert and other lithologic inhomogeneities (such as silt bodies) that provide a weakly connected network through which saturated flow can occur (Solomon et al. 1992). The residaum is thin or non-existent near karst features such as dolines (sink holes), swallets (sinking streans), and solution pan features (Ketelle and Huff 1984). Depth to bedrock varies throughout the Chestnut Ridge Regime, but is usually less than $100 \mathrm{ft}$. 
The Knox Group consists of 2,500 to 3,000 ft of gray to blue-gray, thin- to thick-bedded cherty dolostone with interbeds of limestone (Figure 5). All but the southernmost portion of the Chestnut Ridge Regime is underlain by the Knox Group. The Chickamauga Group overlies the Knox Group disconformably and generally consists of thin- to medium-hedded argillaceous limestone and interbedded shale.

The most pervasive structural features in the Chestnut Ridge Regime are extensional, hybrid, and shear fractures (Solomon et al. 1992). Three major joint orientations are evident: one that roughly parallels bedding, one steeply dipping set that parallels geologic strike, and one steeply dipping set oriented perpendicular to strike (Dreier es al. 1987). Fracture densities ranging from about 1 to 60 per foot have been observed in rock outcrops near the ORNL (Dreier at al 1987; Sledz and Huff 1981). Most fractores are short, ranging from tenths of inches to a few feet in kength (Solomon at al. 1992).

Dissolution of carbonates along fracturts has produced many karst features in the Chestnut Ridge Regime. Smith et al (1983) identified a series of sinkholes along the crest of the ridge that show a promiment alignment parallel to strike. This linear trend may resul from dissolution along a bedding plane or joint set (Ketelle and Fuff 1984; Smith a al al. 1983).

\subsection{Groundwater System}

Solomon ef al. (1992) divide the groundwater system underlying the ORR into two basic hydrogeologic units with fundamentally different hydrologic characteristics: the Knox Aquifer and the ORR Aquitards. In the vicinity of the Y-12 Plant, the Knox Aquifer consists of the Knox Group and the underiying Maynardville Limestone Formation of the Conasauga Group. The remaining formations of the Conasauga Group (collectively referred to in this report as the Conasauga Shales), the underlying Rome Formation, and the Chickamauga Group form the ORR Aquitards.

In general, both the Knox Aquifer and the ORR Aquitards are divided by Solomon at al. (1992) into four parts: (1) the stormflow zone, (2) the vadose zone, (3) the groundwater zone and (4) the aquicinde (Figure 6). The divisions are based on the amount of water transmitted by each subsystem (i.e., flux), which decreases with depth. The flow system is vertically gradational with no discrete boundaries separating the subsystems. However, the bulk permeability of the Knox Aquifer is about ten times greater than that of the ORR Aquitards (Solomon al al. 1992).

Recharge on Chestant Ridge is via vertical percolation through the regolith and probably includes more direct input via sinkholes. Annual recharge has been estimated to be about 2.6 inches, and it is possible that a portion of the recharge results from delayed unsaturated flow (Solomon et al. 1992). Groundwater discharge pathways from the ridge have not been completely 
established, but based on bnowledge of flow in sinilar karst systems and the results of various hydrologic studies in the Chestnut Ridge Regine, springs and seeps located along or across strike are the probable discharge points.

\subsubsection{Stormnow Zone}

Investigations in Bethel Valley and Melton Valley near ORNL show that groundwater occurs intermittently above the water table in the ORR Aquitards in a shallow "stomblow zone" that extends from ground surface to a depth of about $6 \mathrm{ft}$ (Moore 1989). Channels for lateral flow in the stormflow zone include macropores and mesopores, which are connected voids created by various processes, including biochanneling, cracking, and soil particle aggregation (Moore 1989). The stormflow zone is thicker and more permeable in forested areas than in grassy or brushy areas, and is more permeable near the ground surface than at deeper intervals (Moore 1989). Lateral flow in the stomflow zone is intermittent, creating a perched water table lasting only a few days or weeks after rainfall (Wilson et al. 1990). Most groundwater within the stormflow zone is either lost to evapotranspiration or recharge to the water table and the remaining water discharges at nearby seeps, springs, or streams. Detziled studies of the stormflow zone have not been conducted in the Chestnut Ridge Regime. However, as part of the RU/FS in progress for the BCV CA, stormflow tubes installed at six sites (three along the northem flank of Chestnut Ridge) have all showed brief periods of soil saturation in response to rainfall (Science Applications International Corporation 1995). These findings suggest that stormflow also occurs in BCV, although capping of the waste management areas has probably altered the stormflow zone in some areas of the Bear Creek Regime. Additionally, the significance of groundwater flux and contaminant transport in the stormflow zone in BCV is not fully understood, and may not be as great as in Bethel Valley and Melton Valley.

\subsubsection{Vadose Zone}

The vadose zone occurs between the stomflow zone and the water table. The geometric mean depth to the water table beneath Chestmut Ridge is about $100 \mathrm{ft}$. Water is added to the vadose zone by percolation from the stormflow zone and is removed by transpiration and recharge to the water table. The vadose zone is unsaturated except in the capillary fringe above the water table and within wetting fronts during periods of vertical percolation from the stormflow zone (Moore 1989). Most recharge throngh the vadose zone is episodic and occurs along discrete permeable fractures that become saturated, even though surrounding micropores remain unsaturated (Solomon if al. 1992). 
The hydranlic conductivity of the unsaturated residual soils on Chestnut Ridge has beea determined from slug tests in wells located at the United Nuclear Corporation Site (Mishu 1982), and in areas several miles west of the Chestmut Ridge Regime (Woodward-Clyde Consultants, Inc. 1984). Little variation was observed with depth, but conductivities determined by field and laboratory tests varied by approximately two orders-of-magniude for comparable depth intervals. Mean field conductivities ranged from 0.0057 to 0.49 feet per day (ft/d) and mean laboratory conductivities ranged from $2.8 \times 10^{-5}$ to $9.1 \times 10^{-3} \mathrm{ft} / \mathrm{d}$. Resuls of the slug tests are similar to those obtained from infiltrometer tests. Moore (1988) reported a geometric mean hydraulic conductivity of about $0.006 \mathrm{ft} / \mathrm{d}$ for residuun on Chestnut Ridge based on results of infilurometer studies near ORNL reported by Watson and Lummoore (1986) and Wilson and Luxmoore (1988).

The hydraulic conductivity of the residuom overlying the Knox Group varies with the degree of saturation (Luxmore 1982; Daniels and Broderick 1983). Luxmoore (1982) demonstrated that hydraulic conductivity decreases by approximately one order-of-magnitude with a volumetric water content decrease to $90 \%$ of saturation, and two orders-of-magnitude with a volumetric water content decrease to 75\% of saturation. Daniels and Broderick (1983), as summarized in Ketelle and Huff (1984), reported that hydraulic conductivity decreases by roughly one order-of-magnitude relative to maximuen when saturation is $90 \%$, and three ordersof-magnitude relative to maximum when satoration is $75 \%$. Ketelle and Huff (1984) also noted that wide variations in soil permeability occur over short lateral distances. These findings are consistent with observations of permeability variation in residual soils found in other karst areas (Quinlan and Aley 1987).

\subsubsection{Groundwater Zone}

Groundwater below the vadose zone occurs within orthogonal sets of permeable, planar fractures that form water-producing zones within an essentially impermeable matrix. Waterproducing zones commonly develop within a single layer of rock, and have an average thickness (assuming an average dip of 35 ) of 2 ft or less (Dreier et al. 1987). Dissoiution of carbonates has greatly increased permeability of the water-producing zones in the Knox Group and Maynardville Limestone; enlarged fractures and solution cavities in the Maynardville Limestone yield as much as 200 to 300 gallons per minute (gpm) (Shevenell es al. 1993), which is several orders-of-magnitude greater than the typical yield ( $0.25 \mathrm{gpm}$ ) of water-producing zones in the ORR Aquitards (Solomon et al 1992). Additionally, stratiform fractures provide the principal flowpaths in both hydrogeologic units, and groundwater flows primarily in the direction of geologic strike (which may or may not cortelate with the maximum hydraulic grafient) toward springs, seeps, and crosscutting tributary streams (Moore and Toran 1992).

95-069451020796 
Because the frequency, aperture, and connectivity of permeable fractures decrease with depth, the bulk hydraulic conductivity of the groundwater zone is vertically gradational. Most of the groundwater flux occers in a highly permeable zone (the water table interval) within the transitional horizon between regolith and unweathered bedrock; lower flux (and longer solute residence times) oxcurs at successively greater depths in the bedrock. Changes in the geochemistry of the groundwater suggest that active flow in the Conasauga Shales occurs at depths less than $100 \mathrm{ft}$ below ground surface (bgs). Active groundwater flow occurs deeper in the Maynardville Limestone, as indicated by pressure responses to rainfall that are evident in some wells completed at depths of 200 to $400 \mathrm{ft}$ bgs (Science Applications Intemational Corporation 1995). In addition, changes in groundwater geochemistry that occur with depth in the Conasauga Shales are not evident in the Maynardville Limestone; calcium-magnesium bicarbonate groundwater is present in the Maynardville Limestone to depths of 1,000 ft bgs (Dreier et al. 1993). Moreover, decreased permeability with depth in the Maynardville Limestone has not been characterized because hydraulic conductivity tests have been performed primarily in shallow ( $<100 \mathrm{ft}$ bgs) monitoring wells. As noted previously, however, active groundwater flow may occur to deptis of at least $400 \mathrm{ft}$ bgs in the Maynardville Limestone.

Similarly, active groundwater flow occurs deeper within the Knox Group than in the Conasauga Shales. Estimates of the hydraulic conductivity of the intermediate interval in the Knox Group are provided by results of straddke packer tests perforned in three core holes in the Chestnin. Ridge Regime: two at the east end of the regime near the Sediment Disposal Basin and one at the west end of the regime near Industrial Landfill IV. The tested intervals were in the Copper Ridge Dolomite of the Knox Group and were generally less than $600 \mathrm{ft}$ below the ground surface. Calculated hydraulic conductivities ranged from 0.0002 to $3.1 \mathrm{f} / \mathrm{d}$ (King and Hase 1988) and averaged about $0.6 \mathrm{ft} / \mathrm{d}$. The upper population represents more permeable water-producing intervals and the lower population represents kess pernteable matrix intervals.

The hydraulic conductivity of some water-producing intervals in the Knox Gtoup is much greater than indicated by the straddle packer tests owing to an interconnected network of solution cavities and solutionally-enlarged fractures. For instance, groundwater flow rates determined from a tracer test performed by Ketelle and Huff (1984) ranged from about 490 to $1,250 \mathrm{ft} / \mathrm{d}$. Preliminary results of a dye-tracer test performed by Geraghty \& Miller, Inc. (1990b) at the Security Pits indicated flow rates of about 100 to $300 \mathrm{ft} / \mathrm{d}$, although findings of the test were not confirmed by a second test performed at the site (Science Applications International Corporation 1993). However, results of the Ketelle and Huff (1984) and the Geraghty \& Miller, Inc. (1990b) tracer tests are within the range of conduit-flow rates typical in karst terrains (Qutinlan and Ewers 1985). 


\subsubsection{Aquiclude}

The aquiciude is generally matked by the presence of saline water with TDS concentrations of 40,000 to 300,000 milligrams per liter ( $\mathrm{mg} / \mathrm{L}$ ) (Solomon et al. 1992). Information obtained sourheast of the Chestnut Ridge Regime in Melton Valley indicates that the satine water typically cccurs at depths of about 600 to $700 \mathrm{ft}$ bgs (Solomon ets at 1992). The water in the aquiclude is a sodium-, calcium-, and chloride-rich brine that is chemically similar to brines associated with major sedimentary basins (Solomon ts all 1992).

Saline groundwater has not been encountered at depth in the Chestmut Ridge Regime. However, several wells in BCV completed at depths of about 500 to $1,000 \mathrm{ft}$ bgs surface in the Conasauga Shales monitor groundwater with TDS concentrations of several thousand mg/L to several tens of thousand $\mathrm{mg} / \mathrm{L}$. This highly mineralized groundwater may represent a mixing or diffusion zone between fresh and saline groundwater, suggesting that the aquiclnde may be present at greater depth near the Y-12 Plant than in Melton Valley (Solomon et al. 1992).

\subsection{Groundwater Flow Directions}

Directions of groundwater flow in the Chestnut Ridge Regime were evaluated from static water level measurements obtained in April and October 1995. Water levels were measured in 86 monitoring wells during April 1995 (the seasonally high water table) and in 84 wells in October 1995 (the scasonally low water table). Depth-to-water and water-level elevations for each well are presented in Appendix H. Review of these data shows that the average total water level decline from April to October was $6.4 \mathrm{ft}$. The largest decreases (between 10 and $27 \mathrm{ft}$ ) typically occurred in wells located along the crest of Chestnat Ridge in the western part of the Regine. Additionally, the pattem of water level decrease was the same in all wells such that there were no significant changes in groundwater flow directions (i.e., no significant deviation of horizontal hydraulic gradients).

Groundwater elevations in the Chestnut Ridge Regine are illustrated on Figure 7. Monitoring wells were the primary controls for placement of elevation isopleths, but springs and streams also provided additional control. The water table in the Chestnut Ridge Regine generally reflects surface topography and radial groundwater flow directions; strike-parallel gradients range from 0.01 to 0.05 and strike-normal gradients range from 0.03 to 0.07 . The overall trend of groundwater flow in the Chestrut Ridge Regime is easterly, reflecting a general decrease of land surface elevation from west to east. The crest of Chestnut Ridge represents a groundwater divide and the ridge serves as a recharge area. Thus, vertical gradients are downward and flow components to the north and south, away from the crest of the ridge, exist along the length of the 95-00014.020796 
ridge. Principal discharge areas include springs, seeps, and tributaries along the north and south flanks of the ridge. Some discharge directly to Upper East Fork Poplar Creek likely occurs in the northeast portion of the Regime.

Water level hydrographs prepared from measurements made in selected wells at several of the waste management units in the regine are presented on Figure 8. The hydrographs illustrate that water levels fluctuate seasonally and that the fluctuations are generally concurrent and uniform. The hydrographs further illustrate that water levels on the crest of Chestnut Ridge tend to be more responsive to seasonal variations than those near the base of the ridge or within Bethel Valley.

\subsection{Rate of Gronndwater Flow and Contaminant Migration}

The following estimates of groundwater flow and contaminant migration rates are presented to comply with RCRA interim status assessment monitoring requirements. Estimated groundwater flow rates are provided for migration in the residuum and the underlying Knox Groop bedrock. Several methods were used to estimate the groundwater flow rates in both residuom and bedrock. Contaminant migration rates are based on the assumption that contaminants move unimpeded by various retardation processes. Factors that would reduce cransport rates, such as matrix diffusion, degradation, sorption, and chemical precipitation, and factors that may increase transport rates, such as dispersion, were not explicitly considered. Moreover, migration as free product, which may occur independent of the direction and rate of groundwater flow, and vapor-phase transport, a potentially significant mechanism for the introduction of VOCs to the groundwater, also were not considered.

The wide range of potential contaminant migration rates described in the following sections demonstrates the uncertainty in detemining a representative rate of solate transport for this groundwater flow system. Long-term transport of solutes in dual porosity and/or highly heterngeneous media is a complex physical process that is not fully explained by the methods used for estimating migration rates. It is probable that at various times and under various contitions, migration rates throughout the estimated ranges occur; some contaminants move relatively rapidly through the flow system while other more highly attenuated constiments move much more slowly. Moreover, even highly mobile compounds will diffuse into smaller pores from the large condnits, and then, under a reverse concentration gradient, will slowly diffuse back out. Thus, althongh the actual migration rates may vary over several orders of magninude, the apparently limited extent of groundwater contamination in the regime suggests that processes such as matrix diffusion and adsorption are important controlling factors in reducing the rate of contaminant migration relative to groundwater flow velocities. 


\subsubsection{Residaum}

Two methods were used to estimate the rate of vertical groundwater flow and contaminant migration throvgh the residuun overlying the Knox Aquifer: the water-balance method, and the arrival time method. The water-balance method is based on the relationship $v=q / n$, where $v$ is the flow rate, $q$ is the annual recharge rate, and $n$ is the effective porosity. The following assumptions were used: an estimated annual recharge of 2.6 inches per year for the Knox Aquifer (Solomon et al. 1992); a range of zero to 95\% recharge flux through the stomflow zone (Moore 1988); and an effective porosity of 0.0042 (Moore 1989). Under these assumptions, the estimated groundwater flow rates range from 2.6 feet per year (ft/yr) $(0.007 \mathrm{f} / \mathrm{d})$ assuming $95 \%$ subsurface runoff to $51.6 \mathrm{ftyr}(0.14 \mathrm{fv} / \mathrm{d})$ assuming zero subsurface runoff.

Historical water quality data for well 1080 (now plugged and abandoned) were used to determine the contaminant migration rate with the arrival time method. Installed at the Security Pits in CY 1982, voCs were first detected in samples collected from the well in CY 1986. The top of the screened interval for this well was about $160 \mathrm{ft}$ bgs. Using this depth and assuming a 13-year travel time (i.e., the period from initial waste oisposal at the Secturity Pits in CY 1973 to detection of the VOCs in CY 1986), a migration rate of about $12 \mathrm{fV} / \mathrm{yr}(0.033 \mathrm{fvd})$ is calculated. Thus, actual VOC contaminant migration rates appear to be toward the lowef end of the estimated range of groundwater flow rates indicating some attenuation in the subsurface.

\subsubsection{Bedrock}

Two types of groundwater tlow are typical of karst systems: diffuse flow and condmit flow. Rates for diffuse flow in the Knox Group were determined using the modified Darcy equation given by $\mathbf{v}=\mathbf{K} \mathbf{h} \mathbf{h}$, where $\mathbf{v}$ is the migration rate, $K$ is the hydraulic conductivity, $\mathbf{I}$ is the horizontal hydranlic gradient, and $\mathbf{n}$ is the effective porosity. Using the range of hydrautic conductivity test results $(0.002$ to 3.1 fVd) reported by King and Haase (1988) for wells completed in the Knox Grotp, an effective porosity of 0.01 (Smith ef al, 1976), and the range of strikenormal and strike parallel hydranlic gradients reported in Section 4.3, the Darcy velocity for diffuse flow ranges from about $2 \mathrm{f} / \mathrm{yr}$ to about $22 \mathrm{ft} / \mathrm{d}$ for flow perpendicular to strike, and from about 0.7 $\mathrm{ft} / \mathrm{yr}$ to $16 \mathrm{f} / \mathrm{d}$ for strike-parallel flow. Although the modified Darcy equation can be used to calculate average flow velocities in fractured rock (assuming it is an equivalent porous media), actual velocities in individual fractures may vary over orders-of-magnitude, depending on fracture aperture and wall roughness (Freeze and Cherry 1979). Additionally, the value for effective porosity is representative for fractured limestones, but the actual value may be different by mone

95-069:4SA120796 
than an order-of-rnagnitude, which wonld result in an inversely proportional variation in the Darcy velocity.

Migration rates for conduit flow were inferred from the results of dye-tracer tests perfomed on Chestnut Ridge. As noted in Section 4.2.3, results of the Geraghty \& Miller, Inc. (1990b) and Ketelle and Huff (1984) dye tracer tests provide estimates of conduit flow rates in the Knox Group. Although not confirmed by a subsegnent dye test (Science Applications International Corporation 1993), results of the Geraghty \& Miller, Inc. (1990b) tracer test indicated transport velocities of 100 to $300 \mathrm{ft} / \mathrm{d}$. Ketelle and Huff (1984) determined transport rates of about 490 to 1,250 fv/ in their tracer test on Chestnut Ridge west of the Chestnut Ridge Regime.

\subsection{Surface Water Systen}

Surface streams in the Chestnut Ridge Regime lie exclusively on the southern flank of Chestnut Ridge. Five primary tributaries exist within the regime boundaries (Figures 4 and 5): two unnamed tributaries located west and east of Industriai Landfill II; the McCoy Branch drainage basin, which contains the Ash Disposal Basin and Rogers Quarry; the Chestnut Branch drainage basin, which contains Industrial Landfill $\mathrm{V}$ and Construetion/Demolition Landfill VII; and an unnamed drainage basin containing Kerr Hollow Quarry. These strearus are largely intermittent above an elevation of $900 \mathrm{ft}$. Sources of flow to these streans are exclusively runoff, stormflow, and groundwater via baseflow and spring discharge. Baseflow contributions gradually increase along the length of the streams as elevation decreases. Spring discharges represent substantial point contributions to total flow for most of the tributaries. All of the tributaries discharge to Melton Hill Reservoir (Clinch River).

Historical data indicate that MoCoy Branch has been the most heavily inpacted stream in the Chestnut Ridge Regime because of activities at the Ash Disposal Basin. Contaminants related to the Ash Disposal Basin appear to be restricted only to the stream channel immediately downstream of the site (U.S. Department of Energy 1994b). Data collected as part of best management practice programs at other tributaries have not shown conclusive evidence of contamination from upstream sources. Sporadic elevated concentrations of some constituents, particularly barium, have been observed in discharges at Kerr Hollow Quarry.

Two spring stations were sampled in CY 1995 as part of best management practice monitoring. Station CBS-1, a large spring located along Chestnut Branch (Figure 4), was sampled semiannually. Station SCR2.2SP, located below Industrial Landfill II, was sampled during the second quarter. Surface water discharging from Kerr Hollow Quarry will be sampled semiannually in CY 1996 as part of the CERCI.A ROD requirements. 



\subsection{REFERENCES}

Bailey, J.K, 1989. Martin Marietta Energy Systems, Inc. Personal Conmumication to Charlotte Kimbrough, Martin Marietta Energy Systems, Inc. February 16. 1989.

Battelle Columbus Division. 1988. RCRA Facility Investigation Plan, Filled Coal Ash Pond (D112), Oak Ridge Y-12 Plant, Oak Ridge Tennessee. Prepared for Martin Marietta Energy Systems, Inc. (Y/TS-411).

Buty, T.R and H.H. Stoner. 1983. Disposal of United Nuclear Company Materials at the Y-12 Plant. Health, Safety, and Envirommental Affairs Division, Union Carbide Corporation, Y-12 Plant, Oak Ridge, TN.

Daniels, D.E., and G. Broderick. 1983. Results of Moisure-Suction and Permeability Tests on Unsaturated Samples. Oak Ridge National Laboratory (ORN//Sub/83-64764/1).

Dreier, R.B., D.K. Solomon, and C.M. Beaudoin. 1987. Fracture Characterization in the Unsaturated Zone of a Shallow Land Burial Facility. In: Flow and Transport through Fractured Rock. Anerican Geophysical Union Monograph 42, pp. 51-59.

Dreier, R.B., T.O. Early, and H.L. King. 1993. Results and Interpretations of Groundwater Data Obtained from Multi-port Instrumented Core Holes (GW-131- GW-135) Fiscal Years 1990 and 1991. Martin Marietta Energy Systems, Inc. (Y/TS-803).

Evans, R.D. 1955. The Atomic Nucleus. McGraw-Hill, New York, N.Y.

Freeze, R.A. and J.A. Cherry. 1979. Groundwater. Prentioe-Hall, Inc., Eaglewood Cliffs, New Jersey, (pp. 408-409).

Geraghty \& Miller, Inc. 1990a. Comprehensive Groundwater Monitoring Plan for the Department of Energy Y-12 Plant Oak Ridge, Tennessee. Prepared for Martin Marietta Energy Systems, Inc. (Y/SUB/90-00206C/5).

Geraghty \& Miller, Inc. 1990b. A Study of Ground-Water Flow from Chestnut Ridge Security Pits Using a Fluorescent Dye Tracer. Prepared for Martin Marietta Energy Systems, Inc. (Y/SUB/90-00206C/6).

Grutzeck, M. 1987. United Nuclear Corporation's Y-12 Plant Site: Final Report. Pennsylvania State University, Materials Research Laboratory. Prepared for Martin Marietta Energy Systems, Inc. (Y/SUB/86-23729/1).

H\&R Technical Associates, Inc. 1995a. Letter to W.K. Jago, Lockheed Martin Energy Systems, Inc., November 20, 1995.

H\&R Technical Associates, Inc. 1995b. Letter to W.K. Jago, Lockhoed Martin Energy Systems, Inc., December 14, 1995.

H\&R Technical Associates, Inc. 1996a. Letter to W.K. Jago, Lockheed Martin Energy Systerns, Inc., January 9, 1996.

H\&R Technical Associates, Inc. 1996b. Letter to W.K. Jago, Lockheed Martin Energy Systems, Inc., February 9, 1996. 
HSW Environmental Consultants, Inc. 1994. Sampling and Analysis Plan for Groundwater and Surface Water Monitoring at the Y-12 Plant During Calendar Year 1995, Y/SUB/94EAQ10C/4.

Jones, S.B., B.K. Thompson, and S.M. Field. 1995. Updated Subsurface Data Base for Bear Creek Valley, Chestnut Ridge, and Parts of Bethel Valley on the U.S. Department of Energy Oak Ridge Reservation. Martin Marietta Energy Systems, Inc. (Y/TS-881/R3).

Jennings, J.N. 1985. Karst Geomorphology. Blackwell and Oxford, New York (pp. 34-69).

Ketelle, R.H., and D.D. Huff. 1984. Site Characterization of the West Chestnut Ridge Site. Oak Ridge National Laboratory (ORNLTM-9229).

Kimbrough, C. W. and L.W. McMahon. 1988a. RCRA Appendix IX Sampling and Analysis Project at the Oak Ridge Y-12 Plant: Disposal Basin Field Sampling Plan and Field Data. Prepared by Martin Marietta Energy Systems, Inc. (Y/SUB/88-97376/1).

Kimbrough, C. W. and L.W. McMahon. 1988b. RCRA Appendix DX Sampling and Analysis Project at the Oak Ridge Y-12 Plant. New Hope Pond Analytical Data Summary. Prepared by Martin Marietta Energy Systems, Inc. (Y/SUB/88-97376/).

King, H.L., and C.S. Haase. 1987. Subsurface-Controlled Geological Maps for the Y-12 Plant and Adjacent Areas of Bear Creek Valley. Oak Ridge National Laboratory (ORNLTM-10112).

King, H.L., and C.S. Haase. 1988. Summary of Results and Preliminary Interpretation of Hydrogeologic Packer Testing in Core Holes GW-131 Through GW-135 and CH-157. Oak Ridge Y-12 Plant. Prepared for Martin Marietta Energy Systems by E.C. Jordan Company. (Y/TS-495).

King, H.L., C.S. Haase, and D.L. LaRne. 1989. Groundwater Investigation Drilling Program for Fiscal Years 1986, 1987, and 1988 Y-12 Plant, Oak Ridge, Tennessee. Prepared by C-E Environmental, Inc. for Martin Marieta Energy Systens, Inc. (Y/SUB/89-E4371V/2).

Lockheed Martin Energy Systems, Inc. 1995a. Letter from T.R. Butz to Tom Tiesler, TDEC, titled: "Serniannual Statistical Determinations of Groundwater Quality Data from the Keir Hollow Quarry (KHQ), Oak Ridge Y-12 Plant, First, 180-Day Reporting Period for Calendar Year (CY) 1995, July 25, 1995.

Lockheed Martin Energy Systems, Inc. 1995b. Letter from T.R. Butz to Tom Tiesler, TDEC. tited: "Semiannugl Statistical Determinations of Groundwater Quality Data from the Chestnut Ridge Sediment Disposal Basin (CRSDB), Oak Ridge Y-12 Plant, First, 180-Day Reporting Period for Calendar Year (CY) 1995, July 25, 1995.

Lockheed Martin Energy Systems, Inc. 1996a. Letter from T.R. Butz to Tom Tiesler, TDEC, titled: "Semiannual Statistical Determinations of Groundwater Quality Data from the Kerr Hollow Quarry (KHQ), Oak Ridge Y-12 Plant, Second, 180-Day Reporting Period for Calendar Year (CY) 1995, TO BE ISSUED.

Lockheed Martin Etergy Systems, Inc. 1996b. Letter from T.R. Butz to Tom Tiesler, TDEC, titled: "Semiannual Statistical Determinations of Groundwater Quality Data from the Chestnut Ridge Sediment Disposal Basin (CRSDB), Oak Ridge Y-12 Plant, Second, 180Day Reponting Period for Calendar Year (CY) 1995, TO BE ISSUED. 
Lockheed Martin Energy Systems, Inc. 1996c. Annual Report of 1995 Groundwater Monitoring Data for the Kert Hollow Quarry and Chestnut Ridge Sediment Disposal Basin, Y-12 Plant, Oak Ridge, Tennessee, Y/TS-1438.

Luxmoore, RJ. 1982. Physical Characteristics of Soils of the Southern Region Fullerton and Sequoia Series. Oak Ridge National Laboratory (ORNL-5868).

Martin Marietta Energy Systems, Inc. 1988a. Revised RCRA Closure Plan for the Chestnut Ridge Security Pits, Oat Ridge Y-12 Plant, Oak Ridge, Tennessee. (Y/TS-391).

Martin Marietta Energy Systerns, Inc. 1988b. Environmental Surveillance Quality Control Program. (ES/ESH/INT-14).

Martin Marietta Energy Systems, lic. 1989a. Chestnat Ridge Security Pits (D-023), Suramary of Closure Under Rules Goveraing Hazardous Waste Management in Tennessee. (Y/TS-391/2).

Martin Marietta Energy Systems, Inc. 1989b. Closure Plan for the United Nuclear Corporation Waste Disposal Site. (Y/IA-200).

Martin Marietta Energy Systems, Inc. 1992. Y-12 Environmental Restoration Remedial Action Surveillance and Maintenance Program Plan, Oak Ridge Y-12 Plant, Oak Ridge, Tennessee. (Y/ER-50).

Martin Marietta Energy Systems, Inc. 1993. Y-12 Plant Groundwater Protection ProgramGroundwater Monitoring Program Data Management Plan. (Y/SUB/93-TK532C/1).

Martin Marietta Energy Systems, Inc. 1994a. Post-Closure Permit Application for the Chestnut Ridge Security Pits at the Y-12 Plant, Oak Ridge, Tennessee. (Y/ER/SUB/91-ALV96/4, Rev. 2. Angust 1994).

Martin Marietta Energy Systems, Inc. 1994b. Post-Closure Permit Application for the Chestnut Ridge Sediment Disposal Basin at the Y-12 Plant, Oak Ridge, Tennessee. (Y/ER/SUB/91* ALV96/5, Rev. 2. September 1994).

Mishu, L. 1982. Subsurface Analysis of Waste Disposal Facilities at the Y-12 Plant. Prepared for Martin Marietta Energy Systerns, Inc. (Y/SUB/82-24700/2).

Moore, G.K. 1988. Concepts of Groundwater Flow and Occurrence Near Oak Ridge National Laboratory, Tennessee. Oak Ridge National Laboratory (ORNLTM-10969).

Moore, G.K 1989. Groundwater Parameters and Flow Systems Near Oak Ridge National Laboratory. Oak Ridge National Laboratory (ORNL/TM-1 1368).

Moore, G.K and L.E. Toran. 1992. Supplement to a Hydrogeologic Framework for the Oak Ridge Reservation, Oak Ridge, Tennessec. Oak Ridge National Laboratory (ORNLTM12191).

Quinlan, J.F., and T. Aley. 1987. Discussion of a New Approach to the Disposal of Hazardous Waste on Land. Gronndwater (Vol. 25, pp. 615-616). 
Quinlan, J.F., and R.O. Ewers. 1985. Groundwater Flow in Limestone Tertains: Strategy, Rationale and Procedure for Reliable, Efficient Monitoring of Groundwater Quality in Karst Areas. National Symposium and Exposition on Aquifer Restoration and Grounitwater Monitoring Proceedings, National Water Well Association, Worthington, Ohio (pp. 197-234).

Saunders, M.B. 1983. Leachability of Samples from New Hope Pond Disposal Basin, U.S. DOE Y-12 Plant, Oak Ridge, TN. Prepared for Union Carbide Corporation, Nuclear Division (Y/DZ81 Rev 1).

Science Applications Intemational Corporation. 1992. Chestnut Ridge Borrow Area Waste Pile Woik Plan. Prepared for Martin Marietta Energy Systems. Inc. (Y/SUB/91 - 99928C/1).

Science Applications International Corporation. 1993. Final Report of the Second Dye-Tracer Test at the Chestntt Ridge Security Pits, Y-12 Plant, Oak Ridge, Tennessee. Prepared for Martin Marietta Energy Systems, Inc. (Y/SUB 93 - 99928C/Y 10/I).

Science Applications International Corporation. 1995. Remedial Investigation Report for Bear Creek Valley, Preliminary Draft, Rev. 12/4/95. Prepared for Lockheed Martin Energy Systems, Inc.

Shevenell, L.A., R.B. Dreier, and W.K. Jago. 1993. Summary of Fiscal Years 1991 and 1992 Construction. Hydrologic, and Geologic Data Obtained from the Maynardville Limestone Exit - Pathway Monitoring Program. Y/TS-814.

Sledz, J.J., and D.D. Huff. 1981. Computer Model for Determining Fracture Porosity and Permeability in the Conasauga Group. Oak Ridge National Laboratory (ORNLTM-7695).

Smith. D.I., T.C. Atkinson, and D.P. Drew. 1976. The Hydrology of Limestone Tertains. The Science of Speleolopy, London Acadernic Press (pp. 179-212).

Smith, R.E., N.J. Gilbert, and C.E. Sams. 1983. Stability Analysis of Waste Disposal Facilities at the Y-12 Plant. Prepared for Martin Marietta Energy Systems, Inc. (Y/SUB/83$49712 / 1$ ).

Solomon, D.K., G.K. Moore, L.E. Toran, R.B. Dreier, and W.M. McMaster. 1992, Status Report - A Hydrologic Framework for the Oak Ridge Reservation. Oak Ridge National Laboratory (ORNL/TM-12026).

U.S. Department of Energy. 1991. United Nuclear Corporation Record of Decision. IRC No. 910704,0092, June 1991.

U.S. Department of Energy. 1993a. Remedial Investigation Work Plan for Chestnut Ridge Operable Unit 1 (Chestnut Ridge Sectrity Pits) at the Oak Ridge Y-12 Plant, Oak Ridge, Tennessee. (DOE/ORN1-1173\&D1).

U.S. Department of Energy. 1993b. Phase II Sampling Plan for Chestmut Ridge Operable Unit 2 (Filled Coal Ash Pond/Upper McCoy Branch) at the Oak Ridge Y-12 Plant, Oak Ridge, Temessee. (DOE/OR-1045\&D2).

U.S. Department of Energy. 1993c. Postconstruction Report for the United Nuclear Corporation Site at the Oak Ridge Y-12 Plant, Oak Ridge, Tennessee. (DOE/OR/01-1128\&D1). 
U.S. Department of Energy. 1993d. Remedial Investigation Work Plan for Chestnut Ridge Operable Unit 4 (Rogers Quarry/McCoy Branch) at the Oak Ridge Y-12 Plant, Oak Ridge, Tennessee. (DOEKOR-1152\&D1).

U.S. Department of Energy. 1994a. Oak Ridge Reservation Site Management Plan for the Environmental Restoration Program. U.S. Department of Energy Oak Ridge Field Office (DOE/OR-1001/R3).

U.S. Department of Energy. 1994b. Remedial Investigation Report on Chestnut Ridge Operable Unit 2 (Filled Coal Ash Pond/Upper McCoy Branch) at the Oak Ridge Y-12 Plant, Oak Ridge, Tennessee. (DOE/OR-01-1268/V1\&D1).

U.S. Department of Energy. 1995. Feasibility Study for the Y-12 Chestnut Ridge Operable Unit 2. (Filled Coal Ash Pond), Oak Ridge, Tennessee. (DOE/OR/02-1259\&D2, January 1995).

U.S. Environmental Protection Agency. 1988. Laboratory Data Validation Functional Guidelines for Evaluating Organic Analyses. U.S. EPA, Office of Solid Waste.

U.S. Environmental Protection Agency and Tennessee Department of Environment and Conservation. 1988. Letter from J.H. Scartrough, Chief of the Region IV RCRA Branch, and T. Tiesler, Director of the Division of Solid Waste Management, Tenmessee Department of Health and Environment, to RJ. Spence, Y-12 Site Manager, U.S. Department of Energy - Oak Ridge Operations, November 18, 1988.

Watson, K.W. and RJ. Luxmoore. 1986. Estimating Macroporosity in a Forested Watershed by use of a Tension Infiltrometer. Soil Science Society of America, Journal 50 (pp 578-582).

Welch. S.H., C.S. Haase, C.W. Kimbrough, and T.M. Mercier. 1987. Solid Waste Management Unit Information for Y-12 Plant RCRA 3004 (u) Facility Assessment, Volumes I, I, and III. Martin Marietta Energy Systems, Inc. (Y/TS-273).

WiIson, G.V. and R.J. Luxmoore. 1988. Infiltration, Macroporosity, and Mesoporosity Distributions of two Forested Watersheds. Soil Science Society of America, Journal 52 (pp 329 -335).

Wilson, G.V., P.M. Jardine, R.J. Luxmoore, and J.R. Jones. 1990. Hydrology of a Forested Hillslope During Storm Events. Geoderma, Journal 46, pp. 119-138.

Woodward-Clyde Consultants, Inc. 1984. Subsurface Characterization and Geohydrologic Site Evaluation, West Chestnut Ridge Site. Oak Ridge National Laboratory (ORNL/SUR/83. 647641/IVI2). 
APPENDIX A

\section{FIGURES}





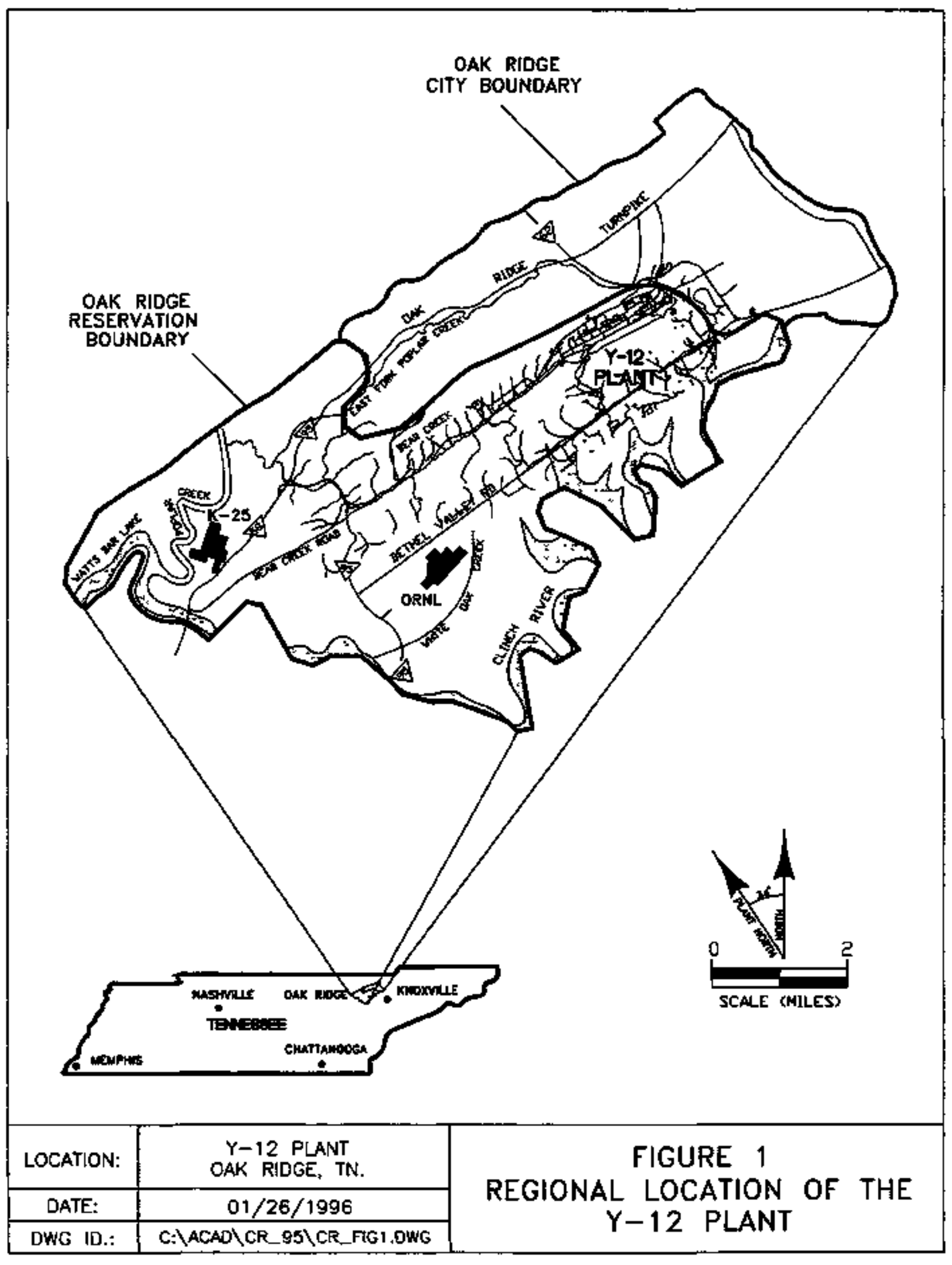





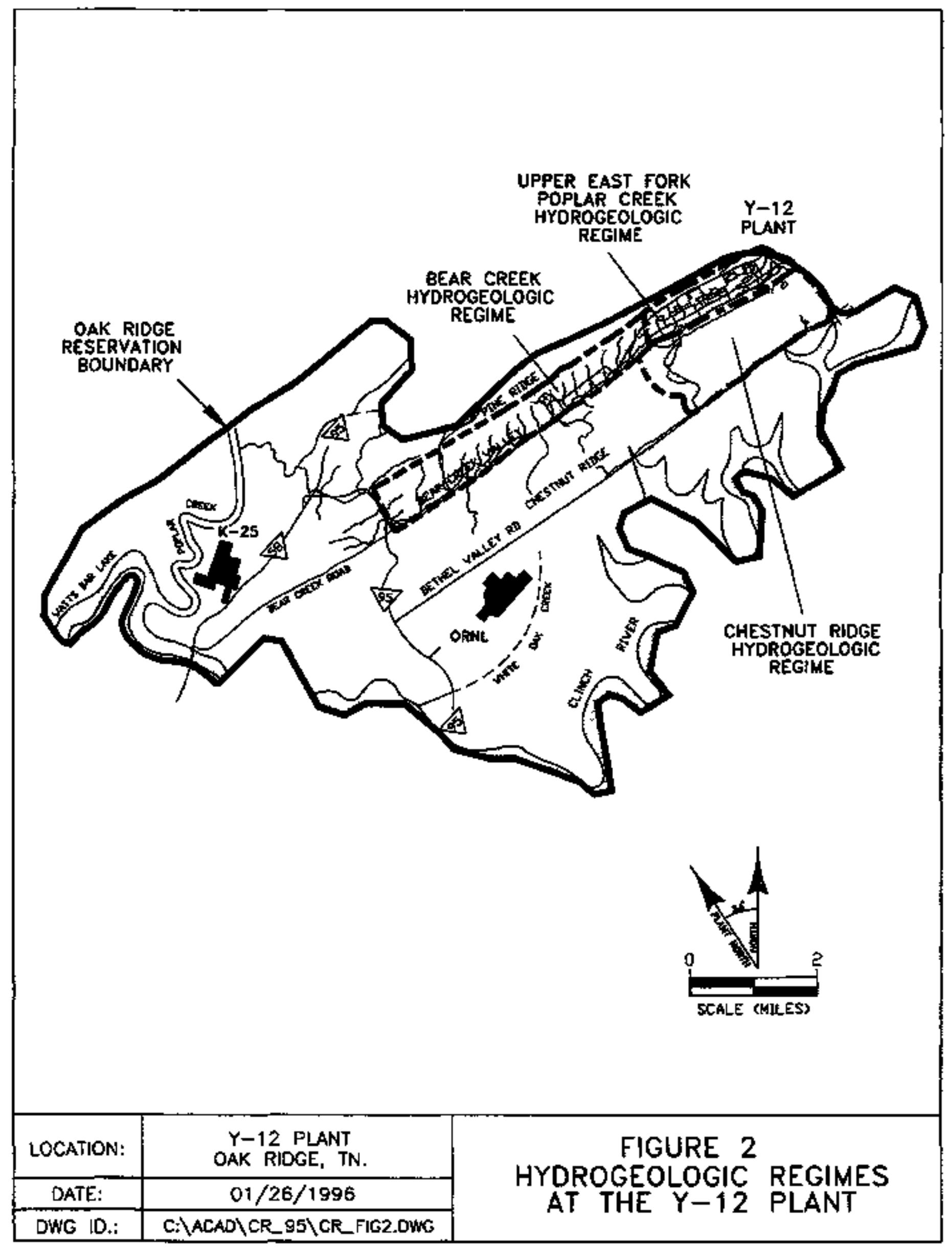




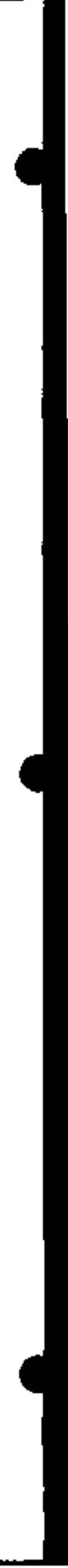




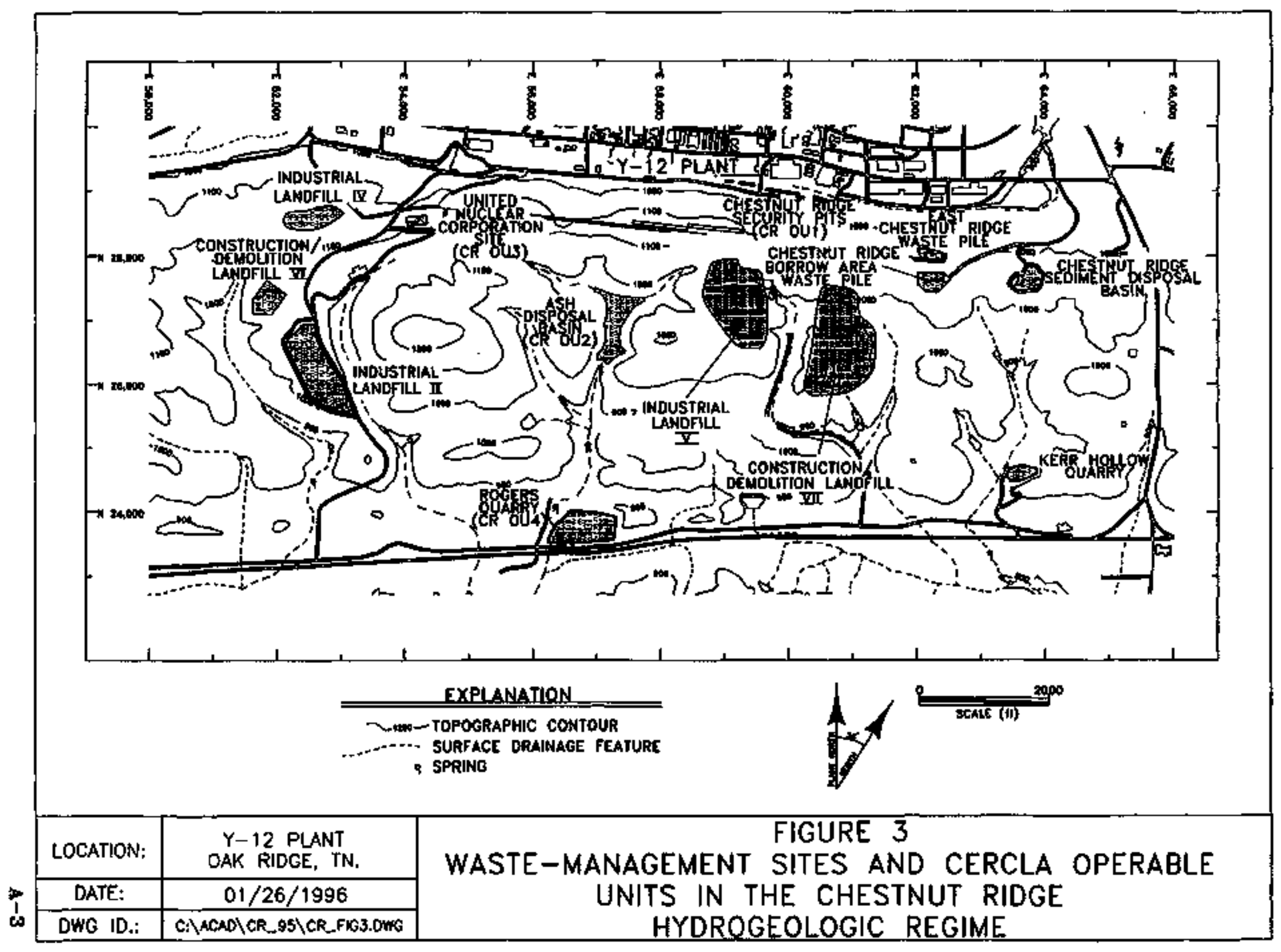




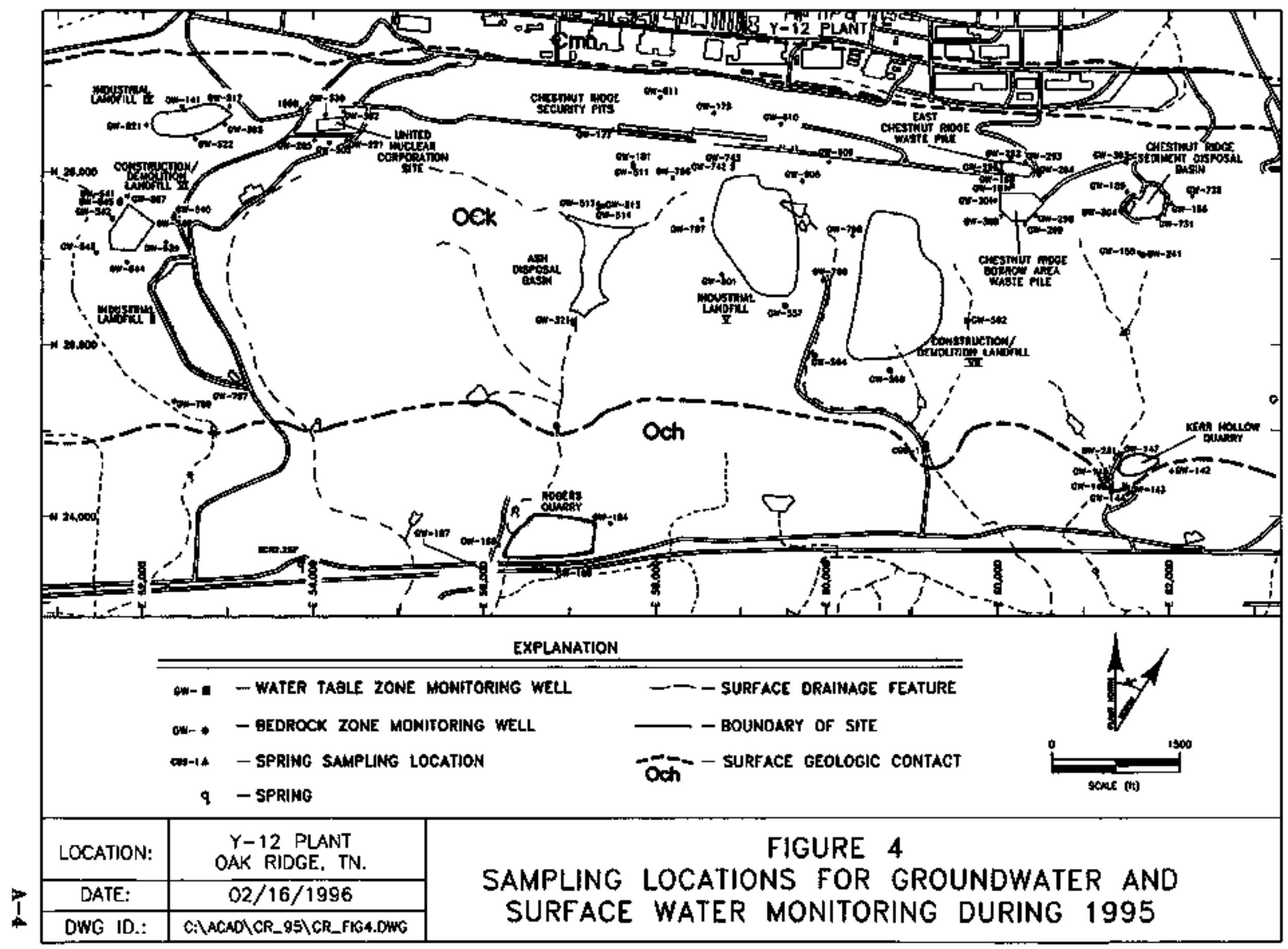





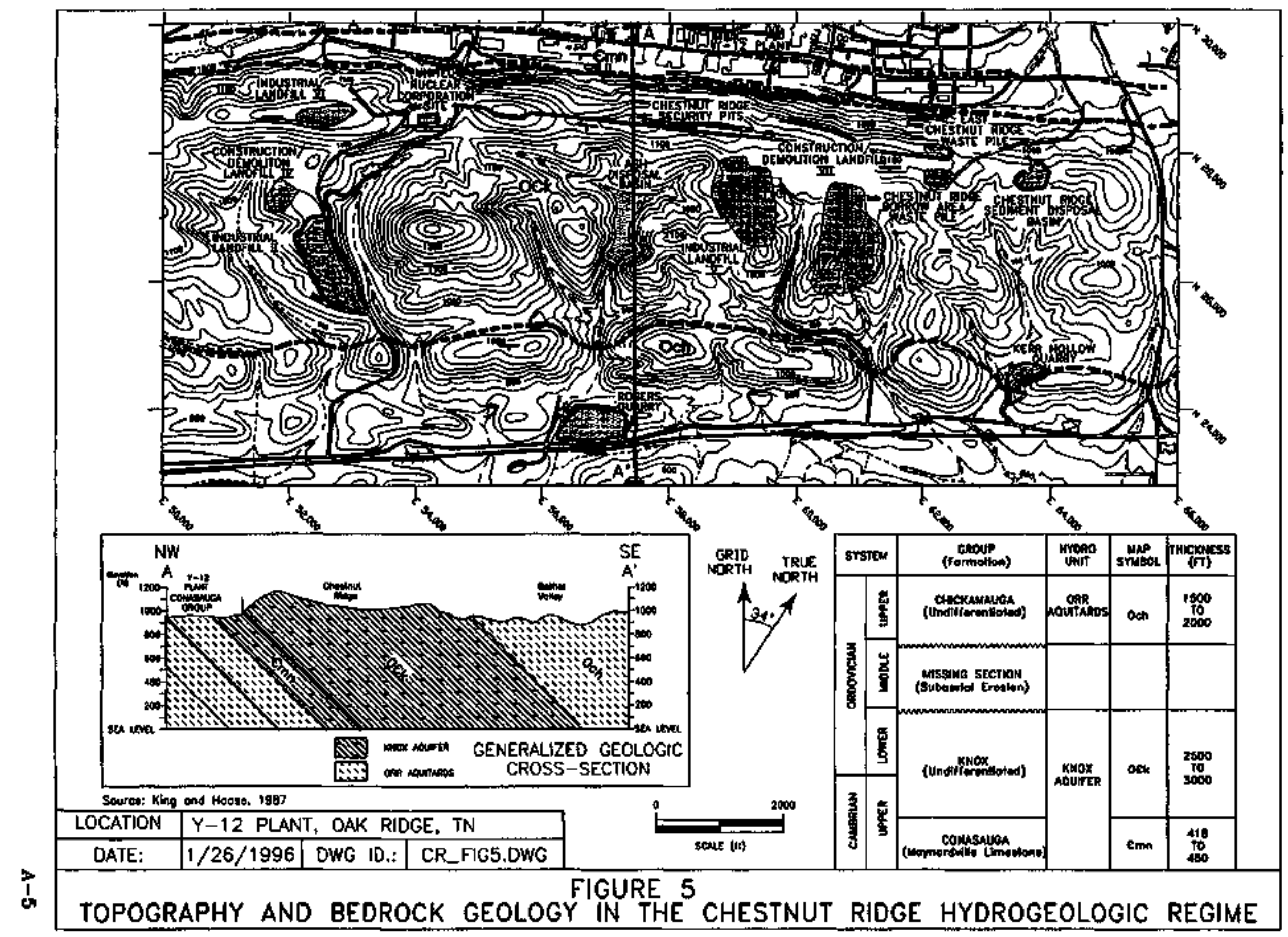




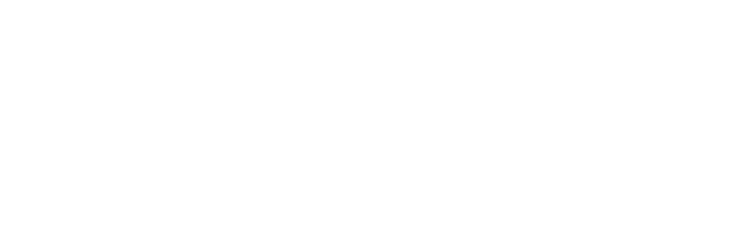


HYDROSTRATIGRAPHIC UNITS

PROPOSED GY SOLOWON af ol. (1992)

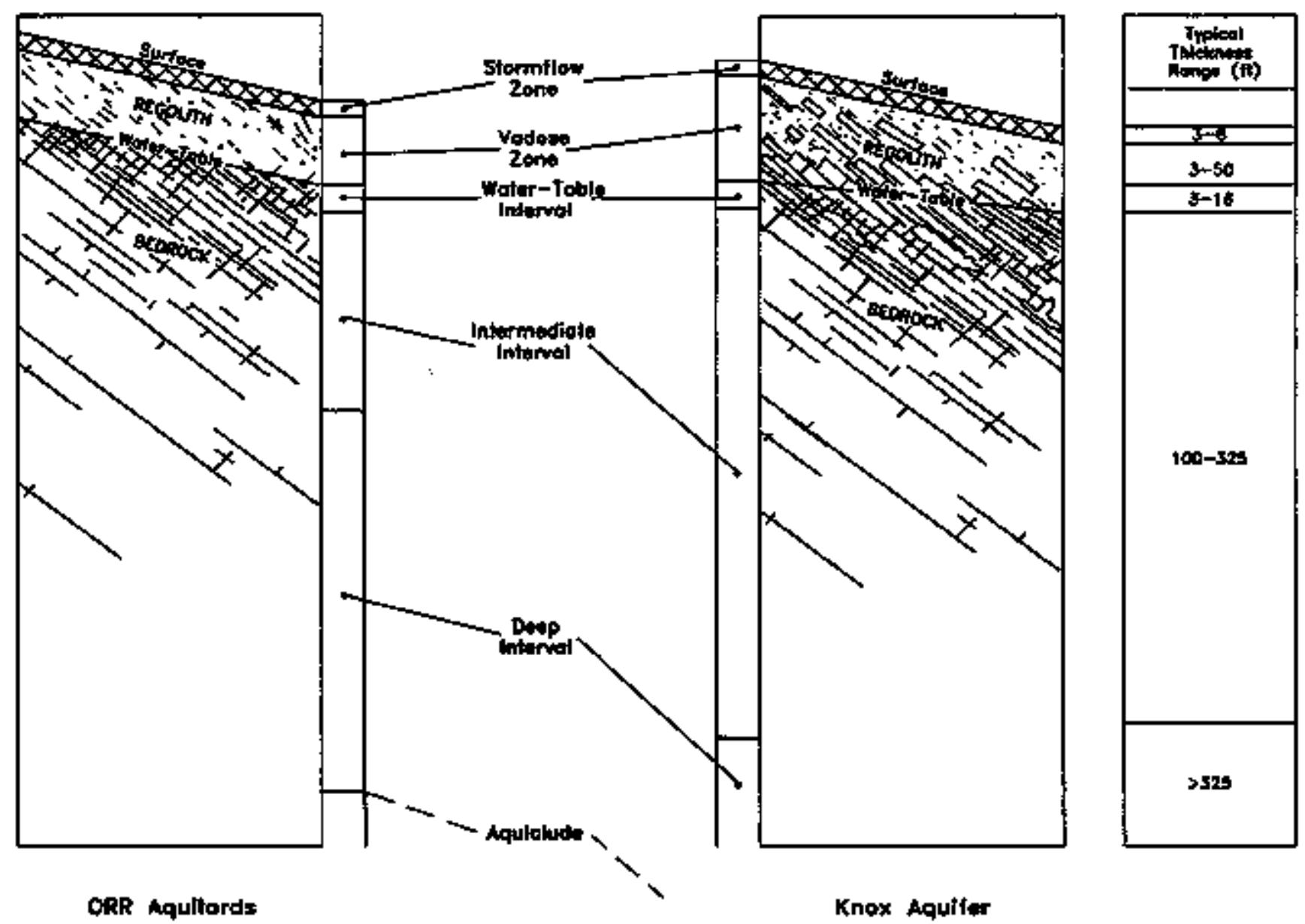

\begin{tabular}{|c|c|c|}
\hline LOCATION: & $\begin{array}{l}\text { Y-12 PLANT } \\
\text { OAK RIDGE. TN. }\end{array}$ & $\begin{array}{c}\text { FIGURE } 6 \\
\text { SCHEMATIC PROFILE OF }\end{array}$ \\
\hline DATE: & $01 / 26 / 1996$ & GRAPHIC UNITS IN THE \\
\hline DWG ID.: & $C: \backslash A C A O \backslash C R \quad 95 \backslash C R$ FIG6.DWG & \\
\hline
\end{tabular}


. 


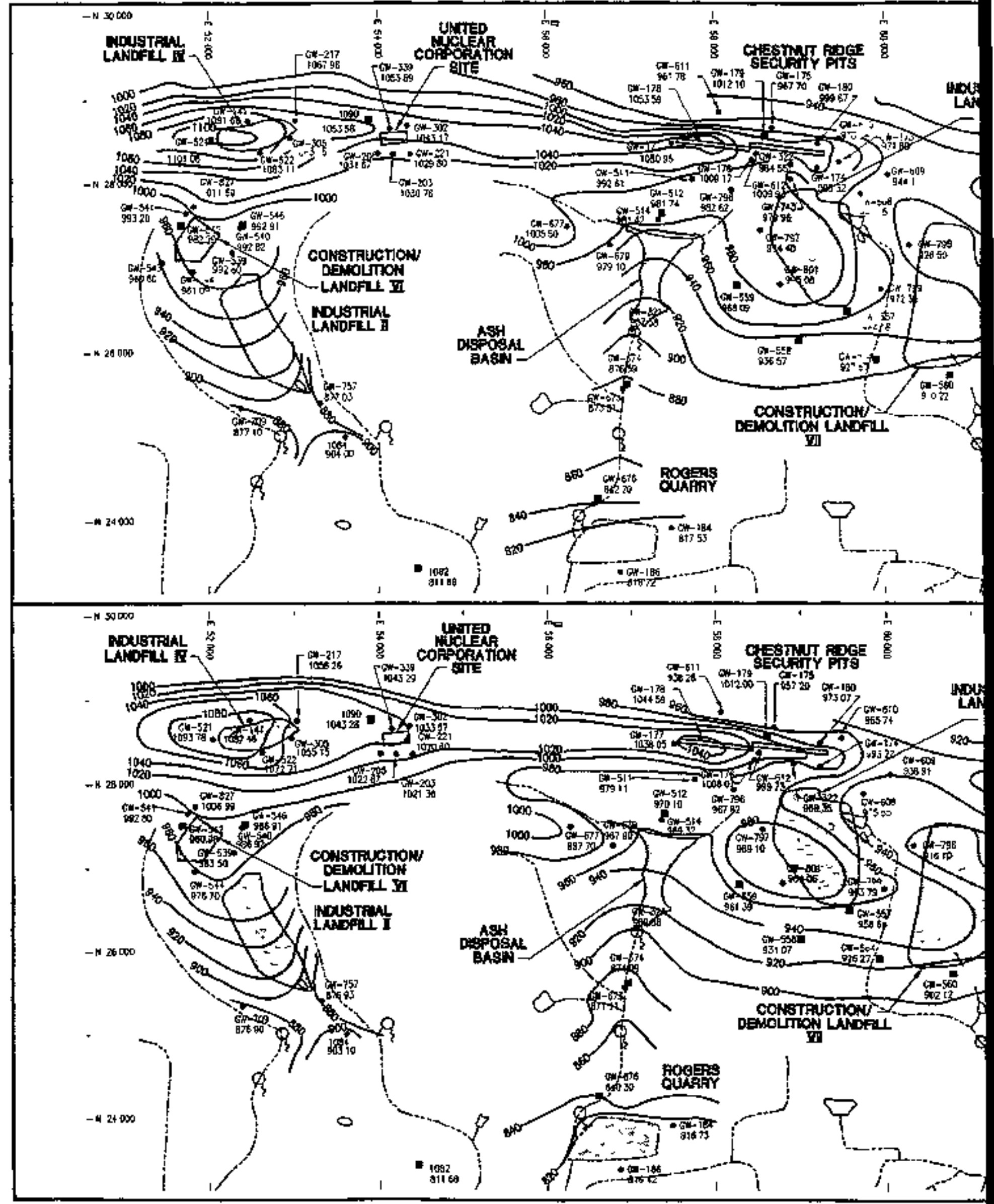




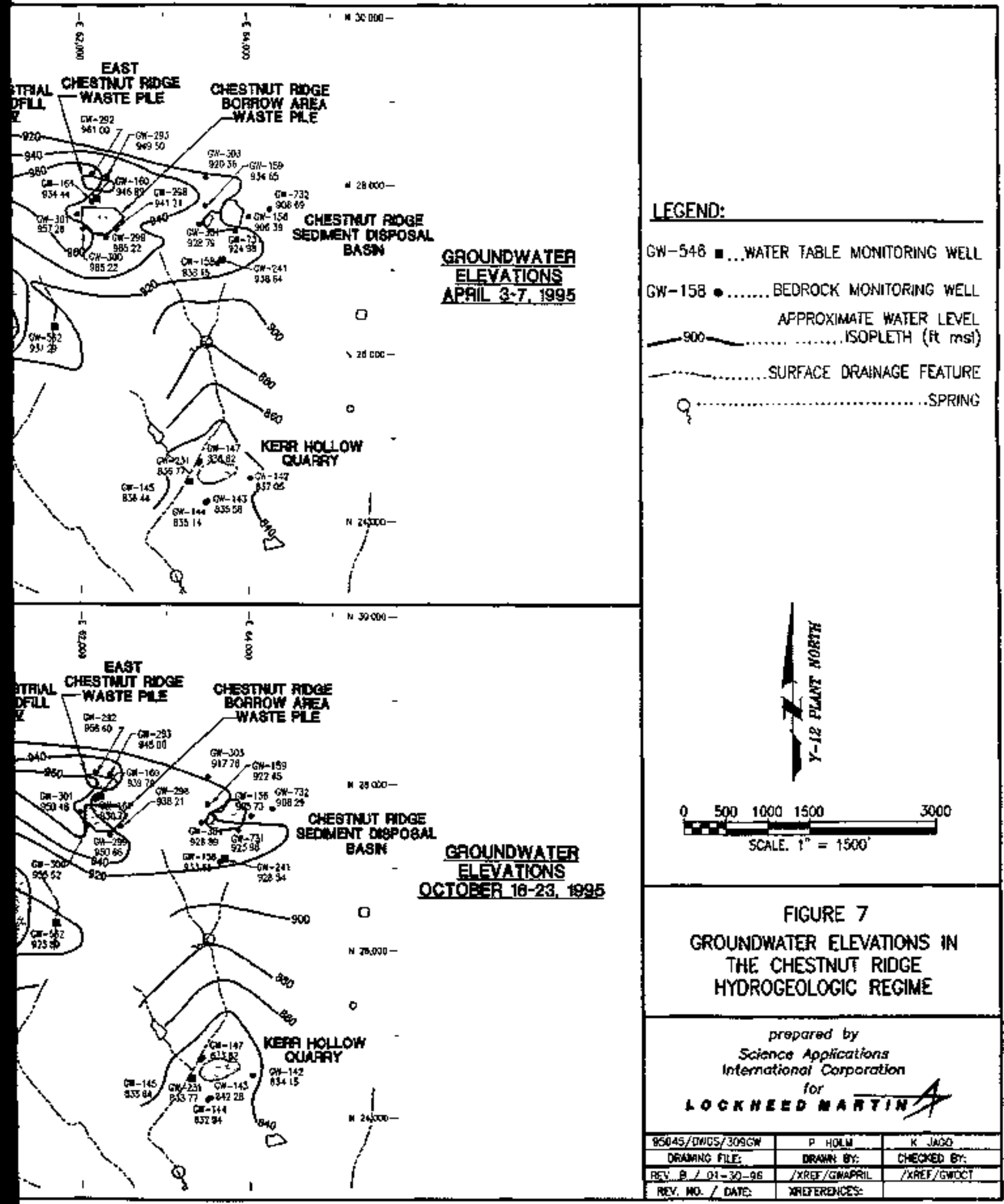




\section{Upgrodient Sites}

Recharge

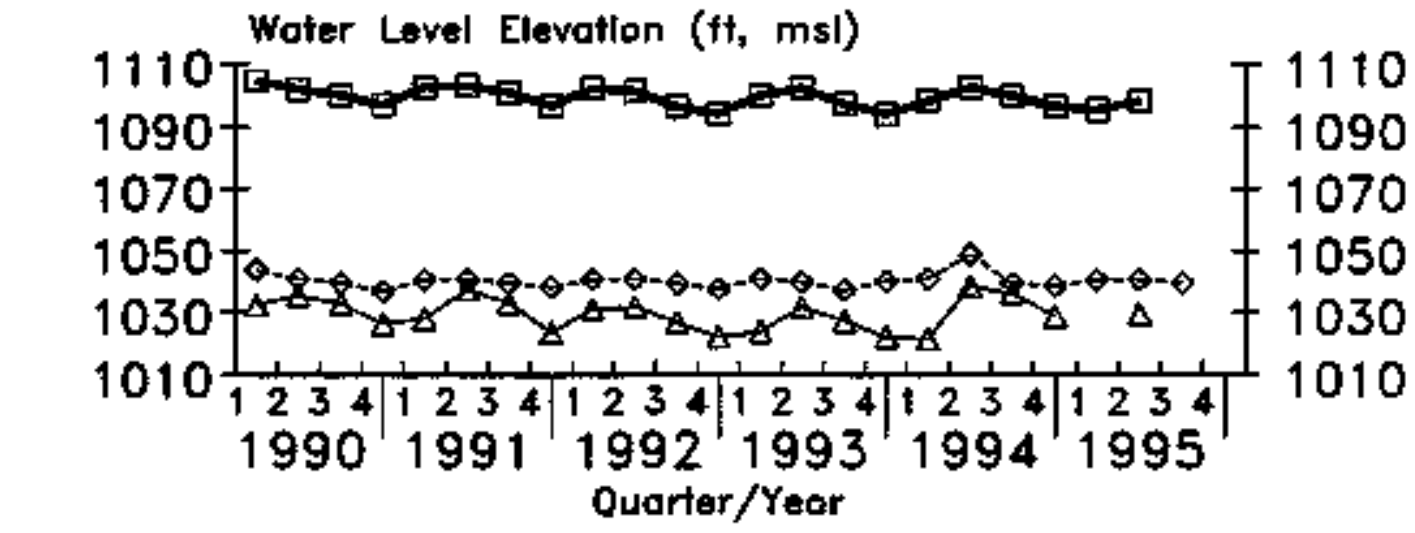

$\rightarrow$ uV (GW-521)

UNCS (GW-221)

$\rightarrow \operatorname{CRSP}(\mathrm{GW}-177)$

Downgradient Sites

Dischorge

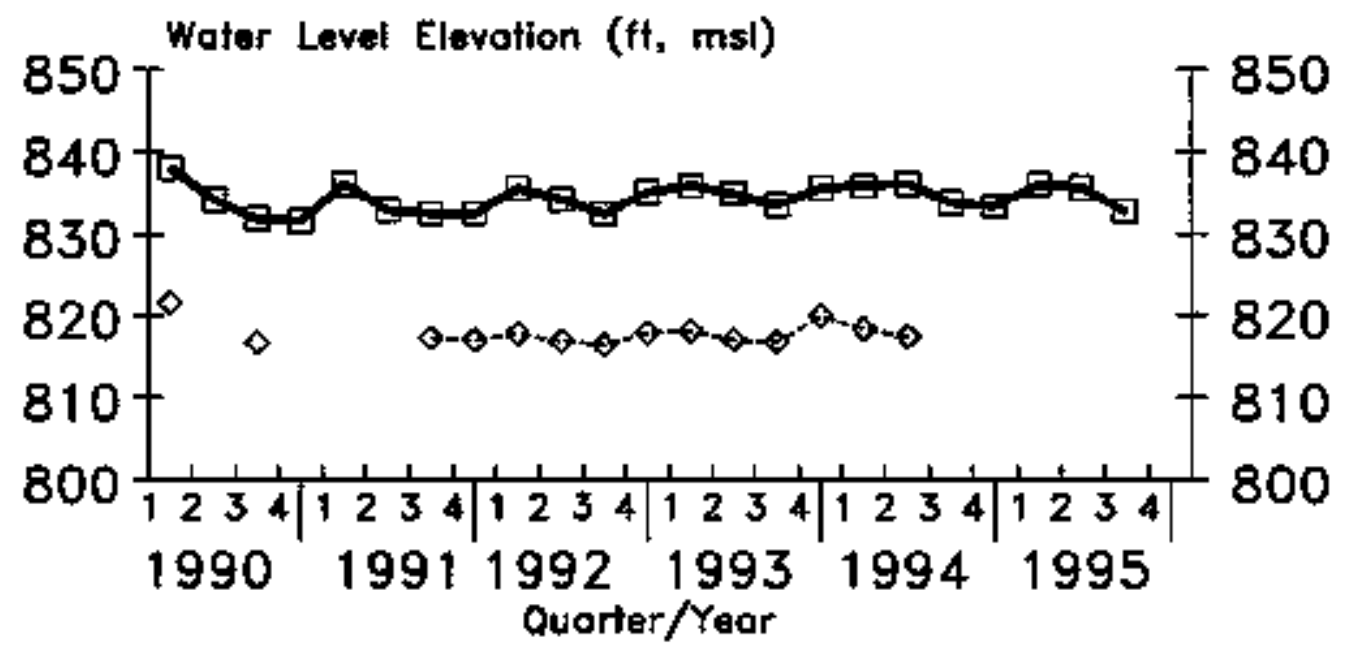

$=$ KHO (GW-144) NON RQ (GW-184)

\section{EXPLANATION}

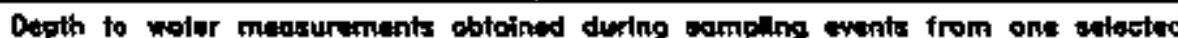

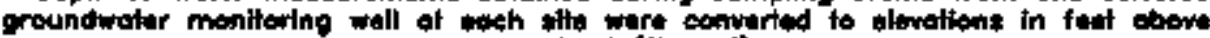
mon soo lom ( $\mathrm{ft}$ mol).

\section{UPGRAOEkT SITES}

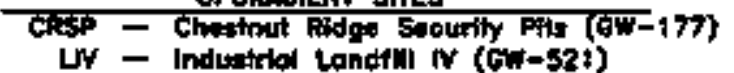

uncs - Unitad Nuntuar Corporation Silk (GW-221)
DOMHGRADIFWT STIES

KHQ - KorT Holiow Quorry (GW-144)

RQ - Ragurs Quarry (GW-184)

\begin{tabular}{|c|c|}
\hline LOCATION: & $\begin{array}{c}\text { Y-12 PLANT } \\
\text { OAK RIDGE, TN. }\end{array}$ \\
\hline DATE: & $02 / 07 / 1996$ \\
\hline DWG ID:: & C: $\backslash A C A D \backslash C R \_95 \backslash C R \_F I G 8 . D W G$ \\
\hline
\end{tabular}

FIGURE 8

HYDROGRAPHS FOR

SELECTED SITES IN THE CHESTNUT RIDGE HYDROGEOLOGIC REGIME 


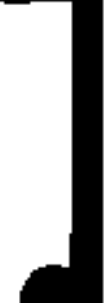




\section{APPENDIX B}

TABLES 


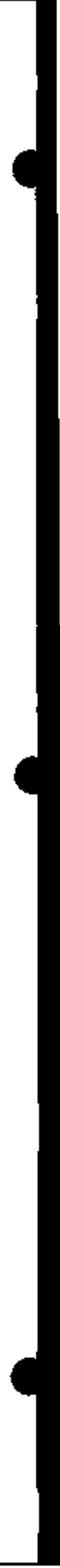


Table 1. Waste-Management Sites and CERCLA Operable Units in the Chestnut Ridge Hydrogeologic Regime

\begin{tabular}{|c|c|c|}
\hline \multirow[b]{2}{*}{ Site } & \multicolumn{2}{|c|}{ Requlatory Classification } \\
\hline & Historica| ${ }^{1}$ & Corrent ${ }^{2}$ \\
\hline Chestuut Ridge Sediment Disposal Basin & TSD Unit & Study Area TSD Unit \\
\hline East Chestnut Ridge Waste Pile & TSD Unit & Sudy Area/TSD Unit \\
\hline Kerr Hollow Quarry & TSD Unit & Saudy Area/TSD Unit \\
\hline Chestnut Ridge Security Pits & TSD Unit & CR OU 01/TSD Unit \\
\hline Ash Dispasal Basin & SWMU & CROU 02 \\
\hline United Nuclear Corporation Site & SWMU & $\mathrm{CR} O \mathrm{OU} 03$ \\
\hline Rogers Quarry & SWMU & CR OU 04 \\
\hline Industrial Landfill II & SWDF & SWDF \\
\hline Industrial Landfill IV & SWDF & SWDF \\
\hline Industrial Landfill V & NA & SWDF \\
\hline Construction/Demolition Landfill VI & N/A & SWDF \\
\hline Construction/Demolition Lamdfill VII & N/A & SWDF \\
\hline Chestmut Ridge Botrow Area Waste Pile & NR & Sudy Area \\
\hline
\end{tabular}

Notes:

1 Regulatory classification before the 1992 Federal Facility Agreenent.

TSD Unit - RCRA-regulated land-based Treatment, Storage, or Disposal Facility.

SWMU - RCRA-regulated Solid Waste Management Unit

SWDF - Solid Waste Disposal Facllity (non-hazardous waste)

NA - Not applicable (new facility)

NR - Not regulated

2 Modified from: Oak Ridge Reservation Site Monagement Plon for the Environmental Restoration Program (U.S. Department of Energy 1994a)

CR OU 01 - Chestuut Ridge Operable Unit 01 (Source Control and Groundwater OU)

CR OU 02 - Chestnut Ridge Operable Unit 02 (Source Control and Groundwater oU)

CR OU 03 - Chestnut Ridge Operable Unit 03 (Source Control and Groundwater OU)

CR OU 04 - Chestnut Ridge Operable Unit 04 (Source Control and Groundwater OU) 
Table 2. CY 1995 Monitoring Program Summary

Monitoring Program = Best-Management Practice Monitoring

$\begin{array}{lll} & \text { Sampling } & \\ & \text { Point } & \text { Location } \\ & & \\ \text { GW-160 } & \text { CRBAWP } \\ \text { GW-161 } & \text { CRBAWP } \\ \text { GW-184 } & \text { RQ } \\ \text { GW-186 } & \text { RQ } \\ \text { GW-187 } & \text { RQ } \\ \text { GW-188 } & \text { RQ } \\ & \text { GW-292 } & \text { ECRWP } \\ \text { GW-293 } & \text { ECRWP } \\ & \text { GW-294 } & \text { ECRWP } \\ & \text { GW-296 } & \text { ECRWP } \\ & \text { GW-298 } & \text { CRBAWP } \\ \text { GW-299 } & \text { CRBAWP } \\ \text { GW-300 } & \text { CRBAWP } \\ \text { GW-301 } & \text { CRBAWP } \\ \text { GW-321 } & \text { ADB } \\ \text { GW } & \text { GW-512 } & \text { ADB } \\ & \text { GW-513 } & \text { ADB } \\ & \text { GW-514 } & \text { ADB }\end{array}$

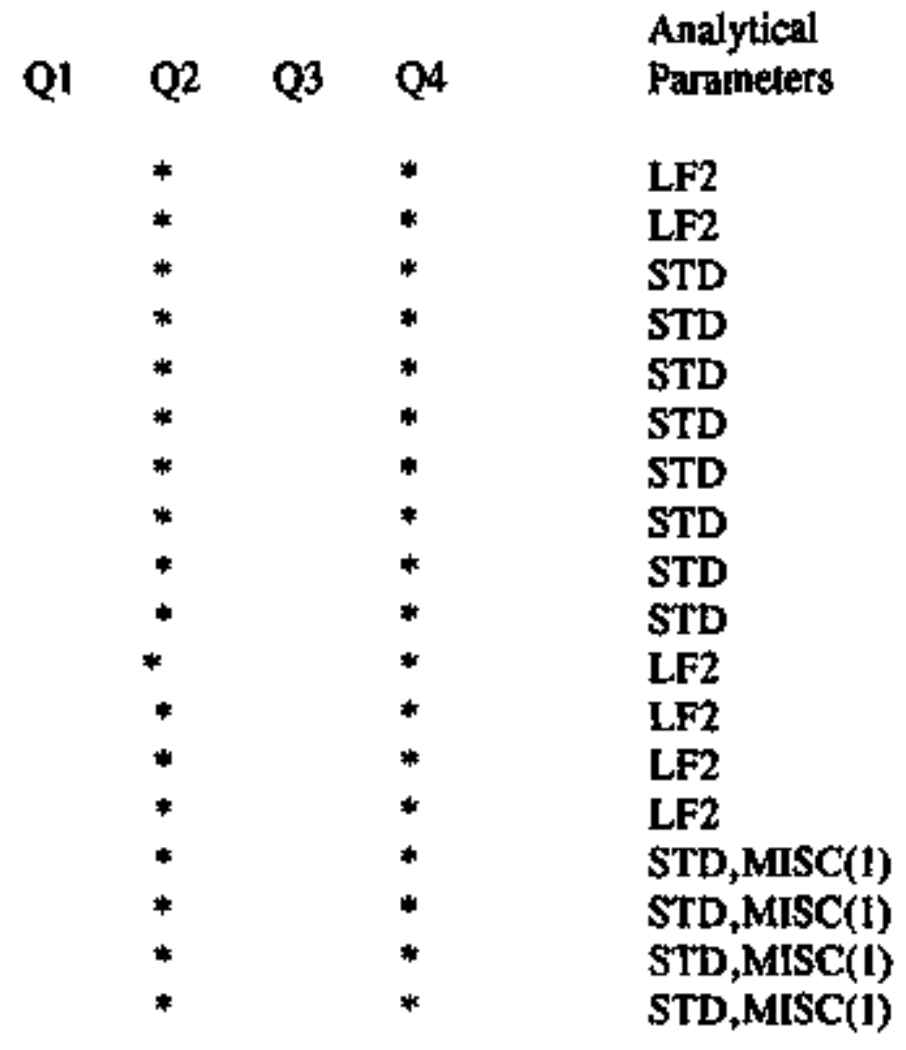

Program

Change 
Table 2, CY 1995 Monitoring Program Summary (cont'd)

Monitoring Program mCERCLA Record of Decision Monitoring

\begin{tabular}{|c|c|c|c|c|c|c|}
\hline $\begin{array}{l}\text { Sampling } \\
\text { Point }\end{array}$ & Location & Q1 & Q2 & Q3 & Q4 & $\begin{array}{l}\text { Analytical } \\
\text { Parameters }\end{array}$ \\
\hline 1090 & UNCS & & * & & * & STD,RAD $(3,5)$ \\
\hline GW-203 & UNCS & & * & & * & STD,RAD $(3,5)$ \\
\hline GW-205 & UNCS & & $*$ & & * & STD,RAD $(3,5)$ \\
\hline GW-221 & UNCS & & * & & * & STD,RAD $(3,5)$ \\
\hline GW-302 & UNCS & & * & & * & STD,RAD(3,5),MET(2) \\
\hline GW-339 & UNCS & & * & & * & STD,RAD $(3,5)$ \\
\hline
\end{tabular}

-Monitoring Program $=$ RCRA Assessment Monitoring

$\begin{array}{lllllll}\text { Sampling } & \text { Location } & \text { Q1 } & \text { Q2 } & \text { Q3 } & \text { Q4 } & \begin{array}{l}\text { Analytical } \\ \text { Parameters }\end{array} \\ \text { GW-175 } & \text { CRSP } & * & * & * & * & \text { STD } \\ \text { GW-177 } & \text { CRSP } & * & * & * & * & \text { STD } \\ \text { GW-181 } & \text { CRSP } & * & * & * & * & \text { STD } \\ \text { GW-511 } & \text { CRSP } & * & * & * & * & \text { STD } \\ \text { GW-608 } & \text { CRSP } & * & * & * & * & \text { STD } \\ \text { GW-609 } & \text { CRSP } & * & * & * & * & \text { STD } \\ \text { GW-610 } & \text { CRSP } & * & * & * & * & \text { STD } \\ \text { GW-611 } & \text { CRSP } & * & * & * & * & \text { STD } \\ \text { GW-742 } & \text { CRSP } & * & * & * & * & \text { STD } \\ \text { GW-743 } & \text { CRSP } & * & * & * & * & \text { STD }\end{array}$


Table 2. CY 1995 Monitoring Program Summary (cont'd)

Monitoring Program=RCRA Int. Status Detect. Monitoring

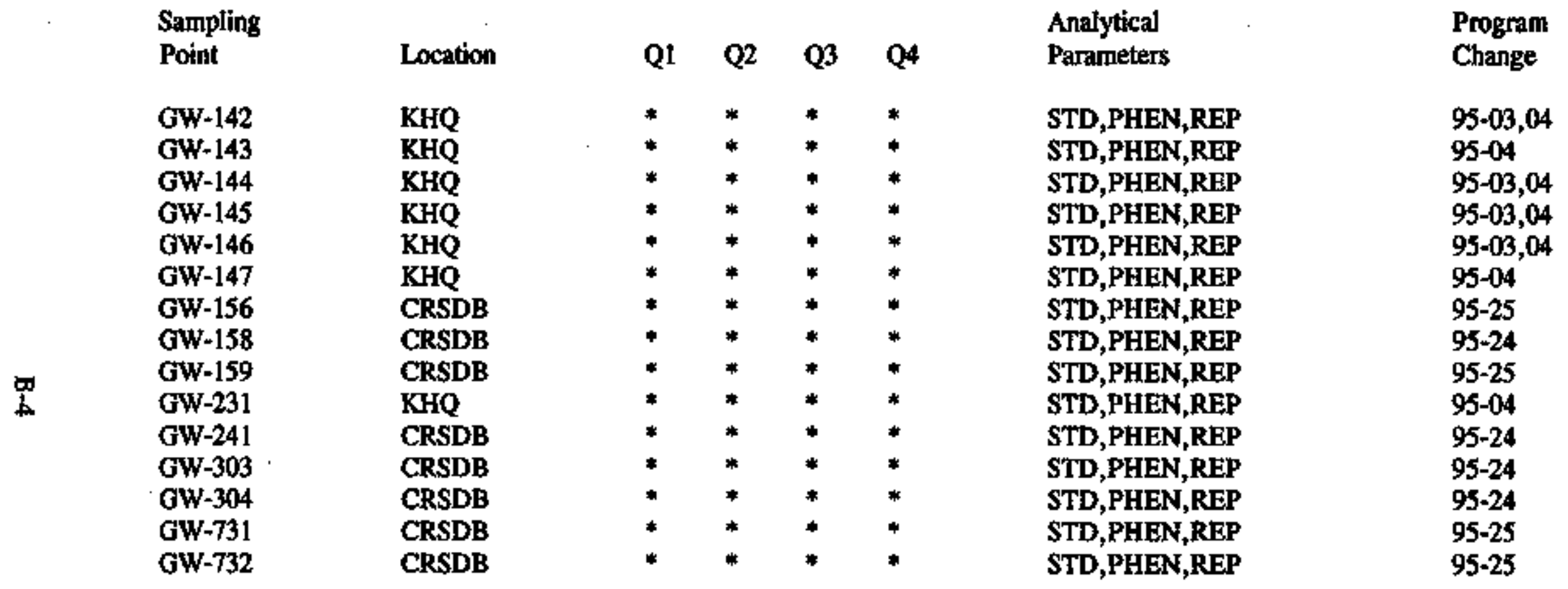


Table 2. CY 1995 Monitoring Program Surnmary (cont'd)

-Monitoring Program =SWDF Detection Monitoring

\begin{tabular}{lll}
\hline Sampling & \\
Point & Location \\
& CBS-1 & LV \\
GW-141 & LIV \\
GW-217 & LIV \\
GW-305 & LIV \\
GW-521 & LIV \\
GW-522 & LIV \\
GW-539 & LII \\
GW-540 & CDLVI \\
GW-541 & CDLVI \\
GW-542 & CDLVI \\
GW-543 & CDLVI \\
GW-544 & CDLVI \\
GW-545 & CDLVI \\
GW-546 & CDLVI \\
GW-557 & LV \\
GW-560 & CDLVII \\
GW-562 & CDLVII \\
GW-564 & CDLVI \\
GW-709 & LII \\
GW-757 & LII
\end{tabular}

$\begin{array}{lllll}\text { Q1 } & \text { Q2 } & \text { Q3 } & \text { Q4 } & \begin{array}{l}\text { Analytical } \\ \text { Parameters }\end{array} \\ & * & & * & \text { LF4,TPH } \\ * & & * & & \text { LF1 } \\ * & & * & & \text { LF1 } \\ * & & * & & \text { LF1 } \\ * & & * & & \text { LF1,RAD(2,3,4,5) } \\ * & & * & & \text { LF1 } \\ & * & & * & \text { LF4 } \\ & * & & * & \text { LF3 } \\ & * & & * & \text { LF3 } \\ & * & & * & \text { LF3 } \\ & * & & * & \text { LF3 } \\ & * & & * & \text { LF3 } \\ & * & & * & \text { LF3 } \\ & * & & * & \text { LF4 } \\ & * & & * & \text { LF4,TPH } \\ & * & & * & \text { LF4,TPH } \\ * & * & & * & \text { LF4,TPH } \\ * & & * & & \text { LF1 } \\ & & * & & \text { LF1 }\end{array}$

Program

Change

95-14

$95 \cdot 02$ 
Table 2. CY 1995 Monitoring Progran Summary (cont'd)

-Monitoring Program=SWDF Detection Monitoring (cont'd)

Sampling

Point

GW-796

GW-797

GW-798

GW-799

GW-801

Location
LV
LV
CDLVII
LV
LV

Q1

Analytical

Parameters

Program

Change 
Table 2. CY 1995 Monitoring Program Summary (cont d)

Addendum $=95-02$

Sampling

Point

GW-545

GW-827
Action

Drop Sampling Point Add Sampling Point
Program

SWDF

SWDF
Sampling Frequency

0 $52 \& 4$
Analytical Parameters

LF3

LF3
Effective

Date

02/14/95

$02 / 14 / 95$

Addendum =95-03

Sampling

Point

GW-142S

GW-144S

GW-145s

GW-146S
Action

Add Sampling Point

Add Sampling Point

Add Sampling Point

Add Sampling Point
Program

DMP

DMP

DMP

DMP
Sampling

Frequency

Q1

Q1

Q1

Q1
Analytical Parameters

MET(1),RAD(3)

MET(1),RAD(3,7)

$\operatorname{RAD}(3,7)$

RAD(7)
Effective

Date

$03 / 30 / 95$

$03 / 30 / 95$

$03 / 30 / 95$

03/30/95

-Addendum $=95-04$

\section{Sampling}

Point

GW-142

GW-143

GW-144

GW-145

GW-146

GW-147

GW-231

\section{Action}

Change Prog.

Change Prog.

Change Prog.

Change Prog.

Change Prog.

Change Prog.

Change Prog.

\section{Sampling}

Frequency

Program

DMP

DMP

DMP

DMP

DMP

DMP

DMP
Q

Q

Q

Q

Q

Q
Analytical Parameters

STD,PHEN,REP,RAD(3,7)

STD,PHEN,REP,RAD (3,7) STD,PHEN, REP,RAD $(3,7)$ STD,PHEN,REP,RAD $(3,7)$ STD,PHEN,REP,RAD $(3,7)$ STD,PHEN,REP,RAD $(3,7)$ STD,PHEN,REP,RAD (3,7)
Effective

Date

$03 / 20 / 95$

03/20/95

$03 / 20 / 95$

$03 / 20 / 95$

$03 / 20 / 95$

$03 / 20 / 95$

$03 / 20 / 95$ 
Table 2. CY 1995 Monitoring Program Summary (cont'd)

-Addendum $=95-06$

$\begin{array}{llllll}\begin{array}{lll}\text { Sampling } \\ \text { Point }\end{array} & \text { Action } & \text { Program } & \text { Sampling } & \text { Effective } \\ \text { SCR2.2SP } & \text { Add Sampling Point } & \text { BMP } & \text { Q1 } & \text { STD,RAD(2) } & \text { Date }\end{array}$

Addendum $=95-14$

\begin{tabular}{|c|c|c|c|c|c|}
\hline $\begin{array}{l}\text { Sampling } \\
\text { Point }\end{array}$ & Action & Program & $\begin{array}{l}\text { Sampling } \\
\text { Frequency }\end{array}$ & Analytical Parameters & $\begin{array}{l}\text { Effective } \\
\text { Date }\end{array}$ \\
\hline $\begin{array}{l}\text { GW-539 } \\
\text { GW-709 } \\
\text { GW-757 }\end{array}$ & $\begin{array}{l}\text { Change Prog. } \\
\text { Change Prog. } \\
\text { Change Prog. }\end{array}$ & $\begin{array}{l}\text { SWDF } \\
\text { SWDF } \\
\text { SWDF }\end{array}$ & $\begin{array}{l}\$ 134 \\
\$ 134 \\
\$ 134\end{array}$ & $\begin{array}{l}\text { LF4 } \\
\text { LF1 } \\
\text { LF1 }\end{array}$ & $\begin{array}{l}07 / 13 / 95 \\
07 / 13 / 95 \\
07 / 13 / 95\end{array}$ \\
\hline
\end{tabular}

Addendurn $=95-24$

\begin{tabular}{|c|c|c|c|c|c|}
\hline $\begin{array}{l}\text { Sampling } \\
\text { Point }\end{array}$ & Action & Program & $\begin{array}{l}\text { Sampling } \\
\text { Frequency }\end{array}$ & Analytical Parameters & $\begin{array}{l}\text { Effective } \\
\text { Date }\end{array}$ \\
\hline GW-158 & Drop Sampling Point & DMP & $\mathbf{0}$ & STD,PHEN,REP & $10 / 01 / 95$ \\
\hline$G W-241$ & Drop Sampling Point & DMP & 0 & STD,PHEN,REP & $10 / 01 / 95$ \\
\hline GW-303 & Drop Sampling Point & DMP & 0 & STD,PHEN,REP & $10 / 01 / 95$ \\
\hline ow-304 & Drop Sampling Point & DMP & 0 & STD,PHEN,REP & $10 / 01 / 95$ \\
\hline
\end{tabular}


Table 2. CY 1995 Monitoring Program Summary (cont'd)

Addendum $=95-25$

Sampling

Point

GW.156

GW-159

GW-731

GW.732
Action

Change Prog.

Change Prog.

Change Prog.

Change Prog.
Sampling

Frequency Analytical Parameters

Q4,X4

Q4,X4

Q4,X4

Q4,X4
Effective

Date

$\begin{array}{llll}\text { PCDM } & \text { Q4,X4 } & \text { FLD,CHEM,MET(1,2),RAD(1) } & 10 / 01 / 95 \\ \text { PCDM } & \text { Q4,X4 } & \text { FLD,CHEM,MET(1,2),RAD(1) } & 10 / 01 / 95 \\ \text { PCDM } & \text { Q4,X4 } & \text { FLD,CHEM,MET(1,2),RAD(1) } & 10 / 01 / 95 \\ \text { PCDM } & \text { Q4,X4 } & \text { FLD,CHEM,MET(1,2),RAD(1) } & 10 / 01 / 95\end{array}$

Addendum $=95-26$

Sampling

Point

GW-732S

Action

Program

Sampling

Frequency

Analytical Parameters

Effective

Date

Add Sampling Point

DMP

Q3

FLD,CHEM,MET(1),RAD(1)

$08 / 14 / 95$

Addendum $=95-30$

Sampling

Point

GW-321S

Sampling

Point

GW-295

\section{Action}

Add Sampling Point

Program

CERCLA

Addendum =95-31

Sampling

Frequency

Analytical Parameters

Effective

Date

Q1

MET(1),Br,Cyanide,RAD(1)

$01 / 10 / 95$ 
Table 2. CY 1995 Monitoring Program Surnmary (cont'd)

\section{SAMPLING POINT:}

\section{EXPLANATION}

$\begin{array}{lll}\text { CBS-1 } & - & \text { Chestnut Branch Spring 1 } \\ \text { GW } & - & \text { Monitoring well } \\ \text { S } & - & \text { Special event sampling in addition to routine monitoring } \\ \text { SCR2.2SP } & - & \text { South Chestnut Ridge Spring, kilometer } 2.2 \\ 1090 & - & 1000 \text { series monitoring well }\end{array}$

\section{LOCATION:}

$\begin{array}{lcl}\text { ADB } & - & \text { Ash Disposal Basin } \\ \text { CDLVI } & - & \text { Construction/Demolition Landfill VI } \\ \text { CDLVII } & - & \text { Construction/Demolition Landfill VII } \\ \text { CRBAWP } & - & \text { Chestnut Ridge Borrow Area Waste Pile } \\ \text { CRSDB } & - & \text { Chestnut Ridge Sediment Disposal Basin } \\ \text { CRSP } & - & \text { Chestnut Ridge Security Pits } \\ \text { ECRWP } & - & \text { East Chestnut Ridge Waste Pile } \\ \text { KHQ } & - & \text { Kerr Hollow Quarry (located in Bethel Valley) } \\ \text { LI } & - & \text { Industrial Landfill II } \\ \text { LIV } & - & \text { Industrial Landfill IV } \\ \text { LV } & - & \text { Industrial Landfill V } \\ \text { RQ } & - & \text { Rogers Quarry (located in Bethel Valley) } \\ \text { UNCS } & - & \text { United Nuclear Corporation Site }\end{array}$

PROGRAM

$\begin{array}{lll}\text { BMP } & - & \text { Best-Management Practice Monitoring } \\ \text { DMP } & - & \text { RCRA Interim Status Detection Monitoring } \\ \text { PCDM } & - & \text { RCRA Post-Closure Detection Monitoring } \\ \text { SWDF } & - & \text { Landfill Detection Monitoring } \\ \text { CERCLA } & - & \text { CERCLA RI/FS Monitoring }\end{array}$

\section{SAMPLING FREQUENCY:}

Q:

Q1:

Q3:

Q4:

S 134:

S 1\&3:

S 284:

0.

X4:
Quarterly sampling

Annual sample to be taken in first calendar quarter Annual sample to be taken in third calendar quarter Annual sample to be kaken in fourth calendar quarter Samples to be taken in first, third and fourth calendar quarters Seniannual samples to be taken in first and third calendar quarters Semiannual samples to be taken in second and fourth calendar quarters

No samples to be taken

Replicate samples to be taken on 4 consecutive days 
Table 2. CY 1995 Monttoring Program Summary (cont'd)

\section{EXPLANATION}

\section{ANALYTICAL PARAMETERS:}

$\begin{array}{ll}\text { STD: } & \text { FLD, CHEM, MET(1), VOC(1), RAD(1) } \\ \text { LF1: } & \text { STD, MET(2,3), VOC(2,3), MISC(1,2) } \\ \text { LF2: } & \text { STD, MET(2), VOC(2), MISC }(1,2) \\ \text { LF3: } & \text { STD, MET(2,3), VOC }(3), \operatorname{RAD}(3,6), \operatorname{MISC}(1,2,3) \\ \text { LF4: } & \text { STD, MET(2,3), VOC }(2,3), \operatorname{RAD}(3,6), \operatorname{MISC}(1,2,3)\end{array}$

Br: Bromide

CHEM: Anions: Alkalinities(cartonate \& bicartonate), Chloride IC, Fluoride SIE, Nitrate Nitrogen, Sulfate

Other: $\quad \mathrm{pH}$, Specific conductance, Total dissolved solids, Total

Cyanide: Cyanide suspended solids, Turbidity

FLD: Field measurements: Depth to water, Dissolved oxygen, pH,Redox,Specific conductance, Temperature

MET(1): ICP: Aluminum, Antimony, Arsenic, Barium, Beryllium, Boron, Cadmium, Calcium, Chromium, Cobalt, Copper, Iron, Lead, Magnesium, Manganese, Molybdenum, Nickel, Potassium, Selenium, Silver, Sodium, Strontium, Thorium, Vanadium, Zinc Plasma Mass Spectrometry Uranium

\section{CVAA Mercury}

MET(2): AAS: Cadmium, Chromium, Lead

MET(3): Thallium

MISC(1): Total organic carbon, Total organic halides

MSC(2): Chemical oxygen demand, Cyanide

MISC(3): Ammonia

PHEN: Phenols

RAD(1): Gross alpha activity, Gross beta activity

RAD(2): $\quad$ Strontium, 99-Technetium, Tritum

RAD(3): 234-Uranium, 235-Uranium, 238-Uranium

RAD(4): 241-Americium, 129-Ladine, 237-Neptunium, 238-Plutonium, 239-Plutonium

RAD(5): Radium

RAD(6): Gamma activity

RAD(7): Strontium

REP: $\quad 4$ replicate samples each for $\mathrm{pH}$, Specific conductance, Total organic carbon, Total organic halides

Total U; Total uranium

TPH: $\quad$ Total petrolenm hydrocarbons 
Table 2. CY 1995 Monitoring Prograw Summary (cont'd)

\section{EXPLANATION}

ANALYTICAL PARAMETERS:

VOC(1):

1,1,1-trichloroethane

1,1,2,2-tetrachloroethane

1,1,2-trichlonothane

1,1-dichloroethane

1,1-dichloroethene

1,2-dictloroethane

1,2-dichlorcethene

1,2-dichloropropane

2-butanone

2-hexanone

4-methyl-2-pentanone

acetone

benzene

bromodichloromethane

bromoform

bromomethane

carbon disulfide

carbon tetrachloride

chlorobenzene

chlorodibromomethane

chloroethane

chloroform

chioromethane

cis-1,3-dichloropropene

ethylbenzene

methylene chloride

styrene

tetrachloroethene

toluene

trans-1,3-dichloropropene

trichloroethene

vinyl acetate

vinyl chloride

xylenes 
Table 2. CY 1993 Monitoring Program Summary (cont'd)

\title{
EXPLANATION
}

\section{ANALYTECAL PARAMETERS:}

$\operatorname{voc}(2):$

\author{
acrolein \\ acrylonitrile \\ 2-chloroethylvinyl ether \\ dibromomethane \\ 1,4-dichloro-2-butene \\ dichlorodifluoromethane \\ ethanol \\ ethyl methacrylate \\ iodomethane \\ trichlorofluoromethane \\ 1,2,3-trichloropropane \\ 1,1,1,2-tetrachloroethane \\ 1,2-dibrono-3-chloropropane \\ 1,2-dichlonobenzene \\ 1,4-dichlorobenzene \\ bromochloromethane \\ cis-1,2-dichloroethene \\ dibromomethane \\ trans-1,2-dichlonothene
}

voc(3): 
Table 3. General Construction Information for Monitoring Welts Sampled During 1995

\begin{tabular}{|c|c|c|c|c|c|c|c|}
\hline \multirow[b]{2}{*}{ Well No. } & \multirow[b]{2}{*}{ Location } & \multicolumn{2}{|c|}{ Aguifer } & \multicolumn{4}{|c|}{ Mogitored Interval } \\
\hline & & Unit & Formation & & Depths (bgs) & Ele & rations (msl) \\
\hline 1090 & UNCS & $\mathrm{AQF}$ & OCk & & - & & - \\
\hline$G W \cdot 141$ & LIV & $\mathrm{AQF}$ & OCk & 141.00 & -156.00 & 1042.50 & $=1027.50$ \\
\hline$G W-142$ & KHQ & $\mathrm{AQF}$ & OCk & 248.50 & -295.00 & 719.79 & - 673.29 \\
\hline GW-143 & KHQ & $\mathrm{AQF}$ & OCk & 205.00 & -253.00 & 706.04 & -658.04 \\
\hline GW-144 & KHQ & $\mathrm{AQF}$ & $\mathrm{OCk}$ & 148.00 & -195.00 & 762.48 & -715.48 \\
\hline GW-145 & $\mathrm{KHQ}$ & $\mathrm{AQF}$ & OCk & 86.00 & - 110.00 & 751.29 & -727.29 \\
\hline GW-146 & $\mathrm{KHQ}$ & $\mathrm{AQF}$ & OCk & 190.00 & -220.00 & 647.00 & -617.00 \\
\hline GW-147 & KHQ & $\mathrm{AQF}$ & OCk & 52.00 & -69.00 & 796.41 & -779.41 \\
\hline GW-IS6 & CRSDB & $\mathrm{AQF}$ & OCk & 146.00 & $-157,00$ & 900.90 & $-\quad 889.90$ \\
\hline GW-158 & CRSDB & $\mathrm{AQF}$ & $\mathrm{OCk}$ & 356.00 & $=441.00$ & 625.24 & - $\quad 540.24$ \\
\hline GW.159 & CRSDB & $\mathrm{AQF}$ & OCk & 146.00 & -157.00 & 902.80 & -891.80 \\
\hline$G W-160$ & CRBAWP & $\mathrm{AQF}$ & OCk & 205.00 & -235.00 & 885.70 & -855.70 \\
\hline GW-161 & CRBAWP & $\mathrm{AQF}$ & OCk & 350.00 & -400.00 & 740.90 & -690.90 \\
\hline GW-I7S & CRSP & $\mathrm{AQF}$ & OCk & 148.30 & -166.70 & 933.60 & -915.20 \\
\hline GW-177 & CRSP & $\mathrm{AQF}$ & OCk & 132.00 & $\cdot 145.00$ & 1023.50 & -1010.50 \\
\hline GW-181 & CRSP & $\mathrm{AQF}$ & OCk & 155.00 & -168.00 & 935.60 & $=922.60$ \\
\hline GW·184 & RQ & $\mathrm{AQT}$ & Och & 101.50 & -130.00 & 823.15 & -794.65 \\
\hline GW-186 & $\mathbf{R Q}$ & $\mathrm{AQT}$ & Och & 142.00 & $-\quad 171.00$ & $686.3 \mathrm{~J}$ & $-\quad 657.31$ \\
\hline GW-187 & $\mathbf{R Q}$ & $\mathrm{AQT}$ & Och & 139.00 & -162.00 & 692.59 & $\Rightarrow 669.59$ \\
\hline GW-188 & $\mathbf{R Q}$ & AQT & Och & 49.00 & $-\quad 68.00$ & 785.33 & -766.33 \\
\hline GW-203 & UNCS & $A Q F$ & $\mathrm{OCk}$ & 144.00 & - 156.00 & 958.30 & - 946.30 \\
\hline$G W-205$ & UNCS & $\mathrm{AQF}$ & OCk & 154.00 & -164.00 & 947.50 & $-\quad 937.50$ \\
\hline GW-217 & LIV & $\mathrm{AQF}$ & OCk & 165.20 & -180.00 & 1009.10 & - 994.30 \\
\hline GW-221 & UNCS & $\mathrm{AQF}$ & OCk & 147.00 & $=158.00$ & 956.40 & $+\quad 945.40$ \\
\hline GW-231 & KHQ & $\mathrm{AQF}$ & OCk & 22.80 & $=\quad 35.00$ & 824.10 & - 811.90 \\
\hline GW-241 & CRSDB & $\mathrm{AQF}$ & OCk & 78.00 & -103.00 & 902.80 & - 877.80 \\
\hline GW-292 & ECRWP & $\mathrm{AQF}$ & OCk & 172.10 & -186.00 & 898.00 & -884.10 \\
\hline GW-293 & ECRWP & $\mathrm{AQF}$ & OCk & 197,00 & $=214.00$ & 864.70 & - 847.70 \\
\hline
\end{tabular}


Table 3. General Construction Information for Monitoring Wells Sampled During 1995 (Cont'd)

\begin{tabular}{|c|c|c|c|c|c|c|c|}
\hline \multirow{3}{*}{$\begin{array}{l}\text { Well No. } \\
\text { GW-294 }\end{array}$} & \multirow{3}{*}{$\begin{array}{l}\text { Location } \\
\text { ECRWP }\end{array}$} & \multicolumn{2}{|c|}{ Aquifer } & \multicolumn{4}{|c|}{ Monitored Interval } \\
\hline & & \multirow{2}{*}{$\begin{array}{l}\text { Unit } \\
A Q F\end{array}$} & \multirow{2}{*}{$\begin{array}{l}\text { Formation } \\
\text { OCk }\end{array}$} & \multicolumn{2}{|c|}{ Depths (bgs) } & \multicolumn{2}{|c|}{ Elevations (ms) } \\
\hline & & & & 113.00 & -128.00 & 968.70 & -953.70 \\
\hline GW-296 & ECRWP & $\mathrm{AQF}$ & OCk & 134.40 & - 147.00 & 953.90 & - 941.30 \\
\hline GW-298 & CRBAWP & $\mathrm{AQF}$ & OCk & 171.10 & - 190.00 & 875.30 & - 856.40 \\
\hline GW-299 & CRBAWP & $\mathrm{AQF}$ & OCk & 153.00 & -168.00 & 898.30 & -883.30 \\
\hline GW-300 & CRBAWP & $\mathrm{AQF}$ & ock & 132.00 & -147.00 & 938.70 & $-\quad 923.70$ \\
\hline$G W-301$ & CRBAWP & $\mathrm{AQF}$ & OCk & 148.50 & -163.50 & 935.40 & $-\quad 920.40$ \\
\hline GW-302 & UNCS & $\mathrm{AQF}$ & $\mathrm{OCk}$ & 121.50 & - $\quad 134.80$ & 1018.10 & -1004.80 \\
\hline GW-303 & CRSDB & $\mathrm{AQF}$ & OCk & 300.00 & - 321.00 & 704.40 & - 683.40 \\
\hline GW-304 & CRSDB & $\mathrm{AQF}$ & OCk & 148.50 & -167.00 & 894.30 & -875.80 \\
\hline GW-305 & LIV & $\mathrm{AQF}$ & OCk & 165.30 & - 179.60 & 1015.80 & -1001.50 \\
\hline GW-321 & ADB & $\mathrm{AQF}$ & $\mathrm{OCk}$ & 84.00 & 98.60 & 839.10 & -824.50 \\
\hline GW-339 & UNCS & $\mathrm{AQF}$ & ock & 101.00 & -114.00 & 1021.20 & -1008.20 \\
\hline GW-511 & CRSP & $\mathrm{AQF}$ & OCk & 140.00 & -153.70 & 950.70 & -937.00 \\
\hline GW-S12 & ADB & $A Q F$ & OCk & 48.00 & -61.00 & 950.99 & - 937.99 \\
\hline GW-513 & $A D B$ & $\mathrm{AQF}$ & OCk & 111.00 & -125.30 & 887.99 & -873.69 \\
\hline GW-514 & $\mathrm{ADB}$ & $\mathrm{AQF}$ & $\mathrm{OCk}$ & 174.00 & - 195.00 & 824.66 & -803.66 \\
\hline GW-521 & LIV & $\mathrm{AQP}$ & OCk & 123.20 & - 136.00 & 1056.30 & -1043.50 \\
\hline GW-522 & LIV & $\mathrm{AQF}$ & OCk & 183.00 & - 195.30 & 989.00 & $-\quad 976.70$ \\
\hline GW-539 & LII & $\mathrm{AQF}$ & OCk & 136.40 & -156.00 & 954.00 & - $\quad 934.40$ \\
\hline GW-540 & CDL VI & $A Q F$ & $\mathrm{OCk}$ & 158.50 & -171.50 & 910.90 & $-\quad 897.90$ \\
\hline GW-541 & CDL VI & $\mathrm{AQF}$ & OCk & 86.70 & -104.50 & 969.10 & -951.30 \\
\hline GW-542 & CDL VI & $\mathrm{AQF}$ & $\mathrm{OCk}$ & 59.00 & 76.50 & 990.00 & $-\quad 972.50$ \\
\hline GW-543 & CDL VI & $\mathrm{AQF}$ & $\mathrm{OCk}$ & 76.20 & $-\quad 93.60$ & 945.00 & -927.60 \\
\hline GW-544 & CDL VI & $\mathrm{AQF}$ & OCk & 91.00 & - 109.30 & 951.50 & - 933.20 \\
\hline GW-546 & CDL VI & $A Q F$ & OCk & 66.20 & - 84.40 & 1003.40 & - 985.20 \\
\hline GW-557 & ILF V & $\mathrm{AQF}$ & OCk & 112.90 & -138.00 & 965.70 & -940.60 \\
\hline GW.S60 & CDL VII & $\mathrm{AQF}$ & OCk & 45.20 & 69.00 & 890.97 & $-\quad 867.17$ \\
\hline GW- 562 & CDL VII & $\mathrm{AQF}$ & ock & 36.00 & 60.00 & 895.86 & -871.86 \\
\hline
\end{tabular}


Table 3. General Construction Information for Monitoring Wells Sampled During 1995 (Cont'd)

\begin{tabular}{|c|c|c|c|c|c|c|c|c|}
\hline \multirow{3}{*}{$\frac{\text { Well No. }}{\text { GW-564 }}$} & \multirow{3}{*}{$\begin{array}{l}\text { Location } \\
\text { CDL VII }\end{array}$} & \multicolumn{2}{|c|}{ Aquifer } & \multicolumn{5}{|c|}{ Mopitored Interval } \\
\hline & & \multirow{2}{*}{$\frac{\text { Unit }}{A Q F}$} & \multirow{2}{*}{$\begin{array}{l}\text { Formation } \\
\text { OCk }\end{array}$} & \multicolumn{2}{|c|}{ Depths (bgs) } & \multicolumn{3}{|c|}{ Elevations (msl) } \\
\hline & & & & 52.00 & 81.00 & 883.12 & ـ & 854.12 \\
\hline GW-608 & CRSP & $A Q F$ & $\mathrm{OCk}$ & 148.00 & - $\quad 220.00$ & 923.00 & - & 851.00 \\
\hline GW- 609 & CRSP & $\mathrm{AQF}$ & OCk & 256.40 & -269.00 & 853.30 & - & 840.70 \\
\hline GW-610 & CRSP & $\mathrm{AQF}$ & OCK & 105.10 & - 117.40 & 951.70 & - & 939.40 \\
\hline$G W-611$ & CRSP & $\mathrm{AQF}$ & OCk & 101.50 & -121.60 & 943.90 & - & 923.80 \\
\hline GW-709 & LII & $\mathrm{AQF}$ & OCk & 68.70 & 80.60 & 835.14 & - & 823.24 \\
\hline GW-731 & CRSDB & $\mathrm{AQF}$ & OCk & 164.00 & $=\quad 178.70$ & 881.80 & 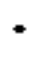 & 867.10 \\
\hline GW-732 & CRSDB & $\mathrm{AQF}$ & OCk & 178.30 & -190.00 & 882.40 & - & 870.70 \\
\hline GW-742 & CRSP & $A Q F$ & OCk & 350.00 & -420.00 & 747.80 & - & 677,80 \\
\hline GW-743 & CRSP & $\mathrm{AQF}$ & OCk & 150.10 & $=161.10$ & 948.60 & - & 937.60 \\
\hline GW-757 & L II & $\mathrm{AQF}$ & OCk & 134,00 & -166.70 & 824.65 & - & 791.95 \\
\hline GW-796 & LF V & $\mathrm{AQF}$ & OCk & 122.90 & $=136.50$ & 925.90 & - & 912.30 \\
\hline GW-797 & LLF V & $\mathrm{AQF}$ & OCk & 118.00 & -134.10 & 938.10 & " & 922.00 \\
\hline GW-798 & CDL VII & $\mathrm{AQF}$ & OCk & 122.00 & - 135.40 & 880.42 & - & 867.02 \\
\hline GW-799 & IIF V & $A Q F$ & $O C k$ & 78.70 & - $\quad 92.00$ & 899.40 & - & 886.10 \\
\hline GW-801 & ILF V & $A Q F$ & ock & 175.80 & -188.90 & 918.02 & - & 904.92 \\
\hline GW-827 & CDL VII & $\mathrm{AQF}$ & OCk & 122.10 & -134.80 & 926.03 & - & 913.33 \\
\hline
\end{tabular}

Fostinotes:

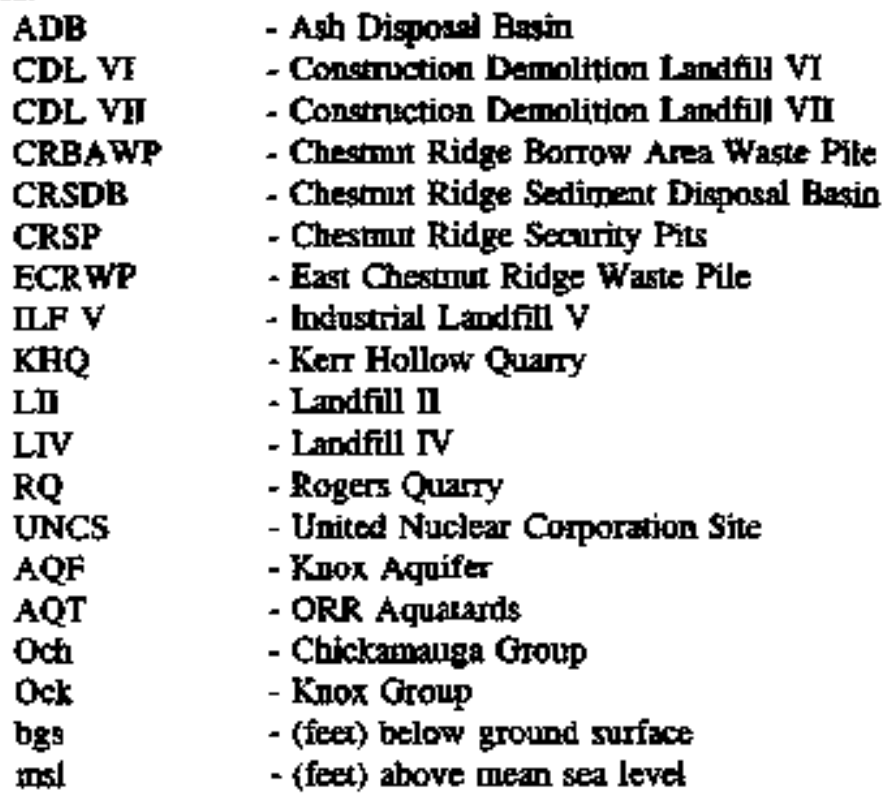


APPENDIX C

MONITORING WELL CONSTRUCTION DETAILS 



\section{EXPLANATION}

LOCATION:

$\begin{array}{rll}\text { ADB } & : & \text { Ash Disposal Basin } \\ \text { CDLVI } & : & \text { Construction/Demolition Landfill VI } \\ \text { CDLVII } & : & \text { ConstructionDertolition Landfill VI } \\ \text { CRBAWP } & : & \text { Chestrut Ridge Bortow Area Waste Pile } \\ \text { CRSDB } & : & \text { Chestnut Ridge Sediment Disposal Basin } \\ \text { CRSP } & : & \text { Chestnut Ridge Security Pits } \\ \text { ECRWP } & : & \text { East Chestnut Ridge Waste Pile } \\ \text { KHQ } & : & \text { Kert Hollow Quarty } \\ \text { LII } & : & \text { Industrial Landfill II } \\ \text { LIV } & : & \text { Industrial Landfill IV } \\ \text { ILFV } & - & \text { Industrial Landfill V } \\ \text { RQ } & : & \text { Rogers Quarry } \\ \text { UNCS } & - & \text { United Nuclear Corporation Site }\end{array}$

GENERAL INFORMATION:

$\begin{array}{rll}\text { Coordinates } & - & \text { Y-12 Plant grid system } \\ \text { Elevations } & - & \text { Feet above mean sea level } \\ \text { Depths } & - & \text { Feet below ground surface } \\ \text { AQF } & - & \text { Knox Aquifer } \\ \text { AQT } & - & \text { ORR Aquitards } \\ \text { B } & - & \text { Bedrock Interval } \\ \text { WB } & - & \text { Weathered Bedrock } \\ \text { U } & - & \text { Unconsolidated } \\ \text { OCK } & - & \text { Knox Group } \\ \text { Och } & : & \text { Chickamauga Group } \\ - & - & \text { Not Applicable or not available }\end{array}$

CASING:

OD (in) - Outside diameter, in inches

Bonehole Diameter - Noninal diameter, in inches Conductor Casing Type:
PVC/ $\# 0$
SS/1304
Polyvinyl chloride, schedule 40
Steel - carton steel
F-25/I-55 - American Petroleum Institate Grade 



\title{
EXPLANATION (cont'd)
}

\section{MONITORED INTERVAL:}

\author{
Top - Depth to top of filter pack or open-hole \\ Bottom - Depth to bottom of filter pack or open-hole \\ Screen Material: \\ PVC/sl - PVC, slotted \\ PVC/sw/.01 - PVC, spiral wound, 0.01 inch slot size \\ SS/sw/.01 - Stainless steel, spiral wound, 0.01 inch slot size \\ SS/sl/.0I - Stainless steel, slotted, 0.01 slot size
}

Length of screen and open hole interval is in feet.

NOTE:

Data Compiled from Updated Substufface Data Base for Bear Creek Valley, Chestnut Ridge, and parts of Bethel Valley on the U.S. Department of Energy Oak Ridge Resernation. (Jones et al. 1995) 


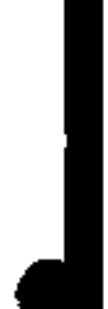


AFPENDIX C

Monitoring Well Constraction Details

\begin{tabular}{|c|c|c|c|c|c|c|c|}
\hline well No. & 1090 & GW-141 & GW-142 & OW-143 & $G W+144$ & GW-I45. & GW-I46 \\
\hline Strinplo Group & CR2 2 & CR I & CR? & $\mathrm{CR} 7$ & $\mathrm{CR} 7$ & CR 7 & CR? \\
\hline loxation & UPCS & LV & KHOQ & KHO & KHQ & KHQ & KHQ. \\
\hline \multicolumn{8}{|l|}{ GENERA1 BNORMATION } \\
\hline Emst Coondimans & 33853.02 & 32463.00 & 69030.00 & 6352200 & 63502.00 & 6266.00 & 63272.00 \\
\hline Noxth Coordinute & 28718.02 & 28755.00 & 24524,00 & 24257.00 & 24255.00 & $24 \$ 41.00$ & 24432.00 \\
\hline Massuring PL Exovtion & 1103.90 & 1186.10 & 970.35 & 913.18 & 913.34 & 840.04 & 838.16 \\
\hline Ground Flerition & $110 \mathrm{~L} 60$ & 1183.50 & 968.29 & 911.04 & 910.48 & 83729 & 837.00 \\
\hline Hydroctratiguntic tint & $A Q F$ & $\mathrm{AOF}$ & $\mathbf{A Q F}$ & $A Q F$ & $A Q F$ & $\mathrm{AQF}$ & $A Q F$ \\
\hline Geologic Form:ticn & oCh & $O C$ & $o c k$ & oct & OCk & OCk & OCk \\
\hline Adgnifer Zone & $W B$ & B & B & $\mathbf{B}$ & $\mathbf{B}$ & $\oplus B B$ & B \\
\hline Wouthered Rock - Depth & - & . & . & . & . & . & - \\
\hline Wealherod Rock - Elovation & $\cdot$ & . & . & . & - & - & - \\
\hline Fresh Rosk - Depth & - & $\$ 7,0$ & . & 18.0 & . & . & 11.0 \\
\hline Freat Rock - Ekration & . & 1129.10 & . & 895.18 & . & . & 827.16 \\
\hline Total Depth Dorted & 96.70 & 156.00 & $2 \$ 5.00$ & 253.00 & 195.00 & 110.00 & 220.00 \\
\hline \multicolumn{8}{|l|}{ SURFACECONDUTCTOR CASING } \\
\hline Conduntor Caring Depth & . & 65.00 & 20.00 & 20.00 & 40.00 & 12,00 & 13.40 \\
\hline Conductor Cuing OD (in) & - & 10.75 & 12.50 & 10.63 & 1250 & 1250 & 1250 \\
\hline Conductor Cusing Type & Dote & st:cl/F25 & PVCillut & PVCl\#40 & PVCH40 & PVCHAT & PVCristo \\
\hline \multicolumn{8}{|l|}{ WELL CASNG } \\
\hline Borwhale Diantiter & 8.00 & 10.00 & 11.00 & 10.00 & 11.00 & [1..60 & 11.00 \\
\hline Well Celis Depth & - & 144.5 & 248.5 & 205.0 & 150.0 & 88.3 & 190.0 \\
\hline Woll Cing OD (in) & 6.50 & 4.50 & 6.62 & 6.62 & 4.50 & 4.50 & 6.62 \\
\hline Well Caning Trpe & PVCHAO & $\mathrm{SS} 3 \mathrm{HOA}^{-1}$ & skodF2s & steelF2s & PVC:A40 & 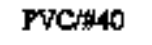 & steel/F25 \\
\hline \multicolumn{8}{|l|}{ MONITORED INTERVAL } \\
\hline Top Morutored loterval - Deqth & . & 141.0 & 248.5 & 205.0 & 148.0 & 86.0 & 190.0 \\
\hline Top Honitored Interwsl - Elcration & - & 104250 & 719.39 & 706.04 & 76248 & 75120 & 647.00 \\
\hline Midpotmi - Deptit & - & 548,5 & 27.8 & 229.0 & 171.5 & 980 & 205.0 \\
\hline Mldpoin - Elsution & , & 1033,00 & 695.54 & 682.04 & 738.98 & 739.29 & 632.00 \\
\hline Botsem Monitoned Iriarval - Depth & . & 156.0 & 295.0 & 253.0 & 195.0 & 110.0 & 230.0 \\
\hline 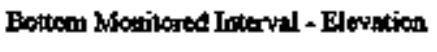 & - & 1027.50 & 673.29 & 658.04 & 715.48 & 727.29 & 617.00 \\
\hline Soun Mattill & PVCsl & SStsow 01 & . & . & PVCisw.01 & PVCisw.01 & - \\
\hline Serean Lenph & - & 10.7 & - & , & 40.0 & 20.0 & . \\
\hline Openolfole longth & - & - & 46.5 & 48.0 & - & - & 30.0 \\
\hline Qpenifolo Diamoter & 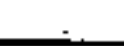 & - & 6.00 & 6.00 & . & . & 6.00 \\
\hline
\end{tabular}


APPENDIX C

Monitoring Well Construction Betatis

\begin{tabular}{|c|c|c|c|c|c|c|c|}
\hline Well No. & $6 \mathrm{~W}-147$ & OWW 136 & GW-158 & GW-159 & GW-150 & CFP-161 & ow-175 \\
\hline Sample Croup & CR 7 & CR 6 & CR 6 & CR6 & $\mathrm{CR} 5$ & CR 5 & CR,3 \\
\hline Lacation & KHQ & CRSDB & CRSDB & CRSDB & CRBAW & CRBAWP & CRSP \\
\hline \multicolumn{8}{|l|}{ GENERAL, INFORMLATION } \\
\hline Eart Coordinats & 63428,00 & 64020.00 & 63640.00 & $634,96.00$ & 62165.00 & 62146.00 & 38686.00 \\
\hline North Coordinate & 24731,00 & 27626.00 & 27069.00 & 27T64,00 & 27803.00 & 27805.00 & 28676.00 \\
\hline Medering Ph Reviliox & $\$ 51.62$ & I049.10 & 983.05 & 1051.20 & 1093.10 & 1003.50 & $10 \$ 4.00$ \\
\hline Ground Elovation & 848.42 & 1046.90 & 981.24 & 1048.80 & 1090.70 & 1090.90 & 1081.90 \\
\hline Hydrostritionphie Init & $A Q P$ & $A Q F$ & $\mathrm{AQF}$ & $\mathrm{AQF}$ & AQR & AOF & $A C B$ \\
\hline Geologic Farmation & OCk & ack & ack & $\infty \times$ & OCk & $\phi c k$ & $\infty k^{\prime}$ \\
\hline Aquifer Zone & wB & B & B & wBSB & $\mathbf{w B}$ & $\mathbf{B}$ & $\mathbf{B}$ \\
\hline Weathered Rook - Depth & . & 84.0 & 87.0 & . & 60.0 & 65.0 & $\$ 6.0$ \\
\hline Westhered Rock - Elepation & . & 955.10 & 896.05 & - & 1033.10 & 1028.50 & 103800 \\
\hline Fresh Rock - Depth & 6.0 & 93.0 & 93.0 & 100.0 & - & $\$ \$ \$ .5$ & 985 \\
\hline Fresh Rock - Eteration & 845.62 & 956.10 & 890.05 & 951.20 & - & 998.00 & 985.50 \\
\hline Tofal Dioth Drilled & 69.00 & 157.00 & 441.00 & 157.00 & 235.00 & 400.00 & 166.70 \\
\hline \multicolumn{8}{|l|}{ SURFACEKCONDUCTOR CASING } \\
\hline Conturetor Cuting Depth & 4.60 & 94.00 & 94.00 & 123.00 & 122.50 & 108.00 & 61.30 \\
\hline Conductor Coxting OD (in) & 10.69 & 10.75 & 10.75 & 10.75 & 12.50 & 12.50 & 10.75 \\
\hline Conductor Cesing Type & PVCJ40 & steol/125 & steel/r25 & Hod/F25 & tseel/25 & steel/25 & unknown \\
\hline \multicolumn{8}{|l|}{ WEL CASING } \\
\hline 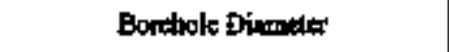 & 10.010 & 8.50 & 10.00 & 8.50 & 11.00 & $11.0,0$ & 9.50 \\
\hline well Ceffars Depth & 59.1 & 147.0 & 356.0 & 147.0 & 205.0 & 350,0 & 150.6 \\
\hline Wêl Caxping OD (in) & 4so & 4.50 & 6.62 & 4.30 & 6.62 & 6.62 & 4.50 \\
\hline Well Casing Type & PtNCHAO & PUCHAD & steatrzs & Ptecras0 & ted/T2S & toel/T2s & Ssfiks04 \\
\hline \multicolumn{8}{|l|}{ MONIFORED NTERVAL } \\
\hline Top Mcesitored heterval - Depth & 52.0 & 146.0 & 356.0 & 146.0 & 205.0 & - 330.0 & 1483 \\
\hline Top Morftered Inervel - Elovation & 796.41 & 900.90 & 625.24 & 90280 & 885.70 & 740.90 & 933.60 \\
\hline Mtipoin + Doplh & 60.5 & 151.5 & 398.5 & 131.5 & 22000 & 375.0 & 157.5 \\
\hline Midpoint - Elevation & 787.91 & 895.10 & 582.74 & 897,30 & 870.70 & 715,90 & 924,40 \\
\hline Boltem Marifered Intermal - Depth & 69.0 & 157.0 & 44.0 & 157.0 & 235.0 & 400.0 & 166.7 \\
\hline Bothom Metricred loterval - Ekevation & 779.41 & 8solo & 540.24 & 891.80 & 855.70 & 690.90 & $\$ 1520$ \\
\hline Screen Mialorial & Pvetswiol & PVCiswiol & . & Pyctowt.01 & - & - & $881 \times 4+01$ \\
\hline Screen Lengh & 15.0 & 20.0 & . & 10.0 & - & - & 15.8 \\
\hline Open-Hole laggh & . & - & 85.0 & . & 30.0 & 50.0 & . \\
\hline Dpeo-Hole Dianoter & & & 6,000 & & 6.00 & 11.00 & \\
\hline
\end{tabular}


APPENDIX C

Moutioring Well Construction Details

\begin{tabular}{|c|c|c|c|c|c|c|c|}
\hline & GW-177 & GW-181 & GW-184 & GW-186 & $G w-187$ & GW-188 & $G+203$ \\
\hline Smpite Grotip & $\mathrm{CR} 3$ & $\mathrm{CR} 3$ & $\operatorname{CR} 9$ & $\operatorname{CR} 9$ & CR9 & CR 9 & CR 2 \\
\hline Loffion & CRSP & CRSP & $\mathbf{R Q}$ & Ro. & $\mathrm{RQ}$ & RQ & UNCS \\
\hline GENERAL BFFORMATTON & & \multirow[b]{2}{*}{57736.00} & \multirow[b]{2}{*}{57475.00} & \multirow[b]{2}{*}{56862.00} & \multirow[b]{2}{*}{$599 \$ 0.00$} & \multirow[b]{2}{*}{56174.00} & \multirow[b]{2}{*}{54190.48} \\
\hline Exst Coxrdinate & 57497.00 & & & & & & \\
\hline Noxth Coordinents & 28483.00 & 2804800 & 23915.00 & 23387,00 & 23393.00 & 23663.00 & 28355.82 \\
\hline Mesencing Ph. Elovation & 115800 & 1003.00 & 927.63 & 831.32 & 834.28 & 897.09 & 1105.30 \\
\hline Ground Elevition & 1153.50 & 1090.60 & 924.65 & 828.31 & 831.39 & 834.39 & 1102.30 \\
\hline 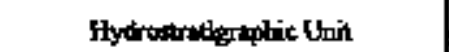 & AQF & $A O F$ & $A Q Q T$ & AQT & AQT & A'T & AQF \\
\hline Geotapic Formation & OCk & OCk & Och & as & Och & och & $\phi \mathbf{x}$ \\
\hline Artifita Zon & wB & $\mathbf{B}$ & B & B & $\mathbf{B}$ & wE & WAB \\
\hline Weathered Rock - Depth & 62.0 & 43. & . & 12.0 & 7,0 & 13.0 & 86.0 \\
\hline Wewthred Rodk - Elrvition & 1096.00 & 1090.00 & , & 819.32 & $\$ 27.28$ & 824.09 & 1019.30 \\
\hline Fresh Rock - Depth & 98.0 & 78.0 & $\mathbf{8 . 0}$ & 145 & 15.0 & 14,0 & 93,0 \\
\hline Frath Rock - Elrvation & 1060.00 & 1015.00 & 919.83 & 816.82 & 819.28 & 823.00 & 1012.30 \\
\hline Toul Depth Drilled & 145.00 & 168.00 & 130.00 & 171.00 & I 62.00 & 68.00 & 156.00 \\
\hline SLRFACECONDIJTOR CASTNG & & & & & & & \\
\hline Consuator Cruins Depth & 82.00 & 102.00 & 8.00 & 14,50 & 7.00 & 15.00 & 94.00 \\
\hline Conductur Casing OD (m) & 10.75 & 10.75 & 10.53 & 10.63 & 10.63 & 10,63 & 10.75 \\
\hline Condintor Casing Type & atioel/F2s & $n t w / 72 s$ & Pros $\# 40$ & PUCHASO & PuC/440 & PVC $\$ 40$ & $\operatorname{al}=1 / 25$ \\
\hline WELL CASDIG & & & & & & & \\
\hline Barahale Diemeter & 8.00 & 9.50 & 10.00 & 10.00 & 10.00 & 10.00 & 8.50 \\
\hline Well Cring Deph & 133.0 & 155.0 & 1005 & 150.0 & 147,0 & 52.5 & $\$ 46.0$ \\
\hline well Cwing OD (in) & 4.50 & 6.62 & 4.50 & 4.30 & 4.50 & 4.50 & 4.50 \\
\hline Well Csing Tye & PVC $\$ 40$ & Tted/F2s & Pvethot & PVCH40 & PVC\#40 & PVCHA & Ptres $\$ 40$ \\
\hline MONITORED INTERVAL & & & & & & & \\
\hline 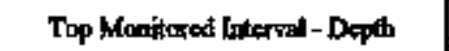 & 132.0 & 155.0 & 1015 & 1420 & 139.0 & $4 \$ .0$ & 144.0 \\
\hline Top Monitored butaval - Elevation & 1023.50 & 935.60 & 823.13 & 586.31 & 692.59 & 785.33 & $\$ 5830$ \\
\hline Mfictpoint - Depth & 1385 & 161.5 & 115.8 & 156.5 & 350.3 & 58.5 & 130.0 \\
\hline Misdpoint - Elevetion & 1017.00 & 929.10 & 8008.90 & 671.81 & 681.09 & 375.83 & 952.30 \\
\hline Botinn Monitored frtarval - Depth & 145.0 & 168.0 & 130.0 & 171,0 & 162.0 & 68.0 & 1560 \\
\hline Bottcon Horitored interval - Elevation & 1010.50 & 922.60 & 794.65 & 65731 & 669.59 & 766.33 & 946.30 \\
\hline Scen Moterit & Pvers & . & PVCtaw & PrCiswon,01 & PYC'sw/, & PvClswi.01 & PVC sqw/At \\
\hline Scrom Lotiogh & 10.0 & - & 20.0 & 20.0 & 15.0 & 15.0 & 10.0 \\
\hline Open-ifide Lauth & . & 13.0 & . & . & . & . & . \\
\hline Opar Hole Diometit & 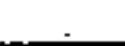 & 6.00 & 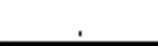 & 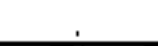 & . & . & . \\
\hline
\end{tabular}


APPENDIX C

Monitoring WeB Construction Detalits

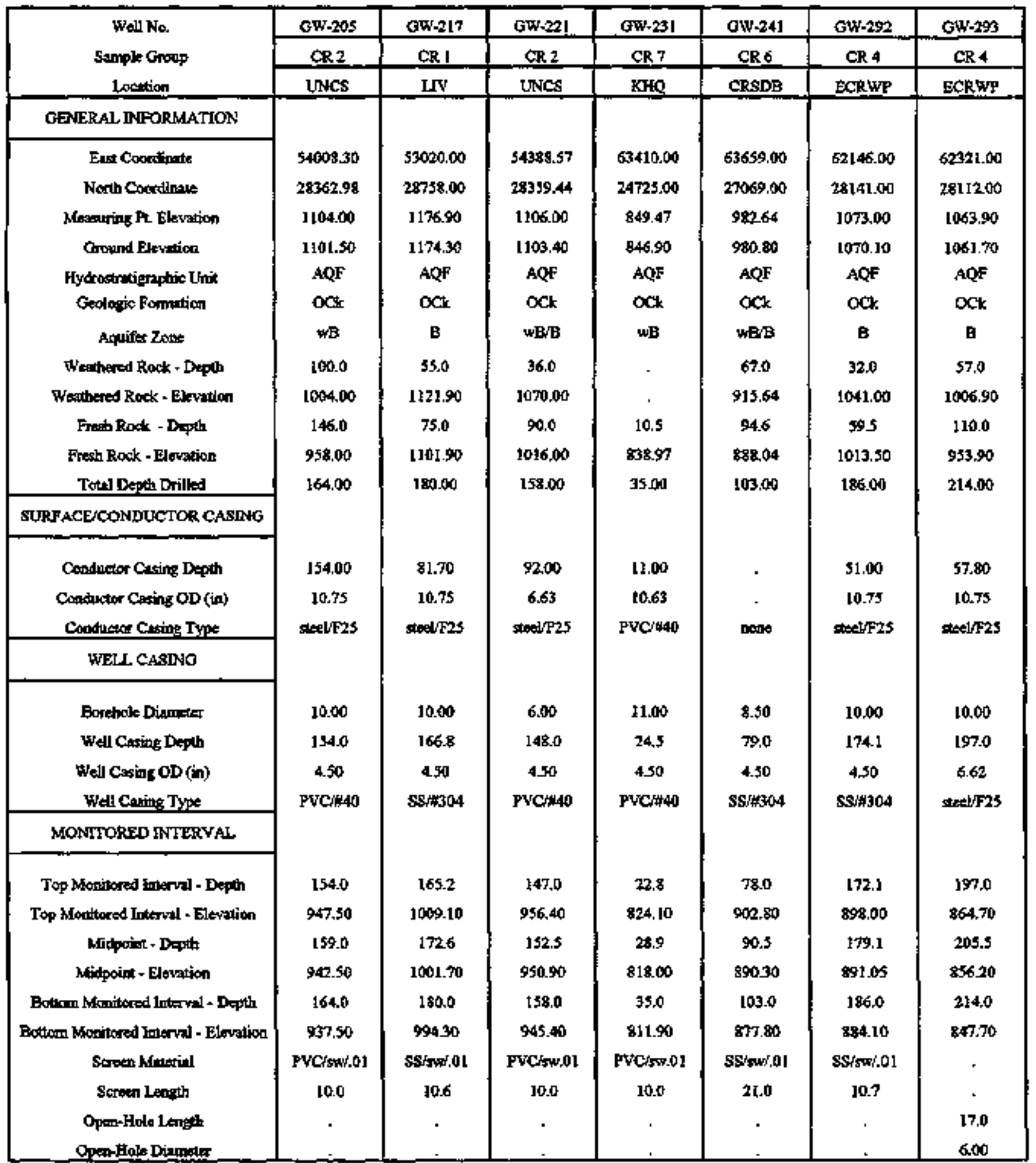


APPENDIX C

Monitoring Well Construction Detsils

\begin{tabular}{|c|c|c|c|c|c|c|c|}
\hline well No. & GW-294 & $C W+296$ & chw-298 & GW-29s & CW-300 & $G(w-30)$ & GW-302 \\
\hline Sranple Grour & CR 4 & $\mathrm{CR} 4$ & CR 5 & CR 3 & CRs & CR.5 & $\mathrm{CR}_{2}$ \\
\hline Lacation & ECRWP & ECRWP & CEBAWP & CRPAWP & CREAWP & CRRAWP & UNocs \\
\hline \multicolumn{8}{|l|}{ GENERAL INFORHATION } \\
\hline East Coostidinte & \multirow{2}{*}{$\begin{array}{l}62483.00 \\
27958.00\end{array}$} & \multirow{2}{*}{$\begin{array}{l}62023.00 \\
27994.00\end{array}$} & \multirow{2}{*}{$\begin{array}{l}67445.00 \\
27495.00\end{array}$} & \multirow{2}{*}{$\begin{array}{l}62319.00 \\
27392.00\end{array}$} & \multirow{2}{*}{$\begin{array}{l}52041.00 \\
27487.00\end{array}$} & \multirow{2}{*}{$\begin{array}{l}61964.00 \\
27662.00\end{array}$} & $\$ 4353.40$ \\
\hline North Coondinsts & & & & & & & 28693.55 \\
\hline Mesosuting in Eievintion & 1083.60 & 1091.00 & 1049.60 & 1053.90 & 1073.10 & 3086.40 & 1141.70 \\
\hline Oround Elovetion & 1081.70 & 108830 & 1046.40 & IfG5130 & $1020 . x$ & 1089,90 & 1139.50 \\
\hline Hydractratigntphic Lail & $M Q F$ & $A Q F$ & $A Q F$ & $A Q F$ & $A Q F$ & $A Q P$ & $A Q F$ \\
\hline Geologic formation & oct & DCK & och & ock & $\mathbf{\infty} \mathbf{x}$ & OCk & OCk \\
\hline Aquifer Zons & $B$ & $\mathbf{B}$ & $\mathbf{B}$ & $\mathbf{B}$ & $\mathbf{B}$ & B & $\mathbf{B}$ \\
\hline Wauthored Rock - Deqth & 62.0 & 67,0 & 650 & 66.0 & 1020 & 94.0 & 63.0 \\
\hline Wenthered Rock - Ekeralion & 1021.60 & 3024.60 & 984.00 & 987.90 & 971.10 & 992.40 & 1678.70 \\
\hline Fresh Rock - Depth & 87.0 & 82.0 & 80.0 & 70.0 & 106.0 & 136.0 & 1020 \\
\hline Fresoh Rack - Elevetín & 996.60 & 1008.00 & 969.00 & 983.90 & \$s7.10 & 950.40 & 1039.70 \\
\hline Tots] Depth Drilled & 128.00 & 147.00 & 190.00 & 168.00 & 147.00 & 163.50 & 136.00 \\
\hline SUTRFACEACONDUCTOR CASING & & & & & & & \\
\hline Confuctor Ceting Depth & 74,50 & 86.50 & 83.30 & 80.00 & 115.00 & 105.000 & 63.80 \\
\hline Conductor Cutig $O D$ (in) & 10.75 & 10.75 & 10.75 & 10.75 & 10.75 & 10.75 & 10.75 \\
\hline Coaduck Cating Typ & steakis & sted/525 & stedF25 & atedIF2s & Aod/F2s & steel/f25 & well \\
\hline WEL 1 CASDNG & & & & & & & \\
\hline Bcrotholo Diameter & 10.00 & 10.00 & 10.00 & 10.00 & 10.00 & 10.00 & 9.50 \\
\hline Wolt Cesing Depth & 117.6 & 136.3 & 176.0 & I55.8 & 134.8 & 151.0 & 124.5 \\
\hline wall Craig OD (im) & 4.50 & 4.50 & 4.50 & 4so & 4.50 & 4.50 & 4.50 \\
\hline Well Cring Type & SSIA304 & $\mathrm{SS} / \mathrm{A3} 04$ & SSN304 & SSI $\$ 304$ & SSH期34 & SSH:Ho4 & SStwo4 \\
\hline MONTIORED LNTERVAL & & & & & & & \\
\hline Tot Mtinitored Intenvil - Depth & 113.0 & 134.4 & $17 \mathrm{l} .1$ & 153.0 & 1320 & 148.5 & 121.5 \\
\hline Top Monitured Intruvl + Elepation & 968.70 & 953.90 & 875.30 & 898.30 & 938.70 & 935.40 & 1018.10 \\
\hline MEdpoirt - Depth & 120.5 & 140.7 & 180.6 & 160.5 & 139.5 & 136.0 & I28 2 \\
\hline Mripoint - Elepation & 961.20 & 947.60 & 8.65 .85 & 890.80 & 931.20 & 92790 & 1015,45 \\
\hline Bottam Matilored herval / Dopth & 1280 & 147.0 & 190.0 & 168.6 & 147.0 & 163.5 & 194.8 \\
\hline Botbun Monitored Lter wal - Elovation & 953.70 & 941.30 & 856.40 & 88930 & 929.70 & 920.40 & 100480 \\
\hline Sareen Materit & stostaw & SS/sout.01 & SStowtol & sstawto1 & SNowito1 & SStswdo1 & SStswer,0l \\
\hline Screen Lenghth & 10.4 & 10.5 & 10.0 & 10.8 & 10.6 & هـ10 & 10.3 \\
\hline Oparthole Lomith & . & . & . & . & , & . & $\cdot$ \\
\hline OperrHolo Diamoler & & & . & &. & & $\cdot$ \\
\hline
\end{tabular}


APPENDEX C

Monitoring Well Construction Detrils

\begin{tabular}{|c|c|c|c|c|c|c|c|}
\hline Well No. & OWF-303 & $054-304$ & GW-30s & 020-321 & CW-339 & GH-S1I & GW-S12 \\
\hline Sumple Group & CR6 & C요 6 & CR $]$ & CR 8 & CR 2 & CR3 & CR 8 \\
\hline Looution & CRSDB & CRSDB & LIV & $A D B$ & tnNCs & CRSP & $A D E$ \\
\hline \multicolumn{8}{|l|}{ GENERAL INFORMATION } \\
\hline Eust Coortinute & 63488.00 & 69425.00 & $\$ 2962.00$ & 57026,00 & $54] 46.52$ & $\$ 7739.00$ & $573 \$ 3.00$ \\
\hline North Cocratipts & 23099.00 & 27545.00 & 28548.00 & 25275,00 & 28658.72 & 28056.00 & 27601.00 \\
\hline Matsuring Ph Elovation & 1007.20 & 1045.50 & 1183.60 & 925.58 & 1124.60 & 1093.20 & 1001.50 \\
\hline Ground Elaradica & 1004,40 & 1042.80 & $118 \mathrm{~L} .10$ & 923.10 & 112220 & 1090.70 & 998.99 \\
\hline Hydrodtatigrephic Unil & $A Q F$ & $A Q F$ & $A Q F$ & $A Q F$ & $\mathbf{A Q F}$ & $A Q F$ & AQf \\
\hline Goologie Formanion & $O C k$ & ock & ock & ock & ock & OCk & $\alpha c k$ \\
\hline Arpoiffer Zoot & B & $\mathrm{B}$ & $\mathrm{B}$ & & $\mathbf{B}$ & B & UwE \\
\hline Wonthered Roct - Depth & 37.0 & 35.0 & $\$ 3.0$ & . & 45.0 & 40.5 & $\$ 5.0$ \\
\hline Wealhered Rock - Elevation & 970.20 & 1010.50 & 1130.60 & . & 1079.60 & Ios2.70 & 94650 \\
\hline Frasb Rock - Depth & t]I. 0 & 92,0 & 84,0 & - & 91.0 & 100.0 & $\cdot$ \\
\hline Fresth Rack - Ekevition & $\$ 9.20$ & 953.50 & 1099.60 & . & 1033.60 & 99320 & . \\
\hline Totul Dopeth Drilled & 321.00 & 167.00 & 179.60 & 98.60 & 11400 & 133.70 & 61.00 \\
\hline \multicolumn{8}{|l|}{ SURFACEKONDUCTOR CASING } \\
\hline Conitustor Caring Depth & 4.80 & 90.00 & 64.00 & 2.200 & 91.00 & 100.00 & . \\
\hline Conduoter Cusing OD (m) & 10.35 & 10.75 & 10.75 & 10.75 & 10.75 & 10.75 & - \\
\hline Conducter Craing Type & Steelf23 & uriknotsin & stelelis25 & stedish & sted & miknown & notse \\
\hline \multicolumn{8}{|l|}{ WELL CASNM } \\
\hline Borebole Dingute & 10.00 & 9.75 & 10.00 & 800 & 9.30 & 9.60 & 9.50 \\
\hline Well Casing Dopech & 300.0 & 156.5 & 168.9 & 87.3 & 103.7 & 1433 & 50.5 \\
\hline Wotl Cming oD (in) & 7.00 & 450 & 4.50 & 450 & 4.50 & 4.50 & 4,50 \\
\hline Well Ceting Type & $\operatorname{step} V$ & ssikso4 & SSWHO4 & ssist34 & $85 / 3304$ & $5 s / 4304$ & $8 \$ 7304$ \\
\hline \multicolumn{8}{|l|}{ MONITORED INIERVAL } \\
\hline Top Monitarad linterval - Depth & 300.0 & 148.5 & 1653 & 84.0 & 101.0 & 140.0 & 48.0 \\
\hline Top Honitored hiterval - Elevetion & 704.40 & 994.30 & 1015.80 & 839.10 & 1021.20 & 950.70 & 980.99 \\
\hline Midpaint - Dopth & 310.5 & 1578 & 1725 & 913 & 107.5 & 146.9 & 54.5 \\
\hline Midpoin - Elenaion & 699.90 & 885.05 & 1008.65 & 891.80 & tot4.7n & 943.85 & 944.49 \\
\hline Bctrom Marikared Irterve - Depth & 321.0 & 167.0 & 179.6 & 98.6 & 114.0 & $I \$ 3.7$ & 61.0 \\
\hline Bottom Monitored therval - Elaustion & 688.40 & 875.80 & 1001.50 & $\$ 2.50$ & 100820 & 93700 & 037.99 \\
\hline Scren Mirtial & - & SStswion & ssitwatol & ssisw.01 & SSiswitol & skiow. 01 & SSIsw \\
\hline Scraen Length & - & 10.0 & 10.7 & 10.7 & 10.3 & 90.0 & 10.0 \\
\hline Opeo-Hole Ithoth & 21.0 & . & . & . & - & . & - \\
\hline Opens-Fyle Diander & 6.00 & . & & & & & \\
\hline
\end{tabular}


APPENDEX C

Monitaring Well Construction Defoils

\begin{tabular}{|c|c|c|c|c|c|c|c|}
\hline \multirow{3}{*}{$\begin{array}{c}\text { Well No. } \\
\text { Sumplo Group } \\
\text { Lopation }\end{array}$} & GH.513 & GW.514 & GW.52! & $\mathrm{GPH}-522$ & GW.539 & GW-540 & GW-541 \\
\hline & CR: & CR 8 & CR 1 & CR 1 & CR 10 & CR 11 & CR IJ \\
\hline & AD旦 & $\mathrm{ADB}$ & LYV & $\mathbf{L V}$ & In & CDL VI & CDL VI \\
\hline \multicolumn{8}{|l|}{ GENERAI, NNFORMATION } \\
\hline Exst Coondinate & \multirow{2}{*}{$\begin{array}{l}57332.00 \\
27607.00\end{array}$} & 57342,00 & $\$ 2040.00$ & 52612.00 & 52278.26 & \multirow{2}{*}{$\begin{array}{l}52371.31 \\
27489.06\end{array}$} & \multirow{2}{*}{$\begin{array}{l}51738.09 \\
27653.53\end{array}$} \\
\hline North Coostinute & & 27575.00 & 28541.00 & 28377.00 & 2719253 & & \\
\hline Newguring Pt Elovation & 1001.40 & 1001.20 & 1182.70 & 117530 & 1095.00 & 3072.10 & 1058.40 \\
\hline Grownd Elevition & 99899 & 998.66 & 1179.50 & \pm 172.00 & $\log 0.40$ & 1069.40 & 1055.80 \\
\hline Hydrodratigraphic Unit & $A Q F$ & $A Q F$ & $A Q F$ & $A Q F$ & $A Q F$ & $A Q F$ & $A Q F$ \\
\hline Geologic Formming & OCK & ock & 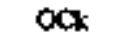 & OCk & OCk & OCk & ock \\
\hline Aqpiiler Zow & B & B & B & $\mathbf{B}$ & B & B & B \\
\hline Weflhmed Rock - Dapth & 67,0 & 44.0 & . & 85.0 & . & 110.0 & 53.5 \\
\hline Wealikered Rock - Elevation & 934.40 & 957.20 & . & 1090.30 & . & 962.10 & 1004.90 \\
\hline Eresh Rock - Dopth & 97.0 & $\mathbf{9 2 . 0}$ & 54.0 & 130.0 & 740 & 150.0 & 69.5 \\
\hline Fresh Roct + Elevation & 90440 & 90920 & 1128.70 & 104530 & 1019.00 & 922.10 & 98890 \\
\hline Tatal Deph Drilled & 125.30 & 195.00 & 136.00 & 195.50 & 156.00 & 171.50 & 104.50 \\
\hline SURFACECONDUCTOK CASDEG & & & & & & & \\
\hline Conduater Casing Depth & 98.00 & 105.00 & 80.50 & 90.tol & 79,00 & 154.00 & 64.10 \\
\hline Conducto: Casing OD (iim) & 10.75 & 10.75 & 10.75 & 10.75 & 10.75 & 10.75 & 10.75 \\
\hline Condustor Caning Typ & unknown & nonknown & utionout & miknownte & Anel & stect & 파요 \\
\hline WELI CASING & & & & & & & \\
\hline Bocibole Diamoter & 9.50 & 9.50 & 9.50 & 9.50 & 9.25 & 9.25 & 9.25 \\
\hline Well Cosing Dapth & 114.8 & 174.0 & 124.9 & 184.6 & 139.8 & 161.2 & 86.8 \\
\hline Wall Cusing OD (in) & 4.50 & 6.62 & 4.50 & 4.50 & 4.50 & 4.50 & 4.50 \\
\hline Wedl Ctsing Typs & SSH304 & IoxlF2s & Ssikso4 & SS/i304 & SS/R304 & ssill304 & ssipso4 \\
\hline MONITORED INTERVAL & & & & & & & \\
\hline Top Monitored Interval - Depth & 111.0 & 174.0 & 123.2 & 183.0 & 136.4 & t58.5 & 86.7 \\
\hline Top Monticted linerval - Elevetion & 887,99 & 824.66 & 105630 & 989.00 & 954,00 & 910.90 & 969.10 \\
\hline Mitipoint - Deptit & 118.2 & 184.5 & 179.6 & 1892 & 146.2 & 163,0 & 95.6 \\
\hline Midpoind - Elevation & 880,84 & 814.16 & 1049,90 & 982.85 & 944.20 & 904.40 & 960.20 \\
\hline Bonken Monitored hiervi - Dephh & 125.3 & 195.0 & 136.0 & 1953 & 156.0 & 171.5 & 104.5 \\
\hline Bottonn Monitared Intarnal - Elewation & 873.69 & 893.66 & 1043.50 & 976.70 & 934.40 & 897.90 & 9513 \\
\hline Sereen Nititid & Sstrow 01 & - & sstowtol & sDrowiot & ssidrol & SSłsllol & SSIStrol \\
\hline Bowen lanph & 10.0 & - & 10.3 & 10.4 & 15.9 & 10.3 & 15.7 \\
\hline Open-Hob Lengh & - & 21.0 & - & . & - & - & . \\
\hline Ogth-Fole Diamoter & & 6.00 & & & & - & \\
\hline
\end{tabular}

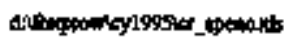


APPENDIX C

Mositoring Well Constructiou Detadls

\begin{tabular}{|c|c|c|c|c|c|c|c|}
\hline & G14-542 & $G w-543$ & GW-344 & GW-S46 & OW-557. & GW-560 & GW.562 \\
\hline Sanple Group & CR [] & CR II & $\operatorname{CR} 11$ & CR 11 & $\mathrm{CR} 12$ & $\mathrm{CR} 12$ & $C R 12$ \\
\hline Locentiog & CDL VI & CDL VT & CDL VI & CDL VI & LᄄF V & CDL VII & CDL VI \\
\hline \multicolumn{8}{|l|}{ GENERAL INFORMATION } \\
\hline Etat Coordinuto & \multirow{2}{*}{$\begin{array}{l}51641.74 \\
27466.22\end{array}$} & \multirow{2}{*}{$\begin{array}{l}31438.48 \\
270 \pi 2.06\end{array}$} & \multirow{2}{*}{$\begin{array}{l}51819.56 \\
26963.22\end{array}$} & \multirow{2}{*}{$\begin{array}{l}52366.34 \\
27473.75\end{array}$} & \multirow{2}{*}{$\begin{array}{l}59519.59 \\
26450.11\end{array}$} & \multirow{2}{*}{$\begin{array}{l}60743.42 \\
23692.28\end{array}$} & 61640.17 \\
\hline North Coordinate & & & & & & & 26276.29 \\
\hline Mesuring PL Elevetion & 1051.60 & 1023.80 & 1045.00 & 1072.20 & 1031.20 & 938.92 & 934.49 \\
\hline Groumd Elovation & 1049.00 & 102120 & 1042.50 & 1069.60 & 1078.60 & 936.17 & 931.86 \\
\hline Hydrostratigntiphic Unut & $A Q F$ & $A Q F$ & AQf & $A C F$ & $\mathrm{AOF}$ & $A Q F$ & AQF \\
\hline Geologic Formation & ock & ock & ock & ock & $o c x$ & OCk & OCx \\
\hline Aqtuifo Zone & $\mathrm{U}$ & $\mathbf{B}$ & B & UtwB & $W w \mathrm{~B}$ & $\mathrm{U} / \mathrm{wB}$ & Uh:B \\
\hline Werlhered Rock - Depth & - & 36.0 & 47.0 & 74.5 & . & . & . \\
\hline Weathered Rock - El-vetion & . & 1007.80 & 99800 & 997.50 & - & - & . \\
\hline Frest Roxk - Depth & . & 370 & 325 & . & $\cdot$ & , & 52.0 \\
\hline Freth Rock + Elevation & . & 986.80 & 992.30 & . & . & . & 88249 \\
\hline Total Derth Drilliod & 77.50 & 94.00 & 110.00 & 84.50 & 139.00 & $11+00$ & 133.00 \\
\hline SURFACENCONDUCTOR CASTNO & & & & & & & \\
\hline Corauctor Caxing Đuth & - & 29.30 & 54,50 & - & gs.tod & - & - \\
\hline Conductic Cusing $O D$ (m) & . & 10.75 & 10.75 & - & 10.75 & . & . \\
\hline Condoctor Cring Iype & nine & steat & steor & nose & stoed & none & nose \\
\hline WEL CASDNG & & & & & & & \\
\hline Bocabole Dirimeta & 9.25 & 9.25 & 9.25 & $9.2 \mathrm{~s}$ & 9.50 & 9.30 & 9.30 \\
\hline Well Congs Deph & 60.8 & 780 & 93.4 & 68.6 & 115.8 & 49.0 & 38.0 \\
\hline Well Casing OD (in) & 4.50 & 4.30 & 4.50 & 4.50 & 4.00 & 4.00 & 4.00 \\
\hline Welt Caging Type & ss/mo4 & S\$\$3 & $\operatorname{sen} 304$ & sst304 & sstuma4 & $8 \$ / 4304$ & SS/A304 \\
\hline MONTIORED INTERVAL & & & & & & & \\
\hline Top Moitlored hterval - Depth & 59.0 & 76.2 & 91.0 & 66.2 & 112.9 & 45.2 & 36.0 \\
\hline Tog Monitored Iflaval - Elovation & $9 \% 0.00$ & 965.00 & 951.50 & 1003.40 & 965.70 & 890.97 & 895.86 \\
\hline Midpoint - Dept & 67.8 & 84.9 & 100.2 & 75.3 & 125.5 & 37.1 & 48.0 \\
\hline Midpoini - Ekevation & 981.29 & 936.30 & 942.35 & 994,30 & 953.25 & 879.07 & 893.86 \\
\hline Bottom Monitared Intruval - Depth & 76.5 & 93.6 & 109.3 & 84.4 & 138.0 & 69.0 & 60.0 \\
\hline Bothom Menitored Interval - Elovation & 972.50 & 927.60 & 933.20 & 985.20 & 940,60 & 867.17 & 871.86 \\
\hline Streen Waterial & sstallor & ssididor & Sstat:01 & ssiansol & sstowtas & ssismt.01 & sognt.os \\
\hline Screen Lorith & 15,7 & 15.6 & 15.9 & 15.8 & 20.0 & 20.0 & 20,0 \\
\hline OpenrHole Langth & . & . & . & . & . & . & . \\
\hline Open-fiolo Disunoter & & . & 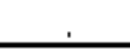 & - & - & - & $=$ \\
\hline
\end{tabular}


APPENDIX C

Monitorits Well Coustruction Detsilg

\begin{tabular}{|c|c|c|c|c|c|c|c|}
\hline Well Na & GW.S64 & GW-608 & GW-609 & GWW-610 & 0W-631 & $0 \% \cdot 709$ & GW-73] \\
\hline Sumple Group & CR 12 & CR3 & $\operatorname{CR} 3$ & CR 3 & $\mathrm{CR}_{3}$ & CR 10 & CR 6 \\
\hline Location & CDL VIl & CCيß口P & CRSP & CRSP & CESP & LII & CRSDB \\
\hline GENERAL INFORMATION & \multirow[b]{2}{*}{59965.28} & \multirow[b]{2}{*}{59724.35} & \multirow[b]{2}{*}{60039.73} & \multirow[b]{2}{*}{5947194} & \multirow[b]{2}{*}{58058.92} & \multirow[b]{2}{*}{52371.88} & \multirow[b]{2}{*}{63863.14} \\
\hline East Cocridinite & & & & & & & \\
\hline North Coordintare & 2587294 & 23889.43 & 2810043 & 28549.31 & 28855.52 & 25344.08 & 27463.63 \\
\hline Mearocing Pt. Elevationt & 937.77 & 1074.00 & 1112.10 & 1059.40 & 1048,40 & 906.60 & 1049.70 \\
\hline Gromed Elevation & 935.12 & $107 \mathrm{~L} .00$ & 1109.70 & 1056.80 & 1045.40 & 903.84 & J045.80 \\
\hline Hydrotentiprophic Unit & $A O F$ & $\mathrm{AOF}$ & $A Q F$ & $A Q F$ & $\Lambda Q F$ & AQF & $A Q P$ \\
\hline Oeologic Fontuation & ock & ock & OCk & $\alpha c$ & OXt & OCh & OCk \\
\hline Aquifer 2 onse & $U W \mathbb{B}$ & $\mathbf{w B S}$ & $\mathbf{w B A}$ & $w \mathrm{~B} B$ & wB/B & $\mathbf{B}$ & B \\
\hline Wenthered Rock - Dopth & . & 113.0 & . & 39.0 & 30.0 & 39.0 & 95.4 \\
\hline Weathered Rock - Elowation & . & 961.00 & . & 1020.40 & 1018.40 & 867.60 & 953.80 \\
\hline Fresh Rask - Depth & 20 & 1400 & I07.0 & 500 & 63.8 & 43.0 & 129.4 \\
\hline Freah Rock - Ekevetiont & 869.77 & 934.00 & IaAs. J0 & 1009.40 & 984.60 & 863.60 & 919.80 \\
\hline Tota1 Deith Dizilled & \multirow[t]{2}{*}{88.00} & 220.00 & 269.00 & 117.40 & 121.50 & 80.60 & 180.40 \\
\hline SURFACEROANUCTOR CASING & & & & & & & \\
\hline Coonductor Casing Deph & \multirow{3}{*}{$\cdot$} & \multirow{3}{*}{$\begin{array}{c}10.75 \\
\text { toed }\end{array}$} & \multirow{3}{*}{$\begin{array}{c}107.00 \\
10.75 \\
5001 / 255\end{array}$} & \multirow{3}{*}{$\begin{array}{c}107.10 \\
10.75 \\
\text { textst's5 }\end{array}$} & \multirow{3}{*}{$\begin{array}{c}75.00 \\
10.75 \\
\text { sos:-5s }\end{array}$} & \multirow{3}{*}{$\begin{array}{c}50.00 \\
11.75 \\
\text { stel/J-55 }\end{array}$} & \multirow{3}{*}{$\begin{array}{c}122.00 \\
11.75 \\
\text { steely-55 }\end{array}$} \\
\hline Contuntor Cacing OD (jn) & & & & & & & \\
\hline Condurtar Cosing Type & & & & & & & \\
\hline WELL CASDNO & & & & & & & \\
\hline Botehole Dianneter & \multirow{4}{*}{$\begin{array}{c}9.50 \\
55.3 \\
4.00 \\
5 S / 13504\end{array}$} & \multirow{4}{*}{$\begin{array}{l}15.50 \\
148.0 \\
10.75 \\
\text { mety } 5.55\end{array}$} & \multirow{4}{*}{$\begin{array}{c}9.50 \\
258.7 \\
4.50 \\
\$ \$ 1 \# 304\end{array}$} & \multirow{4}{*}{$\begin{array}{c}9,50 \\
107.1 \\
4,50 \\
5 S / \$ 504\end{array}$} & \multirow{4}{*}{ 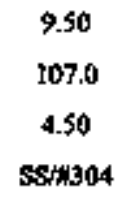 } & 10.60 & 10.60 \\
\hline WeIl Casing Dejth & & & & & & 70.4 & 165.2 \\
\hline Wellacesing OD (n) & & & & & & 4.35 & 4.50 \\
\hline Woil Cening Type & & & & & & SSH 304 & $58 / 18304$ \\
\hline MONITORED INTERVAL & & & & & & & \\
\hline Top Monitoced hataval + Deplh & $\$ 2.0$ & 148.0 & 256.4 & j0s.1 & 101.5 & 68.7 & 164.0 \\
\hline Top Monitored Intarnal - Elovation & 883.12 & 923.00 & 65330 & 951.30 & 943.96 & $\mathbf{8 3 5 . 1 4}$ & 881.80 \\
\hline Midpolate-Deplh & 66.5 & 1840 & 267.7 & 111.3 & 111.6 & 74.7 & 171.4 \\
\hline Midpoin - Elemulion & 868.62 & 887.00 & 847,00 & 944.55 & 933.85 & 829.19 & 874.45 \\
\hline Battom Moritared Interval - Dapth & 810 & 220.0 & 269.0 & 117.4 & 121.6 & 80.6 & 178.7 \\
\hline 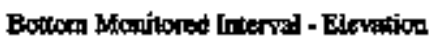 & 854.12 & 851.60 & 840.70 & 939.40 & 923.80 & 82324 & 867.10 \\
\hline Screten Mrateritt & SSisw/01 & . & SBrowtat & ssiswiol & SStrwtol & SSiswtol & $\$ S \sin t .01$ \\
\hline Screan Langalh & 200 & . & 10.3 & 10.3 & 10.0 & 10.0 & 10.0 \\
\hline Openthole Length & . & 720 & . & , & , & , & . \\
\hline Opan-Hole Dinatet & . & 9.90 &. & - &. & . & . \\
\hline
\end{tabular}


APPENDIX C

Monitoring Well Coustruction Detrils

\begin{tabular}{|c|c|c|c|c|c|c|c|}
\hline Well No. & $9 W / 732$ & GW-742 & $6 W-743$ & 0W-757 & GW-7\% & Gw.797 & GWFP \\
\hline Shante Group & CR6 & CR 3 & CR 3 & CR IO & CR 12 & Cat2 & CR t2 \\
\hline Loctation & CRSDB & CRSP & CRSP & $L \Pi$ & LF. V & DFv & CDL VII \\
\hline \multicolumn{8}{|l|}{ GENERAL INFOROLATKON } \\
\hline East Cocrdinute & 64267.74 & 58908.00 & 58908.00 & 5330252 & 38206.40 & 38550.40 & 60309.95 \\
\hline Narth Coondinste & 27716.72 & 28038.00 & 2805600 & 23409.50 & 27923.90 & 27446.60 & 27264.85 \\
\hline Mesturing Pt Elaration & 1064.10 & 1101.00 & 1100.40 & $96 t .43$ & 1052.40 & 1059.80 & 1005.80 \\
\hline Ground Elevelion & 1060.70 & 1097,80 & 1098.70 & 958.65 & 1048.80 & 1056.10 & 100242 \\
\hline Hydrophatigaphic Init & AQF & AQF & $\mathrm{AQF}$ & AQF & $A Q F$ & $A Q F$ & $A Q F$ \\
\hline Geologie Forpmion & $\operatorname{ack}$ & OCK & OCk & $\infty \mathrm{Ck}$ & ock & ock & oxik \\
\hline Aquifier Zone & B & B & B & $\mathbf{B}$ & B & $\mathbf{B}$ & $\mathbf{B}$ \\
\hline Weathered Roek - Depth & 85.0 & 68.0 & 49.0 & 29.5 & 100.0 & 67.1 & 94,4 \\
\hline Wenthered Rock - El=Vution & 979.10 & 1033,000 & 2051,40 & 991.93 & 950.40 & 992.70 & $9 t 1.40$ \\
\hline Pr=h Rock - Depth & 96.0 & 96.0 & 67.5 & 480 & 103.0 & 89.0 & 95.8 \\
\hline Fresh Rock - Elevattogi & 968.10 & 1005.00 & 101290 & 913.47 & 949.40 & 970.80 & 910.00 \\
\hline Total Doch Drillod & 19060 & 420.00 & 161.10 & 166.90 & 139.70 & $I 34.10$ & 135.50 \\
\hline \multicolumn{8}{|l|}{ SURFACEACONDUCTOR CASDG } \\
\hline Conductor Caring Drpth & 100.70 & 92.00 & 82.90 & 16.80 & 107,60 & 95.02 & 99.70 \\
\hline Condexter Casing OD (n) & 11.75 & II.75 & 11.75 & 10.75 & 10.75 & 10.75 & 30.75 \\
\hline Conducta Crsing Type & stex/JJ-55 & ates/25 & $\operatorname{ten} y / 25$ & thed/J-55 & stesl1-35 & teet/J-55 & ated/2-55 \\
\hline \multicolumn{8}{|l|}{ WELI CASING } \\
\hline Bernebole Diameter & 10.60 & 10.60 & 10.60 & 9.62 & 950 & 9.30 & 9.50 \\
\hline woll Casing Dopt: & 173 & 350.0 & 150.2 & 135.5 & 126.5 & 123.5 & 124.5 \\
\hline WoIt Casing OD (in) & 4.50 & 7.00 & 450 & 4.50 & 4,50 & 4.50 & 4.50 \\
\hline well Cusing Tyx & $8 s+404$ & 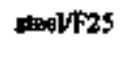 & $3 s$ in:304 & SSSita04 & SS:1304 & Ssinotio4 & $\operatorname{ssin304}$ \\
\hline \multicolumn{8}{|l|}{ WONITOFED NNIERVAL } \\
\hline Top Mocitsited [xerval - Depth & 178.3 & 350.0 & 150.1 & 1340 & 1229 & 118.0 & 1220 \\
\hline Top Monilotod heleval - Eleration & 882.40 & 747.80 & 948.60 & 824.65 & 925.90 & 938.10 & 880,42 \\
\hline Mditpoin - Dapth & 1842 & 385.0 & 155.6 & 150.4 & 129.7 & 126.1 & 128.7 \\
\hline Midpoint - Elovation & 876.55 & 712.80 & 943.10 & 808.30 & 919.10 & 930.05 & 873.72 \\
\hline Buttom Moritured htersal + Depth & 190.0 & 420.0 & 161.1 & 1667 & 136.5 & 134.1 & 135.4 \\
\hline 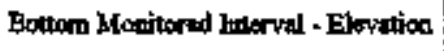 & 870.70 & 677.80 & 937.60 & 79193 & 91230 & 922.00 & 867.02 \\
\hline scroen Mrlerial & Ss/gw/ot & . & $8 \$ \alpha w / 01$ & 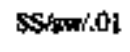 & SSiswida1 & Sstrwiol & SS/SWIOl \\
\hline Sereen latglb & 10.0 & - & 10.1 & 30.0 & 10.0 & 10.0 & 10.6 \\
\hline Oper-Hols Length & . & 70.0 & . & . & . & . & - \\
\hline Openthole Dismoter & & 6.25 & . & & & - & \\
\hline
\end{tabular}


APPSNDLX C

Monitoring Well Constmuction Detolls

\begin{tabular}{|c|c|c|c|}
\hline Wedl Na & Gw-799 & Gư-801 & $G w-827$ \\
\hline Sample Group & CR 12 & CR 12 & CR 11 \\
\hline Location & ILFv & IfF V & CDL VII \\
\hline GENERAL INFORMATION & \multirow[b]{2}{*}{5906120} & \multirow[b]{2}{*}{38779.90} & \multirow[b]{2}{*}{51826.32} \\
\hline Enst Coondinate & & & \\
\hline North Coortinnte & 26745.50 & 26807.80 & $2+721.42$ \\
\hline Mossuring PL Elavation & \multirow{2}{*}{981.09} & \multirow{2}{*}{$\begin{array}{l}1006.96 \\
1093.82\end{array}$} & 1051.39 \\
\hline Ground Eleverticn & & & 1048.13 \\
\hline Hyctodtatigapbic Unil & $A Q F$ & \multirow{2}{*}{. } & $A Q F$ \\
\hline Geolagic Frampion & \multirow{2}{*}{$\begin{array}{c}\text { OCK } \\
B\end{array}$} & & \multirow{2}{*}{$\begin{array}{c}\text { oCx } \\
\mathbf{B}\end{array}$} \\
\hline Aquifer Zooe & & $\mathbf{B}$ & \\
\hline Weathared Rock - Depth & \multirow{5}{*}{$\begin{array}{c}60.8 \\
920.29 \\
62.8 \\
918.29 \\
92.00\end{array}$} & \multirow{5}{*}{$\begin{array}{l}112.50 \\
984.46 \\
113.40 \\
989.56 \\
188.90\end{array}$} & \multirow{5}{*}{$\begin{array}{c}. \\
40.50 \\
1010.89 \\
135.00\end{array}$} \\
\hline Wenthered Rock - Elovulion & & & \\
\hline Freeth Rock - Depth & & & \\
\hline Prowh Rock - Elevelion & & & \\
\hline To4니 Depth Dentied & & & \\
\hline \multicolumn{2}{|l|}{ SURFACEICONDUCTOR CASING } & \multirow{4}{*}{$\begin{array}{c}115.40 \\
10.75 \\
\end{array}$} & \multirow{4}{*}{$\begin{array}{l}43.40 \\
30.73 \\
5 \text { tecel }\end{array}$} \\
\hline Conductor Caning Depth & \multirow{3}{*}{$\begin{array}{c}65.00 \\
10.75 \\
\text { stexp1-55 }\end{array}$} & & \\
\hline Conductor Cusing OD (in) & & & \\
\hline Congutax Cying Tyx & & & \\
\hline WEIL CASING & & & \\
\hline Borehols Diariater & 9.50 & \multirow{4}{*}{$\begin{array}{c}9.87 \\
178.10 \\
450 \\
\$ 8 / * 304\end{array}$} & \multirow{4}{*}{$\begin{array}{c}\$ .87 \\
124.10 \\
4.90 \\
\sin 304\end{array}$} \\
\hline Well Cusing Dejih & 81.0 & & \\
\hline We्ll Caing OD (in) & 4.50 & & \\
\hline Well Casing Type & \multirow[t]{2}{*}{$s s / 4304$} & & \\
\hline MONITORED INTERYAL & & \multirow[b]{2}{*}{175.80} & \multirow[b]{2}{*}{122.10} \\
\hline Top Monitered literval - Bepth & 78.7 & & \\
\hline Top Kknitored Intorval + Elevation & 809.40 & 91802 & 926.03 \\
\hline Midpoinl - Depth & 85.4 & 382,35 & \pm 28.45 \\
\hline Midpoint - Elevatien & 892.75 & 911.47 & 919.68 \\
\hline Bottom Menitored linterral - Depth & 920 & 1880 & 134.80 \\
\hline Bottem Mconitoned lmierval - Flevation & 856.10 & 904.92 & 913.33 \\
\hline Sereen Mtterial & SStronitol & Stawtol & SSfawt.01 \\
\hline Scrom Langth & 10.0 & 1000 & 10.00 \\
\hline Open-Hole Lengh & . & . & . \\
\hline OparfHok Dimeter & & & - \\
\hline
\end{tabular}


APPENDIX D

SAMPLING SEQUENCES 



\section{EXPLANATION}

\section{SAMPLING GROUP:}

Series of sampling locations grouped for data tracking and accounting purposes.

\section{SAMPLE IDENTITY:}

$\begin{array}{rll}\text { Dupe } & - & \text { Field Duplicate Sample } \\ \text { ER } & - & \text { Equipment Rinsate Saraple } \\ \text { FB } & - & \text { Field Blark } \\ \text { GW } & - & \text { Groundwater Monitoring Well } \\ \text { CBS-1 } & - & \text { Chestmat Branch Spring 1 } \\ \text { SCR2.2SP } & - & \text { South Chestnut Ridge Spring 2.2 } \\ 1090 & - & \text { Historical 1000-Series Monitoring Well } \\ \text { RS } & - & \text { Resample }\end{array}$

\section{LOCATION:}

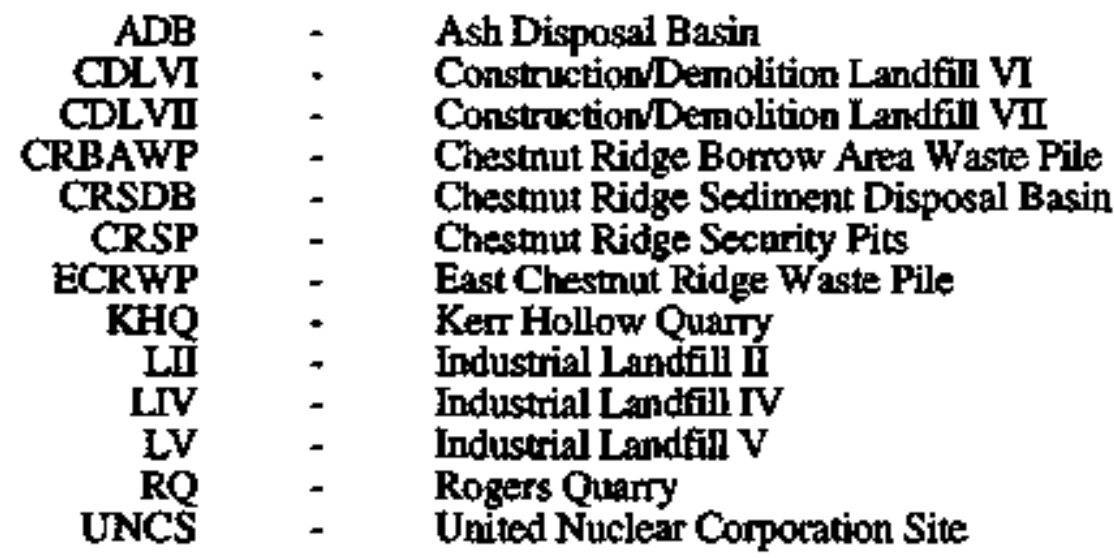

\section{PUMP NUMBER:}

Denotes identification number of gas-piston parnp used to purge the well and collect nonvolatile samples. If pump number is denoted by a " $W$ ", this indicates that the well has a dedicated sampling systern (i.e., Well Wizard).

\section{PURGE TIME:}

The time (in hours:minutes) that groundwater was purged from the monitoring well before samples were collected. If missing (indicated by a ${ }^{n}{ }^{n}$ ), purging took place over more than one day or purging was not required for a location/sample type (t.e. spring station).

\section{NOTE:}

Data compiled from sample tracking data sets (H\&R Technical Associates, Inc. 1995a, 1995b, 1996a, 1996b). 
APPENDIX D

Sampling Sequence, First Quarter 1995

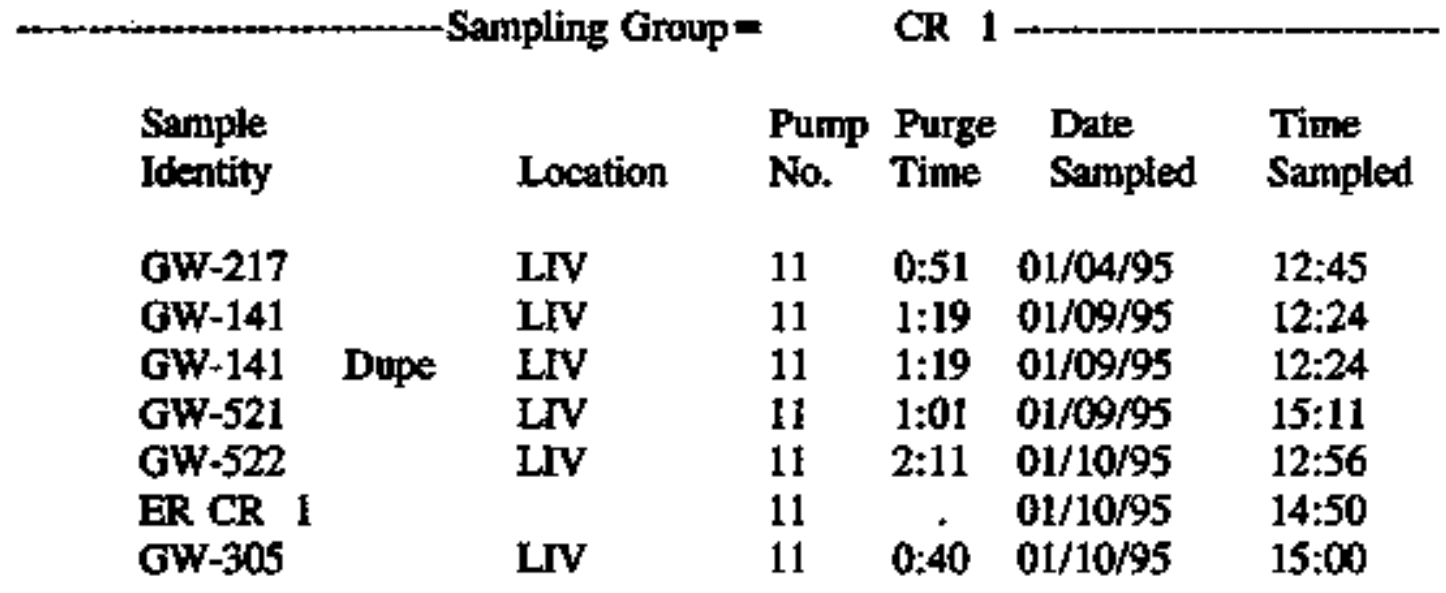

Sampling Growp=

CR 3

Sample

Identity

$\begin{array}{llll} & \text { Pump Purge } & \text { Date } & \text { Time } \\ \text { Location } & \text { No. Time } & \text { Sampled } & \text { Sampled }\end{array}$

GW-181

GW-511

GW-511

$\begin{array}{ll} & \text { CRSP } \\ \text { CRSP } & \text { CRSP } \\ \text { CRSP } \\ \text { CRSP } \\ \text { CRSP } \\ \text { CRSP } \\ \text { CRSP } \\ \text { CRSP } \\ \text { CRSP } \\ \text { CRSP } \\ \text { CRSP }\end{array}$

2 . 01/24/95 15:00

$2 \quad 1: 25 \quad 01 / 24 / 95 \quad 16: 40$

GW-742

$2 \quad 1: 25 \quad 01 / 24 / 95 \quad 16: 40$

GW-743

$2 \quad 1: 25 \quad 01 / 27 / 95 \quad 13: 45$

GW-610

$2 \quad 1: 0901 / 31 / 95$

$11: 19$

GW-608

$2 \quad 1: 14 \quad 01 / 31 / 95$

$15: 19$

GW-177

$2.02 / 02 / 95$

$17: 46$

GW-611

$2 \quad 0: 33 \quad 02 / 13 / 95$

$15: 10$

GW-175

ER CR 3

$2 \quad 0: 20 \quad 02 / 14 / 95$

$11: 00$

$20: 59 \quad 02 / 14 / 95 \quad 14: 00$

GW-609

$\begin{array}{cccc}2 & - & 02 / 15 / 95 & 14: 15 \\ 2 & 2.47 & 02 / 15 / 95 & 14: 22\end{array}$

Sampling Group=

CR 6 .

Sample

Identity

GW-159

GW-304
Location

CRSDB

CRSDB
Pump Purge Date

No. Time Sampled Sampled

11 11

$\begin{array}{lll}0: 36 & 01 / 15 / 95 & 11: 30 \\ 0: 45 & 01 / 15 / 95 & 14: 30\end{array}$


APPENDIX D

Sampling Sequence, First Quarter 1995

Sampling Group=

CR 6 (continued)

Sample

Identity

Location

Pump Purge Date

No. Time Sampled

Time

GW-731

GW-732

GW-732

GW-156

FB CR 6

GW-303

ER CR 6

ER CR 6

GW-241

GW-158

$\begin{array}{cccccc} & \text { CRSDB } & 11 & 0: 56 & 01 / 17 / 95 & 15: 10 \\ \text { CRSDB } & 11 & 0: 45 & 01 / 18 / 95 & 10: 30 \\ \text { CRSDB } & 11 & 0: 45 & 01 / 18 / 95 & 10: 30 \\ \text { CRSDB } & 11 & 0: 13 & 01 / 18 / 95 & 12: 15 \\ & 11 & . & 01 / 19 / 95 & \\ & & 11 & . & 01 / 19 / 95 & 16: 18 \\ & \text { CRSDB } & 11 & . & 01 / 20 / 95 & 11: 30 \\ & & 2 & : & 01 / 20 / 95 & 11: 30 \\ & & 11 & 1: 05 & 01 / 20 / 95 & 11: 35 \\ \text { CRSDB } & 11 & & 01 / 20 / 95 & 11: 40\end{array}$

CR 7

Sample

Identity

GW-142

GW-147

GW-231

GW-143

GW-143

GW.146

GW-144

ER CR 7

GW-145

GW-142

GW-144

GW-146

GW-145

$\begin{array}{lll} & \text { Pump Purge Date } & \text { Time } \\ \text { Location } & \text { No. Time Sampled } & \text { Sampled }\end{array}$

$\begin{array}{cccccc} & \text { KHQ } & 7 & 3: 30 & 01 / 10 / 95 & 13: 45 \\ \text { KHQ } & 7 & 0: 48 & 01 / 15 / 95 & 12: 00 \\ \text { KHQ } & 7 & 0: 43 & 01 / 15 / 95 & 13: 03 \\ & \text { KHQ } & 7 & 4: 10 & 01 / 17 / 95 & 17: 30 \\ \text { KHQ } & 7 & 4: 10 & 01 / 17 / 95 & 17: 30 \\ \text { KHQ } & 7 & & 01 / 17 / 95 & 18: 00 \\ \text { KHQ } & 7 & 2: 37 & 01 / 18 / 95 & 11: 37 \\ & 7 & & 01 / 18 / 95 & 13: 50 \\ \text { KHQ } & 7 & 1: 00 & 01 / 18 / 95 & 14: 00 \\ \text { KHQ } & 7 & 2: 38 & 03 / 20 / 95 & 15: 20 \\ \text { KHQ } & 7 & 2: 24 & 03 / 22 / 95 & 14: 14 \\ \text { KHQ } & 7 & : & 03 / 23 / 95 & 14: 10 \\ \text { KHQ } & 7 & 1: 03 & 03 / 24 / 95 & 10: 45\end{array}$




\section{APPENDIX D}

Sampling Sequence, First Quarter 1995

\begin{tabular}{|c|c|c|c|c|c|}
\hline $\begin{array}{l}\text { Sample } \\
\text { Identity }\end{array}$ & Location & $\begin{array}{l}\text { Pump } \\
\text { No. }\end{array}$ & $\begin{array}{l}\text { Purge } \\
\text { Time }\end{array}$ & $\begin{array}{l}\text { Date } \\
\text { Samplex }\end{array}$ & $\begin{array}{l}\text { Time } \\
\text { Sampled }\end{array}$ \\
\hline GW-321 & ADB & w & $0: 52$ & $01 / 10 / 95$ & $10: 40$ \\
\hline
\end{tabular}

Sampling Group=

CR 10

Sample

Identity

Location

Pump Purge

No. Time Sampled Sampled

GW-539

LII

$10 \quad 0: 43 \quad 01 / 04 / 95$

$13: 15$

GW-709

LII

$10 \quad 0: 42 \quad 01 / 09 / 95$

$11: 40$

GW-709

Dupe

ER CR 10

GW-757

LII

$10 \quad 0: 42 \quad 01 / 09 / 95$

$11: 40$

B $\quad$ - $\quad 01 / 09 / 95 \quad 14: 30$

10 $1: 30 \quad 01 / 09 / 95 \quad 14: 40$

Sampling Group=

CR 13

Sample

Identity

Location

Pump Purge

Time

SCR2.2SP

CRSP

No.

Sampled

Sampled

. $03 / 15 / 95 \quad 11: 00$


APPENDIX D

Sampling Sequence, Second Quarter 1995

Sampling Group=

CR 2

Sample

Identity

Location

Pump Purge Date

No. Time Sampled

Time

GW-203

GW-205

GW-221

UNCS

$5 \quad 1: 45 \quad 04 / 18 / 95$

11:00

GW-221 Dupe

UNCS

$5 \quad 1: 46 \quad 04 / 18 / 95$

$14: 26$

$5 \quad 1: 45 \quad 04 / 19 / 95$

$11: 10$

$5 \quad 1: 45 \quad 04 / 19 / 95$

$11: 10$

GW-339

UNCS

$5 \quad 0: 42 \quad 04 / 19 / 95$

$14: 10$

GW-302

ER CR 2

UNCS

$5 \quad 0: 47 \quad 04 / 19 / 95$

$15: 17$

$5.04 / 20 / 95$

$11: 45$

1090

UNCS

$5 \quad 1: 41 \quad 04 / 20 / 95$

11:51

Sampling Group=

Sample

Identity

GW-511

GW-181

GW-742

GW-743

GW-610

GW-608

GW-177

GW-611

GW-175

ER CR 3

GW-609

GW-609

$\begin{array}{ll} & \\ & \\ & \text { Location } \\ & \text { CRSP } \\ & \text { CRSP } \\ & \text { CRSP } \\ & \text { CRSP } \\ & \text { CRSP } \\ & \text { CRSP } \\ & \text { CRSP } \\ & \text { CRSP } \\ & \text { CRSP } \\ & \\ & \text { CRSP } \\ \text { Dupe } & \text { CRSP }\end{array}$

Pump Purge Date

No. Time Sampled

Time

Sampled

$2 \quad 1: 30 \quad 05 / 03 / 95 \quad 11: 50$

$2 \quad+\quad 05 / 02 / 95 \quad 16: 07$

2 . 05/07/95 9:40

$\begin{array}{llll}2 & 0: 57 & 05 / 07 / 95 & 11: 02\end{array}$

$\begin{array}{llll}2 & 0: 57 & 05 / 07 / 95 & 13: 19\end{array}$

$2+05 / 11 / 95 \quad 12: 00$

$2 \quad 0: 33 \quad 05 / 11 / 95 \quad 14: 43$

$2 \quad 0: 20 \quad 05 / 15 / 95 \quad 13: 20$

$2 \quad 1: 13 \quad 05 / 15 / 95 \quad 16: 15$

2 . $05 / 16 / 95 \quad 12: 30$

$\begin{array}{llll}2 & 3: 03 & 05 / 16 / 95 & 12: 38\end{array}$

$\begin{array}{llll}2 & 3: 03 & 05 / 16 / 95 & 12: 38\end{array}$
Sampling Group*

Sample

Identity

GW-296
Location

ECRWP
Pump Purge Date

No. Time Sampled

5

$0: 48 \quad 04 / 22 / 95$
Time

Sampled

9:48 
APPENDIX D

Sampling Sequence, Second Quarter 1995

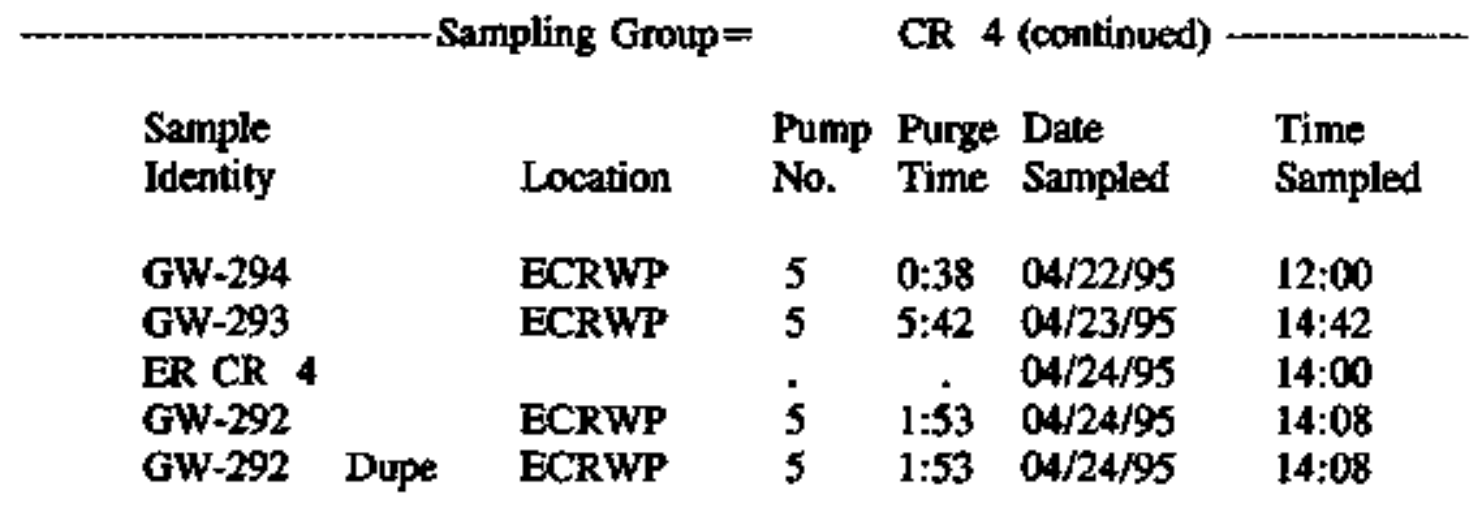

\begin{tabular}{|c|c|c|c|c|c|}
\hline REGIME = & CR & \multicolumn{3}{|c|}{ Sampling Group= } & \multirow{2}{*}{$\begin{array}{l}\text { Time } \\
\text { Sampled }\end{array}$} \\
\hline $\begin{array}{l}\text { Sample } \\
\text { Identity }\end{array}$ & Location & $\begin{array}{l}\text { Pump } \\
\text { No. }\end{array}$ & $\begin{array}{l}\text { Purge } \\
\text { Time }\end{array}$ & $\begin{array}{l}\text { Date } \\
\text { Sampled }\end{array}$ & \\
\hline GW-161 & CRBAWP & B & & $04 / 20 / 95$ & $13: 52$ \\
\hline GW-160 & CRBAWP & 4 & $4: 30$ & $04 / 26 / 95$ & $15 \div 15$ \\
\hline GW-301 & CRBAWP & 4 & $1: 10$ & $04 / 27 / 95$ & $12: 15$ \\
\hline GW-300 & CRBAWP & 4 & $0: 54$ & $04 / 27 / 95$ & $13: 59$ \\
\hline GW.299 & CRBAWP & 4 & $1: 48$ & $05 / 01 / 95$ & $11: 53$ \\
\hline GW-299 Dupe & CRBAWP & 4 & $1: 48$ & $05 / 01 / 95$ & $11: 53$ \\
\hline ER CR 5 & & 4 & . & $05 / 02 / 95$ & 11:15 \\
\hline GW-298 & CRBAWP & 4 & . & $05 / 02 / 95$ & $11: 20$ \\
\hline REGIME $=$ & $\mathrm{CR}$ & \multicolumn{4}{|c|}{ Sampling Group= } \\
\hline $\begin{array}{l}\text { Sample } \\
\text { Identity }\end{array}$ & Location & $\begin{array}{l}\text { Pump } \\
\text { No. }\end{array}$ & $\begin{array}{l}\text { Purge } \\
\text { Time }\end{array}$ & $\begin{array}{l}\text { Date } \\
\text { Sampled }\end{array}$ & $\begin{array}{l}\text { Time } \\
\text { Sampled }\end{array}$ \\
\hline GW-159 & CRSDB & 2 & $0: 43$ & $04 / 06 / 95$ & $18: 15$ \\
\hline GW-159 Dupe & CRSDB & 2 & $0: 43$ & $04 / 06 / 95$ & $18: 15$ \\
\hline GW-304 & CRSDB & 2 & $0: 50$ & $04 / 06 / 95$ & $20: 05$ \\
\hline GW-731 & CRSDB & 2 & $1: 27$ & $04 / 07 / 95$ & $11: 57$ \\
\hline GW-732 & CRSDB & 2 & $0: 58$ & $04 / 07 / 95$ & $14: 36$ \\
\hline GW-156 & CRSDB & 2 & $0: 13$ & $04 / 07 / 95$ & $15: 53$ \\
\hline GW-303 & CRSDB & 2 & . & $04 / 09 / 95$ & $12: 36$ \\
\hline
\end{tabular}


APPENDDX D

Sampling Sequence, Second Quarter 1995

Sampling Group=

CR 6 (continued)

Sample

Identity

Location

Pump Purge Date

Time

FB CR 6

GW-241

ER CR 6

GW-158

$\begin{array}{ccccc} & 2 & . & 04 / 09 / 95 & 14: 47 \\ \text { CRSDB } & 2 & 1: 27 & 04 / 09 / 95 & 14: 47 \\ & 2 & . & 04 / 13 / 95 & 10: 50 \\ \text { CRSDB } & 2 & . & 04 / 13 / 95 & 11: 00\end{array}$

Sampling Group=

CR 7

Sample

Identity

Location

Pump Purge Date

Time

GW-142

GW-147

KHQ

GW-231

KHQ

KHQ

GW-146

GW-143

GW-144

KHQ

KHQ

GW-144

ER CR 7

GW-145

KHQ

No.

Time Sampled

Sampled

KHQ

10

2:58

$04 / 20 / 95$

$14: 30$

$10 \quad-\quad 04 / 21 / 95 \quad 10: 30$

$10 \quad-\quad 04 / 21 / 95 \quad 11: 50$

$10 \quad 1: 29 \quad 04 / 24 / 95 \quad 10: 00$

$10 \quad 4: 50 \quad 04 / 24 / 95 \quad 16: 10$

$10 \quad 2: 41 \quad 04 / 25 / 95 \quad 15: 26$

$10 \quad 2: 41 \quad 04 / 25 / 95 \quad 15: 26$

Dupe

KHQ

in $\quad$ 04/26/95

11:35

$10 \quad . \quad 04 / 26 / 95 \quad 11: 45$

-Sampling Group=

CR 8

Sample

Identity

GW-321

GW-513

GW-512

GW-512

ER CR 8

GW-514
Location

ADB

ADB

ADB

ADB

Dupe

ADB
Pump Purge Date

No. Time Sampled

$5 \quad 1: 55 \quad 04 / 25 / 95$

$5 \quad 2: 36 \quad 04 / 25 / 95$

$5 \quad$ 1:04 04/26/95

$5 \quad 1: 04 \quad 04 / 26 / 95$

$5.04 / 27 / 95$

5

$04 / 27 / 95$
Time

Sampled

13:00

$16: 16$

10:44

$10: 44$

$14: 54$ 


\section{APPENDDX D}

Sampling Sequence, Second Quarter 1995

Sampling Group=

Sample

Identity

GW-184

GW-188

GW-187

ER CR 9

GW-186

GW-186
Location

RQ

RQ

$\mathbf{R Q}$

RQ

Dupe RQ
Pump Purge

No. Time Sampled

$5 \quad 0: 31 \quad 05 / 01 / 95$

$5 \quad 0: 42 \quad 05 / 01 / 95 \quad 14: 45$

$5 \quad 3: 36 \quad 05 / 02 / 95 \quad 12: 51$

$5 \quad$. $05 / 03 / 95 \quad 13: 25$

$5 \quad 4: 00 \quad 05 / 03 / 95 \quad 13: 30$

$5 \quad 4: 00 \quad 05 / 03 / 95 \quad 13: 30$
Sampling Group=

Sample

Identity

GW-540

GW-540 Dupe

GW-546

GW-541

GW-827

GW-542

GW-543

ER CR 11

GW-544
Location

CDLVI

CDLVI

CDLVI

CDLVI

CDLVI

CDLVI

CDLVI

CDLVI
CR 11

Pump Purge

No. Time

Date

Sampled

Time

Sampled

$\begin{array}{llll}10 & 2: 22 & 04 / 04 / 95 & 15: 22\end{array}$

$10 \quad 2: 22 \quad 04 / 04 / 95 \quad 15: 22$

$10 \quad 0: 12 \quad 04 / 05 / 95$

9:55

$10 \quad 1: 02 \quad 04 / 05 / 95$

12:00

15:12

10:00

$10 \quad 0: 15 \quad 04 / 06 / 95$

$\begin{array}{llll}10 & 0: 50 & 04 / 06 / 95 & 11: 35 \\ 10 & & 04 / 06 / 95 & 14-50\end{array}$

$10 \quad 1: 12 \quad 04 / 06 / 95 \quad 14: 57$
Sampling Group=

Sample
Identity
GW-560
GW-560 Dupe
GW-562
GW-564
GW-557
GW-799

Location
CDLVII
CDLVII
CDLVII
CDLVII
LV
LV

7

CR 12

Pump Purge

No. Time

Date

Sampled

Time

Sampled

7

$0: 48$

$04 / 05 / 95$

10:28

$7 \quad 0: 48$

1:06

1:05

$04 / 05 / 95$

$04 / 05 / 95$

$04 / 06 / 95$

$0: 36 \quad 04 / 06 / 95$

1:39 04/06/95
10:28

13:04

11:05

12:56

15:19 


\section{APPENDIX D}

Sampling Sequence, Second Quarter 1995

Sampling Group=

Sample

Identity

GW-798

GW-797

GW-796

ER CR 12

GW-801

CBS-1

CDLVII

LV

LV

LV
CR 12 (continued)

Pump Purge Date

Location No. Time Sampled

$7 \quad 1: 42 \quad 04 / 11 / 95$

1:05 04/11/95 $\begin{array}{lll}7 & 1: 33 & 04 / 12 / 95\end{array}$

$7 \quad 04 / 13 / 95$

$7 \quad 1: 54 \quad 04 / 13 / 95$

$04 / 17 / 95$
Time

Sampled

10:43

14:10

13:33

11:20

11:24

10:02 


\section{APPENDIX D}

Sampling Sequence, Third Quarter 1995

Sampling Group=

CR 1

Sample

Identity

Location

Purnp Purge Date

Time

GW-217

GW-141

GW-521

GW-522

GW-522 Dupe

LIV

No.

Time Sampled

Sampled

ER CR 1

GW-305

Dupe

LIV

10

1:02 07/10/95

$12 ; 00$

$10 \quad 1: 36 \quad 07 / 10 / 95 \quad 16: 06$

$10 \quad 1: 20 \quad 07 / 11 / 95 \quad 15: 28$

LIV

10

$2: 24 \quad 07 / 12 / 95$

$11: 54$

$10 \quad 2: 24 \quad 07 / 12 / 95$

$11: 54$

10

$07 / 12 / 95$

$14: 25$

LIV

$10 \quad 0: 52 \quad 07 / 12 / 95$

$14: 30$

Sampling Group=

CR 3

Sample

Identity

Location

Pump Purge Date

Time

GW-181

GW-181

GW-511

GW-742

GW-743

GW-608

GW-610

GW-177

GW-175

ER CR 3

GW-611

GW-609

ER CR 3

\begin{tabular}{|c|c|c|c|c|c|}
\hline \multirow{13}{*}{ Dupe } & CRSP & 10 & $3: 36$ & $07 / 28 / 95$ & $14: 06$ \\
\hline & CRSP & 10 & $3: 36$ & $07 / 28 / 95$ & $14: 06$ \\
\hline & CRSP & 10 & $1: 10$ & $07 / 29 / 95$ & $10: 00$ \\
\hline & CRSP & 2 & 5:00 & $07 / 30 / 95$ & $13: 58$ \\
\hline & CRSP & 2 & $0: 48$ & $07 / 31 / 95$ & $11: 13$ \\
\hline & CRSP & 10 & $3: 30$ & $07 / 31 / 95$ & $14: 15$ \\
\hline & CRSP & 2 & $0: 48$ & $07 / 31 / 95$ & $14: 48$ \\
\hline & CRSP & 10 & $0: 32$ & $08 / 01 / 95$ & $10: 50$ \\
\hline & CRSP & 2 & 1:06 & $08 / 01 / 95$ & $14: 30$ \\
\hline & & 10 & & $08 / 01 / 95$ & $15: 25$ \\
\hline & CRSP & 10 & $0: 12$ & $08 / 01 / 95$ & $15: 30$ \\
\hline & CRSP & 2 & $2: 39$ & $08 / 01 / 95$ & $18: 09$ \\
\hline & CRSP & 2 & 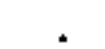 & $08 / 01 / 95$ & $18: 30$ \\
\hline
\end{tabular}

Sampling Group $=$

CR 6

Sample

Identity

GW-159

GW-304
Location

CRSDB

CRSDB
Pump Purge Date

No. Time Sampled Sampled

$2 \quad 0: 45 \quad 07 / 12 / 95$

$2 \quad 0: 50 \quad 07 / 12 / 95$
14:40

$16: 30$ 
APPENDIX D

Sampling Sequence, Third Quarter 1995

Sampling Group = CR 6 (continued)

Sample

Identity

GW-731

GW-732

GW-156

GW-303

GW-303

GW-241

GW-158

ER CR 6

FB CR 6

GW-732 RS $\begin{array}{llll} & \text { Pump Purge } & \text { Date } & \text { Time } \\ \text { Location } & \text { No. Time } & \text { Sampled } & \text { Sampled }\end{array}$

$\begin{array}{lllll}\text { CRSDB } & 2 & 0: 58 & 07 / 13 / 95 & 12: 00\end{array}$

$\begin{array}{lllll}\text { CRSDB } & 2 & 0: 59 & 07 / 13 / 95 & 13: 34\end{array}$

$\begin{array}{lllll}\text { CRSDB } & 2 & 0: 12 & 07 / 13 / 95 & 15: 00\end{array}$

$\begin{array}{lllll}\text { CRSDB } & 2 & \text { - } & 07 / 16 / 95 & 9: 30\end{array}$

$\begin{array}{lllll}\text { CRSDB } & 2 & 07 / 16 / 95 & 9: 30\end{array}$

$\begin{array}{lllll}\text { CRSDB } & 2 & 1: 18 & 07 / 16 / 95 & 11: 43\end{array}$

$\begin{array}{lllll}\text { CRSDB } & 2 & 8: 00 & 07 / 16 / 95 & 17: 45\end{array}$

2 . $07 / 18 / 95 \quad 17: 40$

$2 \quad . \quad 07 / 18 / 95 \quad 17: 45$

$\begin{array}{lllll}\text { CRSDB } & 6 & 0: 48 & 08 / 14 / 95 & 10: 58\end{array}$

Sampling Group=

CR 7

Sample

Identity

Location

Pump Purge Date

No. Time Sampled

Time

GW-142

GW-147

GW-231

GW-143

GW-144

GW-144

ER CR 7

GW-145

GW-146

$\begin{array}{cccccc} & \text { KHQ } & 8 & 2: 28 & 07 / 14 / 95 & 11: 40 \\ & \text { KHQ } & 8 & 0: 28 & 07 / 14 / 95 & 13: 38 \\ \text { KHQ } & 8 & 0: 33 & 07 / 15 / 95 & 9: 23 \\ & \text { KHQ } & 8 & 1: 50 & 07 / 16 / 95 & 12: 30 \\ \text { Dupe } & \text { KHQ } & 8 & 2: 48 & 07 / 16 / 95 & 15: 38 \\ & \text { KHQ } & 8 & 2: 48 & 07 / 16 / 95 & 15: 38 \\ & & 8 & & 07 / 17 / 95 & 12: 10 \\ & \text { KHQ } & 8 & 0: 55 & 07 / 17 / 95 & 12: 15 \\ \text { KHQ } & 8 & 3: 00 & 07 / 17 / 95 & 14: 00\end{array}$

Sampling Group=

CR 10

Sample

Identity

Location

Pump Purge Date

Time

GW-539

LII

No.

Time Sampled

Sampled

8

$0: 50 \quad 07 / 10 / 95$

12:55 


\section{APPENDIX D}

Sampling Sequence, Third Quarter 1995

Sampling Group $=\quad$ CR 10 (continued)

Sample

Identity

$\begin{array}{ll}\text { Pump Purge } & \text { Date } \\ \text { No. Time Sampled }\end{array}$

Location

Time

GW-709

LII

$8 \quad 0: 40 \quad 07 / 10 / 95$

Sampled

GW-709 Dupe LII

ER CR 10

GW-757

LII

$8 \quad 0: 40 \quad 07 / 10 / 95$

$8 \quad . \quad 07 / 11 / 95 \quad 14: 50$

$8 \quad 1: 20 \quad 07 / 11 / 95 \quad 15: 00$ 
APPENDIX D

Sampling Sequence, Fourth Quarter 1995

Sampling Group $=$

CR 2

Sample

Identity

Location

Pump Purge Date

Time

GW-203

GW-205

UNCS

UNCS

No.

Time Sampled

Sampled

GW-221

UNCS

11

1:28 $10 / 07 / 95$

$11: 28$

GW-221

GW-339

Dupe

UNCS

11

$1: 3010 / 07 / 95$

$14: 00$

$11 \quad 1: 30 \quad 10 / 08 / 95 \quad 11: 00$

GW-302

ER CR 2

1090

UNCS

11

$1: 30 \quad 10 / 08 / 95$

$11: 00$

UNCS

$11 \quad 0: 45 \quad 10 / 08 / 95$

$14: 05$

$\begin{array}{llll}11 & 0: 36 & 10 / 08 / 95 & 15: 01\end{array}$

11 . 10/09/95 14:50

UNCS

$11 \quad 1: 46 \quad 10 / 09 / 95$

$14: 56$

Sampling Group=

CR 3

Sample

Identity

GW-181

GW-181

GW-511

GW-742

GW-743

GW-610

GW-608

GW-177

GW-611

GW-175

ER CR 3

GW-609
Location

Dupe

CRSP

CRSP

CRSP

CRSP

CRSP

CRSP

CRSP

CRSP

CRSP

CRSP

CRSP
Pump Purge Date

No. Time Sampled

$\begin{array}{lll}2 & 3: 23 & 11 / 05 / 95\end{array}$

$3: 23 \quad 11 / 05 / 95$

$1: 09 \quad 11 / 07 / 95$

$2: 30 \quad 11 / 09 / 95$

$0: 47 \quad 11 / 13 / 95$

$1: 05 \quad 11 / 13 / 95$

$7: 00 \quad 11 / 16 / 95$

0:33

$11 / 16 / 95$

$0: 14 \quad 11 / 17 / 95$

$1: 15$

$11 / 17 / 95$

$2 \quad$. $11 / 20 / 95$

$2 \quad 2: 42 \quad 11 / 20 / 95$
Time

Sampled

15:53

$15: 53$

$10: 49$

$13: 45$

11:02

$14: 45$

$11: 57$

$14: 30$

$11: 10$

$14: 45$

12:05

12:12

Sampling Group=

CR

Sample

Identity

GW-296
Pump Purge Date

Location

ECRWP
No. Time Sampled

Time

Sampled

$6 \quad 0: 40 \quad 11 / 05 / 95$

$9: 40$ 


\section{APPENDIX D}

Sampling Sequence, Fourth Quarter 1995

Sampling Group=

CR 4 (continued)

Sample

Identity

GW-294

GW-293

ER CR 4

GW-292

GW-292
Location

Pump

No.

ECRWP

ECRWP

ECRWP

ECRWP

$\begin{array}{lccc}6 & 0: 26 & 11 / 05 / 95 & 11: 10 \\ 6 & 3: 24 & 11 / 06 / 95 & 11: 12 \\ 6 & 11 / 06 / 95 & 14: 00 \\ 6 & 1: 37 & 11 / 06 / 95 & 14: 07 \\ 6 & 1: 37 & 11 / 06 / 95 & 14: 07\end{array}$

Sampling Group=

CR 5

Sample

Identity

GW-161

GW-160

GW-160

GW-301

GW-300

GW-299

GW-298

ER CR 5 $\begin{array}{lllll} & \text { Pump Purge } & \text { Date } & \text { Time } \\ \text { Location } & \text { No. } & \text { Time } & \text { Sampled } & \text { Sampled }\end{array}$

$\begin{array}{lllll}\text { CRBAWP } & 6 & 6: 36 & 10 / 31 / 95 & 15: 00\end{array}$

$\begin{array}{lllll}\text { CRBAWP } 6 & 4: 25 & 11 / 02 / 95 & 13: 55\end{array}$

$\begin{array}{lllll}\text { CRBAWP } & 6 & 4: 25 & 11 / 02 / 95 & 13: 55\end{array}$

$\begin{array}{lllll}\text { CRBAWP } & 6 & 0: 22 & 11 / 03 / 95 & 10: 30\end{array}$

$\begin{array}{lllll}\text { CRBAWP } & 6 & 0: 46 & 11 / 03 / 95 & 12: 46\end{array}$

$\begin{array}{lllll}\text { CRBAWP } & 6 & 1: 32 & 11 / 03 / 95 & 14: 52\end{array}$

$\begin{array}{lllll}\text { CRBAWP } & 6 & 0: 50 & 11 / 04 / 95 & 10: 30\end{array}$

$6.11 / 04 / 95 \quad 10: 40$

Sampling Group=

CR 6

Sample

Identity

GW-731-1

GW-159-1

GW-156-1

GW-732-1

GW-732-1 Dupe

ER CR 6

ER CR 6
Location

CRSDB

CRSDB

CRSDB

CRSDB

CRSDB

CRSDB

\section{Pump Purge Date}

No. Time Sampled Sampled

10

7

10

7

7

7

10
1:04 10/23/95

$0: 24 \quad 10 / 23 / 95$

$0: 12 \quad 10 / 23 / 95$

$0.52 \quad 10 / 23 / 95$

$0: 52$

$10 / 23 / 95$

$10 / 23 / 95$

$10 / 23 / 95$
$11: 14$

$11: 30$

13:30

13:32

13:32

15:00

15:15 
APPENDIX D

Sampling Sequence, Fourth Quarter 1995

Sampling Group=

CR 6 (continued)

Sample

Identity

Location

Pump Purge Date

Time

GW-159-2

GW-732-2

GW-732-2 Dupe

GW-156-2

GW-731-2

GW-159-3

GW-732-3

GW-732-3 Dupe

GW-156-3

GW-731-3

GW-159

GW-732

GW-732

GW-156

GW-731

CRSDB
CRSDB
CRSDB
CRSDB
CRSDB
CRSDB
CRSDB
CRSDB
CRSDB
CRSDB
CRSDB
CRSDB
CRSDB
CRSDB
CRSDB

No. Time Sampled

Sampled

B $\quad$. 10/24/95 $10: 15$

B - 10/24/95 11:05

B $\quad$ - 10/24/95 11:05

B $\quad$ 10/24/95 $13: 15$

B $\quad . \quad 10 / 24 / 95 \quad 14: 05$

B $\quad$ - $10 / 25 / 95 \quad 9: 15$

B $\quad$ - $10 / 25 / 95 \quad 9: 45$

B $\quad$. $10 / 25 / 95 \quad 9: 45$

B $\quad$ - 10/25/95 10:30

B $\quad$ - $10 / 25 / 95 \quad 11: 05$

B $\quad$ - 10/26/95 $10 \div 25$

B $\quad$ - 10/26/95 $10: 55$

B . 10/26/95 $10: 55$

B . 10/26/95 13:45

B . 10/26/95 14:20

Sampling Group=

CR 7

Sampie

Identity

Location

Pump Purge Date

Time

GW-142

ER CR 7

GW-147

GW-231

GW-146

GW-143

GW-144

GW-144

ER CR 7

FB CR 7

GW-145

KHQ
KHQ
KHQ
KHQ
KHQ
KHQ
KHQ
KHQ

KHQ

No. Time Sampled Sampled

$\begin{array}{llll}2 & 3: 35 & 11 / 05 / 95 & 14: 40\end{array}$

2 . 11/06/95 $08: 40$

$4 \quad 0: 27 \quad 11 / 06 / 95 \quad 13: 20$

$4 \quad 0: 27 \quad 11 / 06 / 95 \quad 13: 20$

$4 \quad 2: 28 \quad 11 / 08 / 95 \quad 13: 45$

$4 \quad 2: 18 \quad 11 / 09 / 95 \quad 12: 05$

$4 \quad 2: 24 \quad 11 / 14 / 95 \quad 11: 54$

$4 \quad 2: 24 \quad 11 / 14 / 95 \quad 11: 54$

$4 \quad 111 / 15 / 95 \quad 11: 20$

- . 11/15/95 11:30

$4 \quad 0: 55 \quad 11 / 15 / 95 \quad 11: 30$ 
APPENDIX D

Sampling Sequence, Fourth Quarter 1995

Sampling Group*

CR 8

Sample

Identity

Location

Pump Purge Date

Time

GW-321

ADB

No.

Time Sampled

Sampled

GW-321

Dupe

ADB

GW-513

GW-512

ER CR 8

GW-514

ADB

ADB

ADB

$\begin{array}{cccc}8 & 1: 30 & 10 / 26 / 95 & 14: 21 \\ 8 & 1: 30 & 10 / 26 / 95 & 14: 21 \\ 8 & 2: 06 & 10 / 31 / 95 & 14: 11 \\ 8 & 0: 44 & 10 / 31 / 95 & 15: 24 \\ 8 & & 11 / 02 / 95 & 17: 00 \\ 8 & 7: 36 & 11 / 02 / 95 & 17: 06\end{array}$

Sampling Group=

CR 9

Sample

Identity

Location

Pump Purge Date

Time

No. Time Sampled Sampled

GW-184

GW-188

GW-187

GW-187

ER CR 9

GW-186

$\begin{aligned} & \mathbf{R Q} \\ & \mathbf{R Q} \\ & \mathbf{R Q} \\ & \text { Dupe } \mathbf{R Q} \\ & \mathbf{R Q}\end{aligned}$

RQ

$4 \quad 0: 30 \quad 10 / 31 / 95$

10:28

$4 \quad 0: 37 \quad 10 / 31 / 95$

14:19

$4 \quad 3: 22 \quad 11 / 02 / 95$

$12: 45$

$43: 22 \quad 11 / 02 / 95$

$12: 45$

4 . 11/03/95

11:00

RQ

$4 \quad 1: 13 \quad 11 / 03 / 95$

11:08

Sampling Group=

CR 10

Sample

Identity

Location

Pump Purge Date

Time

GW-539

GW-709

LII

No.

Time

Sampled

Sampled

ER CR 10

GW-757

LII

$10 \quad 0: 30 \quad 10 / 06 / 95$

11:05

$\begin{array}{lll}10 & 0: 44 & 10 / 06 / 95\end{array}$

$13: 42$

$10 \quad$. 10/06/95

$15: 50$

GW-757

LII

$10 \quad 1: 13 \quad 10 / 06 / 95$

16:00

$10 \quad 1: 13 \quad 10 / 06 / 95 \quad 16: 00$ 
APPENDIX D

Sampling Sequence, Fourth Quarter 1995

Sampling Group=

CR 11

Sample

Identity

Location

Pump Purge Date

Time

GW-540

GW-540

GW-546

GW-541

GW-827

GW-542

GW.543

ER CR 11

GW-544

Dupe

$\begin{array}{lllll}\text { CDLVI } & 8 & 1: 49 & 10 / 11 / 95 & 11: 59 \\ \text { CDLVI } & 8 & 1: 49 & 10 / 11 / 95 & 11: 59 \\ \text { CDLVI } & 8 & 0: 02 & 10 / 11 / 95 & 14: 30 \\ \text { CDLVI } & 8 & 0: 25 & 10 / 12 / 95 & 13: 45 \\ \text { CDLVI } & 8 & 2: 54 & 10 / 16 / 95 & 13: 04 \\ \text { CDLVI } & 8 & 0: 55 & 10 / 16 / 95 & 15: 45 \\ \text { CDLVI } & 8 & 0: 40 & 10 / 17 / 95 & 13: 25 \\ & 8 & & 10 / 17 / 95 & 15: 05 \\ \text { CDLVI } & 8 & 0: 55 & 10 / 17 / 95 & 15: 10\end{array}$

Sampling Group=

CR 12

Sample

Identity

Location

Pump Purge Date

Time

GW-560

CDLVII

No.

Time Sampled

Sampled

GW-560 Dupe

CDLVII

CDLVI

CDLVII

LV

$\begin{array}{ll}7 & 0: 48\end{array}$

$10 / 06 / 95$

13:10

$7 \quad 0: 48$

$10 / 06 / 95$

$13: 10$

GW-564

GW-557

GW-799

GW-798

GW-797

GW-796

GW-801

ER CR 12

CBS-1

LV

CDLVII

LV

LV

LV

LV
$7 \quad 1: 06$

7

$7 \quad 1: 27$

$7 \quad 0: 24$

$10 / 06 / 95$

14:56

$10: 32$

11:54

$\begin{array}{llll}7 & 1: 41 & 10 / 07 / 95 & 14: 16\end{array}$

$7 \quad 1: 30 \quad 10 / 08 / 95$

$10: 40$

$7 \quad 1: 27 \quad 10 / 08 / 95 \quad 12: 57$

$7 \quad 1: 16 \quad 10 / 08 / 95 \quad 14: 56$

$\begin{array}{lll}7 & 1: 43 \quad 10 / 09 / 95\end{array}$

$12: 13$

$12: 30$

$10 / 09 / 95$

$10: 35$ 
APPENDIX E

GROUNDWATER QUALITY DATA 



\section{EXPIANATION}

SAMPLING PONT:

$\begin{array}{rll}\text { GW } & - & \text { Monitoring Well } \\ \text { CBS.1 } & - & \text { Chestnut Branch Spring 1 } \\ \text { SCR2.2SP } & - & \text { South Chestrut Ridge Spting 2.2 } \\ \text { 1090 } & - & \text { Historical 1000-Series Monitoring Well }\end{array}$

LOCATION:

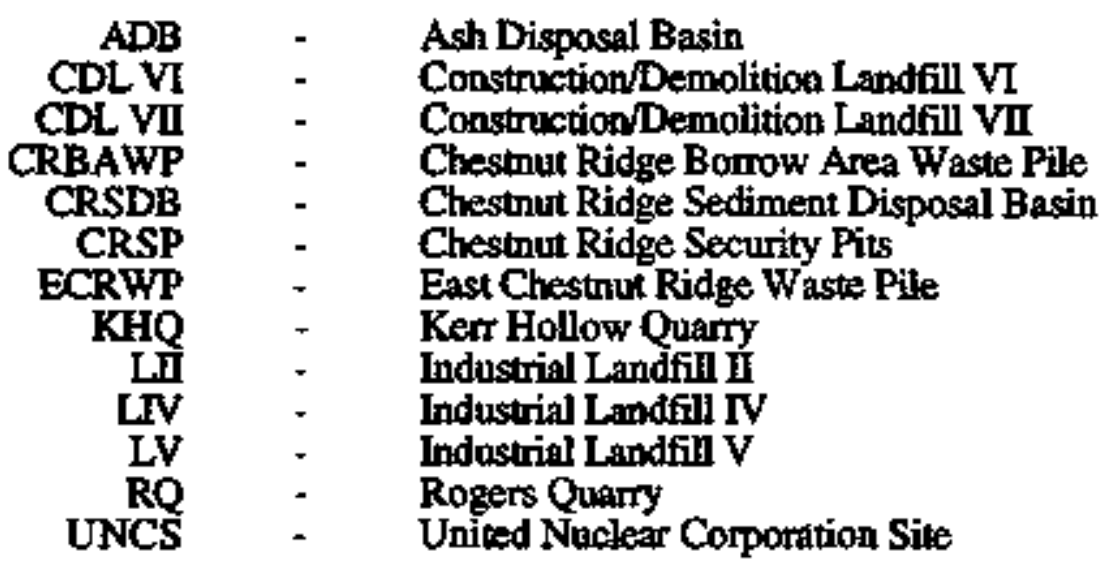

ALL DATA:

Not analyzed or not reported.

ORGANIC COMPOUNDS:

All results in micrograms per liter (ug/L).

METALS, ANIONS, MISCELLANEOUS, AND RADIOCHEMICAL PARAMETERS:

General:

Bicarbonate and carbonate alkalinity reported as $\mathrm{CaCO}_{3}$.

$<\quad$ - Compound analyzed but not detected at the reported minimoun attainable detection limit

DIS - Dissolved Concentration (Filtered Sample)

TOT - Total Concentration (Unfiltered Sample)

TDS - Total Dissolved Solids

TSS - Total Suspended Solids

Sp. Cond. - Specific Condactance 

Method:

$\begin{array}{lll}\text { AAS } & - & \text { Atomic Absorption Spectroscopy } \\ \text { CVAA } & - & \text { Cold Vapor Atomic Absortion } \\ \text { PMS } & - & \text { Plasma Mass Spectroscopy } \\ \text { ICAP } & - & \text { Inductively Coupled Arc Plasma Spectrocopy }\end{array}$

Units:

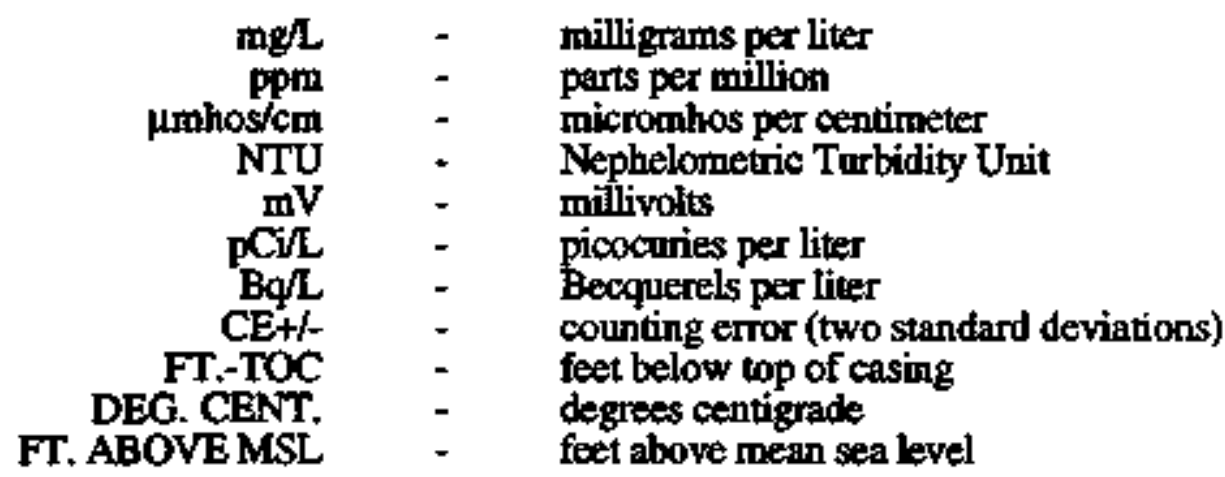




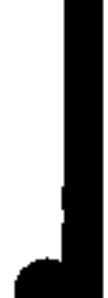




\section{EXPLANATION (cont*d)}

\begin{tabular}{|c|c|c|}
\hline Analyte Class & Qualifier & Explanation \\
\hline \multirow{11}{*}{ Radiomuchides } & $\mathbf{J}$ & Estimated value. \\
\hline & A & Possible detscior contamination. \\
\hline & $\mathbf{E}$ & $\begin{array}{l}\text { Result of analysis is less than MDA, confidence level is less } \\
\text { than } 95 \% \text {. }\end{array}$ \\
\hline & F & Result less than background. \\
\hline & i & Tentatively identified isotope. \\
\hline & $*$ & Duplicate analysis outside control limits. \\
\hline & C & Control analysis outside of control limits. \\
\hline & D & $\begin{array}{l}\text { Spike control limits do not apply, sample activity exceeds spike } \\
\text { activity. }\end{array}$ \\
\hline & $\mathbf{N}$ & Spike recovery not within limits. \\
\hline & $\mathbf{H}$ & $\begin{array}{l}\text { Daughter of uranium isotopes, reported for comparison } \\
\text { purposes only. }\end{array}$ \\
\hline & $\mathbf{G}$ & Gamma photopeak near MDA, poor curve fit. \\
\hline \multirow[t]{6}{*}{ Organics } & $\mathrm{J}$ & Estimated value less than taboratory reporting limit. \\
\hline & B & Compound also detected in the associated laboratory blank \\
\hline & $\underline{\mathbf{U}}$ & Compound not detected. \\
\hline & $\underline{\mathbf{D}}$ & Compound identified at a secondary dilution factor. \\
\hline & $\mathbf{E}$ & Concentration exceeds calibration range of instrument. \\
\hline & s. & Spike recovery not within limits. \\
\hline $\begin{array}{l}\text { ICP and } \\
\text { ICPMS Metals }\end{array}$ & d & Dilution required, reporting limits raised accordingly. \\
\hline \multirow[t]{2}{*}{ AAS Metals } & $s$ & Spike recovery not within limits. \\
\hline & D & Sample contained matrix interferences, reporting limit adjusted. \\
\hline \multirow[t]{2}{*}{ Conductivity } & $\mathbf{L}$ & Sample received with expired holding time. \\
\hline & h & Analysis exceeded holding time. \\
\hline Alkaliaitives & $\underline{H}$ & Analysis exceeded holding time. \\
\hline Nitrate & $\underline{\mathbf{h}}$ & Analysis exceeded holding time. \\
\hline Sulfate & d & Dilution recuired, neporting limits raised accordingly. \\
\hline
\end{tabular}



APPENDIX E.1

TRACE METALS AND ANIONS 



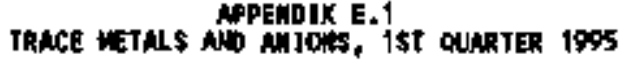

SAMPL [NG POJHT

LOCATIN

DATE SANDLED

TYP

METALs

Mition

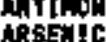

BARIIN

GERTLLLUN

6000:

Chinter

chpistis

chiponiun

CHatentur

conati

copper

IRON

LEA

MHONes LuM

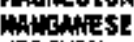

inticting

MoLrsoeimn

MIOKEL

PHOSPHOCGUS

SELEMTU

stLicon

S[LVE

STRONIIU⿴囗十⺝

Thporic

intivice

vampron

AMDDIL

21ucu

A) KaL [N] I $Y$ - HCOS

$r$ Hit $r+c 0 s$

CHLORIDE

UI

HIMONTE HITROOEN

curtat

TOTAL SUSPEROED SOLIDS TUREIDJTY

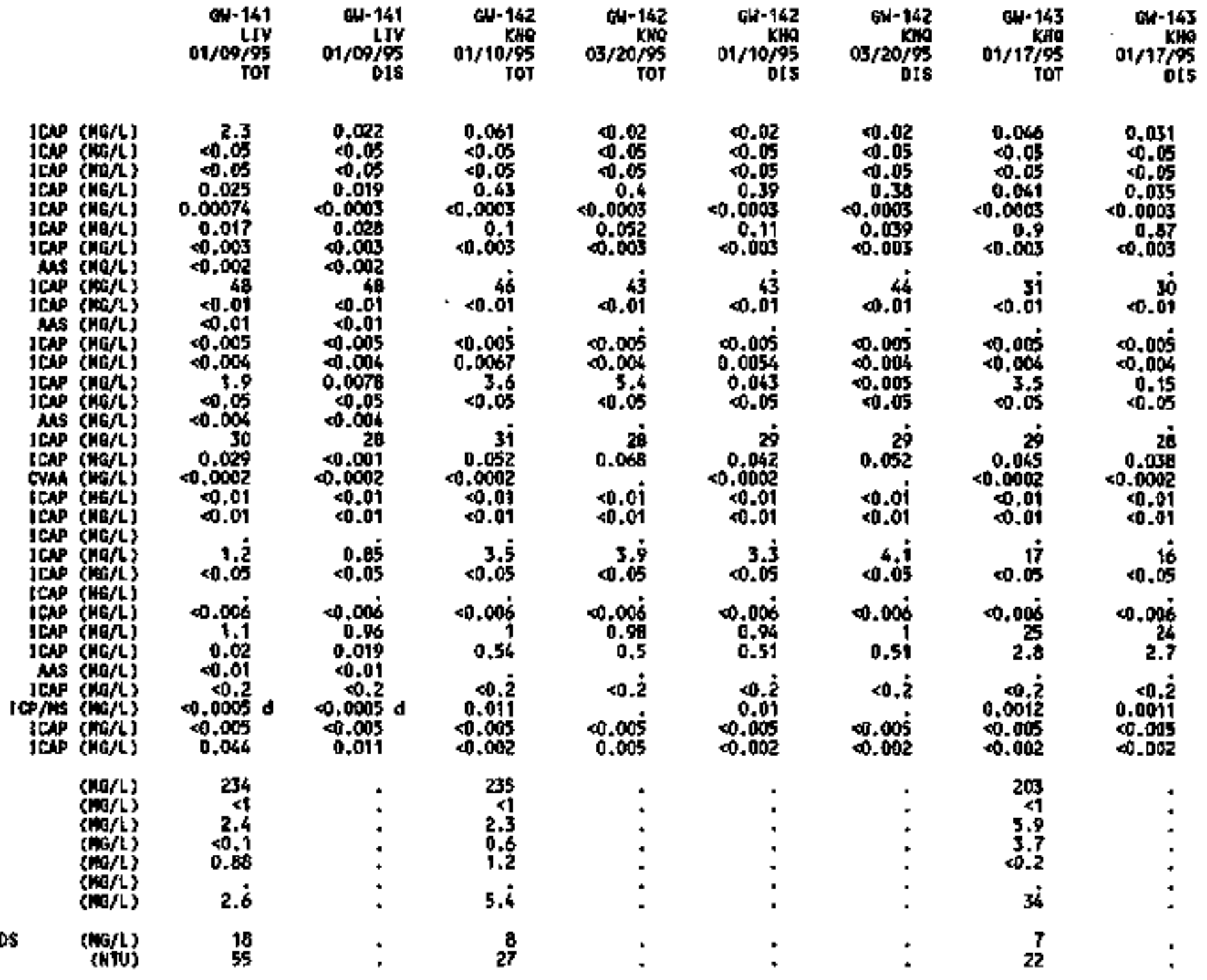




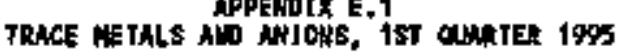

8MPLING ROIHT

LOChTIOH

DATE SAPLLt

DATE

WETALS

Aluitring

ANT JWOFr

ahent

extrutitu

gectrtit

conomintury

CHoNJu

coldut.

Chandiut

Chanium

COAALT

IRONy

LEND

NAROLE]UN

Manchinstse

Nencin

We raberw:

ICKE L

PNOSPHOROUS

Sel Exilu

STEF

SIlico

TIt,VER

STROATIu

THALL IUA

TWORIU

URAYHU

Natalum

ZIHC

AIKALINJTY-HCOZ

ALKalingtr-cot

chlokjos

FLORIDE HITOCEY

Nivonik hITROGE

SULFATE

TOIAL SUSPENDED SOLIDS

TUABIDITY
GN-144 GW-146 GN-144 G.145 G-145 GW+145

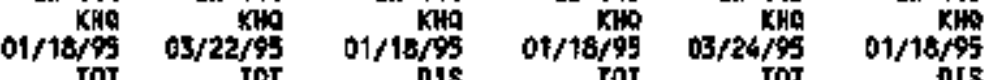

ToT $03 / 22 / 95$ TOT $01 / 10 / 95$

DIS TOT TOT
$6 w+145$
KHo
$03 / 24 / \% 5$
015

CHA 1 146

$01 / 17 / 45$

Gi. 146 cN-140

0.026
20.05
0.05
0.054
$\times 0.0005$
0.058
0.003

40,01

<0. 005

$<.004$

$<0.005$

4

17
$<0.001$

000

$<0.01$

1.5

$<0.05$

$<9.005$

0.087

0.15

$\times 0.05$

0.11

0.3
$\times 0.003$

47
$<0,01$

$+0.005$

0.0042

0.16$$
5
$$

0.015

4.0062

$<0.01$

13
40.05

$<005$

0.006
8.8

$\begin{array}{rrr}0.74 & 0.042 & <0.02 \\ 0.05 & <0.05 & <0.05 \\ <0.05 & 0.05 & <0.05 \\ 0.16 & 0.096 & 0.16 \\ 0.0075 & 0.0003 & 0.00046 \\ 0.26 & 0.31 & 0.21\end{array}$

40.46

44

0.009

0.009
0.0073

0.0073

40.05

0.075

0.09

$\$ .01$

40.12

0.

0.013
7.5
7.3

0.013

0.013

$\$ 0.005$

0. 20.25

40.005

0.0023

\begin{abstract}
40,2
\end{abstract}
$0.01 i$

0.011
0.0095

005

0.0051

40.005

40.05

0.0015

40.007
0.011

20.01

12
$\times 05$

$<0,005$

40.006

43
$\times 0.01$

0.0 .2

0.012

40.005
0.0039
$<0.02$
50.05
$<0.05$
0.16
0.00046
0.21
0.005

$\times 0.005$

$<0.004$

40.005

$<0.0$

35

0,011

$<0.01$

$<0.05$

$\$ 0.006$

4

0.02

4.05
$<0.05$

0,056

0.0003
0.48

20.0403

37
-0.01

0.00

0.005
0.006

0.45

$\$ 0.05$

315
40.015

0.01

$\infty .01$

15
$<0.05$

0.00

0.006
11

$40 . \overline{2}$

$-0.005$

+0.005
+0.002

0,001

0.0014

$+0.002$ is

0.04

0.05

0.065

0.49
$\times 0.0 .3$

0.01

40.005

.

0.42
0.05

0.02

$<0.0002$

(i). 01

$<0.05$

40.006

11
6.9

0.0013

0.0013

4.005
0.0013

229
0.1
2.8

(Hols)

(bG/2)

(Ha/L)

(MG,L)

(Ho/t)

151
4
2.3
0.2
1.6

( $M G$ C)

(itu)

5.3

197
51
11
3
0.66
66
2
6 


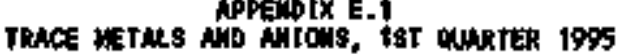

\begin{tabular}{|c|c|c|c|c|c|c|c|c|c|c|c|c|}
\hline \multicolumn{3}{|l|}{$\begin{array}{l}\text { SANPL [NG POJUT } \\
\text { LOSAT IOW } \\
\text { DATE BNIPLE } \\
\text { TTPE }\end{array}$} & $\begin{array}{r}c w+147 \\
\text { k1/ } \\
01 / 15 / 95 \\
\text { ToT }\end{array}$ & $\begin{array}{r}64 \cdot 147 \\
\text { KHa } \\
01 / 15 / 75 \\
015\end{array}$ & 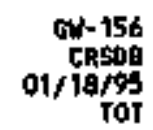 & $\begin{array}{r}04+156 \\
c k 60 \\
01 / 10 / 55 \\
013\end{array}$ & $\begin{array}{r}6 \mathrm{~N}-15 B \\
\text { chspB } \\
04 / 20 / 95 \\
\text { TOT }\end{array}$ & $\begin{array}{r}01-158 \\
\text { ch5os } \\
01 / 20 / 95 \\
015\end{array}$ & $\begin{array}{r}01-159 \\
\text { CRsos } \\
01 / 15 / 95 \\
\text { Tor }\end{array}$ & $\begin{array}{r}0.159 \\
6050 \\
01 / 15 / 95 \\
015\end{array}$ & $\begin{array}{r}0-175 \\
\text { thsts } \\
02 / 14 / 95 \\
\text { T01 }\end{array}$ & $\begin{array}{r}1+175 \\
\text { chsp } \\
02 / 14 / 95 \\
\text { D/s }\end{array}$ \\
\hline \multirow{9}{*}{\multicolumn{2}{|c|}{ 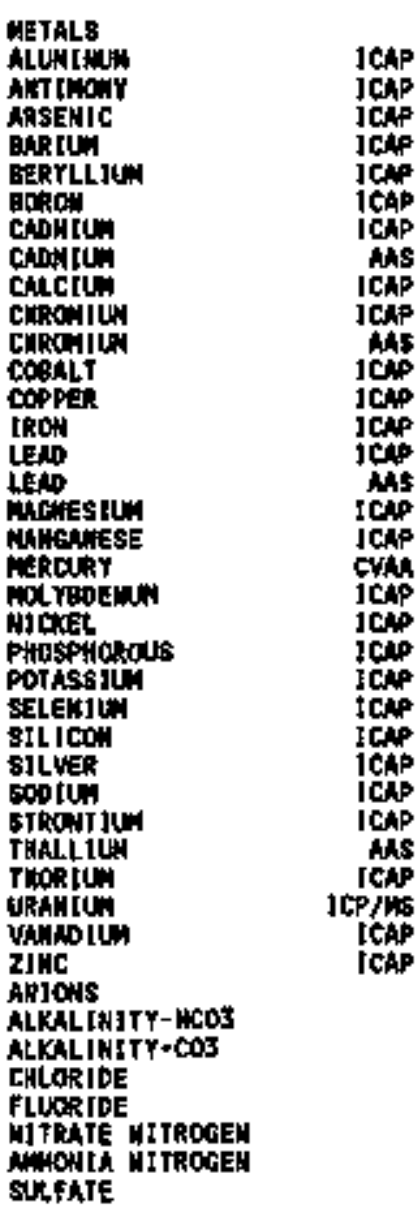 }} & 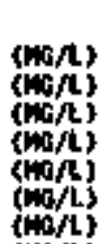 & $\begin{array}{r}0.052 \\
<0.05 \\
\times 0.05 \\
0.077 \\
\times 0.0003 \\
0.048 \\
40.005\end{array}$ & $\begin{array}{r}0.022 \\
<0.05 \\
<0.08 \\
0.083 \\
0.0003 \\
0.004 \\
40.003\end{array}$ & $\begin{array}{r}0.071 \\
0.05 \\
0.05 \\
0.027 \\
0.0003 \\
0.015 \\
0.005\end{array}$ & $\begin{array}{r}0.02 \\
4.05 \\
4.05 \\
0.029 \\
0.0003 \\
0.016 \\
40.003\end{array}$ & $\begin{array}{r}0.042 \\
50.05 \\
0.05 \\
0.047 \\
0.0003 \\
0.016 \\
0.003\end{array}$ & $\begin{array}{r}0.027 \\
<0.05 \\
60.05 \\
0.043 \\
\times 0.0405 \\
0.017 \\
0.003\end{array}$ & $\begin{array}{r}0.37 \\
0.05 \\
0.05 \\
0.012 \\
0.00032 \\
0.014 \\
\times 0.003\end{array}$ & $\begin{array}{r}0.021 \\
40.05 \\
40.05 \\
0.011 \\
<0.0005 \\
0.025 \\
0.003\end{array}$ & $\begin{array}{r}0.027 \\
80.05 \\
40.05 \\
0.039 \\
\times 0.0003 \\
40.006 \\
\times 0.003\end{array}$ & $\begin{array}{r}0.02 \\
40.05 \\
0.05 \\
0.034 \\
40.0063 \\
0.0054 \\
0.003\end{array}$ \\
\hline & & $\begin{array}{l}(m / L) \\
(N / A) \\
(N / L)\end{array}$ & $\times 0.35$ & 80.01 & $\begin{array}{r}74 \\
0.01\end{array}$ & $\begin{array}{r}7 \dot{73} \\
\$ .01\end{array}$ & $\times 0,01$ & $<0,01$ & 0.45 & $\$ 0,01$ & $<0.4 \overline{1}$ & $\$ 0.01$ \\
\hline & & $\begin{array}{l}(w / L) \\
(w / L) \\
(\omega / \mathrm{L}) \\
(\omega / \mathrm{L}) \\
(\omega, / L)\end{array}$ & $\begin{array}{r}40.005 \\
40.005 \\
0.075 \\
<0.05\end{array}$ & $\begin{array}{r}<0.005 \\
0.012 \\
<0.005 \\
40.05\end{array}$ & $\begin{array}{r}40.005 \\
0.011 \\
0.15 \\
\times 0.05\end{array}$ & $\begin{array}{l}<0.005 \\
0.0056 \\
0.005 \\
0.05\end{array}$ & $\begin{array}{r}<0.005 \\
<0.004 \\
2.04 \\
0.03\end{array}$ & $\begin{array}{l}0.005 \\
\times 0.005 \\
0.005 \\
0.05\end{array}$ & $\begin{array}{r}\times 0.005 \\
\times 0.004 \\
0.54 \\
\times 0.05\end{array}$ & $\begin{array}{r}0.005 \\
0.019 \\
0.15 \\
0.05\end{array}$ & $\begin{array}{r}40.005 \\
0.012 \\
0.76 \\
\times 0.05\end{array}$ & $\begin{array}{r}<0.005 \\
0.0091 \\
0.013 \\
0.05\end{array}$ \\
\hline & & $\begin{array}{l}(\omega / L) \\
(\omega / L) \\
(N G / L) \\
(N / L) \\
(\omega / L) \\
(\omega / t)\end{array}$ & $\begin{array}{r}19 \\
0.017 \\
0.0002 \\
40,01 \\
40.01\end{array}$ & $\begin{array}{r}20 \\
+0.001 \\
40.0002 \\
40.01 \\
60.01\end{array}$ & $\begin{array}{r}4 \dot{4} \\
0.016 \\
\times 0.0002 \\
40.01 \\
<0.01\end{array}$ & $\begin{array}{r}4.4 \\
0.0056 \\
\times 0.0002 \\
40.01 \\
40.01\end{array}$ & $\begin{array}{r}2 \dot{2} \\
0.0009 \\
\$ 0.0002 \\
0.01 \\
\$ 0.01\end{array}$ & $\begin{array}{r}22 \\
0.0058 \\
<0.0002 \\
<0.01 \\
<0.01\end{array}$ & $\begin{array}{r}29 \\
0.025 \\
0.0002 \\
80.09 \\
40.01\end{array}$ & $\begin{array}{r}27 \\
0.0029 \\
<0.0002 \\
0.01 \\
0.01\end{array}$ & $\begin{array}{r}0.09 \\
0.0047 \\
\times 0.0002 \\
\times 0.01 \\
\infty 0.01\end{array}$ & $\begin{array}{r}29 \\
0.002 \\
\times 0,0002 \\
0,01 \\
0.01\end{array}$ \\
\hline & & $\begin{array}{l}(w / L) \\
(m, / L)\end{array}$ & $\begin{array}{r}4.5 \\
\times 0.05\end{array}$ & 50,05 & $\begin{array}{r}3 . \dot{0} \\
0.05\end{array}$ & 40.05 & $\begin{array}{r}0,68 \\
\times 0,05\end{array}$ & $\begin{array}{r}0 . \overline{7} \\
40.05\end{array}$ & $\begin{array}{l}0.80 \\
0.05\end{array}$ & $\underset{4,05}{0.65}$ & $\begin{array}{l}0.73 \\
0.05\end{array}$ & 0.75 \\
\hline & & 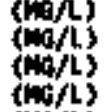 & $\begin{array}{r}0.006 \\
2.6 \\
0.83\end{array}$ & $\begin{array}{r}0.006 \\
2.9 \\
0.96\end{array}$ & $\begin{array}{r}\times .006 \\
2.8 \\
0.034\end{array}$ & $\begin{array}{r}0,000 \\
3,3 \\
0,034\end{array}$ & $\begin{array}{r}0.008 \\
0.59 \\
0.027\end{array}$ & $\begin{array}{r}0.006 \\
0.69 \\
0.005\end{array}$ & $\begin{array}{r}40,00 \dot{4} \\
0.34 \\
0.017\end{array}$ & $\begin{array}{r}<0.00 \% \\
0.59 \\
0.016\end{array}$ & $\begin{array}{r}<0.006 \\
0.014\end{array}$ & $\begin{array}{r}0.006 \\
0.98 \\
0.075\end{array}$ \\
\hline & & $\begin{array}{l}\text { (NG/L) } \\
(N G / L) \\
(N G / L) \\
(N G / L) \\
(N O / L)\end{array}$ & $\begin{array}{r}<0.2 \\
0.0019 \\
0.005 \\
0.092\end{array}$ & $\begin{array}{l}0 . \dot{2} \\
0.0018 \\
00.005 \\
00,002\end{array}$ & $\begin{array}{r}\$ 0 . \overline{2} \\
0.002 \theta \\
40.005 \\
0.012\end{array}$ & $\begin{array}{r}0.2 \\
0.0029 \\
0.005 \\
0.012\end{array}$ & $\begin{array}{l}<0 . j \\
0.001 ! \\
\infty 0.005 \\
0.002\end{array}$ & $\begin{array}{r}\quad 40 . \dot{z} \\
0.0097 \\
40.005 \\
40.002\end{array}$ & $\begin{array}{r}00 . \overline{2} \\
0.0014 \\
40,005 \\
0.0041\end{array}$ & $\begin{array}{r}40 . \dot{z} \\
0.0013 \\
40.005 \\
0.012\end{array}$ & $\begin{array}{r}\times 0.2 \\
0.00956 \\
0.005 \\
0.009\end{array}$ & $\begin{array}{r}\times 0.2 \\
0.00056 \\
40.005 \\
0.002\end{array}$ \\
\hline & & $\begin{array}{l}(w G / L) \\
(N \sigma / L) \\
(N G / L) \\
(N W / L) \\
(N G / L)\end{array}$ & $\begin{array}{l}172 \\
<1 \\
2.4 \\
0.4 \\
1.2\end{array}$ & : & $\begin{array}{r}342 \\
41 \\
2.8 \\
40.1 \\
0,36\end{array}$ & : & $\begin{array}{r}175 \\
\times 1 \\
1.2 \\
<0.1 \\
40.2\end{array}$ & $:$ & $\begin{array}{r}190 \\
\times 1 \\
2 \\
0.1 \\
0.72\end{array}$ & $\vdots$ & $\begin{array}{r}235 \\
\times 1 \\
2.5 \\
0.1 \\
0.68\end{array}$ & $:$ \\
\hline & & $\begin{array}{l}(w / L) \\
(+N / L)\end{array}$ & 4.3 & : & 10 & : & 10 & : & 6.6 & : & 1.5 & : \\
\hline $\begin{array}{l}\text { TOTAL SUSPENDED SOLIDS } \\
\text { TLREIDITY }\end{array}$ & & $\begin{array}{c}(N G / L) \\
(\text { WTU })\end{array}$ & 4.5 & : & 5,3 & : & 8 & : & 1.6 & : & 1 & : \\
\hline
\end{tabular}




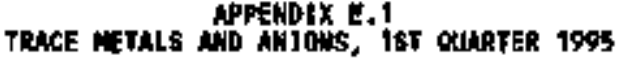

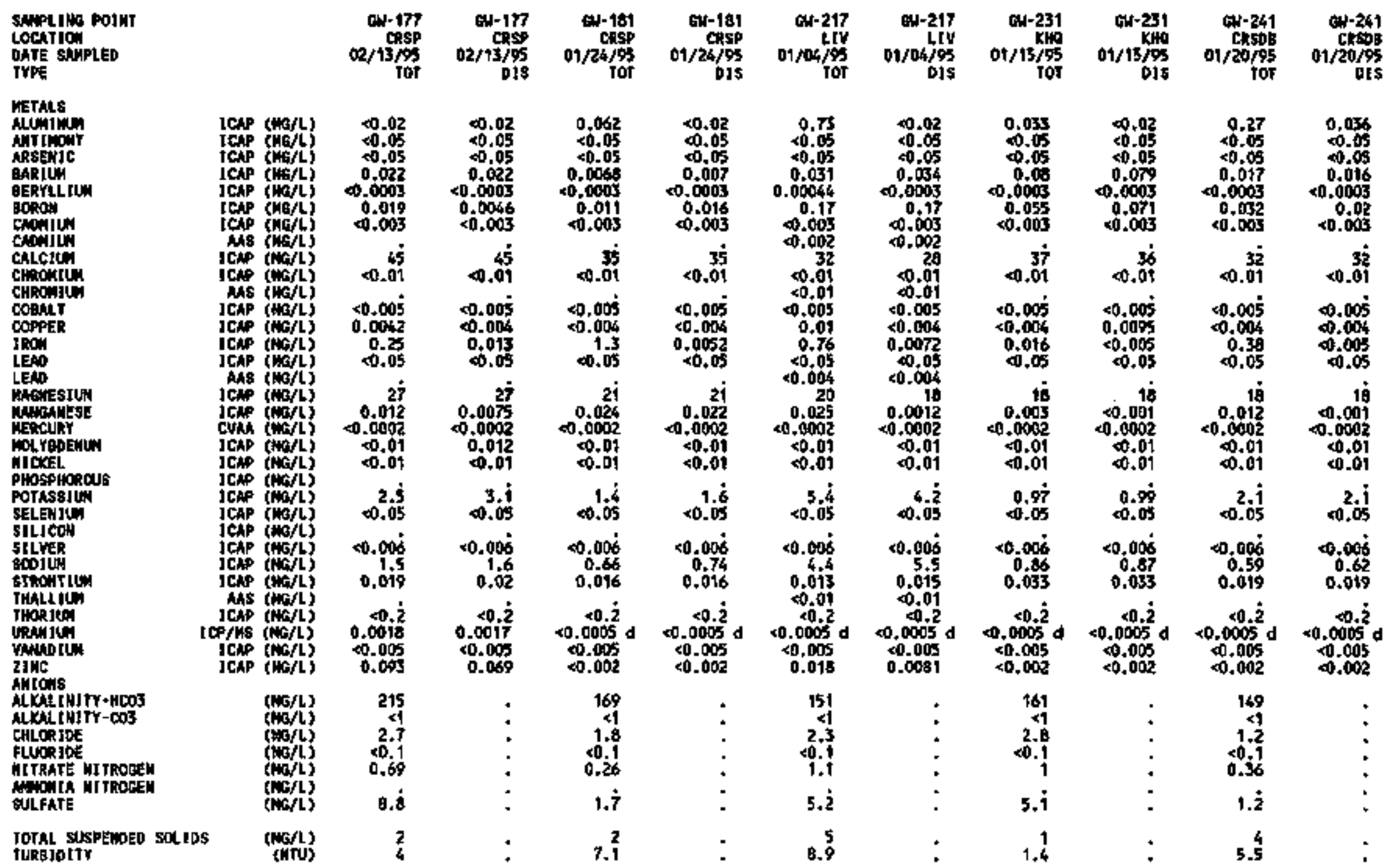




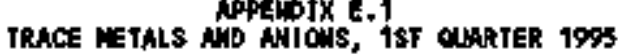

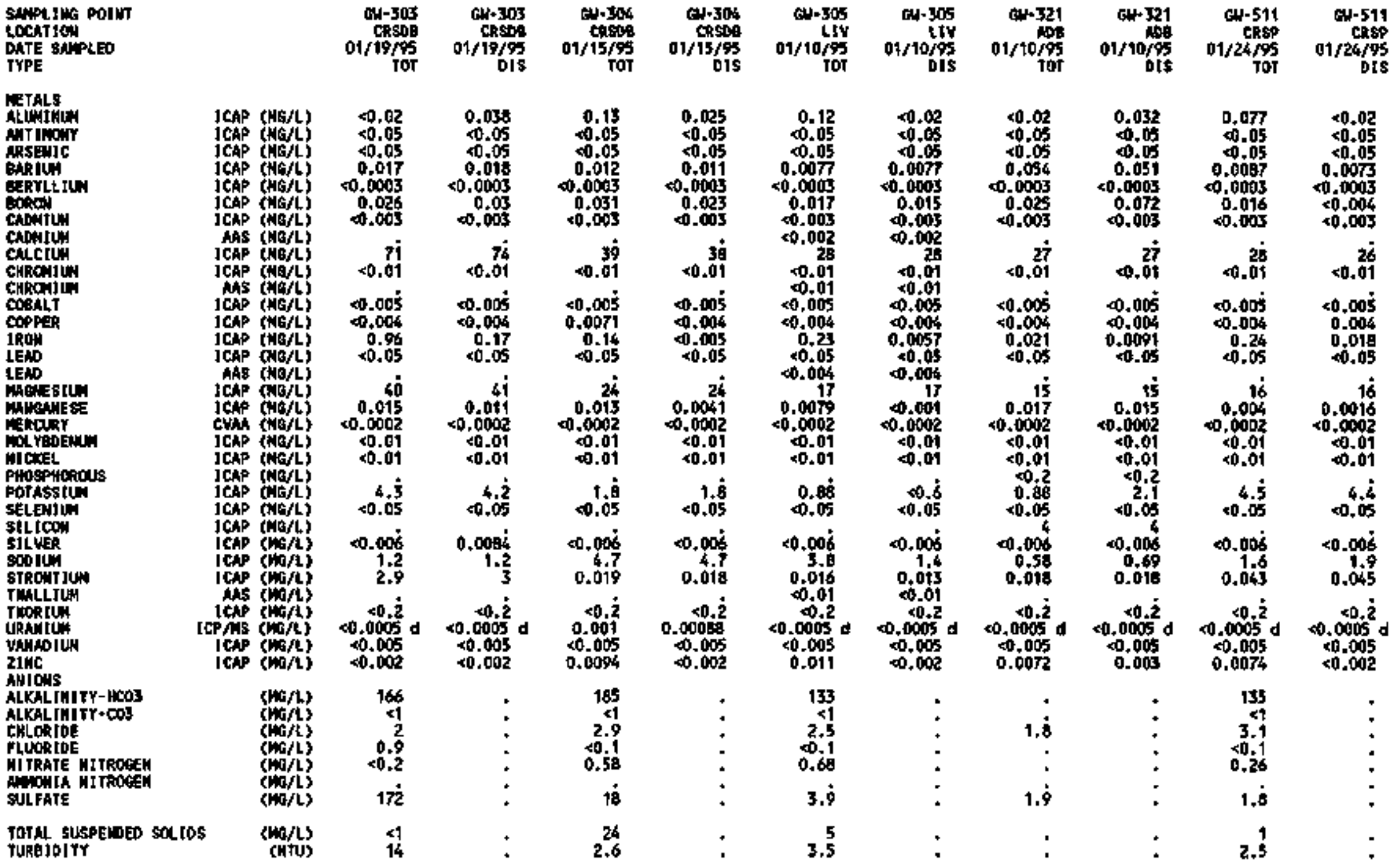




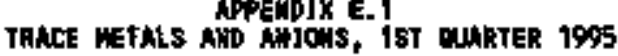

Shlolim pojm

Locktion.

Trpe

METALS

ALUN]

crsetic

agtion

GERYLL1U

goron

chonitus

ChLclin

ChrkanILN

cogALT

COgALT

coprent

LEAD

LEAD

MtCresjum

MHGANESE

Mrosery

NICkel

pliospliogous

POTABSjL

Filenit

SELEMITH

SILVE.

SILVE

STROAT JuM

EHALLIUM

Thon

incingus

wetur

ZinC:

Ancons

AlKats intr-Hog

ALKALIMjTY-cos

ciloribe

Cluporito

MITRAJE HITROCE

Awowt Hutrogen

SULAte

totAl SUSPEMDE SOLIOS

Turka10 TY

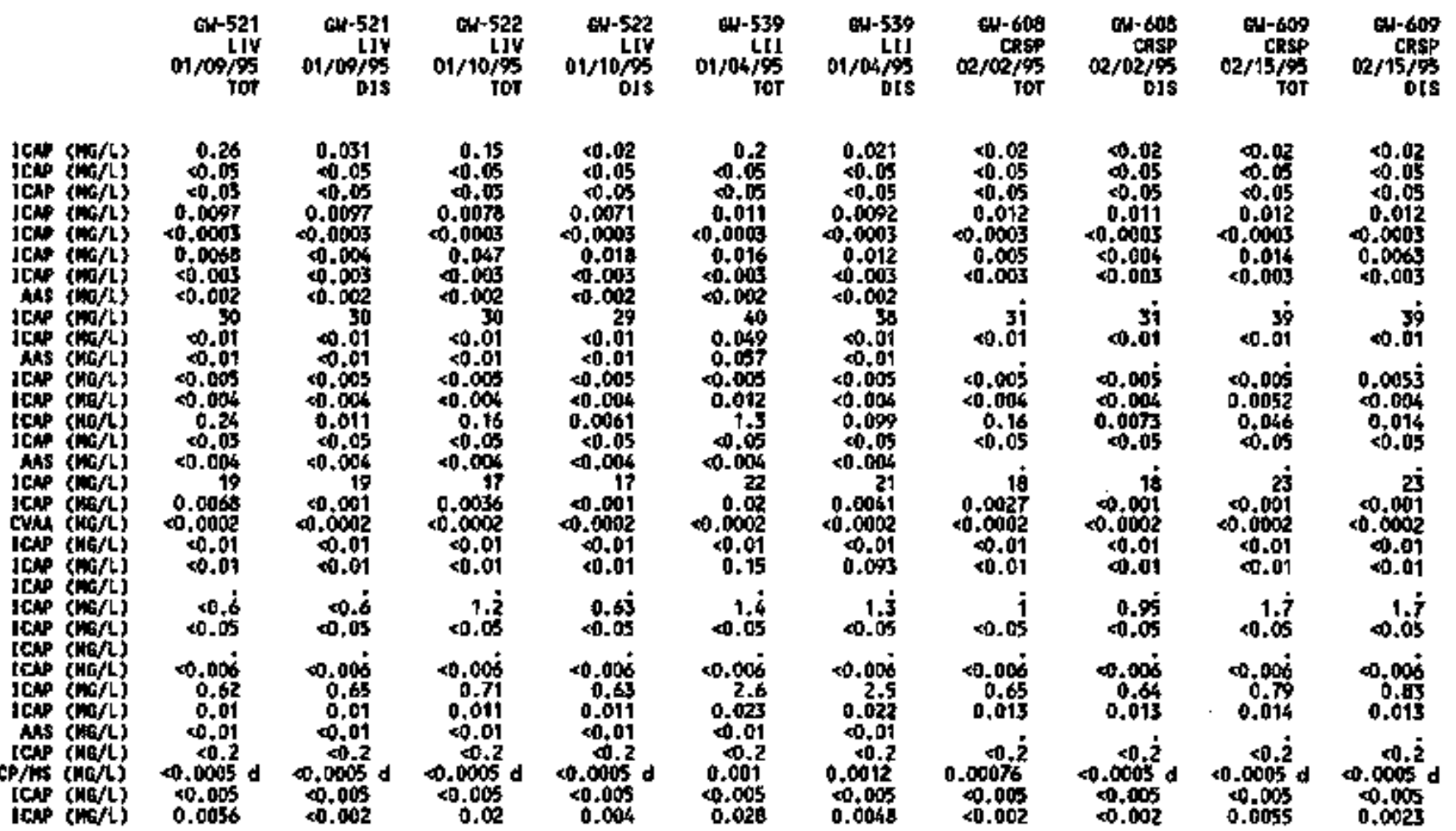

(HG/L)

152

140
21
2.2

$14 \%$

153

196

$(N 15 / L)$

(ML/L)

(Nof $L$ )

(166/1)

2.3
0.1

20.1

2.5

$1 . \dot{5}$

0.2

0.7

(ath

i

2.6

$<0.1$

6.7

3.5

79

1.9

0.3 
TRACE METALS AMD AIIOHB, IST GUARTER 1995

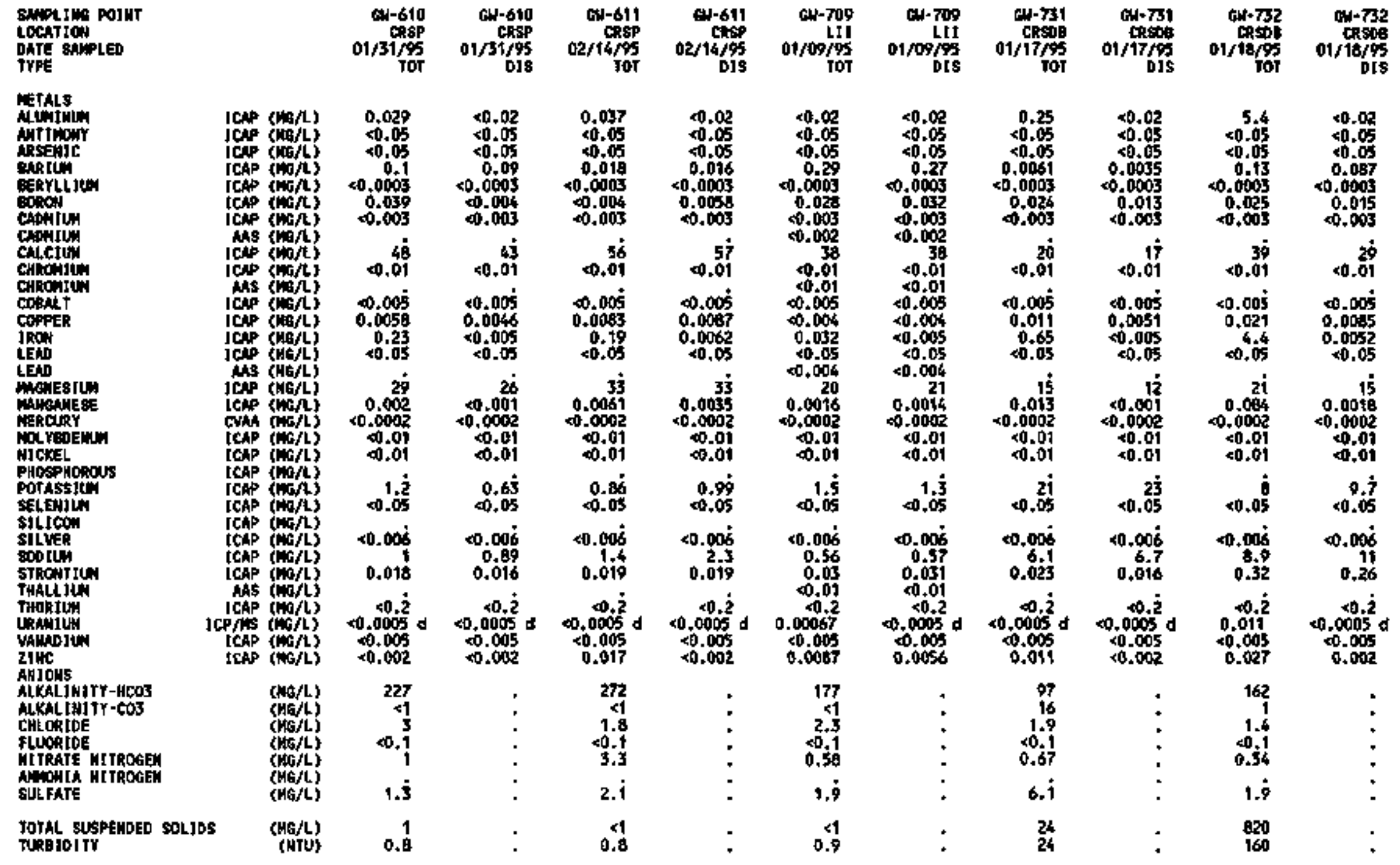


TRACE METALS APPDOHDLE E.1

SNPLING POtHT

LOCATJON

ORTE SNINTED

TYPE

DEThLS

Aluining

Altipinitir

Arsenctiv

Perrintum

Gorow

catoritu

callation

catetuw

Chinowitn

Coght

coppte

IRON

Lent

UEAD

Macinstion

MHGAHESE

mercar

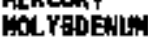

MICKEL

Pliospitands

POTA8sIU

SELENHUN"

SILICD

stuven

TRont rus

tNialtilut

Thoctly'

Maring

ztwc

Anjons

ALKAL HNITY-HCO3

AL KALIH]Tr-COS

CHLORIDE

PLiORIOE

NMPB] MITROE:H

SULFATE

TOFAL SUSPEMDED SOLIDS

TURBIDITY
CH-742
CRsp
$01 / 27 / 95$
TO1

cu-742
chss
$01 / 27 / 95$
018

QW- T43
Cksp
$01 / 31 / 95$
TOT

$c 1-743$
Crsp
$01 / 31 / 95$
018

64-757

01/09/95

[iN-757

osrog/g;

8th2.28P

$03 / 15 / 95$

TOT

tck2.2sp

03/15/45

ots

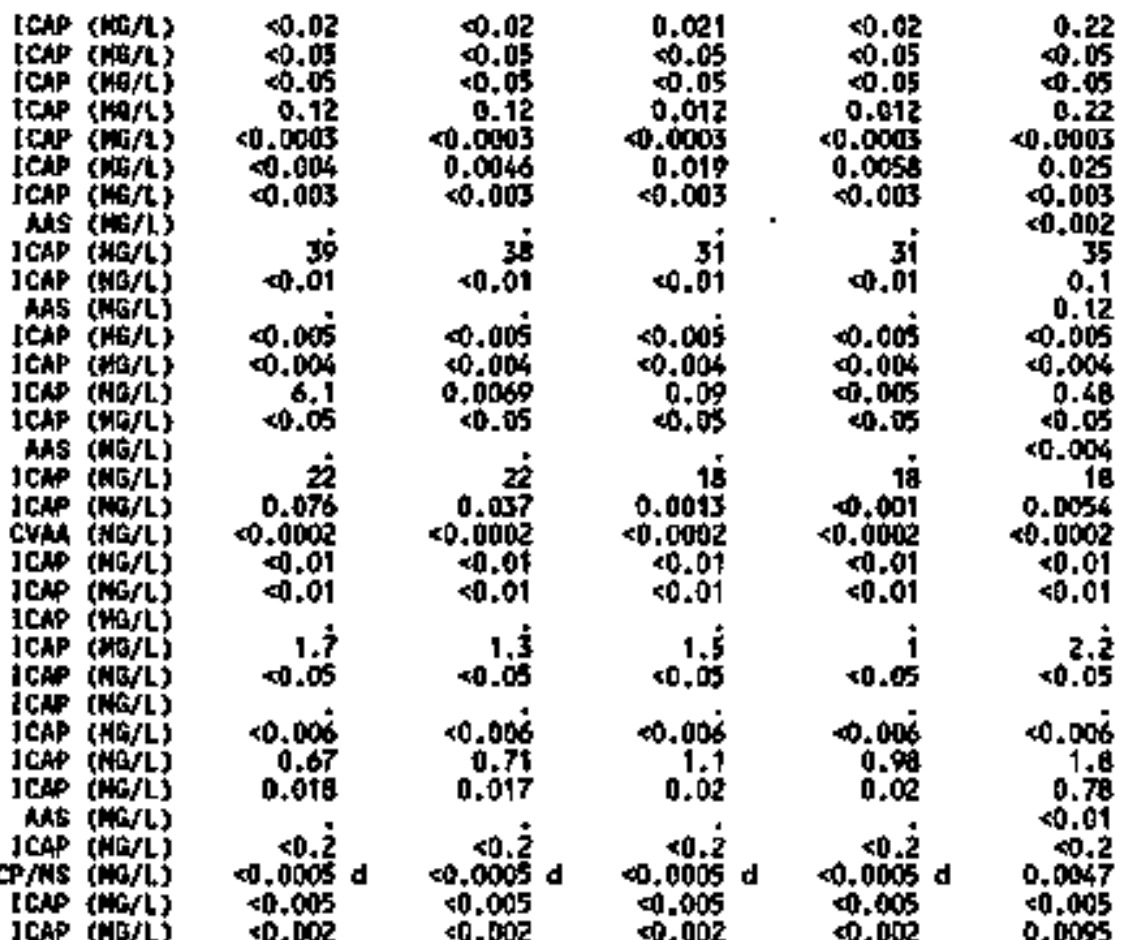

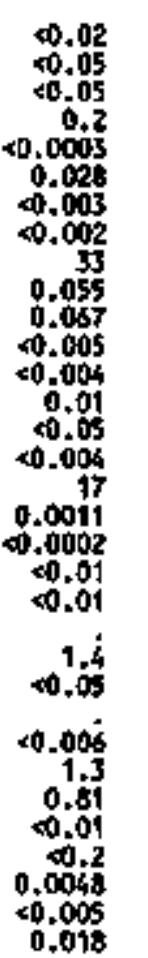

0.099

$<0.05$

0,02

4.0003

0.016
$\times 0.003$

40.47

$<0.005$

0.004

0.25

6.9

0.0002

0.0002

$<0.01$

0.02

$<0.05$

0.023

$<0.0003$

0.027
$\times 6.005$

a.

$4 \mathrm{~B}$
+0.01

$+0.005$

$<0.004$

0.066

i

40.0002

$<0.01$

0.68

0.006 <.0.0

2.

2,5

0.2

.

0.0078

$0.000 . \overline{2}$ d

0.01

143
41
6.5
0.1
2.1
8
3.4


TRACE METAL8 AHD NHIONS, Z1:D OUATER 1995

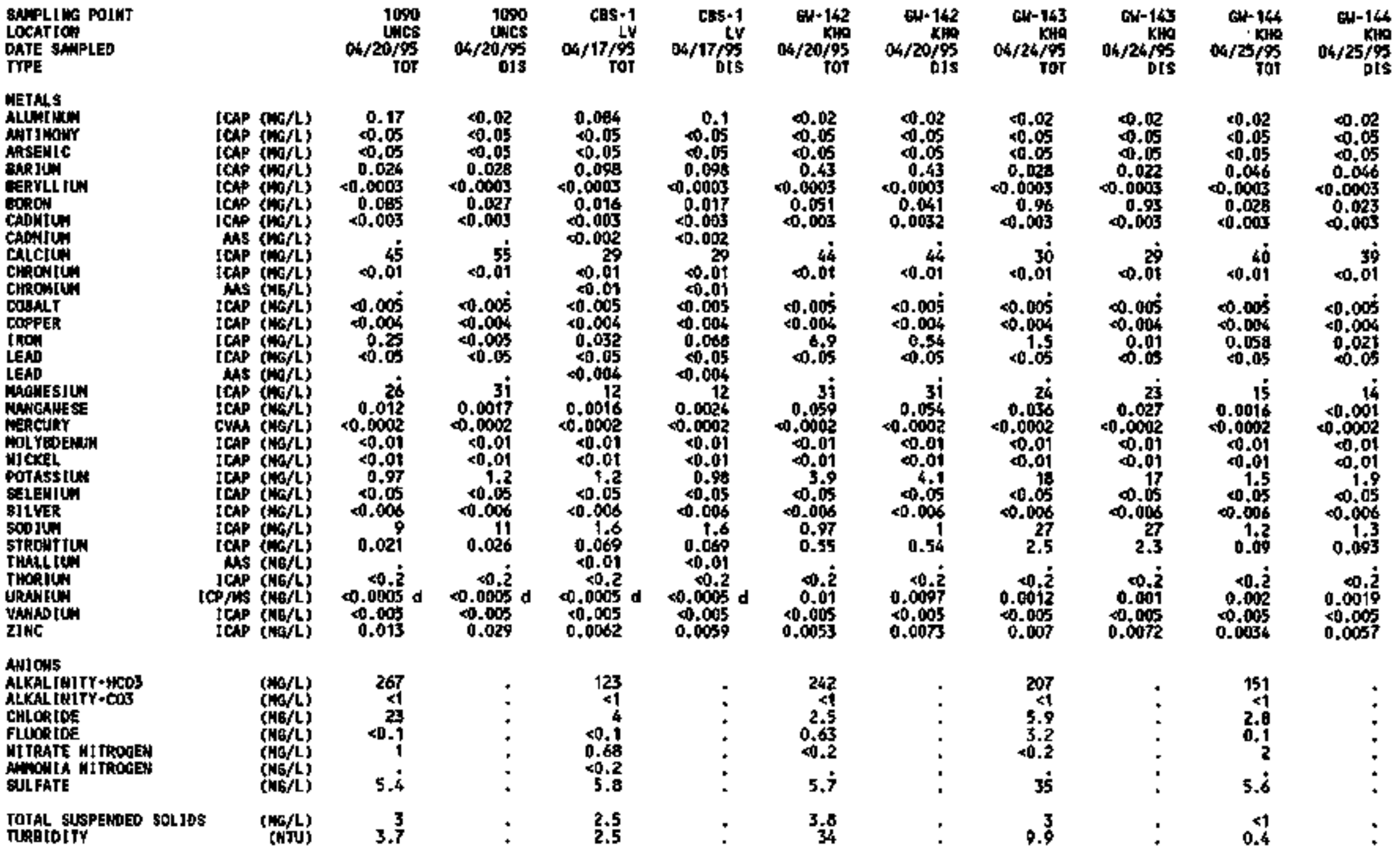


TRACE METALS AHD ANIOWS, aW OAMTER 1995

\begin{tabular}{|c|c|c|c|c|c|c|c|c|c|c|c|c|}
\hline \multicolumn{3}{|l|}{$\begin{array}{l}\text { SNPLIJO POIKT } \\
\text { LOCATJOW } \\
\text { OATE SAHFLED } \\
\text { THPE }\end{array}$} & $\begin{array}{r}014-145 \\
1449 \\
04 / 26 / 85 \\
\text { Tor }\end{array}$ & $\begin{array}{r}6 \mathrm{w}+145 \\
\mathrm{KHa} \\
04 / 26 / 95 \\
\text { DIs }\end{array}$ & $\begin{array}{r}0.148 \\
1010 \\
04 / 24 / 95 \\
\text { To1 }\end{array}$ & $\begin{array}{r}04-166 \\
1619 \\
04 / 24 / 95 \\
015\end{array}$ & $\begin{array}{r}\text { Gis-147 } \\
\text { KHA } \\
04 / 21 / 9 S \\
\text { TDT }\end{array}$ & $\begin{array}{r}0 \mathrm{Or}-147 \\
\mathrm{KHO} \\
04 / 21 / 95 \\
018\end{array}$ & $\begin{array}{r}\text { CN-150 } \\
\text { CRsog } \\
04 / 07 / 95 \\
\text { Tor }\end{array}$ & $\begin{array}{r}\text { GH-156 } \\
\text { CRSDB } \\
04 / 07 / 95 \\
\text { ols }\end{array}$ & $\begin{array}{r}\text { cln- } 158 \\
\text { CRsp: } \\
04 / 13 / 95 \\
\text { ToT }\end{array}$ & $\begin{array}{r}\text { ar-158 } \\
\text { cospos } \\
06 / 13 / 45 \\
\text { ots }\end{array}$ \\
\hline \multirow{5}{*}{ 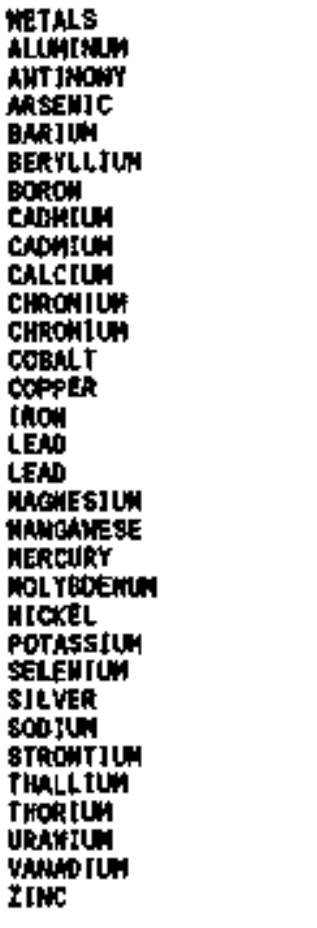 } & \multirow{5}{*}{ 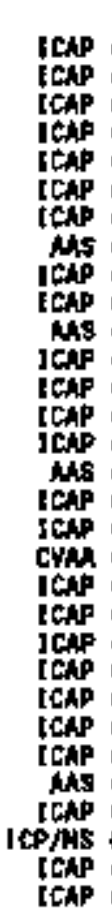 } & \multirow{5}{*}{ 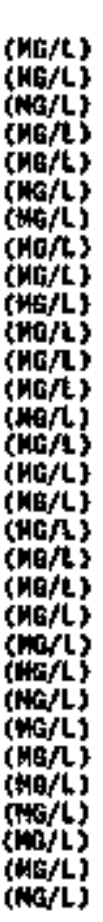 } & $\begin{array}{r}0.066 \\
<0.05 \\
<0.05 \\
0.094 \\
0.0003 \\
0.24 \\
0.003\end{array}$ & $\begin{array}{r}0.026 \\
60.05 \\
\times 0.05 \\
0.057 \\
0.0005 \\
0.25 \\
<0.003\end{array}$ & $\begin{array}{r}6.02 \\
40.05 \\
\times 0.05 \\
0.063 \\
4.0003 \\
40.005\end{array}$ & $\begin{array}{r}0.02 \\
0.05 \\
0.05 \\
0.048 \\
\times 0.0003 \\
0.39 \\
0.003\end{array}$ & $\begin{array}{r}6.3 \\
40.05 \\
0.055 \\
0.25 \\
0.0007 \\
0.002 \\
0.0031\end{array}$ & $\begin{array}{r}80.02 \\
<0.05 \\
20.05 \\
0.081 \\
40.0003 \\
0.4 \\
40.003\end{array}$ & $\begin{array}{r}0.13 \\
0.05 \\
\times 0.05 \\
0.046 \\
\times 0.0003 \\
0.018 \\
0.005\end{array}$ & $\begin{array}{r}0.047 \\
40.05 \\
80,05 \\
0.052 \\
40.0003 \\
0.018 \\
40.003\end{array}$ & $\begin{array}{r}0.059 \\
0.05 \\
0.05 \\
0.007 \\
\times 0.0005 \\
0.005 \\
0.003\end{array}$ & $\begin{array}{r}0.045 \\
40.05 \\
<0.05 \\
0.046 \\
40.0003 \\
0.012 \\
0.003\end{array}$ \\
\hline & & & $\begin{array}{r}4 \overline{7} \\
<0.01\end{array}$ & $<0 . \dot{42}$ & $\begin{array}{r}4 \dot{0} \\
40.01\end{array}$ & $\begin{array}{r}3 \dot{3} \\
\times 0.01\end{array}$ & $\begin{array}{r}7 i \\
<0.01\end{array}$ & $<0,0 t$ & $\begin{array}{r}70 \\
40.01\end{array}$ & $\begin{array}{r}69 \\
<0.01\end{array}$ & 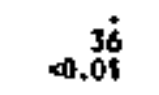 & $\begin{array}{r}3 \dot{6} \\
0.01\end{array}$ \\
\hline & & & $\begin{array}{r}40.005 \\
0.004 \\
0.1 \\
0.05\end{array}$ & $\begin{array}{r}<0.005 \\
+0.006 \\
0.0071 \\
<0.05\end{array}$ & $\begin{array}{r}<0.005 \\
<0.004 \\
0.05 \\
<0.05\end{array}$ & $\begin{array}{r}<0.005 \\
0.004 \\
0.0075 \\
40.05\end{array}$ & $\begin{array}{r}0.0081 \\
0.014 \\
13 \\
=0.05\end{array}$ & $\begin{array}{l}\infty .005 \\
0.004 \\
0.011 \\
<0.05\end{array}$ & $\begin{array}{r}0.005 \\
0.004 \\
0.21 \\
0.05\end{array}$ & $\begin{array}{r}60.095 \\
0.0040 \\
0.011 \\
\times 0.05\end{array}$ & $\begin{array}{r}\infty .005 \\
\infty .004 \\
0.15 \\
0.05\end{array}$ & $\begin{array}{r}40.005 \\
40.004 \\
0.022 \\
40.05\end{array}$ \\
\hline & & & $\begin{array}{r}35 \\
0.01 \\
<0.0002 \\
<0.01 \\
<0.01 \\
+3 \\
0.05 \\
\times 0.006 \\
5.8 \\
7.8\end{array}$ & $\begin{array}{r}34 \\
0.0027 \\
0.0002 \\
60.01 \\
<0.01 \\
72 \\
00.05 \\
0.006 \\
5.9 \\
7.4\end{array}$ & $\begin{array}{r}34 \\
0.0005 \\
40.0002 \\
40.01 \\
0.01 \\
16 \\
40.05 \\
40.006 \\
13 \\
7\end{array}$ & $\begin{array}{r}28 \\
0.0061 \\
<0.0002 \\
0.01 \\
0.01 \\
\times 0.05 \\
\times 0.006 \\
41 \\
4.2\end{array}$ & $\begin{array}{r}26 \\
1.7 \\
0.0002 \\
80.01 \\
0.022 \\
40.05 \\
<0.006 \\
4.2 \\
2.6\end{array}$ & $\begin{array}{r}20 \\
0.0013 \\
0.0002 \\
0.04 \\
40.04 \\
5.5 \\
40.05 \\
40.006 \\
4\end{array}$ & $\begin{array}{r}4 t \\
0.0059 \\
<0.0002 \\
60.04 \\
80.0 t \\
6.0 \\
40.05 \\
0.006 \\
5.3 \\
0.044\end{array}$ & $\begin{array}{r}41 \\
0.0022 \\
0.0002 \\
<0.01 \\
\times 0.01 \\
7.3 \\
40.05 \\
40.006 \\
5.5 \\
0.05\end{array}$ & $\begin{array}{r}20 \\
0.0056 \\
\times 0.0002 \\
40.01 \\
\times 0.01 \\
0.85 \\
\times 10.05 \\
\times 0.006 \\
0.58 \\
0.022\end{array}$ & $\begin{array}{r}20 \\
0.0062 \\
\times 0.0002 \\
0.01 \\
0.01 \\
0.80 \\
00.05 \\
0.006 \\
0.58 \\
0.022\end{array}$ \\
\hline & & & $\begin{array}{r}0 . \dot{2} \\
0.012 \\
0.005 \\
0.0079\end{array}$ & $\begin{array}{r}0 . j \\
0.012 \\
0.005\end{array}$ & $\begin{array}{r}0 . j \\
0.0916 \\
0.005 \\
0.0072\end{array}$ & $\begin{array}{l}<0 . \dot{2} \\
0.0014 \\
20.005 \\
0.0096\end{array}$ & $\begin{array}{l}0 . j \\
0.003 \\
0.02 \\
0.11\end{array}$ & $\begin{array}{r}50, \dot{z} \\
0.0024 \\
50.005 \\
0.0096\end{array}$ & $\begin{array}{r}0 . \dot{2} \\
0.0037 \\
0.005 \\
0.016\end{array}$ & $\begin{array}{r}\times 0.2 \\
0.0037 \\
0.005 \\
0.0054\end{array}$ & $\begin{array}{l}0.00 .2 \\
00,005 \\
0.0054\end{array}$ & 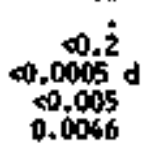 \\
\hline \multirow{2}{*}{\multicolumn{2}{|c|}{ 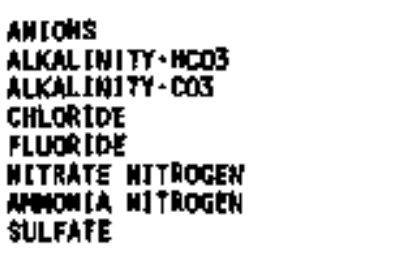 }} & \multirow{2}{*}{ 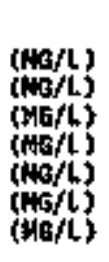 } & $\begin{array}{r}232 \\
<1 \\
9.7 \\
2.4 \\
0.35\end{array}$ & $:$ & $\begin{array}{r}19.8 \\
\times 1 \\
11 \\
2.8 \\
0.5\end{array}$ & : & $\begin{array}{r}169 \\
<1 \\
2.6 \\
0.7 \\
1.1\end{array}$ & $:$ & $\begin{array}{r}353 \\
<1 \\
3.2 \\
<0.1 \\
0.71\end{array}$ & $\vdots$ & $\begin{array}{r}175 \\
1.4 \\
<0.1 \\
<0.2\end{array}$ & $\dot{*}$ \\
\hline & & & 37 & $\dot{.}$ & $7 \dot{0}$ & ; & $5 . \dot{5}$ & $\dot{.}$ & 9.1 & : & $0 . \dot{2}$ & $:$ \\
\hline $\begin{array}{l}\text { TOTM sUSPEIDED SOL IDS } \\
\text { THRBTDtY }\end{array}$ & & $\begin{array}{l}\text { (MB/L) } \\
\text { (Hitl) }\end{array}$ & 4,2 & $:$ & 6.2 & : & 18 & : & 3.8 & 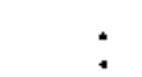 & 1,4 & 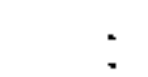 \\
\hline
\end{tabular}


TRACE NETALS AW ANIOHS, 2ND OUARTER 1995

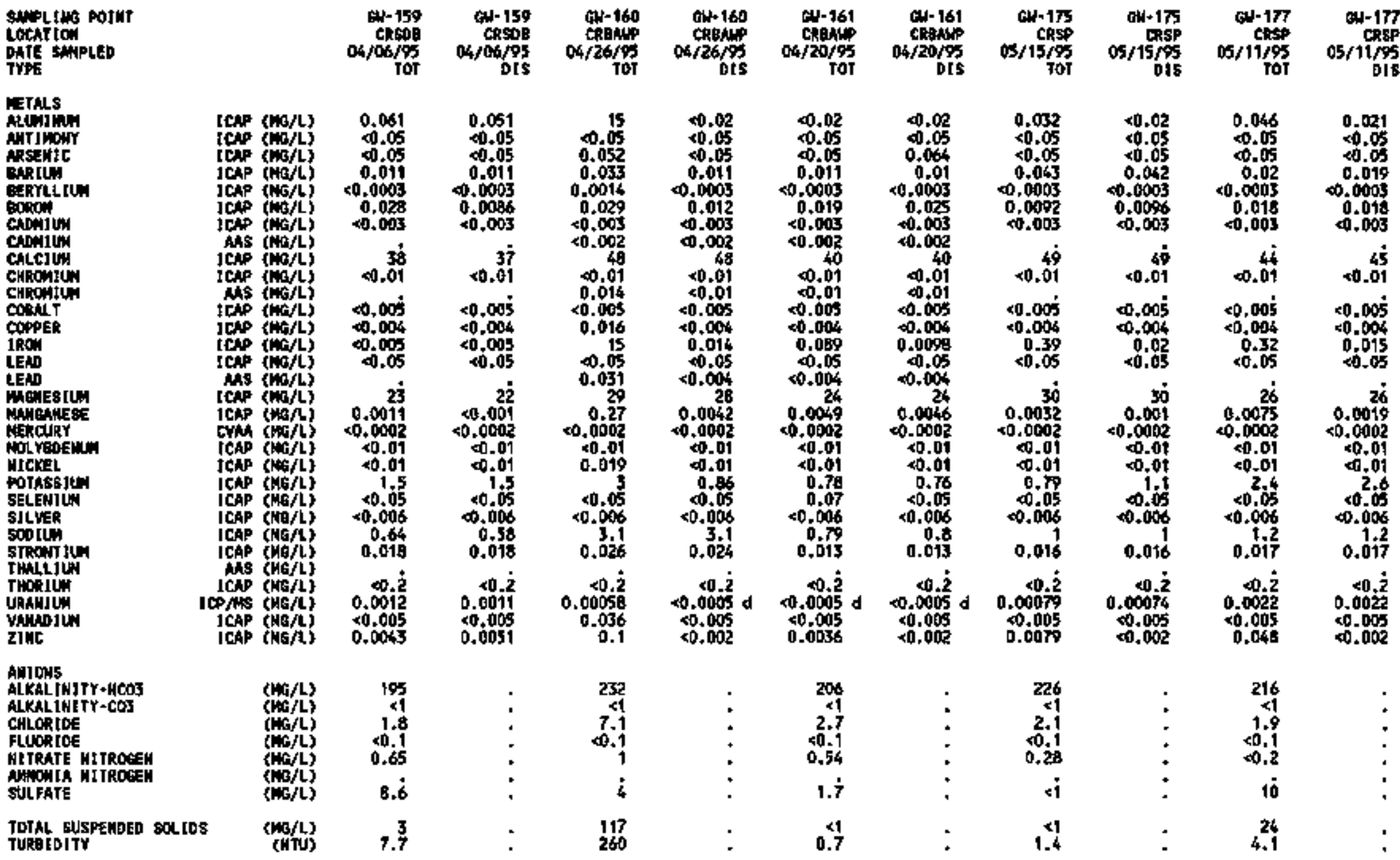




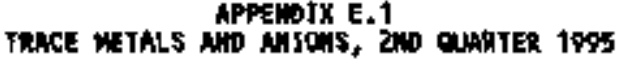

sarpling PothT

Lochtron

DATE SWPLED

\section{metal8}

Altinimin

AHSENIC

Bar IUN

Bexritidu

列

croniun

CALCJU

Chinonjum

CARONJU

coost

copp

LEAP

Hentrstia

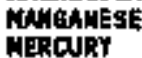

moreorint

ifJext

PDIASSIUY

SELEHIOM

scortew

STROHTIUN

THaty

ipenju

voutantin

7)inc

Nistons

AtKAt JnITY - mos

ALCAL] J I Tr-cos

CHLOR be

FUOR IDE

MIIRATE MITROGEN

HWON IA HJTROGE

SULFATE

TOTAL SULPENDEO SOLIDS

TUREIDITY

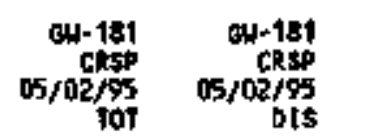

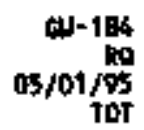

$05-184$
$05 / 01 / 95$
015

CW- 186
Ro
$05 / 03 / 25$
TOT

0,186
$\cos / 05 / 205$
Dis

$6 N-197$
00
$05 / 02 / 53$
$T 0 \%$

187
Ro
Os/02/5s
D1s

$05 / 101 / 95$
Tot

GH- 486 Os/01/

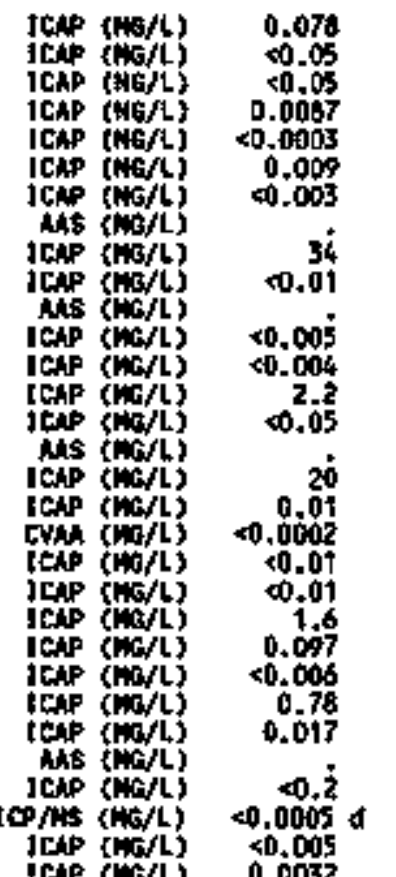

\begin{tabular}{|c|}
\hline $\begin{array}{r}0.022 \\
0.05 \\
0.05 \\
0.0047 \\
40.0005 \\
0.0092 \\
0.003\end{array}$ \\
\hline$\times 0.01$ \\
\hline $\begin{array}{l}<0.005 \\
<0.004 \\
0.0090 \\
<0.45\end{array}$ \\
\hline $\begin{array}{r}19 \\
0.0049 \\
0.0002 \\
\times 0.01 \\
\times 0.01 \\
1.9 \\
0.054 \\
\times 0.006 \\
0.79 \\
0.0+6\end{array}$ \\
\hline $\begin{array}{c}<0, \bar{z} \\
0.0005 \\
\times 0.005 \\
\times 0.002\end{array}$ \\
\hline
\end{tabular}

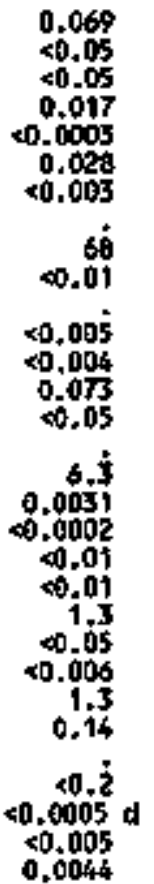

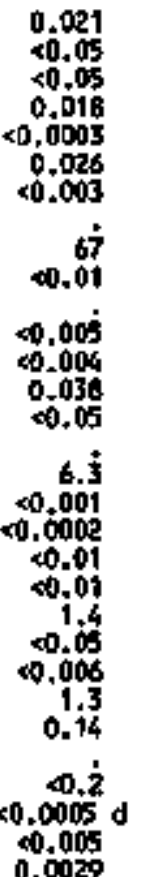

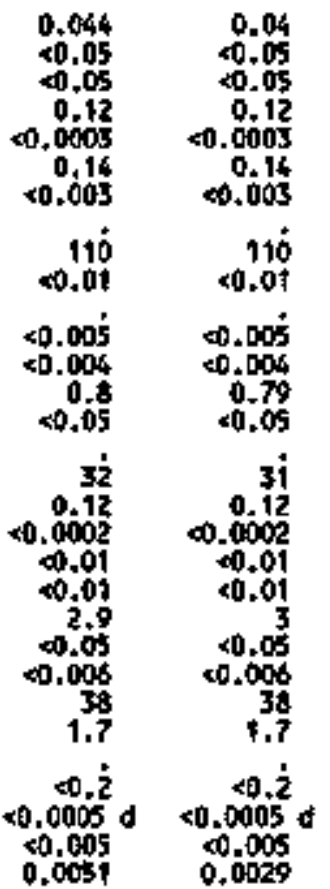

0.047
0.05
0.05
0.27
0.0003
0.54
0.003
40.01
$<0.005$
$<0.004$
0.061
40.05
0.0031
00.0002
$<0.01$
40.01
$\times 0.05$
$<0.006$
140
0.91
$40 . \dot{2}$
0.00050
$<0.005$
0.001

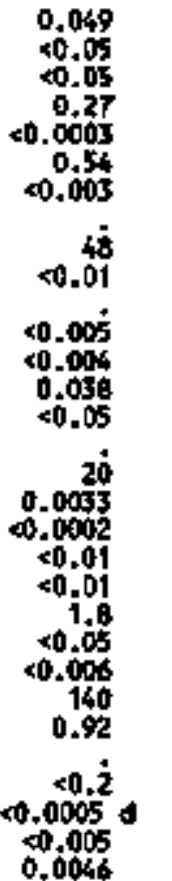

\subsection{7}

$<0.05$

0.036

40.0003

0.13

.003

35

$\infty, 005$

0.004

0.13
$\times 0.05$

24

0.0 .02

$+01$

4.01

40.05

$<0.006$

1

$<0 . \dot{2}$

$0.0005 d$

0.005

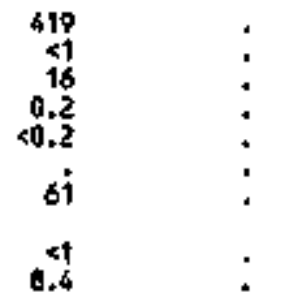

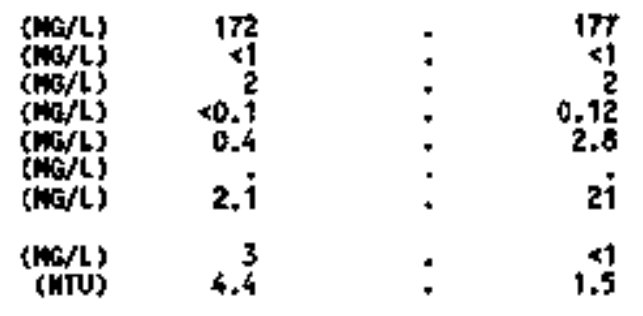

349
41
110
1.1
0.65
24
51
30

207
4
5
0.73
0.37
27
41
2

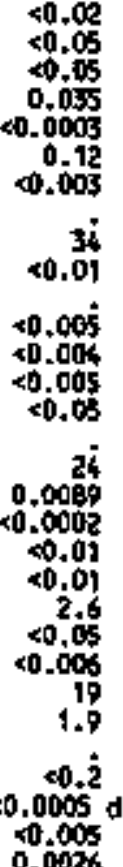




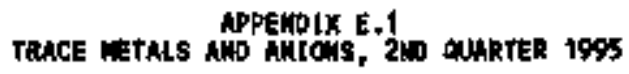

\begin{tabular}{|c|c|c|c|c|c|c|c|c|c|c|c|c|}
\hline \multicolumn{3}{|l|}{ 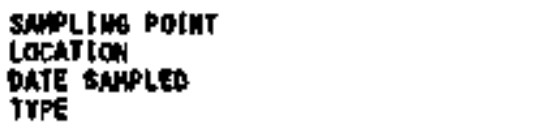 } & $\begin{array}{r}64-203 \\
\text { tocs } \\
04 / 4 / 45 \\
\text { ToT }\end{array}$ & $\begin{array}{r}04-203 \\
0465 \\
04 / 18 / 95 \\
0 \text { [5 }\end{array}$ & $\begin{array}{r}\text { ch-20s } \\
\text { tucs } \\
04 / 1 \frac{105}{\text { ToT }}\end{array}$ & $\begin{array}{r}04-205 \\
\text { uncs } \\
04 / 1 \text { des } \\
\text { ots }\end{array}$ & $\begin{array}{r}6 N-221 \\
\text { Lewcs } \\
04 / 19 / \% 5 \\
\text { T0F }\end{array}$ & $\begin{array}{r}0+221 \\
0405 \\
04 / 19 / 95 \\
\text { DIS }\end{array}$ & 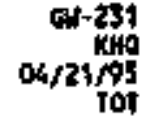 & $\begin{array}{r}014-231 \\
\times 1 \% 0 \\
04 / 21 / 95 \\
015\end{array}$ & $\begin{array}{r}\text { GN-241 } \\
\text { corsts } \\
0,09 / 95 \\
\text { Tor }\end{array}$ & $\begin{array}{r}\text { er-241 } \\
\text { CRsos } \\
04 \text { ro9/9s } \\
\text { ols }\end{array}$ \\
\hline \multirow{5}{*}{\multicolumn{2}{|c|}{ 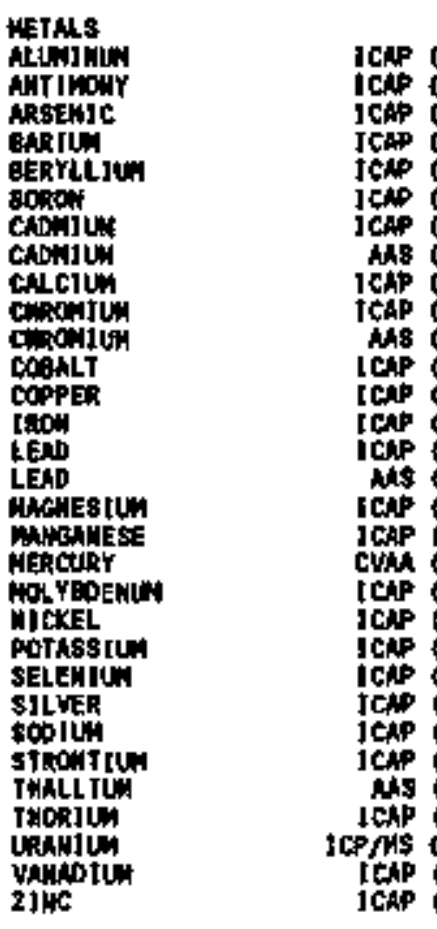 }} & 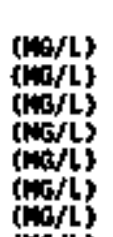 & $\begin{array}{r}0.28 \\
<0.05 \\
\times 10.05 \\
0.0086 \\
\times 0.0003 \\
0.0074 \\
0.003\end{array}$ & $\begin{array}{r}0.02 \\
0.05 \\
40.05 \\
0.0078 \\
<0.0003 \\
0.061 \\
<0.003\end{array}$ & $\begin{array}{r}0.048 \\
0.05 \\
40.05 \\
0.014 \\
80.0003 \\
0.0092 \\
0.003\end{array}$ & $\begin{array}{r}40.02 \\
0.05 \\
0.05 \\
0.014 \\
0.0005 \\
0.034 \\
<0.003\end{array}$ & $\begin{array}{r}\infty .02 \\
00.05 \\
\times 0.05 \\
0.0073 \\
0.0003 \\
0.053 \\
\times 0.003\end{array}$ & $\begin{array}{r}80.02 \\
40.05 \\
+0.05 \\
0.0073 \\
0.0003 \\
0.017 \\
\times 0.003\end{array}$ & $\begin{array}{r}50.02 \\
\times 0.05 \\
0.05 \\
0.081 \\
0.0003 \\
0.0082 \\
40.003\end{array}$ & $\begin{array}{r}0.02 \\
0.05 \\
0.05 \\
0.08 \\
\times 0.0003 \\
0.015 \\
\times 0.003\end{array}$ & $\begin{array}{r}2.5 \\
0.05 \\
0.05 \\
0.022 \\
0.0005 \\
0.013 \\
0.003\end{array}$ & $\begin{array}{r}0.063 \\
20.05 \\
60.05 \\
0.015 \\
0.0003 \\
0.014 \\
\times 0.003\end{array}$ \\
\hline & & $\begin{array}{l}(\mathrm{wC} / \mathrm{L}) \\
(\mathrm{wg} / \mathrm{L}) \\
(\mathrm{w} / \mathrm{h})\end{array}$ & 40.301 & $<0 . \dot{01}$ & $\begin{array}{r}3 \dot{31} \\
40.01\end{array}$ & $\begin{array}{r}31 \\
0.01\end{array}$ & $\begin{array}{r}30 \\
<0.01\end{array}$ & $\begin{array}{r}29 \\
\times 0.01\end{array}$ & $\begin{array}{r}3 \dot{3} \\
\times 0 . \dot{01}\end{array}$ & $\infty .01$ & $\infty . \dot{01}$ & 40.01 \\
\hline & & 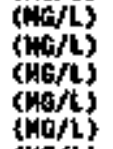 & $\begin{array}{r}<0.005 \\
<0.006 \\
0.47 \\
0.05\end{array}$ & $\begin{array}{r}<0.005 \\
\times 0.004 \\
0.029 \\
<0.05\end{array}$ & $\begin{array}{r}9.005 \\
0.004 \\
0.016 \\
0.05\end{array}$ & $\begin{array}{r}<0.005 \\
<0.004 \\
0.0094 \\
40.05\end{array}$ & $\begin{array}{l}\infty .005 \\
0.004 \\
0.033 \\
0.05\end{array}$ & $\begin{array}{l}\times 0.0055 \\
\times 0.004 \\
0.005 \\
0.05\end{array}$ & $\begin{array}{l}0.005 \\
0.004 \\
0.0061 \\
0.05\end{array}$ & $\begin{array}{r}20.005 \\
<0.004 \\
\times 0.005 \\
<0.05\end{array}$ & $\begin{array}{r}<0.005 \\
<0.004 \\
2.4 \\
0.05\end{array}$ & $\begin{array}{l}20.005 \\
\times 0.004 \\
0.0055 \\
0.05\end{array}$ \\
\hline & & 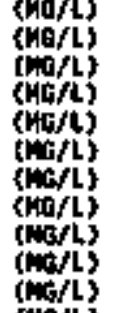 & $\begin{array}{r}17 \\
0.073 \\
0.0002 \\
0.01 \\
0.01 \\
1.1 \\
0.05 \\
0.008 \\
0.52 \\
0.01 t\end{array}$ & $\begin{array}{r}16 \\
0.0022 \\
40.0002 \\
0.01 \\
0.01 \\
0.09 \\
0.05 \\
0.004 \\
0.56 \\
0.011\end{array}$ & $\begin{array}{r}18 \\
0.009 \\
\times 0.0092 \\
0.01 \\
0.01 \\
1.4 \\
00.05 \\
0.006 \\
0.72 \\
0.012\end{array}$ & $\begin{array}{r}18 \\
0.0964 \\
0.0002 \\
\times 0.01 \\
40.01 \\
1.2 \\
\times 0.03 \\
0.008 \\
0.76 \\
0.017\end{array}$ & $\begin{array}{r}18 \\
0.0015 \\
\times 0.0002 \\
40.01 \\
0.01 \\
0.09 \\
80.05 \\
0.006 \\
0.67 \\
0.01\end{array}$ & $\begin{array}{r}17 \\
40.001 \\
<0.0007 \\
60.01 \\
40.09 \\
0.99 \\
20.05 \\
<0.006 \\
0.61 \\
0.01\end{array}$ & $\begin{array}{r}20 \\
40.001 \\
4.0002 \\
0.01 \\
40.01 \\
0.60 \\
0.05 \\
40.008 \\
0.05 \\
0.055\end{array}$ & $\begin{array}{r}20 \\
<0,001 \\
0.0002 \\
40.01 \\
40,01 \\
0.9 \\
<0.05 \\
\times 0.006 \\
0.08 \\
0.035\end{array}$ & $\begin{array}{r}16 \\
0.096 \\
0.9002 \\
40.01 \\
4.01 \\
2.00 \\
40.05 \\
0.006 \\
0.83 \\
0.02\end{array}$ & $\begin{array}{r}15 \\
\$ 0.001 \\
\times 0.002 \\
40.01 \\
0.01 \\
2.3 \\
0.05 \\
\times 0,006 \\
0.07 \\
0.019\end{array}$ \\
\hline & & 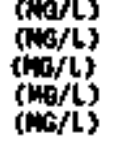 & $\underset{0.0005}{0 . j} d$ & $\begin{array}{c}0 . \overline{2} \\
0.0005 \\
0.005 \\
0.0006\end{array}$ & $\begin{array}{r}40.2 \\
0.00092 \\
50.005 \\
0.00 .7\end{array}$ & $\begin{array}{r}00 . \overline{2} \\
0.00058 \\
0.005 \\
0.007\end{array}$ & $\begin{array}{c}\infty 0.2 \\
\times 0.005 \\
0.005 \\
0.009\end{array}$ & $\begin{array}{c}\infty .0 .2 \\
0.0005 \\
0.0005 \\
0.0012\end{array}$ & $\begin{array}{c}0.0 .2 \\
<0.005 \\
0.0024\end{array}$ & $\begin{array}{c}<0 . \dot{2} \\
<0.0005 d \\
20.005 \\
0.0054\end{array}$ & $\underset{\substack{0.0005 \\
0,0.061 \\
0.018}}{\infty}$ & $\begin{array}{c}0.005 \\
<0.005 \\
0.005\end{array}$ \\
\hline \multirow{2}{*}{\multicolumn{2}{|c|}{ 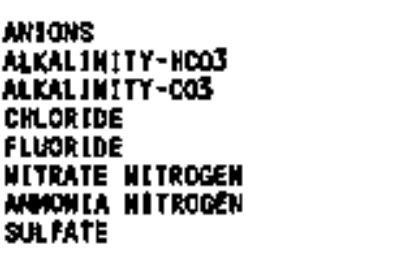 }} & 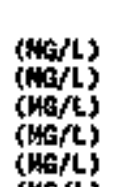 & $\begin{array}{r}138 \\
41 \\
1.9 \\
40.1 \\
1.4\end{array}$ & $\dot{:}$ & $\begin{array}{r}160 \\
11 \\
2.7 \\
40.1 \\
6.3\end{array}$ & : & $\begin{array}{r}151 \\
41 \\
1.9 \\
0.1 \\
0.9\end{array}$ & $\dot{:}$ & $\begin{array}{r}171 \\
61 \\
2.6 \\
\times 0.1 \\
1.1\end{array}$ & $:$ & $\begin{array}{r}144 \\
<1 \\
1.5 \\
\times 0.1 \\
\times 0.2\end{array}$ & $\vdots$ \\
\hline & & $\begin{array}{l}\{(M G / L) \\
\{(M 6 / L)\end{array}$ & $\uparrow, \overline{2}$ & $\dot{.}$ & $6 . i$ & $\dot{.}$ & 1.8 & $\dot{r}$ & $4 . \dot{2}$ & ; & $1 \dot{7}$ & : \\
\hline \multicolumn{2}{|l|}{$\begin{array}{l}\text { TOTML SUSPEEDE SOLJOS } \\
\text { TURBDItY }\end{array}$} & 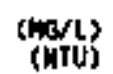 & $\begin{array}{l}5.5 \\
7.1\end{array}$ & : & 2.8 & : & 1 & : & 0.1 & : & $\begin{array}{l}40 \\
50\end{array}$ & ; \\
\hline
\end{tabular}




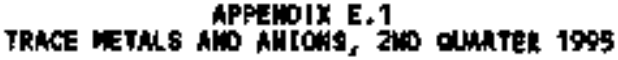

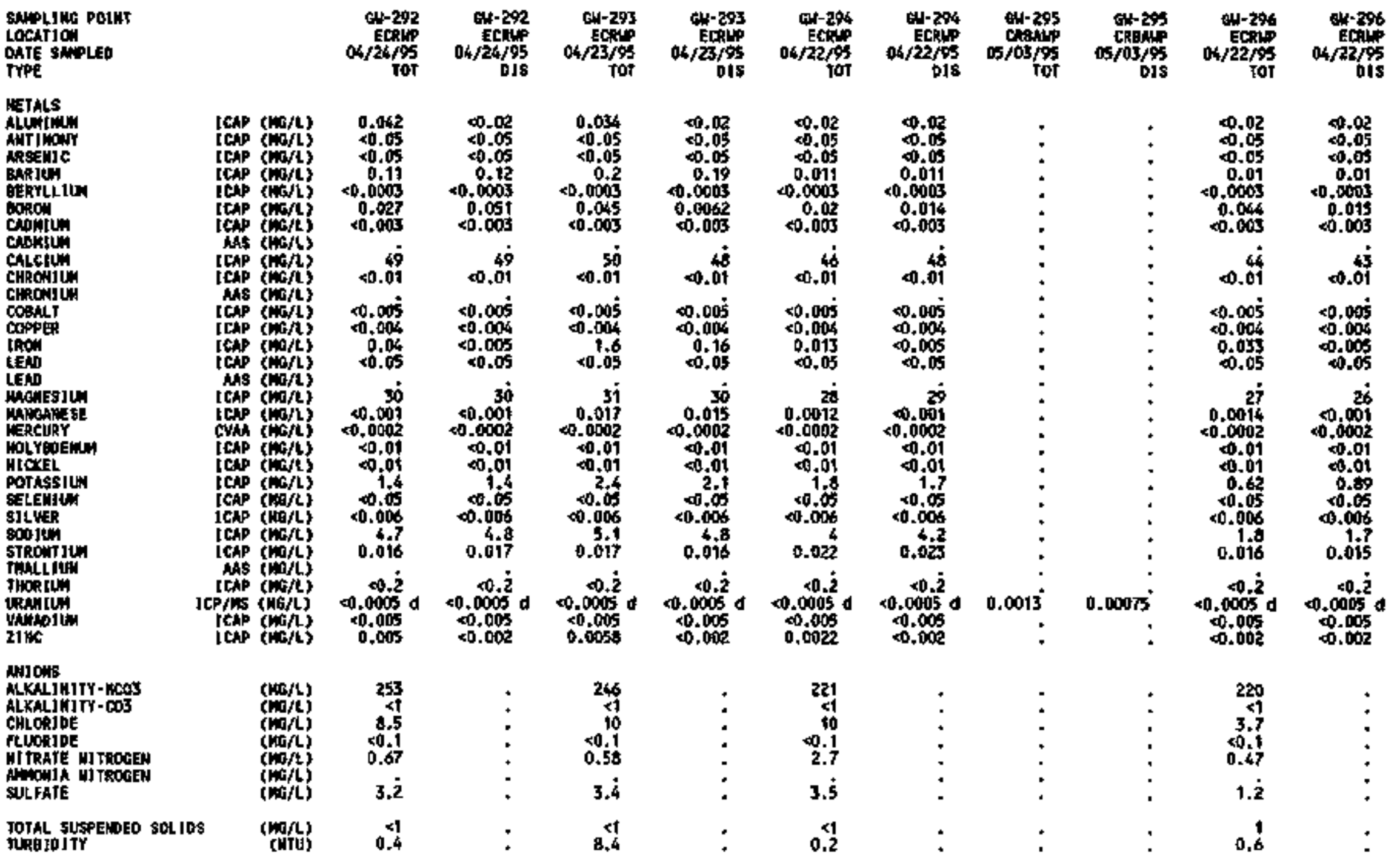




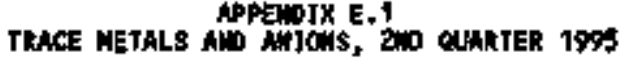

\begin{tabular}{|c|c|c|c|c|c|c|c|c|c|c|c|}
\hline 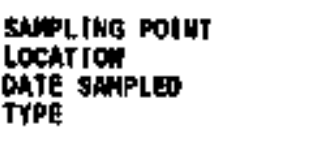 & & 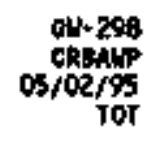 & $\begin{array}{r}\text { ay-298 } \\
\text { CRBMHF } \\
05 / 02 / 95 \\
\text { D1s }\end{array}$ & $\begin{array}{r}\text { ch-299 } \\
\text { chedlp } \\
05 / 01 / 95 \\
\text { Tot }\end{array}$ & $\begin{array}{r}\text { rN-299 } \\
\text { CRBALP } \\
05 / 01 / 95 \\
\text { Dis }\end{array}$ & $\begin{array}{r}6 \mathrm{cW}+300 \\
\text { thathe } \\
04 / 27 / 95 \\
\text { Tot }\end{array}$ & $\begin{array}{r}\text { qu+300 } \\
\text { ckBups } \\
04 / 27 / 95 \\
\text { oIs }\end{array}$ & $\begin{array}{r}\text { ch-301 } \\
\text { chanp } \\
04 / 27 / 95 \\
\text { Tot }\end{array}$ & $\begin{array}{r}\text { CA-301 } \\
\text { CRBALP } \\
04 / 27 / 45 \\
\text { DIs }\end{array}$ & $\begin{array}{r}\text { wh-302 } \\
\text { UHCS } \\
\text { 04/19/95 } \\
\text { Tot }\end{array}$ & $\begin{array}{r}\text { su+30z } \\
\text { vilcs } \\
0419 / 95 \\
\text { Bis }\end{array}$ \\
\hline \multirow[t]{2}{*}{ 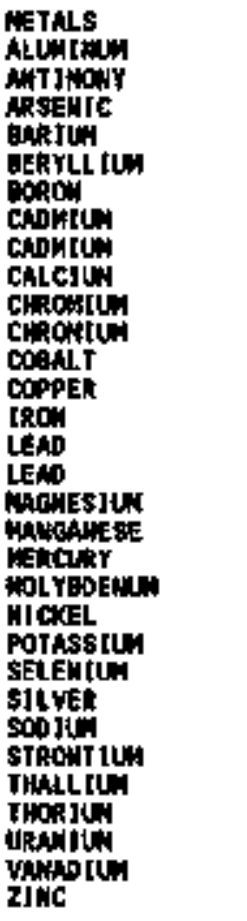 } & \multirow[t]{2}{*}{ 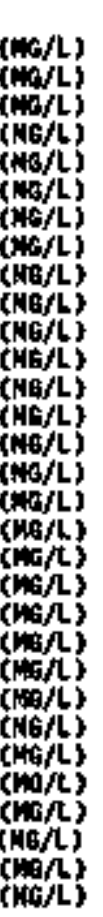 } & 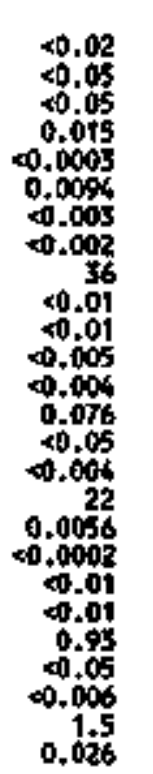 & $\begin{array}{r}0.021 \\
0.05 \\
0.05 \\
0.015 \\
<0.0003 \\
0.064 \\
4.003 \\
40.002 \\
0.01 \\
0.01 \\
0.005 \\
0.005 \\
0.0051 \\
00.05 \\
40.004 \\
0.0044 \\
0.0002 \\
<0.01 \\
20.01 \\
0.95 \\
40.05 \\
40.006 \\
1.5 \\
0.026\end{array}$ & 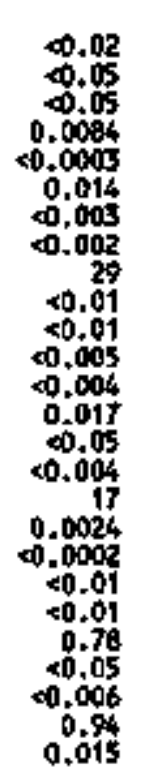 & 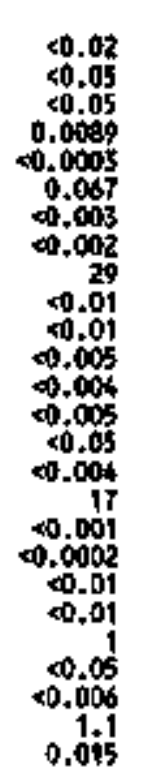 & 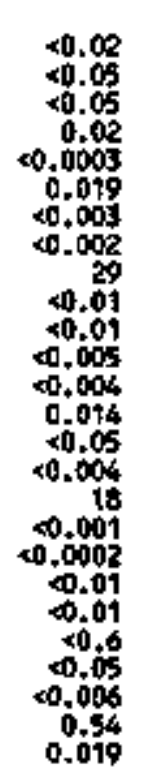 & 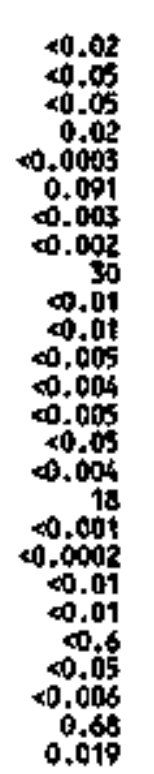 & 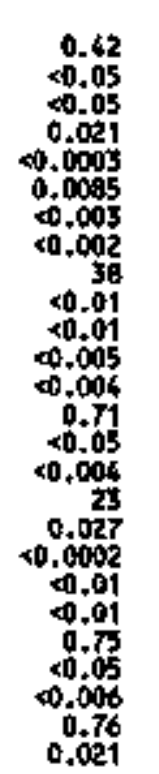 & 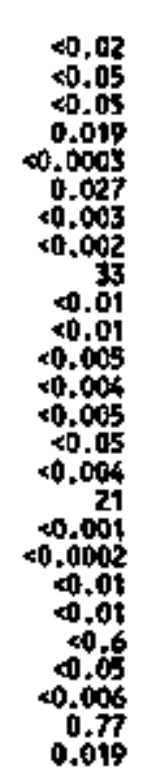 & 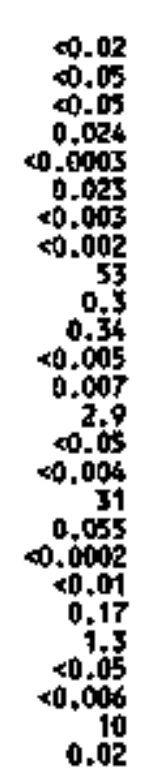 & 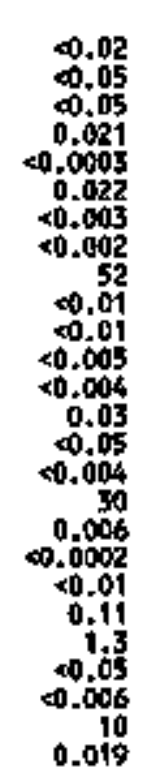 \\
\hline & & $\begin{array}{l}0.0 .2 \\
0.00 t 5 \\
0.005 \\
0.0056\end{array}$ & $\begin{array}{r}0 . \dot{2} \\
0.0016 \\
40.005 \\
0.0006\end{array}$ & $\begin{array}{c}0,0 . i \\
0.005 \\
0.0067\end{array}$ & $\begin{array}{c}40 . j \\
\times 0.0005 \\
0.005 \\
0.0056\end{array}$ & 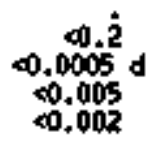 & $\begin{array}{c}00 . \overline{2} \\
4.0005 \\
0.005 \\
0,0044\end{array}$ & $\begin{array}{c}\alpha .000 . \dot{2} \\
0.005 \\
0.0066\end{array}$ & $\begin{array}{l}0.0005 \\
0.005 \\
0.002\end{array}$ & $\begin{array}{c}0 . \dot{2} \\
<0.0005 \mathrm{~d} \\
0.005 \\
0.011\end{array}$ & $\begin{array}{l}0.0005 \\
0.005 \\
0.005\end{array}$ \\
\hline \multirow{2}{*}{ 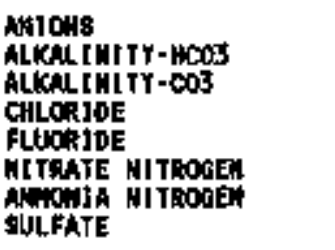 } & 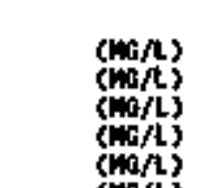 & $\begin{array}{r}170 \\
41 \\
1.6 \\
0.1 \\
0.42\end{array}$ & : & $\begin{array}{r}144 \\
<4 \\
1.7 \\
<0.1 \\
0.37\end{array}$ & : & $\begin{array}{r}145 \\
\times 1 \\
2 \\
<0.1 \\
0.5\end{array}$ & $\vdots$ & $\begin{array}{r}169 \\
41 \\
2.7 \\
0.1 \\
0.64\end{array}$ & $:$ & $\begin{array}{r}245 \\
x 1 \\
26 \\
20.1 \\
1.2\end{array}$ & : \\
\hline & & $6 . \dot{7}$ & : & 3.8 & : & 3.6 & $\vdots$ & 2.8 & : & $\dot{4}$ & . \\
\hline $\begin{array}{l}\text { TOTAL SUSPEIDED SOLIDS } \\
\text { TURBIDITY }\end{array}$ & (No/f) & 19 & $\dot{.}$ & 0.6 & $\dot{0}$ & $<1$ & : & 23 & ; & $\frac{2}{18}$ & . \\
\hline
\end{tabular}




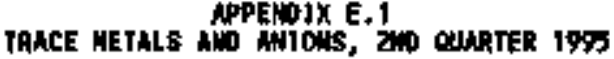

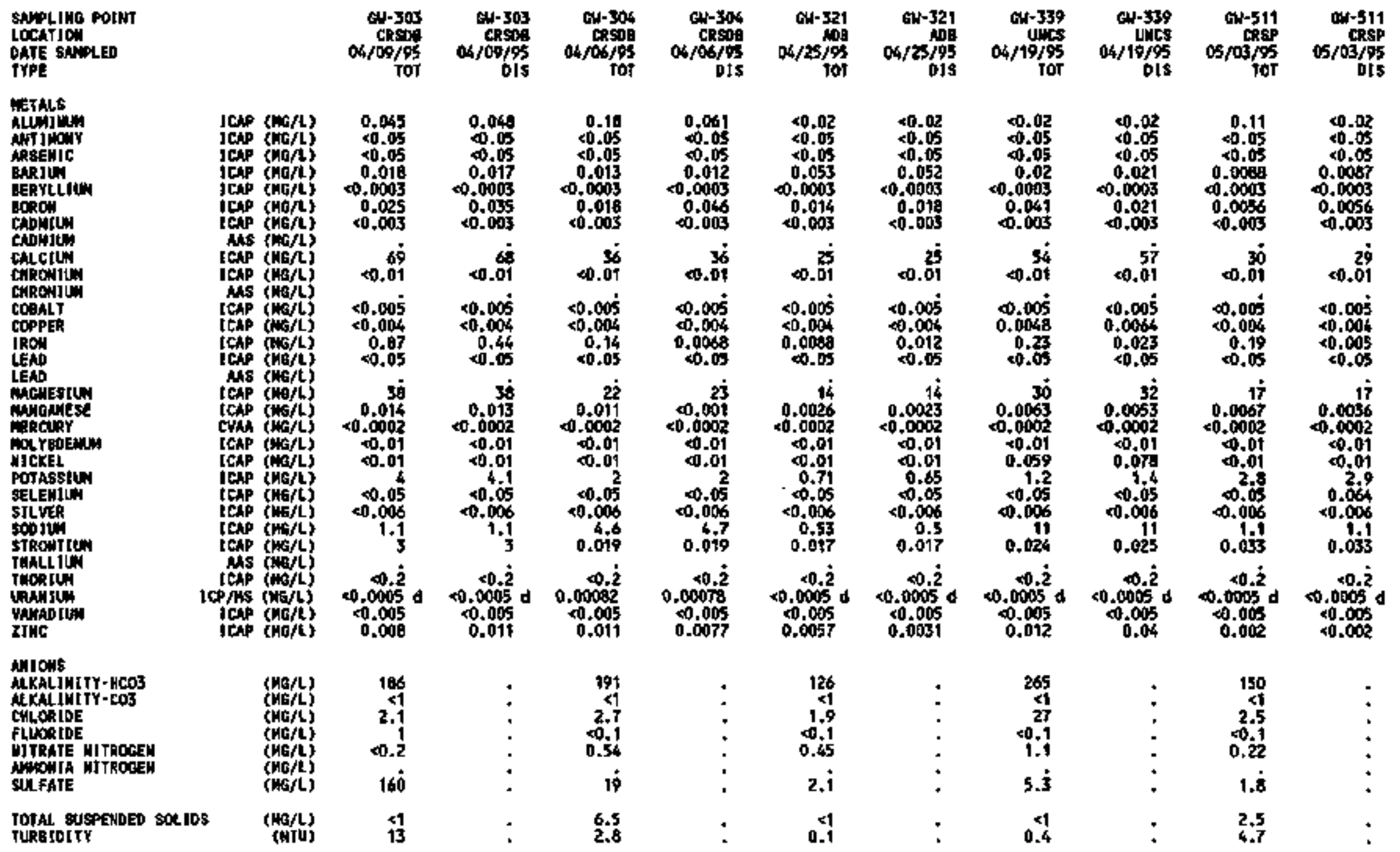


TRACE WEIALS APPE AMJONE, ZNO CUARTER 1995

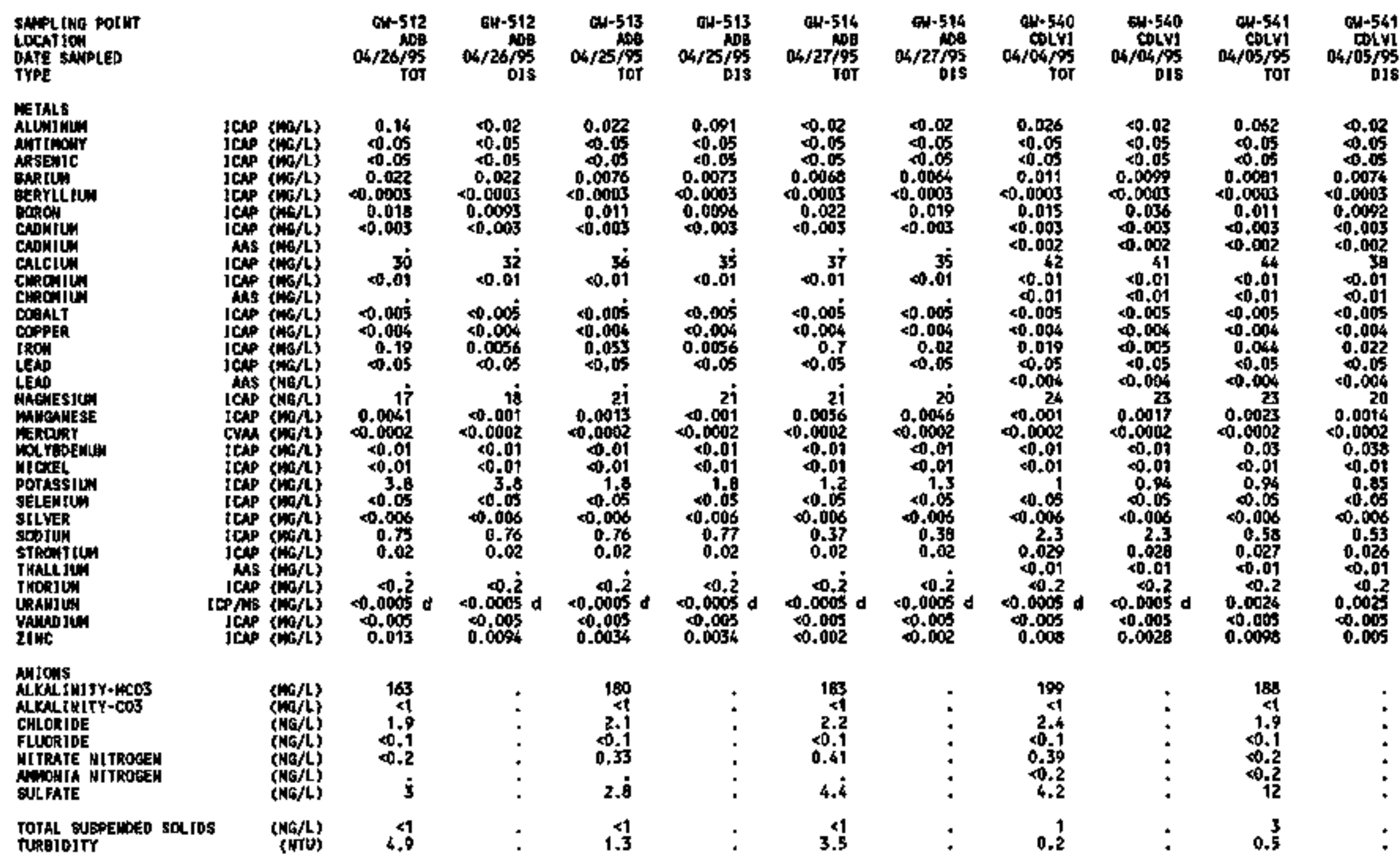




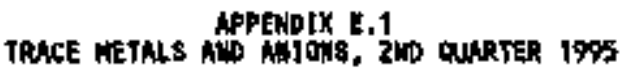

SAYPLIMe Potht

COCATION

DATE SHPLED

TYPE

\section{NeThLS}

Aluwingin

Aitinowr

Anstuc

tritulion

extion

capritus

chltcium

chrowiun

Cinaniti

Comatis

$1 \mathrm{RO}$

LEND

IEND

Mánesju保

Machinese

notruden

NICKEL

POTASSION

ExLENu

s1tven

STROATIIN

Thotion

ctanctu

vantoitu

zike

Nisous

ALKALIKJTY-HCos

ALKAL IWITY- $\cos$

CHLORJDE

HTRATE MITKOFE

Ninila nitrocen

SULATE

TOTAL SUSPEIDEO soltDS

TURAIDITY

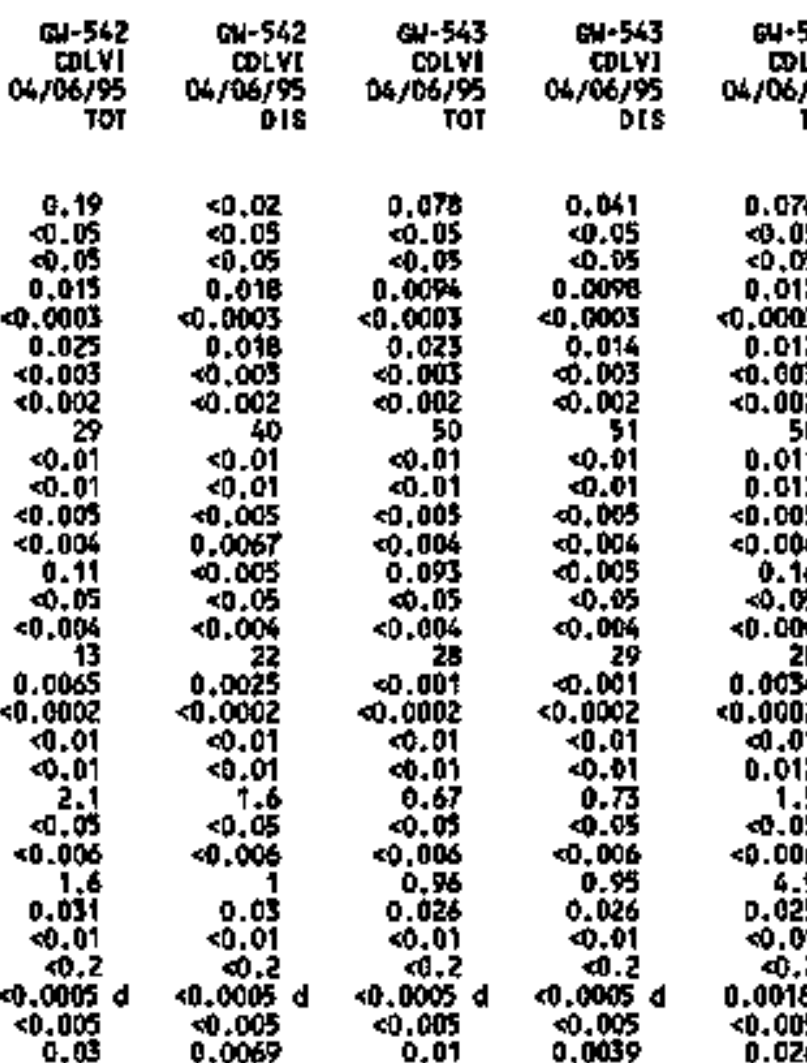

EN-544
coLy]
04/06/95
DIs

4.546
colvi
O4/ $105 / 95$
TOT

ar-546
CDLy1
$04 / 05 / 95$
ors

aw-557
$1 y$
$0400 / 95$
Tot

64-557

ToT

0.059

2.6

JCh (mo/l)

lcap (wo/)

Cos $(1+5 / h)$

jedp $(\omega, L / L)$

JCAP ( $\mathrm{HC} / \mathrm{L}$ )

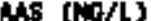

ICAP (Wo/l)

CAP (Nos)

AN (NG/L)

(c)

ictp

jCAP $(\mathrm{NB} / \mathrm{L})$

MS (40/L)

lcep (nor)

CVA (wo/l)

itsp (non)

JCAP (woll)

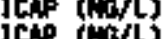

(c)

jotp (wor)

ICAP (No/L)

的

(1)

JCAF (Wrols

$<0,005$

0.0067

\begin{tabular}{|c|c|}
\hline $\begin{array}{r}240 \\
51 \\
3.3 \\
80.1 \\
0.57 \\
0.2 \\
3.3\end{array}$ & ? \\
\hline$<\frac{1}{2}$ & \\
\hline
\end{tabular}

$\$ 0.05$

0.01

$<0,0003$

5.013

$\$ 0.002$

48
$\times 0.01$

40.0

$<0.005$

0.0056

0.01
$\times 0.05$

$\times 0,006$

$<0,003$

$<0.002$

0.01
$\times 0.01$

it

20,05

40.006

0.023

40.

0.0011

$<0,005$

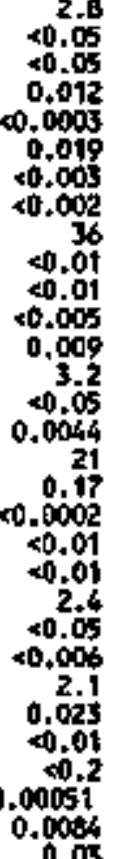

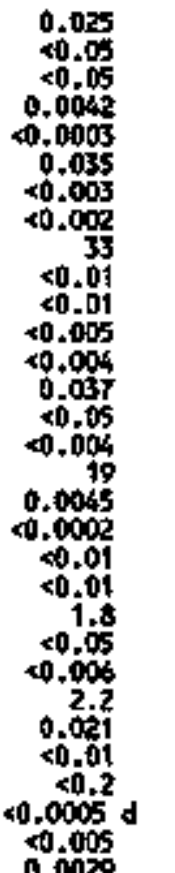

0.05

0.012

40.0003

0.024

$\times 0.002$

<4?

40.01

$<0,005$

$<0,004$

0.2

$\times 0.004$

22

0.0002

$\infty .01$

$<0.01$

0.05

40.006

0,006

0.017

$<0.01$

40.0005 a

0.0007

0.031

0.05

0.012

40.0003

0.153

$<0.002$

57

<0.01

40.005

0.004
$<0.005$

$<0.05$

$<0.004$

$\times 0.001$

40.0002

$<0.01$

- 01

0.05

$<0.006$

0.72

40,01

0.2

0.005

145
$\times 1$
2.4
0.1
0.39
$<0.2$
4.1
62
152

169
41
2
40.1
1.1
$\times 0.2$
1.7
5
4.4




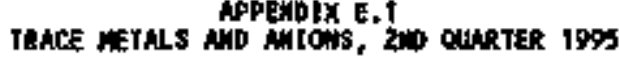

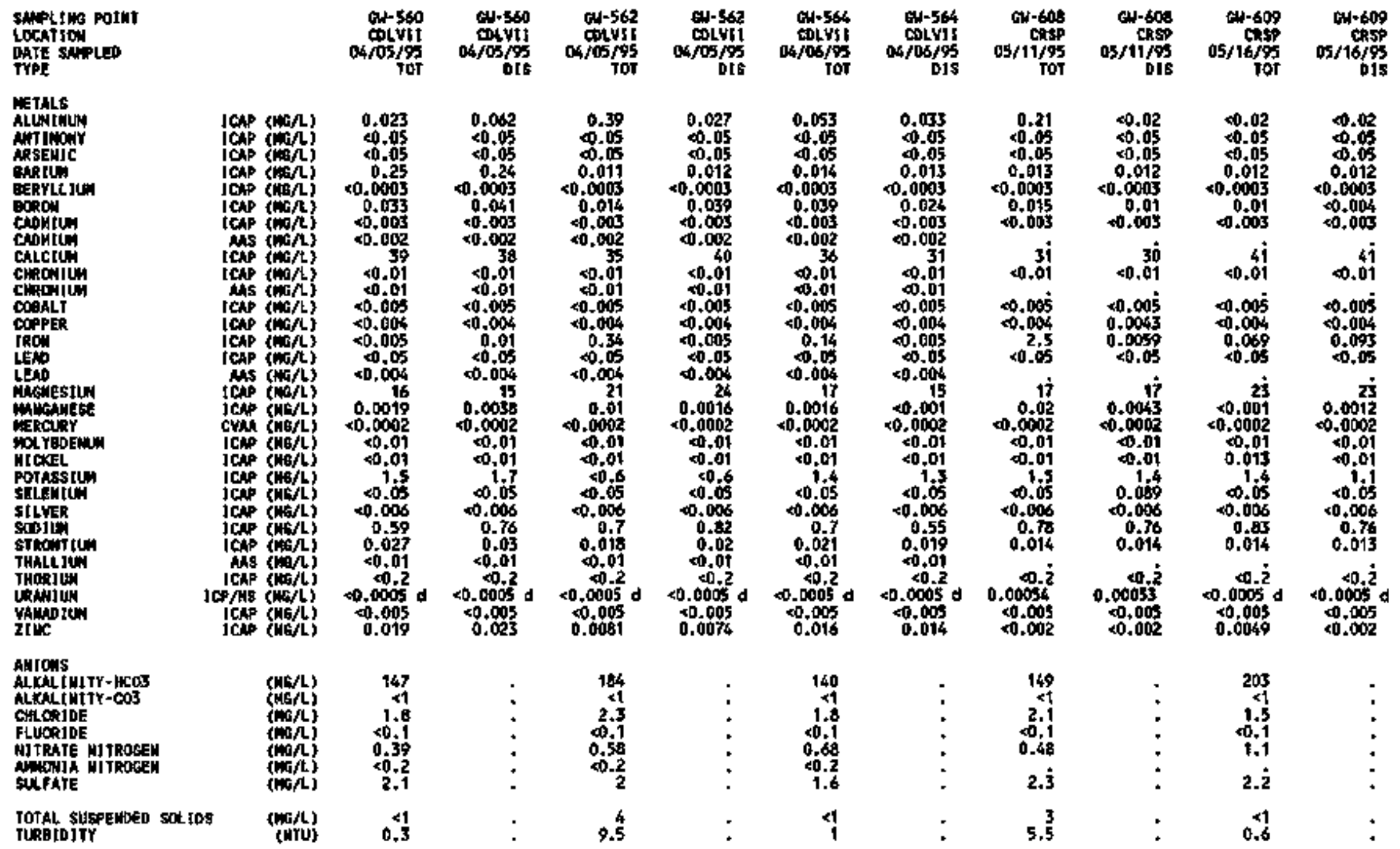




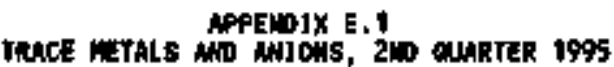

\begin{tabular}{|c|c|c|c|c|c|c|c|c|c|c|c|c|}
\hline 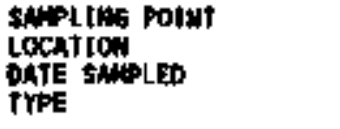 & & & $\begin{array}{r}\text { cit-610 } \\
595 p \\
05 / 07 / 95 \\
\text { ToT }\end{array}$ & $\begin{array}{r}\text { 6N-610 } \\
\text { orsp } \\
05 / 07 / 95 \\
015\end{array}$ & $\begin{array}{r}0,-611 \\
\text { Clsp } \\
05 / 15 / 95 \\
\text { tor }\end{array}$ & $\begin{array}{r}\text { GN-611 } \\
\text { cRsp } \\
05 / 15 / 45 \\
015\end{array}$ & $\begin{array}{r}\text { Gu-731 } \\
\text { CRsog } \\
04 / 07 / 95 \\
\text { ToT }\end{array}$ & $\begin{array}{r}64-731 \\
\text { CR5ps } \\
04 / 07 / 95 \\
\text { 015 }\end{array}$ & $\begin{array}{r}0 \mathrm{H}-732 \\
\text { Cर50B } \\
04 / 07 / 95 \\
\text { Tor }\end{array}$ & 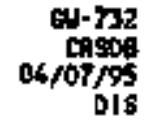 & $\begin{array}{r}01-742 \\
\text { ensp } \\
05 / 07 / 95 \\
\text { TOT }\end{array}$ & $\begin{array}{r}0 \text { du-742 } \\
\text { CRSP } \\
\text { O5/07/\%s } \\
\text { DIs }\end{array}$ \\
\hline \multirow{5}{*}{ 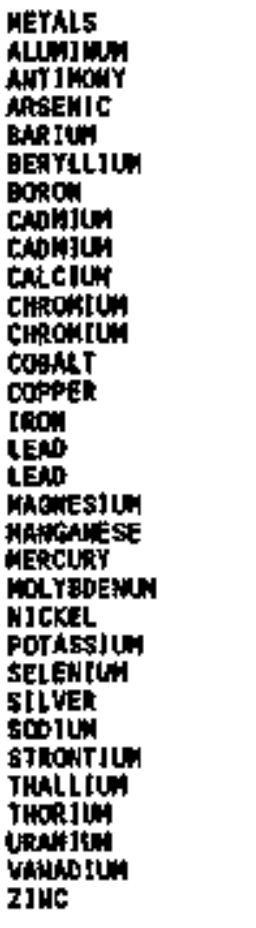 } & \multirow{5}{*}{ 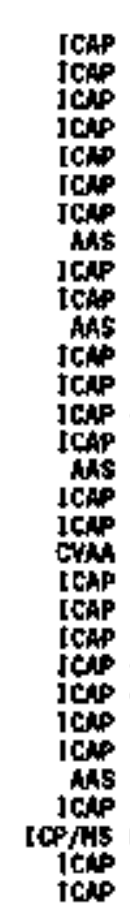 } & \multirow{5}{*}{ 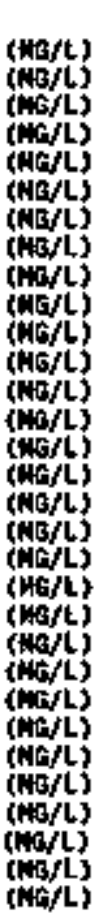 } & $\begin{array}{r}0.027 \\
0,05 \\
0.05 \\
0.11 \\
0.0003 \\
0.0068 \\
40.003\end{array}$ & $\begin{array}{r}0.02 \\
\$ 0.05 \\
0.05 \\
0.11 \\
0.0003 \\
0.01 \\
0.005\end{array}$ & $\begin{array}{r}0.043 \\
0.05 \\
00.05 \\
0.022 \\
00.0003 \\
0.006 \\
40.003\end{array}$ & $\begin{array}{r}0.02 \\
0.05 \\
0.05 \\
0.021 \\
<0.0005 \\
0.0052 \\
40.003\end{array}$ & $\begin{array}{r}0.00 \\
<0.05 \\
<0.05 \\
0.006 \\
<0.0003 \\
0.013 \\
0.003\end{array}$ & $\begin{array}{r}0.024 \\
0.05 \\
\times 0.05 \\
0.0058 \\
40,0003 \\
0.023 \\
0.003\end{array}$ & $\begin{array}{r}14 \\
40.05 \\
\times 0.05 \\
0.14 \\
0.0014 \\
0.034 \\
<0.003\end{array}$ & $\begin{array}{r}0.55 \\
0.05 \\
60.05 \\
0.085 \\
\times 0.0005 \\
0.020 \\
80.003\end{array}$ & $\begin{array}{r}8.02 \\
\times 0.05 \\
\times 0.05 \\
0.12 \\
<0.0003 \\
0.0056 \\
\times 0.003\end{array}$ & $\begin{array}{r}0.02 \\
<0.03 \\
<0.05 \\
0.12 \\
\times 0.0003 \\
80.004 \\
\times 0.003\end{array}$ \\
\hline & & & $\begin{array}{r}47 \\
<0.01\end{array}$ & $<0,4$ & $\begin{array}{r}57 \\
<0.07\end{array}$ & $\begin{array}{r}5 \dot{7} \\
\times 0.01\end{array}$ & $\begin{array}{r}17 \\
0.01\end{array}$ & $\begin{array}{r}1 \dot{17} \\
0.01\end{array}$ & $40 . \overline{35}$ & $\begin{array}{r}25 \\
0.01\end{array}$ & $\begin{array}{r}39 \\
0.01\end{array}$ & ه. \\
\hline & & & $\begin{array}{r}<0.005 \\
<0.004 \\
0.13 \\
\times 0.05\end{array}$ & $\begin{array}{l}0.005 \\
<0.004 \\
0.016 \\
0.05\end{array}$ & $\begin{array}{r}<0.005 \\
<0,004 \\
0.26 \\
20.05\end{array}$ & $\begin{array}{r}<0.005 \\
<0.004 \\
0.016 \\
40.05\end{array}$ & $\begin{array}{r}0.005 \\
0.009 \\
0.10 \\
0.05\end{array}$ & $\begin{array}{l}\infty 0.005 \\
<0.004 \\
<0.005 \\
0.05\end{array}$ & $\begin{array}{r}0.005 \\
0.013 \\
7.5 \\
0.05\end{array}$ & $\begin{array}{r}40.005 \\
0.0041 \\
0.33 \\
40.05\end{array}$ & $\begin{array}{r}\times 0.005 \\
<0.004 \\
0.27 \\
\times 0.05\end{array}$ & $\begin{array}{r}0.005 \\
\times 0.004 \\
0.04 \\
\times 0.05\end{array}$ \\
\hline & & & $\begin{array}{r}27 \\
<0.001 \\
\times 0.0002 \\
0.01 \\
0.01 \\
40.6 \\
40.05 \\
40.006 \\
0.017\end{array}$ & $\begin{array}{r}29 \\
4.001 \\
0.0002 \\
0.01 \\
0.01 \\
0.94 \\
0.05 \\
0.006 \\
1.1 \\
0.017\end{array}$ & $\begin{array}{r}3 \dot{3} \\
0.0043 \\
<0.0002 \\
0.01 \\
<0.01 \\
2.3 \\
<0.05 \\
<0.006 \\
1.9 \\
0.019\end{array}$ & $\begin{array}{r}3 \dot{3} \\
0.0022 \\
0.0002 \\
0.01 \\
40.01 \\
20.65 \\
\times 0.006 \\
2.1 \\
0.019\end{array}$ & $\begin{array}{r}13 \\
0.0024 \\
0.0002 \\
00.04 \\
0.01 \\
12 \\
\times 0.05 \\
\times 0.006 \\
3.9 \\
0.022\end{array}$ & $\begin{array}{r}13 \\
0.001 \\
\times 0.0002 \\
0.01 \\
0.01 \\
147 \\
0.05 \\
0.006 \\
3.7 \\
0.021\end{array}$ & $\begin{array}{r}19 \\
0.1 \\
0.0002 \\
0.01 \\
0.01 \\
8.2 \\
50.05 \\
4.006 \\
10 \\
0.34\end{array}$ & $\begin{array}{r}13 \\
0.0056 \\
80.0002 \\
0.01 \\
40.01 \\
0.8 \\
<0.05 \\
<0.005 \\
12 \\
0.25\end{array}$ & $\begin{array}{r}21 \\
0.021 \\
4.0002 \\
40.01 \\
0.01 \\
0.8 \\
0.071 \\
40.006 \\
0.7 \\
0.016\end{array}$ & $\begin{array}{r}21 \\
0.02 \\
\times 0.0002 \\
80.01 \\
0.01 \\
1.2 \\
40.03 \\
40.006 \\
0.74 \\
0.016\end{array}$ \\
\hline & & & 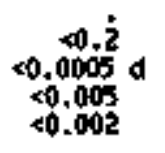 & $\begin{array}{l}40.2 \\
0.0005 \\
0.005 \\
0.002\end{array}$ & $\begin{array}{c}40.2 \\
<0.0005 \\
0.0005 \\
0.0048\end{array}$ & $\begin{array}{c}<0 . \dot{2} \\
<0,005 \\
0.00033\end{array}$ & $\frac{0.0005}{0.005} d$ & $\begin{array}{c}0.0005 \\
20.005 \\
0.01\end{array}$ & $\begin{array}{r}\times 0 . z \\
0.016 \\
40.045 \\
0.074\end{array}$ & $\begin{array}{c}40.2 \\
0.0014 \\
40.005 \\
0.012\end{array}$ & $\begin{array}{l}0.00054 \\
0,005 \\
\times 0,002\end{array}$ & $\begin{array}{c}<0.0025 \\
40.005 \\
\$ 0.002\end{array}$ \\
\hline \multirow{2}{*}{\multicolumn{2}{|c|}{ 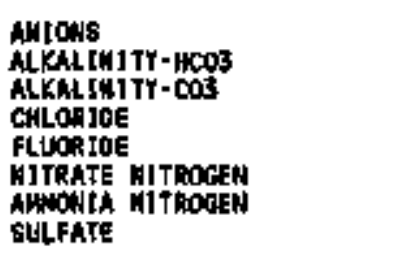 }} & \multirow{2}{*}{ 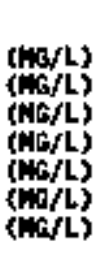 } & $\begin{array}{r}227 \\
41 \\
2.8 \\
0.4 \\
0.81\end{array}$ & $\dot{:}$ & $\begin{array}{r}2.2 \\
41 \\
1.4 \\
0.1 \\
3.3\end{array}$ & : & $\begin{array}{r}101 \\
18 \\
1,8 \\
0.15 \\
0.85\end{array}$ & $:$ & $\begin{array}{r}157 \\
1,6 \\
10.7 \\
0.68\end{array}$ & $:$ & $\begin{array}{r}175 \\
4 \\
1.5 \\
<0.1 \\
<0.2\end{array}$ & : \\
\hline & & & \&i & : & $1 . \dot{5}$ & $\dot{*}$ & $\dot{\sigma}$ & $\dot{*}$ & $2 . \dot{9}$ & $\dot{*}$ & 13 & : \\
\hline $\begin{array}{l}\text { TOTAL SUSPEWED SOL.1DS } \\
\text { TURBJDITY }\end{array}$ & & $\begin{array}{l}\text { (NG/L) } \\
\text { (NTU) }\end{array}$ & $\begin{array}{r}1 \\
1.6\end{array}$ & : & 1.2 & $\dot{E}$ & 2.7 & $\dot{-}$ & $\begin{array}{r}115 z \\
300\end{array}$ & 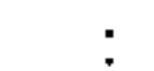 & 2.4 & : \\
\hline
\end{tabular}


TAALE METALS APD AHIOHS, ZWD OAARTER 1995

SAMPL,IME botht

Lochton

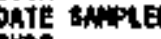

THPE

NEEALS

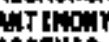

Ansentc

Danjuin

Dofor

ChDHIU.

CADNIUn

catcion

chinoslum

comats

COPPER

crow

tom

Lito

MiCHEs10

NhWcay,se

NERCUKT

moreoeiny

NTCKEL

POTAss]in

SELEHIUN

sicver

Sopjint

TMeLLiLy

ThincLivy

upention

yanablu

2IMC

Anjows

ALKALINJTYY-HCOS

ALKAL IHI TY $\mathrm{COOB}$

CNLORIOE

Ltoriot

N]TRATE HITROEEN

MTDN N MTROSEM

sidfATE

TOTAL SUSPEMDED SOLIDS TUR日IO1TY
GN-743 an-743 GH-796

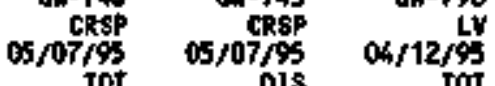

04706
ty
$04 / 12 / 55$
ots

aw-797
Ly
$04 / 11 / 95$
roT

$\begin{array}{rr}64-797 & 04-795 \\ \text { Ly } & c 04 \% 11 \\ 04 / 11 / 95 & 04 / 19 / 45 \\ \text { ofs } & \text { Tol }\end{array}$

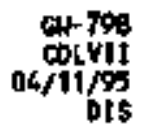

a4-799
$04 / 06 / 95$

Gi-759

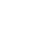

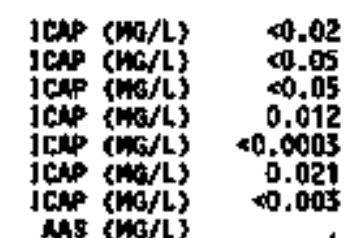

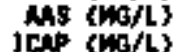

ICAP $(H a / l)$

ICAS ( $(M / \mathrm{N} / \mathrm{L} / \mathrm{L})$

ICAP $(\mathrm{NH} / \mathrm{L})$

Ices $(w / L)$

1Ch $(\mathrm{HW} / \mathrm{L})$

ICH (Wa/L)

ICAP (ing/L)

cVN (W/L)

ICAP (w/L)

$1 \mathrm{cos}(\mathrm{m} / \mathrm{m} / \mathrm{L})$

JCAP $(m / L)$

Icee $\mathrm{mos}$

tcap (noll

ICAP $(\mathrm{Hip} / \mathrm{l})$

ANS (NG/)

ICAP (H6/L)

ICAP $(\mathrm{w} / \mathrm{G} / \mathrm{L})$

40.02

$\$ .05$

012

0.005

0.01

0.005

0.004

0.05

c. 18

0.0002
+0.002

0.01
$\times 0.01$

40.2

0.606

0.019

$<0.0$
$\times 0.0$
40.05
0.01
0.000
0.00

0.00

0.045

0.009

0.096

0,061

0.05

0.05
0.005
$\times 0.0003$

$0.05 \quad 0.05$

$<0.0003 \quad 0.0003$

0.027

$\$ .003$

$\$ 0.002$

$3{ }^{\circ}$

40.01

$\$ .01$

$\$, 005$

0.009

0.05

0.05

$\$ 0.004$

0.0015

$\times 0.002$

$<0,0$

$\infty, 01$
2,7

0.006

1.3

$<0.01$

(

40.2

$\times 0.0005$ a

0.0005

$<0.0005 d$

0.0003

0.005

$\times 0.602$

0.01

$<0.01$

4.005

0.004
$\times 0.005$

$<0.005$

40.05
40.044

0.001

0.0002

$<0.01$

40.01

40,05
$\times 0.006$

1.2

0,046

$\times 0.2$

$+0.0005$

$\times .005$
0.003

0.0084

$<0.0003$

0.056

$\$ 0.002$

34
40.01

0.0

$\$ 0.005$

4004
0.076

0.076

0.004

0.0055

<1.0602

0.01
$\times 0.01$

0.01

$\infty .05$

$+0.006$

0.6

0.01

4.2

$=0.00005 \mathrm{~d}$

i. 0.005

0.000

$\times 0.0005$

0.12

0.003

$<0.002$

40.01

0.0

$<0,005$

0,0047

0.061

$\$ 0,004$

0.20

0.0092

0.0002

0.01

1,2

$\$ 0.006$

0.8

0.02

40.2

$0.0005 \mathrm{~d}$

$<0.005$
0.01

0.036

0.003

40.002

26
4.01

0.01

$\$ .005$

$\$ .004$

0.058

0.004

0.003

0.0027
40.0002

40.01

<.01

1.3
$\times 0.05$

$\infty .006$

0.57

0.015

40.2

0,0005

40,005
0.0054

0.039

0.05

0.05

40.000

0.035

$\$ 0.005$

$\times 0.00$

$\times 0.01$

0.01

$\infty .005$

$\infty .004$

0.005

$\infty .004$

17
-0.001

$<0.0002$

40.06

$\infty, 01$

40,05

$\times 0,006$

0,8

0.016

4.01

143

\begin{tabular}{|c|}
\hline 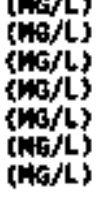 \\
\hline$\left(\begin{array}{c}(W / L) \\
(N T U)\end{array}\right.$ \\
\hline
\end{tabular}

114
11
2
0.1
0.52
40.2
2.1
1
1.5

128
61
3.1
40.1
1
40.2
2.7
41
0.8

4.0005
0.005

$+0.05$

0.0066

0.0005

0.075
$\times 0.005$

$\times 0.002$

27
$\times 0.01$

$<0.01$

0.005

0.004
0.16

$<0.05$

co.cha

0. 16

0.0041
$<0.0002$

40.01
$<0.01$

0.05

20.000

0,79

0.015

$\times 0.2$

$\begin{array}{ll}50.055 & 0.005 \\ 0.016 & 0.0047\end{array}$

159
1.1
1.8
0.7
0.56
80.2
1.7
1.5
2.5

$:$

$\vdots$

129
51
1.8
0.1
7.1
$<0.2$
1.6
3.1
3.2

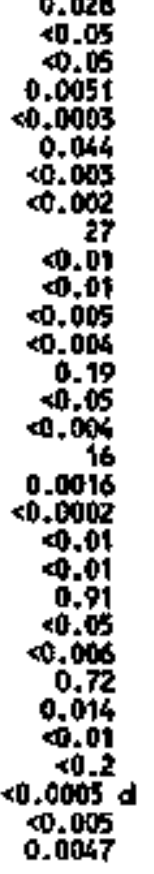




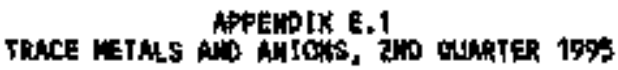

SAMPLIMO POLT

Lockrion

DATE SANOLEO

TYPE

METALS

ALININ⿴囗十)

Niticinic

Arsetilic

BERYLIIN

BoroN

complum

cronitum

calcelum

CHRONIUH

Chenowlu

COBALt

coper

rom

LEN

MGESIII

Mathicse

perectint

inotropeing

Atexel.

Porissiu

StLEN1U.

\$ILVA

sonut

TRRONT 1LN

THLLLIL"

THos tur

unditum

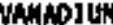

$21 \mathrm{kC}$

ANJONS

MLKALJHLTY-HCOS

ALKAL]HITY-CO3

CHLOR]DE

FUORJE

ANOW] $h$ y]TROEEN

SULFATE

TOTAL SUSPENDEO SOLIDS

TURATDITY

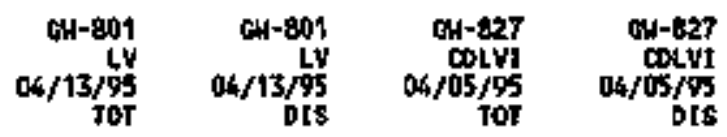

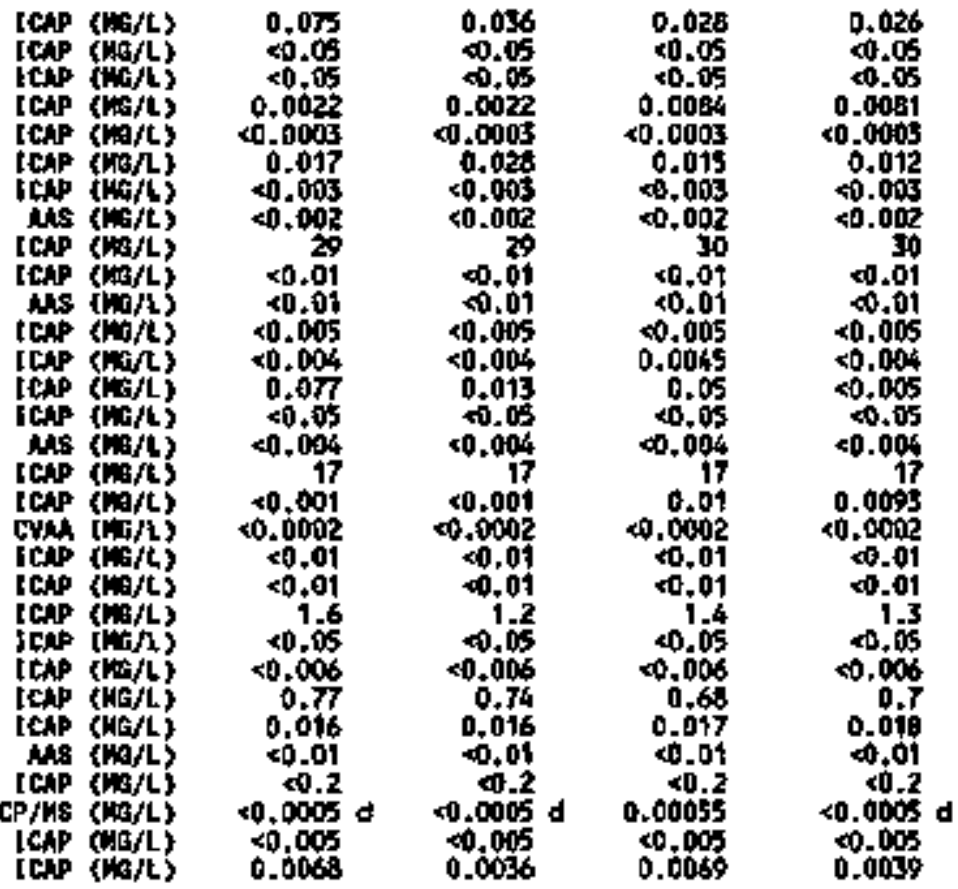

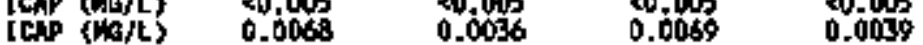

\begin{tabular}{|c|c|c|c|}
\hline $\begin{array}{l}(\mathrm{HG} / \mathrm{L}) \\
(\mathrm{WG/L}) \\
(\mathrm{HG} / \mathrm{L}) \\
(\mathrm{HG} / \mathrm{L}) \\
(\mathrm{NG/L}) \\
(\mathrm{WG/L}) \\
(\mathrm{HG} / \mathrm{L})\end{array}$ & $\begin{array}{r}143 \\
41 \\
2.9 \\
60.1 \\
0.32 \\
0.2 \\
3\end{array}$ & : & $\begin{array}{r}167 \\
<1 \\
1.8 \\
<0.1 \\
0.33 \\
40.2 \\
2.1\end{array}$ \\
\hline $\begin{array}{c}\text { (NG/L) } \\
\text { (NTU) }\end{array}$ & $\begin{array}{r}41 \\
2.5\end{array}$ & 4 & 0.8 \\
\hline
\end{tabular}




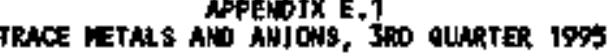

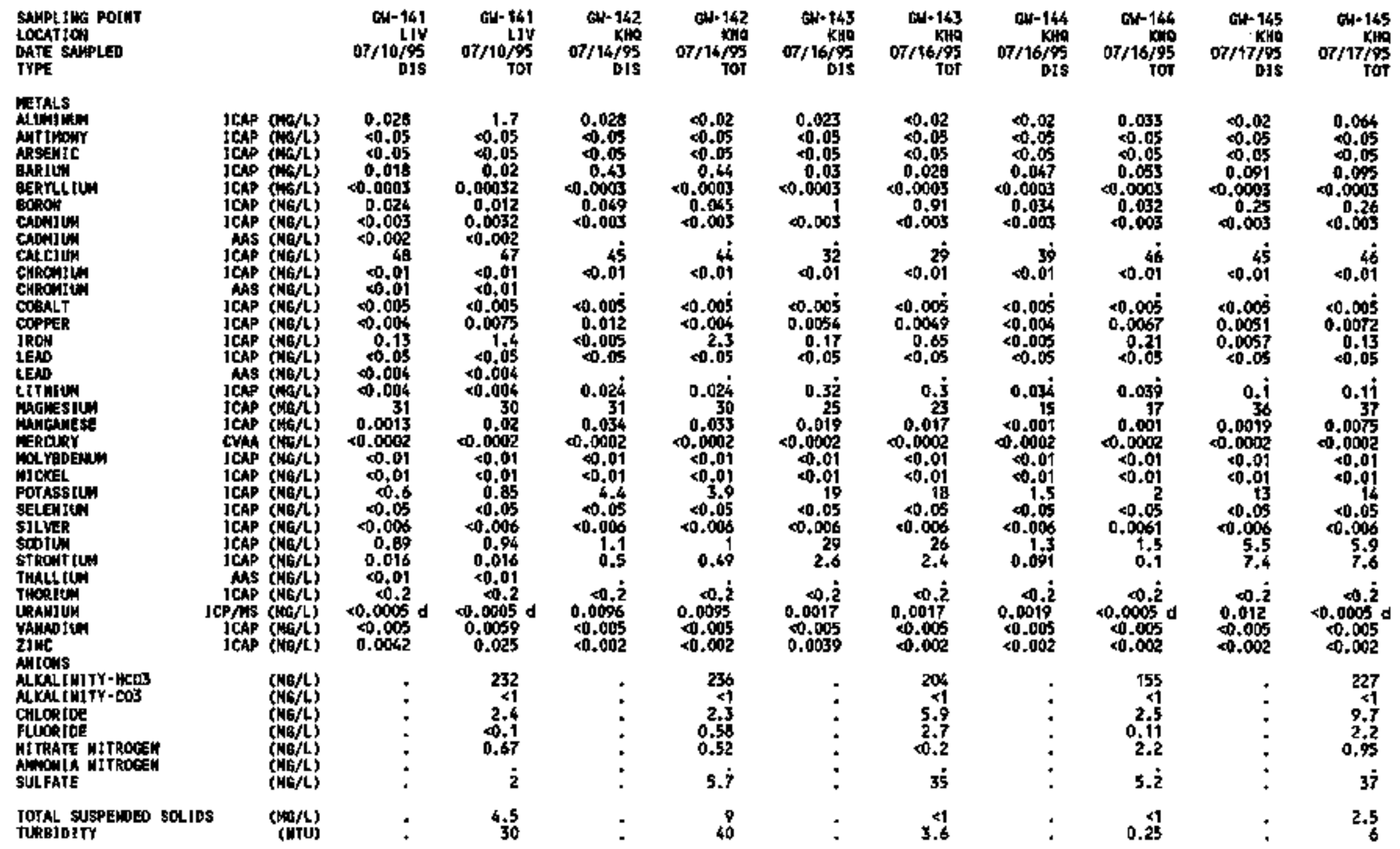




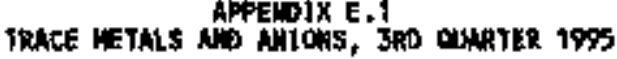

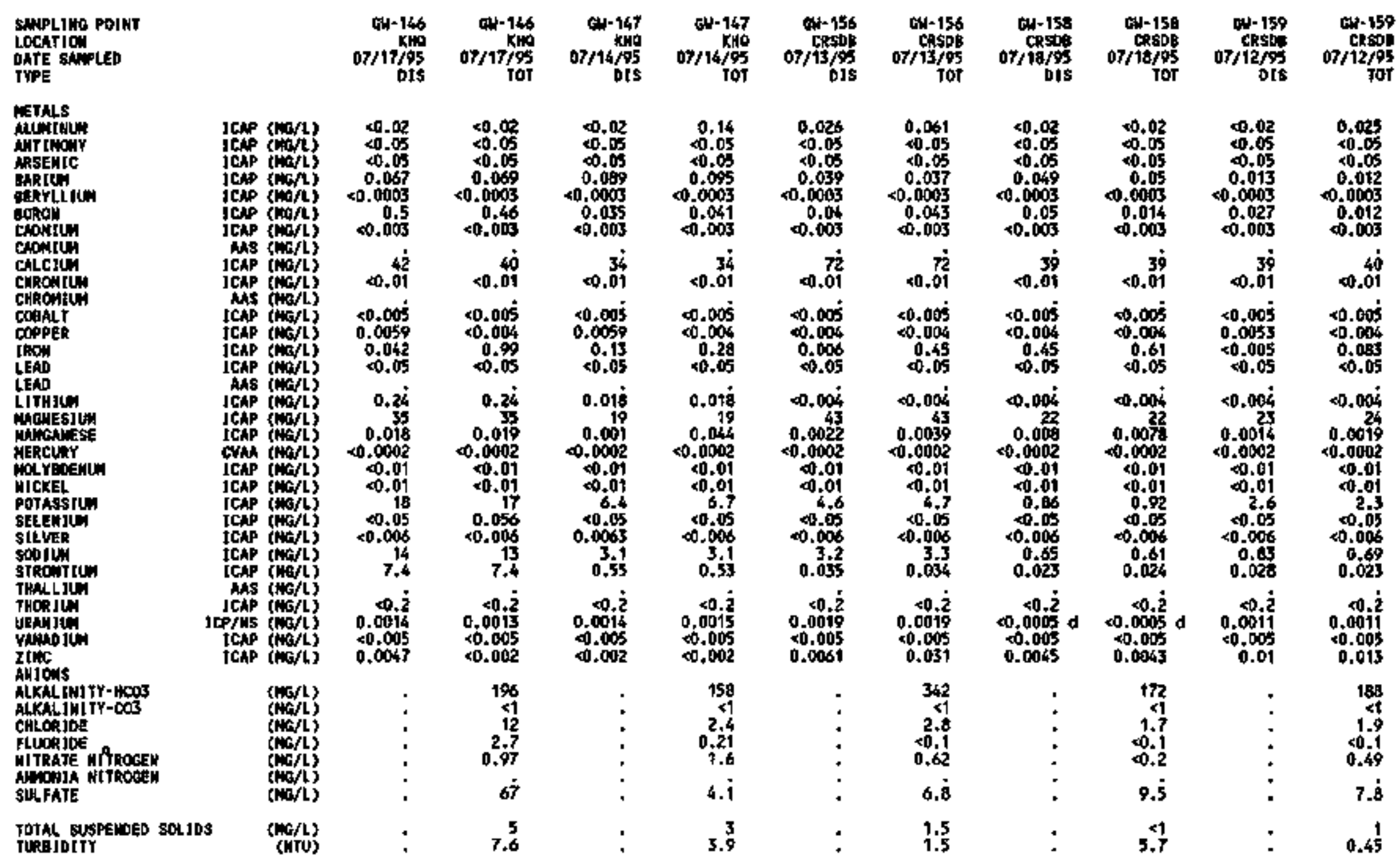




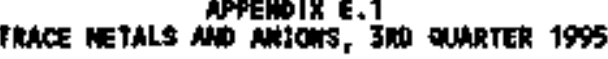

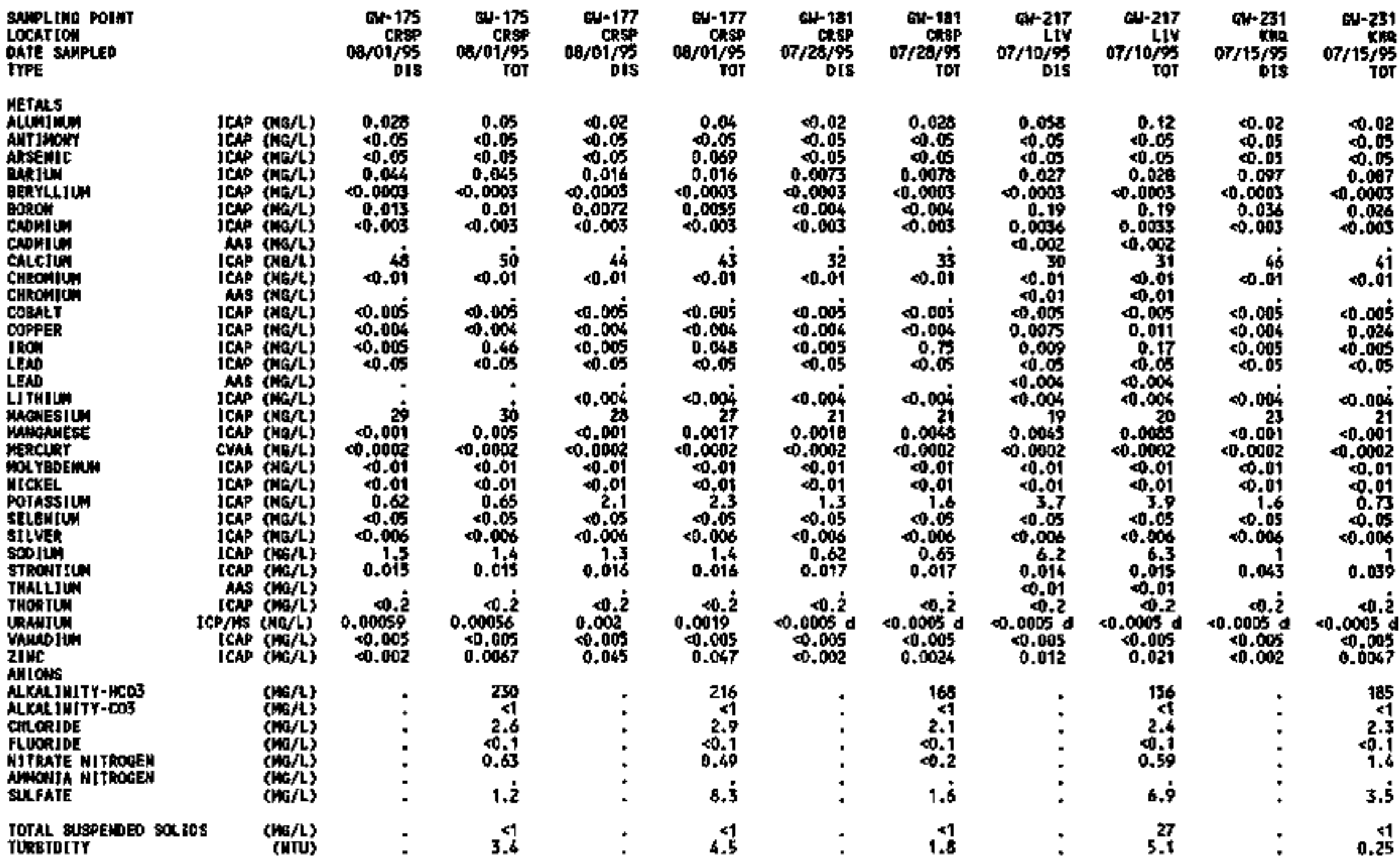




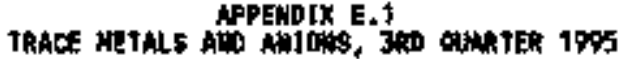

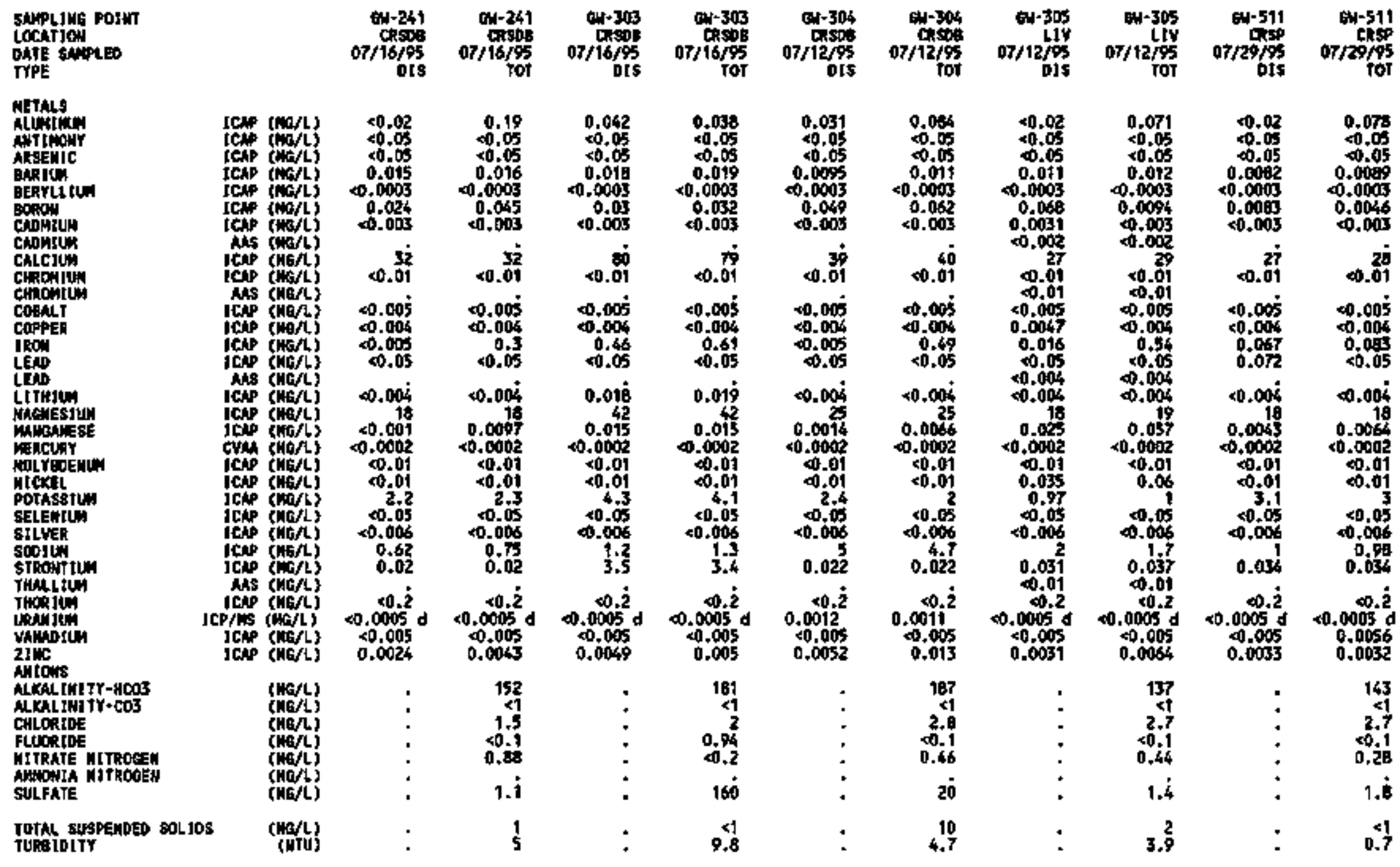




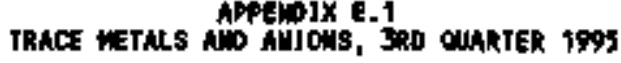

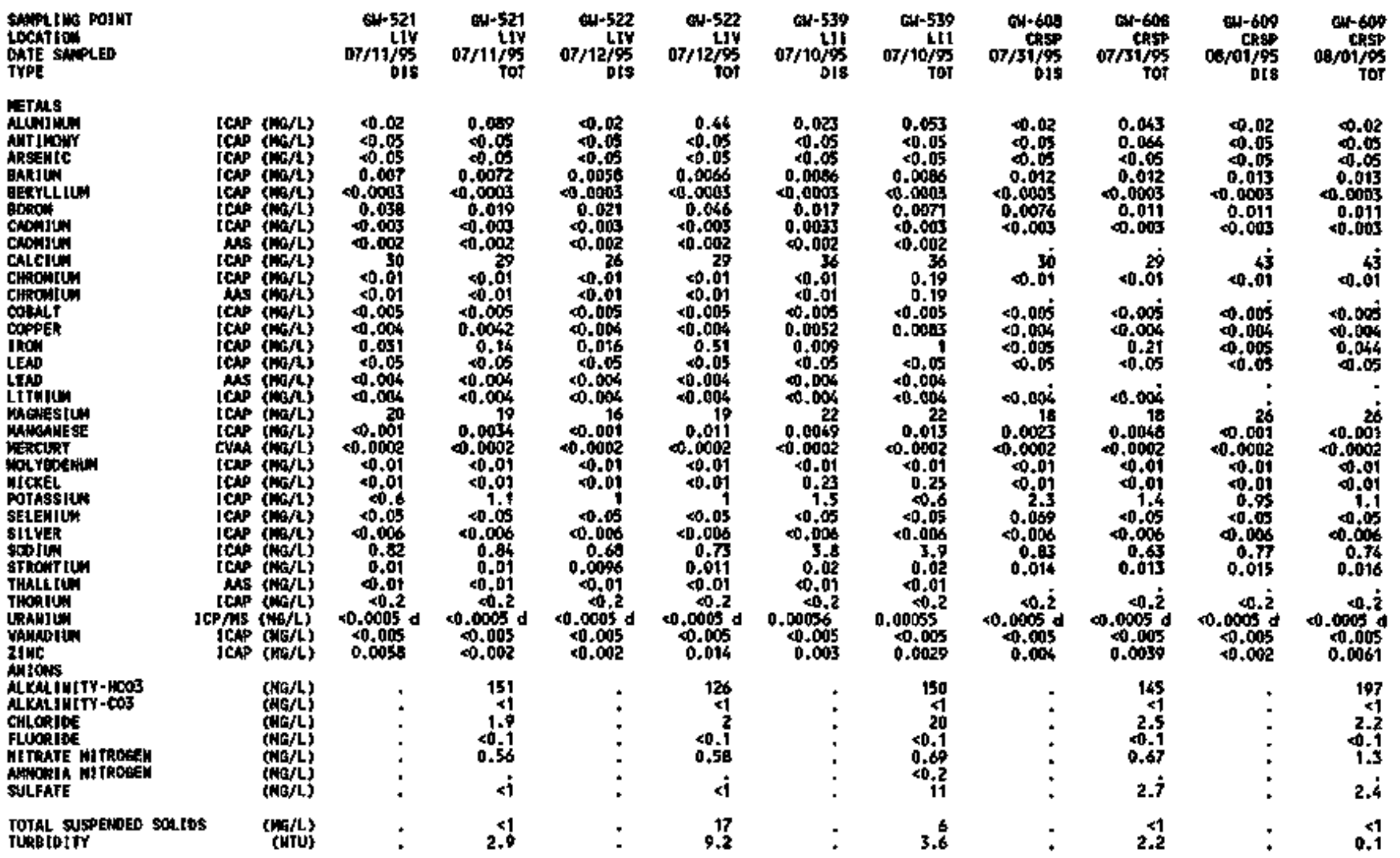




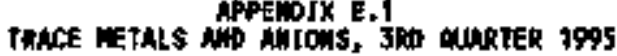

\begin{tabular}{|c|c|c|c|c|c|c|c|c|c|c|}
\hline 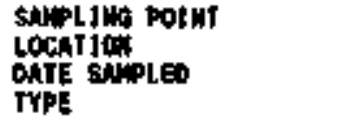 & & & $\begin{array}{r}\text { D. } 610 \\
\text { cRsp } \\
07 / 31 / \% 5 \\
\text { ots }\end{array}$ & $\begin{array}{r}01-610 \\
\text { chs } \\
07 / 1 / 93 \\
\text { ToT }\end{array}$ & $\begin{array}{r}\text { ad-611 } \\
\text { casp } \\
0 / 01 / 05 \\
\text { Dis }\end{array}$ & $\begin{array}{r}\text { ou-611 } \\
\text { ctisp } \\
0.101 / 95 \\
\text { Tor }\end{array}$ & $\begin{array}{r}04-709 \\
611 \\
07 / 10 / 95 \\
018\end{array}$ & $\begin{array}{r}64-708 \\
\text { L]I } \\
07 / 10 / 95 \\
\text { TOT }\end{array}$ & $\begin{array}{r}\text { CW- } 71 \\
\text { CR801 } \\
\text { Or/13/9s } \\
\text { DI8 }\end{array}$ & $\begin{array}{r}04+71 \\
0713 / 65 \\
\text { Tot }\end{array}$ \\
\hline 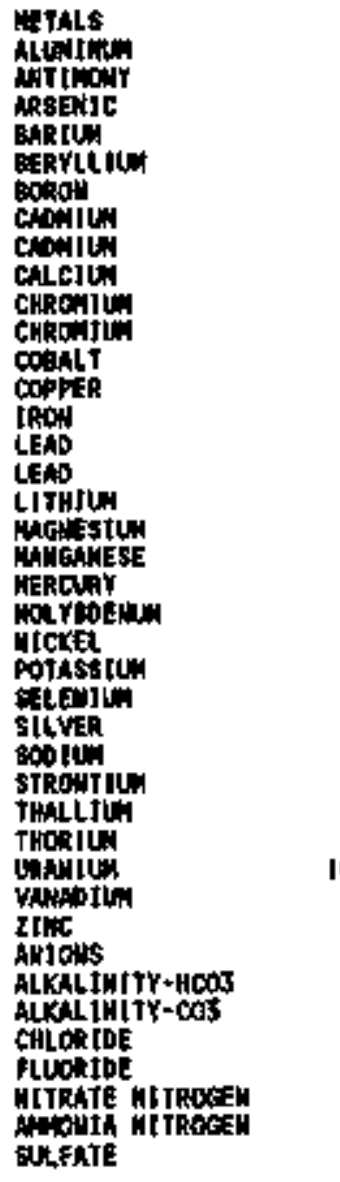 & 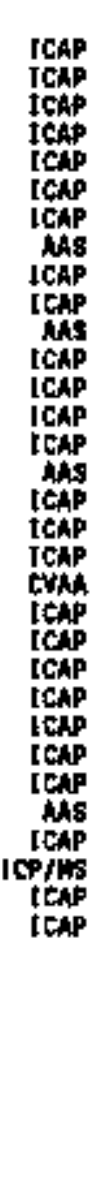 & 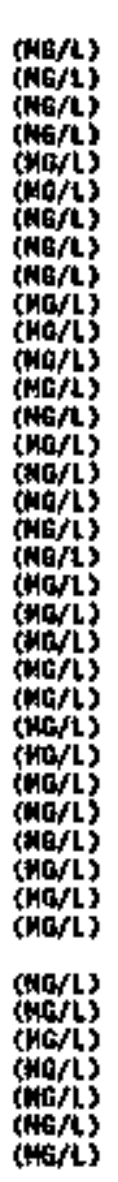 & 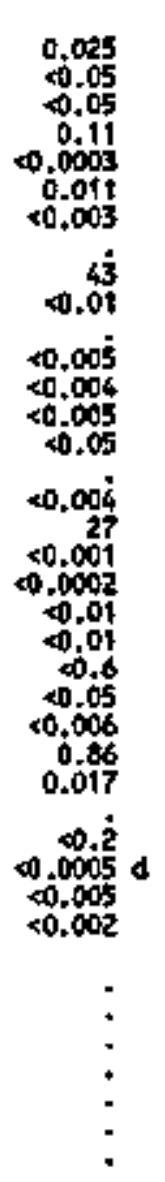 & 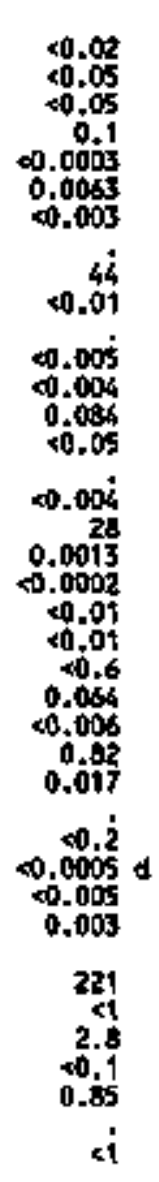 & 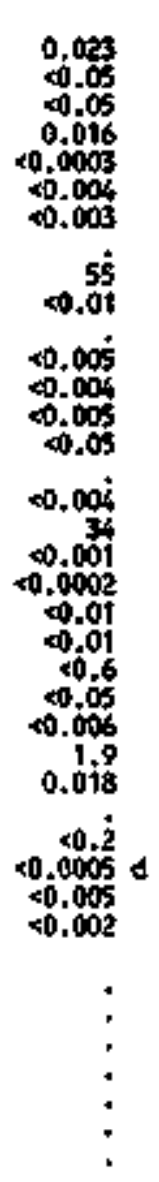 & 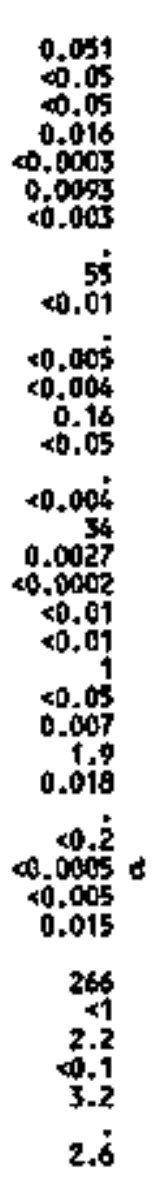 & 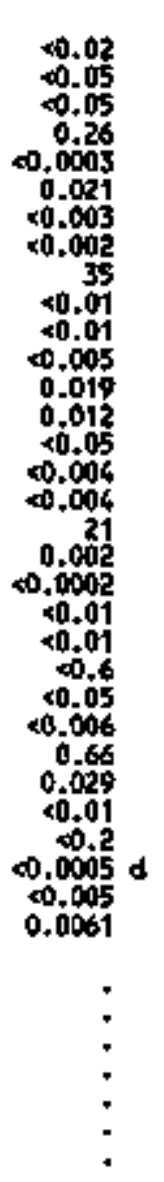 & 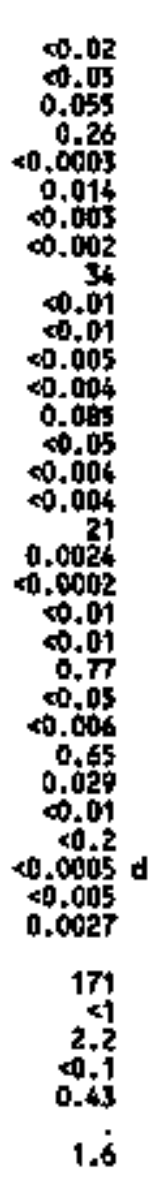 & 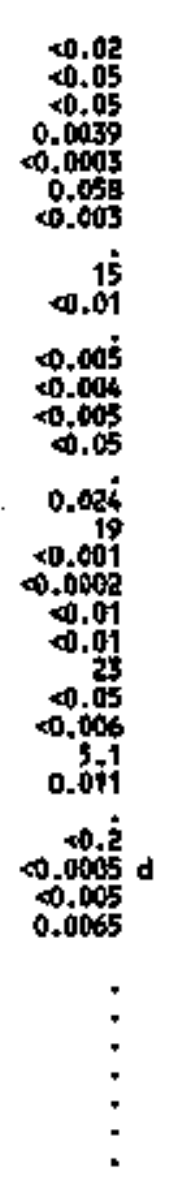 & 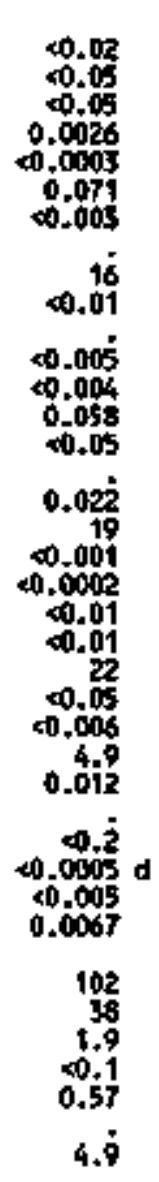 \\
\hline $\begin{array}{l}\text { TOTAL SUSPENDED SOLIDS } \\
\text { TURBIOITY }\end{array}$ & & (Moflus & : & $\begin{array}{r}1 \\
0.5\end{array}$ & $\vdots$ & 1,5 & : & 0,4 & $\dot{7}$ & 0.35 \\
\hline
\end{tabular}


TRAC METALS APDEREDIX E.

APDEAPIX E.1

Sululule potwT

LOCATION

TYPE

METALS

AMT JWAN

Arsenjc

EERYLLIUN

solpis

CADewtü

CADNIUA

cations

crisonitum

coesti

coppt

IROM

LEAD

Lithion

whitum

Mutoune

MERCURY

Wolyotilua

ITREL

porAss]

Secewin

sitvie

STrout IU

inalitur

THORIOA

vathotum

zinc

aylows

aLkat injtr-incos

IIKALAjTY-COS

CHLoRtoe

FLORTDE

MT TPATE FITROEEN

ATHOMIA

toral SuSPEMDE SOLJOS Tuketoity
CU-732
CRspe
$0713 / 95$
oIs

Gi-732
cotspes

$07 / 13 / 95$

an-32

$00 / 11 / 195$

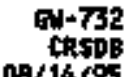

0 op/14/95

an-742

on/ $30 / \%$

G1.742

$07 / 30 / 95$

an-743

$07 / 31 / 95$

사-745

or/st/s

$\begin{array}{rr}0 N \cdot 757 & c 4-757 \\ \text { III } & \text { Lit } \\ 07 / 11 / 93 & 07 / 11 / 95\end{array}$

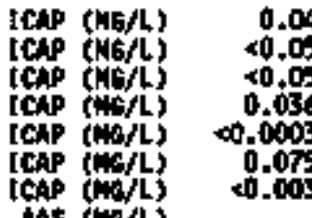

ICAP (MG/L)

ICNP (NG/L)

tosp (war)

icap (toolt)

$\cos (46 / 2)$

icas (Not)

MAP $(N \in / L$

$\cos _{\mathrm{j}} \mathrm{NG/ \textrm {L }}$

jas $(\omega / \mathrm{N}$

CVA (w/L)

ICA ( 6 (

lcep

ine

(che

tCp $\mathrm{NST}$

iche

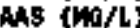

$1 \mathrm{cos}<\mathrm{mo} / \mathrm{L}$

1 (ces

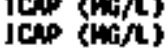

1.8
20.03
00.05
0.072
0.00037
0.027
0.000

2i

0.005

0.007

0.007

0.019

$0.005 \%$

$<0.0002$

$\$ .01$

11

$<0.05$

0.006

00.02
50.03
00.05
0.007
0.0003
0.01
0.003

$\times 0.01$

$\infty .005$

$\$ 0.004$

2,05

0.02

0.0006

40.0002

0.01

0.01
11
$<0.05$

$\$ 0.05$

$+0.006$

$\infty . \dot{2} \quad 0 . \dot{z}$

40.0005 d 0.005

$0.0005 \quad 0.0022$

$\begin{array}{rr}0.02 & 0.02 \\ 0.05 & 20.0 \\ 0.05 & 0.00 \\ 0.0097 & 0.09 \\ 0.0003 & 0.000 \\ 0.012 & 80.00 \\ 0.003 & 40.006\end{array}$

<.

40.005

$<0,005$

$<0.05$

$<0.004$

80,0002

$<0.0002$

$\times 0.01$

1.8
$<0.05$

00.05
$\times 0.006$

$\begin{array}{lll}0.006 & 40.006 & 0.006 \\ 0.93 & 0.79 & 0.65 \\ 0.014 & 0.016 & 0.019\end{array}$

40,01

0.004

$<0.00 \mathrm{i}$

0.0019

40,0002

$\begin{array}{rrr}0.006 & 40.006 & 0.006 \\ 0.93 & 0.79 & 0.65 \\ 0.014 & 0.016 & 0.018\end{array}$

$<0,0005 d$

$\$ .0005 \mathrm{~d}$

0.000

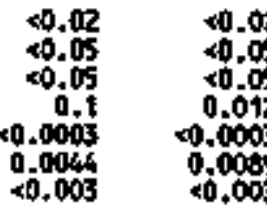

$<0.01$

30
1.0

1.8
0.1

4.

(

(n)

( $\mathrm{Noh} / \mathrm{L}$ )

(MOA)

(nofLs)
$<0.005$

cos

$\$ 0.01$

0.9
0.05

40.006

$\begin{array}{cc}0.0005 \\ 0.005 \\ 0.16 & \begin{array}{c}0.0005 \\ 40.005\end{array} \\ 0.002\end{array}$

$\$ .005$

0.004

0.05

$\$ 0.004$

0.075

$<0,0002$

8.07
80.01

0,05

0.006

0.65

156

$<1$
1.5

4.005

40.004

$<0.05$

$<0.004$

0,08

0.0002
40.01

40.01

4.1
$<0.05$

40.006

0.64

$0.00 .25 \mathrm{~d}$

0.005

169
4
1.7
$<0.1$

$<0.2$

13

t.8

2.8

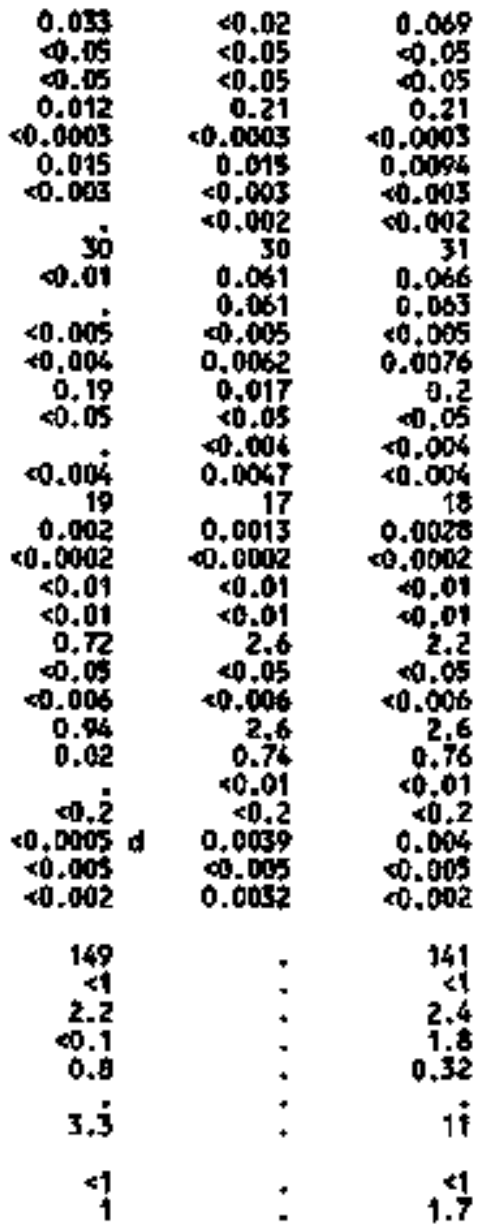

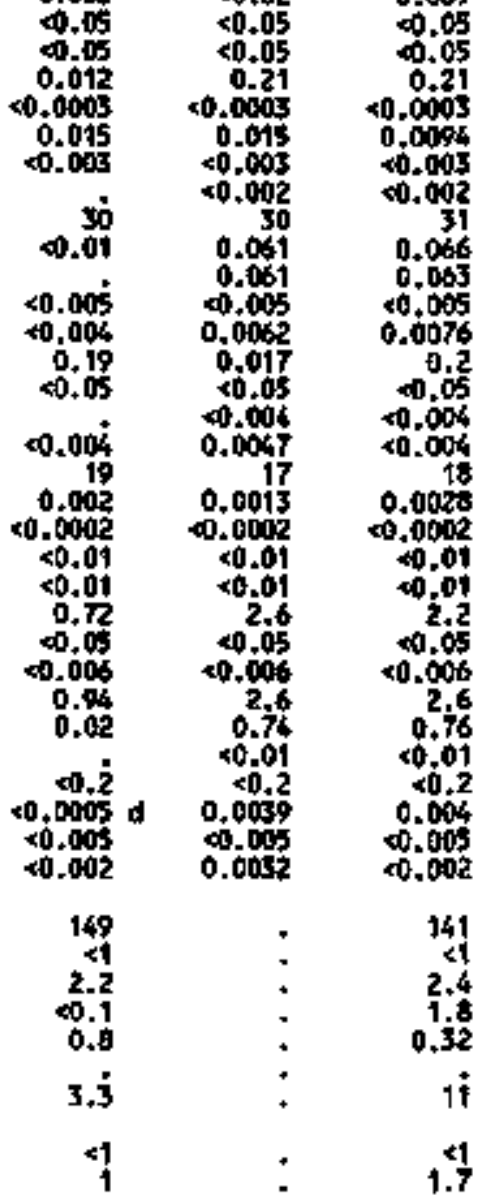




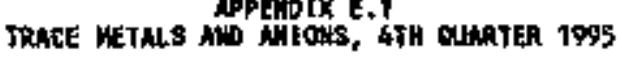

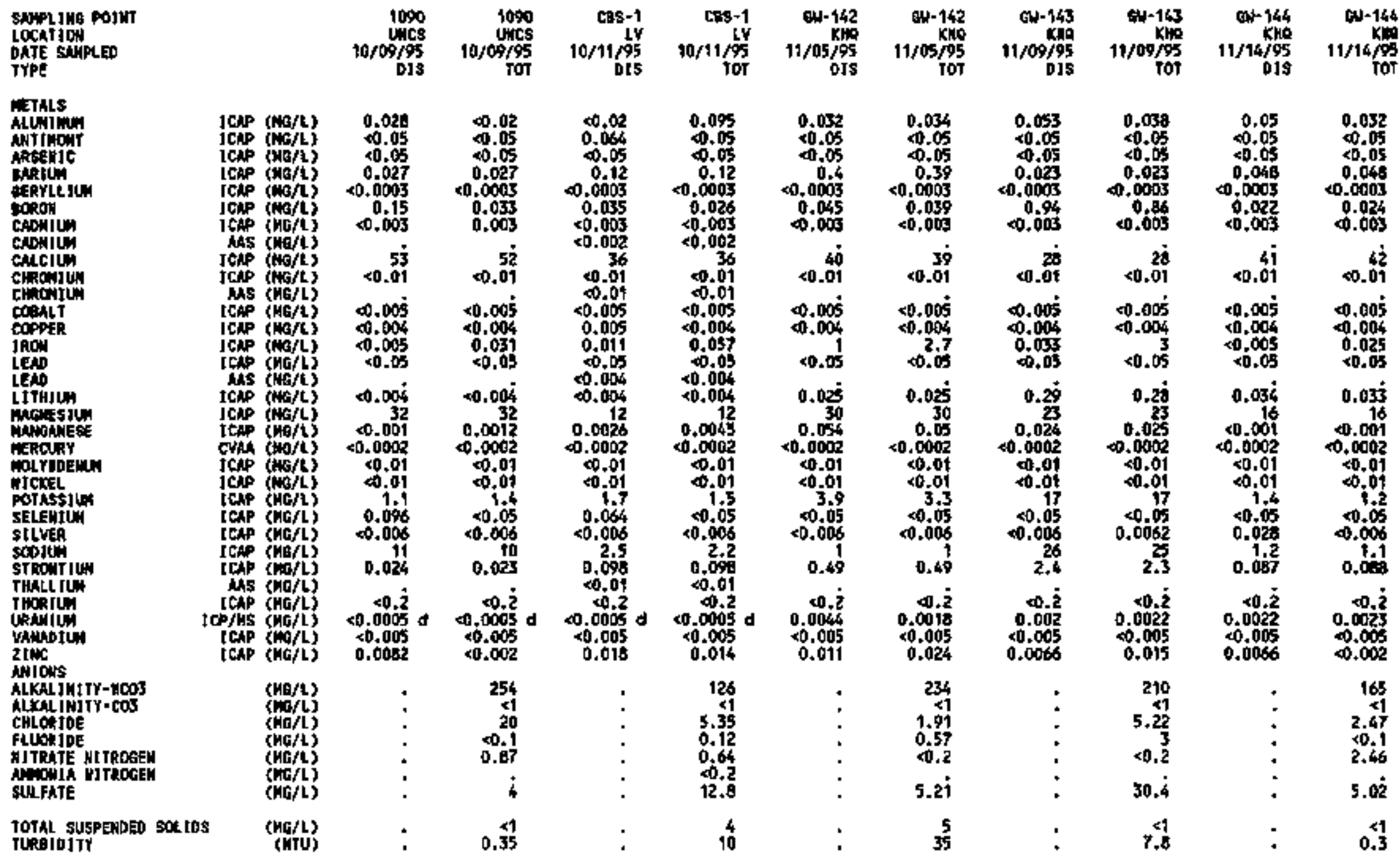




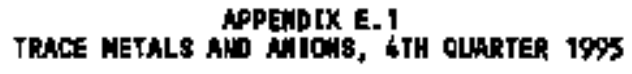

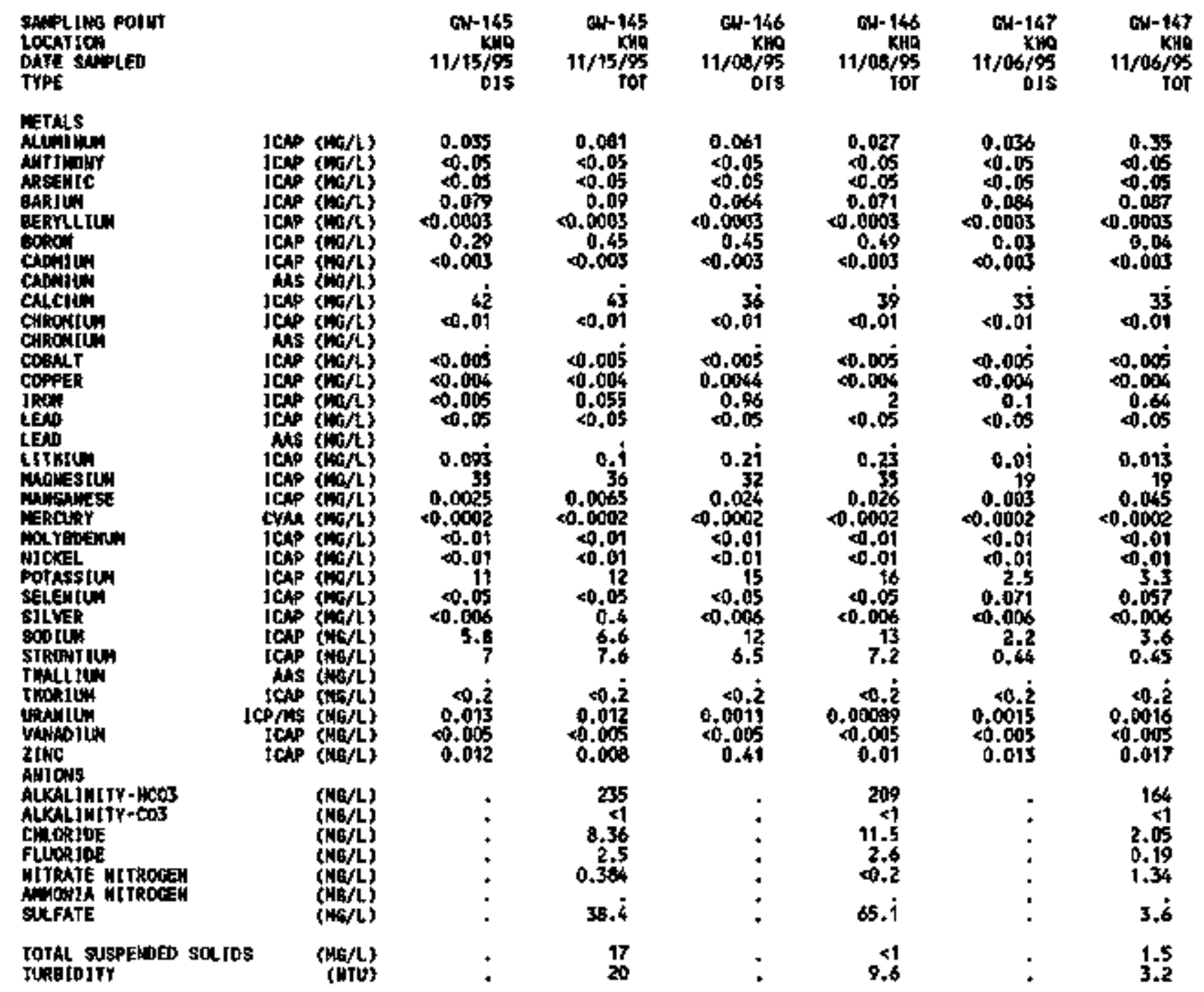




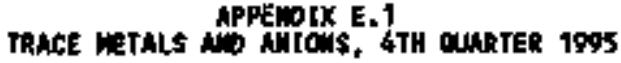

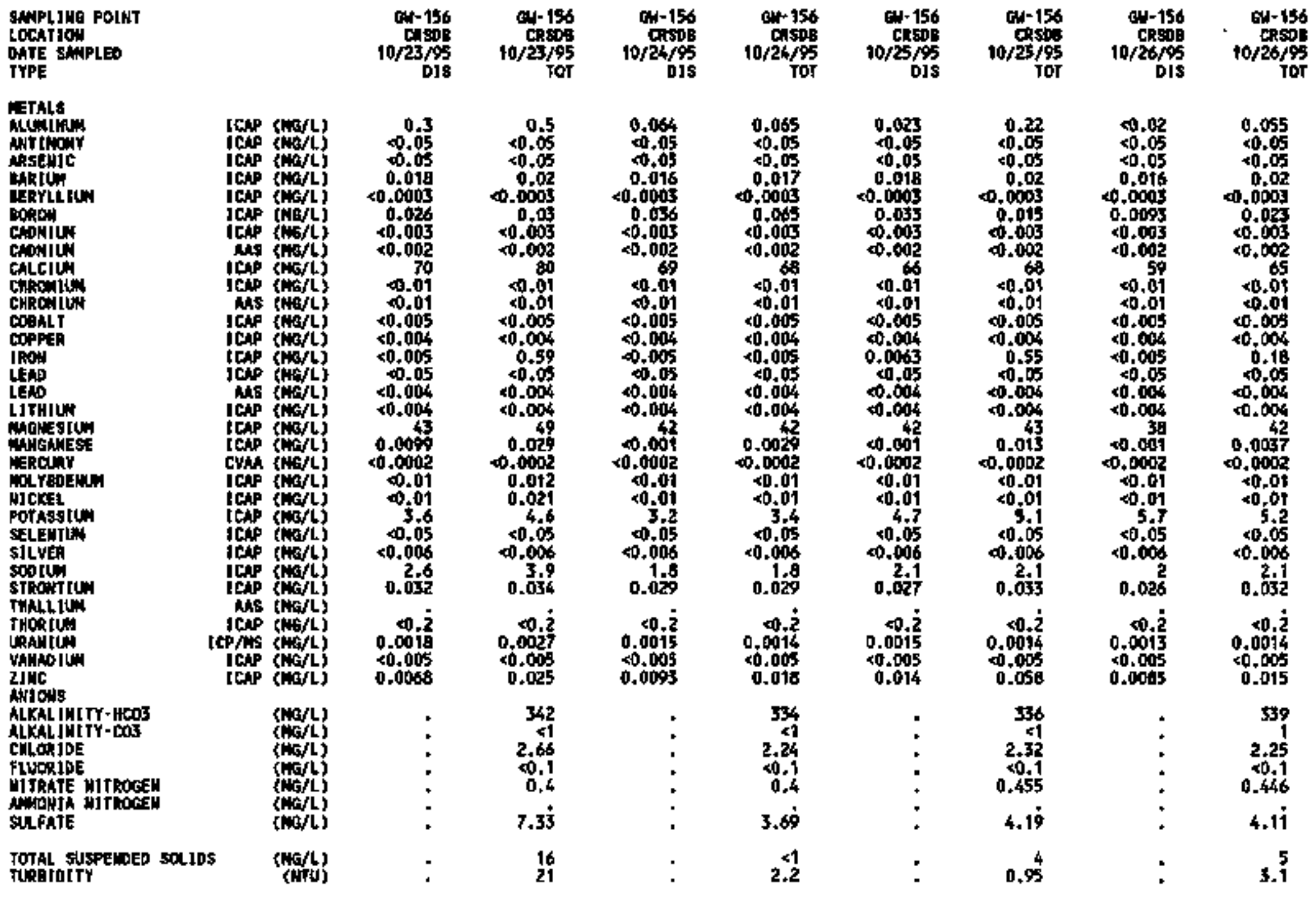




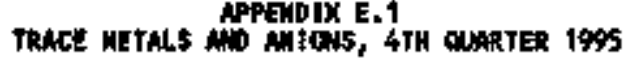

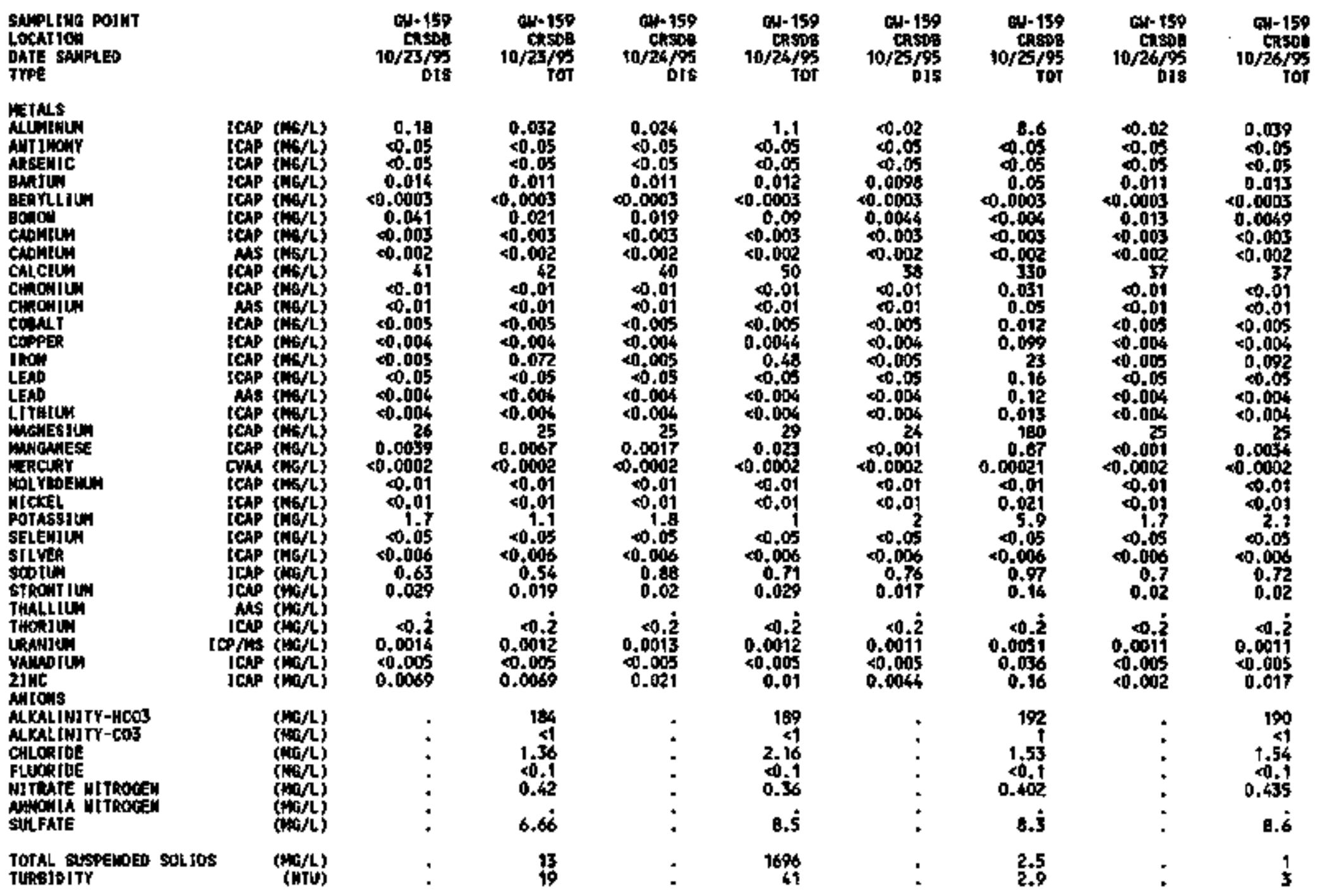


TRACE METhLS APP AHIONS, 4TH CUMRTER 1995

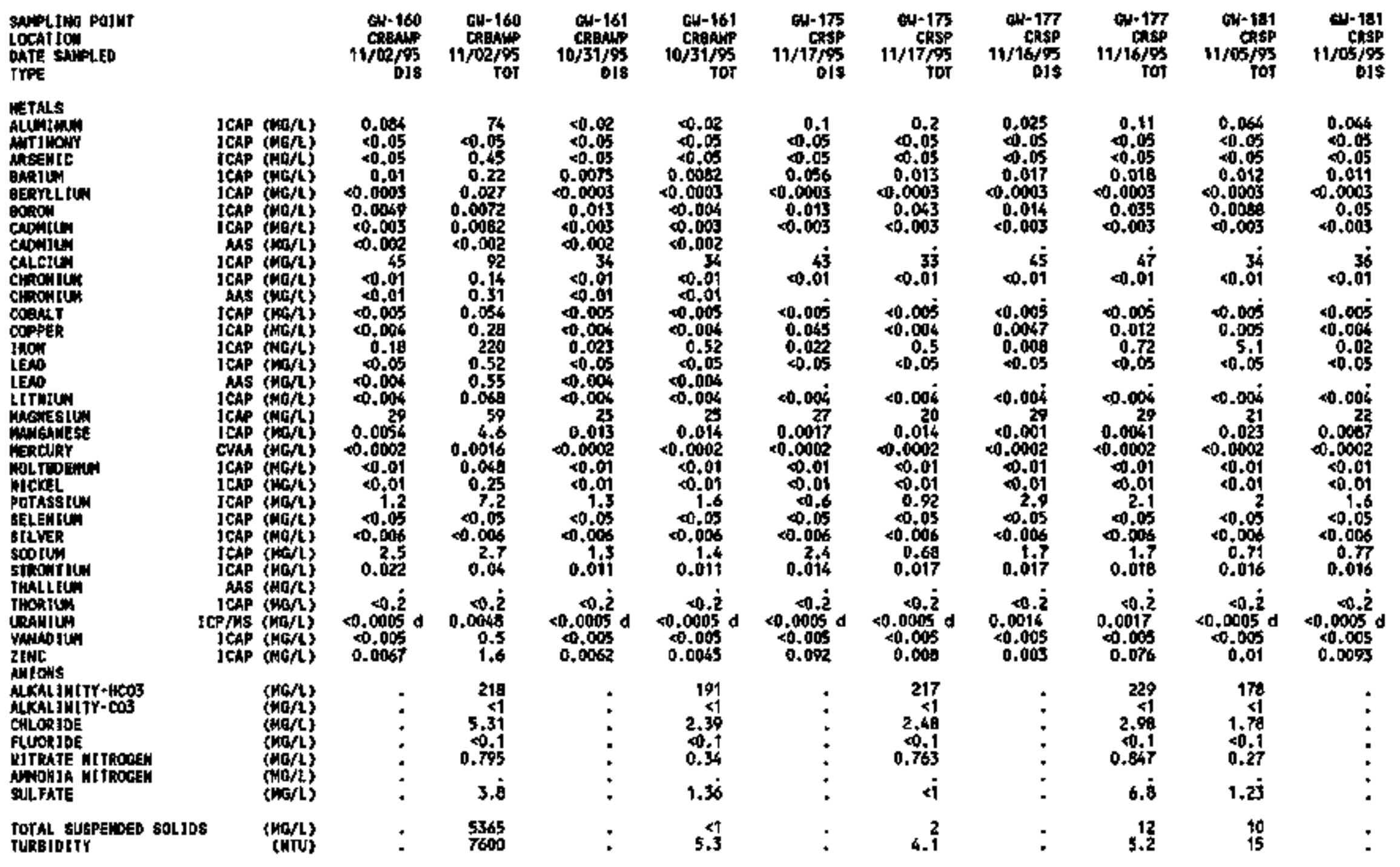




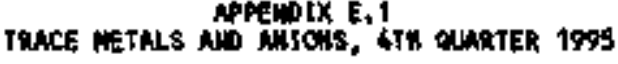

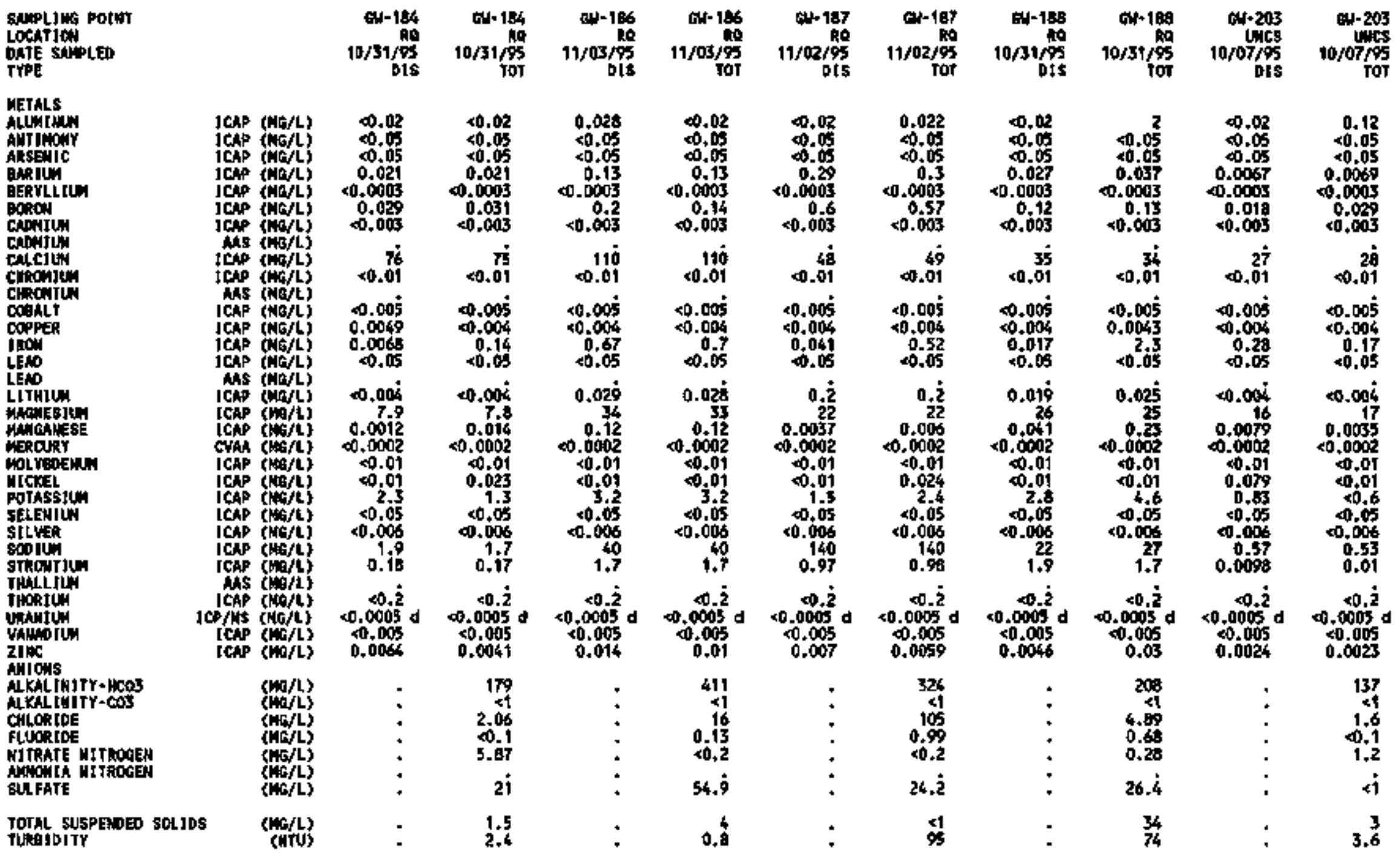




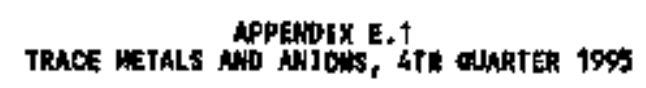

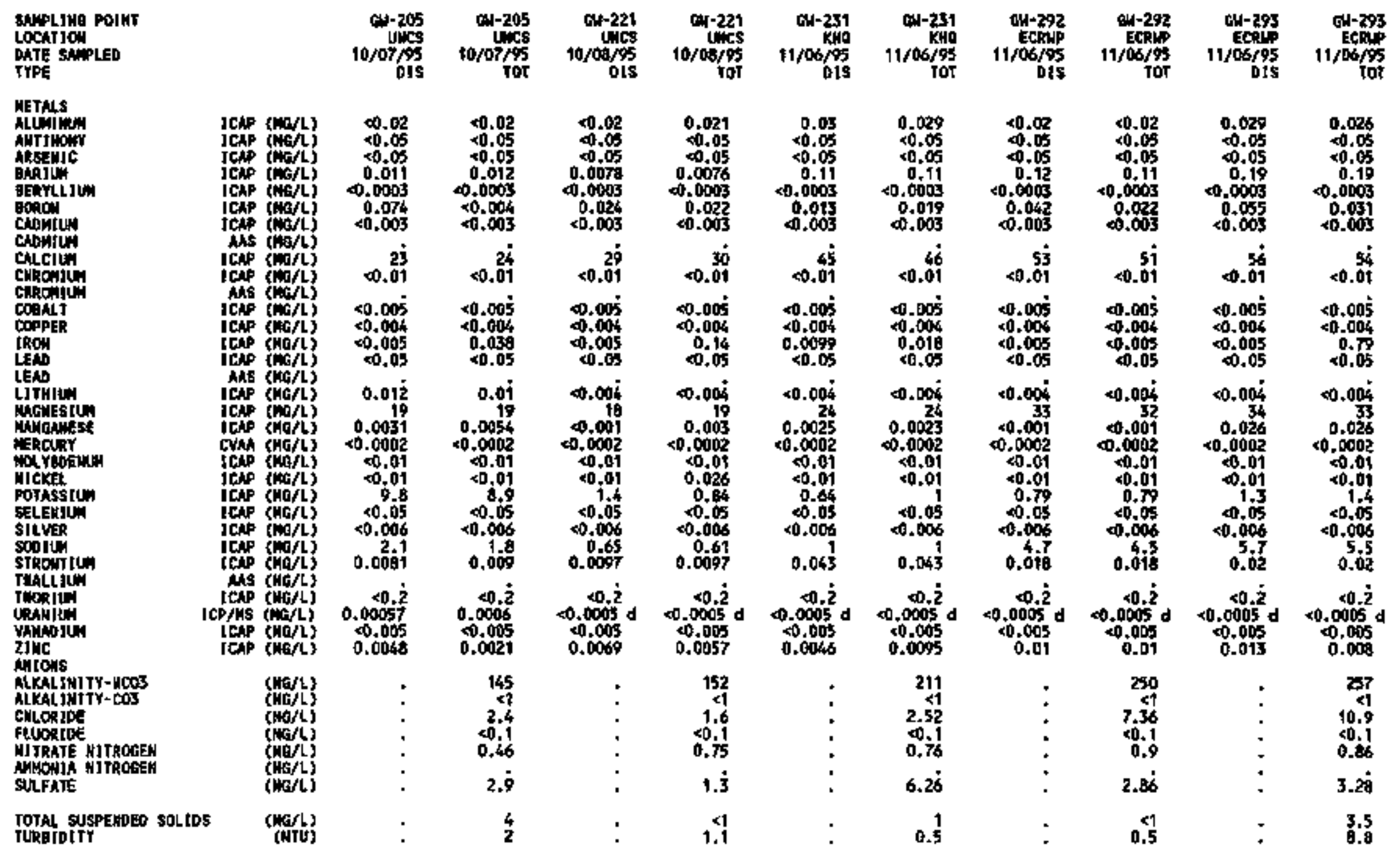




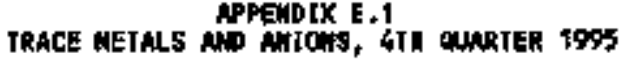

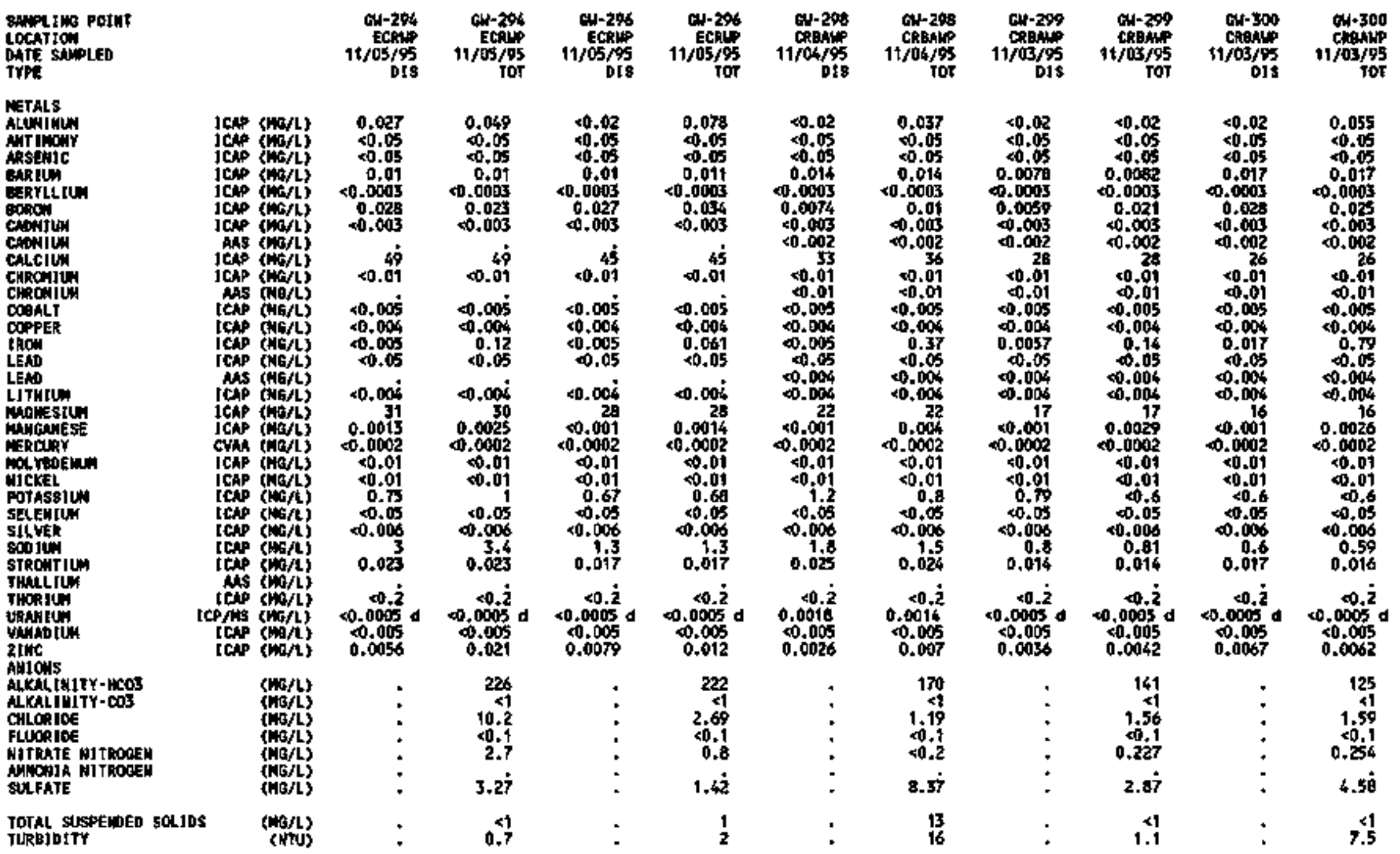




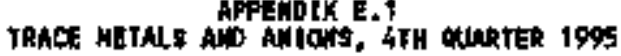

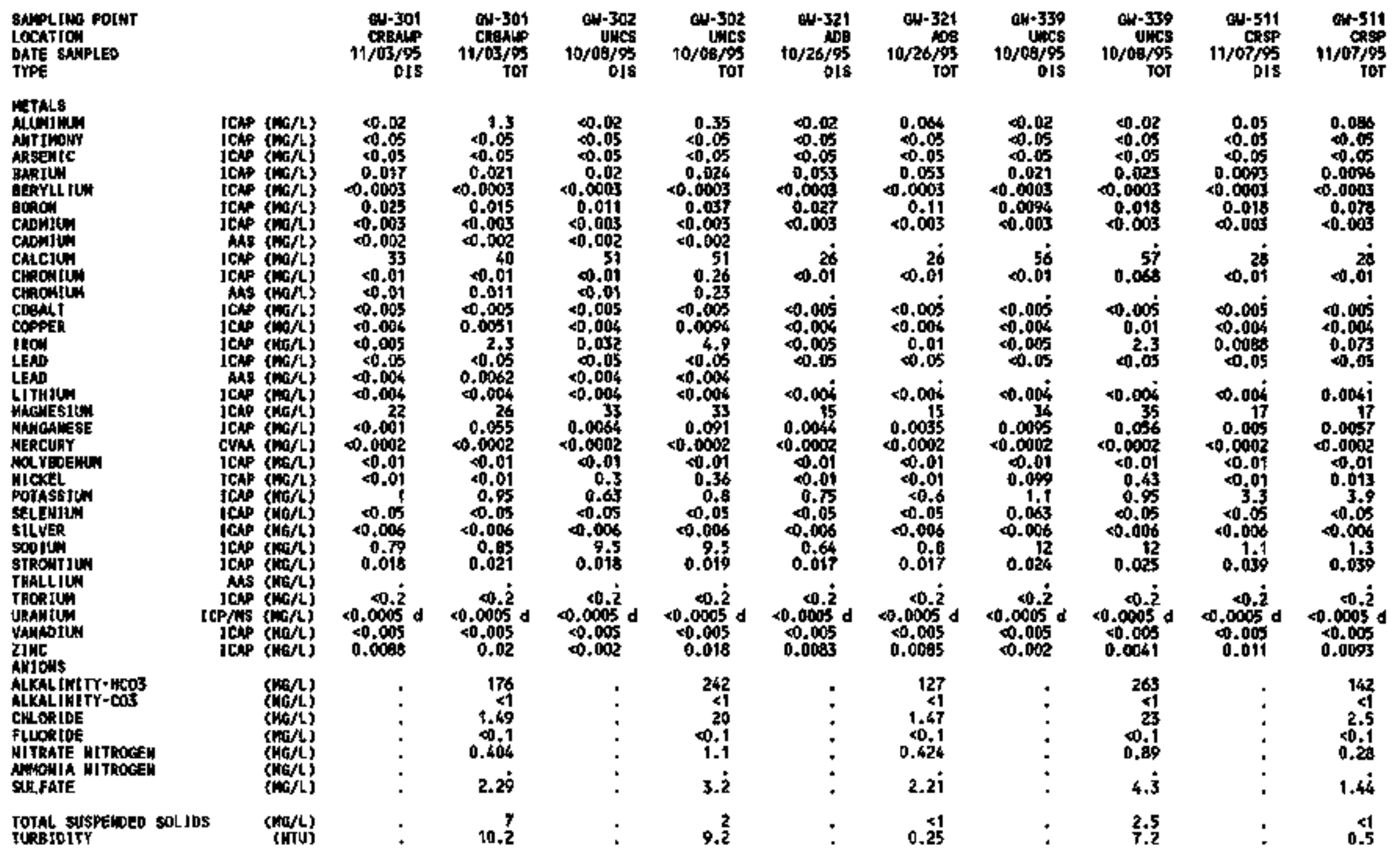




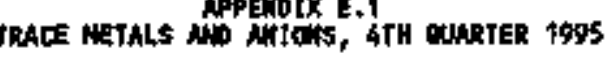

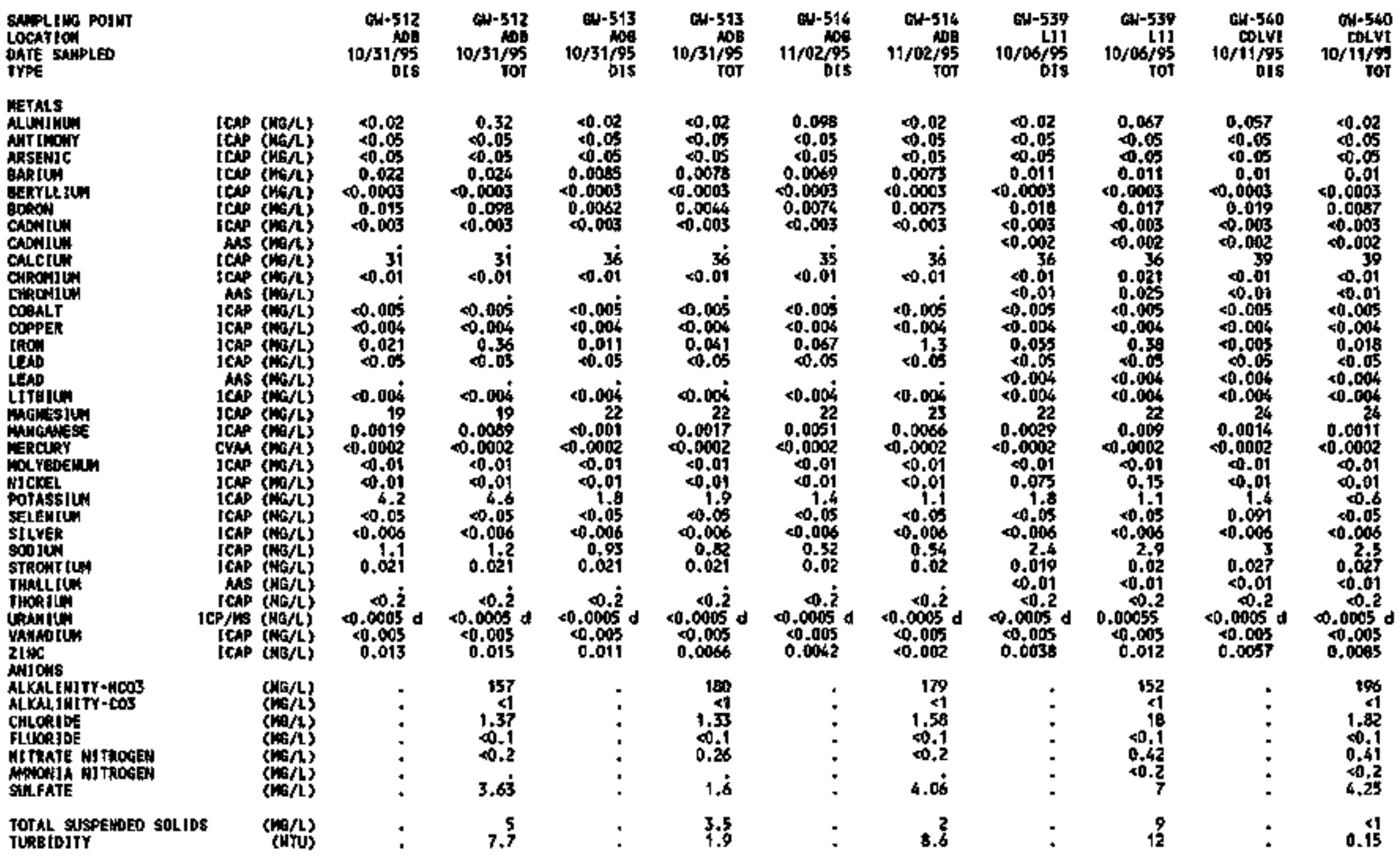




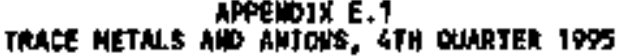

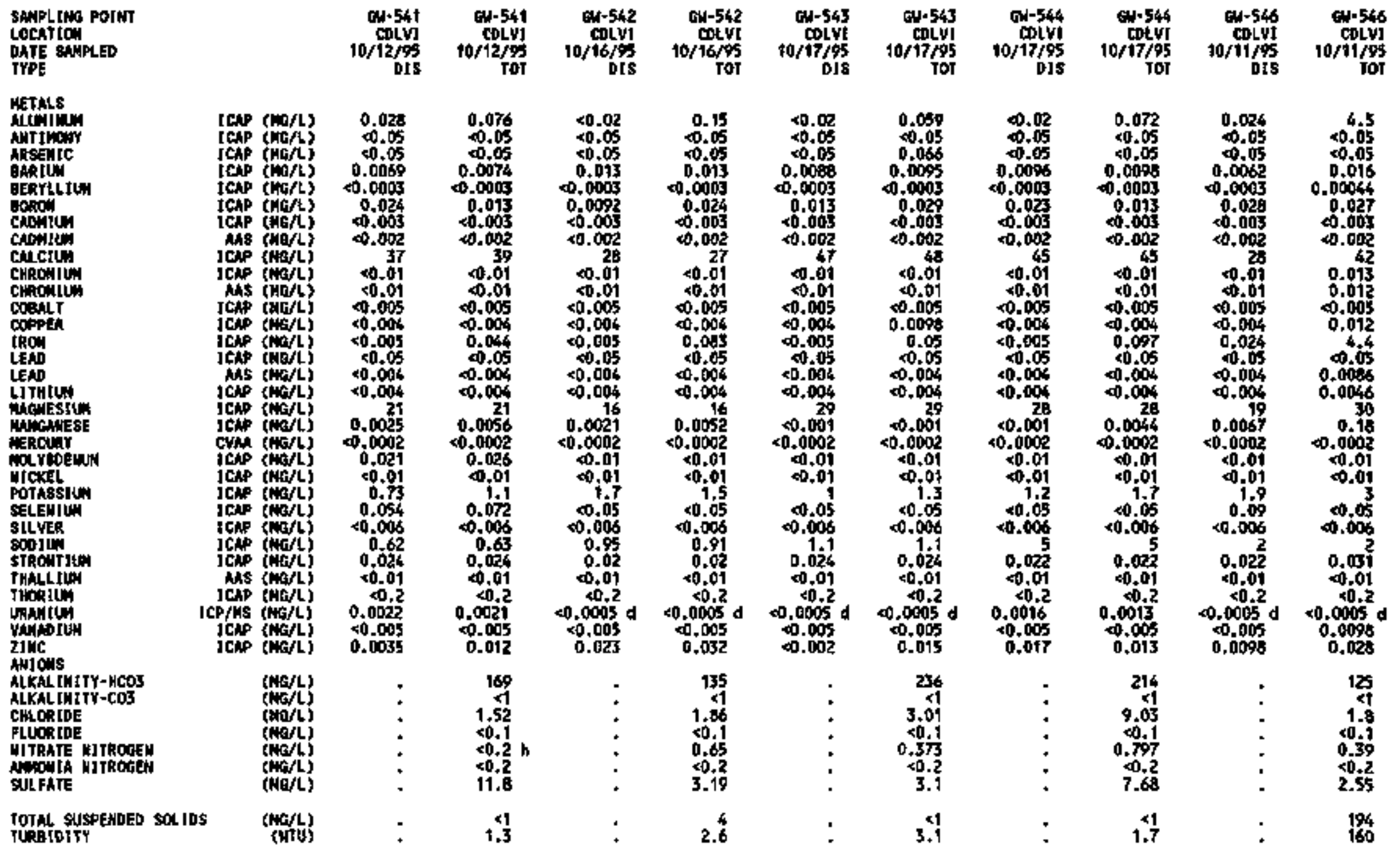


TRACE METALS MUP ANIONS, 4TW OMRTEK 1995

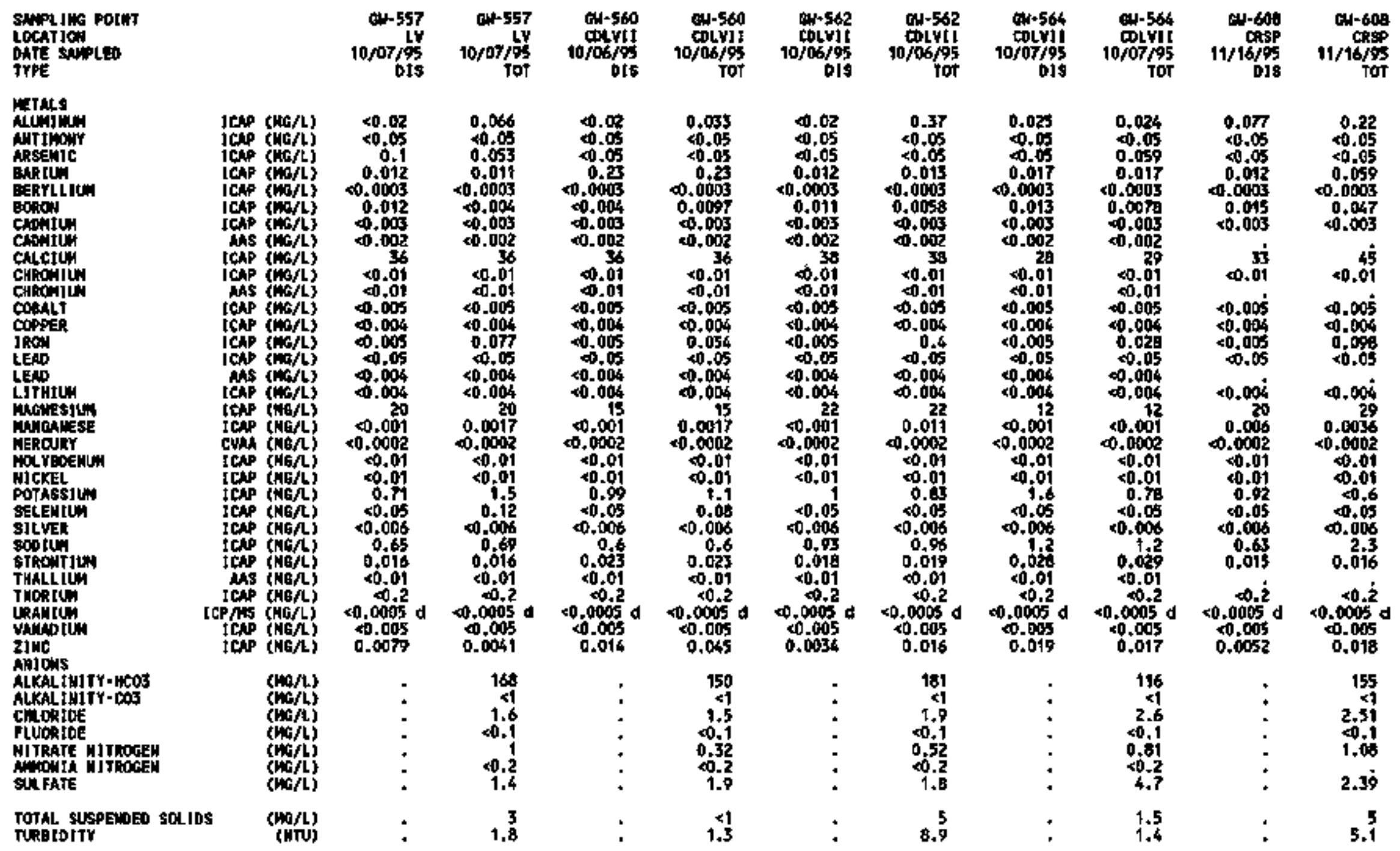


TRACE METALS APO AHIOHS, 4TH OURTER 1995

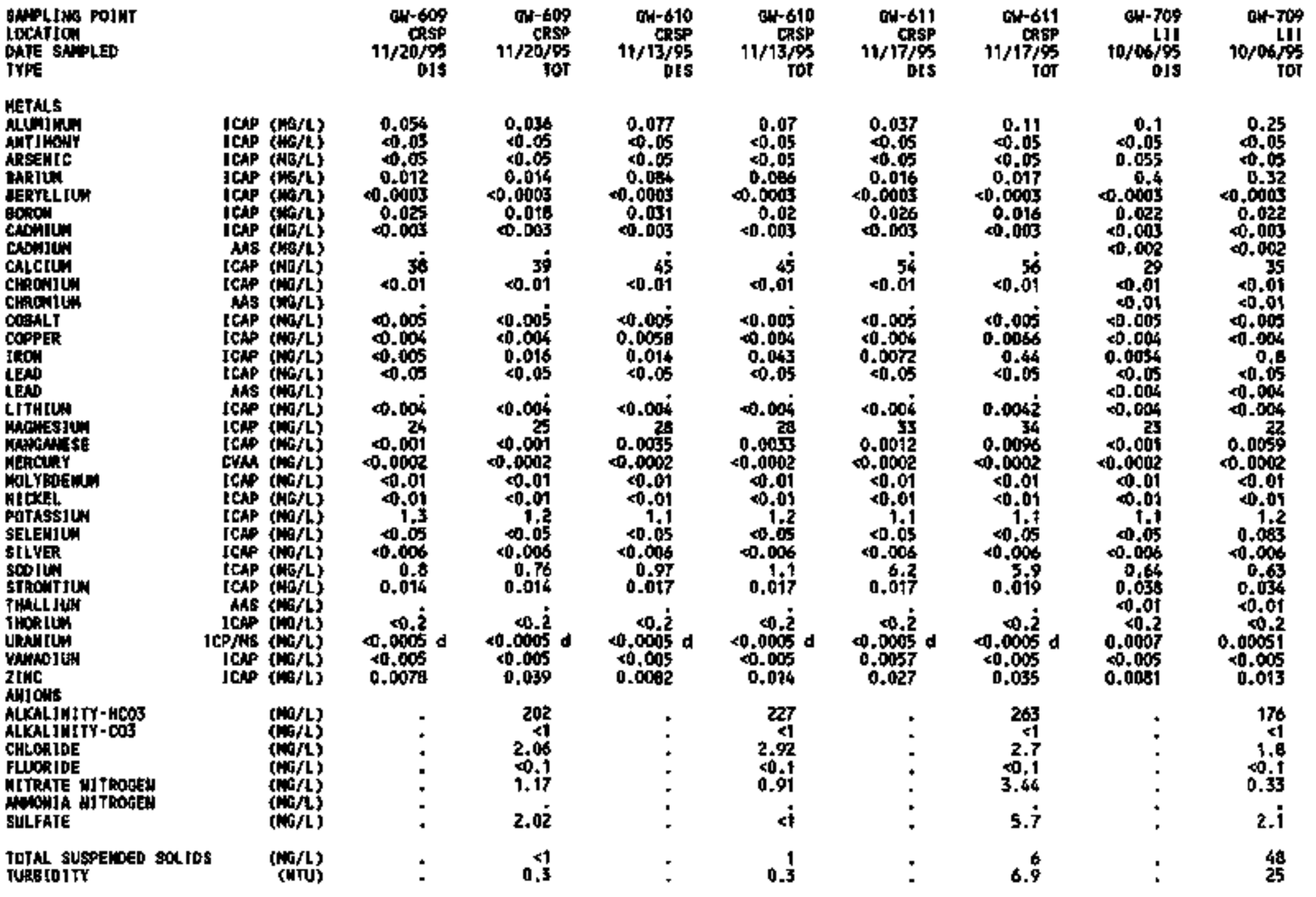


TRACE METALS AMO NHOAS, 4TW CUMTER 1905

SNPLING PDtht

BAFE SHPLED

retals

elinising

int linint

ARsent

GerYLLIU

BerinLL

socis

chowion

chatcjus

CIROAfO

Chichis

coedL?

18GN

LEATHAN

minoliosiu

monstios

minciary

merrabein

H] GXEL

porasstu

selfintu

silyer

stRontum

fMALL Jui

THORJU:

WRAHIU

YAHDiust

2Inc

AlKaLIMtTY- HCOS

ALKAL] Hitr-cos

clitoride

Futotio:

Pi TPTE Hitroren

Manonla HITROEE

SUTFTE

TOTAL SUSPEWEO SOLTDS TURBtajty cha

$0 / 24 / 95$

$0 \times \cdot 75$

cherso:

cis -75

$0 / 24 / 95$

const

10/24/os

cherst

corsts

ch-731

10/25/9s

a.1-731

10/26/95 10/26/95

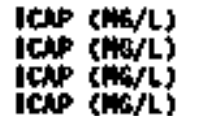

ICAP ( $M / L$ )

JCA (NG/

icte (Nor

Ans $(\mathrm{Noj} / \mathrm{L}$ )

JCAP (NG/L)

JCA

As (Non)

tie

JCH (NG/L)

JCAP (No/L)

ICA $(\mathrm{HO} / \mathrm{L}$

NAS ( $N$ H $/$ L)

ICA $\left(\mathrm{H}_{\mathrm{W}} / \mathrm{L}\right.$

ICAP (NoSh)

jêp ( $\mathrm{NOC} / \mathrm{C}$ )

cren (HGA)

$\cos _{10 \mathrm{~N}}(\mathrm{NG} / \mathrm{L}$

jets ( $\mathrm{N} \mathrm{H} / \mathrm{L}$ )

JCAP (NG/L)

JCAP (NGG/L)

JDe (NO/L)

jowp

ICAP (tath

(ANS $(40 / 5)$

ICD/LS ( $\mathrm{W} / \mathrm{L}$ )

[CAP ( $\mathrm{MO} / \mathrm{L}$ )

0,071

50.05

0.00

4,0003

4.003

4.003
4.002

4.002

$<0.01$

$<0.01$

40.05

0.011

40.05

40.004

4.004

0.0022

$<0.0002$

40.01

4,01

<io.

40.006
2.7

0.36
0.05
0.05

0.0074

$\times 0.0003$

0.023
$\times 0.003$

40.003
+0.402

4.

0.0t3

+0.01
$\times 0.005$

400

0

$<0.05$

$<0.004$

0.00

0.019

40.0002

$<0.0$

40,01

$<0.05$

$<0.00$

0.029

0.075
0.05
0.05

0.0053

$\times 0.000$

0.068
$\times 0.003$

0.007
40.002

4.14

$\infty .0 t$

0.005

40.005

0.005

40.05

50.005

0,000
$<0.007$

40.0602

0.01

6.01

40.12

$<0.006$

0,022

0.15

0.05
$\times 0.05$

0.0094

40003

0.063

4.002

17

$\infty .01$

50.005

0.006

0.45

40.004

0.009
0.0094

$<0.0009$

$\$ 01$
$\$ 0.01$

0.01

$\infty 0.09$

40.006
3.9

$00 . \dot{z}$

$40,000 \dot{2}$

$<0.005$

50.0005
0.005

i

0.029

4.02

0.05
4.05

0.0068
0.0005

0.012
$<0,003$

$<0.002$

12
$\infty 0.01$

$<0.04$

$<0.004$

$<.09$

$\times 0,005$

$<0,004$

0.01

13
$40.00 \%$

$\$ 0.0002$

$\infty .01$

0.01

0.05

4.4

$40,000 . \dot{2} d$

.0005

$\infty, 005$

40,0005

40.0005

0.005

0.35

$<0.05$

0.012

0,0003

0.031
$<0.003$

40.003
$\times 0.002$

15
0.019

0.079
0.019
$<0.005$

0.024

$\times 0.2$

$\times 0.05$
0.0051

0.02

15
0.039

80,0002

$<.01$

$\times 0.01$

$<0.05$

$<0.006$

0.037

0.02

40.05
40.05

0,004

40,0003

0.013
+0.603

40.002

9.4
50.01

401
401

$<0.005$

0,016

40,005

0.004

0.016

13
$\times 0.001$

$<0.0002$

$\$ 0.01$

0.016

40.05

0.005

0.013

$<0 . \dot{2}$

$<0,0005 d$

40.2

20,0005

0,005

0.025

92
10
1.21

0.37

$5.2 \dot{8}$

(No/L

(No/l)

(wo/L)

112
61.26
40.8
0.36
4.85
4.5

30
1.56

1.56
0.0 .1

0.376

5,45

33

0.11

0.05
+0.05

0.005
$\times 0.0003$

$<.0003$

$\rightarrow 00$

$\$ 0,002$

17
0.01

$+0.01$

0.00

.00ss

Q.is

$\infty .004$

0.014

0.015

40.0002

<.01

0.14

0.006

0.006
0.1

$40.0005 \mathrm{~d}$

$<0.605$

84

94
16
1.48

0.418

5.68

511 
TRACE IETALS APPEWIX E.t

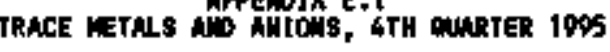

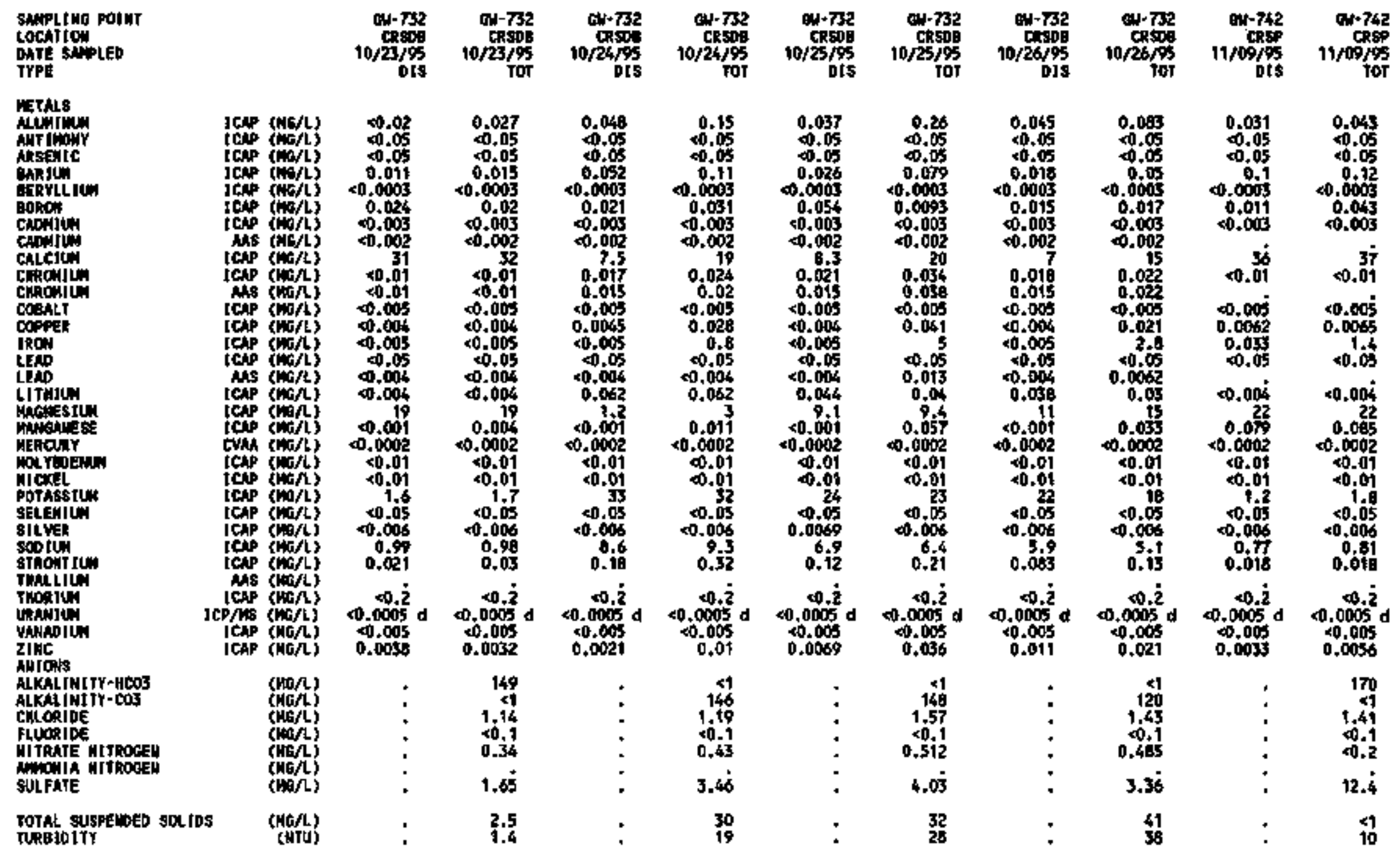




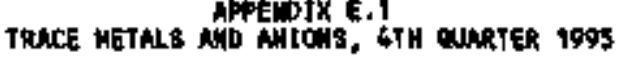

\begin{tabular}{|c|c|c|c|c|c|c|c|c|c|c|c|c|}
\hline $\begin{array}{l}\text { SHMPLINE POIMT } \\
\text { LOCATION } \\
\text { DNEE SANHED } \\
\text { TYPE }\end{array}$ & & & $\begin{array}{r}61-745 \\
\text { Ches } \\
11 / 13 / 95 \\
\text { Dis }\end{array}$ & $\begin{array}{r}04+743 \\
\text { CREP } \\
11 / 13 / 95 \\
\text { TOT }\end{array}$ & $\begin{array}{r}\text { aw-757 } \\
\text { h15 } \\
10 / 06 / 95 \\
\text { bis }\end{array}$ & $\begin{array}{r}00-75 \\
\text { tid } \\
10 / 06 / 95 \\
701\end{array}$ & $\begin{array}{r}0 k+796 \\
\text { iv } \\
\text { 10/00/95 } \\
\text { bis }\end{array}$ & $\begin{array}{r}0 \mathrm{w}-7 \% \\
\text { to/0s/os } \\
\text { Tor }\end{array}$ & $\begin{array}{r}0 w-797 \\
6 y \\
10 / 00 / 65 \\
018\end{array}$ & $\begin{array}{r}0 i-797 \\
1 V \\
10 / 00 / 55 \\
\text { Tor }\end{array}$ & $\begin{array}{r}\text { cin. } 790 \\
\text { colvit } \\
10 / 00 / 95 \\
\text { bits }\end{array}$ & 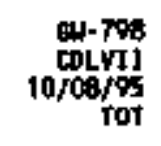 \\
\hline 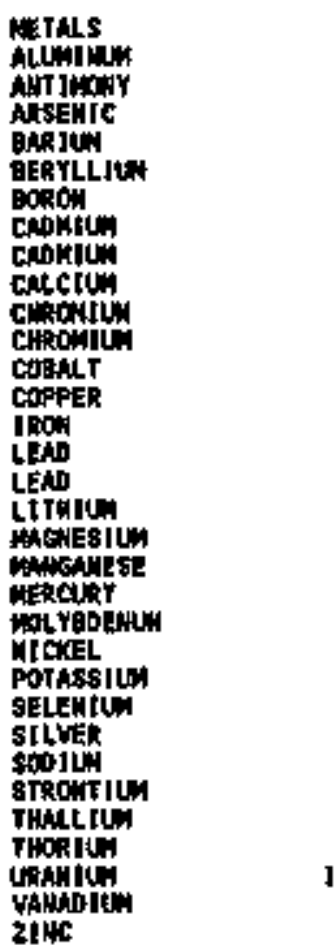 & 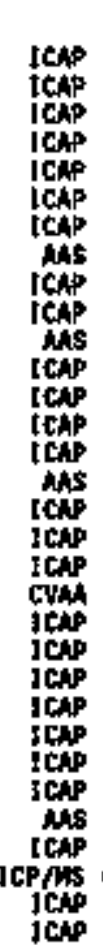 & 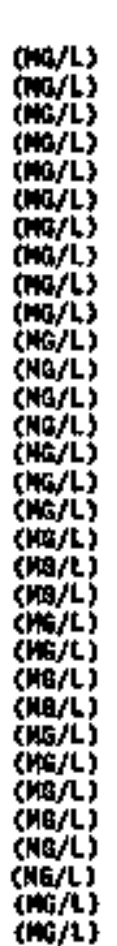 & 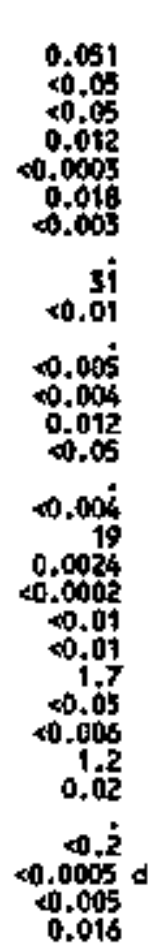 & 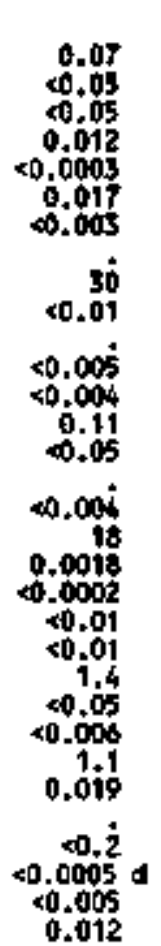 & 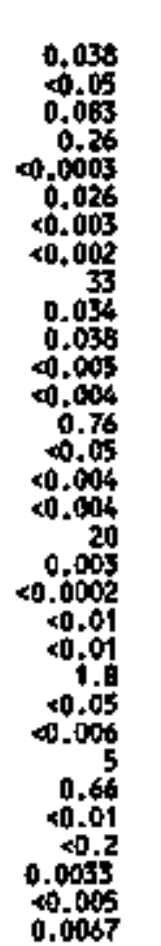 & 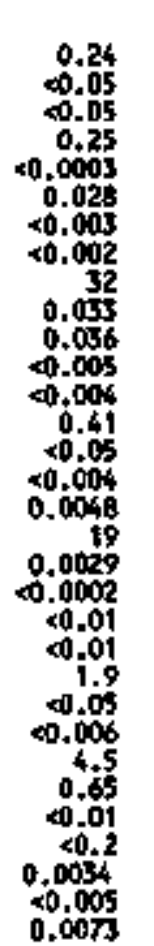 & 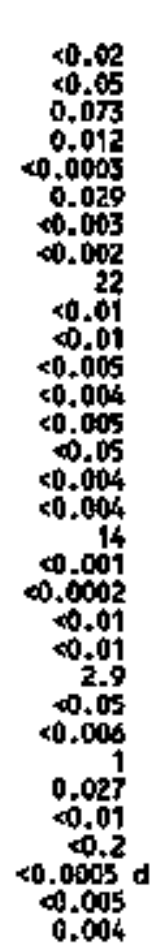 & 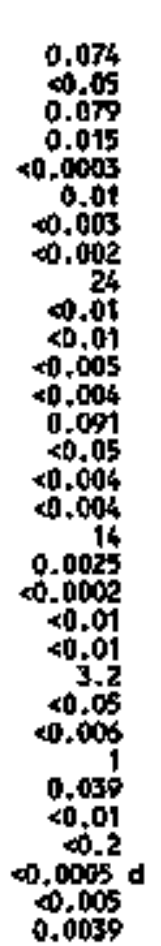 & 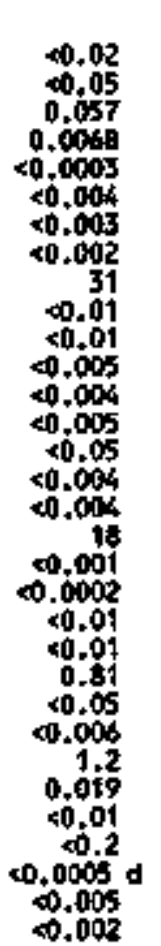 & 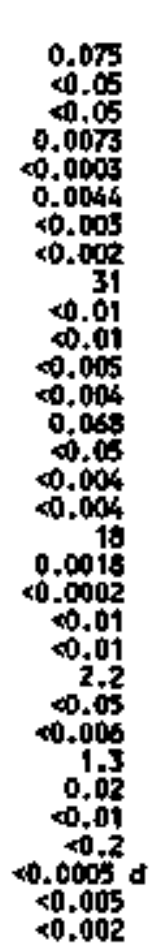 & 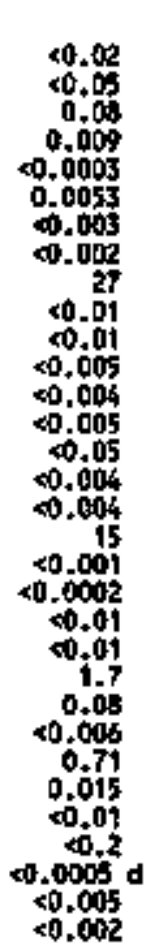 & 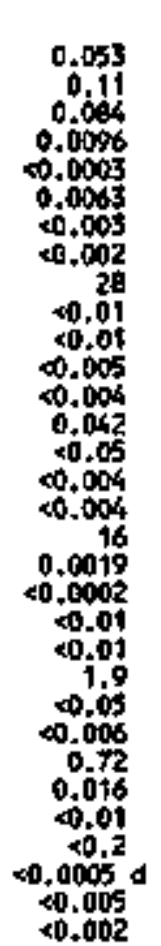 \\
\hline 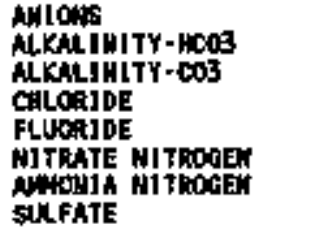 & & 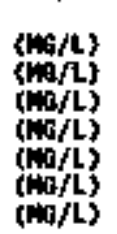 & $:$ & $\begin{array}{l}146 \\
28 \\
2.24 \\
40.1 \\
1.05 \\
3.02\end{array}$ & . & $\begin{array}{r}163 \\
2.4 \\
1.3 \\
0.48 \\
0.24 \\
9.8\end{array}$ & : & $\begin{array}{r}118 \\
<1 \\
1.7 \\
<0.1 \\
0.52 \\
<0.2 \\
1.3\end{array}$ & : & $\begin{array}{r}151 \\
\times 1 \\
1.5 \\
0.1 \\
0.39 \\
0.2 \\
2.4\end{array}$ & $:$ & $\begin{array}{r}133 \\
<1 \\
1.3 \\
40.1 \\
0.8 \\
0.2 \\
2.5\end{array}$ \\
\hline $\begin{array}{l}\text { TAL GUSPEIDEO S } \\
\text { RBID]TY }\end{array}$ & & 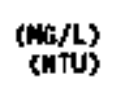 & - & 0.65 & : & 4.8 & : & 4.3 & : & 1.7 & $:$ & 0.65 \\
\hline
\end{tabular}


TRACE WETML APP Mipix E.1

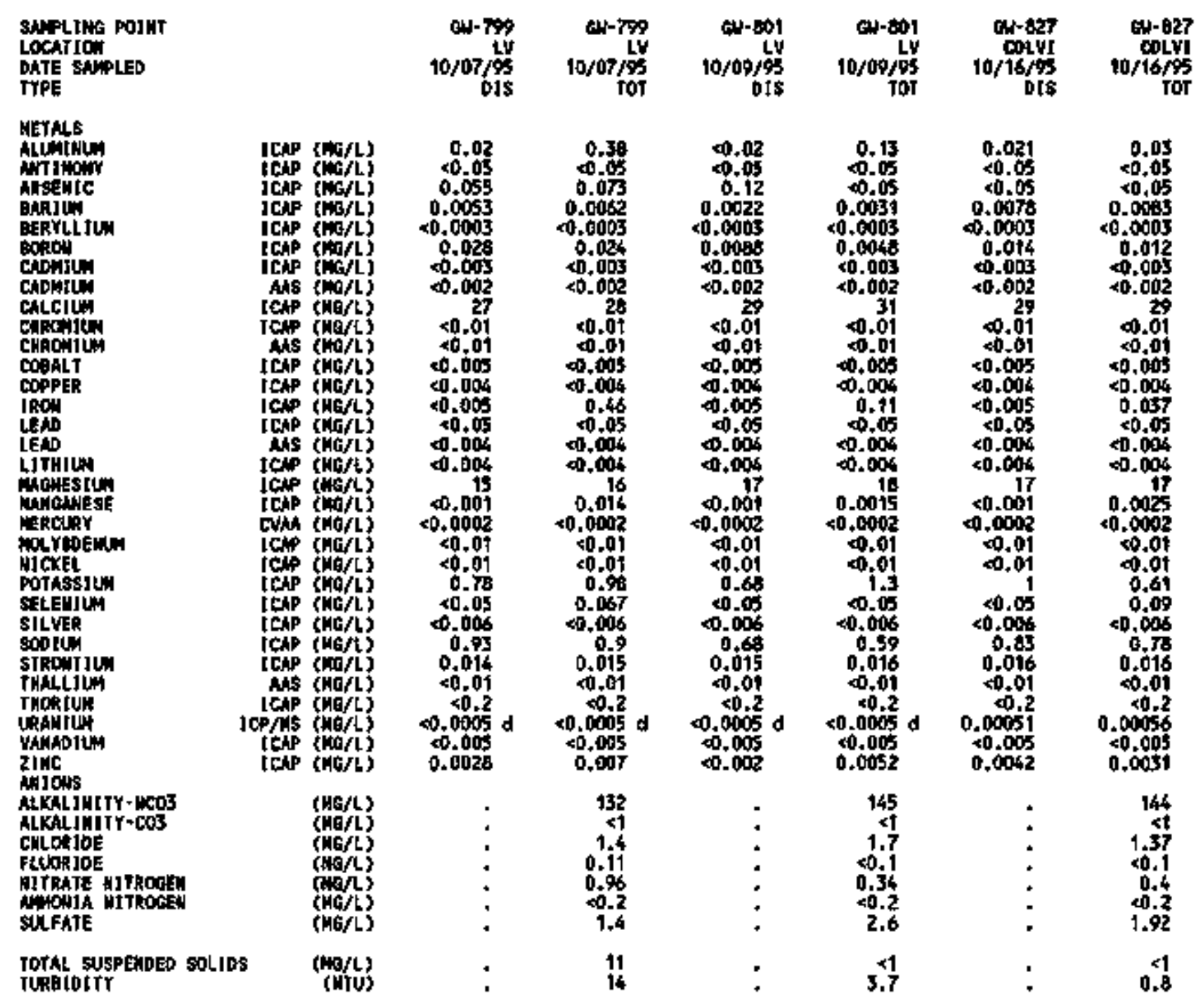




\section{APPENDIX E.2}

VOLATILE ORGANIC COMPOUNDS 


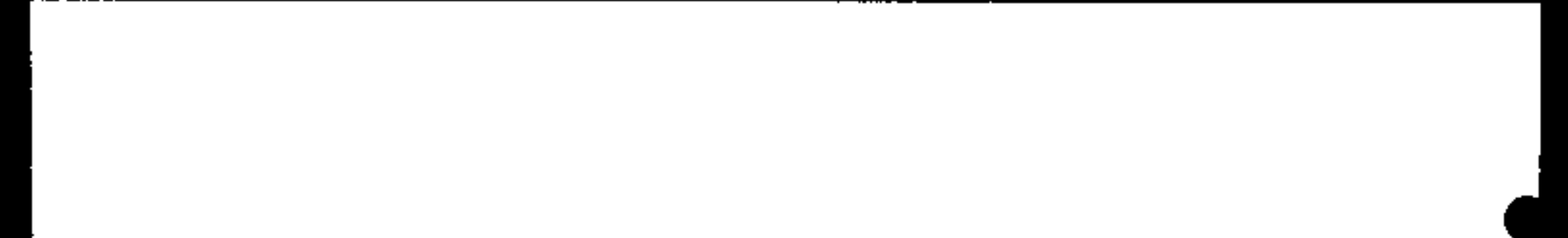




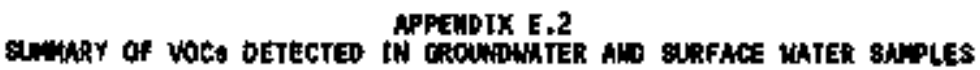

Totol senples

Senples without voc

39

\begin{tabular}{|c|c|c|}
\hline consonso & & $\underset{\text { WURTTER }}{\text { FIRST }}$ \\
\hline 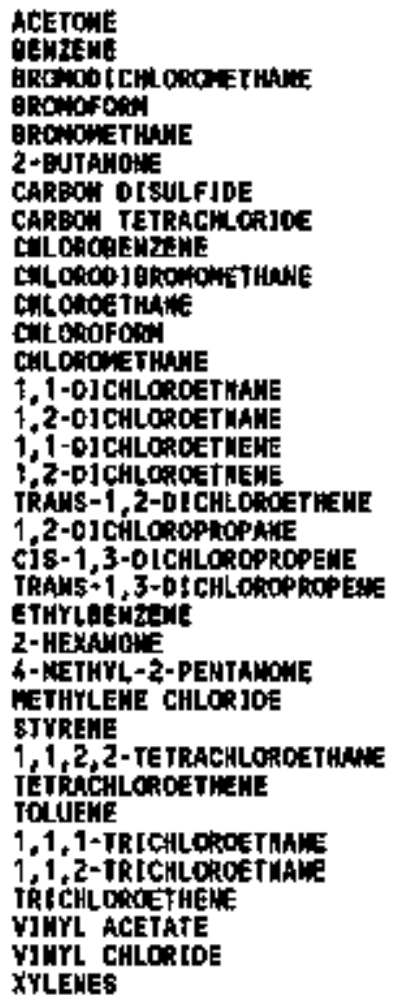 & 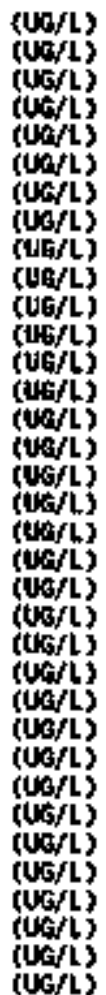 & $\begin{array}{l}5 \\
0 \\
0 \\
0 \\
0 \\
6 \\
0 \\
1 \\
0 \\
0 \\
0 \\
0 \\
0 \\
0 \\
0 \\
3 \\
1 \\
0 \\
0 \\
0 \\
0 \\
0 \\
0 \\
0 \\
0 \\
0 \\
0 \\
4 \\
4 \\
5 \\
0 \\
2 \\
0 \\
0 \\
0\end{array}$ \\
\hline
\end{tabular}


VOLATILE OREAHIC CONFOUXOS, ISt QUARTER 1995

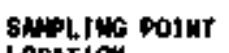
LOChIJOA

DATE SAYPLE

\section{ACETOLE}

BROWOO ICHLORONETHANE BRamfork

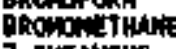

2- IUTAMom

Carpon disuctoe

CAReon TeTkhcheor tbe

CHLOMOSENZEME

CHLOAOD JRROWAETHAME

CHLOROEThANE

chlososos

CHLCRONETHANE

1,1-01CHLOROE THANE

2-DICHLOROETHWNE

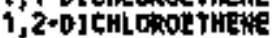

TRANS- t, 2-btCALOROET:ELE

1,2-0tCHLOUGSROPAME

Cj8-1,3-DICILLROPROPENE

JRAn-1,3-01ChLOAXPROPG

ETWYLEEWEENE

S-METHYL-2-DEMTAHDNE

CTIHLEE CALOAIDE

STYRENE

$1,1,2,2$-TETAACHLOROE THAHE

TET TACHLOROE THENE

Tounis:

$1,1,1$-TR R CHL ONOE THAHE

$1,1,2-T R$ I G HLONOETHWHE

FR TCHLORETTHEN

VIMYL ACETARE

XYLEIES

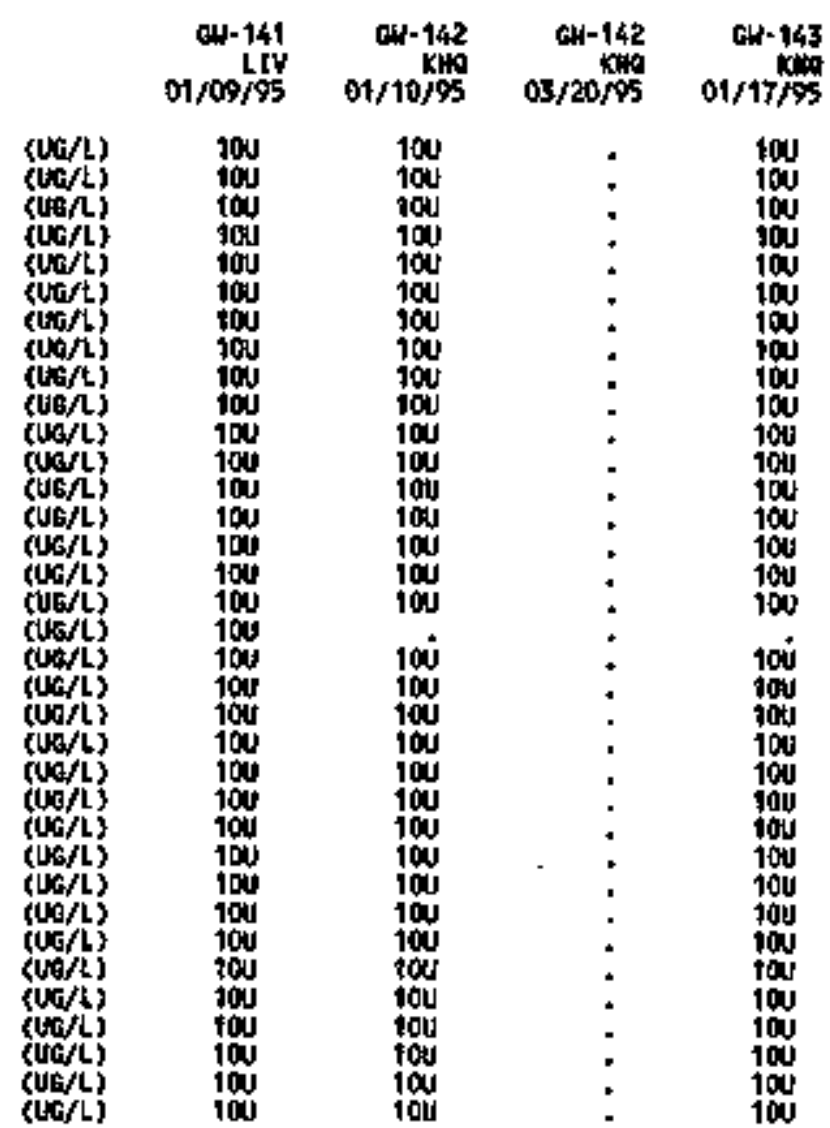

\begin{tabular}{|c|c|c|c|c|}
\hline $\begin{array}{r}a w-144 \\
x+4 \\
01 / 18 / 95\end{array}$ & $\begin{array}{r}0 \times-144 \\
03 / 22 / 95\end{array}$ & $\begin{array}{r}m-1+5 \\
k+19 \\
01 / 18 / 45\end{array}$ & $\begin{array}{r}0 \mathrm{r}-145 \\
\mathrm{KHO} \\
03 / 24 / S 5\end{array}$ & $\begin{array}{r}e N-14 \\
\text { Kit } \\
01 / 17 / 0\end{array}$ \\
\hline $\begin{array}{c}8 \mathrm{Jl} \\
100 \\
100 \\
100 \\
100 \\
0 \\
100 \\
3 \\
100 \\
100 \\
100 \\
100 \\
100 \\
100 \\
100 \\
100 \\
100\end{array}$ & $:$ & $\begin{array}{l}100 \\
100 \\
100 \\
100 \\
100 \\
5 \\
100 \\
100 \\
100 \\
100 \\
100 \\
100 \\
100 \\
100 \\
100 \\
100 \\
100 \\
100\end{array}$ & $:$ & $\begin{array}{l}10 \\
10 \\
10 \\
10 \\
10\end{array}$ \\
\hline $\begin{array}{l}100 \\
100 \\
100 \\
100 \\
100 \\
104 \\
100 \\
100 \\
100 \\
1 . J \\
6 \\
0.00 \\
100 \\
100 \\
100 \\
100 \\
100\end{array}$ & $:$ & $\begin{array}{l}100 \\
100 \\
100 \\
100 \\
100 \\
100 \\
100 \\
100 \\
100 \\
100 \\
100 \\
100 \\
100 \\
100 \\
100\end{array}$ & : & 10 \\
\hline
\end{tabular}

a)-146 a)/aths

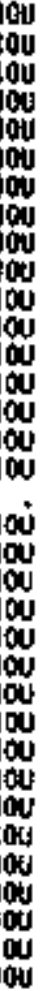




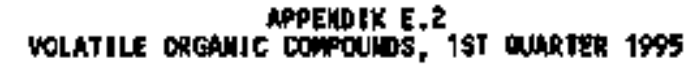

SNeltitc POINT Lochtion

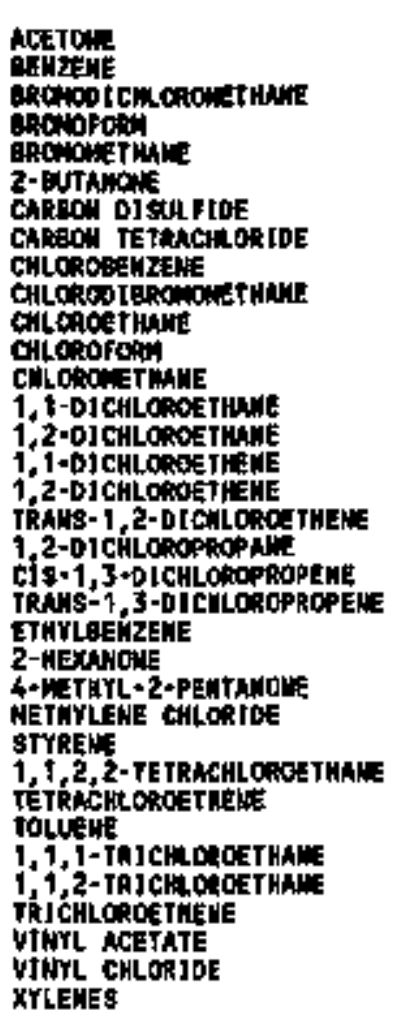

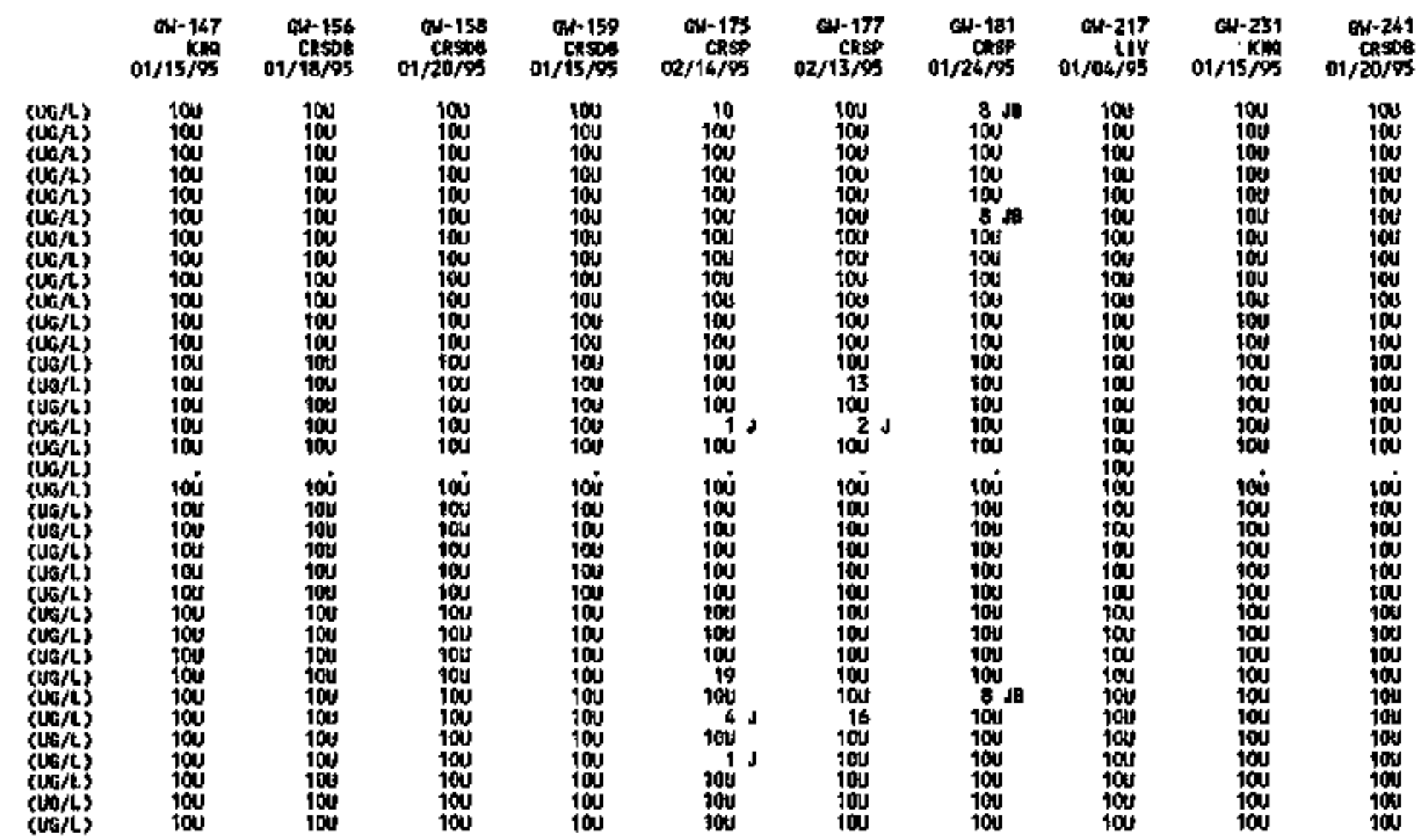




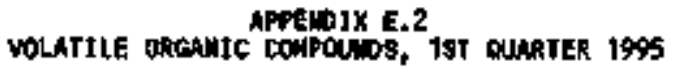

snpr InG pos IIT

Lockt tow

DATE SANPLED

ACETONE

BENREWE

BRCWOFORA

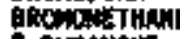

2-6urtinow

CAdigaw ot surfipe

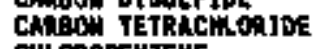

CHLOROSE EIIINE

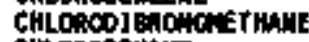

c.imonot thale

chichorofont

CHLondict)

1. 1-6TCHLONGETHANE

1,2-OJCHLONOETHANE

1,1-OICHLOCOETHENE

riris+1 2-ptorionoethele

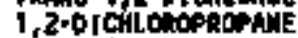

CIS-1,3-OICFLOHOPROPENE

TWAUS-1,3-DICHLOKOPROPEES

ETMLEEM ZW

arianding

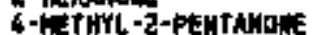

NETHYLENE Cintó IDE

STYRENE

$1,1,2,2$-TETRACHLOAOEthaye TEfRAC LOARETHENE

TOLLEAE

1, 1, 1-TRICHLOROETHAME

1,1,2-TRICHLOROE THAHE

TRICHLOROETHENE

VINYL ALETRTE

VInYL CHAOA to

XYLEWES
$5+304$

o1/ $15 / 65$

100
100
100
100
100
100
100
100
100
100
100
100
100
100
100
100
100
100
100
100
100
100
100
100
104
100
100
100
100
100
100
100
100 $\operatorname{anc} 305$
ity
$01,00 \% 5$

$104-321$
$0170 / 55$

CH-511

(Uor)

(Ua/L)

(UG/

(ua)

cust

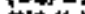

(wt)

(casc

(1)

(tor

(Ut)

(uch

(uts)

(tans

surit

(ut 7 )

(WG/L)

(⿻上丨𣥂巾

(uof

(WW/

uto

(Uayl)

(UE)

(U)

vat

(vo/L)

\begin{tabular}{|c|c|}
\hline $\begin{array}{r}\text { Gu-521 } \\
\text { LIV } \\
\text { ol porss }\end{array}$ & $\begin{array}{r}\text { cu-522 } \\
\text { ltV } \\
01 / 10 / 95\end{array}$ \\
\hline $\begin{array}{l}100 \\
100 \\
100 \\
100 \\
100 \\
100 \\
100 \\
100 \\
100 \\
100 \\
100 \\
100 \\
100 \\
100 \\
100 \\
100 \\
104 \\
100 \\
100 \\
100 \\
100 \\
100 \\
100 \\
100 \\
100 \\
104 \\
100 \\
100 \\
100 \\
100 \\
100 \\
100 \\
100 \\
100 \\
100\end{array}$ & 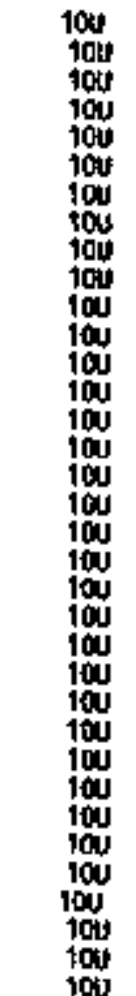 \\
\hline
\end{tabular}

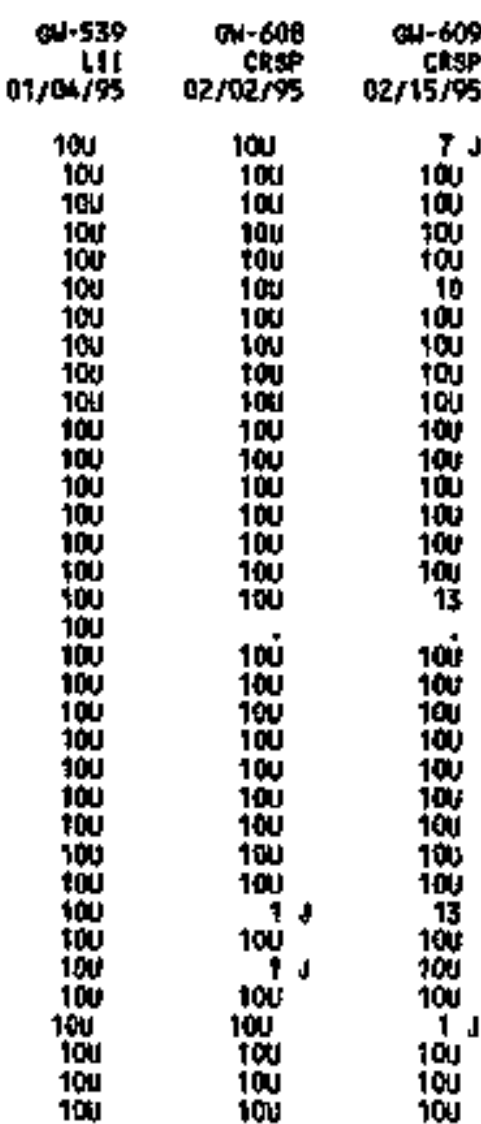


SHipling POIHT LOCATIOH

OATE SAIPLCOO

Actront

OENOZWE

enciofoph

GRQHOFTHANE

2-BuTAMG'S

CAREOH OISULF JOE

Calobooteghowometmane

cincon

chromertunt

$1,1-0$ ICHLoRotinat:

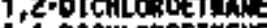

1,1 -

TAHS-1, 2-0JC) ChOETHE

1,2-DICHLOROPROPAME

CI8-1,3-DJCHLOROPROPEWE

GTHFin: the

-HEXAYA

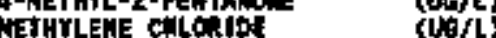

NTHYLE CHLORIDS

$1,1,2,2$-TETRACHLOROETHANE TETRACTLOAOETHENE TOLUEHE

1,1, 1-TRICHLAOETnum

1,1,2-TRICHL OROETRANE

THCHLOROETWEN

VTWYL ACETRTE

VIWYL CHLORIOE

MYLENES

(Worl)

(ligh

(ung

un $/$,

(L) L L )

(ING/L)

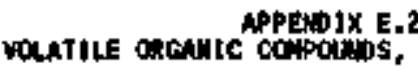

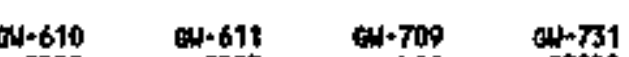
$01 / 31 / 95$

constios

$6+709$

cil- 731

61. 72

014-742

ain:743

a 4075

$01 / 09 / 95$

SCk2.2sp

100 10

$104 \quad 10$

100

100

100

100

100

100

100

100

100

100

100

100

100

100

100

$10 \mathrm{~J}$

100

(t)

(us/t?
$01 / 27 / \%$

100

100
100
10u

10u

105

$30 \mathrm{~s}$

tous

100

tou

100

100

100

100

100

100

104

100

100

100

100

100

100

100

100
100
100
100
104
100
100
100
100
100
100
100
100
100
100
100
100
100
100
100
100
100
100
100
100
100
100
100
100
100
100
100
100
100
100

100
100
100
100
100
100
100
100
100
100
100
100
104
100
104
100
100
100
100
100
100
100
100
100
100
100
100
100
100
100
100
100
100
100
100

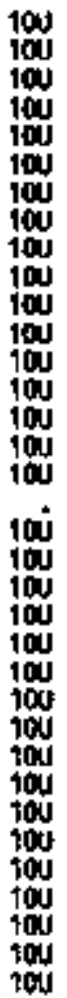




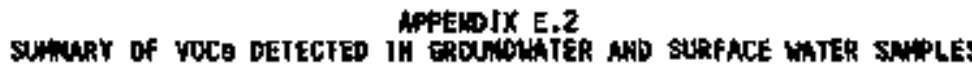

Total sonoles

\begin{tabular}{|c|c|c|}
\hline achas & & $\begin{array}{r}\text { stcown } \\
\text { outiter }\end{array}$ \\
\hline 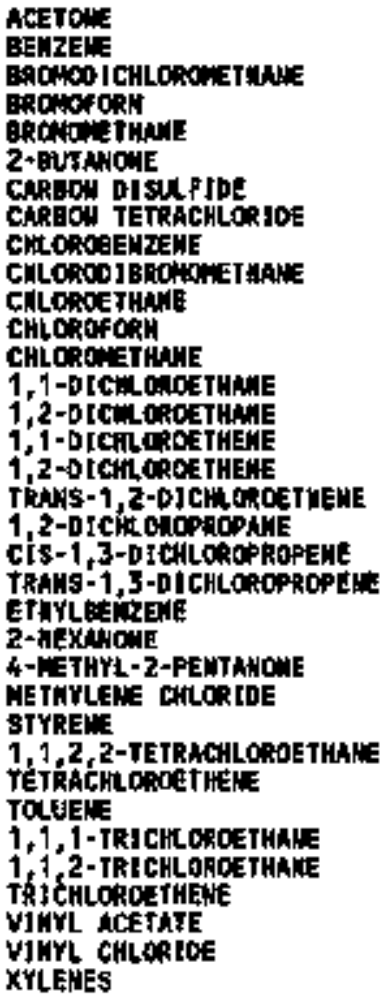 & 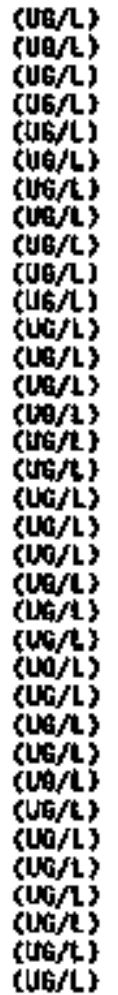 & $\begin{array}{l}5 \\
0 \\
0 \\
0 \\
0 \\
0 \\
0 \\
1 \\
0 \\
0 \\
0 \\
1 \\
0 \\
1 \\
0 \\
1 \\
1 \\
0 \\
0 \\
0 \\
0 \\
0 \\
0 \\
0 \\
6 \\
0 \\
0 \\
2 \\
0 \\
4 \\
0 \\
1 \\
0 \\
0 \\
0\end{array}$ \\
\hline
\end{tabular}


APPgibtx E.2

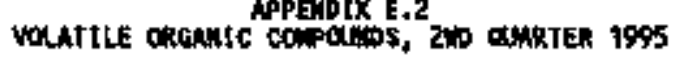

SHALING PotmT LOCATIn

\section{ACETONE}

ECNIZE⿰冫士口E iROWOFOFit

ekginitint

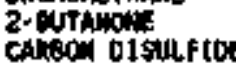

CARed O1sulf toe

CHLOROO IBROMONETHAHE

cHLokOET ThL

CHLOAOFOR.

CHLORONETHAME

$1,2+$ DICHLORDETHAHE

1, 1-DICHLOROETHEHE

TkAts-1 2-DTCHLOtoetweng

1, 2-DICFLOSOPOOPHIF

CIS-1,3-DICHLOROPLOPENE TRANS+1, 3-0 CNLOROPROPEINE

eThruether

4-METHYL-2-PEMTANONE

4-METNYL-2-PENTAiom

arthe CHLORTO

$1,1,2,2$-TETRaphlowoetride TEFHCHLOROETMEN

1,1,1-TR ICNLOROETHAIE

1, 2-TRICHLOROETHAE

The

VIWYL ACETATE

XrLEMES
1090 o4/20/95

cos-1

$04 / 17 /$ 노

$0 N-142$
KHa
$04 / 20 / 95$

ch-143

$06 / 24 / 95$

6N-144

6i- 145

KHO

cor-146

$04 / 24 / 95$

Can-147 $4 / 21 / 95$

c1.15t

$\mathrm{HW}+158$

(UB:L)

(Uof $/$ )

$10 y$

100
100
100
100
100
100
100
100
100
100
100
100
100
100
100
100
100
100
100
100
100
100
100
100
100
100
100
100
100
100
100
100

$\begin{array}{ll}100 & 100 \\ 100 & 100 \\ 100 & 100 \\ 100 & 100 \\ 100 & 100 \\ 100 & 100 \\ 100 & 100 \\ 100 & 100 \\ 100 & 100 \\ 100 & 100 \\ 100 & 100 \\ 100 & 100 \\ 100 & 100 \\ 100 & 100 \\ 100 & 100 \\ 100 & 100 \\ 100 & 100 \\ 100 & 100 \\ 100 & 100 \\ 100 & 100 \\ 100 & 100 \\ 100 & 100 \\ 100 & 100 \\ 100 & 100 \\ 100 & 100 \\ 100 & 100 \\ 100 & 100 \\ 100 & 100 \\ 100 & 100 \\ 100 & 100 \\ 100 & 100 \\ 100 & 100\end{array}$

100
100
104
100
100
100
100
100
100
100
100
100
100
100
100
100
100
100
100
100
100
100
100
100
100
100
100
100
100
100
100
100
100
100

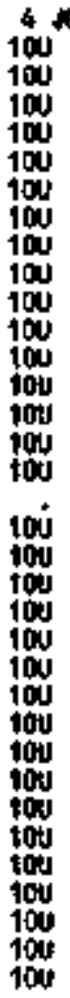

100
100
100
100
100
100
100
100
100
100
100
100
100
100
100
100
100
100
100
100
100
100
104
100
100
100
100
100
100
100
100

100
100
100
100
100
100
100
100
100
100
100
100
100
104
100
100
100
100
100
100
100
100
100
104
100
100
100
100
704
100
100
100

100
100
100
100
100
100
100
100
100
100
104
100
104
100
100
100
100
100
100
100
100
100
100
104
104
100
100
100
100
100
100
100
100
100 


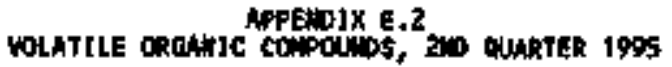

\$HPLING Fotat DATE SNAt Low

\section{ACEFow}

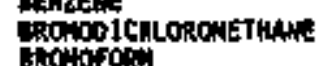

BROWOWETWANE

2+6UTMOHE

CARBOW OISUL F TDE

GARBON YETRACHLORIDE

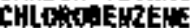

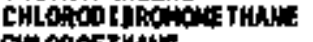

CHeOROE THA

choporoin

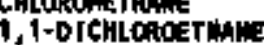

1,2+0 ICHLOROETHA

1, 1 DCH AROETHE

TRALS-1,2-D1CHLOROETHENE

f, 2-DJCILOROPROPNE

C.15-1,3-DICHLONOPHAPCDE.

TRAMS-1, 5-DTCHLOROPROPEWE

ETHYLLEMZEF

4-METUVL-2-PEUt AYowE

HETHYe ENE CHLORTOE

ST MREFE

$1,1,2, z+$ TEIRACHLOROE THane CTRACHLOROETHEUE

1,1,j-TRTCHLOROETHAKE

1,1,2-TRTCHLORFETHAN

TŔCHLORUETHENE

TWYL ACE TATE

XYLEMES

\begin{tabular}{|c|c|c|}
\hline $\begin{array}{r}\text { cu-159 } \\
\text { CRspos } \\
04 / 05 / 95\end{array}$ & $\begin{array}{r}\text { cw-160 } \\
\text { Crestup } \\
04 / 26 / \% 5\end{array}$ & $\begin{array}{r}64-161 \\
\text { CRRAM } \\
04 / 20 / 9\end{array}$ \\
\hline $\begin{array}{l}100 \\
100 \\
100 \\
100 \\
100 \\
100 \\
100 \\
100 \\
100 \\
100 \\
100 \\
100 \\
100 \\
100 \\
100 \\
100 \\
100\end{array}$ & $\begin{array}{l}100 \\
100 \\
100 \\
100 \\
100 \\
100 \\
100 \\
100 \\
100 \\
100 \\
100 \\
100 \\
100 \\
100 \\
100 \\
100 \\
100\end{array}$ & $\begin{array}{l}100 \\
100 \\
100 \\
100 \\
100 \\
100 \\
100 \\
100 \\
100 \\
100 \\
100 \\
100 \\
100 \\
100 \\
100 \\
104 \\
100\end{array}$ \\
\hline $\begin{array}{l}100 \\
100 \\
100 \\
100 \\
100 \\
100 \\
100 \\
100 \\
100 \\
100 \\
100 \\
100 \\
100 \\
100 \\
100 \\
100 \\
100\end{array}$ & $\begin{array}{l}104 \\
104 \\
104 \\
100 \\
100 \\
100 \\
104 \\
104 \\
104 \\
1004 \\
100 \\
100 \\
100 \\
100 \\
100 \\
100 \\
104\end{array}$ & $\begin{array}{l}100 \\
100 \\
100 \\
100 \\
100 \\
100 \\
100 \\
100 \\
100 \\
100 \\
100 \\
100 \\
100 \\
100 \\
100 \\
100 \\
100\end{array}$ \\
\hline
\end{tabular}

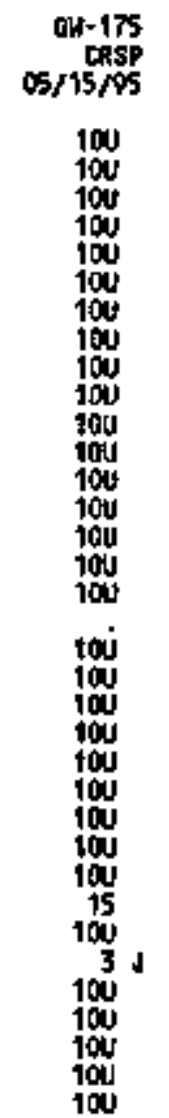

AN-177
cRsp
$05 / 11 / 55$

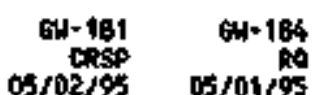

$6 \pi-186$

$05 / 03 / 05$

i. 167

$05 / 02 / 95$

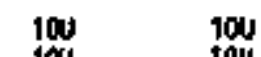

100

(Uo/L)

(us $(\mathrm{s})$

duar

(utift)

(Ut)

(Uin)

(Uavi)

(ugh)

(Uos)

(UGG/L)

(U⿺辶)

(U6)

(Uoll

(115/L)

(166/L)

(utorli

Gof

(ung/L)

(UG/L)

(utor)

(UTI)

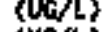

(U⿺辶)

(U⿺辶)

(U⿺辶)

(UG/)

(NG/)

(vo/k)

100
100
104
100
100
100
100
100
100
104
100
100
104
100
100
104
100
100
100
100
100
100
100
100
100
100
100
104
100
100
100
104
104
100

100
100
100
100
100
100
100
100
100
100
104
100
100
100
100
100
100
100
100
100
100
100
100
100
100
100
100
100
100
100
100
100
100
100

100
100
100
100
100
100
100
100
100
100
100
100
100
100
100
100
100
100
100
100
100
100
100
100
100
100
100
100
100
100
100
100
100
100

GH- 18

$05 / 01 / \%$

100
100
100
100
100
100
100
100
100
100
100
100
100
100
100
100
100
100
100
100
100
100
100
100
100
100
100
100
100
100
100 
APPELIX 4.2

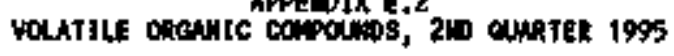

SUMPING POINT

LOFATION

atie sintete

ACETOine

BROM⿻上丨 ICHLOROWETHAYE

Btowofory

BROMNEJHA:

2+chtrinow

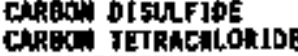

CHR JETRACILONIDE

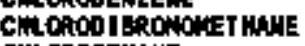

CHLOROETHANE

CHLOROFO丶 in

ciftine

1, 1-OICALOROETHAME

, 2-OICHLOROE THANE

1.DCHLOROCIHE-

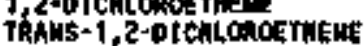

1,2-0]ChLOROPROP WhE

TRAYS-1, 3-DICHLONOPHOPENE

CTivechere

4- HETAYL+2 4 PEUTANONE

M*A.THYL - CHENTMONE

STYREAE

t, 1,2, 2-TETRACHLOROE THANE

TETRACHLOROETHEW

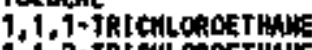

1, 2+TR CHLOROETHAN

TR JCHEOROSTHENE

VIHYL CHLOR TOE

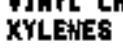

$0 \mathrm{n}+203 \quad \mathrm{EN}+205 \quad \mathrm{WH}-221$

Uanes uncs

10416/95

o. 19/95

64-231

(1)

an-261

CRSDB

Ou-292
Echap
$04 / 24 / 95$

100
100
100
100
100
100
104
100
100
100
100
100
100
100
100
100
100
100
100
100
100
100
100
100
100
100
100
100
100
100
100

$\begin{array}{ll}100 & 100 \\ 100 & 100 \\ 100 & 100 \\ 100 & 100 \\ 100 & 100 \\ 100 & 100 \\ 100 & 100 \\ 100 & 100 \\ 100 & 100 \\ 100 & 100 \\ 100 & 100 \\ 100 & 100 \\ 100 & 100 \\ 100 & 100 \\ 100 & 100 \\ 100 & 100 \\ 100 & 100 \\ 100 & 100 \\ 100 & 100 \\ 100 & 100 \\ 100 & 100 \\ 100 & 100 \\ 100 & 100 \\ 100 & 100 \\ 100 & 100 \\ 100 & 100 \\ 100 & 100 \\ 100 & 100 \\ 100 & 100 \\ 100 & 100 \\ 100 & 100 \\ 100 & 100 \\ 100 & 100\end{array}$

105
100
100
100
100
100
100
100
100
100
100
100
100
100
100
100
100
100
100
104
100
1004
100
100
100
100
100
100
100
100
100
100
104
ㅅ‥203

Eckp

$123 / 95$

100
100
100
100
100
100
100
100
100
100
100
100
100
100
100
100
100
105
100
100
100
100
100
100
100
100
100
100
100
100
100
100
100
100
0 at-296

ECIL"

$5 \mathrm{~T}+29$

os chashos

6u-296

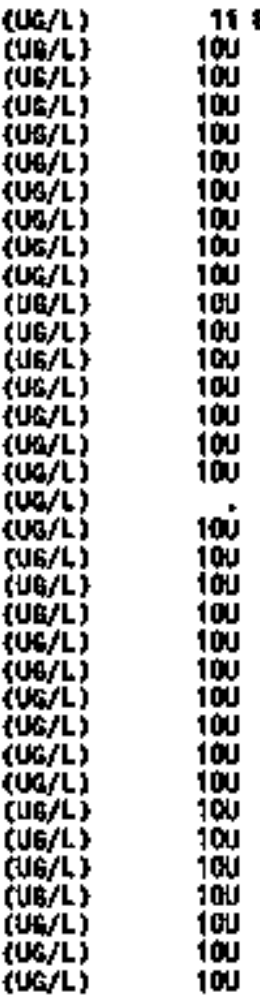

15
100
100
100
100
100
100
100
100
100
100
100
100
100
100
100
100
100
100
100
100
100
100
100
100
100
100
100
100
100
100

100
100
100
100
100
100
1000
100
100
100
100
100
100
100
100
100
1000
100
100
1000
100
100
100
100
100
100
100
100
100 OCDAF

100
100
100
100
100
100
100
100
100
100
100
100
100
100
100
100
100
100
100
100
100
100
100
100
100
100
100
100
100




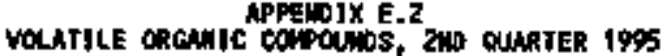

SAMLLJMO POINT

LOCAT JON

DATE sMPLED

Acrion:

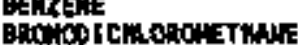

Biphoschat

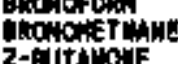

Chapol DIsulf IOE

CArow TETBCHLA

Che r Th

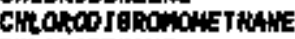

CHLOROETHAYE

CllOROFOCA

CHLOROLETHANE

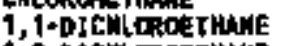

,2-btchtorostmint

1-Dichosotrine

tRAirs-1,2-DJCHLOROE THENE

1, 2-DIC LTEROPROPNIE

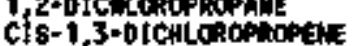

TRMS: 9 3-DICHLORSOROVENE ETHYdoeszan

2-rexthane

4-KETHTL - 2-PENTAMUE

MTHYLENE CHLORIOE

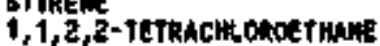
TETRACLODOE THENE

TOLtiele

1, $1,1-T R$ ICHLOHOETMUVE 1,1,2-TR LCHLChOETHAIN

R. CHLORDETHER'

INYL ACETHE

XYLEHES

\begin{tabular}{|c|c|c|c|}
\hline 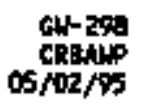 & $\begin{array}{r}\text { cel-zos } \\
\text { crputp } \\
\text { os roi/ss }\end{array}$ & $\begin{array}{r}\text { cu-30o } \\
\text { Crintp } \\
\text { OA/27/9s }\end{array}$ & $\begin{array}{c}\text { CH+30 } \\
\text { CRBAH } \\
04 / 27 / 9\end{array}$ \\
\hline $\begin{array}{l}105 \\
100 \\
100 \\
100 \\
100 \\
100 \\
100 \\
100 \\
100 \\
100 \\
100 \\
100 \\
100 \\
100 \\
100 \\
100 \\
100 \\
100\end{array}$ & $\begin{array}{l}10 y \\
100 \\
100 \\
100 \\
100 \\
100 \\
100 \\
100 \\
100 \\
104 \\
100 \\
104 \\
104 \\
100 \\
100 \\
100 \\
100 \\
100\end{array}$ & $\begin{array}{l}100 \\
100 \\
100 \\
100 \\
100 \\
100 \\
100 \\
100 \\
104 \\
100 \\
100 \\
100 \\
100 \\
100 \\
100 \\
100 \\
100 \\
100 \\
100\end{array}$ & 101 \\
\hline $\begin{array}{l}100 \\
100 \\
100 \\
100 \\
100 \\
100 \\
100 \\
100 \\
100 \\
100 \\
100 \\
100 \\
100 \\
100 \\
100 \\
100 \\
100\end{array}$ & $\begin{array}{l}100 \\
100 \\
100 \\
100 \\
104 \\
100 \\
100 \\
100 \\
100 \\
100 \\
100 \\
100 \\
100 \\
100 \\
100 \\
100 \\
100\end{array}$ & $\begin{array}{l}100 \\
100 \\
100 \\
100 \\
100 \\
100 \\
100 \\
100 \\
100 \\
100 \\
100 \\
100 \\
100 \\
100 \\
100 \\
100 \\
100 \\
100\end{array}$ & $\begin{array}{l}1 \\
1 \\
1 \\
1\end{array}$ \\
\hline
\end{tabular}

64-302 101-303

uncs

Chats

$10 \mathrm{~N}$

100
100
100
100
100
100
100
100
100
100
100
100
100
100
100
100
100
100
100
100
100
100
100
100
100
100
100
100
100
100
100
100
100
100
100
100
100
100
100
100
100
100
100
100
100
100
100
100
100
100
100
100
100
100
100
100
100
100
100
100
100
100
100
100
100
100
100
CNA +304 04/06/95

100
100
100
100
100
100
100
100
100
100
100
100
100
100
100
100
100
100
100
100
100
100
100
100
100
100
100
100
100
100

6.1-32? $04 / 25 / 5$

100
100
100
100
100
100
100
100
100
100
100
100
100
100
100
100
100
100
100
100
100
100
100
100
100
100
100
100
100
100
100
100
100

64-539 on towes

100

100

100

10

10

10.

iou

10

100

190

$\operatorname{lod}$

100

10

100

10i

100

100 cu-511 as $/ 03 / 75$

200
100
100
100
100
100
100
100
100
100
100
100
100
100
100
100
100
100
100
100
100
100
100
100
100
104
100
100
100
100
100
100
100 
Aperiolx 6.2

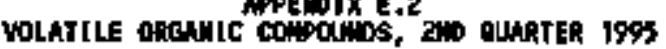

BALPLJME POIMT LOCAIIOH.

\section{ACETONE}

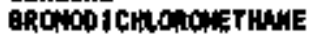

eRONofOAH

Baromintare

2-비새엘

Chktion oj sule io:

CARBO TETHCHL Dejot

CAlo

ctionoo tophint

chonogtin.

Cittorosonk

CNLOROMETMAE

1,1+0JCHLOROETHAHE

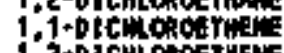

TRRUS-1,2-DICHLOROETMENE

1,2-DICHLOROPAOPAME

cts+1,3+0ICHLOOXPROPENE

ETHFLEEMREHE

2+ HEXMLONE

4-NETHYL-2-PERT AHOW

AETIrLefie chlonide

STruEME

1,1,2-TETRACHLOROETHAUE TETRACHLOROETHENE

TOLUE

$1,1,1$ - FAJ

1, 1, 2-TRJCLOAOE THAT

TK TCHLOAOETHL

VINTL ACETATE

VIAYL CALORIOE

XYLENES

\begin{tabular}{|c|c|c|}
\hline $\begin{array}{r}a .560 \\
\cot 1] \\
04 / 05 / \% 5\end{array}$ & $\begin{array}{r}\text { ou-562 } \\
\text { cDtvIl } \\
\text { ontosros }\end{array}$ & $\begin{array}{r}\text { CW-564 } \\
\text { COLIV] } \\
04 / 66 / 93\end{array}$ \\
\hline $\begin{array}{l}100 \\
100 \\
100 \\
100 \\
100 \\
100 \\
100 \\
100 \\
100 \\
100 \\
100 \\
100 \\
100 \\
100 \\
100 \\
100 \\
100 \\
100 \\
100 \\
100 \\
100 \\
100 \\
100 \\
100 \\
100 \\
100 \\
100 \\
100 \\
100 \\
100 \\
100 \\
100 \\
100\end{array}$ & $\begin{array}{l}100 \\
100 \\
100 \\
100 \\
100 \\
100 \\
100 \\
300 \\
100 \\
100 \\
000 \\
100 \\
100 \\
100 \\
100 \\
100 \\
100 \\
100 \\
100 \\
100 \\
100 \\
100 \\
100 \\
100 \\
100 \\
100 \\
100 \\
100 \\
100 \\
100 \\
100 \\
100 \\
100 \\
100\end{array}$ & 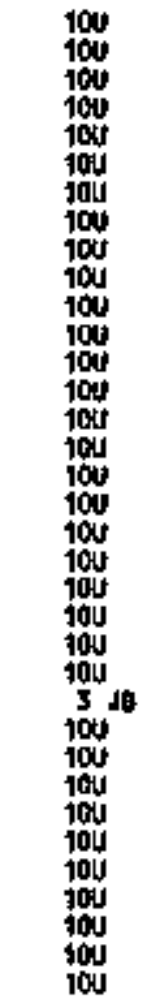 \\
\hline
\end{tabular}

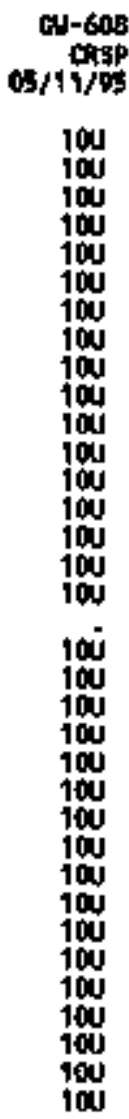

6i-609 os $116 / 5$

100
104
104
100
100
100
100
100
100
104
100
100
100
100
100
100
13
100
100
100
700
100
100
100
100
1001
10
100
100
100
$100 \mathrm{~J}$
100
100
100

Cir-610

cisp

\section{0}

100
100
100
100
100
100
100
100
100
100
100
100
100
100
100
100
100
100
100
100
104
100
100
100
100
100
100
100
100
100
100
100
100

(a)-611 $03 / 15 / 45$

100
100
100
100
100
100
100
100
100
100
100
100
100
100
100
100
100
100
100
100
100
100
100
100
100
100
100
100
100
100

4t) 71 $04 / 07 / 55$

保

100

100

tow

tov

100

100

100

I0U

100
100
100
100

300

$10+$

100

iov

$\operatorname{lox}_{100}$

100

16.

10.

100

10.

tod

iou

10u

\begin{tabular}{|c|c|}
\hline $\begin{array}{r}\mathrm{w}-732 \\
\mathrm{CRSpB} \\
\times / 07 / 95\end{array}$ & 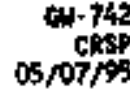 \\
\hline $\begin{array}{l}100 \\
100 \\
100 \\
100 \\
100 \\
100 \\
100 \\
100 \\
100 \\
100 \\
100 \\
100 \\
100 \\
100 \\
100 \\
100 \\
100\end{array}$ & $\begin{array}{l}100 \\
100 \\
100 \\
100 \\
100 \\
100 \\
100 \\
100 \\
100 \\
100 \\
100 \\
100 \\
100 \\
100 \\
100 \\
100 \\
100 \\
100\end{array}$ \\
\hline $\begin{array}{l}100 \\
100 \\
100 \\
100 \\
100 \\
100 \\
100 \\
100 \\
100 \\
100 \\
104 \\
104 \\
100 \\
100 \\
100 \\
100 \\
100\end{array}$ & $\begin{array}{l}100 \\
100 \\
100 \\
100 \\
100 \\
100 \\
100 \\
900 \\
100 \\
100 \\
100 \\
100 \\
100 \\
100 \\
100 \\
100 \\
100\end{array}$ \\
\hline
\end{tabular}




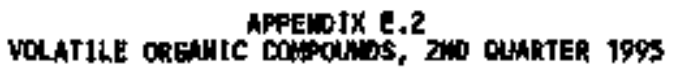

anph IMt POLthy

LOCRT10

Dhte satples

ACE TOWE

BENEEWE

BRoworoin

BRonometwaye

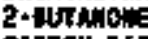

Choㅇ d

CRRBON TETRACHLOR IDE

CHOMOEEM 2 ENE

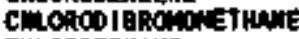

CHCOROETHAN

cherorgen

1,1 -b1ch dot

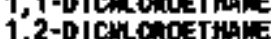

1, 1-bicuchotfinthe

ThNS-1, 2-OTCHLOROETHENE

1,2-0.CHLONOPROPAWE

C[8-1,3-0 ICHLOROPROPENE

ETHYLIEHZEME

2-KEXMNOHE

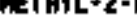

METHYLEW CHLORIDE

STTRENE

1,1,2,2-TETHACHLOROETHAME

TETRACHLOAOETHEHE

TOLEW

T,1, 1-TRITLLOROETHANE

$1,1,2$-TRl chlonoethane

ThtCDOROETHEHE

VIuYt CHLDAjoe

KrLEins ow-743
Chsp
$05 / 07 / 95$

$64-796$
$04 / 2 / 95$

6N-797

$04 / 11 / 95$

ar-79s
colvif
$0411 / 95$

ㅂill-799

$04 / 06 / 95$

다- 501 $04 / 13 / 65$

Q1) 827

(voll) 100

(No/)

(No/)

(ubn

(UOA)

(uif)

(uith)

(Uo/L)

(Uor)

(UOW/L)

(U6)

(ub/t)

(UC/L)

(UW/L)

(Wo/i?

(uorts

(USA)

(U6/L)

(UG/L)

(UG/L)

(UT/L)

(U6)

(Ut:L)

(UD) L

(Uer)

war

(tos)

tures

(LO/L)

(UtolL)

(Uoli)

(uole)

21
100
100
100
100
100
100
100
100
100
100
100
100
104
100
100
100
100
100
100
100
100
100
100
100
100
100
100
100
100
100
100
100

$\begin{array}{cc}100 & 100 \\ 100 & 100 \\ 100 & 100 \\ 100 & 100 \\ 100 & 100 \\ 100 & 100 \\ 100 & 100 \\ 100 & 100 \\ 100 & 100 \\ 100 & 100 \\ 100 & 100 \\ 100 & 100 \\ 100 & 100 \\ 100 & 100 \\ 100 & 100 \\ 100 & 100 \\ 100 & 100 \\ 100 & 100 \\ 100 & 100 \\ 100 & 100 \\ 100 & 100 \\ 100 & 100 \\ 100 & 100 \\ 100 & 100 \\ 100 & 3 \\ 100 & 100 \\ 100 & 100 \\ 100 & 100 \\ 100 & 100 \\ 100 & 100 \\ 100 & 100 \\ 100 & 100 \\ 100 & 100 \\ 100 & 100 \\ 100 & 100 \\ & \end{array}$

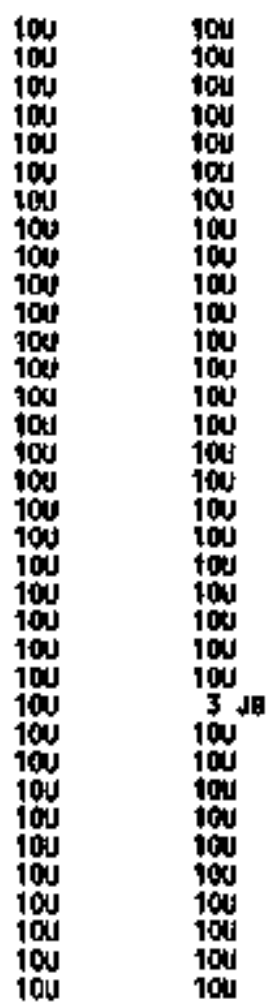




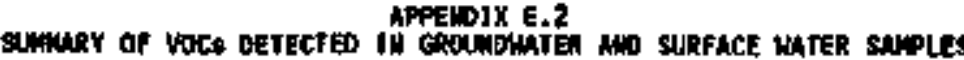

Total saniples

8uiplea without VOC.

Strples with Wack

34
27

\begin{tabular}{|c|c|c|}
\hline conoting & & THARTER \\
\hline 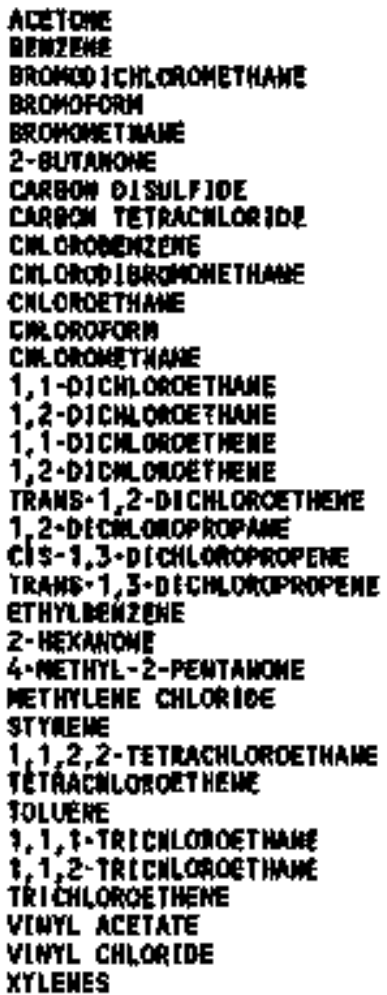 & 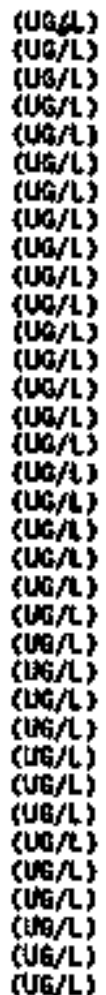 & $\begin{array}{l}0 \\
0 \\
0 \\
0 \\
0 \\
0 \\
0 \\
0 \\
0 \\
0 \\
0 \\
0 \\
0 \\
2 \\
0 \\
2 \\
1 \\
0 \\
0 \\
0 \\
0 \\
0 \\
0 \\
0 \\
0 \\
0 \\
0 \\
4 \\
0 \\
5 \\
0 \\
1 \\
0 \\
0 \\
0\end{array}$ \\
\hline
\end{tabular}




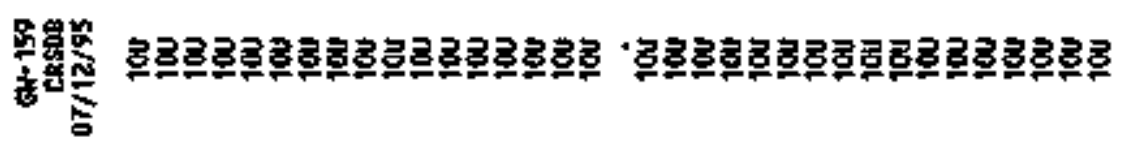

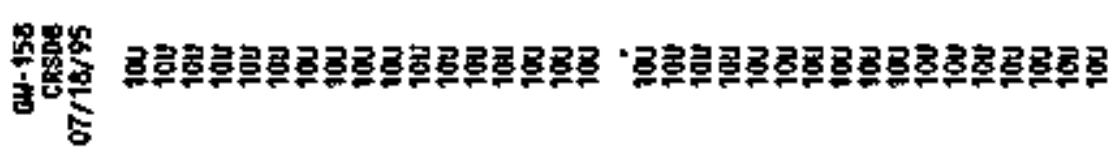

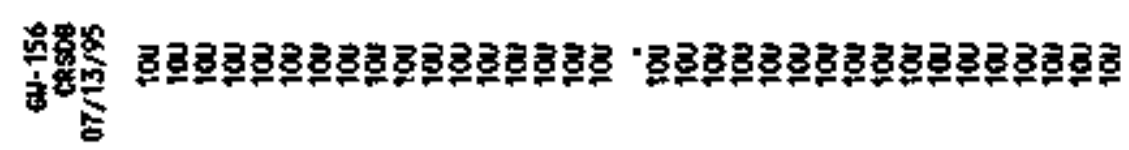

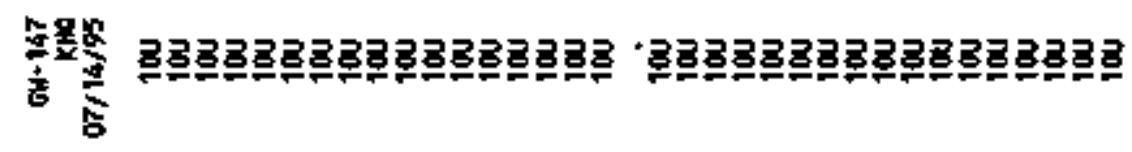

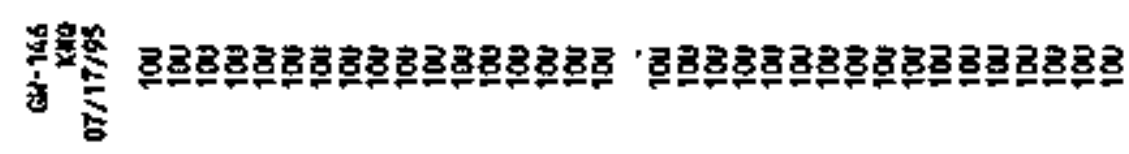

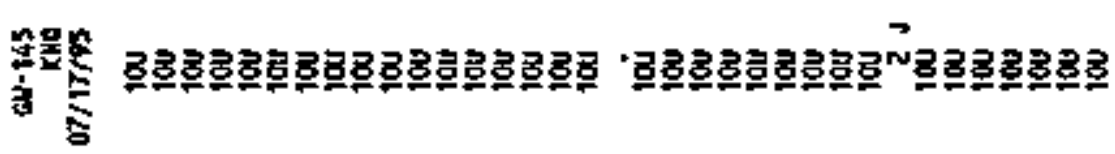

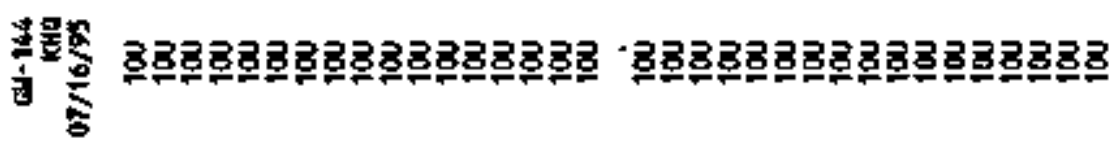

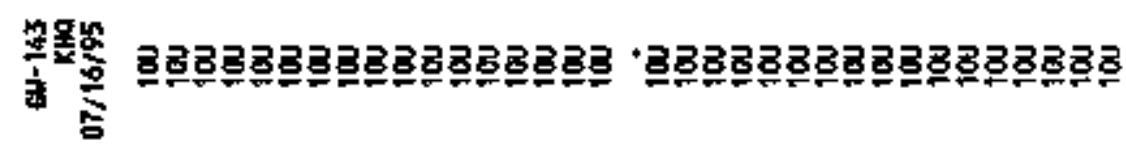

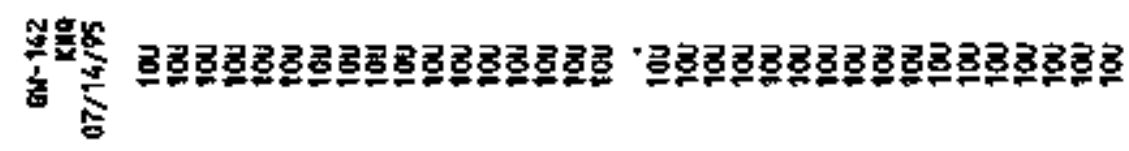

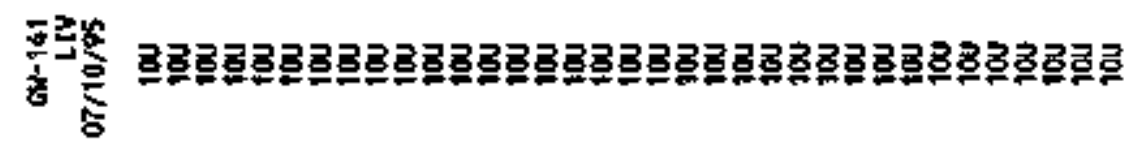

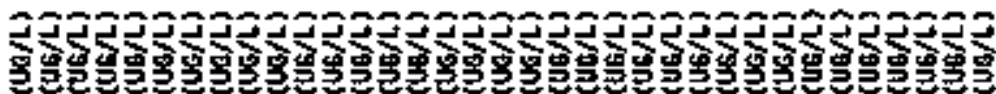

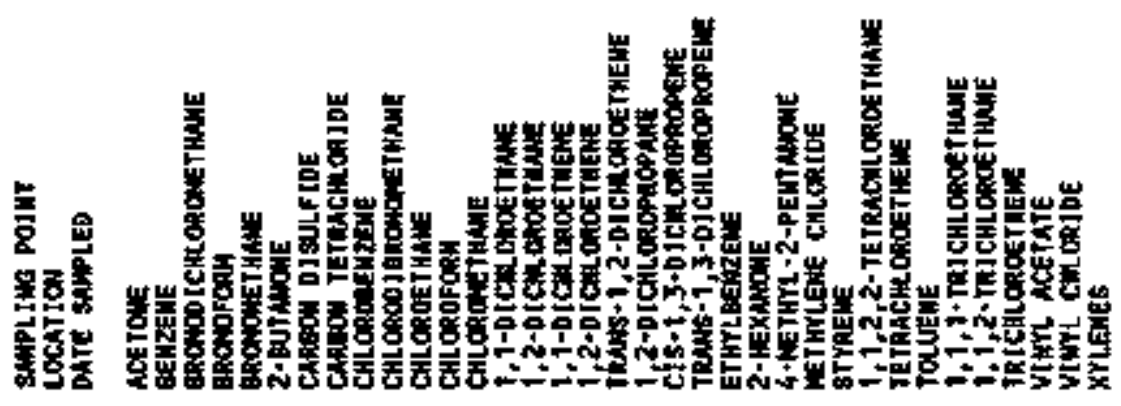




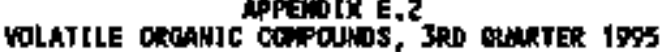

BAPLING FOLAT LochtIon

DATE SUIPLED

ACETON"

SROMOO I CNCAROME ThAn

chonofocin

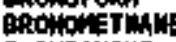

CNRBC. O1STLFIDE

CMAPON TETRAC:LORIDE

Colomogerretic

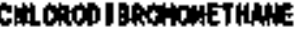

Chorost fink

Cingroropat

Gilonerthis

1,2-0JCHLOBOETHA

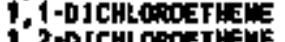

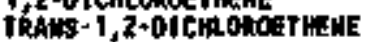

1,2-01 tik oaghopdin

t: $\$-1,3$-ptCHLOROPROPENE

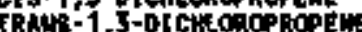

ETMYLEENZEN

2-HEXAlWois

4-AETHYL-2-PESt HONE

EETHLet: CHLORTOE

STYREIE

TÉTPRCHLOEOETHEHE

TOSTE

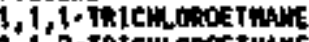

1, 1,2-TRTCHLORGethive

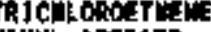

VHYY MCETATE

vinTL CHLosioe

XYLENES

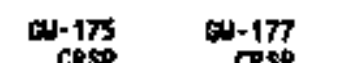

owoins

(Ua/t)

(U⿺/)

(UE/L)

ush

(us

(WoflL)

(US/L)

fwoll

(UG

(LOH/L

(un,

(W)

(L)/L

Lot

(ut/L

chant

(un

uor.

(UG,

$(x) / L$

(tin/t)

wers.

von?

(uon

$(w 0 / \mathrm{L})$

stagk

funf

(Uor)

(J) L

(uf/L)

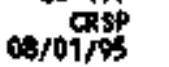

100

tou

100100

iou

100

树

10J $10 \mathrm{H}$

100 100

10u

100

100

100

100

10 i

100

10it 100

100

100

100

100

100

5

100

100

$10 \mathrm{v}$

100

u-181
catsp
$97 / 25 / 95$

जv-217

$07 / 10 / 95$

G.231

จ7/ $15 / 95$

Cu-241
CRsol
o7) $16 / 95$

nt-30s

or Cispos

a)-304

$0712 / \%$

civisy

4I-511

100100100

$100 \quad 100 \quad 100$

tots

100

id

$\operatorname{lox}_{10 .}$

10u

100

id

100

100

100

100

$\begin{array}{cc}100 & 100 \\ 100 & 100 \\ 100 & 100 \\ 100 & 100 \\ 100 & 100 \\ 100 & 100 \\ 100 & 100 \\ 100 & 100 \\ 100 & 100 \\ 100 & 100 \\ 100 & 100 \\ 100 & 100 \\ 100 & 100 \\ 100 & 100 \\ 100 & 104 \\ 100 & 100 \\ 100 & 100 \\ 100 & 100 \\ 100 & 100 \\ 100 & 100 \\ 100 & 100 \\ 100 & 100 \\ 100 & 100 \\ 100 & 100 \\ 100 & 100 \\ 100 & 100 \\ 100 & 100 \\ 100 & 100 \\ 100 & 100 \\ 100 & 100 \\ 100 & 100 \\ 100 & \end{array}$

100
100
100
100
100
100
100
100
100
104
100
100
100
100
100
100
100
100
100
100
100
100
100
100
100
100
100
100
100
100
100
100

100
100
100
100
100
100
100
100
100
100
100
100
100
100
100
100
100
100
104
104
100
100
100
101
100
104
100
100
104
104
100

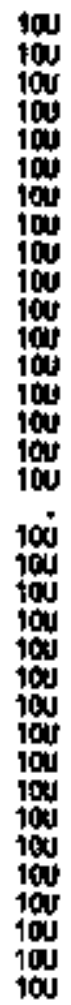

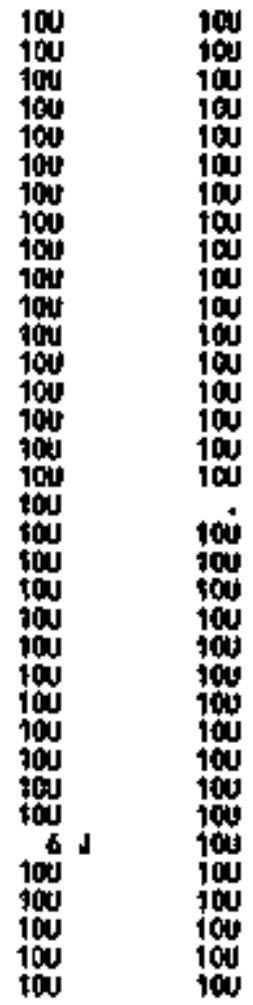




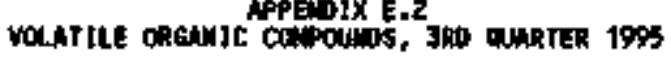

SAMPL, lH: POINT LOCAT TO.

oAte shipled

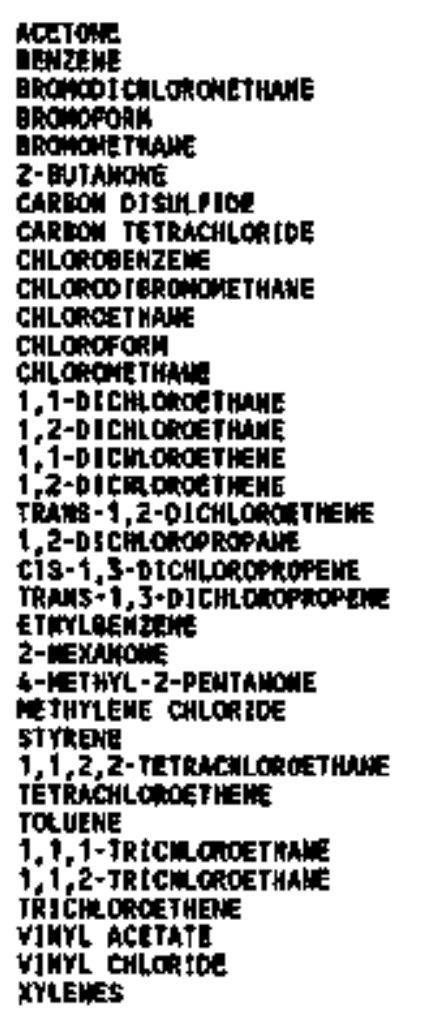

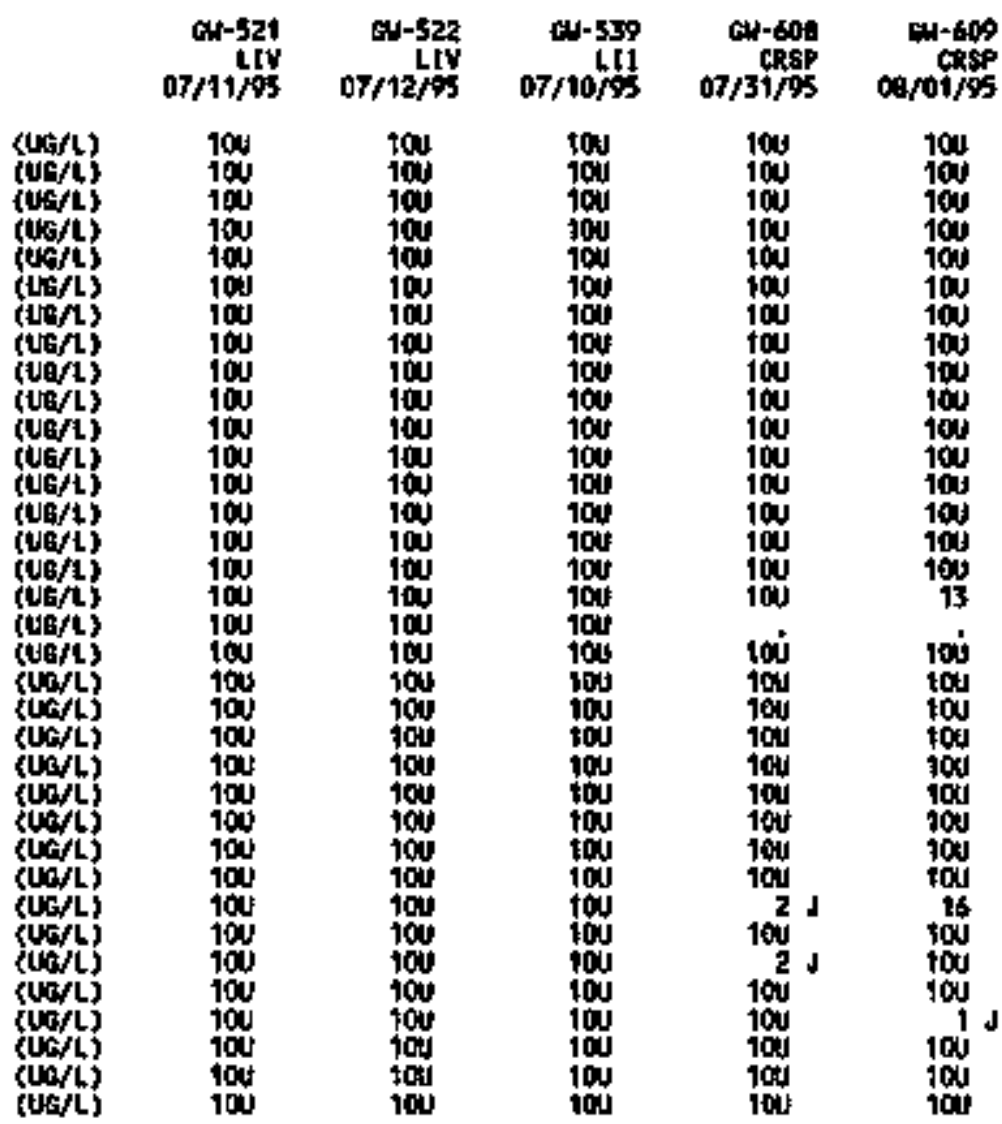

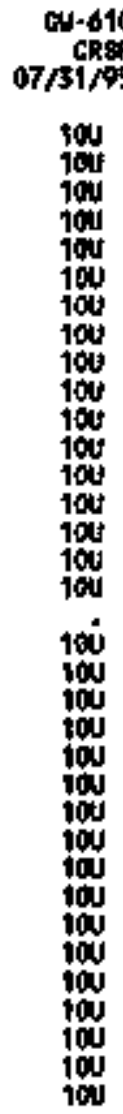

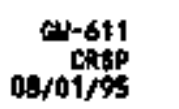

mo-709 $07 / 10 / 9$

100
100
100
100
100
100
100
100
100
100
100
100
2
100
100
100
100
100
100
100
100
100
100
100
100
100
100
100
100

0it - 731 07/13/3095

100
100
100
100
100
100
100
100
100
100
100
100
100
100
104
100
100
100
100
100
104
100
100
100
100
100
100
100
100




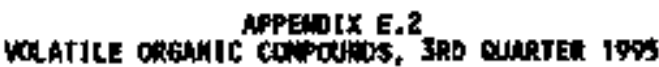

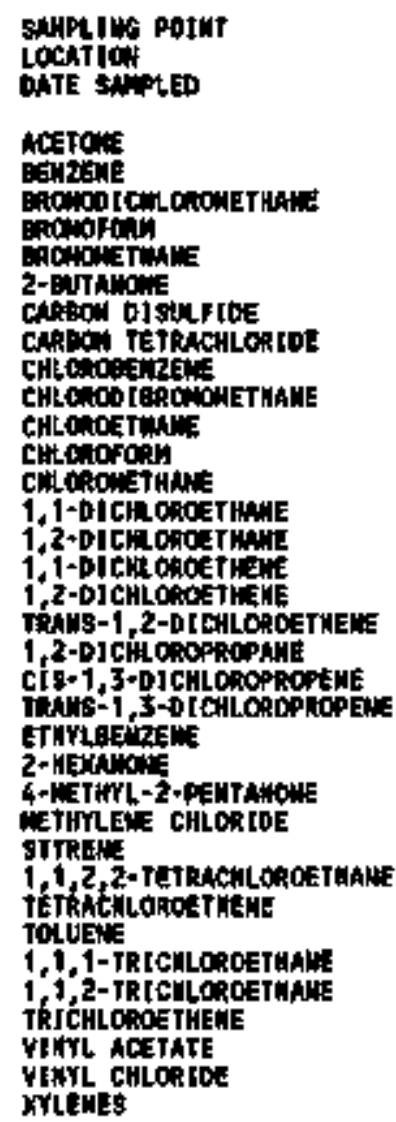

\begin{tabular}{|c|c|c|c|}
\hline & $\begin{array}{r}\text { cysz } \\
\text { chases } \\
07 / 13 / 45\end{array}$ & $\begin{array}{r}0 \mathrm{y}-732 \\
\mathrm{cRs00} \\
\text { ot/14/45 }\end{array}$ & $\begin{array}{r}\text { cw-76 } \\
\text { cins } \\
07 / 30 / 9\end{array}$ \\
\hline$(\mathrm{N}) / \mathrm{L})$ & 100 & 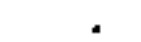 & \\
\hline (U) $/ L)$ & 100 & 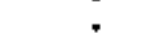 & 1 \\
\hline (UF/L) & 100 & * & 100 \\
\hline $\begin{array}{l}\text { (UtG/L) } \\
\text { (UG }\end{array}$ & 100 & $=$ & 100 \\
\hline (UB/L) & 100 & ; & 100 \\
\hline (una/L) & 104 & . & 100 \\
\hline$(\mathrm{w} / \mathrm{c})$ & 100 & 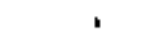 & 100 \\
\hline$(4 \mathrm{~d} / \mathrm{L}, \mathrm{s}$ & 100 & * & 100 \\
\hline (Uth/L) & tow & : & 100 \\
\hline (UQ/L) & 100 & . & $10 \mathrm{~J}$ \\
\hline (US/L) & $10 y$ & $\bullet$ & 100 \\
\hline (US/L) & 100 & - & 100 \\
\hline (1) & 104 & 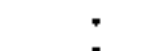 & $\operatorname{loy}_{100}$ \\
\hline$(0, / 5)$ & ito & 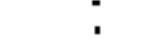 & 100 \\
\hline (US/L) & 107 & • & 100 \\
\hline (dus/l) & 100 & . & tou \\
\hline (1)/4) & 100 & . & 100 \\
\hline$(1+5 / L)$ & 100 & • & 1004 \\
\hline (UtL) & tou & ๘ & iov \\
\hline (UG $/ L)$ & 100 & • & $10 \mathrm{~d}$ \\
\hline (Uo/t) & 100 & : & 10u \\
\hline$\left(U_{5} / \mathrm{l}\right.$ & 100 & . & 1at \\
\hline & 100 & . & 10 \\
\hline & 100 & • & 104 \\
\hline (I) & iou & . & 100 \\
\hline & a & . & tol \\
\hline & 1 & $\cdot$ & 1014 \\
\hline & 10 & . & \\
\hline
\end{tabular}

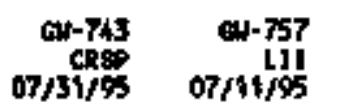

$\begin{array}{cc}100 & 100 \\ 100 & 100 \\ 100 & 100 \\ 100 & 100 \\ 100 & 100 \\ 100 & 100 \\ 100 & 100 \\ 100 & 100 \\ 100 & 100 \\ 100 & 100 \\ 100 & 100 \\ 100 & 100 \\ 104 & 100 \\ 104 & 100 \\ 100 & 100 \\ 100 & 100 \\ 100 & 100 \\ 100 & 100 \\ 100 & 100 \\ 100 & 100 \\ 100 & 100 \\ 100 & 100 \\ 100 & 100 \\ 100 & 100 \\ 100 & 100 \\ 100 & 100 \\ 100 & 100 \\ 100 & 100 \\ 100 & 100 \\ 100 & 100 \\ 100 & 100\end{array}$




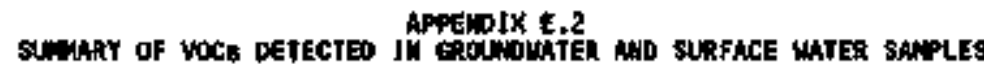

Total somples

tithout MoCs

$\mathbb{3}$

seinples with voc:

\begin{tabular}{|c|c|c|}
\hline Cowround & & $\begin{array}{l}\text { Fountu } \\
\text { ountex }\end{array}$ \\
\hline 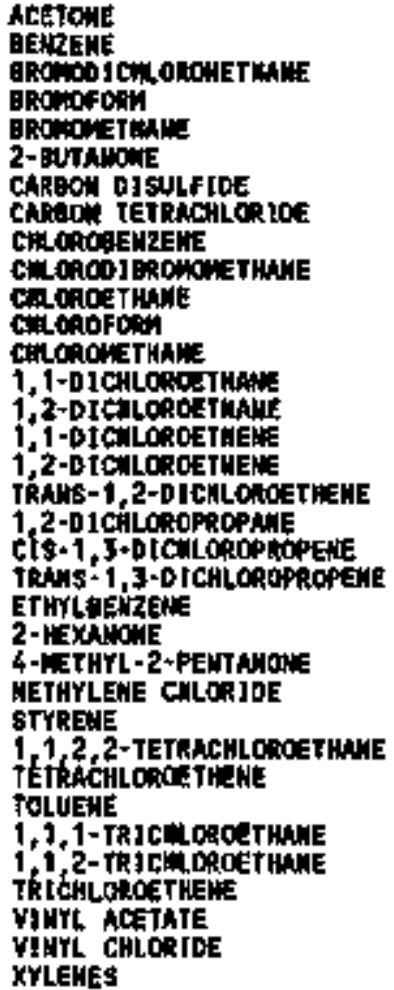 & 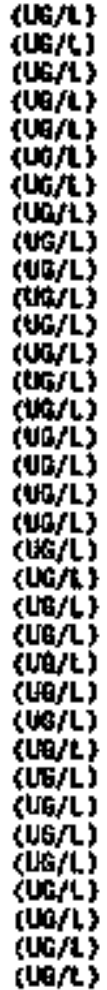 & $\begin{array}{l}1 \\
0 \\
0 \\
0 \\
0 \\
8 \\
0 \\
3 \\
0 \\
0 \\
0 \\
0 \\
0 \\
2 \\
0 \\
2 \\
1 \\
0 \\
0 \\
0 \\
0 \\
5 \\
0 \\
0 \\
2 \\
0 \\
0 \\
3 \\
0 \\
5 \\
0 \\
1 \\
0 \\
0 \\
5\end{array}$ \\
\hline
\end{tabular}


WOLATLE ORGAIC COHDOUX E.2

\begin{tabular}{|c|c|c|c|c|c|c|c|c|c|}
\hline $\begin{array}{l}\text { SAPLING POJNT } \\
\text { LOCATION } \\
\text { OATE SNMPED }\end{array}$ & & $\begin{array}{r}1090 \\
10 / 09 / 5 s \\
10 / 09 / 9\end{array}$ & $\begin{array}{r}\text { Cos-1 } \\
\text { Ly } \\
10 / 11 / 95\end{array}$ & $\begin{array}{r}w-142 \\
11 / 05 / 45\end{array}$ & $\begin{array}{r}6 N-143 \\
11 / 09 / 45\end{array}$ & $\begin{array}{r}a+144 \\
\mathbf{k H a} \\
11 / 14 / 95\end{array}$ & $\begin{array}{r}0 w-145 \\
\mathbf{k W 9} \\
11 / 15 / 95\end{array}$ & $\begin{array}{r}0 * 146 \\
11 / 08 / 95\end{array}$ & $\begin{array}{r}04-147 \\
1010 \\
11 / 06 / 95\end{array}$ \\
\hline 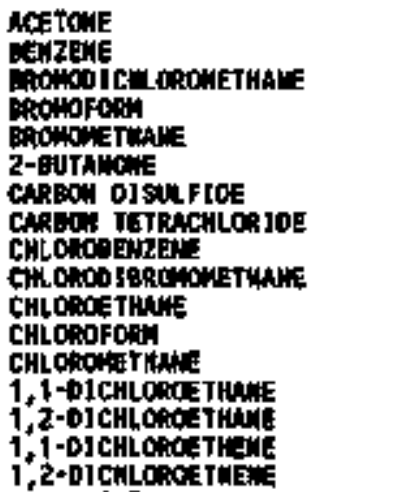 & 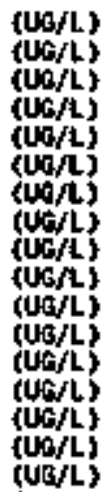 & o & $\begin{array}{l}100 \\
100 \\
100 \\
100 \\
100 \\
100 \\
100 \\
100 \\
100 \\
100 \\
100 \\
100 \\
100 \\
100\end{array}$ & $\begin{array}{l}i_{100} \\
100 \\
100 \\
100 \\
100 \\
100 \\
100 \\
100 \\
100 \\
100 \\
100 \\
100 \\
100 \\
100 \\
100\end{array}$ & $\begin{array}{l}100 \\
100 \\
100 \\
100 \\
100 \\
100 \\
100 \\
100 \\
100 \\
100 \\
100 \\
100 \\
100 \\
100 \\
100 \\
100 \\
100\end{array}$ & $\begin{array}{l}100 \\
100 \\
100 \\
100 \\
100 \\
100 \\
100 \\
100 \\
100 \\
100 \\
100 \\
100 \\
100 \\
100 \\
100\end{array}$ & $\begin{array}{l}100 \\
100 \\
100 \\
100 \\
100 \\
100 \\
100 \\
100 \\
100 \\
100 \\
100 \\
100 \\
100 \\
100 \\
100 \\
100\end{array}$ & 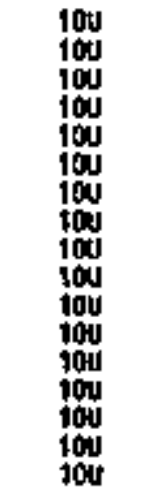 & 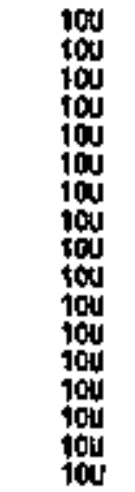 \\
\hline 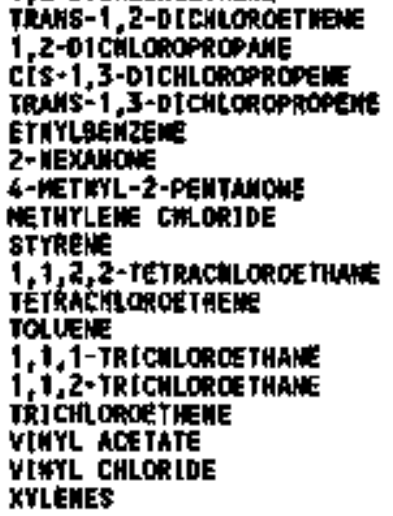 & 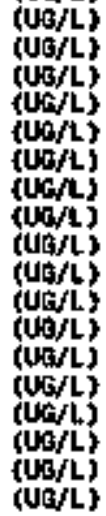 & 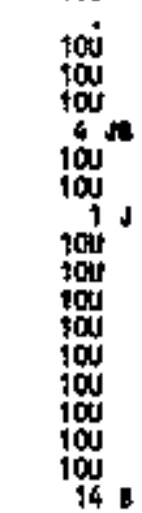 & $\operatorname{lox}_{100}$ & 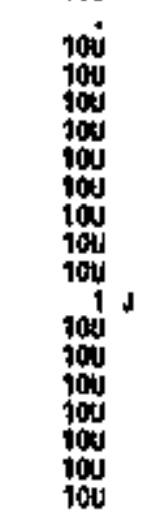 & $\begin{array}{l}100 \\
100 \\
100 \\
100 \\
100 \\
100 \\
100 \\
100 \\
100 \\
104 \\
104 \\
100 \\
100 \\
100 \\
100 \\
100 \\
100\end{array}$ & $\begin{array}{l}100 \\
100 \\
100 \\
100 \\
100 \\
100 \\
100 \\
100 \\
100 \\
100 \\
100 \\
100 \\
100 \\
100 \\
100 \\
100\end{array}$ & 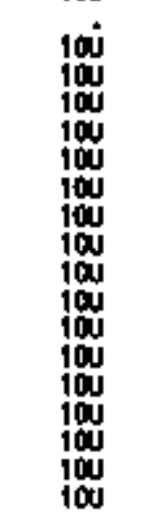 & $\begin{array}{l}100 \\
100 \\
100 \\
100 \\
100 \\
100 \\
100 \\
100 \\
100 \\
1004 \\
100 \\
100 \\
100 \\
100 \\
1000 \\
100 \\
100\end{array}$ & $\begin{array}{l}100 \\
100 \\
100 \\
100 \\
100 \\
100 \\
100 \\
100 \\
100 \\
100 \\
100 \\
100 \\
100 \\
100 \\
100 \\
100 \\
100\end{array}$ \\
\hline
\end{tabular}


APPGidX E.

VOLATILE OREMITC CEPONIDS, 4TM OUARTER 1995

SALPLING POIMT

cochtics

ACFTONE

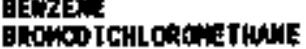

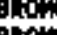

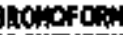

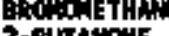

2-60imAinons

CM.

CMAPU TETRACHLORJDE

Clinowew

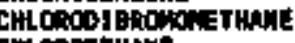

cincosozthay

Cincosofon

CHLOROMTHANE

1,1-0ICHLOAOETWAHE

,201c-lonoETHALE

1, -DichlonoerHe

ThA1 - 2 - 2-0ICHLOMOETHENE

$1,2-01$ chloropophte

CIS-1,3-DTCHLROPROPENE

Tluns-1,

crinterint

2-hexhow -2-PEntaing

- ring-2-Pemange

METHYLLEE CHEORIDE

STYREKE

$1,1,2,2$-TETRACILLOCOETHAHE

TET WACHLOROE TWEME

TOLYeNE

$1,1,1$-ThuCHLOnOETHAN

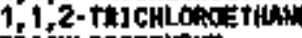

TRtchlolxOen his

Vint Arta

VIUKL CHLORIOE

xrleites
aW. 158

chisos

ㅅ․156

G-156

ail- 156

av-159 nospo $10 / 24 / 05$ tasts $10 / 25 / 95$
211-159 $10 / 25 / 95$

\section{$0+450$} 새요용 $10 / 26 / 5$

a.1. 160

(UG/L)

(JB/ $/$

(U6/ $/$ ?

$(4 \mathrm{H} / \mathrm{L}$

(U⿺辶)

(U⿴囗十)

(wa/

땐ㄱㄴ

(Ue/

(UG/

(U6/)

(409)

(tat

(tor

(U⿺辶⿸丆口

(U⿺辶)

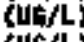

(Wish

(UG）

$(w 6 /)$

(ut/

(usc)

(wes

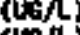

(당

$(150 /)$

(Uar)

(DLW/L)

(UDAㄴㄱ

(UWL

(uby

(uoy.

(ub $/$

(JA)

(UNA)

$\begin{array}{cc}100 & 100 \\ 100 & 100 \\ 100 & 100 \\ 100 & 100 \\ 100 & 100 \\ 100 & 100 \\ 100 & 100 \\ 100 & 100 \\ 100 & 100 \\ 100 & 100 \\ 100 & 100 \\ 100 & 100 \\ 100 & 100 \\ 100 & 100 \\ 100 & 100 \\ 100 & 100 \\ 100 & 100 \\ 100 & 100 \\ 100 & 100 \\ 100 & 100 \\ 100 & 100 \\ 100 & 100 \\ 100 & 100 \\ 100 & 100 \\ 100 & 100 \\ 100 & 100 \\ 100 & 100 \\ 100 & 100 \\ 100 & 100 \\ 100 & 100 \\ 100 & 100 \\ 100 & 100 \\ 100 & 100\end{array}$


ABpEDDIX E.?

WOLATILE OREAIC COPOOHDS, 4TH AMRTER 1995

SAPLING potint

LOCAT TOW

DATE SHAPLED

MCETONE

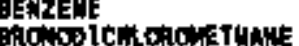

Hinnolch

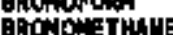

exaninginis

CURBOH OISULFLDE

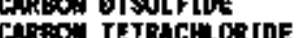

chtor

CHLOROD raponinethuse

CHLOROETHALE

culopofoin

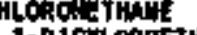

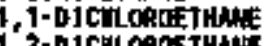

1-01Choc

i, $2+$ DICHLOAOE

ThANS-1,2-0ICHLOFOE TUENE

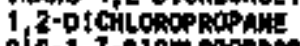

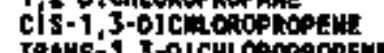

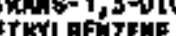

2.

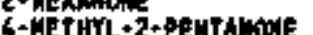

NeTHYLENE CHitondoE

STYRENE

$1,1,2$, 2-TETHACHLOROETHANE

TETHCNLOPOETHENE

1,1,1-TR tehlowäYHMAE

1, 1,7-TRL CHLORGETHANE

R] CHLOROETIENA

1MYL ACEFA스

vIMrL CHLORIDE

XYLENES

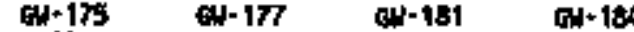

11,170

$1116 / 95$

CRSP
$11 / 05 / 95$

$10 / 31$ \%

$131 / 9$

(U⿺辶)

(UB/L)

(bo/

$(x, y)$

(UAJl)

(UN/L)

(utfli)

cunols

(und

(uin/

cus

(U)

(Wor)

(ust)

(uof

(UG

(U⿺辶)

(ub $/ \mathrm{L})$

(UG)

(Utofl)

(UOC/L)

(uofl

(U⿺辶)

(Lo $/ \mathrm{L}$ )

(una)

(Wo/L)

(us

(us, )

(ung

(UG)

(U)

(us/l)

100
100
100
100
100
100
100
100
100
100
100
100
100
100
100
100
100
100
100
100
100
100
100
104
104
15
100
100
100
100
100
100

100
100
100
100
100
100
100
20
100
100
100
100
17
100
3
100
100
100
100
100
100
100
100
100
100
100
100
20
100
100
100
100
100

100
100
100
100
100
100
100
100
100
100
100
100
100
100
100
100
100
100
100
100
100
100
100
100
100
100
100
100
100
100
100
100
100
100

100
100
100
100
100
100
100
100
100
100
100
100
100
100
100
100
100
100
100
100
100
100
100
100
100
100
100
100
100
100
100
100
100
100

6N-186 $11 / 03$ Rs

cat-1日? $11 / 02 / 85$

100
100
100
100
100
100
100
100
100
105
100
100
100
100
100
100
100
100
100
100
100
100
100
100
100
100
100
100
100
100
100
100
100
100

100
100
100
100
100
100
100
100
100
100
100
100
100
100
100
100
100
100
100
100
100
100
100
100
100
100
100
100
100
100
100
100
100

$4+198$

GN-203

intos

10/31/ 95

100
100
100
100
100
100
100
100
100
100
100
100
100
100
100
100
100
104
100
100
100
100
100
100
100
100
100
100
100
100
100
100
100

100
100
100
100
100
100
104
100
100
100
100
100
100
100
100
100
100
100
100
100
100
100
100
100
100
100
100
100
100
100
100
100
100
100

an-20S tiwcs

100
100
100
100
100
100
100
100
100
100
100
100
100
100
100
100
100
100
100
100
100
100
104
100
100
100
100
100
100
100
100
100
100

UNCS

$0 \mathrm{ros}$

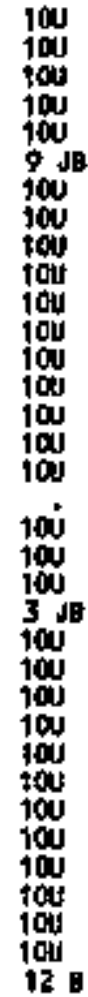




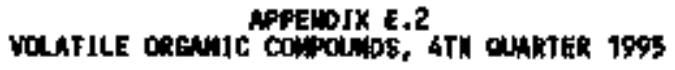

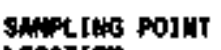
LOCATI IOH

\begin{tabular}{|c|c|}
\hline 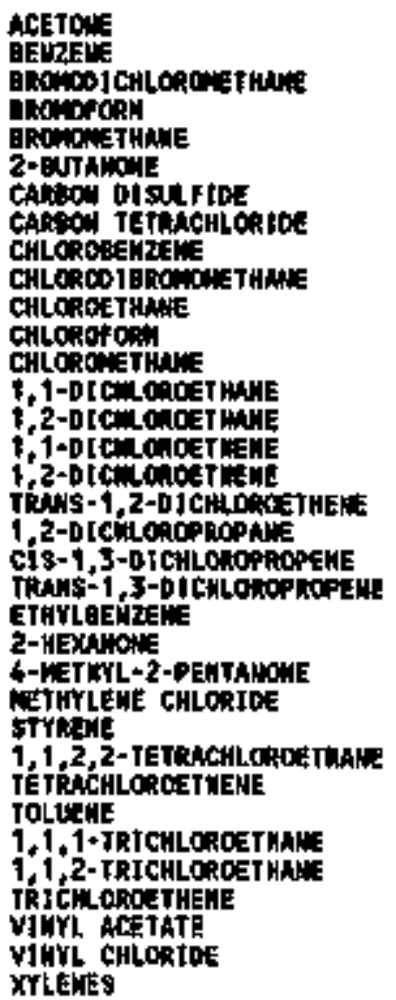 & 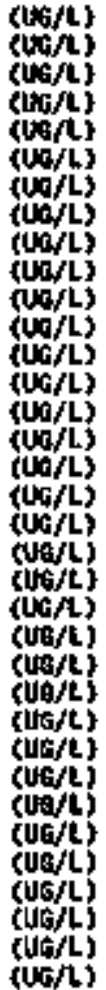 \\
\hline
\end{tabular}

\begin{tabular}{|c|c|c|c|c|c|c|}
\hline $\begin{array}{r}6 \times+231 \\
11 / 0 \times / 95\end{array}$ & $\begin{array}{r}04-292 \\
\text { echip } \\
11 / 06 / \% 5\end{array}$ & $\begin{array}{r}\text { an-293 } \\
\text { tichlop } \\
11 / 06 / 95\end{array}$ & $\begin{array}{r}\text { OA-2944 } \\
\text { ECRIP } \\
11 / 05 / 95\end{array}$ & $\begin{array}{r}\text { aN-296 } \\
\text { ECKTP } \\
11 / 05 / 95\end{array}$ & $\begin{array}{r}\text { ch-29s } \\
\text { chans } \\
11 / 04 / 95\end{array}$ & 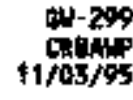 \\
\hline $\begin{array}{l}100 \\
100 \\
100 \\
100 \\
100 \\
10 \\
100 \\
104 \\
100 \\
100 \\
100 \\
104 \\
100 \\
100 \\
100 \\
100 \\
100\end{array}$ & $\begin{array}{l}100 \\
100 \\
100 \\
100 \\
100 \\
100 \\
100 \\
100 \\
100 \\
100 \\
100 \\
100 \\
100 \\
100 \\
100 \\
100 \\
100\end{array}$ & $\begin{array}{l}10 y \\
100 \\
100 \\
100 \\
100 \\
100 \\
100 \\
100 \\
100 \\
100 \\
100 \\
100 \\
100 \\
100 \\
100 \\
100 \\
100\end{array}$ & $\begin{array}{l}100 \\
100 \\
100 \\
100 \\
100 \\
100 \\
100 \\
100 \\
100 \\
100 \\
100 \\
100 \\
100 \\
100 \\
100 \\
100 \\
100 \\
100\end{array}$ & $\begin{array}{l}100 \\
100 \\
100 \\
100 \\
100 \\
100 \\
100 \\
100 \\
100 \\
100 \\
100 \\
100 \\
100 \\
100 \\
100 \\
100 \\
100\end{array}$ & $\begin{array}{l}100 \\
100 \\
100 \\
100 \\
100 \\
100 \\
100 \\
100 \\
100 \\
100 \\
100 \\
100 \\
100 \\
100 \\
100 \\
100\end{array}$ & 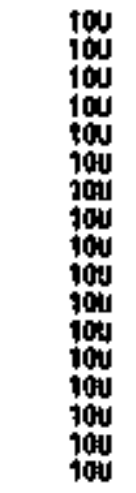 \\
\hline $\begin{array}{l}100 \\
100 \\
100 \\
100 \\
100 \\
100 \\
100 \\
100 \\
100 \\
100 \\
100 \\
100 \\
100 \\
100 \\
100 \\
100 \\
100 \\
100 \\
100 \\
100\end{array}$ & $\begin{array}{l}100 \\
100 \\
100 \\
100 \\
100 \\
100 \\
100 \\
100 \\
100 \\
100 \\
100 \\
100 \\
100 \\
100 \\
100 \\
100 \\
100\end{array}$ & $\begin{array}{l}100 \\
100 \\
100 \\
100 \\
100 \\
100 \\
100 \\
100 \\
100 \\
100 \\
100 \\
100 \\
100 \\
100 \\
100 \\
100 \\
100\end{array}$ & $\begin{array}{l}100 \\
100 \\
100 \\
100 \\
100 \\
100 \\
100 \\
100 \\
100 \\
100 \\
100 \\
100 \\
100 \\
100 \\
100 \\
100\end{array}$ & $\begin{array}{l}100 \\
100 \\
100 \\
100 \\
104 \\
100 \\
104 \\
104 \\
100 \\
100 \\
100 \\
100 \\
100 \\
100 \\
1001 \\
100 \\
100\end{array}$ & $\begin{array}{c}100 \\
100 \\
100 \\
100 \\
100 \\
100 \\
100 \\
100 \\
100 \\
100 \\
100 \\
100 \\
100 \\
100 \\
100 \\
100 \\
100\end{array}$ & $\begin{array}{l}100 \\
100 \\
100 \\
100 \\
100 \\
100 \\
100 \\
100 \\
100 \\
100 \\
100 \\
100 \\
100 \\
100 \\
100 \\
100 \\
100\end{array}$ \\
\hline
\end{tabular}

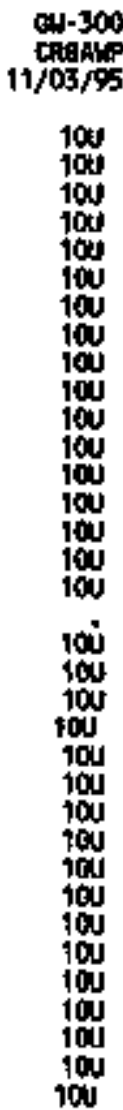

CAH-301
CKEALP
$11 / 03 / 85$

데-J02 $10 / 0 \mathrm{lng}$

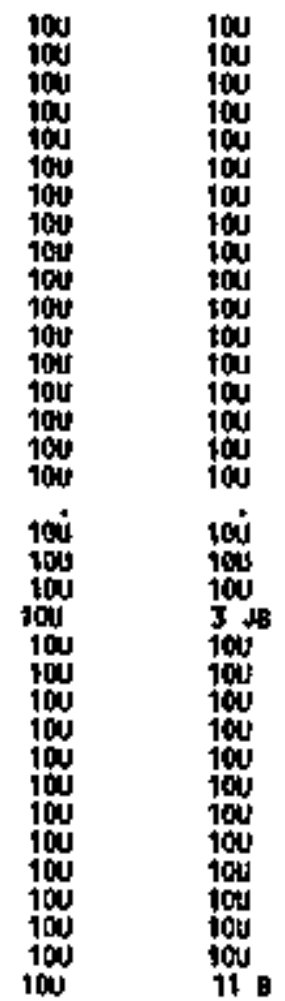




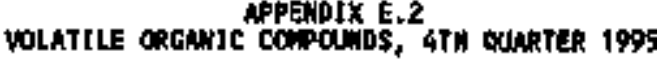

SANPLING POIMT OCATE TOM

\section{MCETOME}

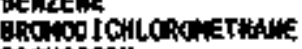

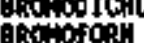

anowowetwaie

ChaBow o[ HUF]

CAROON TETRACHE DaJ

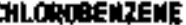

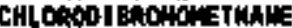

CHLOMETHWHE

CHLOBOfonis

CHLOROMTTANE

T, 1-DICHLOROE THMWE

r-gichlokgrthat

1,2-btchlogatint

TRANS-1, 2-O ICHLOROETHENE

1,2-DJCHLOAOPROPANE

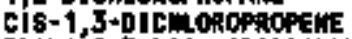

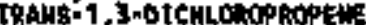

ETHYaemXENE

2+itexhlyow

-HETUYL-2-PEMTALOHE

HeTHTLENE cow ORIOE

STYRENE

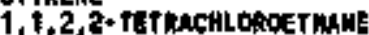

TRRACHLOROETHEA

UJE

1,1,1-TKICHLOROETHAVE

1, 2-TRICHLOROCTHANE

THU CHLORAETHEME

VIMTE ACEIATE

VIMTL CHLR IDE

XYLERES

0
$10 / 26 / 9$
100
100
100
100
100
100
100
100
100
100
100
100
100
100
100
100
$10+$
100
100
100
100
100
100
100
100
100
100
100
100
100
100
100
100
100

6. $00-539$ on-512

$11 / 07 / 95$

$10 / 31 / 58$

an-\$13

$10 / 31 / 95$

$014-514$
$11 / 02 / 95$

1010

contivi

10) $11 / 95$

GN-541

10/12/\%s

$04-542$

100100100

$100 \quad 100 \quad 100 \quad 10 \mathrm{~J}$

(UG/L)

(Uari)

(ubs)

SUG

(난가

(UG /L)

(U⿺辶)

(Uoy)

(No)

(4)

(ud

(ubs)

(usi)

(U⿺辶)

(W6/)

(UG/L)

(t)tith

(W'G

(Ua)

(UG)

(uart?

(top

(uts

Uafi

(tef $L$ )

(U6/L)

totaf $\mathrm{k}$

(t)

(tili) $L$ )

(t)ifl)

UG/L)

(UGe/L)

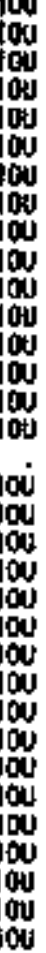

100
100
100
100
100
100
1001
100
100
100
100
100
100
100
100
100
100
100
100
100
100
100
100
100
100
100
100
1000
100
1001
100
100

100
100
100
100
100
100
100
100
100
100
100
100
100
100
100
100
100
100
100
100
100
100
100
100
100
100
104
100
100
100
100
100

100
100
100
100
100
100
100
100
100
100
100
100
100
100
100
100
100
100
100
100
100
100
100
100
100
100
100
100
100
100
100
200
100

100
100
100
100
100
100
100
100
100
100
100
100
104
100
100
100
100
100
100
100
100
100
100
100
100
100
100
100
100
190
100
100
100
100
100

100
100
100
100
100
100
100
100
100
100
100
100
100
100
100
100
100
100
100
100
100
100
100
100
100
100
100
100
104
100
100
100
0

100
100
100
100
100
100
100
100
100
100
100
100
100
100
100
100
100
100
100
100
100
100
100
100
100
100
100
100
100
100
100
100
100
100
100

$10 / 16 / 95$

10.

10

100

10u

tod

ind

100

du

10U

ion

tov

101

100

100

100

100

19!

100

100

10u

10u

100

100

100

100

10U

100 
APPEIDIX E.2

WLATILE DAENIC DOHPOUDS, 4TH OLATER 1995

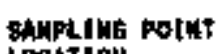
LDCkTIon

DATE SATPLED

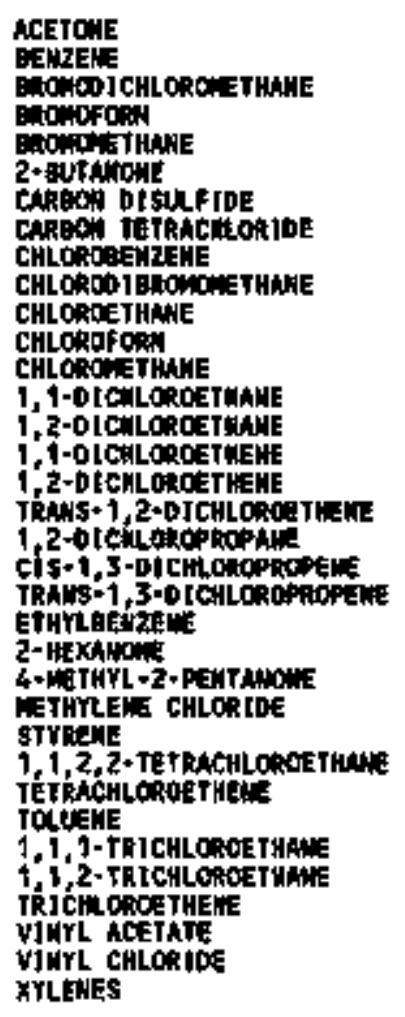

$\cos -543$
CDLYI

(usa)

(Ue/L

cust.

tes/L

(U)'L

(U⿺辶)

(UST/L)

(UIS/L)

(u6/t)

(ue/t)

(ue/c)

(UG/t)

(U)

(us/h

(US/L)

Ua/L

(ugh

(Uo/

suoht

(UW/L)

(Uor)

(Wo/L)

(WT/L)

(tis)

(Ub)

(t)ist

tits

(t)is

(up)

(U) L

WG/L

NG/L

(U)

(U): L

(tốl)

$10 / 17 / 95$

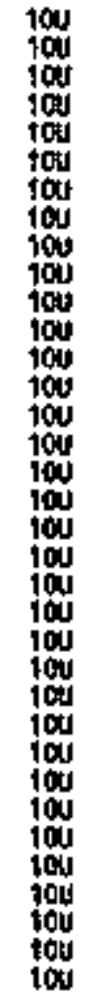

ON-544

aN-546

coly

aw-557 $10 / 07 / 95$ $04+560$
c04vII
$10 / 06 / 95$

colytis

$10 / 06 / 95$

C1I-564
coLv11

toplv11

0N-60s

11/16/\%5

$04-609$ 11/20/95

a4-6t0

100
100
100
100
100
100
100
100
104
100
100
101
104
100
100
100
100
100
100
100
100
100
100
100
100
100
100
100
100
100
100
1001
100
100

100
100
100
100
100
100
100
100
100
100
100
100
100
100
100
100
100
100
100
100
100
100
100
100
100
100
100
100
100
100
100
100
100

$\begin{array}{cc}100 & 100 \\ 100 & 100 \\ 100 & 100 \\ 100 & 100 \\ 100 & 100 \\ 100 & 100 \\ 100 & 100 \\ 100 & 100 \\ 100 & 100 \\ 100 & 100 \\ 100 & 100 \\ 100 & 100 \\ 100 & 100 \\ 100 & 100 \\ 100 & 100 \\ 100 & 100 \\ 100 & 100 \\ 100 & 100 \\ 100 & 100 \\ 100 & 100 \\ 100 & 100 \\ 100 & 100 \\ 100 & 100 \\ 100 & 100 \\ 100 & 100 \\ 100 & 100 \\ 100 & 100 \\ 100 & 100 \\ 100 & 100 \\ 100 & 100 \\ 100 & 100 \\ 100 & 100 \\ 100 & \end{array}$

100
100
100
100
100
100
100
100
100
100
100
100
100
100
100
0
100
100
100
100
100
100
100
100
100
13
100
100
100
100
100
100

100
104
104
100
100
100
100
100
104
100
100
104
104
100
104
100
100
100
100
100
100
100
100
100
100
100
100
100
100
100
1001
100
100 


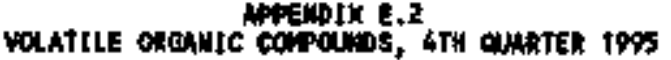

SAPLIII POE:HT

Lochtton

bite strite

ACTrone

gRomotcmonsethne

epownopin

照aiofopin

2.

chentis ot

CAcon TETPh

CAchom

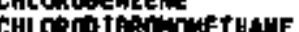

CHLORD THAl'

CHiono

1.1-D IC MODOETHAWE

$1,2+0$ ICTLOROETHAIE

11 -

$12-0$ cte on

THNS-1, 2-DICHLOROETWENE

1, 2-DICHLCHOPROPAHE

c.15-1,3-0ICHLOAOPbOPEN

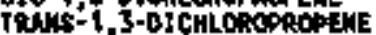

ETHYLEMENE

2-Hexdions

4-NGTHL-2-PEHTNWOM

wethrLewe CHLORIOE

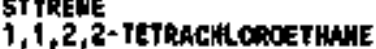

1,2,2- TETRACHLCO

TOLUEA

1,1.1-TRICWLOHOETHWE

richlohor

Tich AcETht:

VIAYL CHLORIOE

Xrtencs

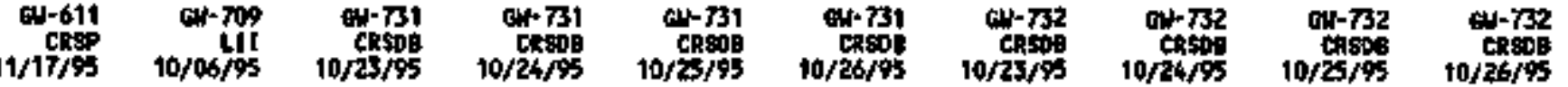

(bis/t)

(wos)

(baft)

(uof

(wo/ㄴ)

$(40 /$ )

(UG

(UC, L)

(U)

(U6/L)

(No/l)

(t)

(I)

(unil

uafi

(

(unfli

(Ut)

(uofl)

(low/h)

(us/

Uat

(ut/ $/$ )

(uth/

(UE/L)

(UaflL)

(Uth/t)

(t)

Lon

Uats

Uas

Uos $L$ ?

(usert)

100
100
100
100
100
100
100
100
100
100
100
100
100
100
100
100
100
100
100
100
100
100
100
100
100
100
100
100
100
100
100
104
100
100

: 
APPEADTX E.?

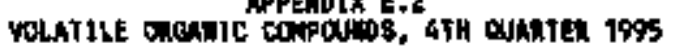

swatine pothT Locht ton

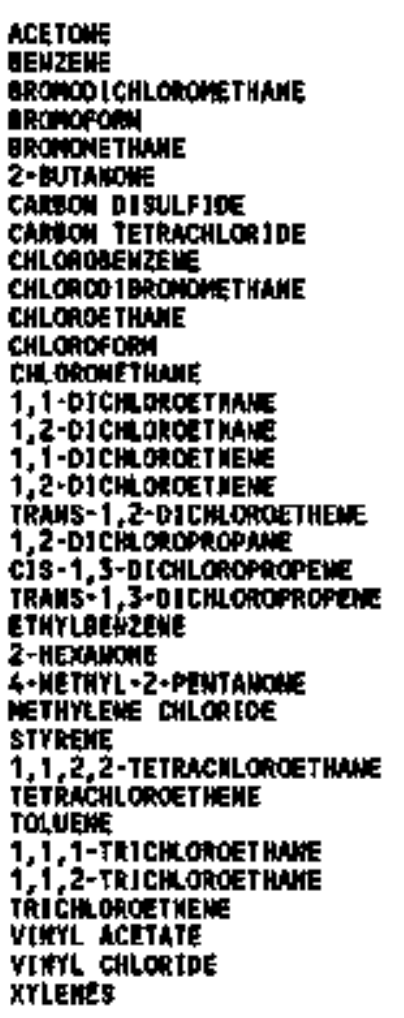

\section{cw-742} $11 / 09 / 95$

(U)

(UA L)

(U) $6 /$ L)

(UA/L)

(UB $/$ L

(UQVIL

UG/L)

Q

(UA'L)

(UACL)

(UG/L)

(U⿴囗十)

(wor

(LW/L)

(Whit)

(uth

cuort

(L) $A$ )

tuef:

(us/t)

cuor.

$x \mathrm{~W} / \mathrm{A}$

(D) $A$ )

(i)

(uof $/$.

(uG/t)

(U⿺辶)

(Wo/t)

(U⿺尢)

(UEA)

LUE/L?

(uort?

(t)

(two/t)

(UG)

\begin{tabular}{|c|c|}
\hline $\begin{array}{l}100 \\
100 \\
100 \\
100 \\
100 \\
100 \\
100 \\
100 \\
100 \\
100 \\
100 \\
100 \\
100 \\
104 \\
100 \\
100\end{array}$ & $\begin{array}{l}100 \\
100 \\
100 \\
100 \\
100 \\
100 \\
100 \\
100 \\
100 \\
100 \\
100 \\
100 \\
100 \\
100 \\
100\end{array}$ \\
\hline $\begin{array}{l}100 \\
100 \\
100 \\
104 \\
100 \\
100 \\
100 \\
100 \\
100 \\
104 \\
100 \\
100 \\
100 \\
100 \\
100 \\
100 \\
100\end{array}$ & $\begin{array}{l}100 \\
100 \\
100 \\
100 \\
100 \\
100 \\
100 \\
100 \\
100 \\
100 \\
100 \\
100 \\
100 \\
100 \\
100 \\
100 \\
100\end{array}$ \\
\hline
\end{tabular}

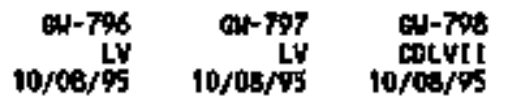

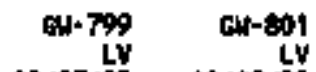
10/00/ Ly

ad-827 ColvI

\begin{tabular}{|c|c|}
\hline $\begin{array}{l}100 \\
100 \\
100 \\
100 \\
100 \\
9510 \\
100 \\
100 \\
100 \\
100 \\
100 \\
100 \\
100 \\
100 \\
100 \\
100 \\
100 \\
100 \\
100 \\
100 \\
100 \\
3 \\
100 \\
100 \\
100 \\
100 \\
100 \\
100 \\
100 \\
100 \\
1 \\
100 \\
100 \\
100 \\
100 \\
100 \\
11 \mathrm{~J}\end{array}$ & 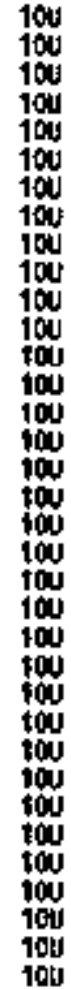 \\
\hline
\end{tabular}


APPENDIX E.3

ADDITIONAL ORGANIC COMPOUNDS 


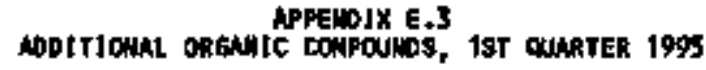

SHipling POINT

Locht ton

DATE SMIPLED

ACHOLE $[$ H

ACKYLONITRILE

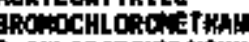

2-CUl OOOETHYLVTHYL ETwE IFonto THAH:

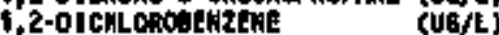

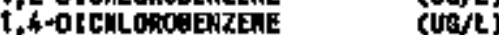

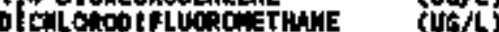

CIS-1,2-D TCHLOROETHEHE ULIL

14-0ICHOO0-2-BITENE (UCL)

Ermantol

TML METHACXILATE

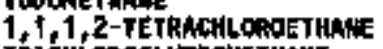

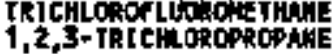

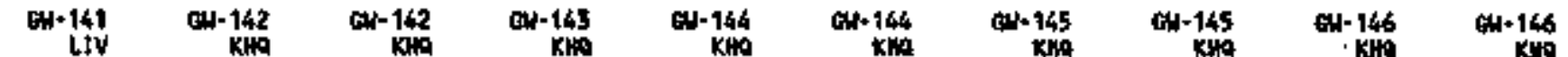

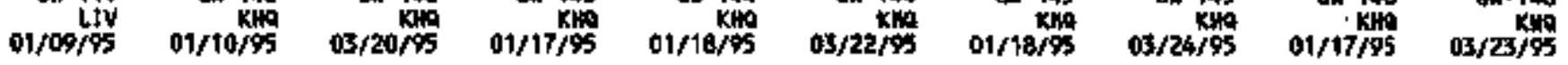




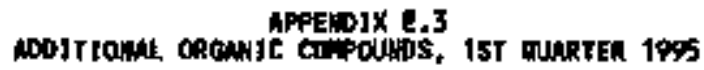

setpime pojut

LOCATSO

DATE SHYPLED

\section{ACPOEELN}

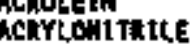

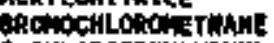

2-CHLOBOFI HTLVIUYL ETHER

1, 2-DLthom-3-CHLOROPROFAM

1.2+0]CHLOROBEZLLE

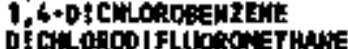

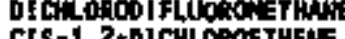

CIS-1,2-D]CHLOROE THER 1,4-DI DLORO-2-BTTEWE EFHAHOL

ETMYL METHACRYLATE

IOODNETHA:FE

$1,1,1,2$-JETRACHL,OROE TMALE

TRICHLOROFLUOAONETHLFE

$1,2,3$ - TR TCHLOROPRDPAHE
CN- 147
ad-156
ㅂ. 150
6. 159
6il-175
c4-177
6w-161
9h+217
(5)-231
84-241

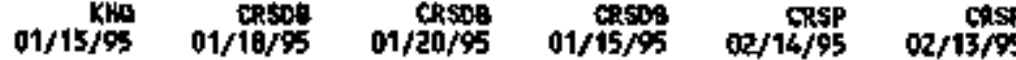
$01 / 24 / 95$
$01 / 04 / \%$ 01/15/95 01/20/9

(UNG/L)

(utill

Quach)

(uchl)

(UT/L)

(uglis)

unat?

(uith

(vath)

(uto $/$ )

(Ut $/$,

tert

Uin/

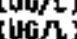

(t)

(US/L)

$\begin{array}{lll}: & : & : \\ : & \vdots & : \\ : & : & : \\ : & : & : \\ : & : & :\end{array}$

$:$




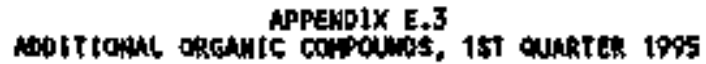

SEPLING POLHT

LOCATION

Ackolet

MCRYLOUITR SLE

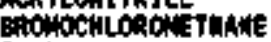

2-ChLOROETHYLYIMTL ETHER

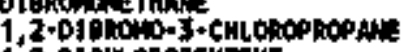

1, 2-biChlomoachzahe

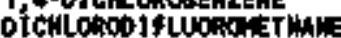

CIS-12-D]CHLOROF J H HE

$1,4-6$ ChL

efranol

ETWYL METHCAYLATE

1, $1,2-T$ TRKACLLOADETHAME

The
GN-305 GW304 cis-305

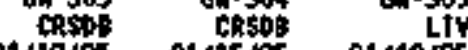

$01 / 15 / 8$
GN-321 6N-511 $01 / 10 / 95001 / 24 / 95$
ON-521

1 [y

$01 / 09 / 5$ aw-522

$01 / 10 / 95$
6N-559 $01 / 04 / 53$ 02 CRSP

cw- 609

(UG/L)

(U6 $/ \mathrm{L}$

(uos)

(uog/t)

(U)

(Ua, $/$ L)

(LG/L)

(U6)

(Wo/t)

$\mathrm{Wa} / \mathrm{t}$

(bo/t

(UG/L)

(UOSL)

(ULCL)

(U6)

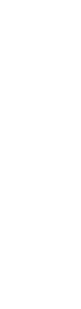

500
500
100
500
100
100
500
500
500
500
500
500

 $02 / 15 / 95$ 


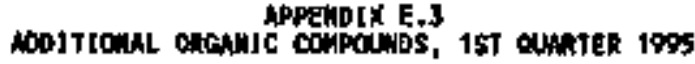

SMly ING POLFT

Locat ion.

DAfE SHipleo

ACAOLEIH

ACRTLitR

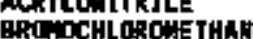

2-CHLOROETHYLYH' ETH DJ BRamomethihl

EN

1,2-DichLOROEEMZEN

1 4+D L CWLOROAENZENE

年

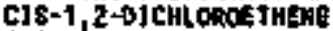

1,4-DL CHLOAD-2-EUTEME

EIthyit.

ETHYL METMACRYLATE

TOO0 ETMME

$1,1,2-$ TETRACHLOROETWANE

Rt CHLORGFLttoRONETHANE

1,2,3-IR TCHLOROPROPAHE

\begin{tabular}{|c|c|}
\hline $\begin{array}{l}+610 \\
\text { thesp }\end{array}$ & $\begin{array}{c}w-611 \\
\mathrm{ces}\end{array}$ \\
\hline
\end{tabular}

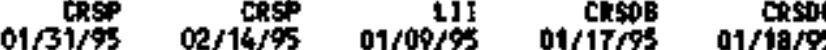
GN-762
CRSP

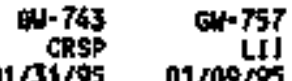
$\operatorname{scn} 2.25$
$05 / 15 / 95$

(uo/L)

(ury

Uof $L$

(Uth

(U6/L)

(U6)

(1) $/ \mathrm{L}$

(40)

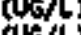

(uns

(UGGL)

(UD, L)

(ubs)

(UOC)

500

Iㄴ)

100

ind

10u

16U

100

ज्ञ

su

5

SU

5

50 
SHMPLIMG porm LOCATION

DAIE SWPLEO

MEROLE1H

MCRYLÓNITRILE

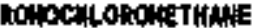

2-CHLOROETMYLIVIME ETHER

IRROnOMETHA

1,2-DIGRCAL-3-CHLONOPAOPAN

1,2-DICHLOQOOUEMZZNE

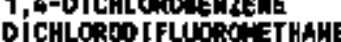

CIS-1, 2-D ICHLONETHEME

1, 4-6 ' 2-D ICHLORO-2-DUTENE

1,4-DiCHLOR0-2- DUTE

ETHan

ETiYL methackritare

SOOONETHANE

$1,1,1,2-T E T R A C H L O R O E$ THAHE

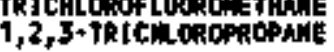

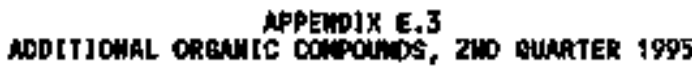

\begin{tabular}{|c|c|c|c|c|c|c|c|c|c|}
\hline $\begin{array}{r}1090 \\
6468 \\
4 / 20 / 95\end{array}$ & $\begin{array}{r}\text { cos-1 } \\
\text { Ly } \\
04 / 17 / 95\end{array}$ & $\begin{array}{r}64-162 \\
\mathrm{KH} \\
04 / 20 / 95\end{array}$ & $\begin{array}{r}5.143 \\
k / 2 m \\
04 / 24 / 95\end{array}$ & $\begin{array}{r}\Delta-144 \\
K 119 \\
04 / 25 / 95\end{array}$ & $\begin{array}{r}\text { ch. } 145 \\
\text { K14 } \\
04 / 26 / 95\end{array}$ & $\begin{array}{r}4 \times 146 \\
\text { Knd } \\
04 / 24 / 95\end{array}$ & $\begin{array}{r}4-147 \\
14 \% \\
04 / 21 / 95\end{array}$ & 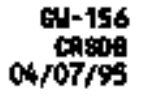 & $\begin{array}{r}0+158 \\
6 / 506 \\
06 / 13 / 95\end{array}$ \\
\hline
\end{tabular}

turest

勿

$\begin{array}{ll}: & : \\ : & : \\ : & :\end{array}$




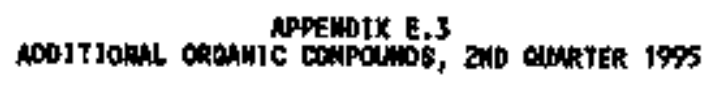

SHMPLIMG POLIT

LOCATIOF

DATE SNMPLE

\section{ACroiejn}

MCRVIOHITRTLE

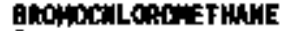

2-CHLOROETHYLVIHYL ETHE:

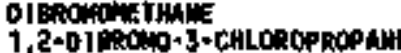

1,2-bichLonosedreane

1, 4-01CHLORoserizeare

Díchlowot FLoget, EThan:

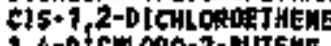

1,4-0 TCWOAO-Z-BUTEHE

ETMMOL

ETHYL METKACMYLATE

1, 1,2-TLTRACHLOROETHAHE

RCHLOROFLOORG, THAME

\begin{tabular}{|c|c|c|c|c|c|c|c|c|}
\hline $\begin{array}{r}\text { ch-15s } \\
\text { CRspa } \\
04 / 06 / \% \%\end{array}$ & $\begin{array}{r}\text { cy-160 } \\
\text { chtansp } \\
04 / 26 / \% 5\end{array}$ & $\begin{array}{r}64-161 \\
\text { Chanto } \\
04 / 20 / 95\end{array}$ & $\begin{array}{r}\text { AN-175 } \\
\text { CRSP } \\
\text { os/15/95 }\end{array}$ & $\begin{array}{r}0.177 \\
\text { chsp } \\
05 / 11 / 95\end{array}$ & 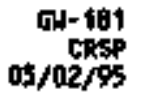 & $\begin{array}{r}94-104 \\
05 / 05 / \% 5\end{array}$ & $\begin{array}{r}\cos 186 \\
05 / 03 / \% 5\end{array}$ & $\begin{array}{r}64-187 \\
05 / 02 / 95\end{array}$ \\
\hline
\end{tabular}

(Ho/h)

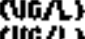

(uti)

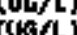

(wo/L)

(UG $/ \mathrm{L}$

U日A

(WG $/$ )

(ush)

(vor)

(NG/L)

(UG/L)

(U⿺辶)

(US/L)

(Uo/L)

(UGAL)

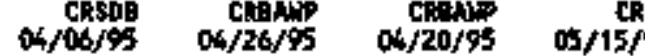

500
500
50
50
50
5
5

500

sol

100

si

50

50

$5 \dot{10}$

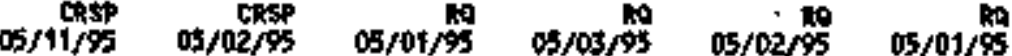


APPENDIX E.3

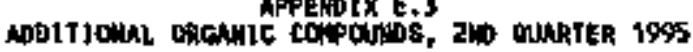

SNPLL1MO DOSHT

DNTE SANPLE

ACROLETH

ACRYLOAI TRJLE

Whabcho:THAME

2-ChLOAOETHYLYHYL ETHE

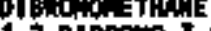

1,2-Uichono:en?ElW

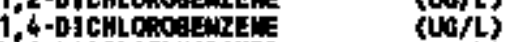

ofchion (No/

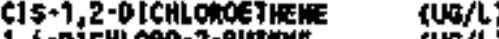

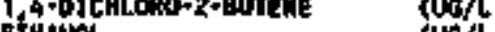

ENW

ETHZ NETGACRYLATE (UG/L

DOCATTME

1,1,2-TETACHLOAOETHANE $\langle\mathrm{UD} / \mathrm{L}$

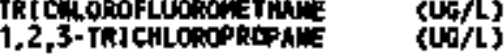

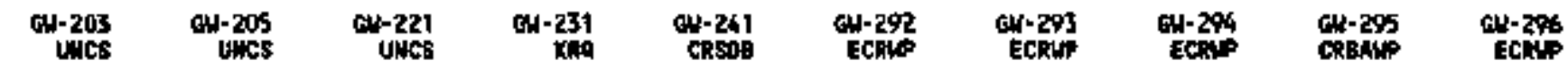

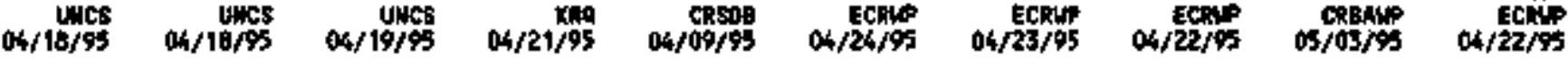




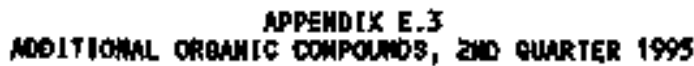

SAnPLIMG POINI

iocition

DATE SANPLED

\section{ACROLE 1II}

ACSYLONJTRJLE

BSONOCHLCHOWE THALE

THALE

CHLOAOETHYLYIHYC ETHER

Digho (Wo/L

2-Dichu GoneuzE

t+Dicm orotenze

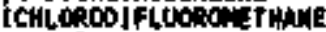

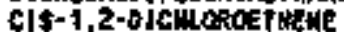

1, 4-OICHLORO-2-gUTEE

Eimand

ETHYL WETMCRYLATE

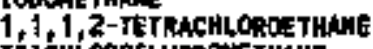

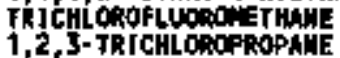

(ats

(with)

(4)

(torstis

cunfli

(L)

(uifl)

(Uol)

\begin{tabular}{|c|c|c|}
\hline $\begin{array}{r}\text { CH-29t } \\
\text { cRBNip } \\
05 / 02 / 5 s\end{array}$ & $\begin{array}{r}\text { ch-299 } \\
\text { cobsipp } \\
05 / 01 / 95\end{array}$ & $\begin{array}{r}\text { UN-390 } \\
\text { CREA4P } \\
\text { Ot/27/95 }\end{array}$ \\
\hline $\begin{array}{l}50 \mathrm{~J} \\
50 \mathrm{~J}\end{array}$ & $\begin{array}{l}500 \\
500\end{array}$ & $\begin{array}{l}\mathrm{sod} \\
\mathrm{sW}\end{array}$ \\
\hline${ }_{50}^{100}$ & ${ }_{\mathrm{x}}^{100}$ & ${ }_{50}$ \\
\hline : & $\vdots$ & : \\
\hline 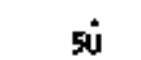 & și & sí \\
\hline 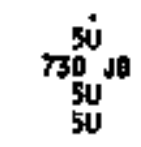 & 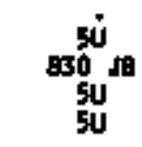 & $\underset{560}{50}$ \\
\hline 武 & sư & si \\
\hline
\end{tabular}

벡-501 에.302 데.303

ches

lincs

Chspes

son

Solu

10:

SU

$5 i$

$19 / 95 \quad 04 / 09 /$

50
500
50
50
50

\begin{tabular}{|c|c|c|}
\hline 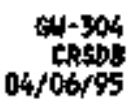 & $\begin{array}{r}04 \times 321 \\
04 / 25 / 95\end{array}$ & $\begin{array}{r}64-339 \\
\text { Jincs } \\
04 / 19 / 95\end{array}$ \\
\hline
\end{tabular}




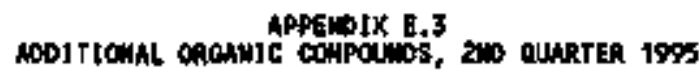

SNYPLING PDLTT

LOCAT 1ON
OATE SAPLD

ADROLEJM

AGRONIIRJLE

ONETHANE

2-CHLORtETAYLVIUYL ETHER

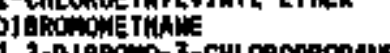

2-DIPROM-3-CHLORO

1,2 -DICHLOROEEHZEHE

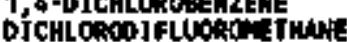

c19-2 2-D

1)

Étion

ETHYL METUACRYLATE

ETri hervach

1, 1,2-TETRACHLORDETHALE

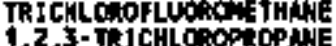

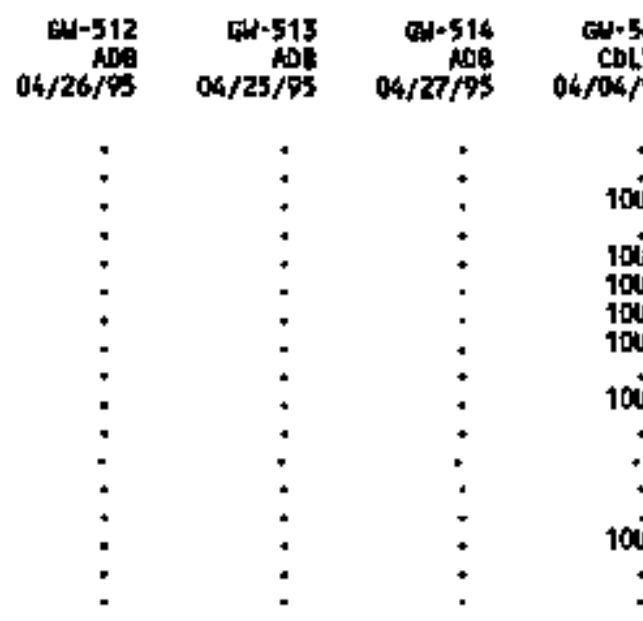

a.5 541 017-56

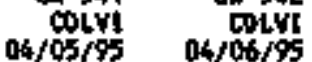

a1-365

CLLI

CDLVI $04 / 06 / 5$

5ut-5as

colvi

611-557

$04 / 06 / \%$

100
100
100
100

$04 / 06 / 95$

 


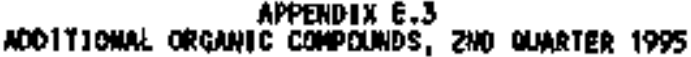

8Amplins polit

LOCRT JON

DATE SANPLED

HGOLEIM

ACRYto t12]LE

AROCHOCHLOHOHETHA

2-CHLOROETHYLYIWY ETYE

OreROWOMETHALE

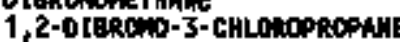

1,2-DICHLOKDEEHZEHE

$14+0$ ICHOROEENZENE

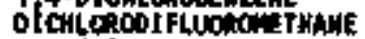

C[S-1,2-D]CHLOUETLE

1,4-DICHLOO-2-But

Etranol

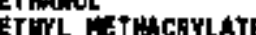

IOODNETHAGE

1,1,1,2-TejRachlocoeshan:

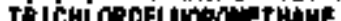

1,2,3-TRICHLOBOPROPAME
Gis-560

colvit

ch-562

$4 / 05 / 95$

( $(\mathrm{H} / \mathrm{C} / \mathrm{L})$

(t) $/ \mathrm{L} / \mathrm{L}$ )

cuterte

(U)

(ub/t)

(Uafl)

(UG/

(und)

(we)

(UG/L)

(UE/

(un

(tio/)

(uG/L)

(avill)

(WG/L)

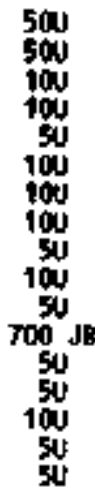

6)-680

an-609

EN-610

an-611 04-731

chisos

an-73

sou

os $111 / 95$

os/16/\% os $107 / 95$

os $/ 15 / 95$

$06707 / 5504107 / 05$

05,070

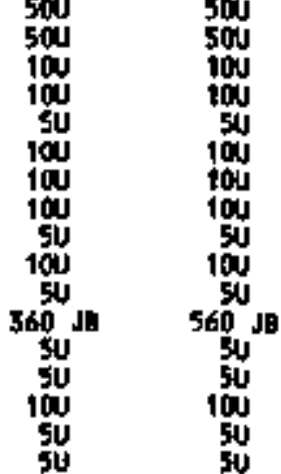


APPEMJX E.3

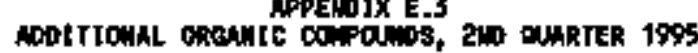

SHPLIME POINT

LOCAT JON

DATE SAMLE

ACHDLEIN

ACRYLONITRLLE

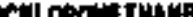

2.CHLORDTHHLVIML ETHE

ROMNETHA:

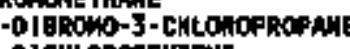

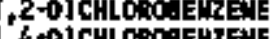

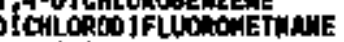

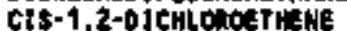

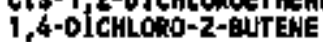

Efranke

EFHYL METHACRYLATE

joomithes

1,1,1,2-TETHenLokocthale

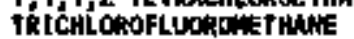

i, 2,3+TRTCHLORJPROPAHE
6N-743 GN7\% W-797 0N-79

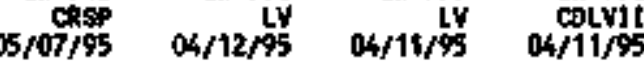

(uth/L)

(uatL)

(uofli)

(Uo/L)
(U⿴囗十)

(uof)

(yods)

(wers

sers

(us

(uon

(uon)

(ut

(40,

(Ua/L)

$\begin{array}{rr}500 & 500 \\ 500 & 500 \\ 100 & 100 \\ 100 & 100 \\ 54 & 54 \\ 100 & 100 \\ 100 & 100 \\ 100 & 100 \\ 54 & 54 \\ 100 & 100 \\ 54 & 54 \\ 100000 & 100000 \\ 50 & 50 \\ 54 & 50 \\ 100 & 100 \\ 50 & 50 \\ 50 & 50\end{array}$

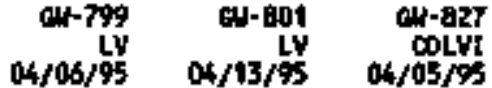



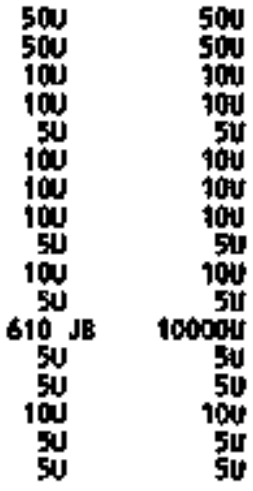

100

100

10

101

100

I0v

:

100 


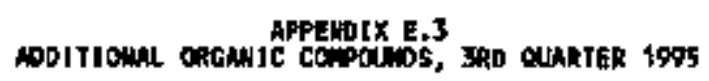

8MNPLING PDINT

LOCATIOM

Date sinfipto

\section{ACKOLE [N}

MCRYLOAN TRELE

BRONOCHLONOHE THAHE

2+CHLOADETHY TVIMTL ETHER

OTEROMONETHWH:

$1,2=01$ CHLOROBEHZENE

1 4*0]CHLOROREEIZEME

ofCHLakoojflyomoctrhane

Crs-1,2-b]cit Donethens

1.4-OICHLOR0-2-Edrtent

ETHAMOL

ETHYL METHACRYLATE

1 BDOHCT Hate

\$, 1, 1,2-TEFhachlonoejakhe

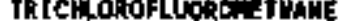

1,2,3-TRICHLOROPROPAN

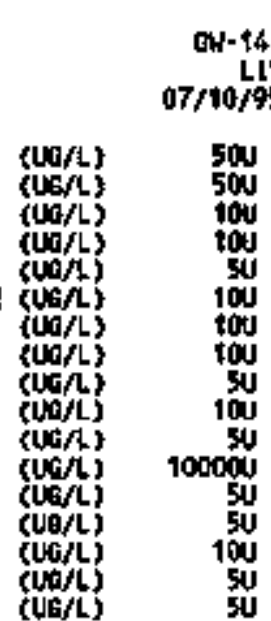

GN-142 GN-143

$07 / 14 / 4507 / 16 / 95$ 6w- 144 07/16/95 a)-145

$07 / 17 / 95$ an- 146
$\times 110$
071795

$9 w-147$
$\times 10$
$07 / 44 / 95$

ㅂ. 156

casts

$04-158$

$7 / 18 / \%$

6N-159

$\cos$

$50 \mathrm{y}$

100

tod

政

tou 


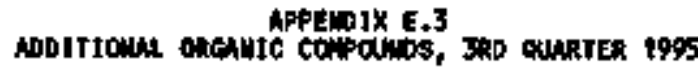

SMPL [WS PO]MT

Lach tow

OATE SWMLLD

ACAOEE IK

2- Cin ofoethry VthYL ETHER

20tchoro 3 -

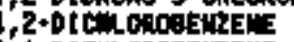

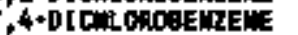

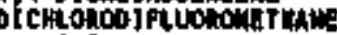

CI8-t, 2 - 0 JCW OEOETHEME

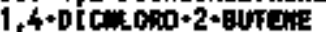

frumbit.

ETHYL WETHACRYLATE

JOOCHE JMAYE

i ti jor

1,2,3-7R

\begin{tabular}{|c|c|c|c|c|c|c|c|c|}
\hline $01 /$ & $\begin{array}{r}\text { an-177 } \\
\text { crgp } \\
00 / 01 / 95\end{array}$ & $\begin{array}{r}\text { GN+191 } \\
\text { CRgP } \\
07 / 28 / \% 5\end{array}$ & $\begin{array}{r}04-217 \\
07 / 10 / 45\end{array}$ & 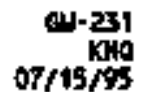 & $\begin{array}{r}\text { ar. } 241 \\
\text { cespes } \\
0716 / 8\end{array}$ & $\begin{array}{r}0.305 \\
\text { chisp } \\
0716 / 9\end{array}$ & 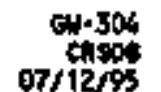 & $\begin{array}{r}\text { 4k-305 } \\
\text { c/Y }\end{array}$ \\
\hline
\end{tabular}

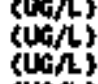

(U⿺辶)

(Ua/t)

(U⿺辶)

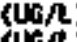

$(46 / 2$

$\{\mathrm{W} / \mathrm{L}\}$

$(\mathrm{U} / \mathrm{h})$

Wh

(U⿻上丨𣥂巾

(บㄷㄴㄱㅗ

(Ua)

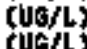

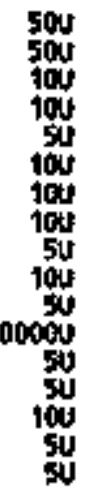




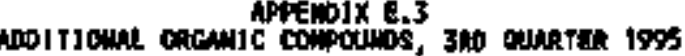

SAnLIHG POtw LOCATLO

OATE SNPLED

ACloLLEty

Acrroontrale

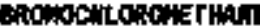

2-CHLOROLTHULYYL ETwER

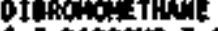

CUG/L

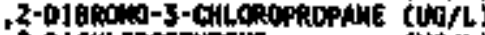

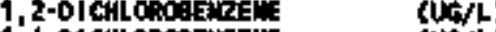

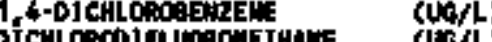

作

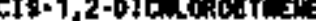

1 4-OICHLOHO-2+BItTHE

crimina

ETLYKL METHACRYLATE

toocinthare

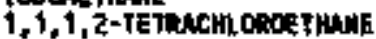

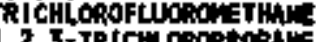

(Ut)

(unth)

(Oo/

(uth/낭
$3-521$
L1y

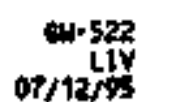

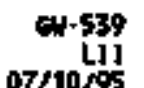

$04-608$

$0731 / 45$

sou

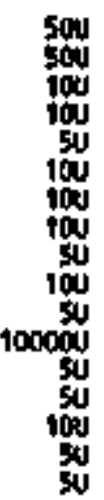





6N-609

corolitos 6in-610

orstris

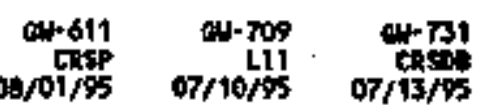

$:$
$:$

$:$ 


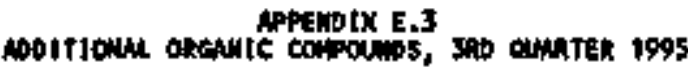

\begin{tabular}{|c|c|c|c|c|c|c|}
\hline 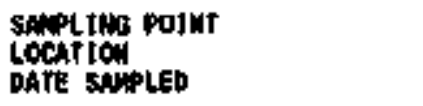 & & $\begin{array}{r}64-732 \\
\text { crispo } \\
07 / 13 / 95\end{array}$ & $\begin{array}{r}a+752 \\
0 / 14 / 05\end{array}$ & $\begin{array}{r}04+742 \\
\text { chsp } \\
07 / 30 / 95\end{array}$ & $\begin{array}{r}\text { an-743 } \\
\text { chsp } \\
07 / 31 / 95\end{array}$ & $\begin{array}{r}64-757 \\
\text { LI5 } \\
07 / 11 / 45\end{array}$ \\
\hline 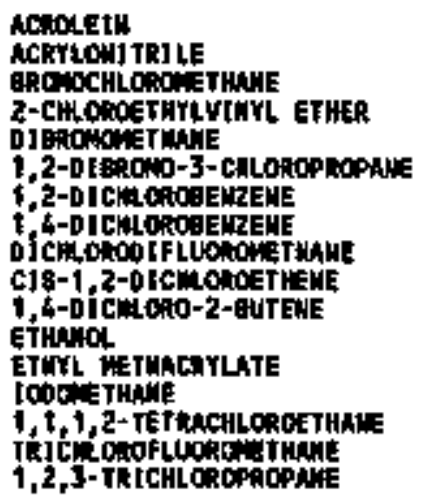 & 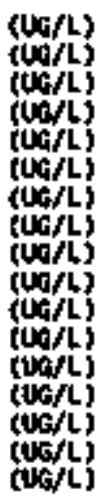 & $:$ & $:$ & $:$ & $:$ & 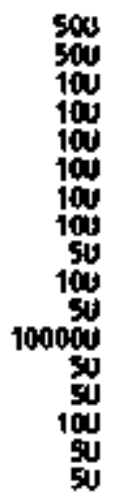 \\
\hline
\end{tabular}




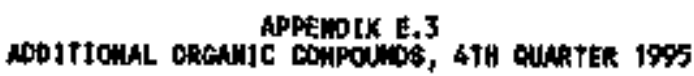

SAPLIME POIHT

Locht tow

DATE SHPLE

ACANLELN

ACRYLONITRILE

Bunochlogontruy

(U6/L)

(US/L)

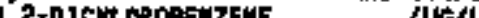

2

Dich onctuon twane

C15-1 2-0 CHLOOCF

140 1 Cu2 0-2 BuTtue

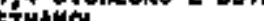

GHTh methacertente

conotrinter

$1,1,1,2$-TETRACHLOAOE THAM:

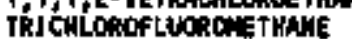

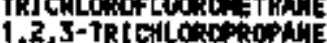

(uting

(watis

\begin{tabular}{|c|c|c|c|c|c|c|}
\hline $\begin{array}{r}109 b \\
\text { uncs } \\
0 / 0 \% / \% 5\end{array}$ & $\begin{array}{r}\text { cos-1 } \\
\text { ty } \\
\text { to/11/ }\end{array}$ & $\begin{array}{r}a w-742 \\
t 1 / 05 / \% 5\end{array}$ & $\begin{array}{r}0 y-143 \\
\mathbf{k H 0} \\
11 / 09 / 95\end{array}$ & $\begin{array}{r}c 1-944 \\
\times 140 \\
11 / 14 / 95\end{array}$ & $\begin{array}{r}\text { AN-145 } \\
\text { KHa } \\
+1 / 15 / 25\end{array}$ & $\begin{array}{r}\omega-146 \\
\text { KHo } \\
\text { 11/08/s5 }\end{array}$ \\
\hline
\end{tabular}

$\begin{array}{ll}: & : \\ \vdots & \vdots \\ \vdots & \vdots \\ \vdots & \vdots \\ \vdots & \vdots \\ \vdots & :\end{array}$

$\begin{array}{lll}: & : & : \\ \vdots & \vdots & : \\ : & \vdots & : \\ : & : & : \\ : & : & :\end{array}$




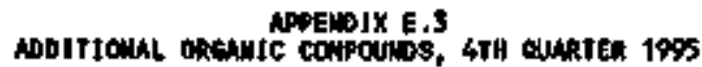

SUPLIFG POLAT

LOCAT1ON

DATE BAHPID

ATROLE]

AtarlonitikiLe

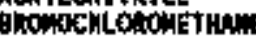

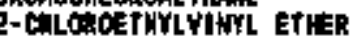

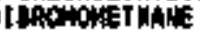

$1,2-0$ TERONO-3-CMOCROPHOPAUE

1, 2-0ICHLOAOAENZTHE

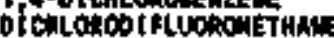

ct5-1

1 is

efrutor

MTHACRYLATE

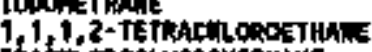

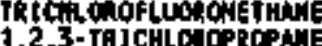

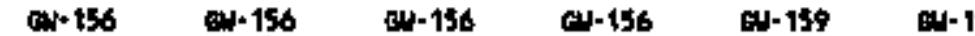

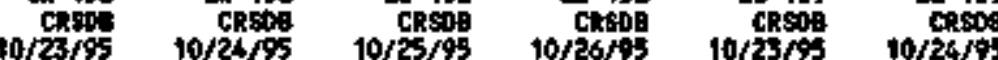

(U6/L)

(U)

NG/L

dust

Ut $\$$

(1)

(

(U)

(US/L)

,

.

(u)

(ut?

(uph

(uoft

.




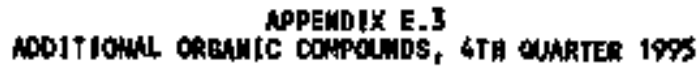

SAnplitis POINT

LOCATIION

OATE SAMPLED

MCRoletw

MCRYLONLTRthe

HOMXCHLOOM TWNE

2-CHLOPOSTHYLIHYL EтHE

DIBRG,

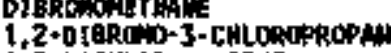

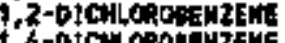

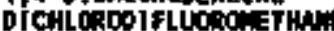

CIS-1, 2-b1chLonoctHEte

4+DICHLOR-2-DUTEUE

tricin

ETHYL RETHACGYHTE

Oboritithe

1, 1,2-TETRACHLOROETHAVE

RJCHLOROFLUONOMETHAHE

CN+175 6U-177 aw-101

chsp casp

$11 / 17 / 95$

$11 / 05 / \% 5$

CN. 184

$10 / 31 / 95$

$018 s$
$11 / 03 / 90$

Qu-107

Ro

cur-18a

10,3110

an-203

$64-205$

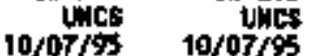

a.221

(uc/L)

(U5/L)

UCA $/ L$

(urt)

cuert?

(undL)

CuG $/$ )

(WG/L)

(Len)

(uo/l)

(uG L

(uto $\mathrm{L}$ )

(Wto $/$ ㄱ

(uth)

(U⿺𠃊)

(UT) 


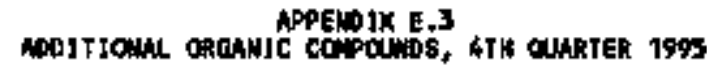

SALPLIN Potut

LOCATIOAN

DHE SHPTED

\section{ACDOELH}

ACRYLONITR ILE

(t)

ETHER

a

(Ute/t?

1,

14 -

DíchomoDisurometHANE

c1)

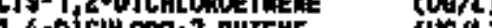

cinina

Gini

CTHY MeTHMCaYLate

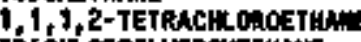

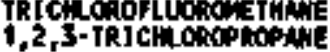

(unch

(us

$(\mathrm{t}, \mathrm{g} / \mathrm{t}$ )

$(16 / \mathrm{t})$

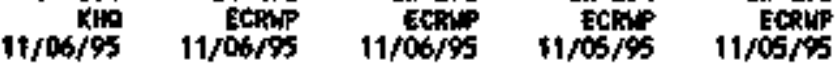

CN-298
CRBMLP
$11 / 04 / 95$

매-299

chesup

$6 \mathrm{~F}+300$

thath

$11 / 03 / 95$

$11 / 03 / 95$

Git301

(1)

$11 / 03 / 95$

떠-302

500
500
50
500
5000
50
50
50

500
500
100
54
50
50
500
50
50
50
50

10/ 0 ats 
NDDTTJOMAL ORGAMIC COWPOULDS, 4TH QUARTER 1958

SHPLINL POLFT

LOCATION

DATE SALPLEO

\section{ACROLE1L}

ACFLCONITRILE

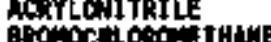

2-CHLCROETHYLITHL ETHE

2rapon

2010

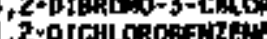

1,2 OICHLOnosenzant

DICHLOROOTFLUOROE THAK

CIS- 1 2-OICHLOROET KESE

(1)

ETHANOL

THYY

TOOML METLACRYLATE

1,1,1,2-TETRACHLOROETWANE

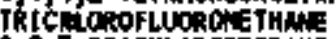

i,2,3-Talchlonophophts

\begin{tabular}{|c|c|c|c|c|c|c|}
\hline $\begin{array}{r}10+321 \\
10 / 26 / 95\end{array}$ & $\begin{array}{r}\text { Au-35s } \\
\text { uwcs } \\
10 \text { rospss }\end{array}$ & $\begin{array}{r}\text { atr-511 } \\
\text { chisp } \\
11 / 0 \% / 95\end{array}$ & $\begin{array}{r}0+512 \\
10 / 3 t / 95\end{array}$ & $\begin{array}{r}\text { Gu-513 } \\
106 \\
10 / 31 / 95\end{array}$ & $\begin{array}{r}c+514 \\
14 / 02 / 95\end{array}$ & $\begin{array}{r}\text { Gi-53 } \\
10 / 06 / 9\end{array}$ \\
\hline . & . & . & . & . & . & \\
\hline ' & - & . & . & . & . & \\
\hline , & - & - & - & - & . & \\
\hline , & . & * & * & . & . & \\
\hline * & , & . & . & . & . & \\
\hline - & - & ' & • & * & * & \\
\hline • & * & . & • & . & , & \\
\hline • & * & - & - & . & . & \\
\hline . & • & . & - & . & . & \\
\hline * & - & • & V & • & . & \\
\hline . & - & - & - & - & . & \\
\hline • & . & ' & . & . & . & 1000 \\
\hline • & - & ' & - & - & - & \\
\hline - & - & . & . & - & . & \\
\hline • & • & ' & . & . & . & \\
\hline - & • & . & - & . & . & \\
\hline , & . & $\cdot$ & - & * & • & \\
\hline
\end{tabular}

cN-540

colvi

coivi

an-542

$10 / 11 / 9$

$0 / 12 / 95$

$10 / 46 / \%$

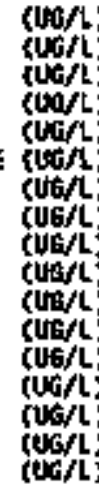




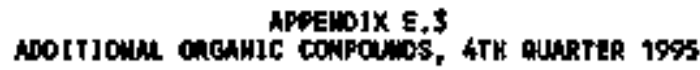

SNPLING POLHI

LOChTLON

ACRYLONITRILE

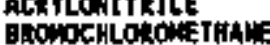

2-CHLOROETHYLIUYL ETHER

DreRow ONEIMANE

1, z-OJCHLOROBEMZENE

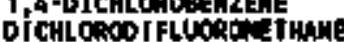

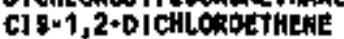

$14+0 j$ CHLORD-2-DUTEK

$1,4+0 j \mathrm{Cr}$

ETWL METHACRYLATE

$1000,1,2+$ TETRACHLOROETHAN:

1,2,3-T, 1 chLoROPAOPAHE

\section{ACROLE]}

GH-543 CH-S44 GU-546

CoLVI COLVI CoLY

6N-55T

10,07t

cur560

cturis

.

(U⿴囗十)

(UG)

(uent)

(UGA)

(uib/

(USD)

(UG/)

(U6/t)

(낙ㄱ

(UF/

(U6/

(U6/)

(U6/

(U6/)

$\begin{array}{rr}500 & 500 \\ 500 & 504 \\ 100 & 100 \\ 100 & 100 \\ 50 & 50 \\ 100 & 100 \\ 100 & 100 \\ 100 & 100 \\ 50 & 50 \\ 100 & 100 \\ 50 & 50 \\ 100000 & 100000 \\ 50 & 50 \\ 50 & 50 \\ 100 & 100 \\ 50 & 50 \\ 50 & 50\end{array}$

$\begin{array}{rr}\text { CDLV1 } & \text { COLY] } \\ 10 / 17 / 9 S & 10 / 11 / 95\end{array}$
CWN+562

cotvI

04-564

선ㄷㄷ

cill-60:

텨-609

44) +610

$10 / 06 / \%$

su

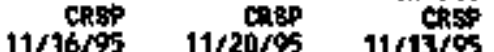

$:$
$:$
$:$
$:$
$:$
$:$


ADOITIONAL OROWIC COApIX E.3

SAHPLJMG PDIST

LOCATIOU

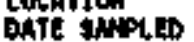

Acratsin

ACRrLOWITRIL

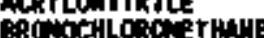

〈UG/L)

ER UG/L)

$1,2+01 \mathrm{Brom}_{0.3}$ -

1.2-bjchlonotanzene

1-05ch

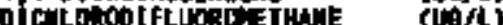

CIs-t 2-btchLO

1+Bich teO-2-日utEUE

ETWTE METWACRTUATE

OoOnetwame

$1,1,1,2-T E$ TRACALOROETHAHE

TRTCMLGOFLUALOMETHAHE

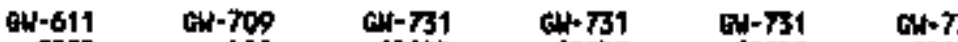

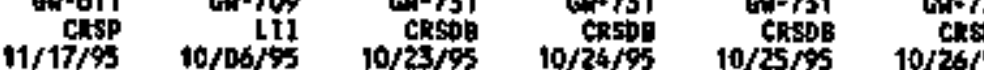

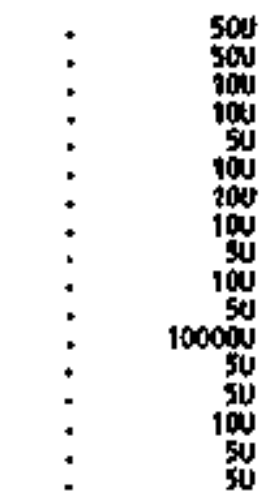

(16)

(us/r.

(UG/t)
64-752 ay-72 6N-732

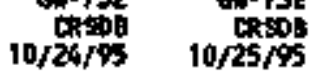

a)- 72

chiso: 
APpentot $x$

\begin{tabular}{|c|c|c|c|c|c|c|c|c|c|c|}
\hline $\begin{array}{l}\text { SANPL1NG POINT } \\
\text { LOCATIOH } \\
\text { OATE SAMPLEO }\end{array}$ & & $\begin{array}{r}\text { wi-742 } \\
\text { Crsp } \\
11 / 09 / 95\end{array}$ & $\begin{array}{r}c 4-743 \\
\text { cksp } \\
11 / 13 / 95\end{array}$ & $\begin{array}{r}01+757 \\
\text { 10/06/95 }\end{array}$ & $\begin{array}{r}6 \mathrm{w}+796 \\
10 / 0 \mathrm{y} / 95\end{array}$ & $\begin{array}{r}t+107 \\
t 0 / 08 / 95\end{array}$ & $\begin{array}{r}\text { ch-79: } \\
\text { chtvt l } \\
10 / 0 \text { stos }\end{array}$ & $\begin{array}{r}0 w-799 \\
\text { Lv } \\
10 / 07 / 95\end{array}$ & $\begin{array}{r}\text { 0.4-601 } \\
10 / 09 / 45\end{array}$ & $\begin{array}{r}\text { cy-az7 } \\
\text { colyt } \\
10 / 96 / 95\end{array}$ \\
\hline 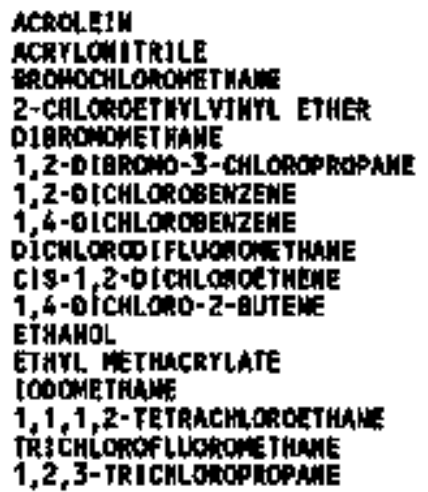 & 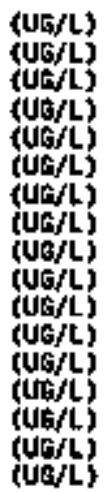 & : & $:$ & 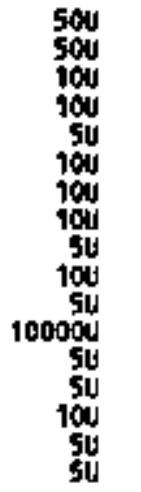 & & $\begin{array}{r}500 \\
500 \\
100 \\
100 \\
50 \\
100 \\
100 \\
100 \\
50 \\
100 \\
500 \\
10000 \\
50 \\
50 \\
100 \\
50 \\
50\end{array}$ & $\begin{array}{r}500 \\
500 \\
100 \\
100 \\
500 \\
100 \\
100 \\
100 \\
50 \\
100 \\
50 \\
100000 \\
50 \\
50 \\
100 \\
50 \\
50\end{array}$ & & 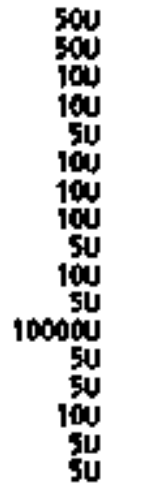 & $\begin{array}{c}: \\
100 \\
100 \\
100 \\
100 \\
100 \\
10 \pm \\
\vdots \\
100 \\
:\end{array}$ \\
\hline
\end{tabular}


APPENDIX E.4

FIELD MEASUREMENTS

AND MISCELLANEOUS PARAMETERS 


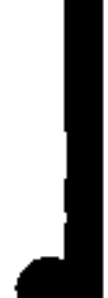




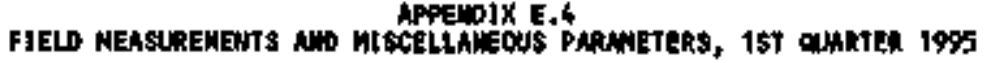

SNAPLIME POINT LOCATION

DATE SANPLED

FIELO MEASLETHERTS

STATIC WHTER LEVEL (FT, GeLCH TOC) WhTER LFVL ELEY, 〈FT, HEONE MSL PH FIEL ANT Cinim DIscolveo ox

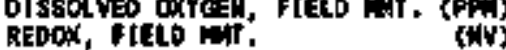

NISCELLLAMFOUS PARMMETERS

BRONTDE

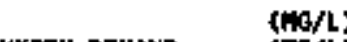

CIEXICAL OKYEEM DENAHD

EYAHIDE

PHENOLS

PH, AEP.

PH, REP. 3

Coing

CONHUCTIYY, DEP,

(3)

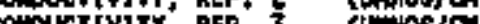

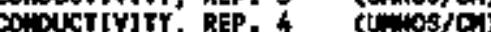

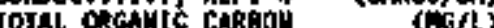

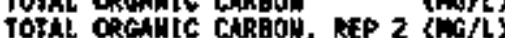

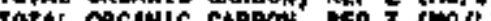

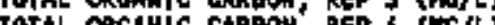

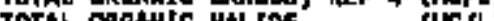

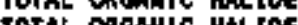

TOTAL CROANIC HLLCE, REP 2 (WO/L

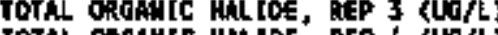

TOTAL ONOAnIC HAIDE, REP 4 (UG/)

orssol sal

rotal sJsPethed sol tos

$(n-1)$

TURB]DITY

(WNU) an-541 G-142

G-142 Gin-143

6.) 163

데 144

ank
$01 / 1495$

ch-144

a4- 165

cur- 145

$6+146$

$03 / 20 / 9 \% \quad 01 / 17 / 9$

$03 / 22195$

$0 t / 18 / 95$

$03 / 24 / 95$

01/17/95

6N+946 $3 / 23 / 95$

$\begin{array}{rr}+94.6 & -133.7 \\ 1091.5 & 836.65 \\ 14.7 & 13.8 \\ 7.3 & 7.8 \\ 414 & 325 \\ 6.5 & 0.5 \\ 166 & 109\end{array}$

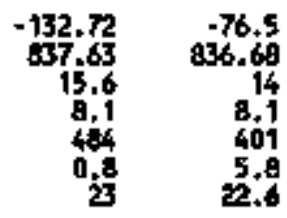

-77.36
935.99
14.2
6
266
10.9
127

-77.75
25.56
16.1
326
5.5
439

-2.47
837.97
14.3
87
476
9.5
170

-3.17
836.87
16.4
8.1
512
7.1
207

$\begin{array}{rr}-96.35 & -113.39 \\ 741.01 & 724.77 \\ 13.8 & 15.3 \\ 9.1 & 7.9 \\ 460 & 575 \\ 8.8 & 0.8 \\ 43 & -39\end{array}$

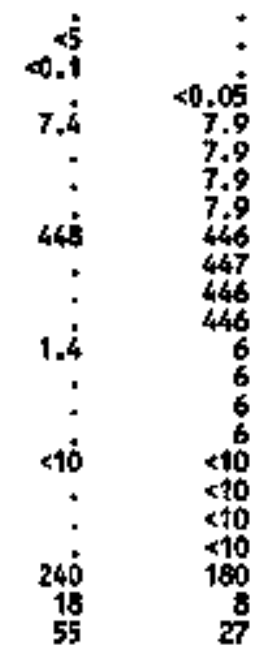

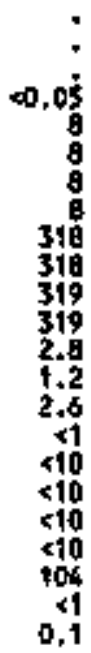

$\vdots$
0.05
8.1
6
8
0.1
$5 \times 9$
532
531
532
1.1
1.8
4.1
41.4
11.4
40
20.2
226
5.2
5.2

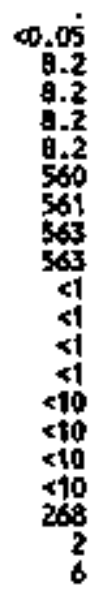




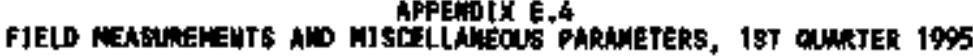

SHPLIM Aojtit

LOCATIAN

DATE SHPLED

F1ELO MEASUHEMENTS

STAE IE WAEA LEVL (FT. BELON TOC) WATER LEVEL ELCV, (FT, ABONE WhTER TEP, FI

Ph, F

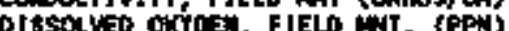
AEDOX, FJEL WIT. (IV)

M]SCELLAMEOUS PAANHETERS

Becmitos

CHEHICAL AXYEEH OENAWD

Crawide

PHEFOL

Py

PH, AEP. 2

PH, NEP, 3

COHouctivitr

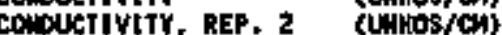

CONDCTHVITY, REF. 3 CUtOS/CH,

CONOUCTIVITY, REF, 4 (UnHOS/CN)

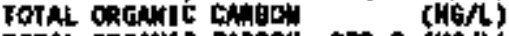

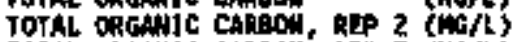

TOFAL DROAHJC CAPBON, REP 3 ( $\mathrm{m} / \mathrm{L}$

TOTAL OAGANIC CARBOH, REP \& ( $\mathrm{MJ} / \mathrm{L})$

TOIAL OROAHJC HALIDE RT (WOFL

TOTAL ORGATC HALIOE, REP 2 (NOS $/ \mathrm{L}$ )

TOTAL ORGAHIC MALIOE, REP 3 (NG/L)

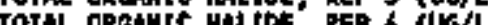

Thato sol

tothe suspemed solos

TOAAL SUSPEMED SOLIDS (

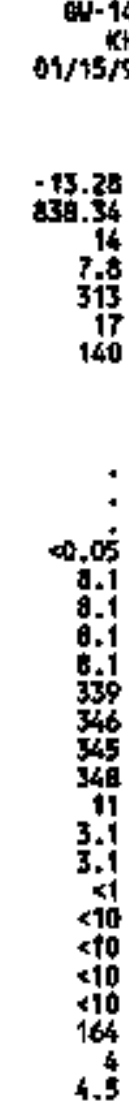

\begin{tabular}{|c|c|}
\hline $\begin{array}{r}\text { Git-156 } \\
\text { cospa } \\
01 / 13 / 95\end{array}$ & $\begin{array}{r}\text { U1-15d } \\
\text { CRSDB } \\
01 / 20 / 95\end{array}$ \\
\hline $\begin{array}{r}-139.89 \\
909.21 \\
13 \\
7.5 \\
590 \\
0.4 \\
208\end{array}$ & $\begin{array}{r}-47.45 \\
955.6 \\
13.3 \\
7.4 \\
409 \\
4.3 \\
177\end{array}$ \\
\hline
\end{tabular}

6) 159

कN-175 an- 177

CRSP
$02 / 13 \% 5$

nN-1 1

ต4-217

61-231 GH-24) $02 / 14 / 95$

01/24/95

$01 / 04 / 95$

$01 / 15 / 95$

$01 / 20 / 4$

\begin{tabular}{|c|c|c|}
\hline $\begin{array}{r}18.25 \\
32.95 \\
13 \\
7.6 \\
37 \\
10.2\end{array}$ & $\begin{array}{r}-117.55 \\
96.45 \\
10.1 \\
7.6 \\
4.5 \\
9.7 \\
167\end{array}$ & $\begin{array}{r}-117.27 \\
1040.73 \\
11.1 \\
0.2 \\
540 \\
17.8\end{array}$ \\
\hline
\end{tabular}

$\begin{array}{rrr}-112.8 & -10.35 & -45.1 \\ 104.1 & 839.12 & 937.54 \\ 11.4 & 14.5 & 12.4 \\ 8 & 7.2 & 7.8 \\ 275 & 295 & 2.7 \\ 10.7 & 7.6 & 5.2 \\ 200 & 116 & 186\end{array}$

9.7
159.9

203

to6

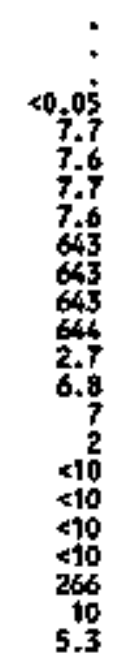

5
0.05
7.9
7.9
7.9
7.9
347
346
347
354
1.2
2.5
3.6
3.5
410
410
410
10
108
8
5

0.5
$<0.1$
0.2
$\vdots$
306
$\vdots$
$\vdots$
$\vdots$
$<10$
$\vdots$
100
3.9

5
0.05
7.8
7.5
7.5
7.5
326
329
327
5.9
3.1
1.6
1
$\times 10$
$\times 10$
410
$\times 10$
146
1.4

\section{b.}

$\begin{array}{rr}8.1 & 6 . \dot{2} \\ 32 i & 306 \\ \vdots & \vdots \\ \vdots & \vdots \\ \vdots & <10 \\ 104 & 10 \dot{5} \\ 7.5 & 8.9\end{array}$

$<.05$
7.9
7.9
7.9
7.9
2.5
2.3
2.3
2.5
7.6
3.4
1.4
3.5
210
510
410
210
136
5.5 


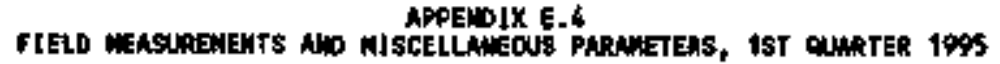

SHeLtes POINT

LOCATIOU SANFL

FTELD HEASHEMEATS

SThTEC MIER LEVEL (FT, BELON FOC) WHTE LEVEL ELEV. (FY. ABONE HS) WTER lEVE ELEV. (FT. ABOHE HA.) po kieto inr. che colscotiven DISSOLED OXYEE, FIEL WAT. (PPN)
REDOX, FIELD WN.

M1sceltameol's patdicters

CHINICAL OCYCEU DERTO CYANIDE Evols

PH, AEP, 2

PH, REP. 3

PH, REP. +

POMn

contuct tvity, aEp. 3

contertyt Ty ap.

Torm onchulc chapo

TOTAL ONCANC CAROON, REF 3 (MB/L)

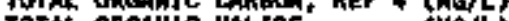

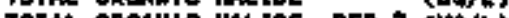

JOFA ORaANIC HALIDE, REP 2 (Uh/L)

TOTA ORGNIC HALIOE, REP 3 (UGJ)

TOTM Onganic kXLIOE, REP 4 (LS/L)

Dissol sulsos

(16:L/L)

TURBiottry

(NTO) 6u-305 Cut-30

CRSOB CASD

GN-305

L1V

4-321

$01 / 10 / 95$

64-511

cur-521

01700705

6N+522

01/ 10\%

64-539

61-60: $02 / 02 / 95$

cill-609

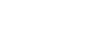

-02.53
924.67
13
7.7
604
4.7

$\begin{array}{rr}.117 .2 & -123.16 \\ 928.3 & 1050.44 \\ 13.7 & 14.1 \\ 7.7 & 8.7 \\ 394 & 222 \\ 11.4 & 9.3 \\ 236 & 162\end{array}$

$\begin{array}{rr}.21 .05 & -100.43 \\ 904.53 & 904.77 \\ 9.5 & 11 \\ 8.5 & 6.5 \\ 162 & 222 \\ 6.9 & 12 \\ 175 & 152\end{array}$

-67.4
1005.3
13.7
270
174

-06.32
107.89
7.7
253
8.0
203

-106
997
10
7.8
325
6.5
123

$\begin{array}{rr}+126.4 & -167.23 \\ 947.6 & 846.87 \\ 13.8 & 13.4 \\ 7.8 & 7.7 \\ 297 & 345 \\ 8.9 & 5.9 \\ 197 & 179\end{array}$

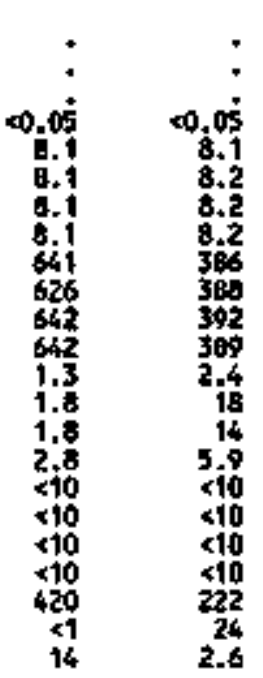

$r 5$
0.1
8
$\vdots$
295
$\vdots$
12
$\vdots$
$\times 10$
$\vdots$
118
6.7

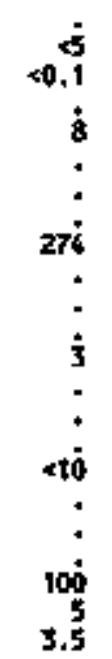

4
0.1
8.2
$\vdots$
368
$:$
41
$\vdots$
40
$\vdots$
40
70
34

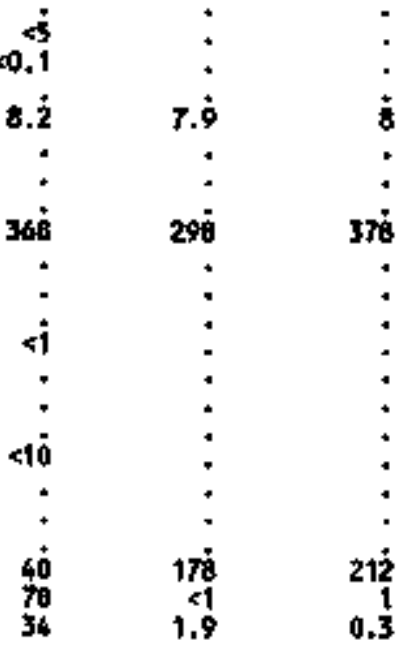




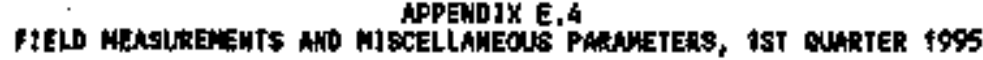

SANLIHE POLTT

LOCATJON

DATE SHMPLEO

FIELD RASURET:ENTS

STATSE WHER LEVEL (FT. BELOW TOC) WATER LEVEL EEEV. CFT. AEONE WOLS PH, FIELD inT.

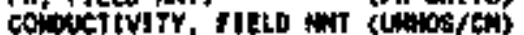

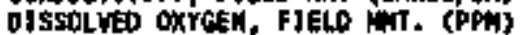

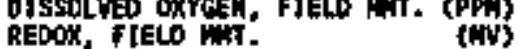
(av) 130

HISCELLAMEOUS PARHETERS

BDONIDE

\section{CTANIDE} PIEINOLS

PH

PH, REP, 2

PA, REP. 3

DA REP, 6

DOHWLCT JVIY, AEP.

CONDUCT JVITY, MEP: 3

CONDUCT JVITY, AEP. 4

Toth orcentc chroot

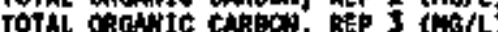

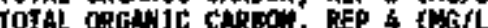

TOTAL GRGNic HALIDE fUo/

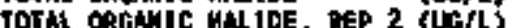

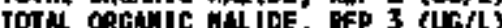

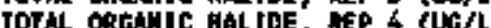

DI580I VEo sol Jos

TOT STISPERED sol tos

(Wots)

TURBDDITY

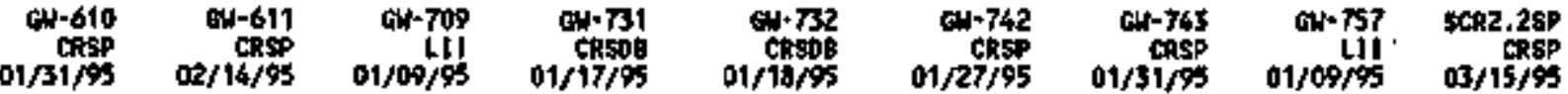

$\begin{array}{rrrr}-78.9 & -96.87 & -28.74 & -124.4 \\ 900.5 & 949.53 & 877.86 & 926.8 \\ 13.2 & 11 & 12.5 & 13.6 \\ 7.7 & 7.4 & 6.4 & 10.1 \\ 302 & 478 & 290 & 261 \\ 139 & 10.1 & 6.8 & 5.8 \\ 139 & 172 & 175 & 159\end{array}$

$\begin{array}{rr}+155.95 & -126.25 \\ 908.15 & 972.75 \\ 13.5 & 11.5 \\ 8 & 8.1 \\ 307 & 29 \\ 7.4 & 9.2 \\ 130 & 104\end{array}$

-126.3
976.1
73.3
8
258
8.6
165

83.77
67.66
14.5
8.2
27
6.4
$11 \%$

11
6.7
570
6.9

\begin{tabular}{|c|c|c|c|}
\hline V & - & & . \\
\hline$=$ & $\star$ & $<0.1$ & , \\
\hline 7.8 & 7.7 & A & $<0.05$ \\
\hline- & 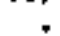 & . & g \\
\hline • & - & . & 9.1 \\
\hline 633 & $540^{\circ}$ & $33{ }^{\circ}$ & 231 \\
\hline * & • & . & 234 \\
\hline - & - & - & 237 \\
\hline : & • & 8 & $<1$ \\
\hline - & . & 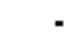 & $<1$ \\
\hline - & . & * & 2.1 \\
\hline • & - & 10 & 2.1 \\
\hline & 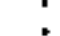 & 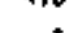 & $<10$ \\
\hline & 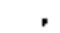 & * & $<10$ \\
\hline 210 & $27 \dot{s}$ & $15 \hat{0}^{\circ}$ & $<t 0$ \\
\hline & $<$ & $\times 1$ & 24 \\
\hline & & 0.9 & 76 \\
\hline
\end{tabular}

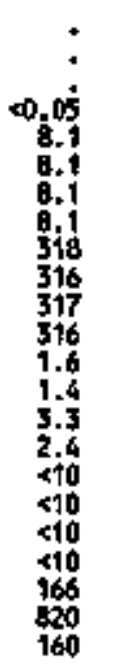

$\vdots$
7.6
345
$\vdots$
$\vdots$
$\vdots$
189
2
15

$\vdots$
7.9
$\vdots$
294
$\vdots$
$\vdots$
$\vdots$
186
1.4

0.5
0.5
$\vdots$
285
$\vdots$
7.8
$\vdots$
40
140
2.3

7.9

316

224
3.4 
APPENDIX E-4

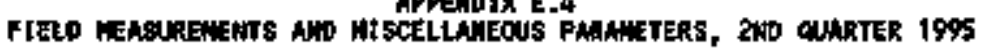

SNAPEIW POIBT

LOChTton

DATE SWPLLt

FIELU MEASUREMENS

STRTIC MATER LEVEL (FT. HELOW TOC) NATE LEVES ELEV (FT HOWE HSL? WATE LEVET ELEV (FT, ABOWE HSL) PH, FTELD BNT. PH, FIELD

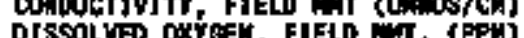

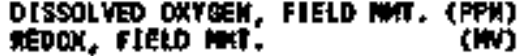

Mrsceqtitureous phodveters

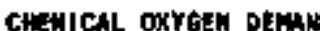

\section{Cranin}

PN, AEP. 2

PW. REP. 3

POin, REP. 4

COADCrivity

con

COYDUCTIYITY, REP. 4

TOTAL ORgivité chation

REP 2 (NB/L)

REP 3 (WT/L)

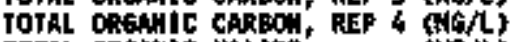

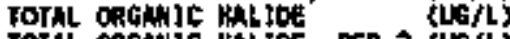

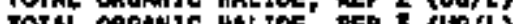

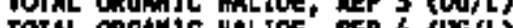

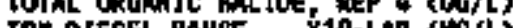

TPF OrESEL RANG XIO-LA KW/L

FOTAL EUSPENDE SOLDS

(nist)

TuRstoitY
$04 / 20$

cos-1

GU- 142

Etw+ 143

口N+144

侜-145

$\lim _{140}$

[5N+146

$04 / 20 / 25$

$04 / 26 / 95$

$04 / 25 / 95$

$04 / 26 / 95$

$04 / 24 / 95$

C.N+147

GN-156

CNit-15s

Cropo

-63.97
1039.93
18.2
7.5
968
9.7
124

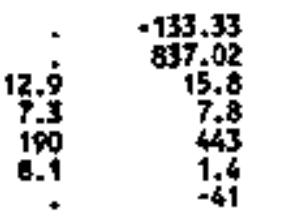

70.35
834.83
14.2
8.2
481
3.7
-19

-78.65
83.69
15.2
7.9
317
3.9
166

-4.44
035.6
16
8
516
4.9
152

-154.7
653.41
13.4
8.4
4.5
6.4
133

-15.5
035.77
15.2
6.2
305
143

$\begin{array}{rr}-142.72 & .45 .51 \\ 906.38 & 957.54 \\ 17 & 15 \\ 7.4 & 8 \\ 735 & 321 \\ 5.3 & 916 \\ 205 & 937\end{array}$

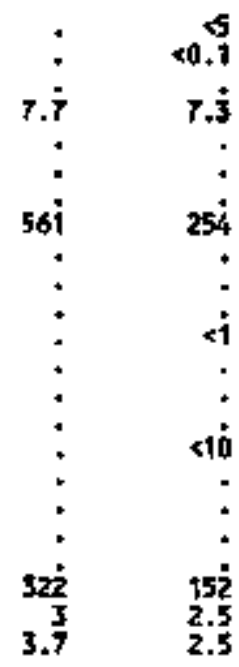

0.05
0.1
8.1
8.1
8.1
474
4.5
475
475
3
3
3
4.5
410
17
$\times 10$
$\times 10$
275
3
9.9
0.05
8.1
8
8
8
512
510
512
512
2.7
4.5
7.3
4.1
410
210
510
410
276
22
4.2

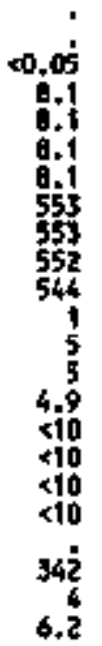

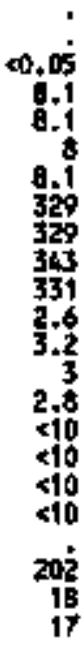

5
20.05
7.5
7.4
7.5
619
630
621
621
13
16
19
22
$\times 10$
210
410
$\times 10$
300
3.8

40.05
7.8
7.8
7.8
7.8
335
334
73
334
2.3
2.8
4.6
2.2
510
$<10$
510
$<10$
190
1.4 


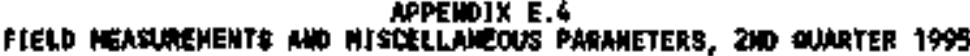

SAHPLIMO POJNT

LOCATION

DhiE SWPLED

FIELL MEASLRELENTS

STATIC WATER LEVEL (FT. BELOU TOC) LATER LEVEL ELEV. (FT. ABOVE HST)

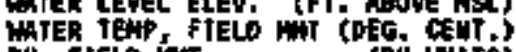
PH, FIELO INT.

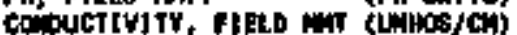

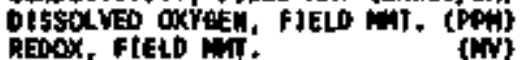

MISCELLHEOUS PARAMETERS

CHEHTCAL OXYESM DEMAND Crakitoe

Prainces

PH, REP, 2

PH, REP, 3

PH, REP. 4

CONOUCTYYTY, REP.

COMUCFVIT, REP.

ToTAl oichilc cakeoj

MEP 2 (WO/L)

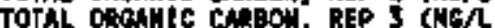

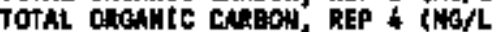

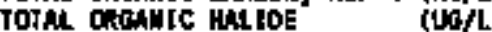

TOTAL ORtGMIIC HALIDE, REF 2 (UEAL)

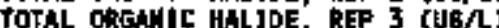

TOTAL Oncente HALIOE REP 4 (UTH

TPI DIESEL RAHE $\times 10-10 \mathrm{~B}$ (HOR)

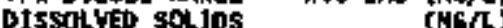

Toral strpentes sol

TURA]0ITY

\begin{tabular}{|c|c|c|}
\hline $\begin{array}{r}\text { y-159 } \\
\text { cksos } \\
\text { o4totos }\end{array}$ & 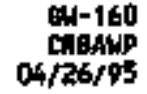 & $\begin{array}{r}\text { CN-161 } \\
\text { CRP/15p } \\
04 / 20 / 95\end{array}$ \\
\hline $\begin{array}{r}+116.52 \\
934.85 \\
14.9 \\
7.6 \\
370 \\
3.2 \\
132\end{array}$ & $\begin{array}{r}-148 \\
945 \\
15.2 \\
7.4 \\
458 \\
7.5 \\
118\end{array}$ & $\begin{array}{r}-160.1 \\
933.4 \\
15.8 \\
7.6 \\
376 \\
73.4\end{array}$ \\
\hline
\end{tabular}

en- 17

$6+17$

Git-181 $\quad \mathrm{N}+12$

c4. 186

n+1 187

$05 / 02 / 95$

6x-108 05/11/\%5 05/02195

$05 / 01 / 9$

$05 / 03 / 95$

$05 / 01 / 25$

19.36
64.64
16.7
8.2
418
8.3
947

$\begin{array}{rr}-117.15 & -106.15 \\ 1090,55 & 96.85 \\ 17.9 & 14 \\ 7.9 & 7.9 \\ 465 & 316 \\ 1.7 & 16.8 \\ 117\end{array}$

-119.23
817.4
14
7.7
394
2.6
168

-14.2
817.12
16.5
7.1
69
0.4
.76

-16.75
816.05
15.4
7.6
938
0.6
.290

-20.6
816.69
15.6
0.1
467
4.7
.104

\begin{tabular}{|c|c|c|c|}
\hline & $<0.1$ & $\begin{array}{r}5 \\
<0.1\end{array}$ & : \\
\hline $\begin{array}{r}0.05 \\
7.9\end{array}$ & 7.6 & 7.7 & 8 \\
\hline 7.9 & & ‘ & : \\
\hline & & ' & : \\
\hline & 437 & 373 & 399 \\
\hline 370 & - & ' & * \\
\hline 378 & 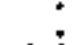 & 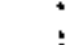 & $\vdots$ \\
\hline & 4.6 & $<1$ & . \\
\hline$<1$ & * & * & " \\
\hline+1 & & 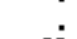 & 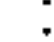 \\
\hline 0 & रा & $<10$ & \\
\hline & - & . & • \\
\hline 10 & : & : & • \\
\hline 26 & $40 \dot{2}^{\circ}$ & $22 \dot{8}$ & $27^{\circ}$ \\
\hline & $\begin{array}{l}117 \\
260\end{array}$ & $\begin{array}{l}<1 \\
0.7\end{array}$ & 1.4 \\
\hline
\end{tabular}

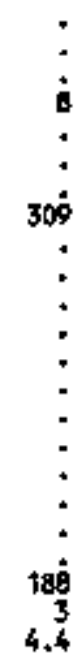

$\vdots$
7
387
$\vdots$
$\vdots$
$\vdots$
$\vdots$
252
1.5

7.
$\vdots$
854
$\vdots$
$\vdots$
$\vdots$
535
6.4

8.2

$\vdots$
$\vdots$
435
$\vdots$
$\vdots$
$\vdots$
276
4
2 


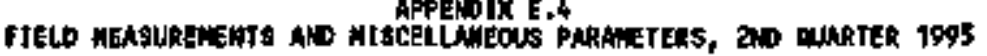

SAPLING DOINT

tOCAT 에

DATE SAMPLED

FtELO MASARETENTS

STATIC WAFR LEVEL (FT, BELON TOC)

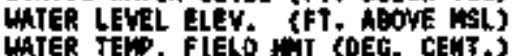
OII FJELD WrT. (PH UNITS)

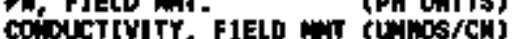
DISSO VED OXÝCEA, FIELD MIT. (PFM) D]SFOL VED ONYEE, FIELD IMT. (PPH)

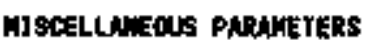

CHEAICAL OXYOEM DEKAMO cranje

Pris

PH, REP. 2

PH, REP. 3

PH, REP, 4

conotictivity ats

conding, Res

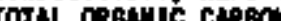

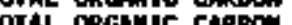

TA

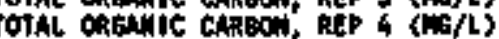

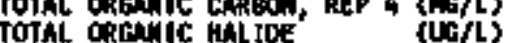

TOTAL OReAtic HALIDE, AEP 2 (WO/L)

TOTAL ORENHIC HALIOE, REP 3 (WO/L)

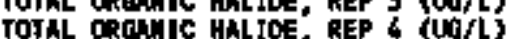

TPH DIESEL RAMOE X10-LN (WH'L

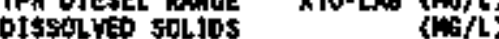

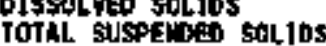

(mo/h)

TuRbibity
611-203

Uimes

$04 / 18 / 95$

64+221 :J-231

0.231
1210

64-261

0400105

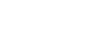

$\begin{array}{rr}r 5.1 & -74.45 \\ 1050.2 & 1029.55 \\ 96 & 16.5 \\ 0.1 & 346 \\ 257 & 3.4 \\ 5.5 & 3.4\end{array}$

$\begin{array}{rr}-76.69 & -13.55 \\ 1029.32 & 45.92 \\ 17 & 13.9 \\ 8 & 7.7 \\ 268 & 280 \\ 6.9 & 2.1 \\ 157 & 190\end{array}$

-45
937.64
16.3
7.7
253
6.3
.00

+112.5
960,25
13.4
7.5
530
6.9
203

-195.2
968.7
13.8
7.8
451
5.6

-97.75
985.65
7.9
73
438
9.4
206

$\begin{array}{rr}-143.2 & -117.75 \\ 949.51 & 975.25 \\ 14.9 & 14.6 \\ 8.4 & 7.8 \\ 836 & 2000 \\ 33 & 7.9 \\ -116 & 202\end{array}$

$15 i$
5.5
7.1



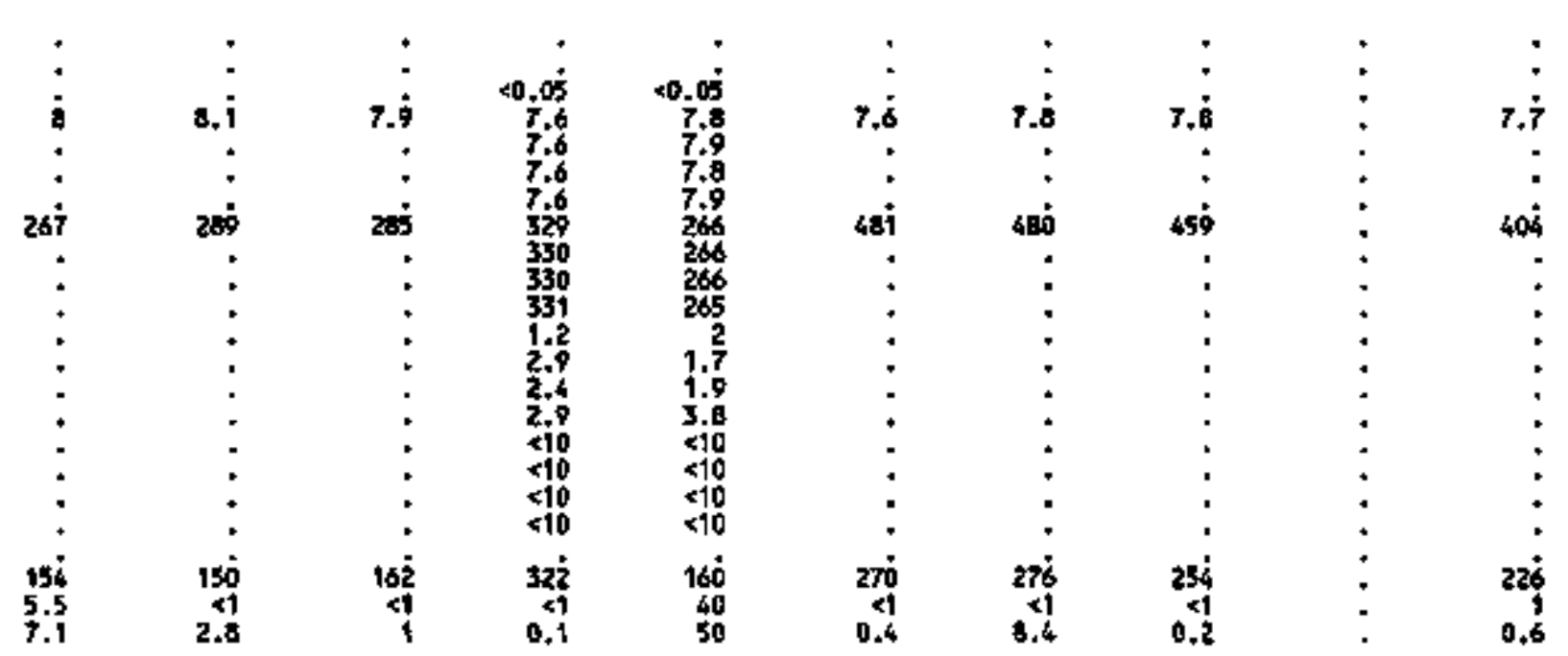

a $\quad 20+293$ an-29\%

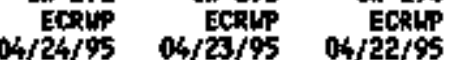

Giv-295 CR:alp

매-296

07279 


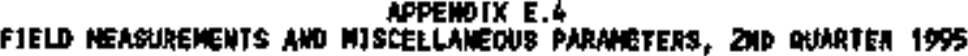

SHet IMG POLINT

LCA'TOW

DArt: akypleo

FIELD MEASUREGEUTS

STAY IC MIET LEVEL (FT. BEL TOC) WATER LEVEL ELEN, (FT. ATOWE HSL) MATER TEN, FIELD RNT QDEG. CEHT.

PH, FTELD :

PH, FIELD Git.

DISSOLVE OXFOEN, fIELO INIT. (PPW)

REDOK, FIELD WNT,

(Wv)

NISCELLUHEOUS PARNTTERS

thencCal farten denung

CYAntDe

PHElO

PH, REP.

PH, REP. 3

PH, REP,

DEF. 2 (WNOOS/CW)

cONDLYIVITY, RE, 3 (UNHOS/CW)

crit onte

Tor a $(H / L)$

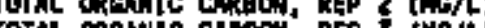

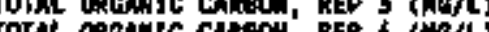

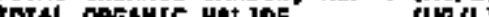

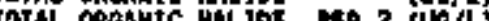

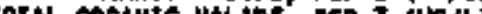

TOT

D

DISSOLVto SOLjos

TOTAL SUSPEEDEO SOLIDS

$(+N / L)$

inll.

\begin{tabular}{|c|c|c|c|c|c|c|c|c|c|}
\hline 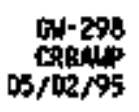 & 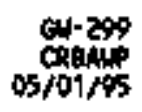 & $\begin{array}{r}0,300 \\
04 / 27 / 68\end{array}$ & $\begin{array}{r}\text { CW-301 } \\
\text { CRBdIP } \\
04 / 27 / \% 5\end{array}$ & $\begin{array}{r}64-302 \\
\text { uncs } \\
04 / 19 / 5 s\end{array}$ & $\begin{array}{r}\text { Gu-303 } \\
\text { cRsod } \\
0, / 09 / 93\end{array}$ & $\begin{array}{r}\text { cy-304 } \\
\text { ckspe } \\
\alpha / 06 / 9 s\end{array}$ & $\begin{array}{r}04-321 \\
04 / 25 / \%\end{array}$ & $\begin{array}{r}0.339 \\
\text { unuc5 } \\
04 / 19 / 85\end{array}$ & $\begin{array}{r}0+511 \\
\text { cksp } \\
05 / 03 / \% 5\end{array}$ \\
\hline $\begin{array}{r}-109.3 \\
939.7 \\
13.4 \\
8 \\
316 \\
2.7 \\
179\end{array}$ & $\begin{array}{r}-97.15 \\
956.75 \\
14.4 \\
7.8 \\
25.6 \\
5.2 \\
67\end{array}$ & $\begin{array}{r}110.12 \\
962.9 \\
14.5 \\
7.4 \\
304 \\
5.5 \\
235\end{array}$ & $\begin{array}{r}-132.65 \\
953.75 \\
17.9 \\
8.1 \\
342 \\
9.5 \\
155\end{array}$ & $\begin{array}{r}-100.92 \\
1040.78 \\
16.4 \\
7.6 \\
502 \\
4.9 \\
112\end{array}$ & $\begin{array}{r}-86.92 \\
920.29 \\
15.0 \\
7.7 \\
694 \\
0.5 \\
.121\end{array}$ & $\begin{array}{r}-117.24 \\
928.26 \\
14.8 \\
8.1 \\
370 \\
3.8 \\
158\end{array}$ & $\begin{array}{r}+18.15 \\
907.43 \\
14.5 \\
8.1 \\
28 \\
4.6 \\
159\end{array}$ & $\begin{array}{r}-74.35 \\
1050.25 \\
16.6 \\
7.4 \\
5.6 \\
3.2 \\
12.9\end{array}$ & $\begin{array}{r}-105.78 \\
997.42 \\
14.3 \\
8.2 \\
313 \\
19.6 \\
157\end{array}$ \\
\hline
\end{tabular}

\begin{tabular}{|c|c|c|}
\hline $40 . t$ & $\begin{array}{r}8.9 \\
<0.9\end{array}$ & $\begin{array}{r}<5 \\
<0.1\end{array}$ \\
\hline 0.2 & 8 & 7.5 \\
\hline - & - & - \\
\hline • & • & ' \\
\hline $31 \frac{1}{3}$ & 261 & $260^{\circ}$ \\
\hline • & • & • \\
\hline ¿ & " & * \\
\hline$\dot{2}$ & सं & 2.7 \\
\hline • & " & - \\
\hline • & $\vdots$ & - \\
\hline סוא & ס艹 & <10 \\
\hline • & • & • \\
\hline$\star$ & 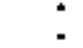 & 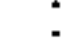 \\
\hline $\begin{array}{r}\text { tod } \\
190 \\
90\end{array}$ & $\begin{array}{l}270 \\
<1 \\
0,6\end{array}$ & $\begin{array}{r}166 \\
<1 \\
1\end{array}$ \\
\hline
\end{tabular}

\begin{tabular}{|c|c|}
\hline$\times 5$ & * \\
\hline$\dot{8}$ & 7,5 \\
\hline , & • \\
\hline ' & - \\
\hline $300^{\circ}$ & 528 \\
\hline - & • \\
\hline : & " \\
\hline 2.5 & , \\
\hline t & . \\
\hline V & . \\
\hline فं & . \\
\hline . & - \\
\hline V & • \\
\hline & \\
\hline $\begin{array}{r}192 \\
23 \\
25\end{array}$ & $\begin{array}{r}188 \\
2 \\
18\end{array}$ \\
\hline
\end{tabular}

0
0.05
7.8
7.8
7.8
7.8
648
648
647
649
3
1.4
2.5
$\times 10$
$\times 10$
$\times 10$
$<10$
446
11
13

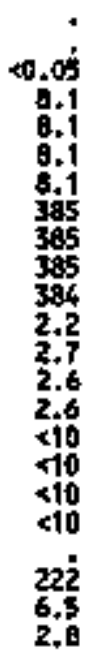

8.1
238
$\vdots$
1.8
$\vdots$
$<0$
$\vdots$
140
0.1

$7 . \dot{5}$

•.j.

sos

273

.

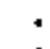

,

(1)

is

268 
APPEIDIX E.4

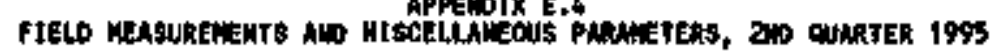

SAYPL1HG POHAT

LOCATIOH

FJELD NEAGRERTS

STATIC UATER LEVEL \{FT. BELON TOC) WATER LEVEL ELEV, (FT. ABOWE MSL WRTER LEVEL ELEV, KFT, ABONE N8L, PH. FIELo thr. cictive

(ains)

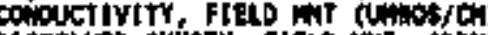

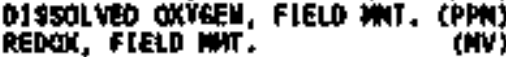

M]SCELLANEOUS PARANETERS

thenical orresy bertho CYANiDE PHEWOL

PH, REF.

PH, REP. 2

PH, REP. 3

PH, AEP.

conouctrity ats,

COMDUCTIVITY, REP. J

con

TOTAL onem CAm

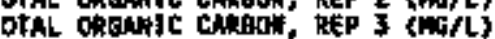

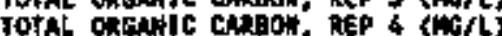

OTAL OROAIC MLIDE RE SUAL)

TOTAL GRoutc HLIDE, REF 2 CNOL

TOTAL GREAIC WLIDE, REF 3 (UO/L)

TOTA ORGAIC WLIDE REF 4 (W6/L)

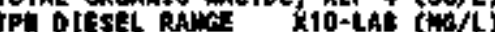

(No/L)

Tuspatoen solids

(n)

TURBIOJTY

(Nit)

\section{4บ-512}

0.4/26/

$04 / 25 / 9$

con-514

C니-54

Colv1

coly

ㅁ.N542

colvI

EN-543 CoLYI

an-54

Dovi

cN-546

colv1

GN-557

$04 / 06 / \%$

45.86
961.14
15.8
5
450
4.7
160

$\begin{array}{rr}-79.3 & -109 \\ 992.9 & 972.7 \\ 12 & 15 \\ 5.4 & 7.9 \\ 770 & 316 \\ 5.7 & 0.8 \\ 165 & 176\end{array}$

$\begin{array}{rrr}-21.9 & -21.8 & -21.10 \\ 979.7 & 978.6 & 0.02 \\ 14.8 & 16 & 15.8 \\ 7.2 & 7.8 & 7.8 \\ 290 & 343 & 362 \\ 5.4 & 5.7 & 3.6 \\ 220 & 165 & 1.32\end{array}$

$\begin{array}{rr}-79.35 & -65.15 \\ 992.75 & 993.25 \\ 15.2 & 14.8 \\ 7.7 & 9.1 \\ 374 & 331 \\ 3.9 & 2.1 \\ 148 & 1.32\end{array}$

-69.37
98.25
14.3
278
4.8
174

+63.35
960.45
14.7
7.4
417
5.5
143

0.5
7.8
$\vdots$
366
$\vdots$
$<1$
$\vdots$
410
$\vdots$
20.
0.2

0.1
7.8
$\vdots$
358
$\vdots$
$<9$
$\vdots$
$\times 10$
$\vdots$
300
0.5

0.1
7.4
$\vdots$
355
$\vdots$
3.4
$\vdots$
80
$\vdots$
20.5
1.3

$\times 0.1$
7.7
$\vdots$
44
$\vdots$
3
$\times 10$
$\vdots$
25
5

0.5
5
$\vdots$
44
$\vdots$
4
$\vdots$
40
$\vdots$
$\vdots$
262
1.5
2

45
$\times 0.1$
8.1
$\vdots$
200
$\vdots$
2.4
$\vdots$
410
$\vdots$
172
152

-109
972.2
75
7.9
316
0.8
176

45
40.1
7.9
$\because$
326
$\vdots$
$\vdots$
$\vdots$
$<10$
$\vdots$
190
5
4.4

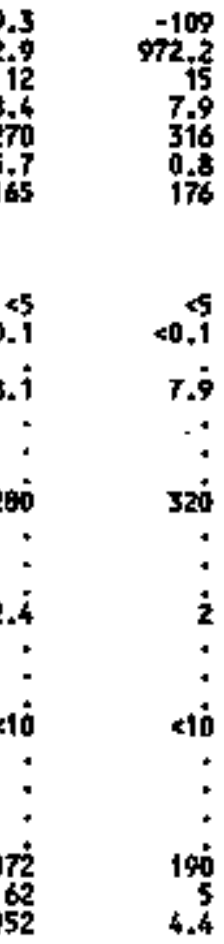

20

$\therefore \quad 3$

2
14 


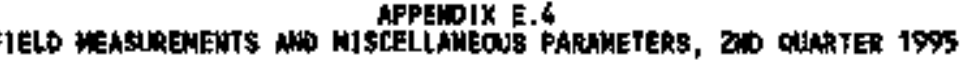

\begin{abstract}
SHePLInG POLNT
LOCATION
\end{abstract}

DATE SAPLED

FIELD REASARTENT

STAIJC WATER LFVEL (FT. EELON TOC) WATJR LEVET ELEV, (FT. ABOV NSL) WhTER TENP, FIELO WII (DSOC. CENT. PH, FIElP imT, OISSOLED OXYCEN FIELO RT. (PFW REOOX, FIELO WIT.

MISCELLMWOUS PARAMETERS

CHEHTCAL OXYGEN DEMAND cranjpe

PH

PH, REP.

PH, REP. 3

Chat, Ret 4

Contctivit REP, 2

CONDUCTIVITY, ALP; 3

CONDUCTEVITY, REP. 4

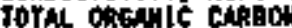

PEP ? (M,

ToTAL ORENUIC CANGOM, REP 3 ( $\mathrm{WG} / \mathrm{L}$ )

TOTAL DRENIC CARBOW, REP 4 (NG/L)

TOTAL OAGAnIC HalDE (Wo/L)

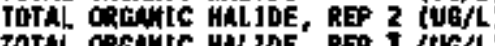

roThl Onevitc HuloE.

TOIAL DRCANIC HAL TDE

DISsalven sol tos

TOTAL SUSPEDE SOLIDS

TUR:10]TY

(no/l)

\begin{tabular}{|c|c|c|c|c|c|c|c|c|c|}
\hline $\begin{array}{r}64-560 \\
00 L v 1] \\
04 / 05 / 45\end{array}$ & $\begin{array}{r}\text { cid-56z } \\
\text { cotvi } \\
04 / 05 / 95\end{array}$ & $\begin{array}{r}\text { cu-564 } \\
\text { coLvi] } \\
04 / 06 / 45\end{array}$ & $\begin{array}{r}\text { or-609 } \\
\text { cossp } \\
\text { OS/11/5s }\end{array}$ & $\begin{array}{r}\omega N-609 \\
\text { CRsp } \\
\text { os/16/05 }\end{array}$ & $\begin{array}{r}04-610 \\
\text { CRsp } \\
\text { os } 107 / \% 5\end{array}$ & $\begin{array}{r}\text { N-611 } \\
\text { CRisp } \\
05 / 15 / 95\end{array}$ & 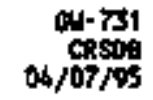 & $\begin{array}{r}\text { on-739 } \\
\text { consos } \\
04 / 07 / \% 5\end{array}$ & $\begin{array}{r}4 H-742 \\
05 / 07 / 5 s\end{array}$ \\
\hline $\begin{array}{r}-24.79 \\
910.13 \\
13.2 \\
7.6 \\
276 \\
6.5 \\
164\end{array}$ & $\begin{array}{r}-3.46 \\
931.03 \\
13.5 \\
359 \\
6.1 \\
152\end{array}$ & $\begin{array}{r}-10.02 \\
927.75 \\
14.6 \\
7.7 \\
263 \\
12 \\
164\end{array}$ & $\begin{array}{r}-131.8 \\
942.9 \\
15.5 \\
4.7 \\
294 \\
8 \\
96\end{array}$ & $\begin{array}{r}-966.87 \\
945.23 \\
16.2 \\
7.7 \\
436 \\
5.9 \\
151\end{array}$ & $\begin{array}{r}-82.43 \\
076.97 \\
15.1 \\
7.7 \\
3 \% 9 \\
5.9 \\
137\end{array}$ & $\begin{array}{r}-94.76 \\
40.62 \\
17 \\
7.9 \\
560 \\
9.1 \\
\uparrow 37\end{array}$ & $\begin{array}{r}-124.37 \\
924.23 \\
15.8 \\
9.1 \\
246 \\
3.8 \\
135\end{array}$ & $\begin{array}{r}157.45 \\
006.63 \\
16.3 \\
0.4 \\
314 \\
5.5 \\
114\end{array}$ & $\begin{array}{r}-15.8 \\
92.12 \\
14.2 \\
7.7 \\
3.6 \\
1.5 \\
160\end{array}$ \\
\hline
\end{tabular}

\begin{tabular}{|c|c|c|}
\hline $\begin{array}{r}5 \\
0,7\end{array}$ & 40.1 & $\begin{array}{r}14 \\
<0.1\end{array}$ \\
\hline 7.8 & 7.8 & 7.7 \\
\hline . & • & • \\
\hline ' & - & \\
\hline $2 \pi$ & 340 & 267 \\
\hline . & . & • \\
\hline ' & - & . \\
\hline 1.3 & $1 . \dot{8}$ & 1.5 \\
\hline . & - & , \\
\hline - & - & $\theta$ \\
\hline र & $\times 10$ & ه' \\
\hline * & - & • \\
\hline & - & \\
\hline $\begin{array}{r}0.0137 \\
140\end{array}$ & 0.0081 & 0,0716 \\
\hline & & \\
\hline
\end{tabular}

$:$
$\vdots$
279
$\vdots$
$\vdots$
$\vdots$
$\vdots$
$\mathbf{1 7}$
5.5

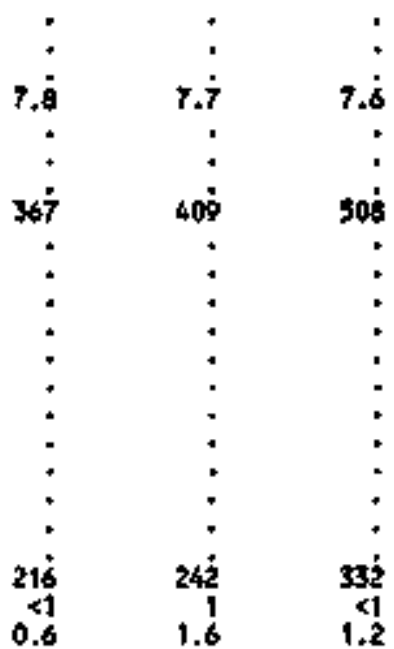

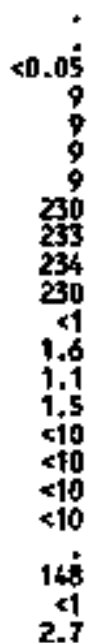

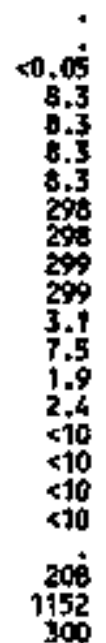

$33 \%$

.

$$
:
$$$$
:
$$

19

${ }_{<1}^{199}$ 


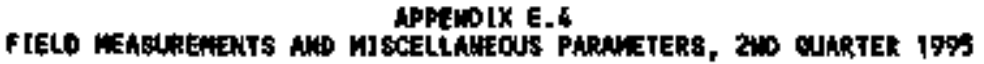

SNMPE ING POLHT

LOCATION SWIEO

FIELD REALULERERTS

STATIC MATER LEVEL. (FT. BELON TOC) WTATER IEVEL ELEV. (FT. ABONE NAS WATER TES FJEL WT CDEG. Cat. OH FIELO in. Ci ficl Cât DISSOLVE OXTGE, FJELD WII. (PPH) (PP)

Cor-743

ches

a)- 47

aty

can-790

colvt

c1) 799 $04 / 12 / 9$

-65.75
994.07
19.3
0.5
293
2.7

74.7
931.1
15.4
9.1
245
3.8
100

6h-801 $04 / 13 / 95$

CAH-207

cotvi

MISCELLMEOUS PNRUETERS

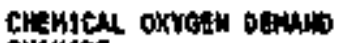
Crayioe

PHEMO

PH, REP.

PH, REP. 2

PH, 4

contcisytr

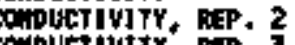

condouct ivity ReP. 4

rojat onowic tarbon

AEP 2 (N)

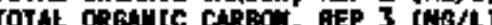

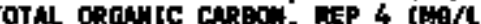

TOTA onctinc Hor IDE

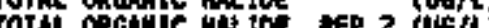

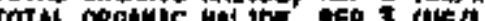

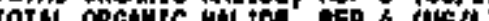

TPH DIESEL DAHCE

8souren rou

$\begin{array}{rr}-124.2 & -71.15 \\ 976.2 & 981.25 \\ 14.8 & 14.1 \\ 7.9 & 8.8 \\ 244 & 212 \\ 6.8 & 7.8 \\ 158 & 176\end{array}$
-8.98
972.11
15.3
6.1
242
160

-703.45
993.51
13.7
8.2
267
3.9

$\begin{array}{rr}71.15 & -65.75 \\ 91.75 & 99.07 \\ 14.1 & 19.5 \\ 8.6 & 0.5 \\ 212 & 29 \\ 7.9 & 2.7 \\ 176 & 150\end{array}$

\begin{tabular}{|c|c|c|c|c|c|c|}
\hline$\vdots$ & $<0 . t$ & 45 & $<0.1$ & $\infty .5$ & 80.7 & 40.5 \\
\hline$\dot{\boldsymbol{s}}$ & $0 . \dot{6}$ & 8 & $7 . \dot{8}$ & 7.9 & i & פ.9. \\
\hline • & 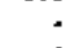 & $*$ & - & . & . & - \\
\hline • & • & - & * & * & • & * \\
\hline 276 & $215 \dot{ }$ & $20 \dot{3}$ & 248 & $250^{\circ}$ & $27 \dot{2}$ & $27 \dot{4}$ \\
\hline • & • & - & - & - & + & + \\
\hline$\star$ & $\dot{-}$ & - & * & + & ' & • \\
\hline : & $1 . \dot{z}$ & i & 2 & $1 . \overline{4}$ & $i$ & $2 . \dot{3}$ \\
\hline . & - & - & - & * & • & . \\
\hline 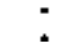 & - & $\cdot$ & . & \pm & : & : \\
\hline 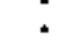 & $<10$ & $\times 10$ & $<10$ & $<+0^{\circ}$ & $<10$ & $<10$ \\
\hline : & : & : & : & * & - & • \\
\hline " & • & • & • & • & • & $\bullet$ \\
\hline$\vdots$ & & & 0.058 & : & $\vdots$ & ; \\
\hline $\begin{array}{r}169 \\
1\end{array}$ & $\begin{array}{r}144 \\
1.5\end{array}$ & $\begin{array}{l}184 \\
1.5 \\
2.5\end{array}$ & $\begin{array}{l}164 \\
0.8\end{array}$ & $\begin{array}{r}164 \\
\times 1 \\
3.2\end{array}$ & $\begin{array}{r}160 \\
<1 \\
2.5\end{array}$ & $\begin{array}{r}160 \\
<1 \\
0.8\end{array}$ \\
\hline
\end{tabular}

45
7.8
$\vdots$
248
$\vdots$
$\vdots$
$\vdots$
40
$\vdots$
0.0584
164
0.8

0.1
7.9
250
$\vdots$
1.4
+
+10
$\vdots$
16
3.1

$+39.65$

101154

7.18

375 


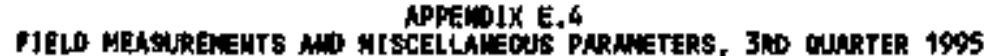

SNIPLING POTYT

LOCATION

DATE SANPLED

FLELO MEASIRERENTS

STATIC WhTER LEVEL (FT. BELON TOC) HATEK LEVEL ELEV. (FT. ABONE HSL) WATER TEP, FJELD NIT (OEO. CEHT) PH, F JElD Fint.

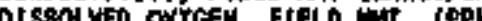
ReD FIELO (ini)

MISCELLNECUNS PhanETERS

EHEAICAL OXYGEN DENAM

CrinjoE

of

PH, REP.

PH, REP. 2

PII, REP. 4

conoctuvety

conpuctivity, Dep

comprivtry tep.

conbuctuvitY, REP.

TOTAL orestic caboo

( REP 2 (WE/C)

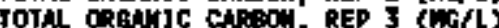

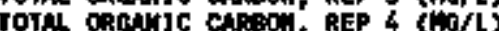

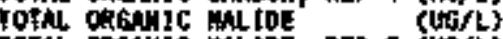

TOTHL ORGHic MLIDE, REP 2 CUST

TOTAL ORENIC WLIDE, REP 3 (Ut:

TOIAL ORGhic MLIDE: REP 4 (UG/L)

Dissoling sol IDs

TOTAL SUSPEEWOED SOALCS

(HG/

TUR日Diti

\begin{tabular}{|c|c|c|c|c|c|c|c|c|c|}
\hline $\begin{array}{r}141 \\
\text { L14 } \\
07 / 10 / 95\end{array}$ & $\begin{array}{r}61-142 \\
K H 6 \\
07 / 14 / 95\end{array}$ & $\begin{array}{r}01+143 \\
k+16 \\
07 / 16 / \% 5\end{array}$ & $\begin{array}{r}64-146 \\
\mathrm{KW0} \\
07 / 16 / 95\end{array}$ & $\begin{array}{r}5+145 \\
\text { KHa } \\
07 / 17 / 95\end{array}$ & $\begin{array}{r}G W+146 \\
K W d \\
07 / 17 / 65\end{array}$ & $\begin{array}{r}0 N-147 \\
\times 140 \\
07 / 44 \% 5\end{array}$ & $\begin{array}{r}\text { ow. 156 } \\
\text { cksos } \\
07 / 13 / 55\end{array}$ & $\begin{array}{r}\text { cor } 136 \\
07 / 18 / 95\end{array}$ & $\begin{array}{r}\text { CH. } 159 \\
\text { CRsts } \\
07 / 12 / 95\end{array}$ \\
\hline $\begin{array}{r}-95.75 \\
100.35 \\
16.3 \\
7.2 \\
429 \\
6.9 \\
140\end{array}$ & $\begin{array}{r}-13.57 \\
233.83 \\
17.1 \\
7.2 \\
443 \\
1.8 \\
127\end{array}$ & $\begin{array}{r}80.13 \\
83.05 \\
17.2 \\
8.2 \\
402 \\
1.5 \\
1.1\end{array}$ & $\begin{array}{r}+80.68 \\
832.66 \\
17.2 \\
5.1 \\
3.3 \\
5.2 \\
179\end{array}$ & $\begin{array}{r}-6.53 \\
83.51 \\
78.1 \\
7.9 \\
509 \\
4.7 \\
146\end{array}$ & $\begin{array}{r}-97.0 \\
740.36 \\
17 \\
8 \\
519 \\
2.1 \\
155\end{array}$ & $\begin{array}{r}+17.9 \\
033.72 \\
19.4 \\
7.8 \\
3.5 \\
0.5 \\
172\end{array}$ & $\begin{array}{r}-143.14 \\
905.96 \\
19.8 \\
7.3 \\
570 \\
9.2 \\
210\end{array}$ & $\begin{array}{r}-47.63 \\
935.42 \\
17.6 \\
7.6 \\
3.07 \\
9.5 \\
.109\end{array}$ & $\begin{array}{r}-117.66 \\
953.54 \\
78.1 \\
7.5 \\
343 \\
5.3 \\
140\end{array}$ \\
\hline
\end{tabular}

$\begin{array}{rr}\times 5 & \\ <0.1 & 40.05 \\ 7.4 & 7.9 \\ \vdots & 7.8 \\ 432 & 7.7 \\ : & 440 \\ 1.9 & 439 \\ : & 5.7 \\ 410 & 3.5 \\ : & 1.1 \\ : & 410 \\ 250 & 410 \\ 4.5 & 208 \\ 30 & 40\end{array}$

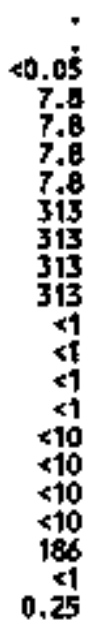

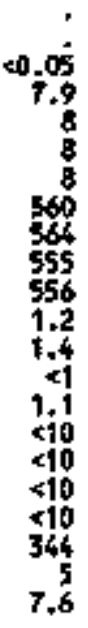

0.05
8.1
8.1
8.2
9.3
313
314
313
313
2.8
2.4
3.1
51
$\times 10$
210
410
$\times 10$
192
3
3.9

5
40.05
7.4
7.5
7.6
7.4
516
619
619
619
5
4.3
4.2
810
510
510
110
355
1.5
1.5

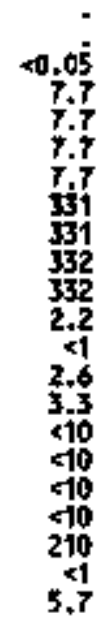

0.05
7.8
7.6
7.9
7.8
366
5.9
371
371
4.1
3.9
1.2
410
40
40
40
222
0.45 
NPDEID IX E.4

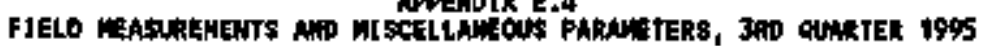

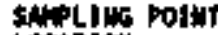

Lochtion

DATE MUAPLE

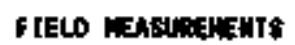

STATIC MATER LEVE 〈FT, OELEN TOC) WRTER LEVEL CLE. (PT. ADOVE NOL WATER TEP, FJEL Wit (0E, LENT.)

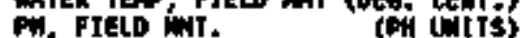

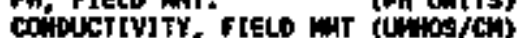

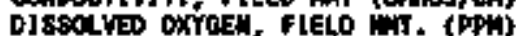

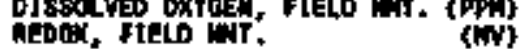
MISCaLingous Parineters

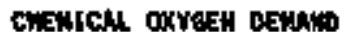
CYMADE

PH

PH, R.T.?

PH, hes. $\frac{2}{3}$

PH, BEF, 4

CondDicivity REP.

COMDUTU, REP.

CONDCr.

TOTAL Gonitc CAhor

(Who

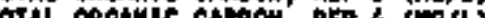

ToTh geton

(U)

TOTAL gonoentC MALIDE, REP a (U6)

THA gomanc

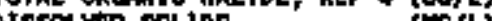

TOTAL SUSPEKDED SOLIDS

(WW/L)

TURGIDITY

\begin{tabular}{|c|c|c|}
\hline $\begin{array}{r}c+175 \\
06 / 01 / \% s\end{array}$ & $\begin{array}{r}0 a+17 \\
\text { chsp } \\
00 v 01 / 95\end{array}$ & $\begin{array}{r}4-181 \\
\text { cisp } \\
07 / 28 / 95\end{array}$ \\
\hline $\begin{array}{r}123.75 \\
960.25 \\
17.3 \\
7.7 \\
376 \\
9.4 \\
172\end{array}$ & $\begin{array}{r}-110.4 \\
1639.6 \\
7.7 \\
7.7 \\
428 \\
2.2 \\
124\end{array}$ & $\begin{array}{r}-110.2 \\
90.8 \\
16.6 \\
7.7 \\
32 \\
5.7 \\
94\end{array}$ \\
\hline
\end{tabular}

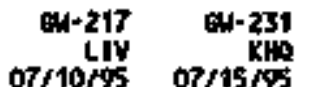

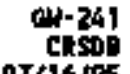

civis

ay-304

chistios

cis-305 $07 / 12 / 95$

ed-511

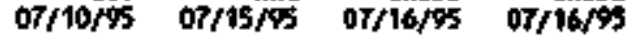

-118.04
927.46
17.3
34.8
5.2
153

$\begin{array}{rr}-125.33 & -112.9 \\ 1058.27 & 90.5 \\ 17.8 & 15.4 \\ 8.1 & 8.9 \\ 24.3 & 257 \\ 8.7 & 7.5 \\ 119 & 12 .\end{array}$

$\begin{array}{rr}\$ 14.37 & -15.8 \\ 1062.53 & 835.67 \\ 16.7 & 14.8 \\ 8.2 & 7.5 \\ 204 & 351 \\ 10.2 & 1.1 \\ 140 & 160\end{array}$

$\begin{array}{rr}-50.55 & -85.57 \\ 932.09 & 918.65 \\ 16.5 & 15.5 \\ 7.7 & 7.6 \\ 260 & 613 \\ 6 & -16 \\ 39 & -165\end{array}$

\begin{tabular}{|c|c|}
\hline $\begin{array}{r}+5 \\
\infty .1\end{array}$ & \\
\hline b. $\dot{2}$ & 40,0 \\
\hline 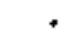 & \\
\hline ' & $\%$ \\
\hline 308 & 7. \\
\hline • & \\
\hline • & 3 \\
\hline 2.7 & \\
\hline " & \\
\hline$<\dot{0}$ & \\
\hline ' & 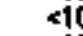 \\
\hline . & \\
\hline $17 \dot{6}$ & 1 \\
\hline $\begin{array}{r}27 \\
5.1\end{array}$ & \\
\hline
\end{tabular}

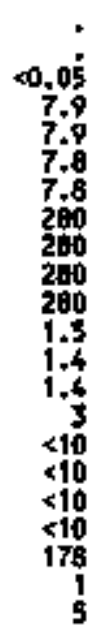

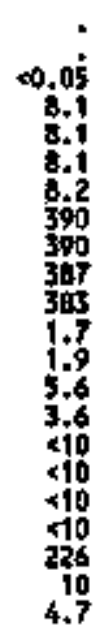

\begin{tabular}{|c|c|}
\hline 4.1 & $\ddot{\prime}$ \\
\hline B.1 & $\mathrm{s}, \dot{z}$ \\
\hline • & . \\
\hline 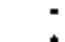 & : \\
\hline 266 & $266^{\circ}$ \\
\hline - & : \\
\hline & $:$ \\
\hline $1 . \dot{8}$ & • \\
\hline * & 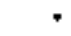 \\
\hline & : \\
\hline$<10$ & " \\
\hline & ; \\
\hline $17^{*}$ & $=$ \\
\hline $\begin{array}{r}138 \\
3 \\
3.9\end{array}$ & $\begin{array}{r}166 \\
41 \\
0,7\end{array}$ \\
\hline
\end{tabular}


APPENDIX E-4

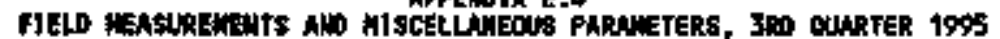

SAmPLING POJHT

LOCATION

DATE SAMPLiD

\section{FIELD MANHEETIS}

STATIC WhTER LEVEL (NT. BELON TOC) WHTER LEVLL ELEV. (IT. MOONE WSL)

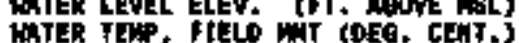

PH, telo ter.

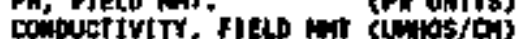

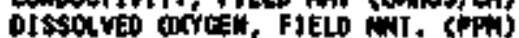
REDOX, FJEL WII.

hiscellangous PMRNETERS

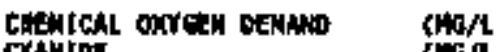
Erinutpit

PHEMOL

PH, DEP. 2

PH, DEP. 2

PI. AP. 4

comptivity, Ras.

COMUCT JVITY, REF, 3

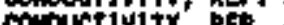

Total orenic chtoon.

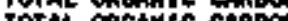

2 int

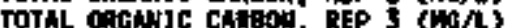

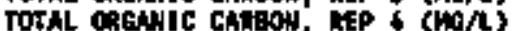

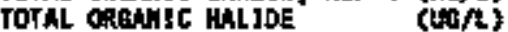

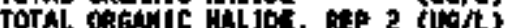

Total aRenilc Hal toe, nep $3\left(\omega_{0} / L\right)$

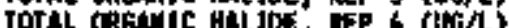

olssous solibs

TOTAL SHSPEWEO SOL 10 S

(Nof

TURB]DIT

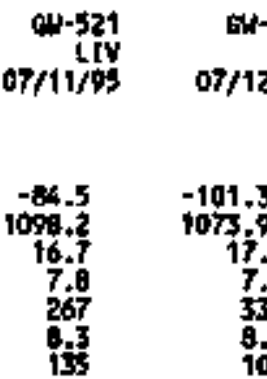

Cin-539
ift
$0710 / 95$

aij-600
chs

6)- 009

GN-610

$07 / 10 / 95 \quad 07 / 31 / 95$

Oovo1/45

$07 / 31 / 5$

car-611

oor C1/\%s

410

or/ $10 / 4$

c나-731

CDSDB

-106.06
086.94
16.3
275
6.6
223

-137.75
936.25
16.9
7.7
296
2.6
140

-172.37
939.76
16.2
7.6
344
6.1
145

-51
968.4
17
7.5
34
6.2
122

-107.02
941.38
17.4
7.4
4.3
2.3
160

-29.77
876.13
77.1
236
5.1
190

-124.56
926.64
18
9.4
24.4
124

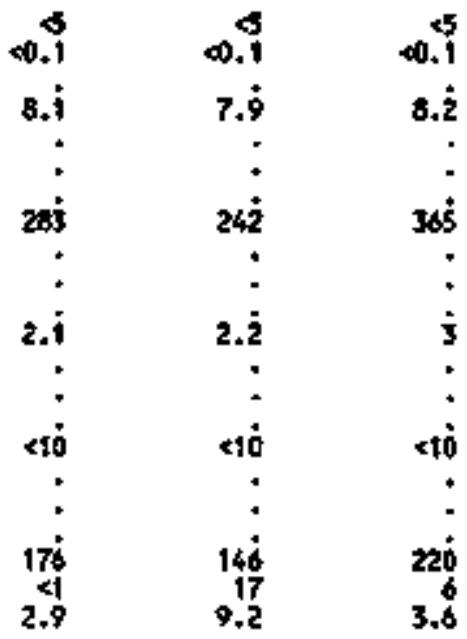

0.15
8.2
$\vdots 35$
$\vdots$
$\vdots$
$\vdots$
+10
$\vdots 20$
3.6

4
0.7
8
$\vdots$
325
$\vdots$
3.2
$\vdots$
410
$\vdots$
200
0.4

0.05
9.4
9.3
9.2
9.5
2.00
271
265
260
1.2
1.3
1.2
1.1
410
510
$\times 10$
$\times 10$
182
0.35 


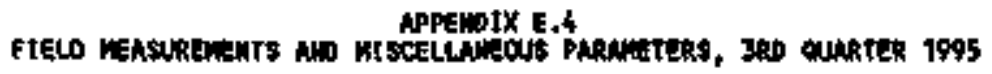

sNot lws porwt

tocht to"

WATE SUIPLEO

pletb intastherents

STATIC WhTER LFUEL (FT. BCtad TOC) WhER LEVA ELEV. (FT. MOVE ML)

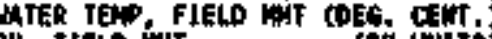
PH, Ftelo îT

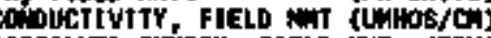

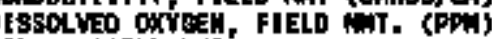
Retoox, fletto int (NW)

MISCELLAMEOUS PARAETERS

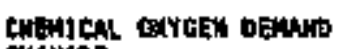
CrantoE MENOLS

PN. REP. 2

Pit, REP. 2

PW, NEP. 4

colouctivity

conderivity, MEP,

COMDCCIVIIY, NEP. 3

rotal boauje takion

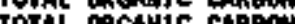

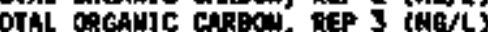

TOTAL DeAnIC CARBOH, TSP 4 (NGL)

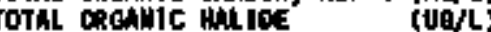

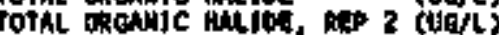

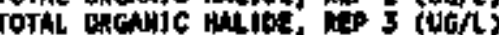

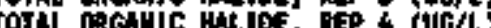

bissolver soulos

TOTAL SUSPEIDED SOL 10 S

(twa/c)

TIAB[D]TY

(inc/

(NTU)
CAN-752

aI- 752

CEspo

세-742

44-76

$07 / 30 / 95$

$07 / 31 / 9$

CU.757

ort/3/4s

-156.55
907.55
16.6
9.6
224
5.6
109

-157.7
906.4
16
7.9
29.
6.5
158

+135.9
96.1
18.6
755
1.2
.53

$\begin{array}{rr}-135 & -64.65 \\ 967.4 & 076.75 \\ 17 & 17.1 \\ 7.6 & 8.4 \\ 261 & 263 \\ 7.1 & 5.4 \\ 175 & 144\end{array}$

.08
$\times 0.05$
10
10
10
10
252
232
235
231
1.6
1.5
2.2
2.4
270
210
210
$2 \neq 0$
130
1055
250

$\vdots$
8.2
8.2
8
85
270
27
279
$\vdots$
$\vdots$
$\vdots$
$15 i$
2.8

$\vdots$
83
327
$\vdots$
$\vdots$
$\vdots$
196
11
13

$\begin{array}{rr}\vdots & 6.4 \\ 7.5 & 8.4 \\ \vdots & \vdots \\ 205 & 294 \\ \vdots & \vdots \\ \vdots & 2.4 \\ \vdots & <10 \\ 152 & 16 . \\ 4 & 1.7\end{array}$ 


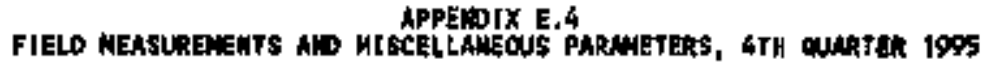

SHPLILE POJUT

LoGhiton

DATE SARPLED

FTELO REASUEWEHFS

SEATC MIER LeVtLL \{fT. EELON TOC) MTER LEVEL ELEV, (FT, ADOVE IESL) WhTER TENP FIELD MAT KOEG. CENT. (ant. Phint

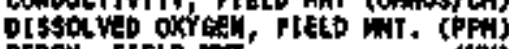
REDOX, FIELD int:

MTSCELLNESOUS PMAMETERS

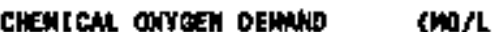

GTANIOE

PMEMOLS

PH, Rap.

PH, KEP. 3

PH, REP 4

contrits

colpur,

conduCT IVITY, REP, \&

tot $\mu$ oncante corkon

REP 2 (HS

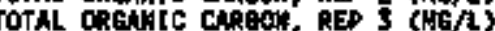

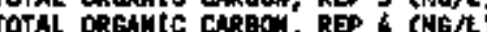

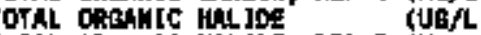

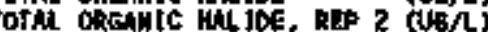

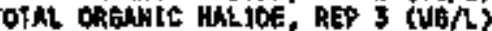

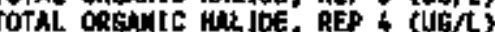

TPH DIESA MNGE X10-LAB MLA

10.0

TOTAL SUSPEELE SOL

$(45 / L)$

TuRBIDitY

$(\mathrm{NG} / \mathrm{L})$

(MTU)

\begin{tabular}{|c|c|}
\hline $\begin{array}{r}1090 \\
\text { Untes } \\
10 / 09 / 9 \%\end{array}$ & $\begin{array}{r}\text { Cas-1 } \\
\text { Ly } \\
10 / 11 / 95\end{array}$ \\
\hline $\begin{array}{r}-59.7 \\
1044.2 \\
16.2 \\
7.2 \\
491 \\
1.5 \\
146\end{array}$ & $\begin{array}{r}15.0 \\
6.4 \\
695 \\
4.0 \\
203\end{array}$ \\
\hline
\end{tabular}

162
$K 119$

as- 14

$19100 / \%$

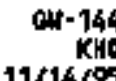

ci-145

4) 146 cH-147

$11 / 14 / \% 5 \quad 11 / 25 / 95$

$11 / 00 / 95$

$11 / 06 / 95$

-70.35
934.93
14
8.2
482
1.1
137

-77.32
836.02
11.4
7.8
407
4.8
173

-3.4
036,84
12.8
6.1
516
8.7

$\begin{array}{rr}-71.87 & -16.57 \\ 766.29 & 85.05 \\ 13.1 & 13.3 \\ 7.9 & 8.2 \\ 556 & 305 \\ 1.7 & 13.0 \\ 237 & 160\end{array}$

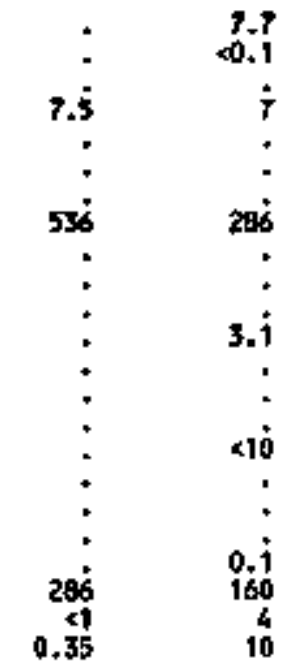

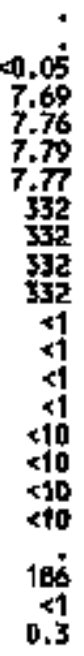

4.05
0.11
0.12
8.12
8.14
319
319
319
318
1.1
1.9
1.8
410
410
510
$\times 10$
168
1.5
3.2 


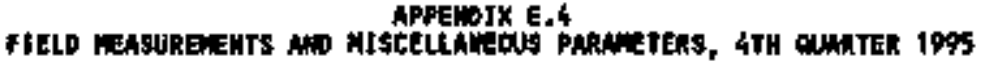

sevpling potkr

Locht10

DATE SNIPLO

FIELb MEAsuretizWTS

STATIC WhTER LENEL (FT, BLLO TOC WATER LEVEL ELV. CET. HONE NSt MTER LENEL ELV. PH FIELD LN ? Ph. FIELC

(PH nins

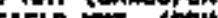
DISBOLVE OXYKEA, FIELD im. (PPW)

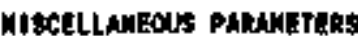

CHERICN OKYGEM DEMAND Crantoe PHEWOLS

PH, REP. 2

PH, DEP. 3

PH, REP. 4

cowouriviry

COADUCTIVITY, REP. 2

CQ, DNDCTIVITY, REP. 3

COTAL ONGAHÍ CARBOH

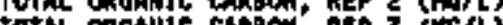

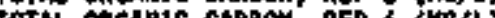

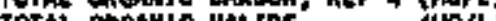

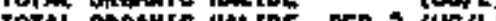

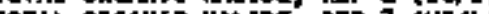

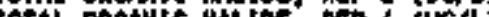

DP

TPH OJESEL RANGE XIO-LA (WW

pissolve son los

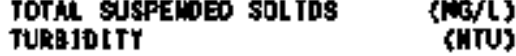

chos

eN-150

chisp

au-156

chiste

cit-156

GN-159

c.159

10/23/95

10/24/95

cal 159

Cas -150

$a n-160$

chrople

(t) 161

-143.42
905.6
15
7.5
606
6.3
106

$\begin{array}{rr}-143.6 & +145.45 \\ 905.5 & 905.65 \\ 14.4 & 7.4 \\ 7.3 & 7.4 \\ 559 & 550 \\ 5.2 & 5.5 \\ 158 & 158\end{array}$

-163.45
905.65
15
7.3
580
5.2
196

-123.55
927.65
14.4
8
340
5.9
148

-143.8
907.4
14.5
7.8
34.5
6.8
112

-138.95
912.5
13.5
74
74
7.4
163

-134.75
916.45
13.9
7.6
492
6.6
116

-150.35
42.75
75.3
7.5
408
6.3
05

-162.7
930.8
14.5
7.9
352
3.3
167

$\begin{array}{rr}\vdots & : \\ 7.4 & 7.4 \\ \vdots 25 & 595 \\ \vdots & \vdots \\ \vdots & \vdots \\ : & \vdots \\ 304 & 366 \\ 16 & 2.1 \\ 21 & 2.2\end{array}$

$\vdots$
596
$\vdots$
$\vdots$
$\vdots$
$\vdots$
336
0.95

$\vdots$
7.4
$\vdots$
605
$\vdots$
$\vdots$
$\vdots$
$\vdots$
350
3.1

$\vdots$
7.9
$\vdots 35$
$\vdots$
$\vdots$
$\vdots$
$\vdots$
736
19
19

$\vdots$
7.8
$\vdots 35 i$
$\vdots$
$\vdots$
$\vdots$
$16 \%$
169

$\vdots$

359
$\vdots$
$\vdots$
$\vdots$
$\vdots$
216
2.5
2.9

$\vdots$
78
$\vdots$
$\vdots$
$\vdots$
$\vdots$
$\vdots$
$18 \dot{1}$
$\frac{1}{3}$

45
0.1
7.46
$\vdots 18$
418
3.3
$\vdots$
$\times 10$
$\vdots$
730
5365
7600

6.4

7.94

:

355 


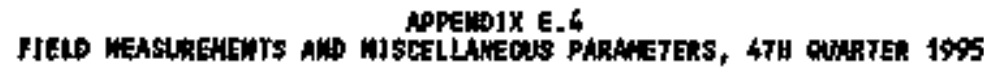

SNPLIMG POLAT

LOCAFIOW

DATE SAIPLED

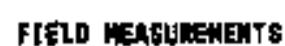

STATIC WhIER LEWEL (FT. BEL OA TOC)

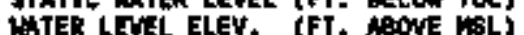
WTER LEMTL. ELEV, (FT, NBONE WSL) Pi FleIn f

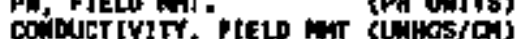

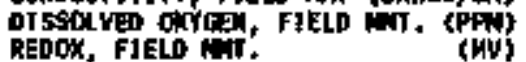
(bv)

MISCELLLNEOUS PARANLTEAS

CHENICAL OKCYEeK DEMAND Cranide

PHENOL

PH, WEP. 2

PH, REP. 3

PH, REP. 4

COADUCT JVITY, KEP,

COMUCTUITY, REP, 3

con'olut jVITY' RE.

Torm ontaylc congon

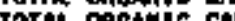

(a) 3 (N)

Total orentite carbon, REP 4 cho/t

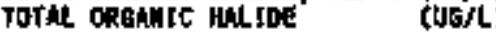

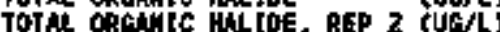

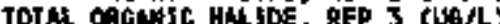

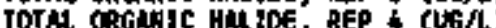

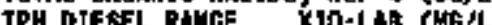

DISFOLVED SOLJOS X

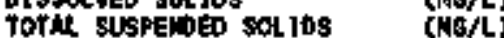

TURBIDITY

\begin{tabular}{|c|c|c|c|c|c|c|c|c|c|}
\hline $\begin{array}{r}\text { cu-175 } \\
\text { casp } \\
14 / 17 / 65\end{array}$ & $\begin{array}{r}\text { cu-177 } \\
\text { cksps } \\
11 / 16 / 95\end{array}$ & $\begin{array}{r}\text { vir-181 } \\
\text { CRSp } \\
11 / 05 / 95\end{array}$ & $\begin{array}{r}6 N-184 \\
10 / 31 / 95\end{array}$ & $\begin{array}{r}186 \\
11 / 03 / 95\end{array}$ & $\begin{array}{r}\text { but } 107 \\
11 \text { roess }\end{array}$ & $\begin{array}{r}\text { an-186 } \\
10 / 31 / 95\end{array}$ & $\begin{array}{r}6 u-203 \\
\text { vics } \\
\text { to/07/45 }\end{array}$ & 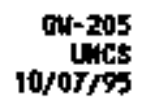 & $\begin{array}{r}\text { a-221 } \\
\text { Lacs } \\
10 / 08 / 05\end{array}$ \\
\hline $\begin{array}{r}-122.5 \\
961.75 \\
13.3 \\
7.5 \\
370 \\
9.5 \\
165\end{array}$ & $\begin{array}{r}-197.0 \\
1040.2 \\
10.9 \\
7.9 \\
414 \\
12.1 \\
175\end{array}$ & $\begin{array}{r}-113.12 \\
979.89 \\
13.8 \\
8 \\
309 \\
4.8 \\
49\end{array}$ & $\begin{array}{r}-109.76 \\
817.68 \\
13.6 \\
7.5 \\
414 \\
4.5 \\
171\end{array}$ & $\begin{array}{r}-14.26 \\
817.06 \\
13.0 \\
7.1 \\
8.0 \\
9.2 \\
-40\end{array}$ & $\begin{array}{r}-19.55 \\
814.75 \\
16.2 \\
7.6 \\
9.54 \\
0.8 \\
-275\end{array}$ & $\begin{array}{r}-20.45 \\
816.64 \\
15.5 \\
89 \\
436 \\
7.5 \\
-26\end{array}$ & $\begin{array}{r}-85.15 \\
1022.15 \\
15.9 \\
7.8 \\
247 \\
0.5 \\
107\end{array}$ & $\begin{array}{r}-41.7 \\
1022.3 \\
16 \\
8.1 \\
262 \\
0.4 \\
176\end{array}$ & $\begin{array}{r}-8.9 \\
1021.1 \\
15 \\
7.7 \\
2.42 \\
2.3 \\
134\end{array}$ \\
\hline
\end{tabular}

\begin{tabular}{|c|c|c|c|c|c|c|}
\hline & & & & & & \\
\hline - & • & . & • & • & $\cdot$ & - \\
\hline & , & $=$ & * & . & • & * \\
\hline $7.7 \dot{7}$ & 7,86 & 7.62 & $7.5 \dot{2}$ & 7.18 & 7.76 & a. 05 \\
\hline • & . & - & . & * & . & + \\
\hline • & - & • & . & . & . & ' \\
\hline $19 \dot{\circ}$ & $42 \dot{i}$ & $3 \dagger \dot{9}$ & $42 \pi$ & $B \overline{0}$ & $1000^{\circ}$ & $450^{\circ}$ \\
\hline • & ' & • & . & . & . & . \\
\hline • & . & . & . & . & . & ' \\
\hline • & • & ' & - & $\cdot$ & - & , \\
\hline • & • & ' & • & • & $\cdot$ & . \\
\hline • & • & $\cdot$ & - & • & • & ' \\
\hline 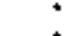 & : & $\dot{ }$ & $\dot{ }$ & ' & E & * \\
\hline$\star$ & $\star$ & $:$ & $\dot{-}$ & ; & i & ; \\
\hline - & . & . & • & . & - & . \\
\hline & , & ' & • & • & r & , \\
\hline * & 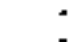 & ' & 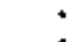 & : & : & • \\
\hline $190^{\circ}$ & $12 \dot{0}$ & $14 \dot{4}$ & 250 & $53 \dot{2}$ & $\sin$ & 324 \\
\hline $\begin{array}{r}2 \\
4.1\end{array}$ & $\begin{array}{r}12 \\
5,2\end{array}$ & $\begin{array}{l}10 \\
15\end{array}$ & $\begin{array}{l}1.5 \\
2.4\end{array}$ & 0.8 & $\begin{array}{l}\mathbf{1} \\
\mathbf{5}\end{array}$ & $\begin{array}{l}34 \\
74\end{array}$ \\
\hline
\end{tabular}

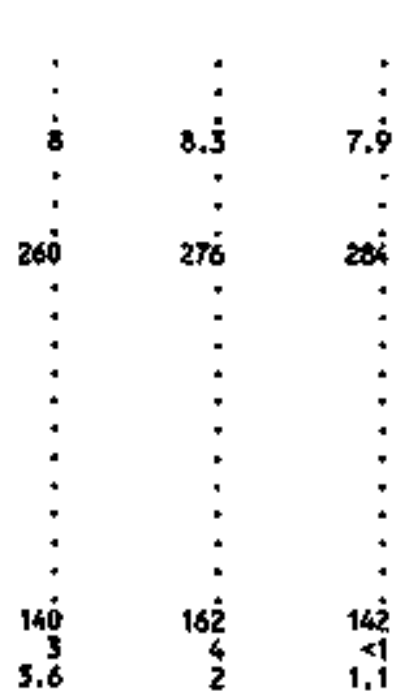


APPEVID TK E.

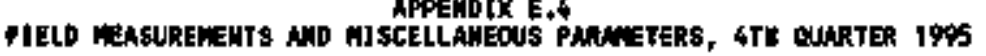

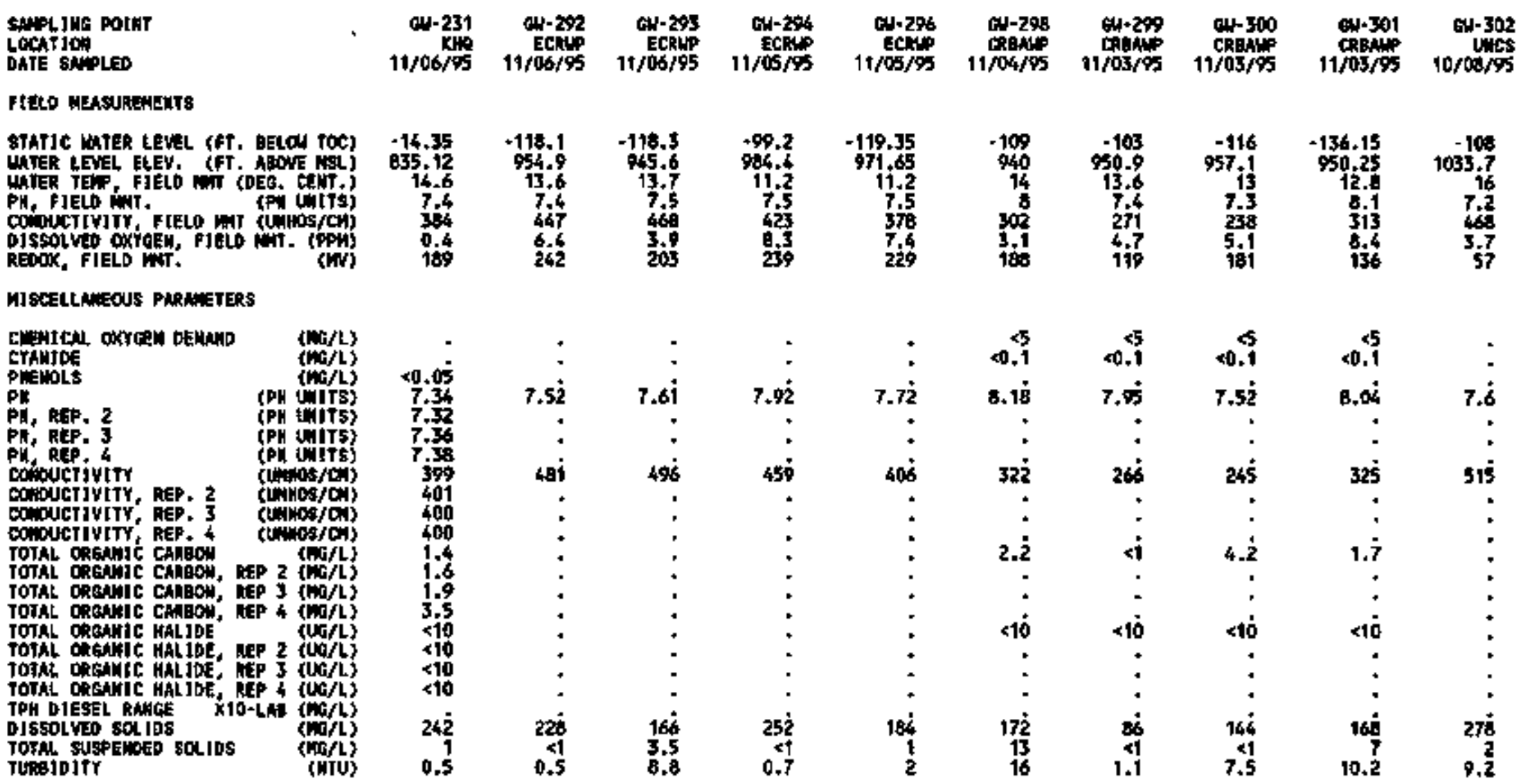


APPENDIX E.4

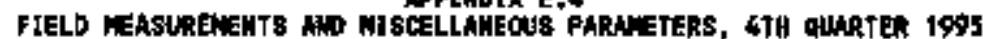

SHPLIMS AOIIT

LAChTIO:

DATE SANPLE

FIELD MEASUMENEATS

STATIC WATER LEVEL (FT. BELOH TOC) WHTER LEVEL ELEV. (FT. MOONE MEL)

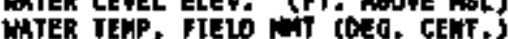

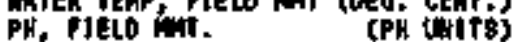
coivintut

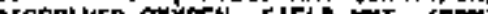

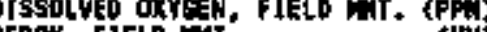
JELD MT. (NV)

MISCELLAHEOUS PARNETERS

chemteal oryed penthib CYAHIDE PHENOCS

PH, NtP,

PH, 政: 3

Pl, NEP. 4

Contuctivity, RLP, 7

COMDUCTIVITY, REP; 3

MXCTIYITY, REP.

Total ondute cakson

BEP 2 (METL)

CARDON, DEP 3 (WW/L)

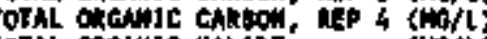

(UO)

O

TrA Go

RPH OIEBEL RAME

T

TOTAL SUSPEIDEO sOl. IDS

(n6/t)

TOTAL SUSPEIDEO SOL. IDS (HG/L)
TURBIDLTY

\begin{tabular}{|c|c|c|c|c|}
\hline $\begin{array}{r}44-321 \\
10 / 26 / 95\end{array}$ & $\begin{array}{r}010-379 \\
10 / 06 / 95 \\
0\end{array}$ & $\begin{array}{r}\text { GN511 } \\
\text { cksp } \\
11 / 07 / \% 5\end{array}$ & $\begin{array}{r}0 k-512 \\
10 / 31 / 45\end{array}$ & $\begin{array}{r}G W-513 \\
109 \\
10 / 31 / 95\end{array}$ \\
\hline $\begin{array}{r}-22.67 \\
905.91 \\
14.5 \\
7.9 \\
22.4 \\
6.4 \\
143\end{array}$ & $\begin{array}{r}180.25 \\
1044.35 \\
16 \\
7.2 \\
499 \\
3.2 \\
18\end{array}$ & $\begin{array}{r}+116.55 \\
979.65 \\
13.7 \\
8.1 \\
257 \\
5.6 \\
174\end{array}$ & $\begin{array}{r}-30.34 \\
971.16 \\
14.5 \\
7.3 \\
281 \\
4.1 \\
183\end{array}$ & $\begin{array}{r}-30.02 \\
970.58 \\
14.5 \\
7.7 \\
20.8 \\
5.2 \\
157\end{array}$ \\
\hline
\end{tabular}

tril

5) 540

CDLVI

All-54t

colvi

6r-542

$10 / 06 / 95 \quad 10 / 11 / 95$

$10 / 469$

109.3
983.6
18
33
0.7

$\begin{array}{rr}-94.8 & -65.72 \\ 997.2 & 99.68 \\ 14.8 & 15.5 \\ 7.5 & 7.7 \\ 331 & 297 \\ 4.3 & 9.6 \\ 97 & 110\end{array}$

-70.45
989.15
15.3
6.8
244
5.4

0.25

0.25

\begin{tabular}{|c|c|c|c|}
\hline ' & • & • & - \\
\hline ' & • & • & • \\
\hline 7.8 & $\mathbf{s}, \mathbf{2 i}$ & $7.5 \dot{2}$ & 7.92 \\
\hline ' & • & . & • \\
\hline , & • & ' & • \\
\hline 558 & 263 & 297 & $32 \dot{9}$ \\
\hline - & • & - & ' \\
\hline - & • & . & - \\
\hline - & : & $1 . \dot{4}$ & 1.6 \\
\hline - & : & $1+4$ & 1.0 \\
\hline • & * & . & 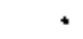 \\
\hline - & 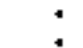 & $<\theta^{\circ}$ & 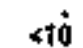 \\
\hline . & V & . & - \\
\hline ' & • & • & \\
\hline . & • & * & \\
\hline $\begin{array}{l}14 \dot{2} \\
2.5 \\
7.2\end{array}$ & $\begin{array}{r}162 \\
\times 1 \\
0.5\end{array}$ & $\begin{array}{r}214 \\
7.7\end{array}$ & $\begin{array}{l}206 \\
3.5 \\
1.9\end{array}$ \\
\hline
\end{tabular}

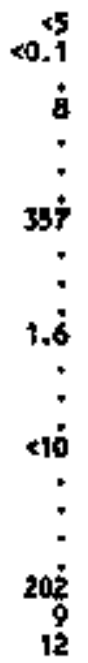

0.1
0.5
367
$\vdots$
2.2
$\vdots$
$\vdots$
$\vdots$
$25 ;$
0.15

45

45

7.19

7.05

330

239

3.4

เะ่

:

2.5
7.2

1.9

142
2
B. 6

240
$\times 1$
1.3

36
.6 
8NHPLING POINT

LOCATION

DATE SAPLE

FIELO WEAsUrearatts

STATIC whTE LEVEL (FT. ARLOW TOC) WHTE TEWEL ELEV, (FT ABOWE WSL)

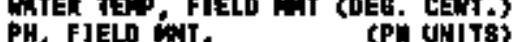
PH, FJELO MNT.

(4)

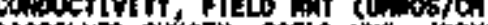

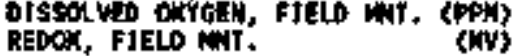

\section{NISCELLMEOUS PARNETEFS}

CrentCal. OXYEEN BEMAto

CYAMTOE
PHENOLS

PII, NEP.

PN, AEP. 3

Pr, REP: 4

Coinuctivitr

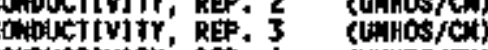

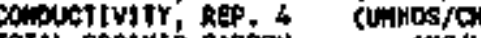

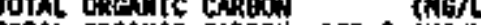

PolAL Oatanic CAmbon, ReP 2 (NB/L)

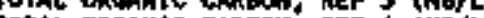

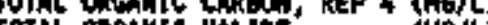

TOTAL ONMAC Huld

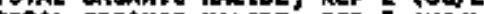

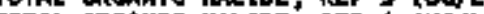

TOTAL CANOANIC HULIDE, REP 4 (UCL)

TPH DIESEL RAME XIO-CAO KNO/L

TSOLE Sillos

TOTAL SUSPENDE SOLIOS

TURBTDJTY
AppeIDIX ES

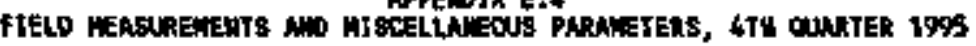

\begin{tabular}{|c|c|c|c|c|c|c|c|c|c|}
\hline $\begin{array}{r}0 \mathrm{~N}-543 \\
\text { cpLy } \\
10 / 17 / 455\end{array}$ & 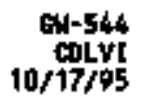 & $\begin{array}{r}\text { ow-546 } \\
\text { colvi } \\
10 / 11 / 95\end{array}$ & $\begin{array}{r}601557 \\
\text { L4 } \\
10 / 07 / 58\end{array}$ & $\begin{array}{r}04+560 \\
004 y 1 t \\
10 / 06 / 95\end{array}$ & $\begin{array}{r}\text { Gu-s62 } \\
\text { colvit } \\
10 / 06 / 93\end{array}$ & $\begin{array}{r}\text { ch-566 } \\
\text { cptv1 } \\
10 / 07 / 95\end{array}$ & $\begin{array}{r}\text { ar-608 } \\
\text { chsp } \\
11 / 16 / 95\end{array}$ & $\begin{array}{r}0 \mathrm{donp} \\
\text { cksp } \\
11 / 20 / \% 5\end{array}$ & $\begin{array}{r}04-610 \\
\text { Corsp } \\
11 / 13 / 9\end{array}$ \\
\hline $\begin{array}{r}-63.94 \\
95 \% .86 \\
15.8 \\
7.2 \\
39.8 \\
5.8\end{array}$ & $\begin{array}{r}-67.1 \\
977.9 \\
15 \\
7.8 \\
501 \\
3.6 \\
124\end{array}$ & $\begin{array}{r}+8.7 \\
907.5 \\
18.4 \\
8.4 \\
200 \\
1.9 \\
112\end{array}$ & $\begin{array}{r}-122.56 \\
95.64 \\
14.8 \\
7.6 \\
2 \% 4 \\
7.8 \\
167\end{array}$ & $\begin{array}{r}-36.25 \\
902.67 \\
1.5 \\
7.5 \\
259 \\
7.2 \\
378\end{array}$ & $\begin{array}{r}-10.25 \\
924.24 \\
13.2 \\
7.6 \\
306 \\
5.9 \\
200\end{array}$ & $\begin{array}{r}11.32 \\
926.45 \\
14.6 \\
6.7 \\
274 \\
6.6 \\
209\end{array}$ & $\begin{array}{r}-132.95 \\
941.05 \\
13 \\
175 \\
8.5 \\
297\end{array}$ & $\begin{array}{r}-168.05 \\
942.25 \\
14.4 \\
7.9 \\
162 \\
5.8 \\
159\end{array}$ & $\begin{array}{r}-80.93 \\
978.57 \\
13.2 \\
6.5 \\
400 \\
7.7 \\
163\end{array}$ \\
\hline
\end{tabular}

\begin{tabular}{|c|c|c|c|c|c|c|}
\hline $\begin{array}{r}6.1 \\
<0.1\end{array}$ & $<0,1$ & $\begin{array}{r}45 \\
0.1\end{array}$ & $\times 0.1$ & $\begin{array}{r}7.3 \\
\times 0.1\end{array}$ & $\begin{array}{r}7 \\
<0.1\end{array}$ & $\begin{array}{r}\$ 5 \\
40.1\end{array}$ \\
\hline 7.6 & $7 . \dot{9}$ & 8.4 & $7 . \dot{9}$ & B.1 & 8.1 & $7 . \dot{2}$ \\
\hline - & - & • & • & • & • & . \\
\hline . & • & . & • & - & • & - \\
\hline $429^{\circ}$ & $4 \dot{5}$ & $25 \dot{2}$ & $31 \dot{3}$ & 285 & 339 & $23 \hat{7}$ \\
\hline * & * & • & * & • & • & - \\
\hline - & • & * & + & • & • & - \\
\hline $2 . \dot{7}$ & 2.5 & $2 . \dot{2}$ & 2.4 & $3 . \dot{z}$ & $1 . \overline{7}$ & 1 \\
\hline - & v & • & - & $=$ & $=$ & - \\
\hline : & i & : & $:$ & $\star$ & : & : \\
\hline$<1 \dot{0}$ & $<10 \dot{0}$ & $\times 10$ & $<10 \dot{0}$ & र10 & <10 & $<10$ \\
\hline : & * & * & * & : & ; & r \\
\hline • & * & * & - & - & * & - \\
\hline & $\vdots$ & : & $\vdots$ & $0 . \dot{1}$ & 0.1 & 0,1 \\
\hline $\begin{array}{r}258 \\
31 \\
3.1\end{array}$ & $\begin{array}{r}175 \\
1.7 \\
1.7\end{array}$ & $\begin{array}{l}176 \\
196 \\
160\end{array}$ & $\begin{array}{r}150 \\
3 \\
1.6\end{array}$ & $\begin{array}{r}152 \\
113 \\
1.3\end{array}$ & $\begin{array}{r}194 \\
5.9\end{array}$ & $\begin{array}{l}128 \\
1.5 \\
1.4\end{array}$ \\
\hline
\end{tabular}

$7.8 \dot{6}$

:

291

7.79

370

$\begin{array}{rr}\vdots & \vdots \\ 570 & \\ 370 \\ \vdots & \vdots 13 \\ \vdots & \vdots \\ \vdots & \vdots \\ 202 & 92 \\ 0.5 & 0.3\end{array}$




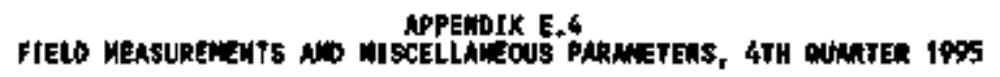

THPLING POIHT

LOCATIOH

DATE SULPLE

FIEID MEASUREAEHTS

STAIIC MIER LEVEL (ET, OELON TOC) WHTER LEVLL FLEV. (ST ABONE WBL) MTER TENP, FI

PH, FELD WIT. (PH UIIS)

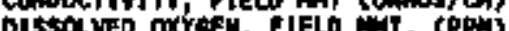

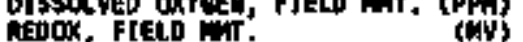

MISCELLAMEOUS PARANETERS

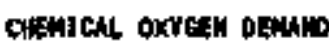

Cranibe

PHellor

PH Rep.

PH, Rept $\frac{2}{3}$

PH, Re; 4

COMULCTIVITY, REP.

comour

Tothl organte toreom

Tora oxtate topon

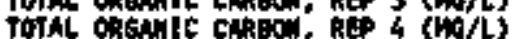

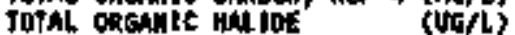

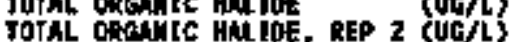

TOTAL ORGMIC HML SGE, RES 3 ULG/L)

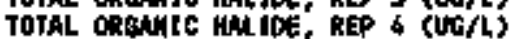

TPM DIESEL RHIGE X10-LNB CHG/L

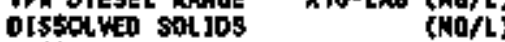

TOTAL SIISPEIDDD SOLIDS MNG

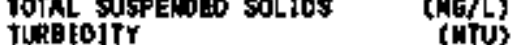

\begin{tabular}{|c|c|c|c|c|c|c|c|c|c|}
\hline $\begin{array}{r}0 N-611 \\
\text { CAsp } \\
11 / 17 / \% 5\end{array}$ & $\begin{array}{r}\text { aw-709 } \\
\text { LID/ }\end{array}$ & 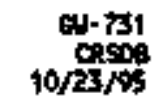 & $\begin{array}{r}\text { aw-751 } \\
\text { crestos } \\
10 / 24 / \% 5\end{array}$ & 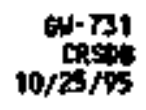 & $\begin{array}{r}4-731 \\
\text { Castol } \\
10 / 26 / 95\end{array}$ & $\begin{array}{r}04-78 \\
10 / 23 / 95 \\
1030\end{array}$ & $\begin{array}{r}\text { cu-73R } \\
\text { ceksos } \\
10 / 24 / 95\end{array}$ & $\begin{array}{r}\text { at-732 } \\
\text { Crspg } \\
10 / 25 / 95\end{array}$ & 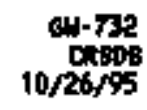 \\
\hline $\begin{array}{r}-193.93 \\
944.47 \\
11.5 \\
7.7 \\
526 \\
71 \\
165\end{array}$ & $\begin{array}{r}-20.63 \\
877.97 \\
15.6 \\
8.2 \\
290 \\
0.7 \\
114\end{array}$ & $\begin{array}{r}-125.21 \\
923.99 \\
14.1 \\
8.7 \\
222 \\
4.9 \\
166\end{array}$ & $\begin{array}{r}-124.15 \\
925,05 \\
14,5 \\
9 ; 6 \\
228 \\
4.4 \\
178\end{array}$ & $\begin{array}{r}-123.5 \\
95.9 \\
13.9 \\
9.3 \\
219 \\
4.7 \\
149\end{array}$ & $\begin{array}{r}-125.15 \\
924.05 \\
15 \\
0.4 \\
215 \\
44 \\
155\end{array}$ & $\begin{array}{r}-157.8 \\
906.3 \\
14.2 \\
8.1 \\
261 \\
5.7 \\
143\end{array}$ & $\begin{array}{r}157.7 \\
906.4 \\
14.3 \\
11.4 \\
327 \\
52.2 \\
307\end{array}$ & $\begin{array}{r}-157.75 \\
906.35 \\
13.5 \\
10.6 \\
250 \\
127 \\
127\end{array}$ & $\begin{array}{r}157.6 \\
906.3 \\
14 \\
11.2 \\
274 \\
6.1 \\
124\end{array}$ \\
\hline
\end{tabular}

\begin{tabular}{|c|c|c|}
\hline & 0.1 & . \\
\hline $7.5 j$ & $8 . \dot{z}$ & 8.7 \\
\hline & : & • \\
\hline 496 & $33 \dot{z}$ & 220 ' \\
\hline * & • & * \\
\hline & 1 & • \\
\hline & 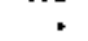 & $\theta^{\prime}$ \\
\hline & $\cdot$ & - \\
\hline & $<10^{\circ}$ & : \\
\hline & $\dot{0}$ & : \\
\hline & $\cdot$ & \\
\hline $\begin{array}{l}28 \dot{8} \\
6.8\end{array}$ & $\begin{array}{r}17 i \\
48 \\
25\end{array}$ & $\begin{array}{r}150 \\
4.5 \\
13\end{array}$ \\
\hline
\end{tabular}

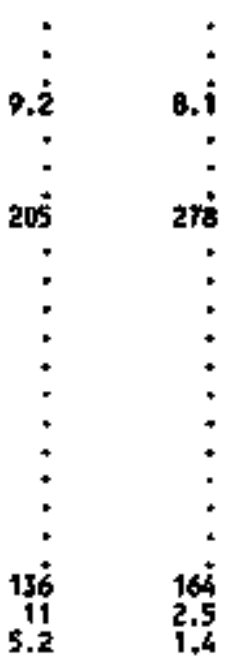

$11 . \dot{2}$

11,5

:

36i

$:$

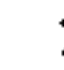

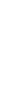

$\frac{130}{32}$
10.2
214
$\vdots$
$\vdots$
$\vdots$
$\vdots$
51
4 
APDEIDIX E.4

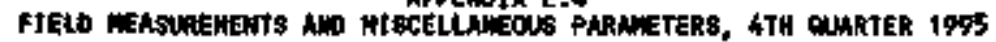

SAHPLIME POTUT

LOCAT JOW

DCTE HAPLO

6N-74

cks

ON-743
CRSP
$11 / 13 / 45$

cit-75?

eu- 79

ty

C4- 797

$10 / 001 \%$

biv- 796

10/0a/ss

cis-799

$6 \mathrm{G}+801$

toroges

G1-827

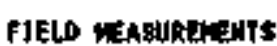

STATIC WATER LEVEL (FT. BELD TOC) WHTE LEVEL ELEY. (FT, MONE HSL)

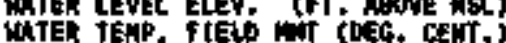

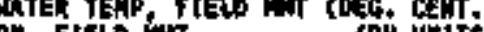

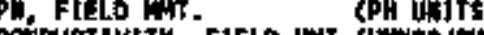
Co DISSOLVED OXYGEA, FJELD WIT, (PPN)
HEDOX, FIELO WI.

$-136.72$

13.8

365

2.5

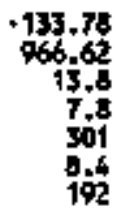

-93.4
870.03
16.2
8.3
311
0.5
145

$\begin{array}{rr}93.76 & -70.37 \\ 96.64 & 909.43 \\ 14.7 & 15.2 \\ 7.8 & 7.6 \\ 200 & 266 \\ 9.1 & 8.7 \\ 205 & 240\end{array}$

.83 .91
921.99
15.2
7.4
239
7.5
235

16.66
96.63
14.7
7.7
227
6.3
216

-112.51
94.45
14.1
7.5
2.5
79.5

.44 .56

1006.8

14.1
7,9

M]SCELLAMEOHS PARANETERS

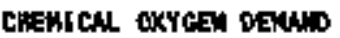
critiloe

PHEMDL

PH, REP.

PH, REP. 3

PH, REP 4

conpuct IVIT, nep,

conDuctivity, AEP. 3

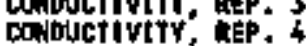

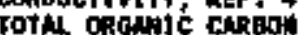

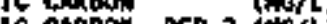

T

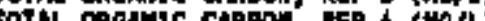

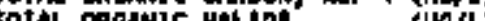

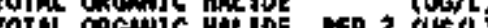

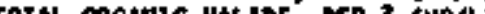

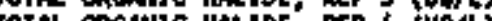

TPU DES

Price RA

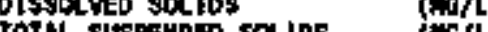

IUR日toify (nTU)

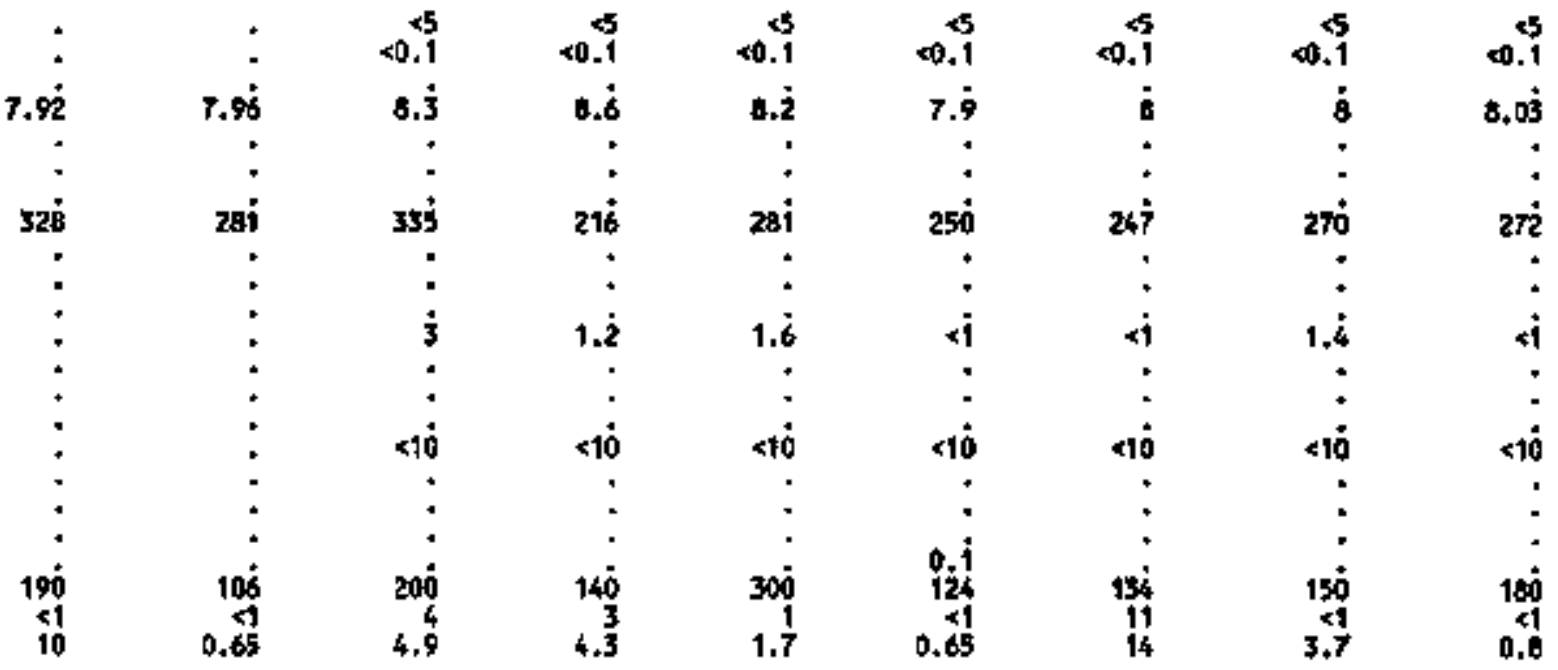



APPENDIX E.5

RADIONUCLIDES 

APPEDDIX E.5

RADLWUCLIDES, IST CUARTER ISAS

strot.1MG potNT LOCkT1ON

OATE SANPLES

CROSS ALPKA cOLHT JHo eRRO Geoss BETh

colvting ekRo

RaDtOMarltoEs

241-Nicktc1Lis COWNTIME EAROR i3T-CEstun

COUNTIN ERROR

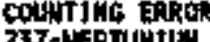

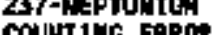

counting enron

20-PLn

courp

CDint

courrind

covractinis

COMning ERTOR

Rejum

COAntus ERROA

rinom

CORROA

97-TEChin TH

consing erka

cothif ine that

TRtI1L

IRtIIly

COWT Mng ERO

234-1 RAN

contring Enton

235-URANTUN

COWTIMG ERRTR

COE-URAhIUM,
6)-141 b14

143

SN-164

ant 144

तn. 144

cN-145

$\mathrm{EN}=145$

CIN-145

$03 / 24 / 9$

$\pi N+146$

KHO 1/17/s

$01 / 10 / 9$

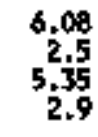

3.06
1.8
18.2
3.7

3,23
1.6
5,45
2.6

$-0.0 \frac{1.3}{2.5}$

5,35 LIV KHO CH-142

c4-142 C4-143

cid-146

$-0.00528$

(241-Aurel/f)

eRcil

(PCI/L)

xio- LAB (BO/L)

(129-100)

(PCI)/L

(2)-neprumin)

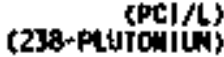

(PCI/L)

(239-pturowius)

¿PROTACTING]

(10+LAg (Bo/L)

\{RNo]tin\}

(PE]/L)

(stront

(PC) $/$ )

(\$9-TECMATI11)

$(231+234+1$ (PCI/L)

xto-LAt $(B) / L)$

(TRITIN)

(234-URCI/L)

(PCI/L)

(235-URANIUN

(238-LRAMLL) $\begin{array}{rr}: & 14.1 \\ : & 3.5 \\ \vdots & 16.7 \\ & 3.6\end{array}$

3.23
1.6
14
3.3

$03 / 23 / 4$

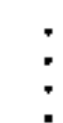

1.36 
APPENDIX E.5

RADCOMUCL JOES, 18T OUARTER 199S

BANPLING POJMT

LOAATION, Silpo

GROSS ALPWA

CodnTINO ERKO anoss Beth

coustils Eason

RADTOMUCLIDES

241-ABRICtUn COUNT JNG ERRO Y37-LEStIH

com-

courifng ERron tount tue etho

count

23-P Und

23o-PLuTanth

colv allu

coun rive cha

cormitio eaho

courin encos

CONTIHG ERROR

IrRonT

coxir ER

P-iectic

cour

coutring ERTOR

TRITIU,

contit IHo ERTOR

234- URAHIU,

CONIT TMG ERROO

25-LRALIOA

COLNTIMG ERRO

COUHTJUG ERROR 01, $15 \mathrm{r}^{\mathrm{K}}$

cisio

crsog

GN-15:

art 17

CRsP $01 / 20 / 45$ of $/ 15 / 4$

1.33
1.4
-1.04
2.5

crsp

$0+181$

$0 \mathrm{c} / 13 / 45$

CRSP

대 217

104105

cu-23

(DC1/A)

(EROSS ALPHA)

2.47
1.4
5.6
2.6

1.4
2.4
2.4
2.4

2.01
1.5
3.39
2.5

2.35
1.6
1.4
2.4

3.22
1.9
2.34
2.7

0.644
1.1
2.2
2.4

0.803
0.06
6.04

1.13
0.90
1.14

$01 / 20 / 95$

(241-MER[C](L)

(PC1/L)

(137-CESIL)

X10-LAB (BO/L)

(129-)100)

(237-nePTHint(L)

(PCI/L)

(Dowr(w)

(239-PUTIOHIL)

(PCt/s)

(PROAncint(Li)

(piorie

(PCI)

(stroutjo

(PCI/L)

(96-TECHKETIUN)

(PCI/L)

$x 10-1$ (nOR(u)

TRET[in]

(ACt $/$ )

(235-tuRAntuy)

(PCI/L)

(238-uRdeitio) 
APPEWIX E. 5

RAOJOUCLIDES, 18T OUMTER 19SS

SHFing Pojut LOCATIOH DHTE SAIPLED

eross MPHA COUNITIHB EREOR GROSS BETA COUUT THO ERROA GAD IONUCC IDES

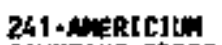
COUNT tNG ERROA 337-ceslit

counT LNG ERRON 129-torin:

coUUTING ERROA 237-NepTun COUITING ERRO 23-plUTokJ chin s. colving ench PatAT JMIU CONHTHG ERMOR Renting

cenift the ERROA Sintrivs

couit ING ERhor

9-TECAMETIUA

CWin cen

Cont

colnt two ERROK

couitring ekath

234-U1CNHIU,

covnring entor

235 (n) N

collin this EAROR

238-UlthHum

\begin{tabular}{|c|c|c|c|c|c|}
\hline & $\begin{array}{r}61+303 \\
\text { colspo } \\
01 / 19 / 95\end{array}$ & $\begin{array}{r}\text { ai-304 } \\
\text { cksoe } \\
01 / 45 / 95\end{array}$ & $\begin{array}{r}\text { aw-305 } \\
\text { ily } \\
01 / 10 / 95\end{array}$ & $\begin{array}{r}04-321 \\
01 / 10 / 95\end{array}$ & $\begin{array}{r}\text { OH-511 } \\
\text { CRsp } \\
01 / 26 / 95\end{array}$ \\
\hline $\begin{array}{r}\text { (PC1/) } \\
\text { (GRoss ALPHA) } \\
\text { (PC1/L) } \\
\text { (mRoss HETA) }\end{array}$ & $\begin{array}{r}2.14 \\
1.4 \\
4.83 \\
2.5\end{array}$ & $\begin{array}{l}1.33 \\
1.5 \\
2.52 \\
2.5\end{array}$ & $\begin{array}{r}0.754 \\
0.234 \\
2.6\end{array}$ & $\begin{array}{r}0.155 \\
1.7 \\
-1.99 \\
2.3\end{array}$ & $\begin{array}{r}0.631 \\
7.1 \\
3.92 \\
2.6\end{array}$ \\
\hline
\end{tabular}

(241-AYERT/L)

241-AER C CIUH

$(\mathrm{PC}] / \mathrm{L})$

x10-LA3 (BO/L)

(139-10Diuk

(PCI/L

(237-MEPTUNIUN)

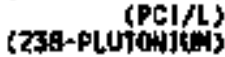

(PG) $(4)$

Tain

(PC1)

(PROTACT [A]U

(

(RODJT)

(PC1)

(strow

(99-techlat)

(PCI/L)

K1+234-THORIUN

$\times 10-\mathrm{LB}(\mathrm{BO} / \mathrm{L})$

TRitis

(PCI/L

(254-CRANI)

(PCI

(235-IRANILW)

(236-URAI/L)

\begin{tabular}{|c|c|c|c|c|}
\hline $\begin{array}{r}\text { an-521 } \\
\text { LIV } \\
01 / 09 / \% 5\end{array}$ & $\begin{array}{r}w-522 \\
\text { LSV } \\
01 / 10 / \phi s\end{array}$ & $\begin{array}{r}04-535 \\
\text { t1d } \\
01 / 04 / 95\end{array}$ & $\begin{array}{r}\text { c4-609 } \\
\text { chsp } \\
02 / 02 / 95\end{array}$ & $\begin{array}{r}0 W+609 \\
\text { CRPSP } \\
02 / 15 / \% 5\end{array}$ \\
\hline $\begin{array}{r}-0,0.0578 \\
1 \\
2.63 \\
2.5\end{array}$ & $\begin{array}{r}-0.167 \\
1.2 \\
-0.996 \\
2.5\end{array}$ & $\begin{array}{r}2,86 \\
1,5 \\
3,59 \\
2.6\end{array}$ & $\begin{array}{r}-0.51 \\
1.3 \\
2.0 .8 \\
2.6\end{array}$ & $\begin{array}{r}0.505 \\
1.2 \\
-1.57 \\
2.5\end{array}$ \\
\hline
\end{tabular}

0.739

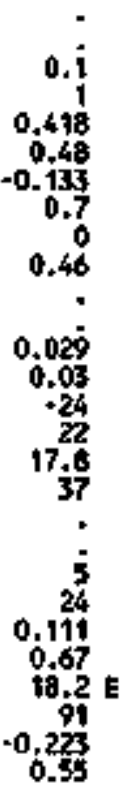


RADICAHCLIDES, I8T QUARTER 1995

SANPLJMe POINT LOCAT 10N

DATE BNAPL

Gooss ilpath COUNTILG EARO

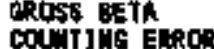

EAbIOWUCL IfES

241-Nititictun 137-CEsiu

COUNIMG ERROR 129-1C0IVE

cOUmitut ERROR

237-WEPTUWIUM

COUNTING ERROA

236-PLUTON] 내

coumtus ENROk

239-DLITONIU:

COUNTITE EREO

PROTACTJUIL

canting exhos

entury

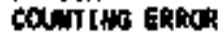

\$Thowt1un

COLNT tilo ERROR

QS-LEChNETIU:

CONTIHE ERROR

231+234-THog1 L4

COUnTJus Eldion

inttsus

COUNT IMO ERROP

24-1 Anutum

COUjtIHe Ebron

35. -

COUNT IMG ERRO

238-4RMulus

COUWTIUS ERROA

\begin{tabular}{|c|c|c|c|c|c|c|c|c|c|}
\hline & $\begin{array}{r}04+610 \\
\text { CRSp } \\
01 / 31 / 95\end{array}$ & $\begin{array}{r}\text { an- } 611 \\
\text { chsp } \\
02 / 14 / 95\end{array}$ & $\begin{array}{r}\text { ow-70p } \\
\text { LII } \\
01 / 00 / \% 5\end{array}$ & $\begin{array}{r}\text { GN-731 } \\
\text { cRsos } \\
01 / 17 / 95\end{array}$ & $\begin{array}{r}\text { M-732 } \\
\text { Crisos } \\
\text { os } / 18 / 95\end{array}$ & $\begin{array}{r}\text { an-742 } \\
\text { thisp } \\
04 / 27 / 95\end{array}$ & $\begin{array}{r}\text { mi-743 } \\
\text { cksp } \\
01 / 31 / 95\end{array}$ & $\begin{array}{r}01+757 \\
\operatorname{liJ}^{01 / 09 / 95} \\
\text {. }\end{array}$ & $\begin{array}{r}\text { scanz.zsp } \\
\text { cksp } \\
05 / 15 / 98\end{array}$ \\
\hline 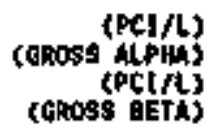 & $\begin{array}{r}0.754 \\
2.1 \\
4.79 \\
2.9\end{array}$ & $\begin{array}{r}1.03 \\
1.4 \\
-0.485 \\
2.5\end{array}$ & $\begin{array}{r}-0.00469 \\
1.2 \\
-0.757 \\
2.5\end{array}$ & $\begin{array}{r}0.97 \\
1.2 \\
19.4 \\
3.9\end{array}$ & $\begin{array}{r}34.1 \\
27.4 \\
6.6\end{array}$ & $\begin{array}{r}6.42 \\
2.0 \\
4.22 \\
2.8\end{array}$ & $\begin{array}{r}3.23 \\
2.3 \\
3.29 \\
2.8\end{array}$ & $\begin{array}{r}0.663 \\
1.4 \\
1.92 \\
2.7\end{array}$ & $\begin{array}{r}0.924 \\
2.9 \\
1.63 \\
2.9\end{array}$ \\
\hline
\end{tabular}

(247-MERCl(L)

$(P C I / 1)$

(137-CEsIL)

K10-LAs (BO/A)

(129-100tin)

(237-1EPTUA'fin)

(PCIA)

(23:-PtuTonrun

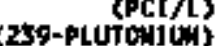

(PC) $/$ )

(PRotact (nli,

X10-LAs (Ba/c)

(RADIUN)

(PCT/L)

(PCI/L)

(9P-tectiwetiuH)

(231+234-7PCI

XTO-LAB (Ba/L)

(TRITIU)

(PCt)

(234-tranius)

(PCI)

(235-trehlitw)

(238-IRANTLG) 


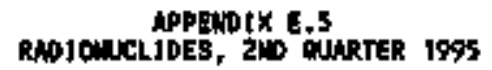

SWP twa polm

Locht ton

CROS: ALPUA

COUIJ He ERROK COWHIMG ERRON COHATIJIS ERROR

RADTOMULLIDES

\section{I37-CH:Stuk}

COSN JHe ERoto C0-POTA8SJU con CoTitjus ERRo: Rrojum Emon Couitine ERroh STrowTIU

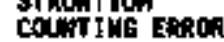
251+254-THeRIU court us eneos Th- 140 coumt 6 Eliten

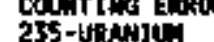

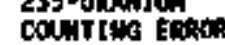
C)

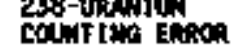

\begin{tabular}{|c|c|c|}
\hline $\begin{array}{r}1090 \\
\text { Uics } \\
0, / 20 / 95\end{array}$ & $\begin{array}{r}\text { cas-1 } \\
\text { Ly } \\
04 / 17 / 95\end{array}$ & $\begin{array}{r}E+142 \\
k 40 \\
04 / 20 / \% s\end{array}$ \\
\hline $\begin{array}{r}2.57 \\
1.7 \\
3.29 \\
2.5\end{array}$ & $\begin{array}{r}-0.165 \\
0.90 \\
0.165 \\
2.5\end{array}$ & $\begin{array}{r}6,34 \\
2.4 \\
10.2 \\
3.1\end{array}$ \\
\hline - & $61 \mathrm{E}$ & . \\
\hline - & $\$ 5$ & * \\
\hline : & ${ }^{*}$ & " \\
\hline : & $\underset{2200}{1670 \mathrm{HE}}$ & \\
\hline $\begin{array}{l}0.025 \\
0.024\end{array}$ & -200 & \\
\hline . & - & $-2,8$ \\
\hline , & $-213^{\circ} \mathrm{HA}$ & 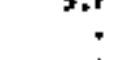 \\
\hline $\begin{array}{r}0.563 \\
0.39 \\
-0.955 \\
13 \\
0 \\
0.2\end{array}$ & $\begin{array}{r}0.309 \\
0.36 \\
-4.57 \\
20 \\
0 \\
0.41\end{array}$ & $\begin{array}{l}4.4 \\
0.95 \\
5.04 \\
7.3 \\
3.05 \\
0.78\end{array}$ \\
\hline
\end{tabular}

Gu-165 09124,105

(4) 146 arike

B. 145 $04 / 25 / 65 \quad 04060$ Gi- K46 aw- 147 6x-156 Cisos $\begin{array}{rr}4.39 & 2.29 \\ 2.2 & 1.5 \\ 15.7 & 4.59 \\ 3.5 & 2.7\end{array}$ $\begin{array}{rr}9.95 & 4.51 \\ 14.7 & 2.2 \\ 3.5 & 16.1 \\ & 3.6\end{array}$ $04 / 21 / 95$ $04 / 07 / 25$

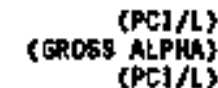
(choss aETA) 1.54
5.17
2.7 2.96 $04 / 13 / \%$

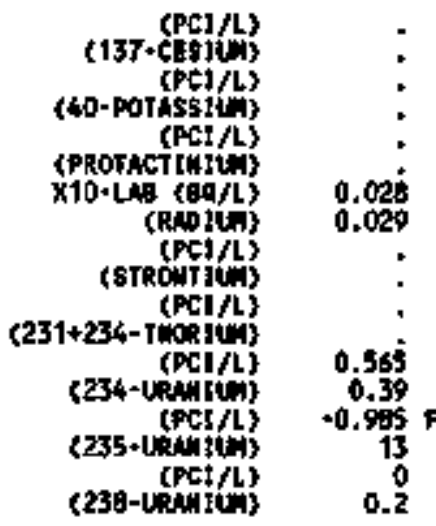

$\begin{array}{rr}09.4 & \vdots \\ 170 & \vdots \\ \vdots & \vdots \\ 7.43 & 0.697 \\ 6.3 & 5.6 \\ 1.01 & 9.07 \\ 0.64 & 2 \\ -5.21 \text { F } & 0.116 \\ 0.736 & 13 \\ 0.53 & 5.7\end{array}$

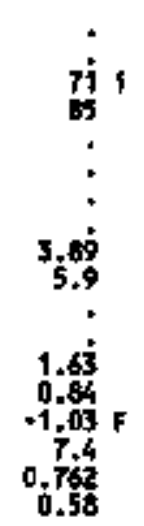




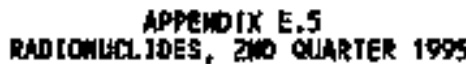

SAPLIN POIMT

LOCNT to

DATE SNUFLEO

Go0s8 M.WA

COLNT IW ERAOR Gposs teth

CONTHA ERIOA

Rubtowuclioes

137-ceston

40-patassiun

count 1lis ERemon

pDort

CAUMTIUS ERFOR

cotrit tur eRpor

stowit ruy

COUWT IW ERPOO

21.234-rHe IU

coin

$24=0$ int

comint the ERRo

cas-1'suris

cortitin erion

23.

cowting Ekho

\begin{tabular}{|c|c|c|c|c|c|c|c|c|c|c|}
\hline & $\begin{array}{r}61-154 \\
\text { CRsog } \\
04 / 06 / 95\end{array}$ & $\begin{array}{r}\text { CW-160 } \\
\text { CRBAMP } \\
04 / 26 / \% 5\end{array}$ & $\begin{array}{r}\text { ON-161 } \\
\text { ORBAHP } \\
04 / 20 / \% S\end{array}$ & $\begin{array}{r}\omega-175 \\
\text { chesp } \\
05 / 15 / 55\end{array}$ & $\begin{array}{r}0.17 \\
\operatorname{cosp} \\
05 / 11 / 05\end{array}$ & $\begin{array}{r}G-181 \\
\text { CRSP } \\
05 / 02 / 75\end{array}$ & $\begin{array}{r}04-194 \\
05 / 01 / 65\end{array}$ & $\begin{array}{r}04-186 \\
\text { Ro } \\
05 / 03 / 45\end{array}$ & $\begin{array}{r}0+187 \\
05 / 02 / 95\end{array}$ & $\begin{array}{r}\text { cin-18: } \\
05 / 401 / 45\end{array}$ \\
\hline 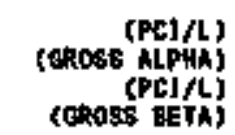 & $\begin{array}{r}5.71 \\
2.5 \\
3.79\end{array}$ & $\begin{array}{r}6.75 \\
4.8 \\
12.9 \\
6.6\end{array}$ & $\begin{array}{r}1.66 \\
2.3 \\
0.743 \\
2.9\end{array}$ & $\begin{array}{r}-0.361 \\
1.1 \\
1.74 \\
2.5\end{array}$ & $\begin{array}{r}4.72 \\
2.4 \\
4.16 \\
2.8\end{array}$ & $\begin{array}{r}1.76 \\
2 \\
3.67 \\
2.8\end{array}$ & $\begin{array}{r}-0.324 \\
1.7 \frac{2}{3}\end{array}$ & $\begin{array}{r}-1.91 \\
2.4 \\
-0,000 \\
3\end{array}$ & $\begin{array}{r}-1,44 \\
2.04 \\
0.0917 \\
3\end{array}$ & $\begin{array}{r}0 \\
2.2 \\
6.57 \\
3.3\end{array}$ \\
\hline (PCI/L) & . & * & • & - & $\bullet$ & . & • & * & 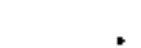 & \\
\hline (137-CEs) & . & . & . & * & . & . & . & . & $:$ & \\
\hline (PC1/L) & . & . & . & . & . & . & . & - & . & \\
\hline (40-potigstim $\}$ & . & - & • & . & . & . & . & . & . & \\
\hline (PCt/L) & . & * & - & . & - & . & . & . & - & \\
\hline (PROTACT]N[I) & - & . & . & - & . & - & . & ? & . & \\
\hline x10-tis (Be, & - & - & - & - & - & - & • & - & . & \\
\hline (abibtum) & . & - & - & . & - & . & . & . & - & \\
\hline (PC, $1 / \mathrm{s})$ & * & - & - & - & - & - & - & - & . & \\
\hline (STROAUTUî) & - & - & . & - & - & - & . & - & . & \\
\hline (PC] $/)$ & . & + & . & * & - & . & . & . & . & \\
\hline 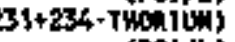 & - & - & - & - & . & - & • & - & . & \\
\hline (PCI/L) & , & - & - & - & • & $\bullet$ & $\cdot$ & - & - & \\
\hline (234-URANIUA) & - & - & - & - & - & - & • & - & - & \\
\hline (PC1/L) & : & - & ; & $*$ & * & - & $\cdot$ & - & • & \\
\hline $\begin{array}{r}\text { (235-LRNAlus) } \\
\text { (PC1)/L) }\end{array}$ & ; & $:$ & : & : & : & : & ; & $\dot{*}$ & $\vdots$ & \\
\hline 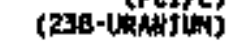 & $:$ & ? & . & . & . & : & 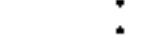 & : & : & \\
\hline
\end{tabular}




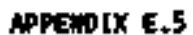

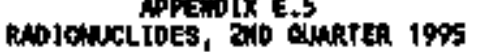

SANAIMG POIMT

LOCAT JAM

EROSS ALPHA

COUNT IMS ERROR

cross beth

COUNTJWG ERROR

Raptouticl thes

$137-C E S 114$

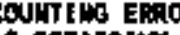

coHTITS ERRO

PRDIACTIMIU,

Cowis ing ERo

cojuin Exad

colinf IU6 ERRO

COUNIJU ERRO

351+234-THOF1U

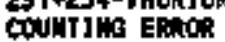

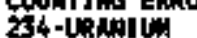

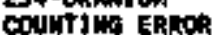

235-uithin

CONNTIS ERROA

zati-uRduliu

COUNT Itw ERitor
W-221 Oin-231 W-2

Uucs UwCs

Git-2B1

congs

ECRM

$04 / 16 / 95$ of/19/9

$\uparrow .01$
1.3
3.28
2.5

2.58
5.58
3.1

2.82
-0.531
2.7

nN-205

ECRtP

1.284

Eckis

W-295

CRBAMP $05 / 03 / 95$

an-2\%

(GROSS ALPHA) (PCI/A)

0.989
1.2
3.86
2.6

1.76
1.3
2.75
2.5

0.901
1.2
1.04
2.4

+.66
2.1
7.68
3.1

0.306
2.1
1.16

2.44
$: \quad-1.57$
2.8
(137-CEClA)

(40+Pothssite)

(PCl/)

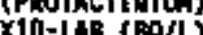

(RADIu)

(PDI)

(strontitw)

(PC1/L

(PCI/L)

(234-0

(25s-uRditw)

(PG)/

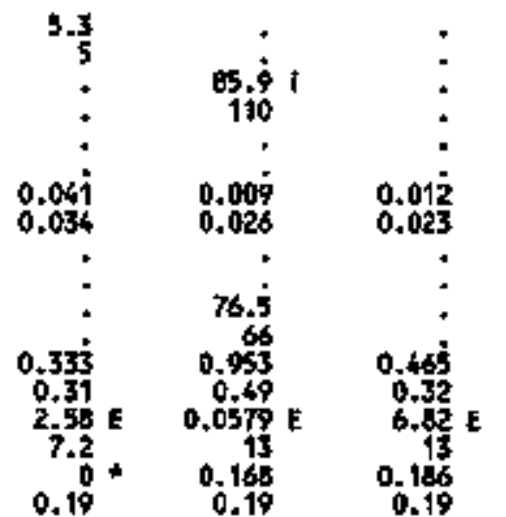




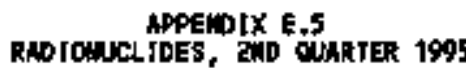

FAPLIMO Polnt LOCAT IOW

DATE Sthel

\section{GROSS MPWA} COUNTIMC ERHOR cross gesta RADIOHuCL JDES

\section{7-CESEIN}

COUMTIME ERRO

40-P0TAss]1.

COUNTINO ERKO

PROTACT [B]

COLWTIUS ERRO

Retion

COUWIHS ERTO

STROHTIU

toupi jus edxo?

231+736-THA It:

COUNTING ERAOH

234-(N)NIIU

COUNTHS ERRO

235-LPAH] t:

Countits ERen

żB-lentius

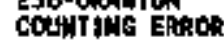

\begin{tabular}{|c|c|c|c|c|c|c|c|c|c|c|}
\hline & 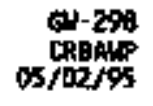 & $\begin{array}{r}\text { w.299 } \\
\text { chenp } \\
\text { osroils }\end{array}$ & 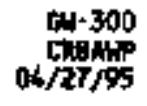 & $\begin{array}{r}\text { GN-30t } \\
\text { CRBAMP } \\
04 / 27 / 95\end{array}$ & $\begin{array}{r}64-302 \\
102 \\
04 / 19 / 45\end{array}$ & $\begin{array}{r}6 N-303 \\
\text { cospos } \\
04 / 09 / 95\end{array}$ & $\begin{array}{r}\text { an-304 } \\
\text { CRsog } \\
06 / 06 / 95\end{array}$ & $\begin{array}{r}a+321 \\
06 / 25 / 6 \%\end{array}$ & $\begin{array}{r}0,339 \\
\text { UlWes } \\
04 / 19 / 95\end{array}$ & $\begin{array}{r}\text { UW-511 } \\
\text { CRSPP } \\
05 / 03 / 95\end{array}$ \\
\hline $\begin{array}{r}\text { (PCL/L) } \\
\text { (cRoss (ALHA) } \\
\text { (PCl/L) } \\
\text { (CRoss BeTA) }\end{array}$ & $\begin{array}{c}-0.296 \\
2.2 \\
2.23 \\
2.6\end{array}$ & $\begin{array}{l}06 \\
2.1 \\
4.66 \\
2.8\end{array}$ & $\begin{array}{r}0.995 \\
7.6 \\
2.72 \\
3.1\end{array}$ & $\begin{array}{r}2.83 \\
2.2 \\
1.25 \\
3\end{array}$ & $\begin{array}{r}1.64 \\
7.5 \\
2.74 \\
2.4\end{array}$ & $\begin{array}{r}0.198 \\
3.34 \\
3\end{array}$ & $\begin{array}{r}1.20 \\
1.8 \\
2.12 \\
2.9\end{array}$ & $\begin{array}{r}-0.609 \\
1.6 \\
4.81 \\
3.2\end{array}$ & $\begin{array}{r}1,3 \\
3.5 \\
2.03\end{array}$ & $\begin{array}{l}6.81 n \\
2.6 \\
2.79 \\
2.7\end{array}$ \\
\hline (PCL/L) & $*$ & • & $*$ & $*$ & $*$ & * & " & ${ }^{\prime}$ & 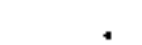 & 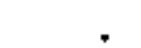 \\
\hline (137-CEstin) & . & - & • & - & • & . & . & - & . & . \\
\hline (PCI/L) & + & - & • & - & • & • & - & v & - & - \\
\hline (40-POTnsstiv) & * & . & * & - & * & - & . & - & . & . \\
\hline (PCI/L) & 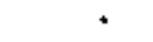 & $\cdot$ & • & - & • & • & • & - & - & - \\
\hline (PROTACTt MILI) & * & * & • & - & ins & - & - & - & & . \\
\hline 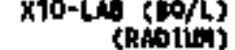 & * & $\cdot$ & $\cdot$ & - & 0.000 & * & - & - & 0.004 & - \\
\hline (PCI/L) & $:$ & 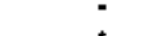 & : & : & & - & - & - & & * \\
\hline (STRONTIII) & : & : & $:$ & - & * & - & * & - & - & - \\
\hline (PC1/L) & : & : & $\vdots$ & : & : & : & $:$ & : & : & $\because$ \\
\hline$\neq 1+2+4-1 m_{0}(t)$ & : & . & . & ; & & . & . & 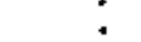 & & 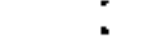 \\
\hline (PcL/L) & - & . & . & . & 0,399 & . & . & - & 0.355 & . \\
\hline RHIIUT) & * & - & . & . & 0.32 & . & - & - & 0,3 & - \\
\hline (PC] $(L)$ & - & - & - & - & $-1,53 \mathrm{~F}$ & - & - & • & -1.27 & • \\
\hline (235-URAw]UT) & - & - & ‘ & - & 0.040 & 2 & * & - & 13 & • \\
\hline 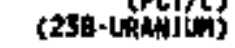 & ; & $:$ & $\vdots$ & $\vdots$ & 0.1 & $\vdots$ & $\vdots$ & $\dot{ }$ & 0.14 & $\vdots$ \\
\hline
\end{tabular}


APPENDIX E.5

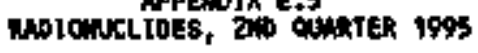

SWFLLLE POINT Lothto:

DRTE setipteo

choss ALPUA

CONTIHE ERRON

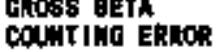

RADIDUCLIDES

137-CESTIM

COWTIHE ERROR

40-POTASSJL

CONTINA ERTLR

PRotheTINu

ChN

Rrojum

COUFT LAB EARQR

stronr [l]

COCHTMG EAROA

231+244-7HORIU

CQMII I

2f4-1.nuktus

255 - L

255-Unmeru

Conin mon

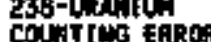

(PC]/L)
(EROSS ALPHA)
(PC]/L)
(OROSS BETA)

GN-512 Bar-513 04/26/95 04/25/95 04/27/\% $04 / 04 / 95 \quad 04 / 05 / 95$ 0,514
$04 / 27 / 5$

EN-540

1.11
3.28
3.1

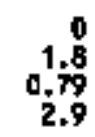

1.55
0.176
2.9

$\begin{array}{rr}0 & 2.61 \\ 1.6 & 2.4 \\ 1.2 & 1,37 \\ 2.8 & 3\end{array}$

CDLI $04 / 06 / 95$

coLvI

coLvI

DivI

$01+546$

CLIVI

6)-557 $04 / 06 / 05$

(PCI/L)
(1377-(ESIL)
(PCI/L)

$(\mathrm{PCI} / \mathrm{L})$
$(40+\mathrm{PDThSs})$

(PRotacífiltu)

XIO-LAB (BaA)

(RAD ION)

(8TROAntion

(231+234- (FCI / )

(234-unnitu)

(235-LRAI (P)

(230-unet/flu)

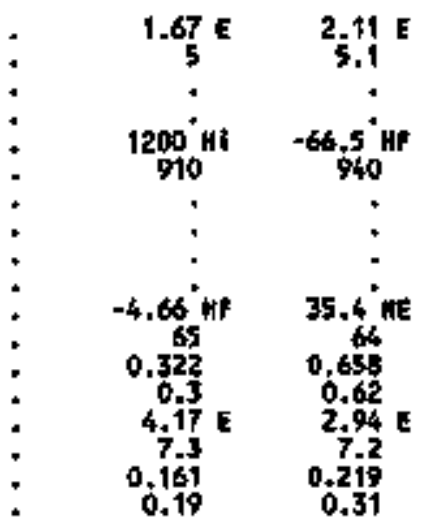

1.18
1.6
1.19
2.5

0.919
1.6
-0.0659
2.8

2.47
1.9
1.58

$4 . \% 6$
3.2
11.1
3.6

0.140
$-1,4$

1.11
2.7

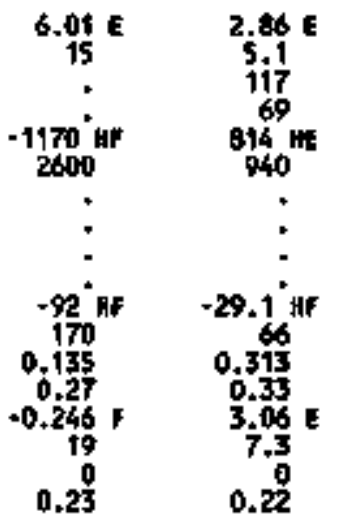

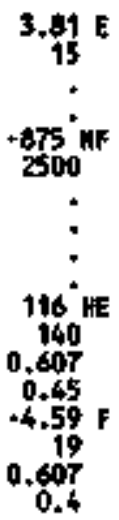

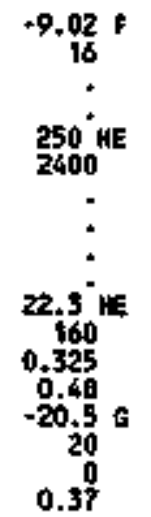




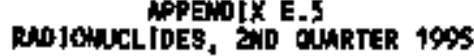

SAPL tho PORT Lochtton

DAtE SNive

GRess alpuh corrt twe ERlto ckoss eET Colint the ERLok RMOLONuCLIDES

\section{7-cestun}

Co-p

Counting ERhor

PRoTact bi:

covirjus Ethor

pebtu

courtius Evero

STAOHTHU

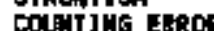

23t+234-tHOR IU:

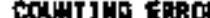

zk-1

CoUnitus $\mathrm{x}$.

235-104is]

cotititus gepo

27-10 ANity

COUNTIA ERAR

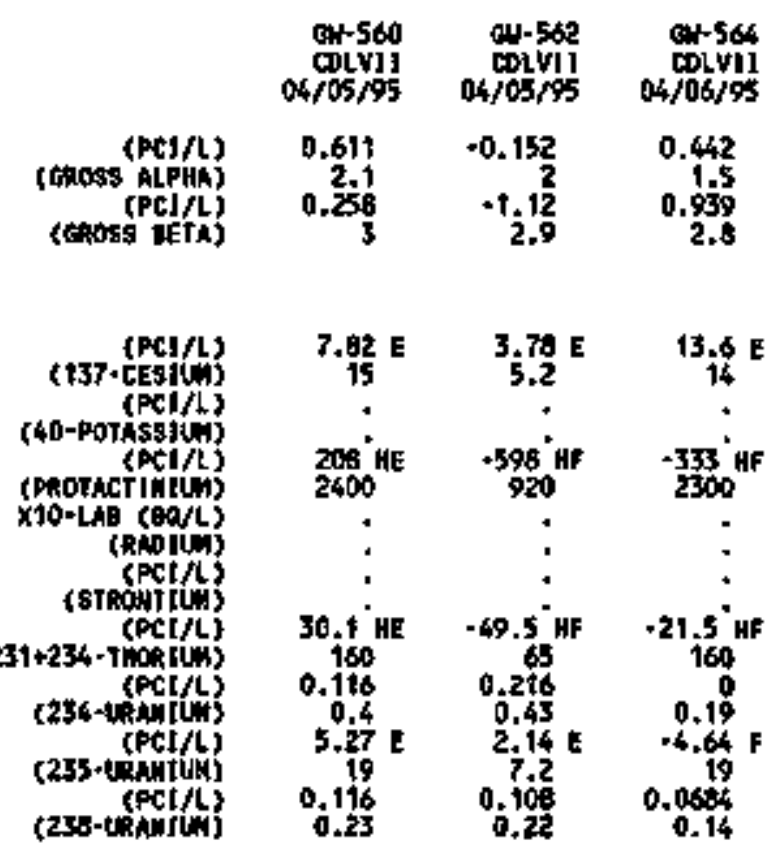

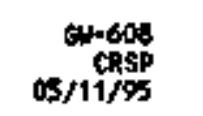

GN+609

$05 / 16 / 95$

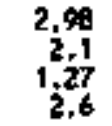

0.872
1,5
1,49
2,5

CN+610
chisp
os 07005

ar-611
cosp

ratsi

an/07/95

tal-752

of $107 / 95$

0.619
1.37
2.6

1,81
1.7
3.14
2.6

0.615
1.6
9.47
3.3

91
20
59.8
31

6it-742 05 rot/s 6.34
2.65 
suplitu point

tochticy

DATE SNUPLED

choss ILPHA

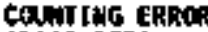

choss atth

conit the ERROA

RAOTOUNCLIDES

137-CESIOM

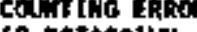

40.porasin

com

colvaris

RAoIU Ex

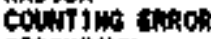

COUNT ING ERROA

c.

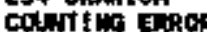

zas-utentu

cotist I Ex ERR

23B-URAMrUW

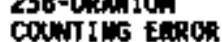

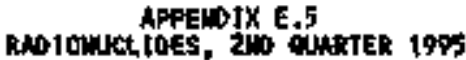

, 20 9urTER

$-743$

$6+745$

$05 / 07 / 95$

G-79 $\quad$ Bu-79

$04 / 12 / 9$

$04 / 11 / 95$

ate 798

क्षiY1]

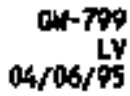

ax-801

난

GI-B27

$\begin{array}{rr}0.32 & -0.166 \\ 1.1 & 0.59 \\ 1.85 & 1.59 \\ 2.6 & 2.6\end{array}$

0.326
1.1
1.17
2.5

0.852
1.48
2.8

0.164
1.1
1.54
2.5

0.649

GROSS ALPH)

(CROSS BETA)

2.3
4.06
2.8

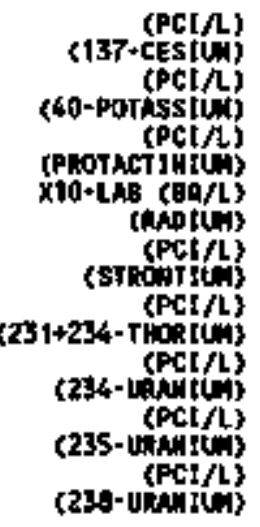

1.78

$1100^{\circ}$

1100

84

$\dot{0}$

2.77
0.441

0.441

$7.3 \mathrm{E}$

7.3
-0.221

0.40
$9.22 \mathrm{~F}$

$.500^{\circ} \mathrm{HF}$

2500

151
140
0.251
0.35
-1.69
-0.231
0.52
$-0.864$

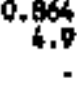

$-16.6 \mathrm{HF}$

240

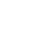

$52.9^{\circ} \mathrm{HF}$<smiles>[As]</smiles>

1.45

7,3

0.0749

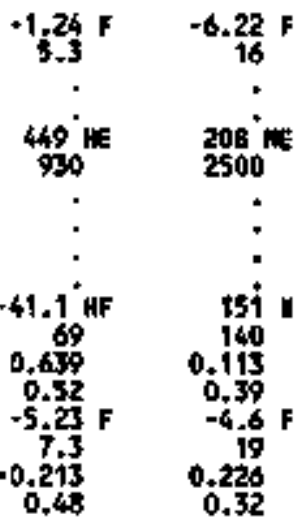




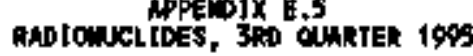

SArplthe POIMT LOCATrON

ONTE SHPLED

GROSS ALPHK

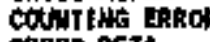

Ghoss Bath

COtNTIHE ERRO

RAO IOHKCLIDES

241-ANEkIC]UN

COUNT THA ER

contus

129-1CBItE

COWNTJHS ERROR

237+NEPTUN]UA

cDNAT two bahor

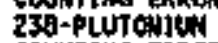

COUNTINO ERRO

239-FUTOWIy

castitus

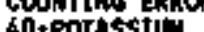

40+Porassicar

comict

court

conis enso

conTIHE ERRO

cant tin ERhon

Q ictur

$331+234+t$ ton

cat

TRATitit End

34-URaniun

OUNT1:3 ERRON

5S-Lixylum

chin

COUTTHB ERROA

(PC)/L)

(PC)/L)
(AROSS ALPHh) (aross GeTh)

$\begin{array}{rr}04+141 & 04-142 \\ 115 & k+49 \\ 07 / 10 / 95 & 07 / 14 / 95\end{array}$

\begin{tabular}{|c|c|c|}
\hline $\begin{array}{r}0,143 \\
k+10 \\
07 / 16 / 95\end{array}$ & $\begin{array}{r}c w-144 \\
\mathbf{k} 10 \\
07 / 16 / 95\end{array}$ & $\begin{array}{r}0.145 \\
\text { nat } \\
07 / 17 / 85\end{array}$ \\
\hline
\end{tabular}

0146
$k 110$
$07 / 17 / 95$

$\begin{array}{rr}1.7 & 6.62 \\ -0.553 & 1.9 \\ 2.8 & 8.44\end{array}$
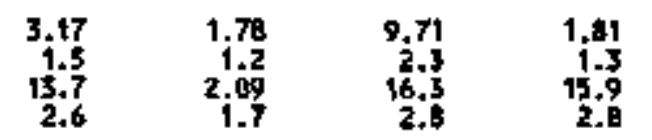
(24t-NERJCJ(L)
(PC1/L)
(137-CES1LW]
$\times 10-1 A 8(\mathrm{BC} / \mathrm{L})$

(129-100 IHE)

(237+NIPTLIItw)

(2EB

(PCI/L)

39-PUTOWIU)

(P0-Potassitt)

(PCI/L)

(10,

X10.

X10.chs

(smouflus)

(acj/h)

(PC1/L)

$x \rightarrow 0-1$ -

(TR(T)in)

(234-LRANI)

(PC1/L)

(235-LRAN]

(PCI)

(236-URALISL)

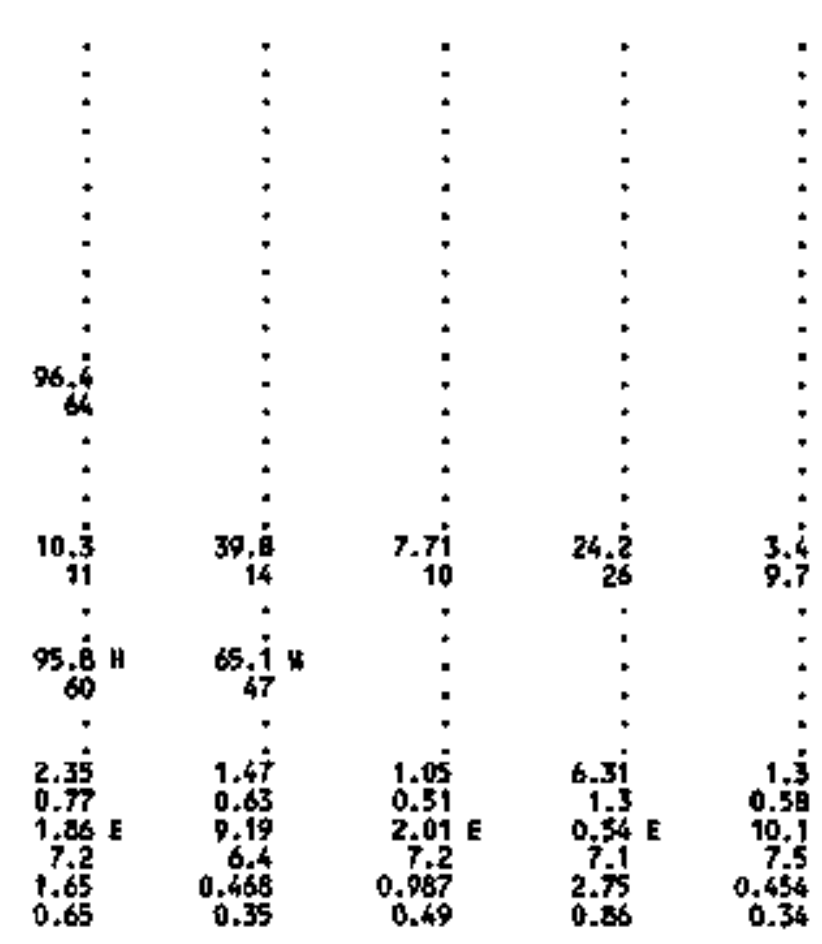

\begin{tabular}{|c|c|c|c|}
\hline $\begin{array}{r}\text { 나-147 } \\
\text { Kug } \\
07 / 14 / 95\end{array}$ & $\begin{array}{r}c 4-156 \\
\operatorname{cosp4} \\
07 / 13 / 9\end{array}$ & $\begin{array}{r}\text { en-15s } \\
\text { Crsats } \\
\text { o7/18/95 }\end{array}$ & $\begin{array}{r}6 N+159 \\
\text { CRSDA } \\
07 / 12 / 45\end{array}$ \\
\hline $\begin{array}{r}2,36 \\
1.5 \\
4,51 \\
1.9\end{array}$ & $\begin{array}{l}3.13 \\
1.45 \\
6.5 .1 \\
2.1\end{array}$ & $\begin{array}{r}1.85 \\
1.8 \\
1.49 \\
1.9\end{array}$ & $\begin{array}{l}0.942 \\
0.93 \\
0.201 \\
1.9\end{array}$ \\
\hline
\end{tabular}




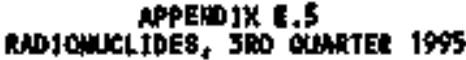

SHPLING POIMT LOChTtON

OATE SAMPLED

Cross MPH

Cont ils than

cobitIin EARD

Rerownajoes

241-ANERICIUN

coming stan

I37-cestui

CoxinTine ERino:

12\%-1001!

connifuc Expor

237-Nepruntu.

coll

as-purontar

D.

239.punc

Cantin tha

40-porkssit

conting ERRo

pot

conritic thes

Rour

cantios then

rhonr tum

ConTin Emo

g-ischin

cus

cour

CONm ERTor

Thiriun

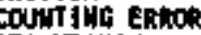

234-LRAMIU

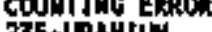

235- LRANIU

COUTIJUG ERKO

230-IRANIUW a-trs

ciltis:

ches casp

Casp
$09101 / \% 5$

6N-18 $07 / 20 \% 95$

1.33
-0.0986
2.6

1.49
1.7
1.54
2.5

-0.167
0.549
2.5

15.-217

$07 / 10 / 9$

的-231

an- 24

trise

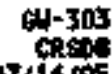

$61+304$

of 12 istos

0.791
1.85
3

0.0765
0.00
0.25
1.6

1.49
1
0.828
1.9

1.65
1.2
2.94
1.9

1.63
1.1
0.485
1.9

LIY

$07 / 12 / 2$

0.1.51 or $29 / 95$

0.621
+.7
4.3

$-0.326$

0.313

(241-Nererict)

(PC) $/ 5)$

(137-cestu

10-LA (c)

(129-101,

(PG]/L)
(237-HEPTLW]UA)

(PC]/L)

(25B-olvtomtin)

(239-fuvtowis)

(40-porisst(4)

(PCI/L)

(PROTACTIM IU⿴囗十)

xio-La says

(PCI/

STROnTIte.

(PE]/L

(PCI/L)

Si+cFs-inokt

10-4s (\%o/l)

(TRition)

(234-URANI (N)

(PE) $L$

(235-URAN]

(23s-Lendt(L) 
ANDTOWUCL IOES, 3 DO OUARTER 1905

Surytio POJuT Locatton

DATE SHMPL

cocoss ALPHA

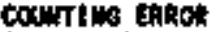
6h0ss IEth

MBJONKLIDES

241-NAEREC]W COMIT IW: ERRO j37-CESIUT

court int takor 129-100]HE

COUNTITG ERRO

237-HEPJuHJu

COWTIMG ERRD

336-PLUTONIUM

com-P

CONT ItG ERPO

40-P0TA8s]

countint epito

PROTAC InIUA

Coljit] EN ERO

DenII

coulutius baron

STROAITHA

CantuHs emon

\$9-FECHEET ILH

courtima thon

$231+234$ - J100210

COUNT JWG ERROA

TRITJU

CONTH ER ERAO

23-Litantur

coming

count

Z30-unditur

COUHI IAG ERROA

\begin{tabular}{|c|c|c|c|c|c|c|c|c|c|}
\hline & $\begin{array}{r}64+521 \\
\text { Lty } \\
07 / 11 / 95\end{array}$ & $\begin{array}{r}01-523 \\
115 \\
07 / 32 / 45\end{array}$ & 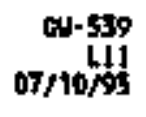 & $\begin{array}{r}04.600 \\
\text { chsp } \\
07 / 31 / \% 5\end{array}$ & $\begin{array}{r}\text { cu-609 } \\
068 \\
04 / 01 / 95\end{array}$ & $\begin{array}{r}a 4-610 \\
\text { CRsp } \\
07 / 3 i / 25\end{array}$ & $\begin{array}{r}\text { cy-611 } \\
\text { cisp } \\
0001 / \% \text { s }\end{array}$ & $\begin{array}{r}04-709 \\
07 / 10 / 95\end{array}$ & $\begin{array}{r}\text { n-731 } \\
\text { cospos } \\
07 / 13 / \% 5\end{array}$ \\
\hline 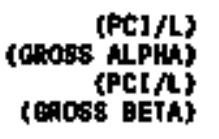 & $\begin{array}{r}0.774 \\
1.7 \\
1.23 \\
2.9\end{array}$ & $\begin{array}{r}0.313 \\
1.7 \\
2.9\end{array}$ & $\begin{array}{r}0.302 \\
3.17 \\
3\end{array}$ & $\begin{array}{r}1.27 \\
1.6 \\
-1.86 \\
2.4\end{array}$ & $\begin{array}{r}0.466 \\
1.5 \\
0.94 \\
2.5\end{array}$ & $\begin{array}{l}0.84 \\
1,3.5 \\
1.5\end{array}$ & $\begin{array}{r}0.057 \\
1.7 \\
1.64 \\
2.5\end{array}$ & $\begin{array}{l}1.62 \\
1.32 \\
0.34\end{array}$ & $\begin{array}{r}0.0867 \\
0.75 \\
14.6 \\
2.8\end{array}$ \\
\hline
\end{tabular}

(241- $(\mathrm{PC}(\mathrm{s})$

$0.767 \mathrm{~J}$

(137-

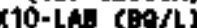

(13)-100,

(237-MEPTLSTIA)

(238-PLUt:

(P.:[A)

(239-putiontin)

(40-POT (PCt/L)

(AC1/L)

(PRotactinjus

$\times 10-10 \mathrm{c}$ (80/L

X10-LA (Rdolin)

(5TRO)

(D)

(99-TEChis

(231+234-T)

(Thuтriti)

(PCLI/C)

(234- Hisaytitis)

(235-U) (PCLAL)

$$
\text { (PCI/A) }
$$

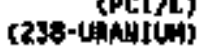

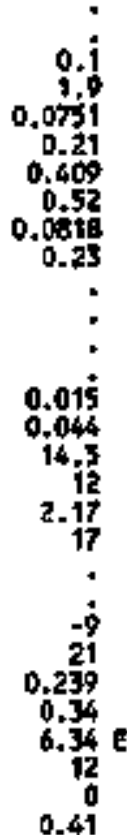


AppeindX E.5

RAb tonucl IDES, 3AD cUNTE 1995

9MPLIME POIAT

Locut to.

DAIE gNFL

conss NLPNA COUIT the ERMOR CowTh ERT cooss 最F

RNojomuctJbes

241-NERICItin CQUnthe ERAOR 137-CESII

CBDi? tNo ERAN

COUiN Jis ERRD 237-NePTu Itin colnin Ca-pUTON1U. covin

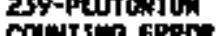
conrsu ekron 40+P0TA89tu partariteln COUNTING ERROR

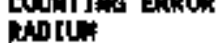

ERhor:

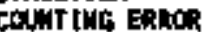
9\%-TECHNETIUN

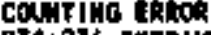
231.234-inas Coming exion Thitrun

COMTIMG ERROO 24. (1) CountJing ER ERo 235. Virhy

Conitur COWTIMG ERROR al-732 Cad-732 or/13/9s os chitist (PCI/)
(emoss HLPHA)
(PCI/L)
(EROSS BETA) 6.49
11.8
2.6 0.65
1.3
2.83
2.6 41- 74 CR5P an+76s 品早 or/
-0.165
1.3
0.0707
2.4

6,58
2.2
-0.0412
2.5

배-757 offilts 6.39
2.2
7.9
3

(261-MCI/L)

\&PCl/ chto-

$x+0-2000 / 4$

(129-100]

(337-LEPTU]

(23s-plut (PC)

(23s-DLutowin)

(239-pLUTOa]U)

(40-por (PCt/

(60-porassiu

X10-LA

(tet

(PCI/L

(9)-iEchinetion

(23 4 (PCL $/ \mathrm{L})$

X10-EAB (BA/L)

(TRj]

(PCLI

(235-uinutur)

(23o-unditu) 
Aablonucl foes, 4TH QuAkTER 1995

SANAtIBO DOINT LOCAT JOA

DATE SANPLE

CROSS MPHA cooss ofth

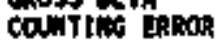

RAOIOMULLIDES

137-CESJU

CDW

40-pOThssjuy

Courtin erro

poothct Jutus

COINTHC ERRO

RaDINW

CONAT IAG ERARO

COWHIIIG ERROC

2314236- FW+RIt

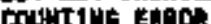

204-1)

COMIIUG Ephio

235-URAlula:

coint 1 Hs ER

cT.

Cointring Ekion

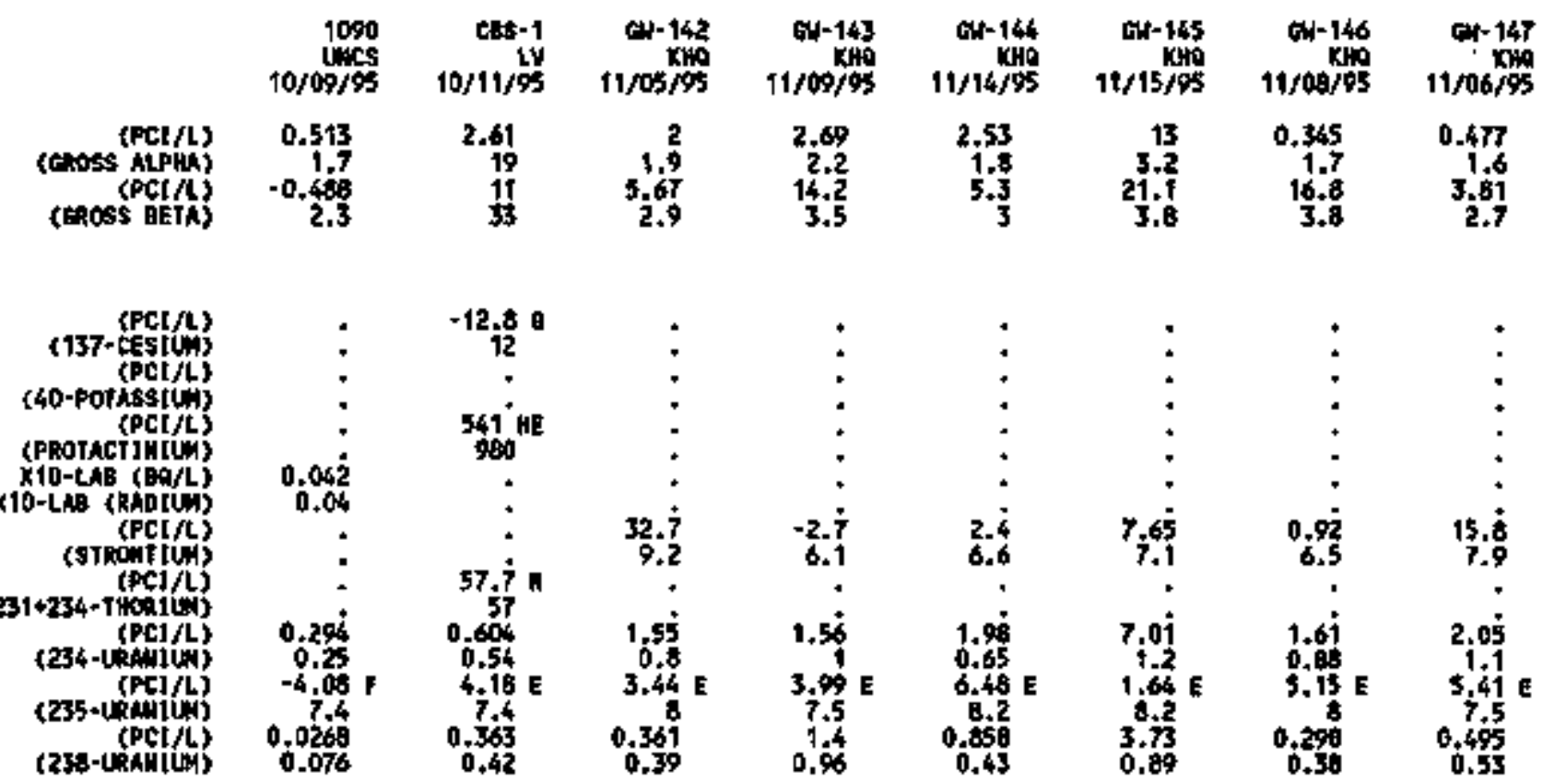




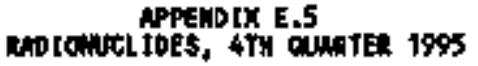

SAMPLING potht tont to

DATE gavipleo

cRoss AlpH

CONTHo terRo

Gross reth

COUnt to ERROM

AND JoIUCL toES

$137+$ cestius

CONHIIU ERRO

40-potassin.

CONTIS ERRO

PROTACT [H]CM

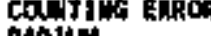

Ato1네

Conititiva ERRO

SThowTtun

CrumTin EnRo

31-304-Tingli

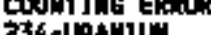

234-UaAniun

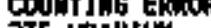

23s-Lahin

cowitring ences

Coukt jut Ebot

\begin{tabular}{|c|c|c|}
\hline & $\begin{array}{r}c 156 \\
\text { casps } \\
10 / 2 \% / 55\end{array}$ & $\begin{array}{r}156 \\
10 / 24 / \%\end{array}$ \\
\hline 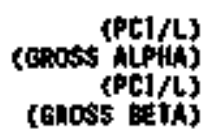 & $\begin{array}{r}2.27 \\
1.6 \\
4.32 \\
2.7\end{array}$ & $\begin{array}{r}2.8 \\
1.7 \\
3.13 \\
2.6\end{array}$ \\
\hline
\end{tabular}

cu-156

데-136

c44-159 $10 / 25 / 95$

CRsps

catsin

costo

10/24/4

AN-159

cit-150

100

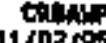

0.522
1.8
5.88
2.7

$\begin{array}{rr}1,62 & 1,85 \\ 2.3 & 1.4 \\ 6.52 & 2,28 \\ 3 & 2.5\end{array}$

1.70
1.4
2.53
2.6

1.14
1.8
1,17
2.4

-1.18
1.6
13.1
3.6

39.4
7.4
34.2
5.4

$10 / 31 / 95$

(ocl/s)
(t57-cesilus)
(fCI/L)

(40-porassium)

(P.:OTACI/L)

X10-Lat $(\mathrm{BO} / \mathrm{L}$

r10-Lá (Roblum)

cotrocitllis)

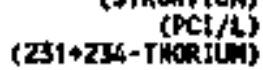

(PCI/A)

(23)-undution)

(235-1Ramuis)

(23a-4)(Ristic) 
SAPLIN POINT

LOCRT JOA

DATE SANPLED

cotoss AlP

CONTS STO ERROA

anoss BET

COCHT IHE ERIOR

RatawelioES

137-CESTUM

Co-

cacirting eseon

potatctrilin

COUNT ING ERROP

aty

calurtita ERROR

sicourte"

Cout tor eRnor

31+234-THOAIU,

CotivT 1HG EkAOA

234-Lint

COUMTIME ERRO

235-LAAH]t:

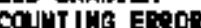

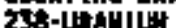

COUT IME ERROR

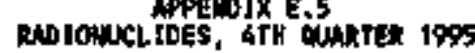

\begin{tabular}{|c|c|c|c|c|c|c|c|c|c|c|}
\hline & $\begin{array}{r}\text { UN-175 } \\
\text { cRsp } \\
11 / 17 / 45\end{array}$ & $\begin{array}{r}w-177 \\
\text { wsp } \\
11 / 6 / \% 5\end{array}$ & $\begin{array}{r}\text { sw-18f } \\
\text { CRsp } \\
11 / 03 / 95\end{array}$ & $\begin{array}{r}61-984 \\
70 / 31 / 95\end{array}$ & $\begin{array}{r}\text { GN- } 180 \\
11 / 03 / 98\end{array}$ & $\begin{array}{r}\text { GD-187 } \\
\text { RD } \\
11 / 02 / 95\end{array}$ & $\begin{array}{r}04-904 \\
10 / 31 / 95\end{array}$ & $\begin{array}{r}64-205 \\
\text { wes } \\
10 / 07 / 55\end{array}$ & $\begin{array}{r}61-205 \\
101+5 \\
10 / 07 / 95\end{array}$ & 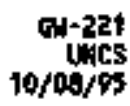 \\
\hline 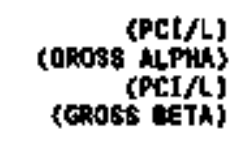 & $\begin{array}{r}-0.51 \\
1.3 \\
0.163 \\
2.6\end{array}$ & $\begin{array}{r}1.9 \\
1.9 \\
2.22 \\
2.7\end{array}$ & $\begin{array}{r}1.7 \\
0.958 \\
2.9\end{array}$ & $\begin{array}{r}0.629 \\
1.7 \\
1.5 \\
3\end{array}$ & $\begin{array}{r}2.35 \\
2.6 \\
1.15 \\
3\end{array}$ & $\begin{array}{r}3.26 \\
3.2 \\
2.35 \\
3.2\end{array}$ & $\begin{array}{r}0.961 \\
4.67 \\
3.2\end{array}$ & $\begin{array}{r}0.324 \\
1.4 \\
0.311 \\
2.3\end{array}$ & $\begin{array}{r}-0.655 \\
1.5 \\
5.09 \\
2.7\end{array}$ & $\begin{array}{r}-1,14 \\
1.2 \\
2,31 \\
2.5\end{array}$ \\
\hline$(137+\operatorname{ces}(1)$ & 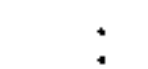 & $\dot{2}$ & 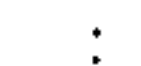 & $\dot{.}$ & * & : & 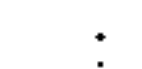 & : & 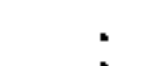 & 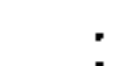 \\
\hline (PCI/L) & . & ; & : & ; & : & : & : & $\dot{0}$ & ; & $\vdots$ \\
\hline (40-PAThsstiut) & 1 & - & - & - & - & . & $*$ & . & . & + \\
\hline & : & : & $:$ & : & : & $:$ & : & · & - & - \\
\hline$\times 10+\mathrm{L} / \mathrm{s}(\mathrm{ba} / \mathrm{L})$ & 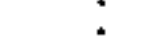 & . & 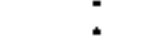 & , & ; & $\dot{7}$ & 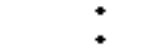 & 0.007 & 0.014 & 0.009 \\
\hline 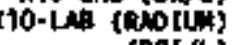 & . & . & . & + & . & : & $\dot{*}$ & 0.03 & 0.031 & 0.062 \\
\hline (PCL/A) & - & - & - & - & . & • & . & . & : & 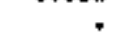 \\
\hline (STaOntIUA) & - & - & - & - & - & - & - & . &. & • \\
\hline (PCI/L) & - & - & - & - & - & . & • & + & - & - \\
\hline $31+234=$ THok164) & • & - & • & . & - & - & - & & & \\
\hline (234-LPC1 $(L)$ & : & : & - & : & - & * & - & 0,303 & & 0,263 \\
\hline 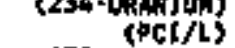 & $\dot{\bar{c}}$ & ; & $:$ & - & $\therefore$ & $\therefore$ & $\dot{-}$ & $-1.51 \mathrm{~F}$ & 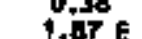 & $2,24 \mathrm{E}$ \\
\hline (235-LRinitis) & . & : & . & : & ; & : & ? & 6.2 & 0.2 & \\
\hline$(\mathrm{P} \neq 1 / L)$ & - & . & . & * & . & - & - & 0 & 0.106 & 0,105 \\
\hline (238-URntivin) & - & - & . & - & - & . & - & 0.17 & 0.15 & 0,15 \\
\hline
\end{tabular}


APPERDIX E.5

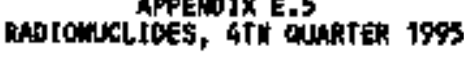

SHupline pount LOCASION

SAPrond

Cross HLPU

conin Al ERro?

COUNT WHG ERRO

RHP IOUUCLIDES

137-CESIOM

COUNT JMO ERROR

$40+$ porAss]

certictur

contrino enton

COUnTHO ERNon

Rayim

COUNITISC ERMOA

sinn

COUNT JWG ERNOR

$251+234-T h a n$ iun

COUiTIno ERko

34-urahiu

con r encon

Zss-10xhin

COUNIJG ERIO

coutrimo ERRO

\begin{tabular}{|c|c|}
\hline & 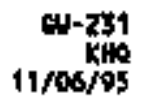 \\
\hline 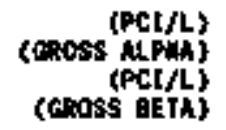 & $\begin{array}{r}0.622 \\
1.7 \\
0.697 \\
2.5\end{array}$ \\
\hline 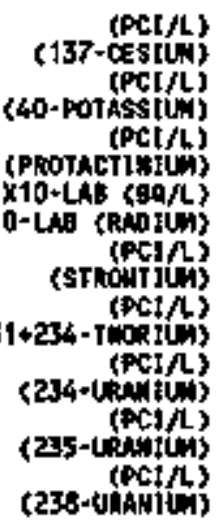 & $\begin{array}{l}1.01 \\
0.71 \\
5.77 \mathrm{E} \\
7.6 \\
0.44 \\
0.46\end{array}$ \\
\hline
\end{tabular}

\section{at.202}

cu- 29

aw-29:

ECRUP

oiv296

91-298

chiskin

chantip $11 / 06 / 55$

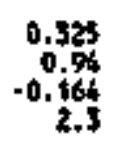

0.358
0.97
-0.16

0.253

0.516
0.308
2.4

2.61
1.6
1.4
2.4

0.164

$-0.163$

1.8
1.75
2.6

$-0.596$

1.3
0.482
2.6

Cllanip

Cㄴ-302

orotios

0.47
1.
2

1.72
1.8
2.6

$+0.354$

$-0.00906$

2.3

0.048

0.036

71

7.6

$0.500^{\circ}$

6.32

6.7

0.202 
APPENDIX ES

RADJOWCL TDES, 4TH OUNRTER 1995

SHapl ING pornt

Locktion

DATE satpe E

gross Alpik

codstims EAme

choss ieth

GAD]OWUCL [DES

137 -cescum

count thi ER:

coming

pooract thiti

courn ters

RADIU,

COUntINO ERRO

STRONTII

Uit Int ERRO:

covit1 ER jum

ConTin ER.

COUNTHA ERROM

contring

toturitut thos

ctoring

COUHTIHS ERROR

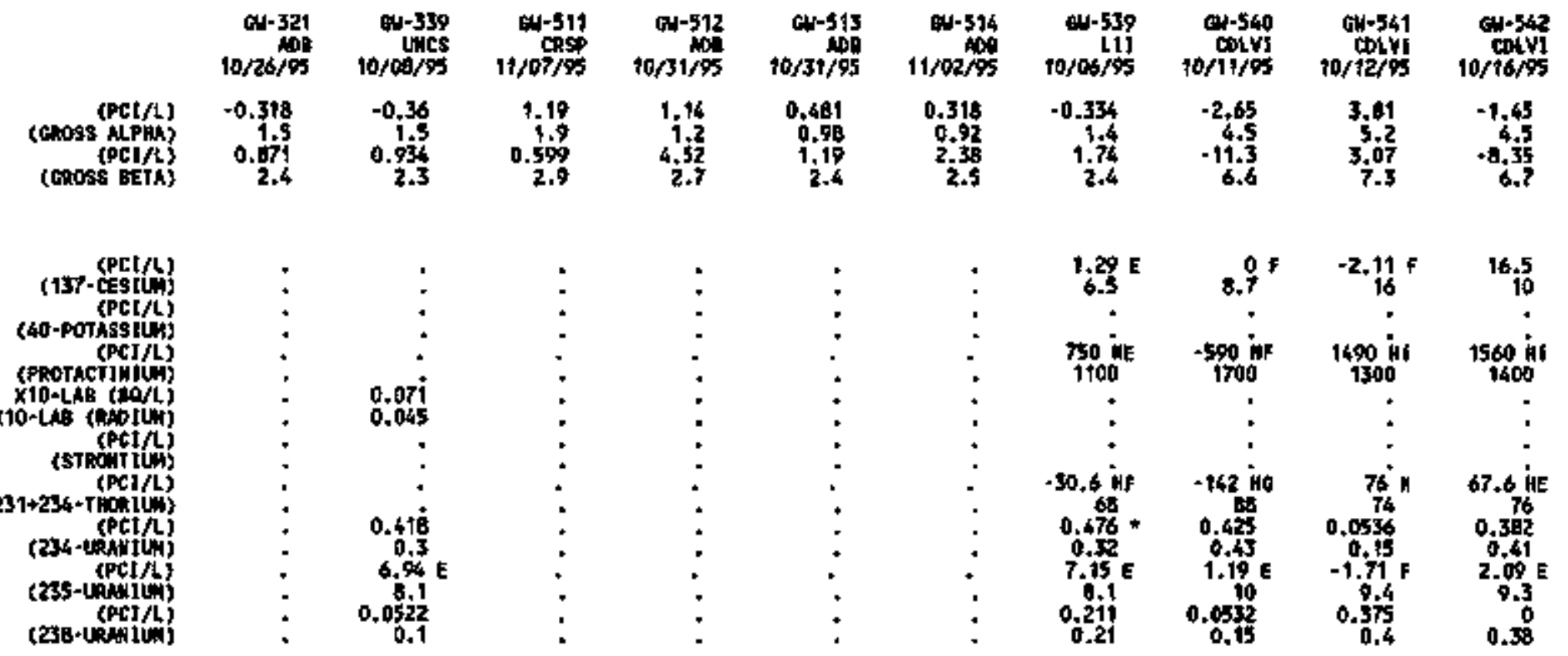


APDEIO IX $E$

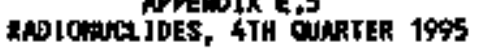

SANPLLHG POIMT LOCATlán

DATE SATPLE

choss AtPu

Colwiting ERRBCh

colinting ERRot

AnDighUCLIDES

137-CESIUN

COUNIING ERROA

40-porkss]1,

CONintwa ERRE

Pkotict InIL

COUntino ERRO

Rondu

COUnTung Earor

STROiTJu

231+234-71608tun

CONATIMG ERROA

25T-URAMTUN

COH,IIMG ERROR

235-uRAtin

CONGITIMG ERkM

238-URAHIO

DOWTHE EARDR

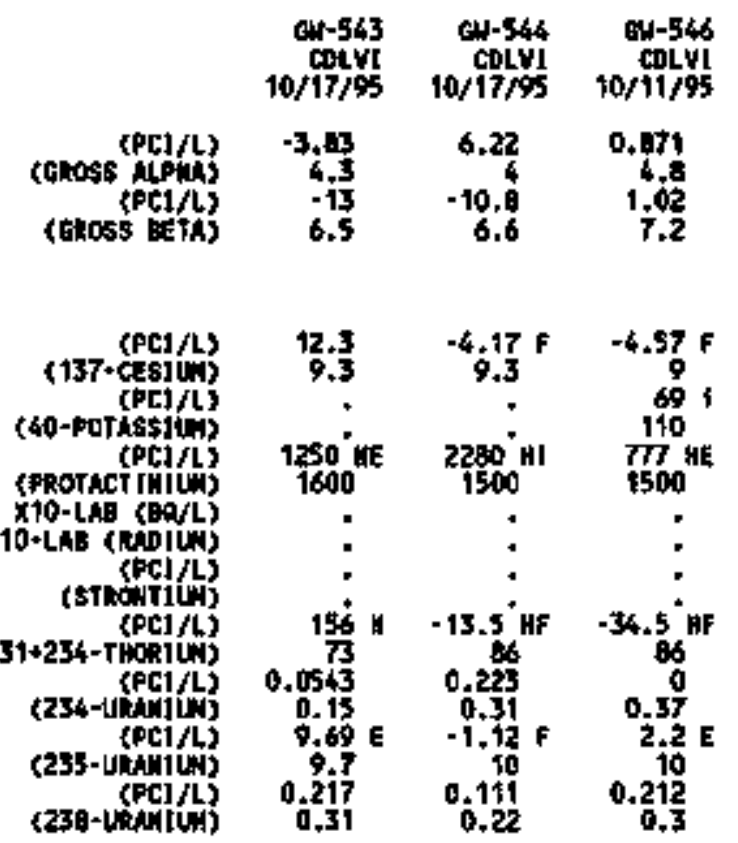

ew-560 10,060145

can-562 10\%06/95

chist4 10/07/s

01-609 CRSP
$16 / 95$

64-600 $11 / 20 / 95$

ct)-610 $0 / 07 / 45$

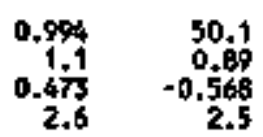

0.641
0.91
-1.32
2.5

0.329
1.5
1.5
2.7

$-0.00075$

.00078
1.4
1.16
2.7

-0.00071
0.0777

2.5

$-6.28$

$1099^{\circ}$ HT $-24^{\circ}$

$-0.585 F$
13
-24
$1100^{\circ}$

$-7.43 *$

325 'HE

$-3,1 \mathrm{~F}$

ano

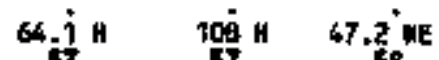

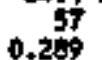

0.249
0.26

0.26
-3.25

0.57 E

0.267 - 0.187

0.187
0.2
7.11

0.0534

0.0535
0.11

$132^{\circ} \mathrm{HE}$

0.2

0.11

105

0.162

0.19 
RUDJONUCLIOES, 4TH GIANTER 1995

SHAP ING Polut LOCATION

DATE SNIPLED

GROS: AlpHA

codilit tiw entros

ChOS ExTh

RaOlouxuloes

$137+\mathrm{CES1}$

Col

COUNT IRO ERFOR

PFotret Injt

CONT1:日 then

poloju

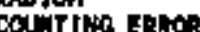

8Thont tu.

COUII ING ERHOR

$231+234-T$ COAI

countikt thon

24-10

bouting Easo

235-10huld

Dountille ExeO

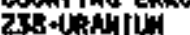

Coumtitic ERroe

\begin{tabular}{|c|c|c|c|c|c|c|c|c|c|c|}
\hline & $\begin{array}{r}n+611 \\
\text { thsp } \\
11 / 17 / \% 5\end{array}$ & $\begin{array}{r}0 w-709 \\
\text { L1] } \\
10 / 06 / 95\end{array}$ & $\begin{array}{r}\text { CR-731 } \\
\text { CRspo } \\
10 / 23 / 95\end{array}$ & $\begin{array}{r}04-731 \\
\text { Crsos } \\
10 / 26 / \% 5\end{array}$ & $\begin{array}{r}\text { cas1 } \\
10 / 25 / 95\end{array}$ & $\begin{array}{r}4-71 \\
10 / 26 / \% 5\end{array}$ & $\begin{array}{r}\text { cutraz } \\
\text { Chsto } \\
10 / 23 / 95\end{array}$ & $\begin{array}{r}\text { anstor } \\
10 / 24 / \%\end{array}$ & $\begin{array}{r}04-732 \\
6 \times 809 \\
10 / 26 / 95\end{array}$ & $\begin{array}{r}0.732 \\
10 / 26 / 95\end{array}$ \\
\hline 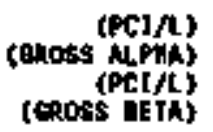 & $\begin{array}{l}0.867 \\
1.7 \\
0.156 \\
2.6\end{array}$ & $\begin{array}{c}5.73 \\
16 \\
11.3 \mathrm{c} \\
2.3\end{array}$ & $\begin{array}{r}0.321 \\
0.93 \\
3.65 \\
2.7\end{array}$ & $\begin{array}{r}2.65 \\
1.6 \\
11.4 \\
3.3\end{array}$ & $\begin{array}{r}-0.343 \\
1.5 \\
12.5 \\
3.3\end{array}$ & $\begin{array}{r}-0.174 \\
1.5 \\
2.39 \\
2.7\end{array}$ & $\begin{array}{l}1.51 \\
1.31 \\
2.31 \\
2.6\end{array}$ & $\begin{array}{r}2.76 \\
2.7 \\
2 ., 4 \\
4.8\end{array}$ & $\begin{array}{r}-0.517 \\
1.5 \\
2.1 \\
4.4\end{array}$ & $\begin{array}{r}-1.08 \\
17.7 \\
17.5 \\
3.9\end{array}$ \\
\hline
\end{tabular}

(137-CEST(L)

(PCI/

(40+Porassitw)

(P)oTActiH (L)

x10-1

x10-L

(PCl/L)

(PCl/L)

(PCI/L)

(235-URMulut)

(2):- (PC)/ 
BADIOACLIDES, 4TM CUARTER 1995

SAHPLINS pallit LDERTIOA

tripte

Gross MPM

COUWT IRG ERROR GROSB gETh

coumtot geror

RAD townet tots

t37-ctsin

COUNTIHG EARON

40+POIASSIU.

cbortin

Phorincrinch

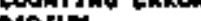

COWNTHE ERROR

COUNT WO ERRO

231+234-THoitio

$231+234-110$ and

234-uph int

cousting Etach

235 -(MkANILA

colnit Ho Ekto

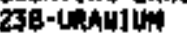

CONTING ERTOR

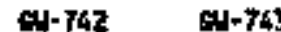

$11 / 09 / 95$

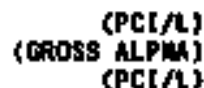

(c)osety

$\begin{array}{cc}5.01 & 0.023 \\ 2.4 & 1.6 \\ 4.72 & 13.4 \\ 3.1 & 3.6\end{array}$

(137-cestus)

(13) (PCI)

(40+potissiua)

SPCl/L

(10-1)

$x$ -

X70-LAB (RADJLA)

(sradint

(231+236-1 (PC]/L)

(PC: $/ \mathrm{h}$

(234+1JRAk]UN)

(2035-19t)

(40. -

(238-uRarilu)

$10 / 06 / 95$ 10/09/95

$\begin{array}{lr}7.17 & 0.402 \\ 9.57 \mathrm{c} & -1.06 \\ 25 & 2.5\end{array}$

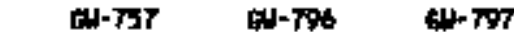

47-

10/09/

1.66
1.3
0.709
2.6

CotvI1

$10 / 00 / 5$

1.13
1.1
1,13
2.7

0.844
0.99
-0.497
2.6

GN-790

Ly

H. 80

4

$10 / 09 / 9$

0,159
0.75
-0.07

CoLY:

10/16/95

-2.05
-9.21

2.5

6.7

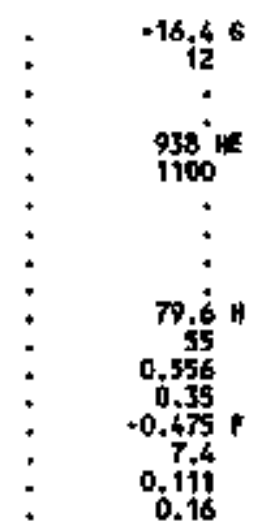

0.322

$-11$

$-6.97$

$-260^{\circ} \mathrm{if}$

$57^{+}$HE $553^{\circ} \mathrm{HE}$

1100

:

$71 . \dot{H}$ H 51.4 HE

52

0,105

3,04 E

$0.7+5$

0.567

0.25

7.6

0.024

57 ye
57
0.155
0.18
3.96
7.5
0.18
$-2.18 \mathrm{~F}$

$950^{\circ} \mathrm{ke}$ 9:11 $130 \mathrm{He}$

$69 . \overline{7} \mathrm{~W} \quad+43.7^{4} \mathrm{HF}$

0.443

4.94

0.08 .6

0.16 

APPENDIX F

FIELD DUPLICATE DATA 



\section{EXPLANATION}

SAMPLING POINT:

$\begin{array}{rll}\text { GW } & - & \text { Monitoring Well } \\ \text { CBS-1 } & - & \text { Chestnut Branch Spring } 1 \\ \text { SCR2.2SP } & - & \text { South Chestnut Ridge Spring 2.2 } \\ 1090 & - & \text { Historical 1000-Series Monitoring Well }\end{array}$

LOCATION:

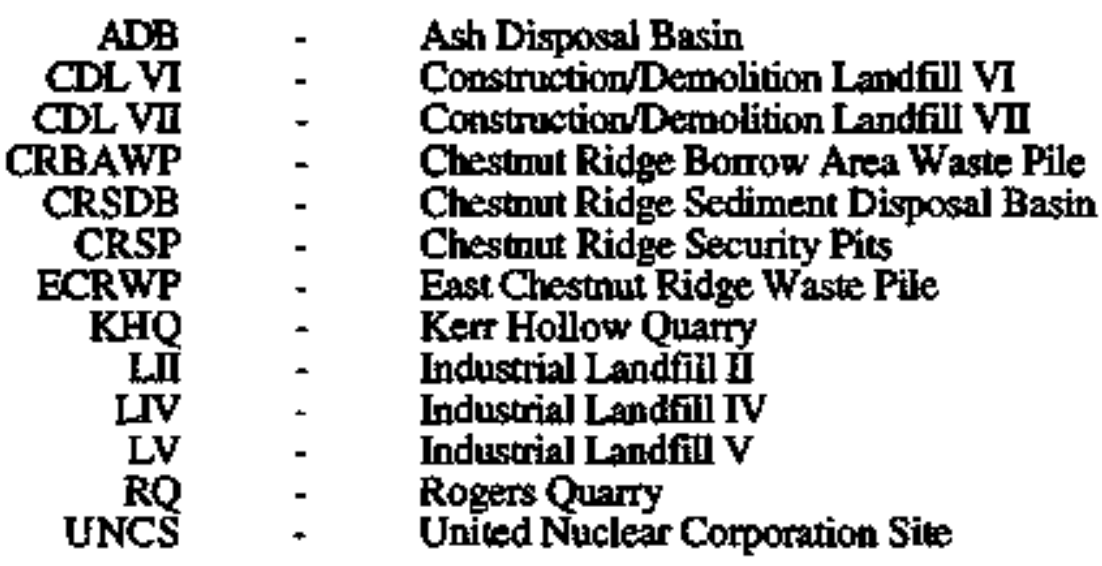

ALL DATA:

Not analyzed or not reported.

\section{ORGANIC COMPOUNDS:}

All results in micrograms per liter (ugl L).

METALS, ANIONS, MISCELLANEOUS, AND RADIOCHEMICAL PARAMETERS:

General:

Bicarbonate and carbonate alkalinity reported as $\mathrm{CaCO}_{3}$.

$<\quad$ - Compound analyzed but not detected at the reported minimum attainable detection limit

DIS - Dissolved Concentration (Filtered Sample)

TOT - Tacal Concentration (Unfiltered Sample)

TDS - Total Dissolved Solids

TSS - Total Suspended Solids

Sp. Cond. - Specific Conductance 



\section{EXPLANATION (cont'd)}

Method:

$\begin{array}{rll}\text { AAS } & - & \text { Atomic Absortion Spectroscopy } \\ \text { CVAA } & - & \text { Cold Vapor Atonic Absorption } \\ \text { PMS } & - & \text { Plasma Mass Spectroscopy } \\ \text { ICAP } & - & \text { Inductively Coupled Anc Plasma Spectrocopy }\end{array}$

Units:

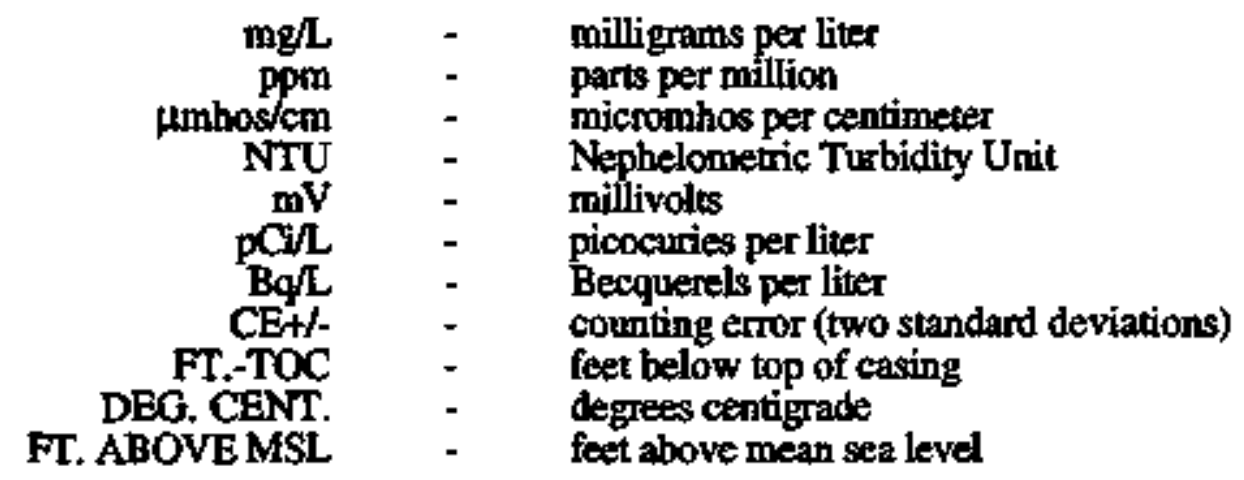



EXPLANATroN (cont'd)

\begin{tabular}{|l|c|l|}
\hline Analyte Class & Qualifer & \\
\hline & & \\
\hline Radionuclides & J & Estimated value. \\
\hline & A & Possible detector contarnination. \\
\hline & E & $\begin{array}{l}\text { Result of analysis is less than MDA, confidence level is less } \\
\text { than 95\%. }\end{array}$ \\
\hline & F & Result less than background. \\
\hline & i & Tentatively identified isotope. \\
\hline & * & Duplicate analysis outside control limits. \\
\hline & C & Control analysis outside of control limits. \\
\hline & D & $\begin{array}{l}\text { Spike control limits do not apply, sample activity exceeds spike } \\
\text { activity. }\end{array}$ \\
\hline & N & Spike recovery not within limits. \\
\hline & H & Daughter of uranium isotopes, reported for comparison \\
purposes only.
\end{tabular}




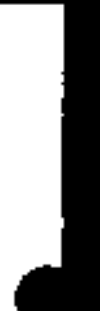




\section{APPENDIX F.1}

TRACE METALS AND ANIONS 



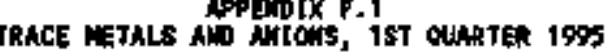

SANPLING POLNT

DATE SNPLED

TYPE

FThLs

AHT timowy

ARSENIC

BARIIN

DeRYLLILA

BOROM

CADitu-

cephiun

Calciution

changontun

COAALT

COPPE

tRON

LEAD

MACHESJU:

Nüans

MeRtury

MOLTE:AEMM

MTCKEL

PHOSPHOitots

orasstur

SELLE:ILI.

stlicon

SILVE

strourtum

STFOnT tum

The

Thensing

rentint

(1)

into

ALXALin TTY-HCOs

ALKALIMITY- $\cos$

CHLOAJDE

FLUOAJDE

MITRATE NITROSEN

AWONJA NJTROSEH

gllFAt

TOTAL SUSPEVEO SOLLS TUREtotfy cut 14

$01 / 09 / 95$

$04-141$
L1)
$0109 / 05$
61s

an. 143

$1 / 17 / 95$

6. 443

01/17\%

aW+511

$01 / 24 / 45$

ch-511

$01 / 24 / 95$

ca-709

$100 / 5$

6w-709 6N-732

1/09/95 $01 / 10 / 9$

64-732

CRSD

DIS

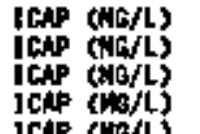

1.6
40.05

0.024

4.024

0

0.000

0.7

JCAP (Nib/L) 0.0003

jCP $(6 / 6)$

AN (in/L)

ICNP (NG/L)

JCAP $\left(w^{3} / \mathrm{L}\right)$

ids $(1 \mathrm{k} / \mathrm{L}$

ICNP $\mathrm{kW}$ (W/L)

JCAP (W.

JCA $(\mathrm{m} / \mathrm{L}$

ins ( $\mathrm{kW}$.

ICAP (NGL

ICA (m/L

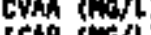

ichp (wo/t)

teap (nov)

leap (nort)

Ichp $(m / 2)$

teAp (inchs

ICAP (WW/t)

ICA

ICtp (wist)

MAs ( $10 / \mathrm{c} / \mathrm{t})$

texp (wo/t)

ICAP (ma)

ICAP ( $10 / 4)$

$\infty .003$

$<0.002$

$\infty .01$

0.01

c.005

$\infty .05$

$<.004$

0.026

40,0002

0.01

1.4

4,05

4.006

$0.01 \%$

0.01

$<0.0005$ d

0.006

0.07

$\$ 0.05$

0,043

40.001

0.011

$<0.003$

$\times .002$

4.01

0.01

$<0.005$

$<0.005$

$+0.05$

80.004

$\times 0.001$

$\infty .0002$

$<0.01$

1,1

$<0,008$

40,008

0.02

40.01
40.2

$<0,0005 d$

$<0,005$
$<, 002$

0.96

33

$<0,01$

$\times 0.005$

0.0075
3.9

$<0,0$

$3 i$

0.002

$\$ 0.01$

0.01

18

0.049

4.05

0.035

$\infty .0003$

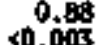

30

$<0$

$<0.000$

0.15

0,

0.039

40.01

40.01

$<0,05$

0.006

4.006

24
3.7

0.0019

0.6011

0.008

$0 . \dot{2}$

0.00

0.005
40,002

0.066

4.0

0.000

40,0003

0.024
40.003

$\times .01$

4.005

$\infty .005$

0.15

16

0.0029
0.0012

$<0.01$

$<0.01$

4.02

40.05
0.05

0.007

$<0.004$

$<0.003$

0.26

0.005

$<0.005$

$\$ 0.005$

$\times 0.05$

16

0.0015

0.0002

40.01

4.5
$\times 0.05$

4.4
$<0.05$

$<0.0006$

0

0.006
4.9

$4 \dot{2}$

$\infty .2$

$<0.005$

40.005

40,002

15

(H)/L

(NG)

(nhy

239
25
2.4

204
5.9
3.6

154
3.1

50.1
0.26

1.0

(NGS $L$ )

(NG/L)

(nTu)
0.032
0.05

$<0.05$

0.0003

0.022

$<.002$

$\$ 0.002$

40.01

$<0.01$

$\infty .005$

0.025

4,05

21
0.0019

40.01

\$0.01

1.1
0.05

$<0,006$

0.006

0.05

$\times 0.2$

0.0005 a

$<0,005$

0.004

178

4.1
0.59

$1 . \dot{9}$

0.5
$\$ 0.02$

$\$ 0.05$

0.26

0.00

0.011

4.002

4.002

$<0.01$

40.01
4.005

20.004

0.0051

$<0.05$

4.004

0.0017

40.0017

0.01

11.4

4.9
60.05
$<0.05$
0.12
0.0003
0.025
$<0.005$

20.01

$<0,005$

0.005

0.004

22

0.076

$<10002$

$<0.01$

7.6

0.006

0.006

0.03

$<01$

$<0.0005$ o

0.005

0.0059

0.006

B.1

$40 ;$

$0.00 \%$

$<0.005$ 


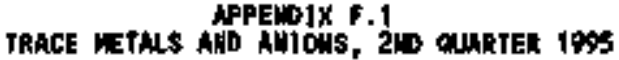

\begin{tabular}{|c|c|c|c|c|c|c|c|c|c|c|c|c|}
\hline 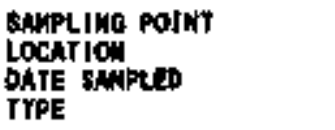 & & & $\begin{array}{r}c u-144 \\
\text { K100 } \\
04 / 25 / 95 \\
\text { Tot }\end{array}$ & $\begin{array}{r}04+146 \\
\text { X109 } \\
04 / 25 / 25 \\
\text { DI5 }\end{array}$ & $\begin{array}{r}\text { ad- } 159 \\
\text { CRsion } \\
04 / 06 / 95 \\
\text { T01 }\end{array}$ & $\begin{array}{r}0 w-159 \\
\text { Casnog } \\
04 / 06 / 95 \\
\text { DJs }\end{array}$ & $\begin{array}{r}64-186 \\
\text { Ra } \\
05 / 03 / 95 \\
\text { Tot }\end{array}$ & $\begin{array}{r}04+186 \\
05 / 03 / 95 \\
\text { Dis }\end{array}$ & $\begin{array}{r}\text { an-221 } \\
\text { 1wcs } \\
0,19 / 95 \\
\text { Tor }\end{array}$ & $\begin{array}{r}04-221 \\
\text { utces } \\
\text { os/49/45 } \\
\text { DIs }\end{array}$ & $\begin{array}{r}\text { Gy-292 } \\
\text { ECRLP } \\
04 / 24 / \% 5 \\
\text { ToT }\end{array}$ & $\begin{array}{r}64-292 \\
\text { ECHT } \\
04 / 24 / 95 \\
\text { ols }\end{array}$ \\
\hline \multirow{5}{*}{ 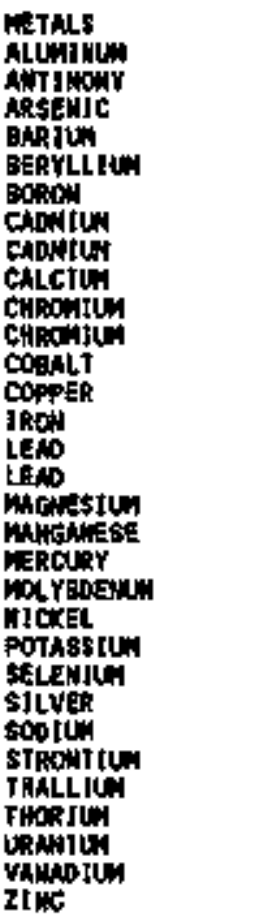 } & \multirow{5}{*}{ 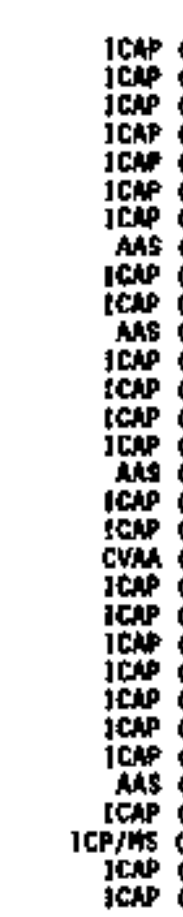 } & \multirow{5}{*}{ 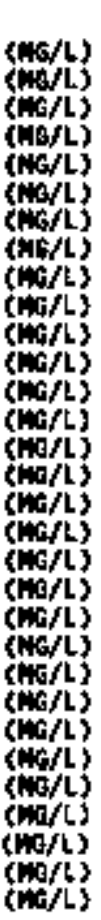 } & $\begin{array}{r}0.02 \\
0.05 \\
40.05 \\
0.047 \\
\times 0.0003 \\
0.037 \\
40.005\end{array}$ & $\begin{array}{r}0.02 \\
40.05 \\
0.03 \\
0.040 \\
40.0003 \\
0.023 \\
0.003\end{array}$ & $\begin{array}{r}0.13 \\
40.05 \\
60.05 \\
0.011 \\
\times 0.0003 \\
0.017 \\
40.003\end{array}$ & $\begin{array}{r}0.19 \\
00.05 \\
<0.05 \\
0.012 \\
<0.0003 \\
0.022 \\
\times 0.033\end{array}$ & $\begin{array}{r}0.054 \\
0.05 \\
0.05 \\
0.12 \\
\times 0.0003 \\
0.14 \\
40.003\end{array}$ & $\begin{array}{r}0.045 \\
40.05 \\
40.05 \\
0.12 \\
<0.0003 \\
0.14 \\
0.003\end{array}$ & $\begin{array}{r}0.031 \\
40.05 \\
\times 0.05 \\
0.007 \\
\times 0.0005 \\
0.056 \\
\times 0.005\end{array}$ & $\begin{array}{r}0.02 \\
0.05 \\
<0.05 \\
0.0074 \\
<0.0003 \\
0.018 \\
0.005\end{array}$ & $\begin{array}{r}40.02 \\
\times 0.05 \\
\times 0.05 \\
0.11 \\
0.0003 \\
0.041 \\
<0.005\end{array}$ & $\begin{array}{r}\$ 0.02 \\
<0.05 \\
0.05 \\
0.12 \\
0.0003 \\
0.11 \\
\times 0.000\end{array}$ \\
\hline & & & $\begin{array}{r}40 \\
<0.01\end{array}$ & 40.01 & 0.01 & $\begin{array}{r}40 \\
<0.01\end{array}$ & 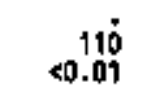 & $\begin{array}{r}110 \\
40.01\end{array}$ & $<0.01$ & $\infty .01$ & $\$ 0.01$ & $\$ 0 . \ddot{\theta 1}$ \\
\hline & & & $\begin{array}{r}\times 0.005 \\
00.004 \\
0.017 \\
\times 0.05\end{array}$ & $\begin{array}{r}40.005 \\
0.004 \\
0.0079 \\
4.05\end{array}$ & $\begin{array}{r}<0.005 \\
<0.006 \\
0.17 \\
<0.05\end{array}$ & $\begin{array}{r}0.005 \\
0.005 \\
0.21 \\
40.05\end{array}$ & $\begin{array}{r}0.005 \\
80.004 \\
0.79 \\
\times 0.05\end{array}$ & $\begin{array}{r}40.005 \\
<0.004 \\
0.79 \\
80.05\end{array}$ & $\begin{array}{r}\times 0.005 \\
\times 0.004 \\
0.056 \\
\times 0.05\end{array}$ & $\begin{array}{l}40.005 \\
40.004 \\
0.0072 \\
10.05\end{array}$ & $\begin{array}{r}0.005 \\
0.0046 \\
0.051 \\
0.05\end{array}$ & $\begin{array}{r}<0.005 \\
0.005 \\
0.0063 \\
00.05\end{array}$ \\
\hline & & & $\begin{array}{r}15 \\
00.001 \\
0.0002 \\
0.01 \\
0.01 \\
2.2 \\
40.05 \\
0.006 \\
1.3 \\
0.093\end{array}$ & $\begin{array}{r}15 \\
\times 0.001 \\
0.0002 \\
40.01 \\
0.04 \\
2.1 \\
40.05 \\
0.006 \\
1.3 \\
0.096\end{array}$ & 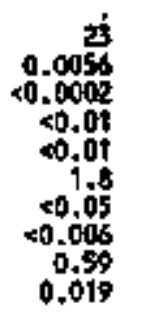 & $\begin{array}{r}2 \dot{4} \\
0.0058 \\
<0.0002 \\
<0.01 \\
<0.01 \\
0.05 \\
0.006 \\
0.62 \\
0.02\end{array}$ & $\begin{array}{r}3 \dot{3} \\
0.12 \\
0.0002 \\
0.01 \\
0.01 \\
2.9 \\
40.05 \\
0.006 \\
37 \\
1.6\end{array}$ & $\begin{array}{r}\frac{3 x}{0.12} \\
4.0002 \\
0.01 \\
00.01 \\
2.6 \\
<0.05 \\
<0.006 \\
37 \\
1.6\end{array}$ & $\begin{array}{r}1+9 \\
0.0012 \\
<0.0002 \\
20.01 \\
<0.01 \\
<0.05 \\
<0.006 \\
0.66 \\
0.01\end{array}$ & $\begin{array}{r}10 \\
40.001 \\
\times 0.002 \\
40.01 \\
40.01 \\
\times 0.05 \\
0.006 \\
0.6 \\
0.01\end{array}$ & 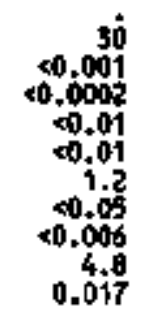 & $\begin{array}{r}31 \\
40.001 \\
0.0002 \\
40.01 \\
40,01 \\
1.4 \\
0.054 \\
40.006 \\
0.017\end{array}$ \\
\hline & & & $\begin{array}{r}0 . \overline{2} \\
0.002 \\
0.005 \\
0.006\end{array}$ & $\begin{array}{r}0.2 \\
0.0021 \\
0.005 \\
0.0054\end{array}$ & $\begin{array}{r}40.2 \\
0.0013 \\
<0.005 \\
0.013\end{array}$ & $\begin{array}{r}0.2 \\
0.0012 \\
40.005 \\
0.012\end{array}$ & $\begin{array}{c}\infty 0 . \overline{2} \\
\alpha .0005 \mathrm{~d} \\
0.005 \\
0.0024\end{array}$ & $\begin{array}{c}<0 . \overline{2} \\
20.0005 \\
0.005 \\
0.0024\end{array}$ & $\underset{0.0005}{0.2} d$ & $\begin{array}{c}0 . \overline{2} \\
0.0005 \mathrm{~d} \\
0.0059\end{array}$ & $\begin{array}{c}40 . \dot{2} \\
0.0005 \\
0.003 \\
0.0039\end{array}$ & $\begin{array}{c}40.000 . \dot{2} \\
0.005 \\
40.002\end{array}$ \\
\hline \multirow{2}{*}{\multicolumn{2}{|c|}{ 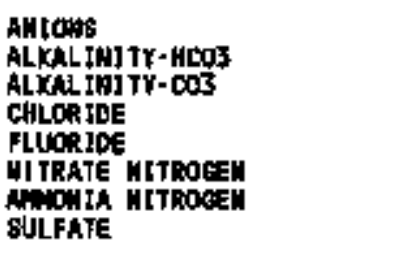 }} & \multirow{2}{*}{ 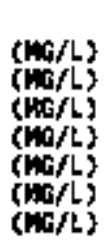 } & $\begin{array}{r}151 \\
24 \\
2.5 \\
0.1 \\
2\end{array}$ & $:$ & $\begin{array}{r}196 \\
<1 \\
2.2 \\
0.1 \\
0.68\end{array}$ & : & $\begin{array}{r}418 \\
41 \\
16 \\
0.7 \\
0.2\end{array}$ & : & $\begin{array}{r}153 \\
<1 \\
1.9 \\
00.1 \\
0.91\end{array}$ & $:$ & $\begin{array}{r}253 \\
<1 \\
8.1 \\
0.1 \\
0.66\end{array}$ & $\dot{*}:$ \\
\hline & & & $5 . \dot{6}$ & $\dot{.}$ & $9 . \dot{5}$ & : & ద் & $\dot{2}$ & 1.8 & $\dot{.}$ & $3 . \dot{2}$ & : \\
\hline $\begin{array}{l}\text { TOYAL SUSPELOED \$OLJOS } \\
\text { TUREIDITY }\end{array}$ & & (HF/L) & 0.4 & $\vdots$ & 4.6 & $\vdots$ & 9.5 & : & $<1$ & $\dot{ }$ & 0.4 & $\overline{8}$ \\
\hline
\end{tabular}


TRACE NETALS AUb Hitous, ZND CUARTER 1995

SHAPLIWG POIMT

LOCATIOA

DATE SPMPLE

TYPE

METALS

NuT join

NRSEMTC

BAnjuN

BERYLLIUN

OORON

coniun

chonilus

CALCIUN

ChROAIU,

E.TALA

coopet

Inom

LEAD

Letho

anctive

Fictor

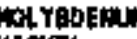

pothlsstun

stentor

stlver

stiont su:

THALLJU

1miniva

Yheroty

AHIons

ALthethItr-HoOs

I Kat IUTY-Cos

CHLORIbE

FLOORJE

HuOH H Trocen

SULFATE

TOTAL SUSPEADE SOLJDS TURB IDITY

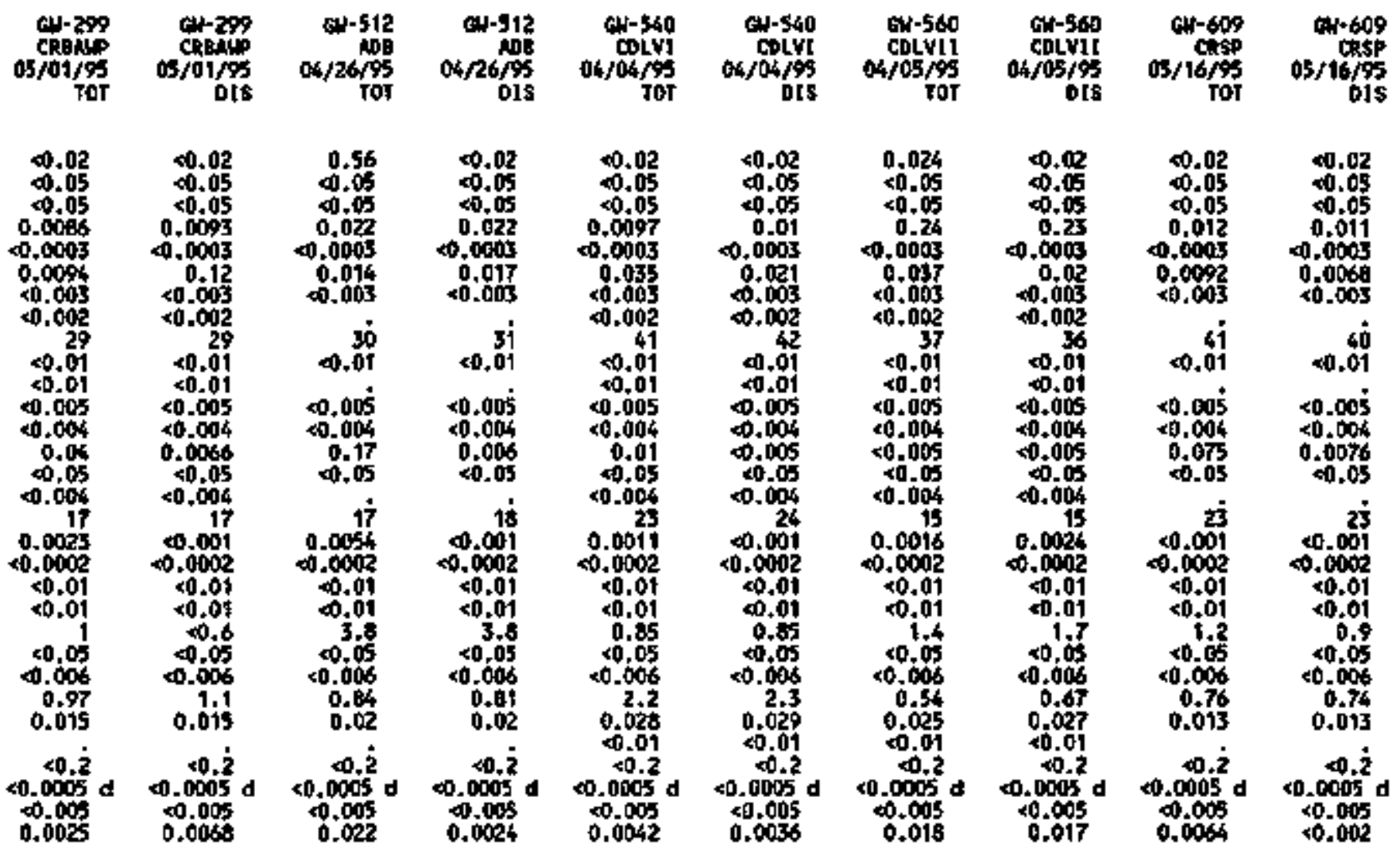

163
41
1.9
40.1
$<0.2$
3.1
2.5
4.5

198
2.1
2.4
00.1
0.38
40.2
4.2
$\times 1$
0.2

148
1.9
00.1
0.39
0.2
2,1
0.1
0.3
205
1.5
$<0.1$
1.1
2.2
0.4 


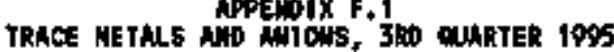

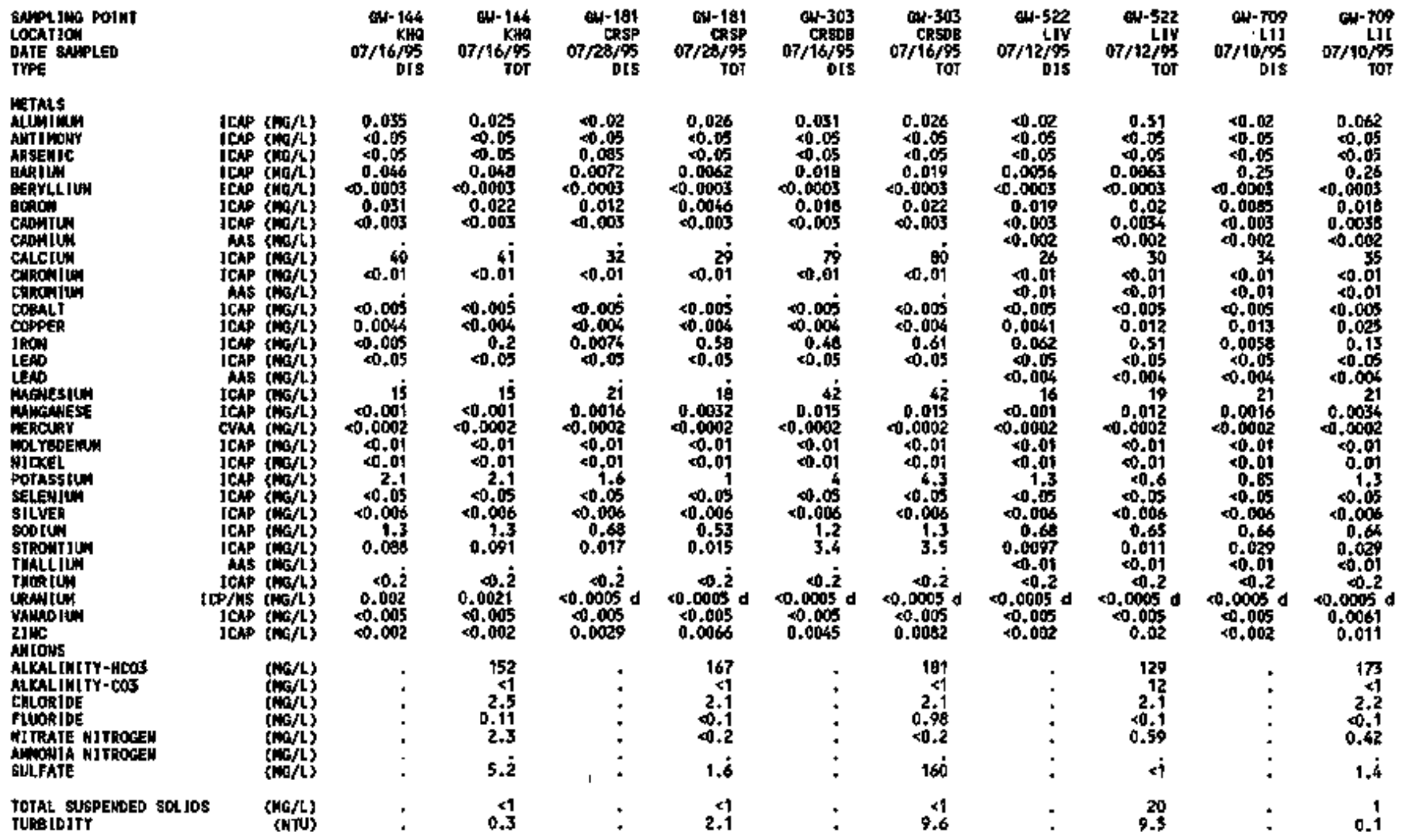




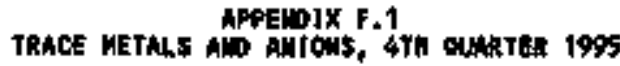

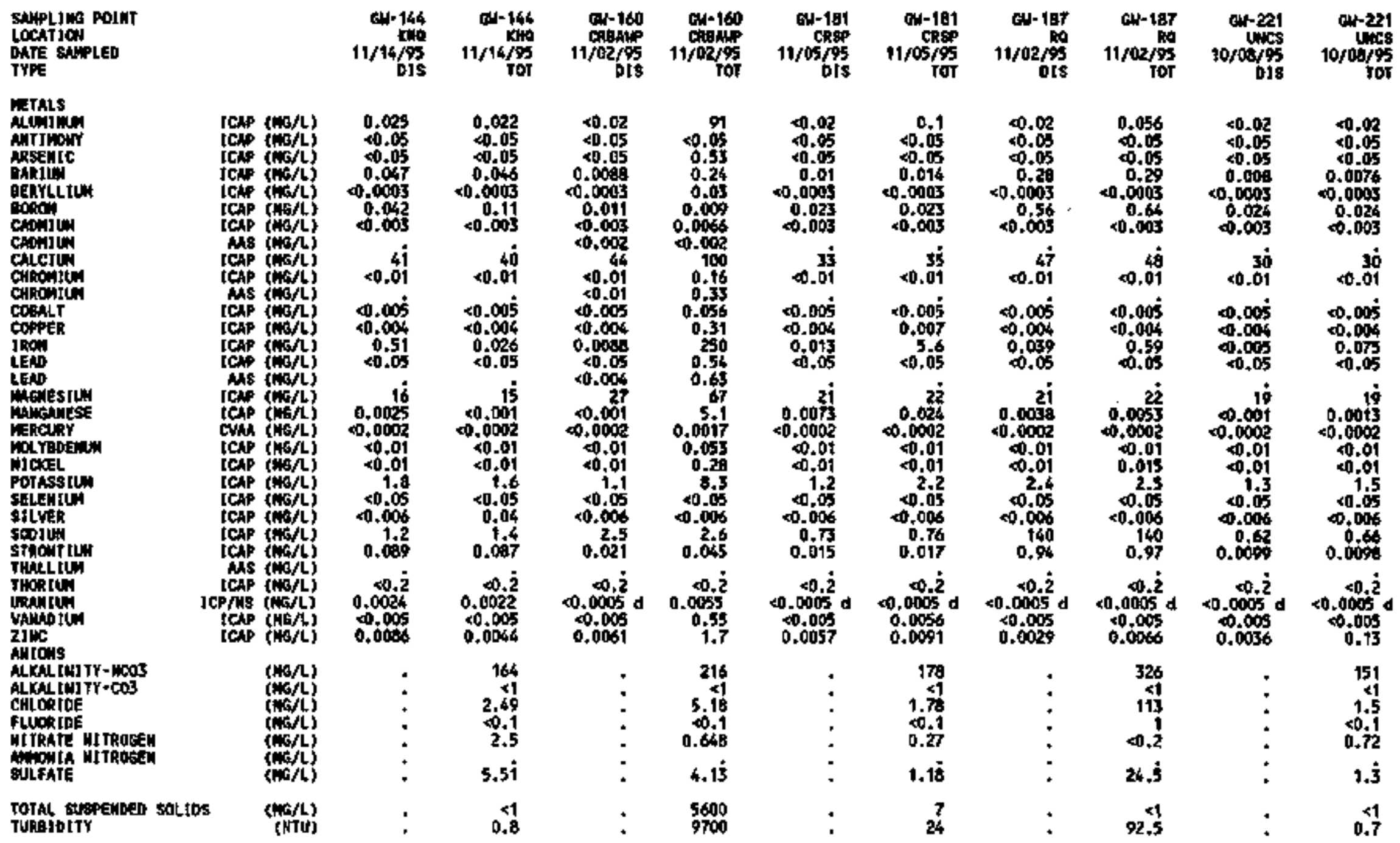




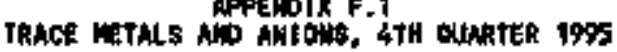

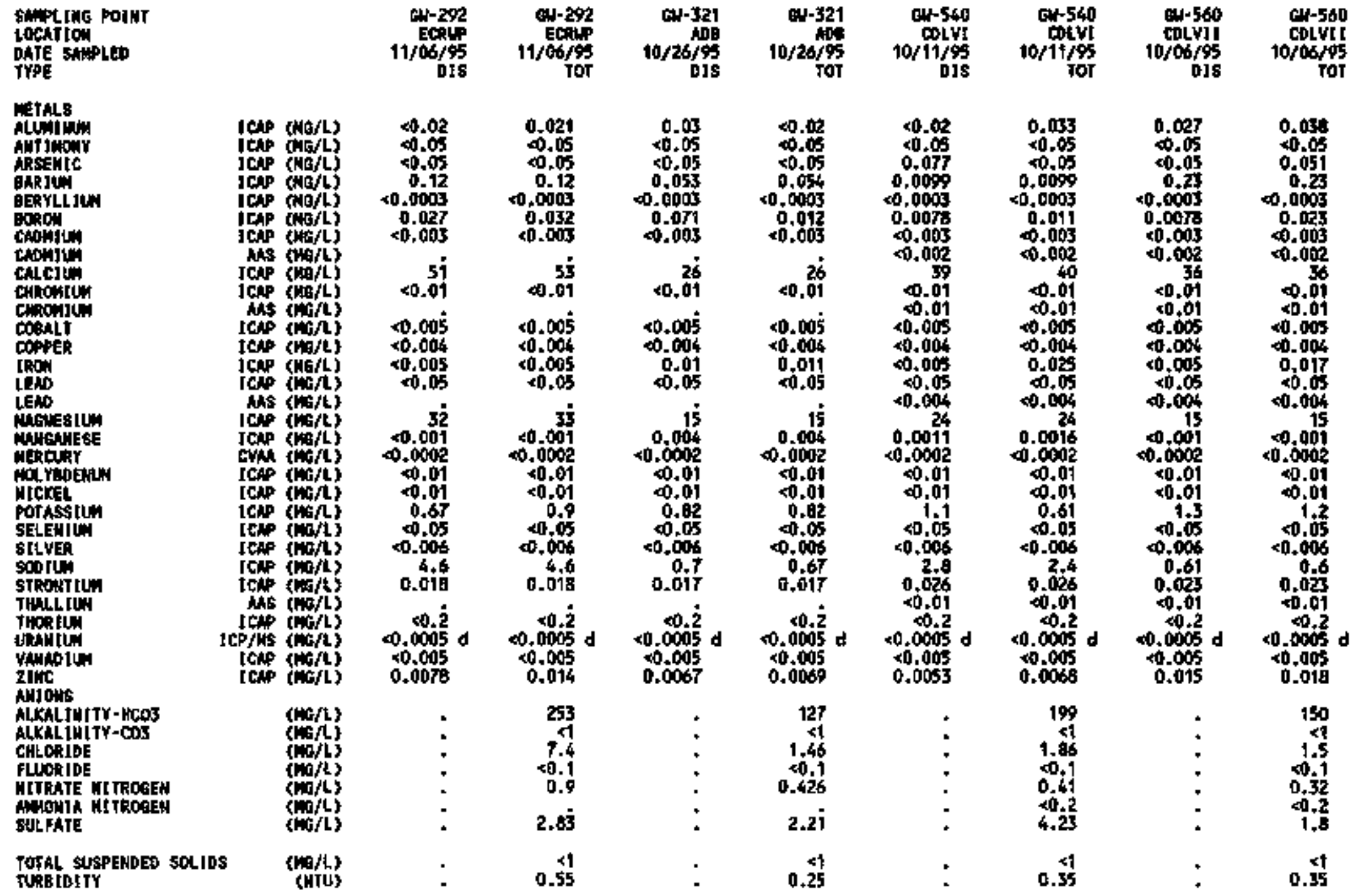




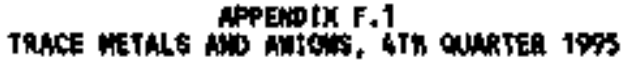

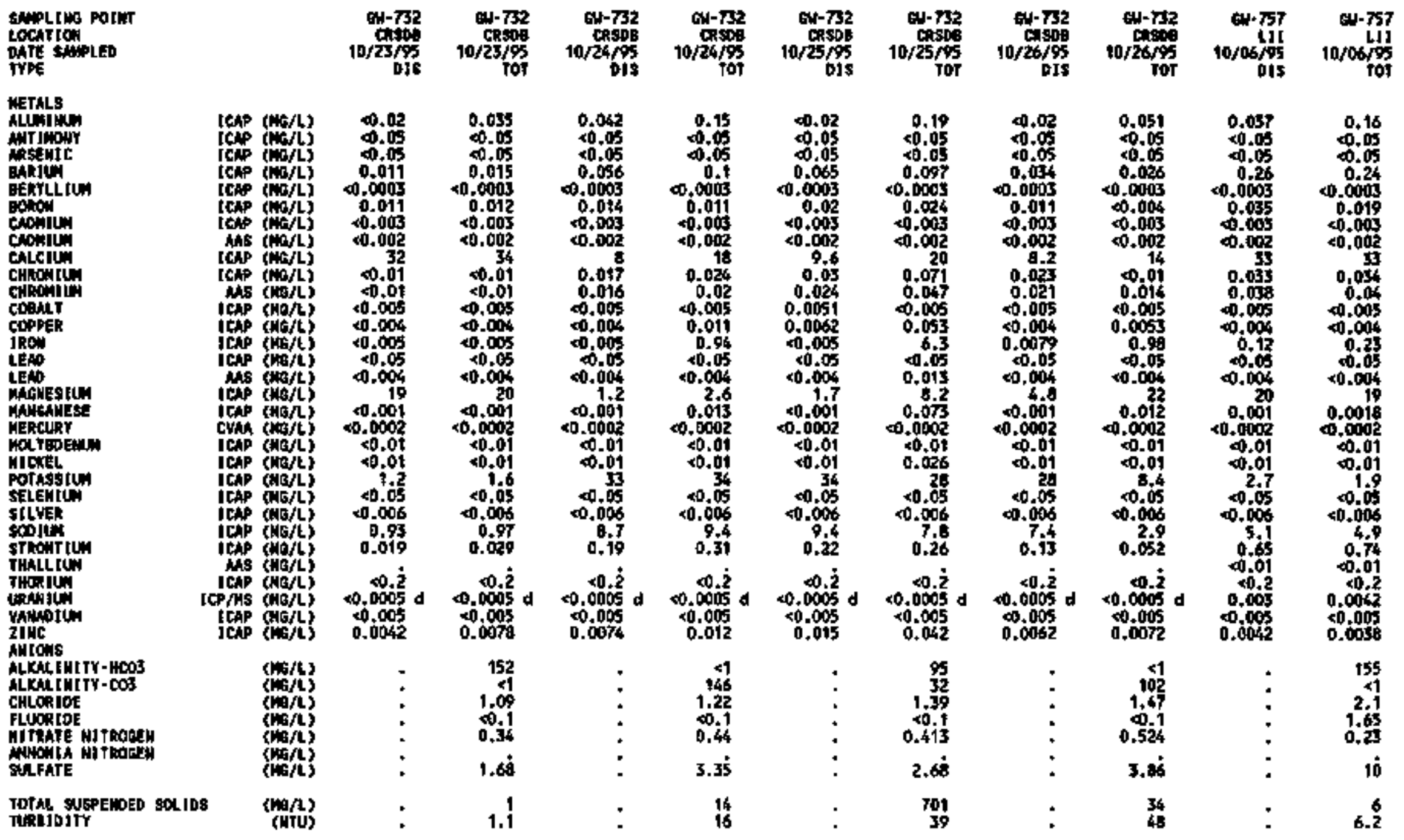





\section{APPENDIX F.2}

VOLATILE ORGANIC COMPOUNDS 

APEIDTX F.

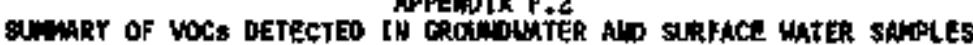

Total semples

Serpl wo without wor.

5emples with voc:

conpouth

ACETOU:

BROmLOLLLLORONETHA:E

Bacmofogis

monistith

CANThNowE

CAREON TETAMCHLORIDE

CHLOONOE TETRACH:

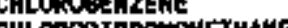

chlontros

CHLotocthati

CHLOparom

1-DrC The

17-DTCHLOROETHAM

-

, 1-O CONLOBOETLELE

Thans-1,2+0|CHLOROETHEN

1,2-0t ćlLokOPLOPAHE

cls-1,3-0ICHLotopropene

TRAns? 1, 3-0]CHLOROPAOPENE

ETlodizene

4-P.THYL-2-PEnTANOWE

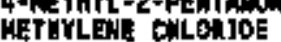

STroeve

STrREME

$1,1,2,2$-TETRACHe

rouvent

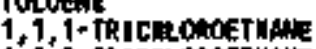

$1,1,2$ - TRICALOCOCTHAN

TRICHLOROETEE

$\checkmark$ MCETAIE

VINTE ChLondDE

XYLEWES

(WorL)

(UAF)

(UG/)

(UG/L)

(ubs)

(bis)

(unc)

(U⿺辶)

(1)

(U⿺辶)

(uin.

(4)

(x)

(uar)

(uth

(uich

(ut.

(ubs)

(uofl.

(ub/

(4)

(u)

(us

(t)

(t)is

(4)

(4)

(us

(u단

(uort

U5/L

(N)'L

(t) $/$ L

(UCA $/ \mathrm{L}$ )

alARTER

1
0
0
0
0
1
0
0
0
0
0
0
0
0
0
0
0
0
0
0
0
0
0
0
0
0
0
0
2
0
0
0
0
0
0 


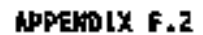

MOLATILE OACABIC COMOUNDS, IST OUARFER 19\%

\begin{tabular}{|c|c|c|c|c|c|c|}
\hline $\begin{array}{l}\text { SAMPLING POINT } \\
\text { DATE SWHFD }\end{array}$ & & $\begin{array}{r}\text { on-141 } \\
\text { Liv } \\
01 / 00 / 9 s\end{array}$ & $\begin{array}{r}64 \cdot 143 \\
\times / 49 \\
01 / 17 / 95\end{array}$ & $\begin{array}{r}0 w+511 \\
\text { CRsp } \\
01 / 24 / \% 5\end{array}$ & $\begin{array}{r}0 w+709 \\
\text { Lif } \\
01 / 09 / 95\end{array}$ & $\begin{array}{r}\text { cw+732 } \\
\text { cisbs } \\
01 / 18 / 95\end{array}$ \\
\hline 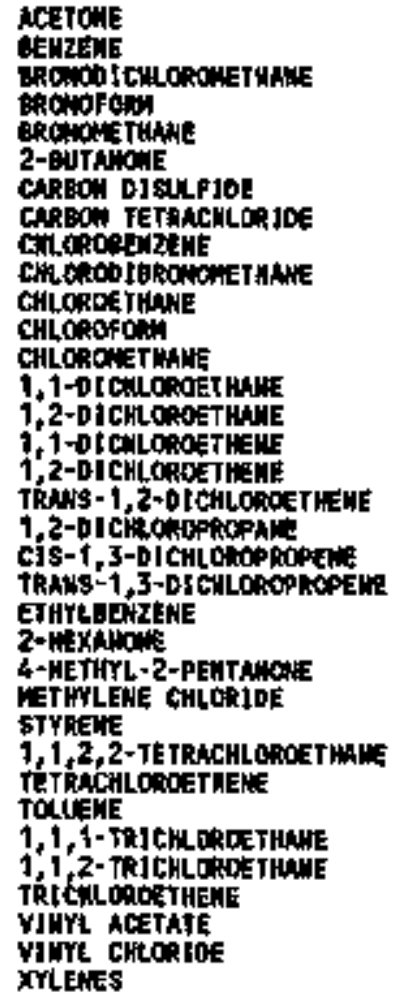 & 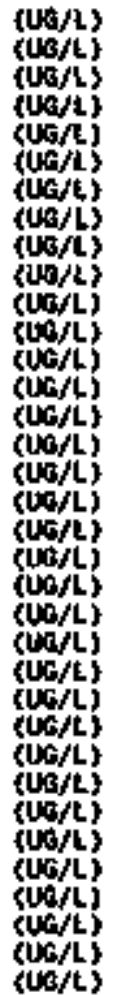 & 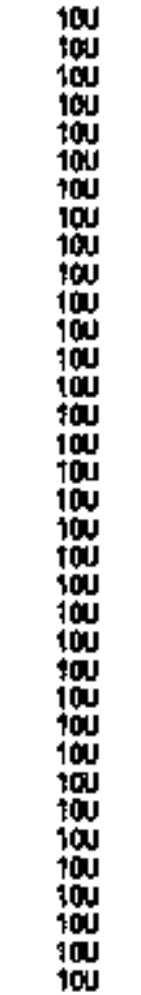 & 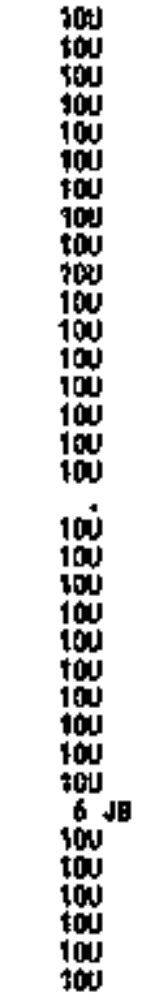 & 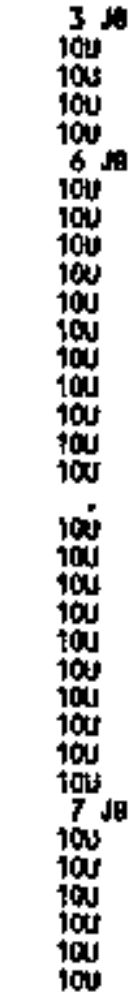 & $\begin{array}{l}100 \\
100 \\
100 \\
100 \\
100 \\
100 \\
100 \\
100 \\
100 \\
100 \\
100 \\
100 \\
100 \\
001 \\
100 \\
100 \\
100 \\
100 \\
100 \\
100 \\
100 \\
104 \\
100 \\
100 \\
100 \\
100 \\
100 \\
100 \\
100 \\
100 \\
100 \\
100 \\
100 \\
100 \\
100\end{array}$ & $\begin{array}{l}100 \\
100 \\
100 \\
100 \\
104 \\
100 \\
104 \\
100 \\
100 \\
100 \\
100 \\
100 \\
104 \\
100 \\
100 \\
100 \\
100 \\
100 \\
100 \\
100 \\
104 \\
100 \\
100 \\
100 \\
100 \\
100 \\
100 \\
100 \\
100 \\
100 \\
100 \\
100 \\
100 \\
100\end{array}$ \\
\hline
\end{tabular}


APPEND $[X \neq .2$

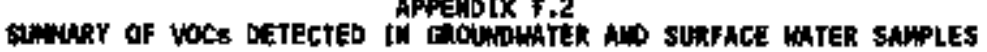

Total sinples

witheut vace

somples with voc.

\begin{tabular}{|c|c|c|}
\hline compouln & & $\begin{array}{l}\text { SECOH' } \\
\text { OIARTER }\end{array}$ \\
\hline 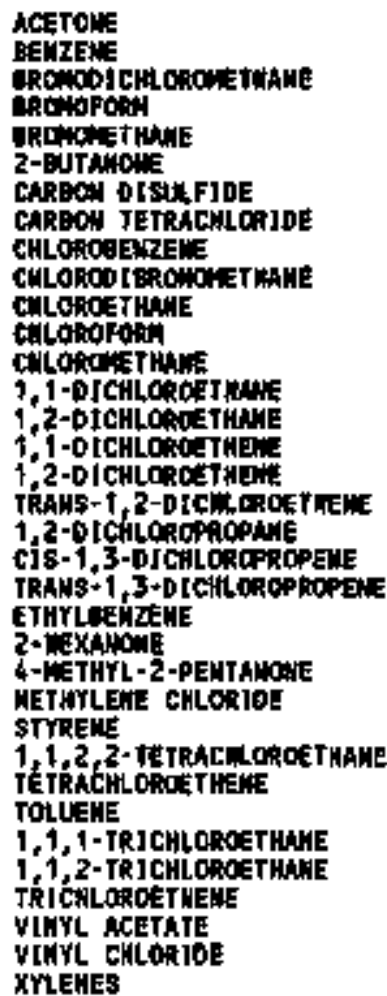 & 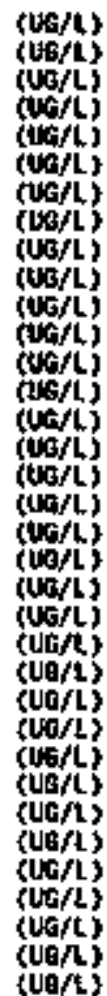 & $\begin{array}{l}1 \\
0 \\
0 \\
0 \\
0 \\
0 \\
0 \\
1 \\
0 \\
0 \\
0 \\
1 \\
0 \\
0 \\
0 \\
0 \\
0 \\
0 \\
0 \\
0 \\
0 \\
0 \\
0 \\
0 \\
0 \\
0 \\
0 \\
1 \\
0 \\
0 \\
0 \\
1 \\
0 \\
0 \\
0\end{array}$ \\
\hline
\end{tabular}




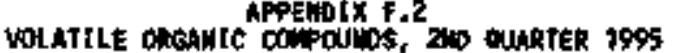

SNPLtWo Dtint DOCAT TOW

\section{ACETONE}

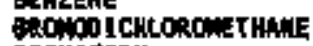

Brokofopis

Bawometmas

2-6ythons

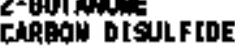

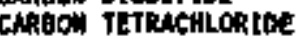

CHE OOOSE MENE

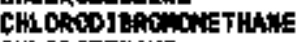

CILGROETHANE

CuloRorony

1,1-0.1CHLOROETHA

1,-DICHLDNOETHAWE

'2-DICHLOAOThE

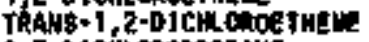

1,2-D1CHLCOOPROPAME

cís-1,3-01EHLosopropesne

(2.A45:1,3-01)

$2+15 x$ Nitions

4+NETOYL - 2-PERT LOONE

STMYEEY CHLORIOE

STYMENE

1,1,2,2-TETRACHLOROETHANE

TET MCHLO OEETHEWE

TOUE⿰亻⿻

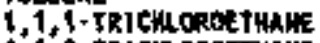

f,1,2-tKICIMCROLTHAME

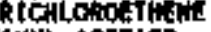

1UYL ACETATE

VthrL CHCOR TOE

XYLEHES

\begin{tabular}{|c|c|c|}
\hline $\begin{array}{r}0 \mathrm{~W}-144 \\
\mathrm{KHO} \\
125 / \%\end{array}$ & $\begin{array}{r}\text { an-159 } \\
\text { caspos } \\
04 / 06 / 25\end{array}$ & $\begin{array}{r}\mathrm{CH}-18 \\
\mathrm{R} / 03 / 9\end{array}$ \\
\hline $\begin{array}{c}45 \\
100 \\
100 \\
100 \\
100 \\
100 \\
100 \\
4 \\
100 \\
100 \\
100 \\
100 \\
100 \\
100 \\
100 \\
100\end{array}$ & 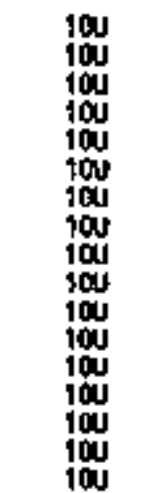 & $\begin{array}{l}100 \\
100 \\
100 \\
100 \\
100 \\
100 \\
100 \\
100 \\
100 \\
100 \\
100 \\
100 \\
104 \\
104 \\
104 \\
104 \\
100 \\
100\end{array}$ \\
\hline $\begin{array}{l}100 \\
100 \\
100 \\
100 \\
100 \\
100 \\
100 \\
100 \\
100 \\
100 \\
100 \\
100 \\
100 \\
100 \\
100 \\
104 \\
100\end{array}$ & $\begin{array}{l}100 \\
100 \\
100 \\
100 \\
100 \\
100 \\
100 \\
100 \\
100 \\
100 \\
100 \\
100 \\
100 \\
100 \\
100 \\
700 \\
100\end{array}$ & 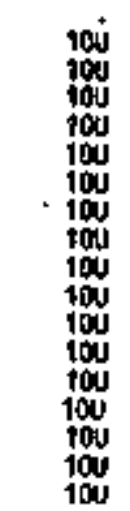 \\
\hline
\end{tabular}

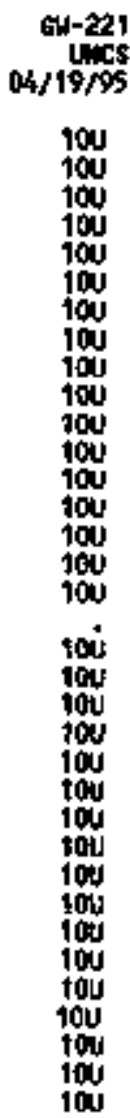

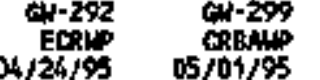

$\begin{array}{cc}100 & 100 \\ 100 & 100 \\ 100 & 100 \\ 100 & 100 \\ 100 & 100 \\ 100 & 100 \\ 100 & 100 \\ 100 & 100 \\ 100 & 100 \\ 100 & 100 \\ 100 & 100 \\ 100 & 104 \\ 100 & 104 \\ 100 & 100 \\ 100 & 100 \\ 100 & 100 \\ 100 & 100 \\ 100 & 100 \\ 100 & 1004 \\ 100 & 100 \\ 100 & 100 \\ 100 & 100 \\ 100 & 100 \\ 100 & 100 \\ 100 & 100 \\ 100 & 100 \\ 100 & 100 \\ 100 & 100 \\ 100 & 100 \\ 100 & 100 \\ 100 & 100 \\ 100 & 100 \\ 100 & 100 \\ 100 & 100\end{array}$

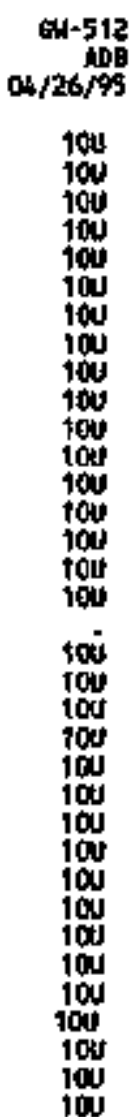

0.560
0.0610
$0405 / 55$

Iov

$\operatorname{lom}_{100}^{100}$

tow tow

iou 100

10u

100

tol

100

100

10u

100

100

100

100

100

100

10

10

100

10

10.

15

10

$10 \mathrm{t}$

100

100

1001
$-609$

$6 / 9$

ov

100

100

10v

104

언

I0.

100

100

10i

104

讨

tou

100

100

100

100

19

IAN

100

에

iou 
APPEIDIX F.2

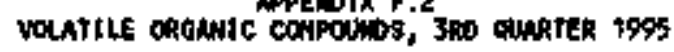

SAMPLI LOS POINT

DACAT SAR

ACETONE

aELZENI

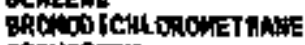

ofDibrorin

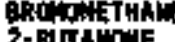

CAREOW DJsthe Ibe

CAREO TETRMCHLÓn!OE

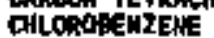

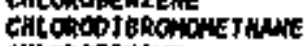

CHLDitosthN

CHLOHOFOAH

Chlociofon

chi-olctiono

1,2-DICHLGIOETHAME

$1+$ T-OTCHLOOSTHEW

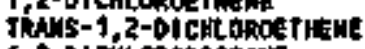

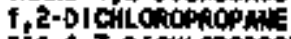

CIs-1,3-0ICHLOROPROPEnE

Torum

EThLeicen

4-HETHYL - 2-PENTANONE

METHYLAE chlostó

GTYRENE

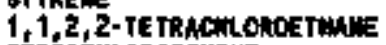
TETHACH OOOEETHEME

3.1,1-TRICHL COOETHNe

t, 1,2+ TRTCHLOSOETHNNE

TRICHLOROETHENE

VMT MCETAIE

Vtturl CALORJDe

Xrlenes

$$
07 / 16 / 95
$$$$
\text { ar-14a }
$$

GI-18

cat-303

$07 / 28 / 98$

$07 / 6 / 95$

G1.522

C[y

$07 / 12 /$

GN-709

$07 / 10 / 95$

\begin{tabular}{|c|}
\hline 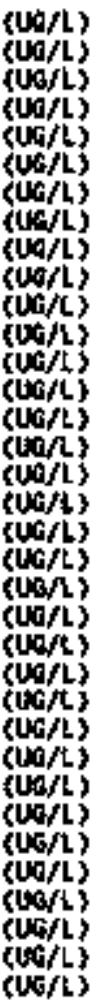 \\
\hline
\end{tabular}

100
100
100
100
100
100
100
100
100
104
104
100
100
100
100
100
100
100
100
100
100
100
100
100
100
100
100
100
100
100
100

100
100
100
100
100
100
100
100
100
104
100
100
100
100
100
100
100
100
100
100
100
100
100
100
100
100
100
100
100
100
100
100

100
100
100
100
100
100
100
100
100
100
100
100
100
100
100
100
100
100
100
100
100
100
100
100
100
100
100
100
100

10u

10J

tou

100

100

100

100

10u

101

ios

100

tow

100

104

tout

100

100

100

$10 \mathrm{U}$

tos

100

104

100

$10 \mathrm{v}$

100

100

tou 


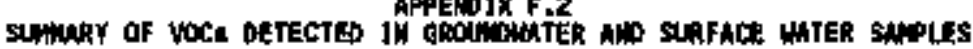

Total Sacples

sempled without voc.

semptes with voco

conpouit

FOMRTh

ACETOH:

Belintive

BRONCO TCHLORONETHAWE

thoworopis

Blolwonthine

2-AUTAHONE

CARBO DI SULF TOE

CARGOW TETRHCHLORTDE

CHLOAOBEHZEX

CHLORDOIBRG

CHLOA E THAH

CHLOROFOWN

CHLOROAETHA:

1, 1-0 LCHLOAOETMAN

1,2+DICMOndeтHAN

1, 1-Dic Loenctitiatia

1,2-Dicitooseth:

TRM18-1,2-OICHLOROETHENE

1, 2-DICILOAOPROPHE

Cj5-1,3-0I ChLOAOPDOPEFt

TRAHS-1,3-DICALOROPAOPEAR

ETHTL- EU2ENE

2- HeXONOWE

4-HETHYL-2-PENTAMONE

RETHYLEAE CruOtide

STrRENE

$1,1,2$ 2-TETMACHLOROETMAHE

TETRMCHLOROETHEN

TOLUEkE

1,1,1-TR]CHLOAOLETHANE

1,1,2-TRJCHLOCOETHALE

TRICHLOROE THEME

VIMTL ACCTATE

VIHYL CHLORIDE

XYLENES

(U6/L)

(UG/L)

(US/L)

(U6/L)

(uth

(U6)

(wo/l.

(UOB $/$

(Wof

(WG/L)

(U6)

(tor/t

(UG/L)

(uo/

(uta/

(us

(UG/L)

(ve/L

(UG/L)

(U⿺尢丶

(t)/

(t) $/ 4$

(UT/

U6/

(UtG/L)

0
0
0
0
0
3
0
1
0
0
0
0
0
0
0
0
0
0
0
0
0
1
0
0
1
0
0
0
0
0
0
0
0
0
0
1




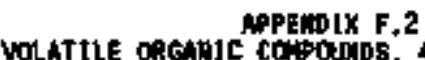

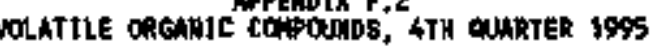

simblitio polut tochtol

ACETone

OROWOOLCHLLRONETHANE

akonoroat

GRONOWETMA:

2-

CAnow OISAB FIOE

CAREA TETPACHLOR10E

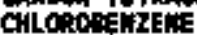

CHLOROOJ日ROMNTHE

CHLORO S THM

CHLOROFO

CHLOConethe

1, 1 otcillotartmine

1,2-otehlonoetMane

1, 1-DICHLOROETHENE

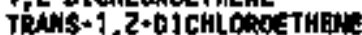

1,20lchlorgontoper

CIS-1,3-0 tonlótoprotpent:

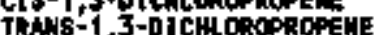

ETWLLEARZEHE

z-HXXimo

9-METHFL - 2-PEUTA

Ge THYLEE CHLOAIOE

tirn:

1,1,2,2-TETRAChL OEOE THANE TETRACWLONOET HEME

T, i,1-TRLC'LOROETHAY

1, 1,2-Fi J CHLLOROETHAME

TR ICHLOBOE THEW

VIML ACLTATE

VIMYL CHLOR TOE

XYLENES

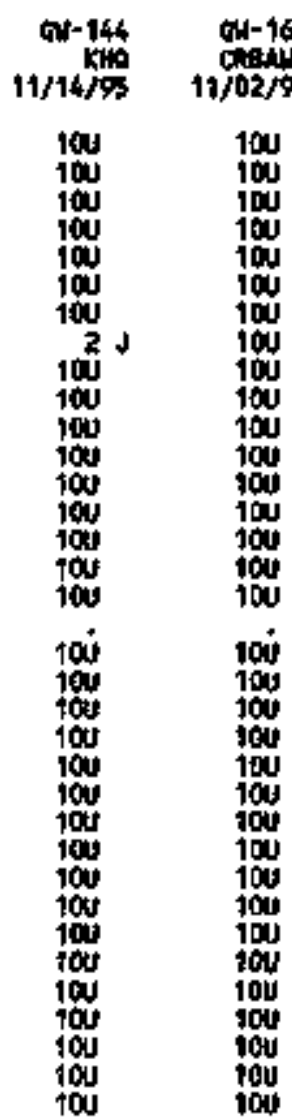

\section{CW-194} $11 / 05 / 05$

10u 100

100
100
100
100
100
100
100
100
100
100
100
100
100
100
100
100
100
100
100
100
100
100
100
100
100
100
100
100
100
100

100

104

100

$10 \mathrm{~s}$

$10 \%$

104

10

100

100

10 d

100

100

100

100

100

100

100

$10 \mathrm{~s}$

104

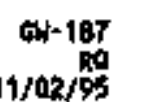

10/0

100

100

100

100

100 an-293

$11 / 2045$

100
100
100
100
100
100
100
100
100
100
100
100
100
100
100
100
104
100
100
100
100
100
100
100
100
100
100
100
100
100
100
6.321
$0 / 26 / 95$ 6h-540 GN-540 10ri1/s 10/06/

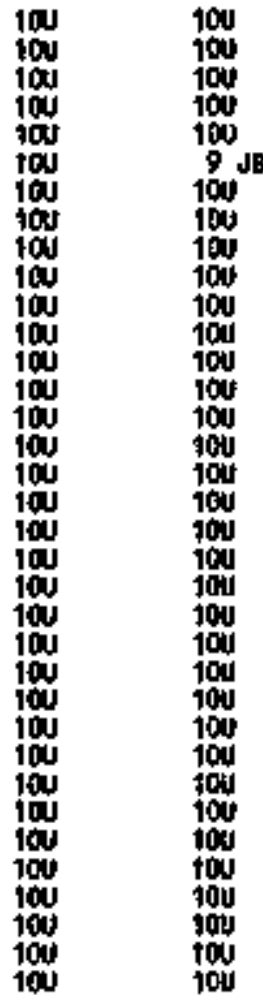




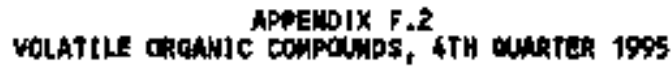

satplas Pothr

Dockit lon

ACETOM

QANZEIL

Prowocin.

2-Butritats

CARSON OISULFIOE

CARBOW TETRACHLRIDE

Chon

Chtowint binowet Hune

chich

Chlonoronis

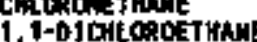

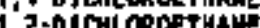

1: - chlokorinake

1,

TRAHS-1, 2-DICW ONOETHENE

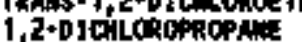

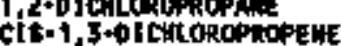

TRAYS 1, 3-DICHLOROPAOPENE

ETMYLEEMERE

2-NGxhome

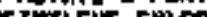

$1,2,2$-TETHACHLOROETHAN

TERYC, LOROETHE'

1,1,1-TATCHLOROE THAK

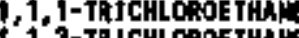

r.

vint atrite

viur chlon

Frlentes

KYLEME

\begin{tabular}{|c|c|c|c|}
\hline $\begin{array}{r}64-72 \\
\text { thatsod } \\
10 / 23 / 95\end{array}$ & $\begin{array}{r}\text { CN-752 } \\
\text { CRSDB } \\
10 / 24 / 98\end{array}$ & 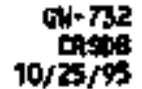 & $\begin{array}{r}c 1-72 \\
\operatorname{cospo} \\
10 / 26 / 95\end{array}$ \\
\hline
\end{tabular}

(uent)

tow/L)

$(\mathrm{W} / \mathrm{l}$ )

(uorls)

(uofli)

(uofls

(UL: L

suth

SUG/L)

(uof

(uols.

(x)

(ustis

Uath

(uth/L)

(uoll

(ua $/$ )

(U6/t)

(vis

(ven

(UG/L)

(ut)

(tot

w

(In)/b)

(uon.

(4)

(usf?

(usf)

uch

UG/

wh/

(uns

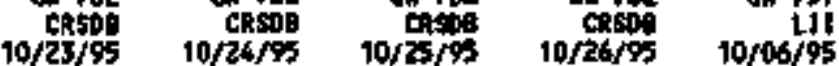

\begin{tabular}{|c|c|c|c|c|}
\hline * & - & * & • & $10 \mathrm{~J}$ \\
\hline • & * & 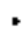 & * & $10 \mathrm{~J}$ \\
\hline , & - & . & - & 100 \\
\hline - & - & . & . & 100 \\
\hline - & • & • & • & 100 \\
\hline • & 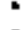 & - & $\star$ & \\
\hline - & " & • & • & 100 \\
\hline • & i & • & " & 100 \\
\hline * & : & $\cdot$ & : & 100 \\
\hline : & . & . & . & inu \\
\hline * & . & . & . & 100 \\
\hline . & * & . & * & 100 \\
\hline • & - & - & - & 100 \\
\hline • & • & • & • & 100 \\
\hline • & • & * & • & $10 \mathrm{~N}$ \\
\hline • & - & * & * & 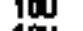 \\
\hline . & • & . & • & J \\
\hline • & - & . & * & $10 \mathrm{~J}$ \\
\hline • & . & • & - & 104 \\
\hline * & • & . & • & $10 \mathrm{~J}$ \\
\hline . & • & • & * & 100 \\
\hline . & . & • & - & \\
\hline . & • & * & • & 100 \\
\hline ' & • & - & ' & 701 \\
\hline ' & . & • & . & tou \\
\hline ' & * & • & $\cdot$ & tou \\
\hline ' & . & • & . & 101 \\
\hline - & • & • & • & tou \\
\hline . & • & • & • & 104 \\
\hline . & . & * & • & tow \\
\hline . & • & • & . & $10 \mathrm{U}$ \\
\hline ' & • & . & . & \\
\hline . & - & - & . & y \\
\hline , & • & . & . & 100 \\
\hline
\end{tabular}



APPENDIX F.3

ADDITIONAL ORGANICS 

APPEDix F 5

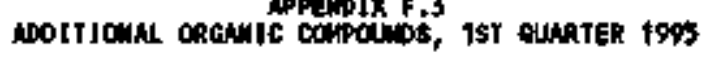

SANP IM FOINT

LOCkTIOI

DOCATION

\section{ACROE EIV}

ACRTCONI ERILE

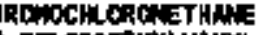

2-C.COCOETHYVIWTL ETHER BT

12-OJ

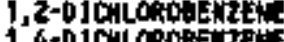

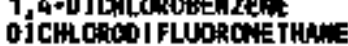

C]S*1, 2-0ICHLOROETHEME

$1,4 \rightarrow 0 j$ CHLOA-2+ENTENE

EFHOL

ETWRL WETHACRTLATE

$1, t, 1,2$-TETRACH OAOETHALE

Tachor

$1,2,3+1 R]$ incoeopropine

$$
\text { GN-141 CW-163 ON-511 }
$$

LIV $01 / 09 / 25 \quad 01 / 17 / \% 5 \quad 01 / 24 / 65$

6w-709

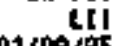

CN-732

fuofls

soll

sov

(lite

$0 \mathrm{~s} / \mathrm{L}$

(W)

(usfl)

(uth $($ L)

(uAft)

(U⿺辶)

(UaG)

(U⿺辶)

suart

(ves)

(U6)

(No/L

(vefli)

: $:$

500
500
100
100
500
100
100
50
100
500
500
50
100
500
50

01/ $18 / 45$ 


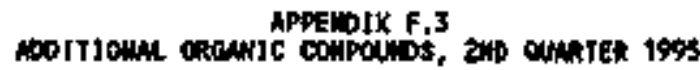

SWPLIME POINT

Lochtion

DAfE SUPLE

\section{ACroceIH}

ACRYONITKLE

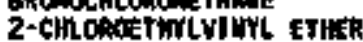

1,2-D) Bhom-3-chlonopontre

2-DiCHCOROAENZZE:

1. 4-DICHLCROCENZENE

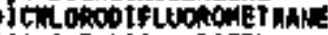

ti\$-1,2-btchlohoETWENE

1, 4-DfCHLOAO-2-ButETE

ETHALC:

ETHYL FETHACXYLATE

COOCNE THAME

1, 1,1, 2-TETHACHLOROCTMAKE

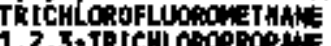

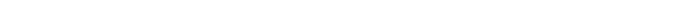

\begin{abstract}
Cas
\end{abstract}




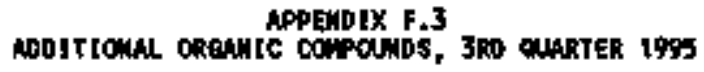

\$NPLIWo POIMT

LOCAT]国

DATE SWPLED

\section{ACROLEIF}

ACRVLONITRILE

BFOWOCHLOBOWETHAN

2-CHLOBOETHYLYJUYL, ETMER

cuerc

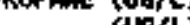

$14-0$ con

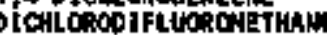

CIS-1,2-bICHLOROETMENE

1. 4-oj́cNeDRo-2-BUTENE

Etriniol

ETHYr methacerlaje

coongth

$1,1,1,2$-TETRACHOMOETHAN

R ICHLOHOFLUOPOHETHAE

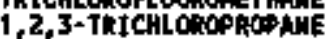

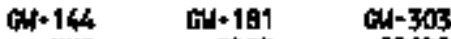

KHQ CRLA

$07 / 16 / 95$

$07 / 25 / 8$

Git.522

서.709

(wo/f)

.

500
500
100
100
100
100
100
500
100
5000
500
500
500
500

500
500
100
100
500
100
100
504
500
100000
50
50
100
50
50 


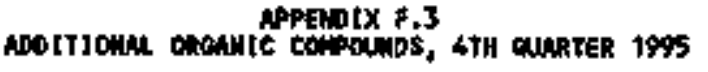

\begin{tabular}{|c|c|c|c|c|c|c|}
\hline $\begin{array}{l}\text { SHPL INS POLAT } \\
\text { LOGATION } \\
\text { DATE SWIPEO }\end{array}$ & & $\begin{array}{r}04.72 \\
\text { ckspos } \\
10 / 25 / \% 5\end{array}$ & $\begin{array}{r}1 \mathrm{~N}-732 \\
10 / 24 / \% 5\end{array}$ & $\begin{array}{r}\text { cin-732 } \\
\text { cosses } \\
10 / 25 / 95\end{array}$ & $\begin{array}{r}04-732 \\
\text { cos } \\
10 / 26 / 95\end{array}$ & $\begin{array}{r}04+757 \\
\text { tit } \\
10 / 06 / 95\end{array}$ \\
\hline 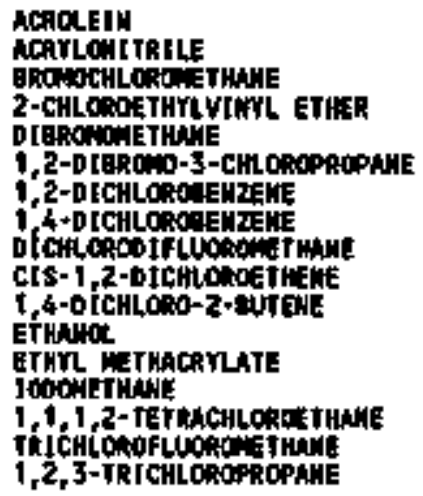 & 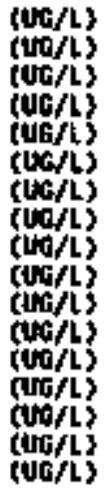 & $:$ & $:$ & $\begin{array}{l}: \\
\vdots \\
\vdots \\
\vdots \\
\vdots \\
\vdots \\
:\end{array}$ & : & 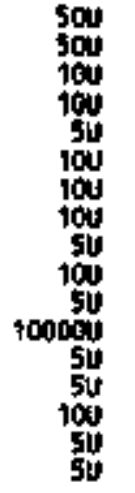 \\
\hline
\end{tabular}



APPENDIX F.4

FIELD MEASUREMENTS

AND MISCELLANEOUS PARAMETERS 



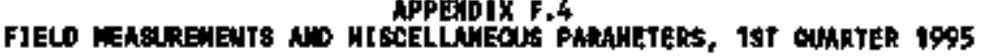

SAMLIH6 PStM"

tocht ton

OATE SWMLED

FIELO MEASTRELENTS

SIAIIC WATEA LEVE (FI. HELOW IOC) WTER LEVEL ELEV. (FT, MONE NG)

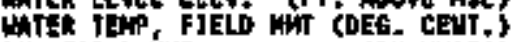
PH, FtEID init.

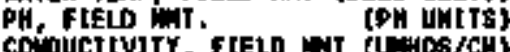

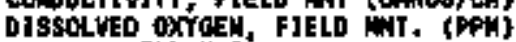

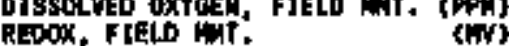

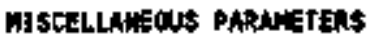

QRONIOE

ChENICW DXYGEN DENAD

CTNHIDE

PVEviots

PK PEP.

PA., REP. 3

REP. 4

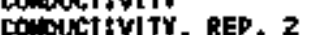

COmolvitr. REP. 3

combt

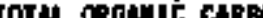

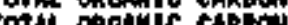

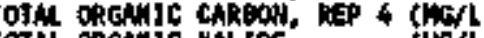

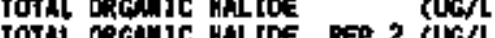

TOTAL o

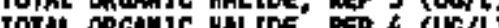

ISSO VED sOL IDS

TOTAL SUSPEWOE SOL IDS

(nt)

TURBIDITY
6N-141 GN-143 GN-511 GN-709 Nㅏ-732

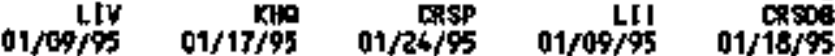

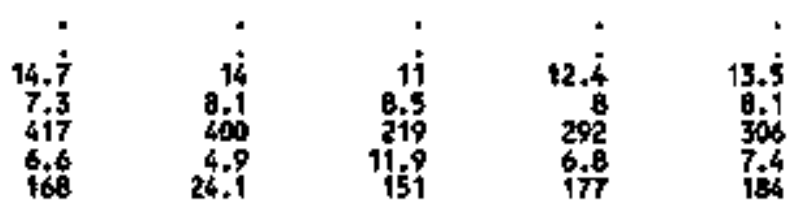

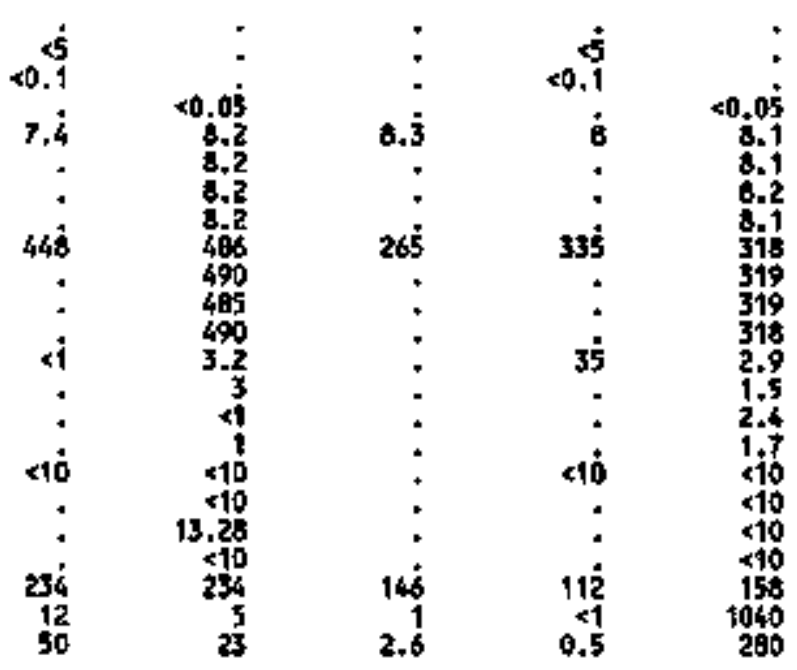




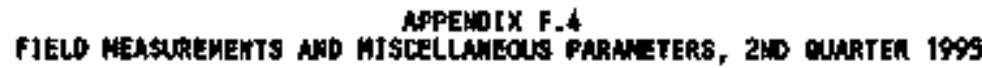

SNMLING BOJHT

LOCATIOH

DATE SHPLED

FIELD FEASREEENTS

STATIC MATER LEVEL (FT. BELOM TOC WHTR LEVEL ELEV. (FT. ABOWE HSL WRTER TEN, FIELD WI (OEO. CELT. PH, FIELO INT.

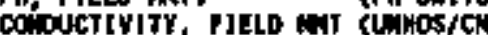

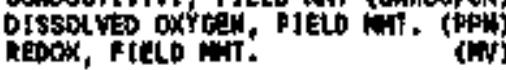
(ini)

MISCELLNGOUS PAMNHETERS

CMEUICAL DXYCEN DENAMD

Cranidos

PH

PU, REP. 2

PH, REP. 3

conpuctivity

COHOLCIVIIY, REP. ?

CONDLCTIVITY, REP. 3

COHDUCTIVITY, REP 4

s

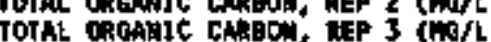

TOTAL OROANIC CMAON, REP 4 (Wo/L)

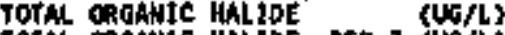

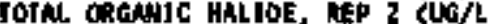

TOTAL OROAHIC HALLOE, NEP I (UG/L

TOTAL ORGNH]C HAL IOE; AEP 4 (UG/L)

TPH DIESEL RANGE X10-LAS (MOAT)

OI $\$ 50$ WVD SOL to5

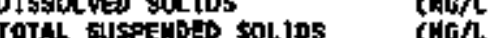

Thkajotiy

(NTO)

04/2

\section{‥1. 159}

$6 n+186$

0 a/os/9s

as/os/s

Eu-22t

a14-292

CRAP

CH-290
CRBHip
OS/01/95

$a t-512$
$04 / 26 / 95$

cu-540

CW-560

colvit

4. 600

an/24/

-116.52
934.68
74.8
7.0
360
3.3
128

-16.2
817.12
15.6
7.1
939
0.4
+82

-76.68
1029.32
16.9
8
249
6.9
159

-112.75
960.25
13.4
7.5
461
6.5
185

-97.15
956.75
14.4
7.9
25.8
5.2
61

-21.8
$97 \%$
14.8
7.2
287
5.4
216

-79.55
992.75
15.3
7.7
378
4
152

-26.79
910.13
13.3
7.6
274
8.7
164

+166.87
045.23

6.87
5.23
46.2
7.7
440
5.8
151

\begin{tabular}{|c|c|c|c|c|}
\hline & - & $=$ & + & $<5$ \\
\hline 40.05 & . & , & + & $\infty, 1$ \\
\hline & 7.7 & $7 . \dot{9}$ & 7.6 & 8 \\
\hline 7.9 & . & . & • & . \\
\hline & ; & : & * & \\
\hline 300 & $656^{\circ}$ & $28 \dot{5}$ & $480^{\circ}$ & 262 \\
\hline 72 & ' & . & - & - \\
\hline 375 & - & $\bullet$ & - & * \\
\hline 2.2 & ; & . & 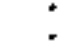 & $<1$ \\
\hline$\overline{\mathbf{z}} . \overline{2}$ & . & . & . & \\
\hline 1.2 & - & . & + & ๑ \\
\hline 8 & - & • & + & in \\
\hline c10 & ; & 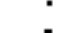 & ; & (1) \\
\hline & , &. & . & 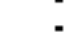 \\
\hline & . & . & . & \\
\hline 4 & $540^{\circ}$ & 176 & 278 & $1 B \bar{B}$ \\
\hline 4 & $\begin{array}{l}1.5 \\
1.4\end{array}$ & $\begin{array}{r}+1 \\
1\end{array}$ & 0,4 & 0.5 \\
\hline
\end{tabular}

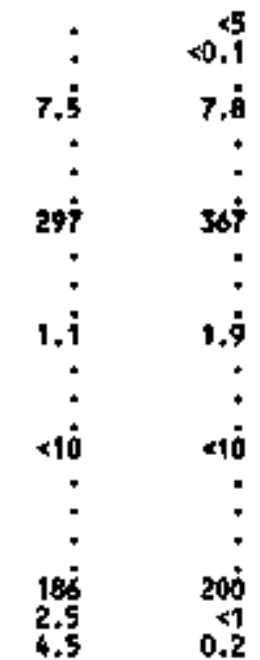

0.1
0.1
7.8
276
$\vdots$
1.7
$\vdots$
$<10$
$\vdots$
0.0225
159
0.3

3.8

0.05
7.9

7.9

701

0,6

0,5

0.2

216
+1 


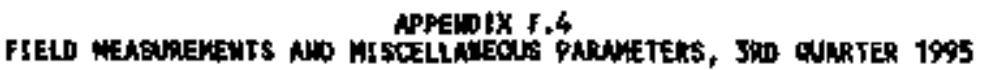

SAPLLLHO POTKT

LOCAT tow

OATE SATOLES

F tELL MEASULENEKTS

\$TATIC WHEK LEVEL (Ft, GELOU TOC) MTER LEVEL ELEY. (FT. MAONE MSL

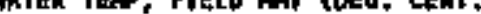
PH, FIELL ÂIT

( m Dissalved oxfCEN, FtELD inT. (PPH)

\section{AT SCELLAREOUS PARAtidTER5}

CNajichl OXYCEN DEWUN

Crawbe

PH, REP.

PH, REP. 3

PH, REP. 4

compuct jvITY

CONDLCT VITY, REP,

CONDUCI JVITY, REP. S

covoct WVIT, REe.

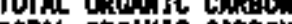

REP $7 \mathrm{H} / \mathrm{h}$

, REP 2 (W)

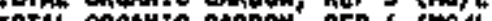

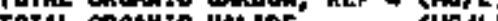

TOIAL GREMTE HMIDE

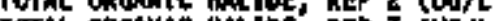

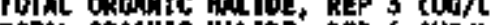

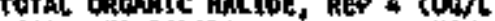

DIS3OLVE SOLIOS

TOTAL SUSPEADED SOLJOS

(n)

TURBIDCTY

(WTV)
Giv-146

$07 / 16 / 15$

ㅂ. 1 -181

CRSP
$07 / 28 / 95$

ck-303

o7/16/95

0.522
114
$07 / 92 / 85$

GN-709

of $110 / 45$

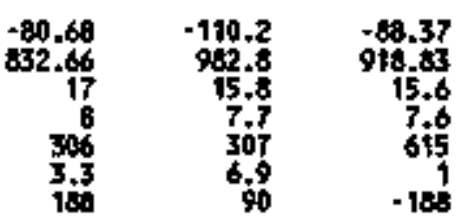

101.35
1073.95
16.7
7.4
329
6.5
176

$-29.77$

E76, 17

25

5,5

\begin{tabular}{|c|c|c|c|c|}
\hline 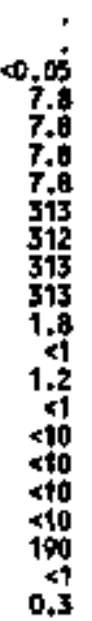 & $\begin{array}{r}\vdots \\
7.62 \\
\vdots \\
320 \\
\vdots \\
\vdots \\
\vdots \\
\vdots \\
198 \\
2.1\end{array}$ & $\begin{array}{r}\dot{0} \\
\infty .05 \\
7.7 \\
7.7 \\
7.7 \\
7.7 \\
6491 \\
6491 \\
649 \\
649 \\
4.7 \\
3.5 \\
3.1 \\
3.3 \\
410 \\
510 \\
510 \\
\times 10 \\
474 \\
41 \\
9.6\end{array}$ & $\begin{array}{r}55 \\
0.1 \\
\dot{8} \\
241 \\
\vdots \\
1.9 \\
\vdots \\
410 \\
\vdots \\
102 \\
20 \\
9.5\end{array}$ & $\begin{array}{r}5 \\
<0.1 \\
\vdots \\
50 \\
\vdots \\
\vdots \\
\vdots \\
\end{array}$ \\
\hline
\end{tabular}




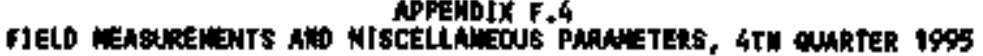

SWHP.1W Dolw

LOCKTIOM

DATE SAPLED

\section{F IELD MEASURERTS}

STAT 1C WHER LEVEL (FT. BELON TOC) WATER LEVEL ELEV, (FT. ABOVE MSL) WTER TEMP, FIELD WNT (DEO, GEHT.

PH. FJELD imT.

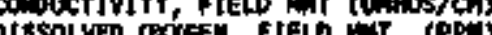

Defor

MJSTELLAIEOUS PARNETERS

CHEIICAL OKYCEN DENA

Crintioe

PMENC:

PH, REP.

PH, REP. 3

PH, ALP, 4

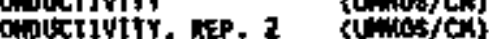

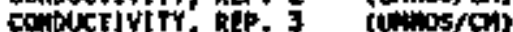

cantity

ToTh socinic capon

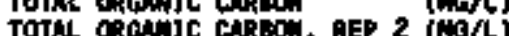

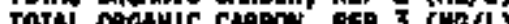

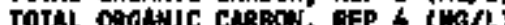

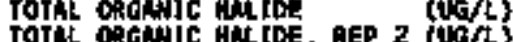

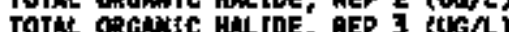

MAC IDE, AEP (

ISSOLVD sCLtOS

TOJUL stspentos sot IDS

TOTREIDITY

\begin{tabular}{|c|c|c|c|c|c|c|c|c|}
\hline $\begin{array}{r}\text { CN-144 } \\
\text { 12Ho } \\
11 / 14 / 95\end{array}$ & $\begin{array}{r}\text { CH-160 } \\
\text { CBantP } \\
11 / 02 / 45\end{array}$ & $\begin{array}{r}04-101 \\
\text { CR5s } \\
14 / 05 / 95\end{array}$ & $\begin{array}{r}\text { SN+157 } \\
R 0 \\
11 / 02 / 95\end{array}$ & $\begin{array}{r}04-221 \\
\text { dawtso } \\
10 / 00 / 85\end{array}$ & 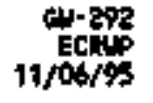 & $\begin{array}{r}c 1 \cdot 321 \\
408 \\
10 / 26 / 285\end{array}$ & $\begin{array}{r}\text { ar-540 } \\
\text { cotvt } \\
10 / 11 / 95\end{array}$ & $\begin{array}{r}\text { cw-560 } \\
\text { codve } \\
10 / 06 / 95\end{array}$ \\
\hline $\begin{array}{r}-77.37 \\
836.02 \\
11.6 \\
7.7 \\
346 \\
5.1 \\
1 / 6\end{array}$ & $\begin{array}{r}-150.35 \\
942,75 \\
15.2 \\
7,6 \\
412 \\
6.3 \\
101\end{array}$ & $\begin{array}{r}-113.12 \\
079.88 \\
13.0 \\
32 \\
4.9 \\
40\end{array}$ & $\begin{array}{r}.19 .55 \\
816.75 \\
16.1 \\
7.6 \\
975 \\
0.5 \\
.275\end{array}$ & $\begin{array}{r}+84.9 \\
1021.8 \\
15.1 \\
7.6 \\
267 \\
2.1 \\
135\end{array}$ & $\begin{array}{r}-148.1 \\
96.9 \\
13.6 \\
7.4 \\
444 \\
6.5 \\
244\end{array}$ & $\begin{array}{r}+22.67 \\
908.91 \\
14.0 \\
7.9 \\
228 \\
6.3 \\
140\end{array}$ & $\begin{array}{r}-84.9 \\
987.2 \\
14.8 \\
7.6 \\
53 \\
4.3 \\
90\end{array}$ & $\begin{array}{r}-36.25 \\
002.67 \\
15.7 \\
7.5 \\
2.5 \\
7.7 \\
365\end{array}$ \\
\hline
\end{tabular}

0
40.05
7.75
7.76
7.76
7.84
352
332
338
332
$<1$
41
41
41
$<10$
$<10$
410
410
176
41
0.6

45
$\times 0.1$
7.5
$\vdots$
415
$\vdots$
$4 . \dot{5}$
$\vdots$
$<10$
$:$
540
5000
9700

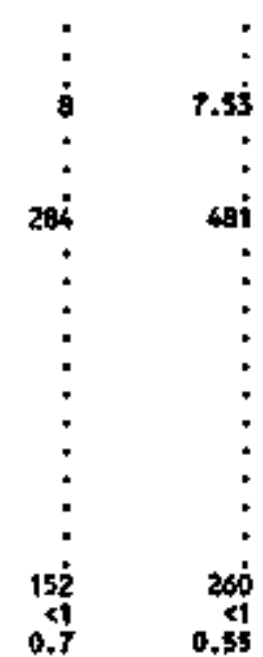

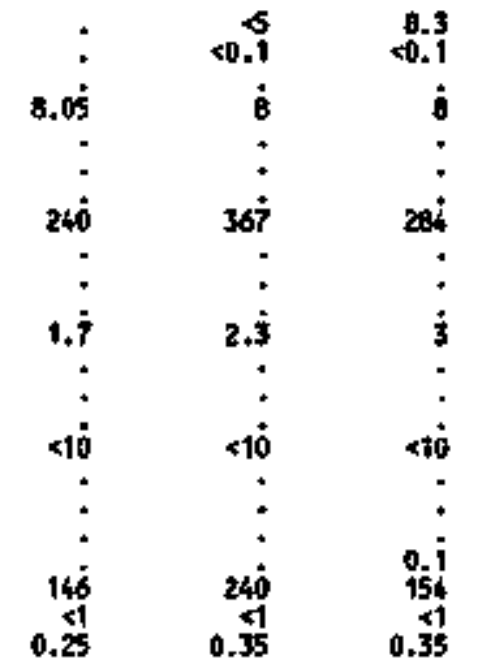




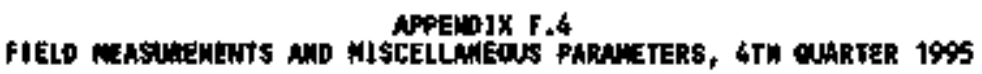

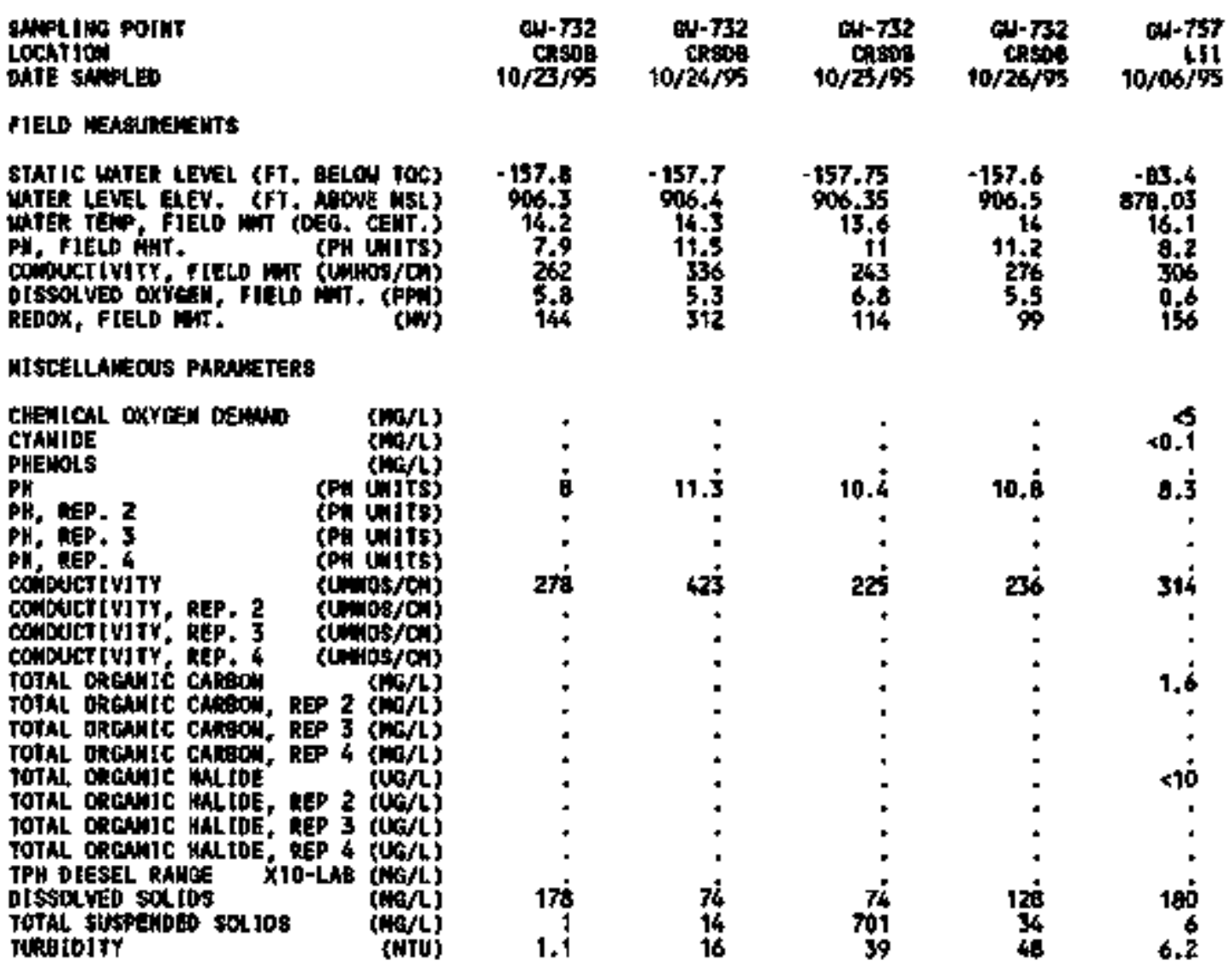





\section{APPBNDIX F.5 \\ RADIONUCLIDES}



APpendx $F .5$

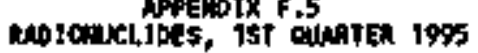

SHAPLJWE POIHT LOCATJA

LOCAT JAT SNAPLD

onoss ALPy:

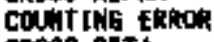

coumtint ERROR

RAOJOWCL IOES

241-ArekICtun

CQunt tus ERROB

CaUt IMB ERRO

129-[ODI lie

contitut ekror

237-UEDTUIU⿴囗十⺝

COMTING EREOA

238-PLUTON]

con

239-purant

coning ERton

cetrum In ERnon

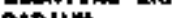

COCNFIM ERER

COANT ING ENROA

9-rechertim

Car

coyring

TRitiu tan

234

Corit

COUNT IN ERROR

2ug-Lenty

couvfing Ekhoo
Car-14

triv

a) 143

$01 / 17 / 95$

EN-511

CRsp

can+ 709

1/00/95 01/te/

(PELA)
(Gnoss ALPHA)
(PC1//)

$-6,00488$

-0.39
2.5

2.47
1.4
18
3.7

0.792
1.2
3.76
2.5

0.331
-0.0121
2.6

29.1

(choss BETA\}

(261-ANERC]/L)

(PC) $(L)$

137-CES1(u)

X10-LAB (BQ/L)

(129-100]HE)

(PC1/L)

(PC) $(L)$

(focis

(239*PLuTOH)

(P)

(PRCT/L)

(RADIL)

(PC)/L)

(STAOHT (1)

(PCI/ $t$ )

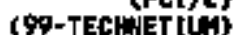

(PCIA)

x10-1

Treitivis

tpct

(PCI/

235-LRAnlim)

(PCI/L)

\{350-URAHIUA? 


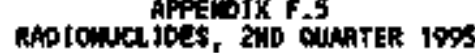

SAPLING POJHT LOCATJON

DATE \&ATLLE

Gross ALPH COUHT INE ERRO choss bEth cotint tus ERros

RAD LOWUCL IDES

137-GES1U,

CONTING ERROA

40-Potinssila

covit tios erion

DOTACT IHIJ

COUNTIMB ERROR

ADI:

coinfints encos

TROMTIU,

COUint]10 ENROR

$231+23$ - THOR JUN

COUMFIHO ERROR

234-LRAIII

colyting throe

255-Ldatulu|

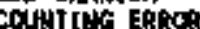

238-t d

COUMTJNG ERROR

\begin{tabular}{|c|c|c|c|c|c|c|c|c|c|c|}
\hline & $\begin{array}{r}0 N+144 \\
k+1 \\
0, / 25 / 95\end{array}$ & $\begin{array}{r}a+159 \\
\text { CRsop } \\
04 / 06 / 95\end{array}$ & $\begin{array}{r}a v-186 \\
05 / 03 / 28\end{array}$ & $\begin{array}{r}04-221 \\
\text { wilcs } \\
04 / 19 / 95\end{array}$ & $\begin{array}{r}4+292 \\
0 / 24 / 95\end{array}$ & $\begin{array}{r}\text { cu-290 } \\
\text { cotant } \\
05 / 04 / 95\end{array}$ & $\begin{array}{r}\text { W-512 } \\
04 / 26 / 55\end{array}$ & $\begin{array}{r}\text { en-540 } \\
\text { cou,vi } \\
04 / 04 / 55\end{array}$ & $\begin{array}{r}m-560 \\
04,11 \\
0405 / \% 5\end{array}$ & $\begin{array}{r}w-600 \\
\text { thsp } \\
05 / 6 / 9 \% 5\end{array}$ \\
\hline 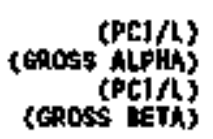 & $\begin{array}{r}1.69 \\
1.4 \\
3.07 \\
2.5\end{array}$ & $\begin{array}{r}0.649 \\
1.7 \\
2.74 \\
2.9\end{array}$ & $\begin{array}{r}+2.7 \\
1.5 \\
3.1\end{array}$ & $\begin{array}{r}0.324 \\
1 \\
3.23 \\
2.5\end{array}$ & $\begin{array}{r}1.68 \\
2.1 \\
2.48 \\
3.1\end{array}$ & $\begin{array}{r}-0.427 \mathrm{c} \\
2.1 \\
5.65 \mathrm{C} \\
2.8\end{array}$ & $\begin{array}{r}-0.155 \\
1.7 \\
-0.263 \\
2.9\end{array}$ & $\begin{array}{r}0 \\
0.050 \\
2.7\end{array}$ & $\begin{array}{r}2.99 \\
2.4 \\
1.45 \\
3\end{array}$ & $\begin{array}{r}0.532 \\
1.4 \\
3.23 \\
2.6\end{array}$ \\
\hline
\end{tabular}

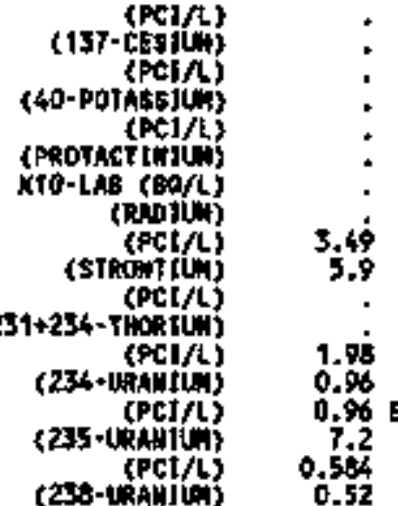

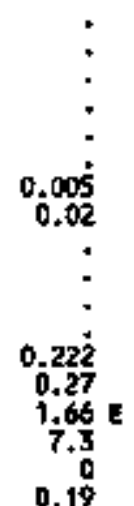

:

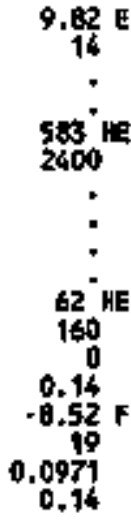

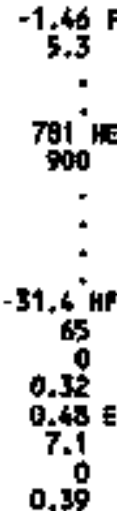


APPEND IX F.5

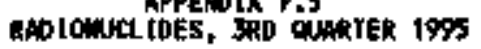

SNPLJHG POIHT Locht1ot

DATE SUPLED

CROSS NUPHA

Dount thi ERica

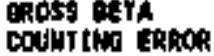

MotoinctioEs

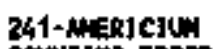

COUNTIFA ERROR

137-CEStLN

cotut the ERROR

129+3 Jojlite

COUnTING ERRA

237-HEPTUWIUN

COUNT IMB ERROR

230-PLUTONIOH

coynttwo exhom

239+PuUTO:日1

couint to eRROR

40-Aothssion.

count JW ERRor

Phoractinius

COUNTJWB ERROA

RADIun

CAuTIMG ERROR

STAONTHW

CONTIHE ERROR

צ-TECHIETtL

CONHTH ER EROR

231 +234-THOR IU

CaANTIMS ERROR

ThITIU

COANIIHS ERROA

23'4-URAHICH

COUWTHB ERROR

$235-1$ RAMTU,

calkt IHO ERKO

COUNT IRG ERRO

\begin{tabular}{|c|c|c|c|c|c|}
\hline & $\begin{array}{r}64-144 \\
1410 \\
07 / 16 / 95\end{array}$ & $\begin{array}{r}\text { w-19t } \\
\operatorname{cosep} \\
07 / 20 / 5\end{array}$ & $\begin{array}{r}\text { GN-303 } \\
\text { CR:08 } \\
07 / 16 / \% 5\end{array}$ & $\begin{array}{r}\text { tur-522 } \\
\text { Liv } \\
07 / 12 / 95\end{array}$ & $\begin{array}{r}04-709 \\
4 t 1 \\
07 / 10 / 95\end{array}$ \\
\hline 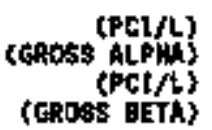 & $\begin{array}{r}1,62 \\
1.1 \\
2.53 \\
1.8\end{array}$ & $\begin{array}{r}-0.00702 \\
5.3 \\
+.27 \\
2.6\end{array}$ & $\begin{array}{r}2.35 \\
1.3 \\
2.5\end{array}$ & $\begin{array}{r}3.13 \\
2.1 \\
6.15 \\
3.2\end{array}$ & $\begin{array}{r}1.67 \\
1.2 \\
5.95 \\
2.8\end{array}$ \\
\hline
\end{tabular}

(241-Awétict)

(241-MEAtctin)

(PCI/L)

10. 10 (

$\times 10 \div$ LAB $(09 / 2\}$

रदि

(237-MEPTLititus)

SPCI/L\})
(236-PLUTOWIL)

(PCI/L)
(239.PLUfourus)

(40-porisc]flu

(pcis)

(Prothatulus)

(PROTACTJKILA)

$\times 10-L 8$ (RAD $/ \mathrm{L}$ )

(RTDL)

(stront It:s)

(PCI/L)

(99-tECAl

(231+2) (PC1/L)

$\times 10-\operatorname{Ln}(\mathrm{cos} / \mathrm{L})$

(TRITIE)

(PCI/L)

(234-URANIUN)

(235-URA (P)IL)

(PCI/L)

(230-URANIL) 


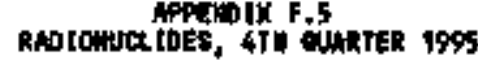

SAMpl1 Ho Po1ht

tochitow

DATE SALPE

Guoss ALPHK

COUHTIME ENhD

obos 8 BETA

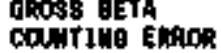

RADSOWCLEES

137-CE8 [UW

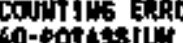

40-PिA

countis Encion

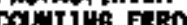

GADIU

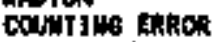

5 TROATIIUA

COUNTIUS ERROR

$231+234$-THORIO

COUIT Jing ERROR

$234=1$ R

Colintitu EkRA

235-URAHIT

COUWT L ERROR

CONIIIIS ERROR

\begin{tabular}{|c|c|c|c|c|c|c|c|c|c|}
\hline & $\begin{array}{r}610-144 \\
11 / 14 / 95\end{array}$ & $\begin{array}{r}c 4-160 \\
\text { chlonis } \\
11 / 02 / 95\end{array}$ & $\begin{array}{r}\text { an+181 } \\
11 / 05 / 9 s\end{array}$ & $\begin{array}{r}64-157 \\
1+1 / 02 / 56\end{array}$ & $\begin{array}{r}\text { at-221 } \\
\text { uncs } \\
10 / 0 \mathrm{~s} / 9 \mathrm{~s}\end{array}$ & $\begin{array}{r}10292 \\
\text { ECRRp } \\
11 / 06 / \% 3\end{array}$ & $\begin{array}{r}4-371 \\
10 / 26 / 95\end{array}$ & $\begin{array}{r}\text { or-540 } \\
\text { obLy) } \\
10 / 11 / 95\end{array}$ & $\begin{array}{r}0.560 \\
\cot 10 / 15 \\
10 / 06 / 95\end{array}$ \\
\hline $\begin{array}{r}\text { (pctfl) } \\
\text { (enass MLPHA) } \\
\text { (bClfl) } \\
\text { conoss BETA) }\end{array}$ & $\begin{array}{r}3.52 \\
1.9 \\
2.8\end{array}$ & $\begin{array}{r}46.2 \\
8.2 \\
45 \\
6.4\end{array}$ & $\begin{array}{r}0.901 \\
1.9 \\
0.420 \\
2.9\end{array}$ & $\begin{array}{r}0.67 \\
2.6 \\
4.68 \\
3.3\end{array}$ & $\begin{array}{r}-0.49 \\
1.5 \\
1.05 \\
2.4\end{array}$ & $\begin{array}{r}1.38 \\
-0.643 \\
2.3\end{array}$ & $\begin{array}{r}-1,47 \\
1.5 \\
-2.37 \\
2.5\end{array}$ & $\begin{array}{r}-1.47 \\
4.6 \\
+5.63 \\
6.9\end{array}$ & $\begin{array}{r}0.489 \\
0.87 \\
-0.818 \\
2.5\end{array}$ \\
\hline 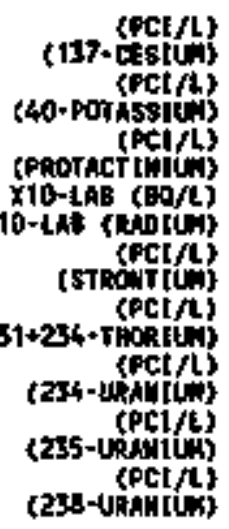 & $\begin{array}{c}\vdots \\
\vdots \\
\vdots \\
5.6 \\
6.8 \\
79.8 \\
52 \\
2.39 \\
0.72 \\
5.31 \mathrm{E} \\
6.53 \\
0.753 \\
0.4\end{array}$ & $:$ & $:$ & $:$ & $\begin{array}{r}\vdots \\
\vdots \\
\vdots \\
0.3 \\
0.034 \\
\vdots \\
\vdots \\
0.456 \\
0.3 \\
-0.0645 \\
6.3 \\
0 \\
0.18\end{array}$ & $:$ & $:$ & $\begin{array}{c}-5.16 \\
j 5 \\
12700^{\circ} \mathrm{HE} \\
1400 \\
\vdots \\
\vdots 2.2 \mathrm{HE} \\
76 \\
0.38 \\
-2,47 \mathrm{~F} \\
9.6 \\
0.35\end{array}$ & $\begin{array}{c}-2.81 \mathrm{~F} \\
13 \\
\vdots \\
1000 \\
\vdots \\
20.6 \mathrm{HF} \\
0.131 \\
0.17 \\
0.255 \mathrm{E} \\
7.5 \\
0.18\end{array}$ \\
\hline
\end{tabular}


APPEDIX F.5

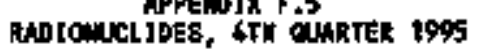

SNPLING POtkT

LOCATION

DATE ANATE

GLOSS ALPH

COLWTHE ERMR

cotoss BETA

COtHT INA ETROA

RAbTouncLloes

$137+$ CEsJu

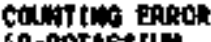

40 -portss lL

COSTITS ERROR

PRotactintu

conting earon

DAortin

COUNTING ERAOR

STRO⿻肀二T IUW

COUNT IAP ERROR

$231+234$ -

CoUNTy Ex

4-Ualun

COUNT 1WE ERRO

235-Uphantus

DOU.ITHB ER

COUNTING ERHOR

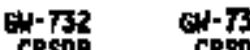

CRSDB
$10 / 23 / 95$
$10 / 24 / 95$
(cooss $(P C) / L)$
conoss Alpin

(CROSS BETA)

-0.640
1.4
2.8
2.5

0.841
1.1
27.2
4.7

0.479

1.7
23.5

-0.665
1.7
7.45
3.7

4I-732

cesps

10/06/95

(PCt/ $L$ )

(137-CEStL)

(40-p0tasstum

(PCL)

D

(FC] 7 )

Tho, J(t)

(231+224-THOR]

(PC]/L

(234-0aㅔ J)

(P)

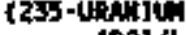

(PC) $/$ ) 



\section{APPENDIX G \\ RADIOLOGICAL CHEMISTRY DATA SUMMARY}




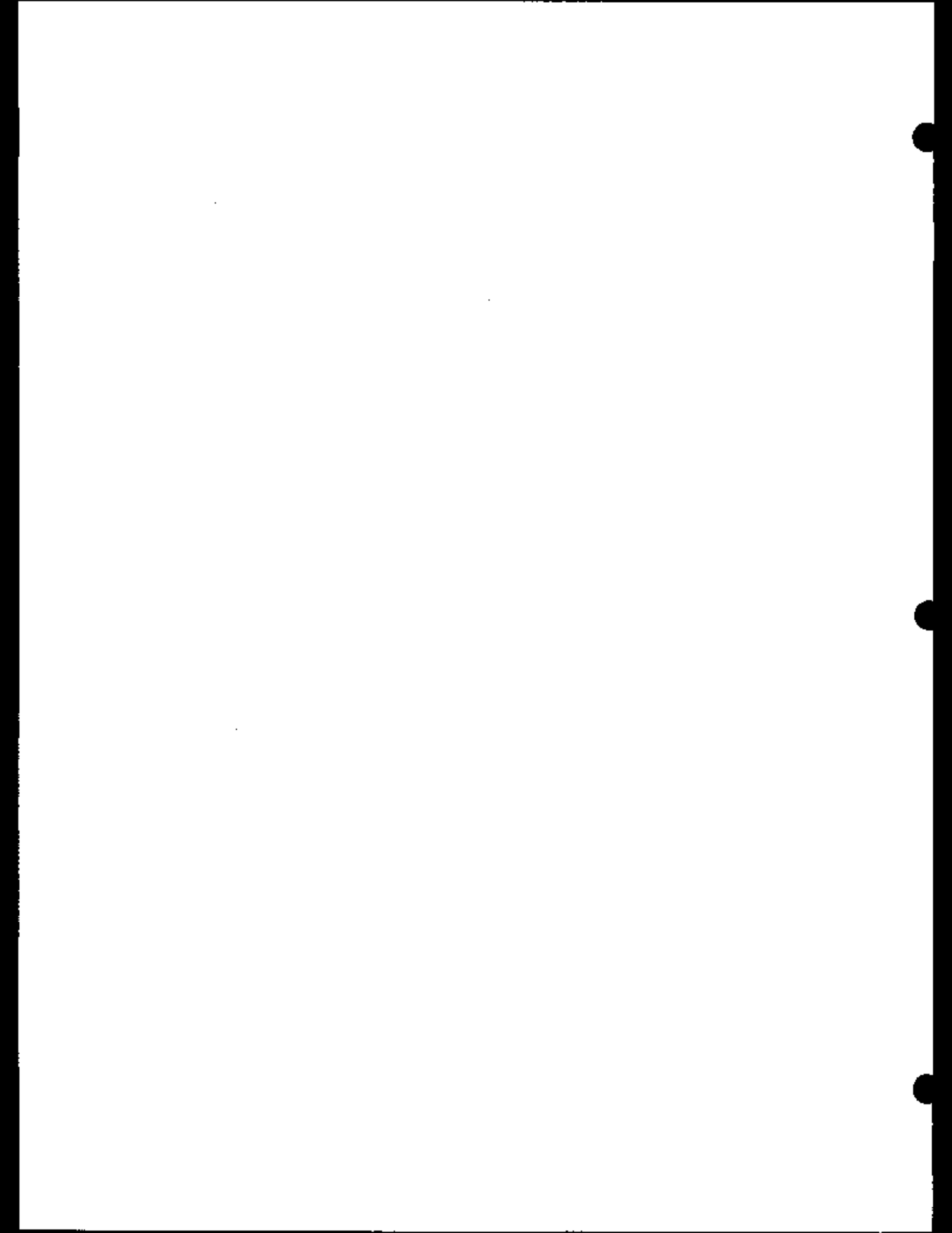




\section{EXPLANATION}

SAMPLING POINT:

$\begin{array}{rll}\text { GW } & - & \text { Monitoring Well } \\ \text { CBS-1 } & - & \text { Chestnut Branch Spring } 1 \\ \text { SCR2.2SP } & - & \text { South Chestnut Ridge Spring 2.2 } \\ 1090 & - & \text { Historical 1000-Series Monitoring Well }\end{array}$

\section{LOCATION:}

\begin{tabular}{|c|c|c|}
\hline $\begin{array}{r}\text { ADB } \\
\text { CDLVI } \\
\text { CDL VII } \\
\text { CRBAWP } \\
\text { CRSDB } \\
\text { CRSP } \\
\text { ECRWP } \\
\text { KHQ } \\
\text { LII } \\
\text { LIV } \\
\text { LV } \\
\text { RQ } \\
\text { UNCS }\end{array}$ & - & 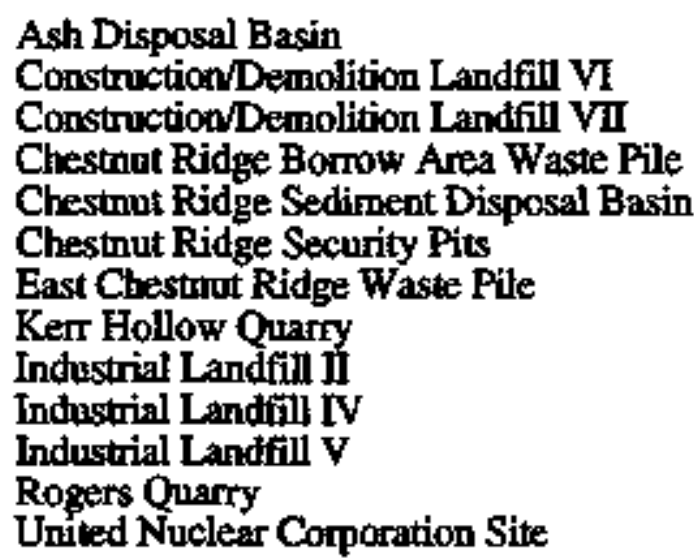 \\
\hline
\end{tabular}

NOTES:

$\begin{array}{lll}\mathrm{pCi} / \mathrm{L} & -\quad & \text { picoCuries per liter } \\ \mathrm{CE}+1 \cdot & - & \text { Counting Error (two standard deviations) } \\ . & \text { Not sampled }\end{array}$

Mean counting error (e) calculated using the following formula from Evans (1955):

$$
e=\sqrt{\frac{E_{1}^{2}}{n^{2}}+\frac{E_{2}^{2}}{n^{2}}+\frac{E_{3}^{2}}{n^{2}}+\cdots}
$$

where $E_{1}, E_{2}, \ldots E_{x}$ are the individual errors, and $n$ is the number of observations. 

APPENDIX 6

Grose Alpho \& Bross Bete Activity bute sumory, 1995

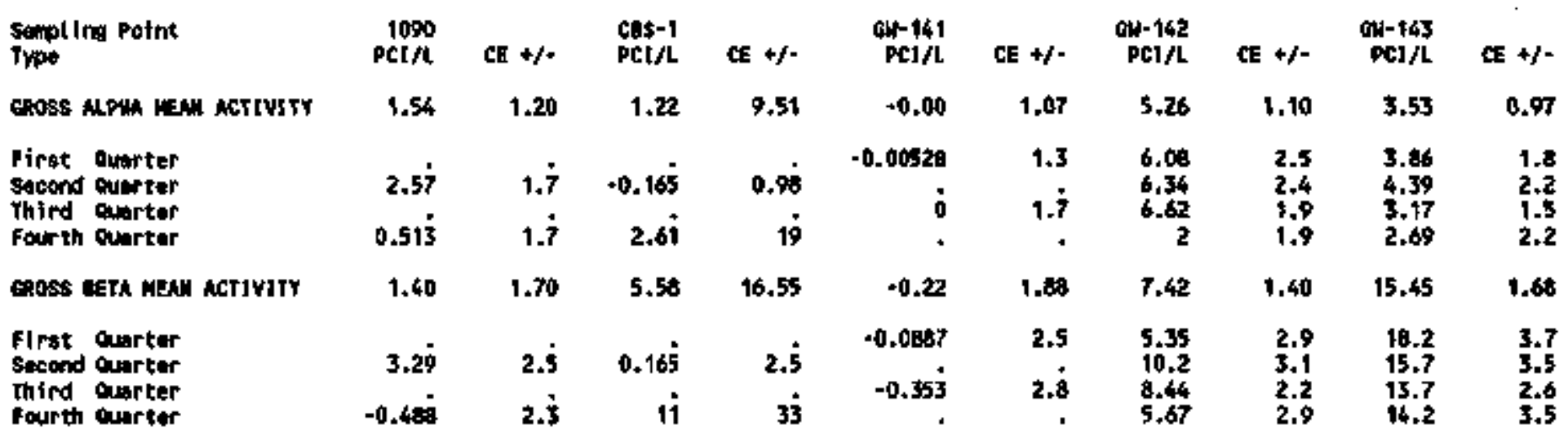


APDENDIX $\mathrm{C}$

arose Alpha geos Bats ictivity Dote sumary, 1995

\begin{tabular}{|c|c|c|c|c|c|c|c|c|c|c|}
\hline $\begin{array}{l}\text { seppl ing Point } \\
\text { Type }\end{array}$ & $\begin{array}{l}\mathrm{GH}-144 \\
\mathrm{PC}] / 4\end{array}$ & $\operatorname{CE}+t=$ & $\begin{array}{l}\text { OU-145 } \\
\mathrm{PCI} / \mathrm{L}\end{array}$ & $\mathrm{CE}+/=$ & $\begin{array}{r}\text { Pu-146 } \\
\text { PCI/L }\end{array}$ & $\mathrm{CE}+/$ & $\begin{array}{c}\text { GH-147 } \\
\mathrm{PCI} / \mathrm{L}\end{array}$ & $\mathrm{C}=+/ *$ & $\begin{array}{l}\mathrm{N}+156 \\
\mathrm{PCJ} / \mathrm{L}\end{array}$ & $\mathrm{Ct}+/-$ \\
\hline qROSS MhPH NEN ACTIVtTY & 2.46 & 0.77 & 11.68 & 1.52 & 2.47 & 0.67 & 1.71 & $0.7 t$ & 2.28 & 0.97 \\
\hline $\begin{array}{l}\text { Firet arter } \\
\text { second gurter } \\
\text { Third Quarter } \\
\text { Fourth Quarter }\end{array}$ & $\begin{array}{r}5.45 \\
4.59 \\
2.09 \\
5.3\end{array}$ & $\begin{array}{r}2.6 \\
2.7 \\
1.7 \\
3\end{array}$ & $\begin{array}{l}16.7 \\
14.7 \\
16.3 \\
21.1\end{array}$ & $\begin{array}{l}3.6 \\
3.5 \\
3.5 \\
3.8\end{array}$ & $\begin{array}{r}14 \\
16.1 \\
15.9 \\
16.8\end{array}$ & $\begin{array}{l}3.5 \\
3.6 \\
2.6 \\
3.8\end{array}$ & $\begin{array}{l}5.6 \\
5.17 \\
4.51 \\
3.81\end{array}$ & $\begin{array}{l}2.6 \\
2.7 \\
1.9 \\
2.7\end{array}$ & $\begin{array}{l}2.27 \\
9.84 \\
4.85 \\
6.52\end{array}$ & $\begin{array}{l}2.4 \\
3.4 \\
2.1\end{array}$ \\
\hline
\end{tabular}


APEDID $G$

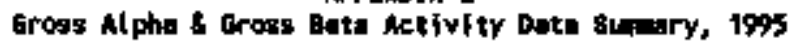

\begin{tabular}{|c|c|c|c|c|c|c|c|c|c|c|}
\hline Sompling point & $\begin{array}{l}\text { m+15s } \\
\operatorname{PCI} / \mathrm{C}\end{array}$ & CE $+/$ & $\begin{array}{c}\mathrm{CH} \cdot 159 \\
\mathrm{PCI} / \mathrm{L}\end{array}$ & $C E+/-$ & $\begin{array}{l}6 \mathrm{~N}-160 \\
\mathrm{PCI} / \mathrm{L}\end{array}$ & $C E+/-$ & $\begin{array}{c}G N-161 \\
O S I / L\end{array}$ & CE $+/$ & $\begin{array}{l}\operatorname{as}-175 \\
\mathrm{Pcl} / \mathrm{C}\end{array}$ & $\boldsymbol{\alpha}+/-$ \\
\hline Goss MLPH NEAH ACTJVITY & 2.16 & 1.06 & 1.86 & 0.87 & 23,07 & 4.41 & 0,03 & 1.37 & 0.45 & 0.70 \\
\hline $\begin{array}{l}\text { Efrst buarter } \\
\text { second guarter } \\
\text { thi rd guarter } \\
\text { fourth guarter }\end{array}$ & $\begin{array}{r}2.01 \\
2.62 \\
1.85 \\
.\end{array}$ & $\begin{array}{l}1.5 \\
2.5 \\
1.1 \\
.\end{array}$ & $\begin{array}{r}2.35 \\
5.71 \\
0.942 \\
-1.18\end{array}$ & $\begin{array}{r}1.6 \\
2.5 \\
0.93 \\
1.6\end{array}$ & $\begin{array}{l}6.73 \\
39.4\end{array}$ & $\begin{array}{r}4 . \dot{8} \\
7 . \dot{4}\end{array}$ & $\begin{array}{r}1.64 \\
-0.00594\end{array}$ & $\begin{array}{l}2 . \dot{3} \\
1 . \dot{5}\end{array}$ & $\begin{array}{r}1.33 \\
-0.361 \\
1.33 \\
-0.5 !\end{array}$ & $\begin{array}{l}1.6 \\
1.1 \\
1.7 \\
1.3\end{array}$ \\
\hline Enoss geth MEN MCTJVITY & 2.84 & 1.47 & 4.62 & 1.40 & 23.55 & 4.26 & 1,54 & 1.98 & 0.19 & 1.25 \\
\hline 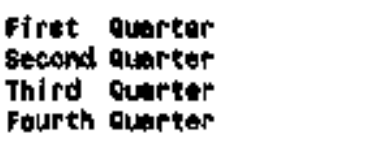 & $\begin{array}{r}3.39 \\
3.65 \\
1.69 \\
.\end{array}$ & $\begin{array}{r}2.5 \\
3.1 \\
1.9 \\
.\end{array}$ & $\begin{array}{r}1.4 \\
3.79 \\
0.201 \\
13.4\end{array}$ & $\begin{array}{r}2.4 \\
3 \\
1.9 \\
3.6\end{array}$ & $\begin{array}{l}12.9 \\
34.2\end{array}$ & $\begin{array}{l}6.8 \\
5.4\end{array}$ & $\begin{array}{r}0.743 \\
2.35\end{array}$ & $\begin{array}{l}2 . \dot{9} \\
2 . \dot{7}\end{array}$ & $\begin{array}{r}-1.04 \\
1.74 \\
-0.0986 \\
0.163\end{array}$ & $\begin{array}{l}2 \\
2 \\
2\end{array}$ \\
\hline
\end{tabular}


APPENDIX

Grote Alphe 6 Gross Bets Activity Date sumpry, $19 \%$

\begin{tabular}{|c|c|c|c|c|c|c|c|c|c|}
\hline$\underset{\text { type }}{\text { sulpe polnt }}$ & $\begin{array}{l}\text { en-177 } \\
\mathrm{FCl} / \mathrm{L}\end{array}$ & $\mathrm{CE}+/-$ & $\begin{array}{l}\text { an-1a1 } \\
\text { PCt/l }\end{array}$ & $c t+7$ & $\begin{array}{c}\text { eN+194 } \\
\text { PC1/L }\end{array}$ & 战 & $\begin{array}{l}4+186 \\
P S 1 / L\end{array}$ & CE $4 /$ & $\begin{array}{r}\operatorname{es} 187 \\
\mathrm{PCt} / \mathrm{L}\end{array}$ \\
\hline GRSSB ALPH MEN NCTIVITY & 2.83 & 1.00 & 0.55 & 0.78 & -0.48 & 1.31 & 3.21 & 1.7 & 0.91 \\
\hline $\begin{array}{l}\text { Firet querter } \\
\text { second aunrter } \\
\text { thl rd quarter } \\
\text { fourth duarter }\end{array}$ & $\begin{array}{l}2.34 \\
4.16 \\
1.56 \\
2.22\end{array}$ & $\begin{array}{l}2.7 \\
2.8 \\
2.5 \\
2.7\end{array}$ & $\begin{array}{r}2.2 \\
3.67 \\
0.549 \\
0.458\end{array}$ & $\begin{array}{l}2.4 \\
2.8 \\
2.5 \\
2.9\end{array}$ & $\begin{array}{l}1 . \dot{7} \\
1.38\end{array}$ & $\begin{array}{l}3 \\
3\end{array}$ & $\begin{array}{r}-0.0008 \\
1.15\end{array}$ & $\begin{array}{l}5 \\
3 \\
5\end{array}$ & $\begin{array}{r}0.0917 \\
2.3 \overline{5}\end{array}$ \\
\hline
\end{tabular}


APDEDIX 6

Grons Alphe 1 Grose bate Activity oute Sumery, 1095

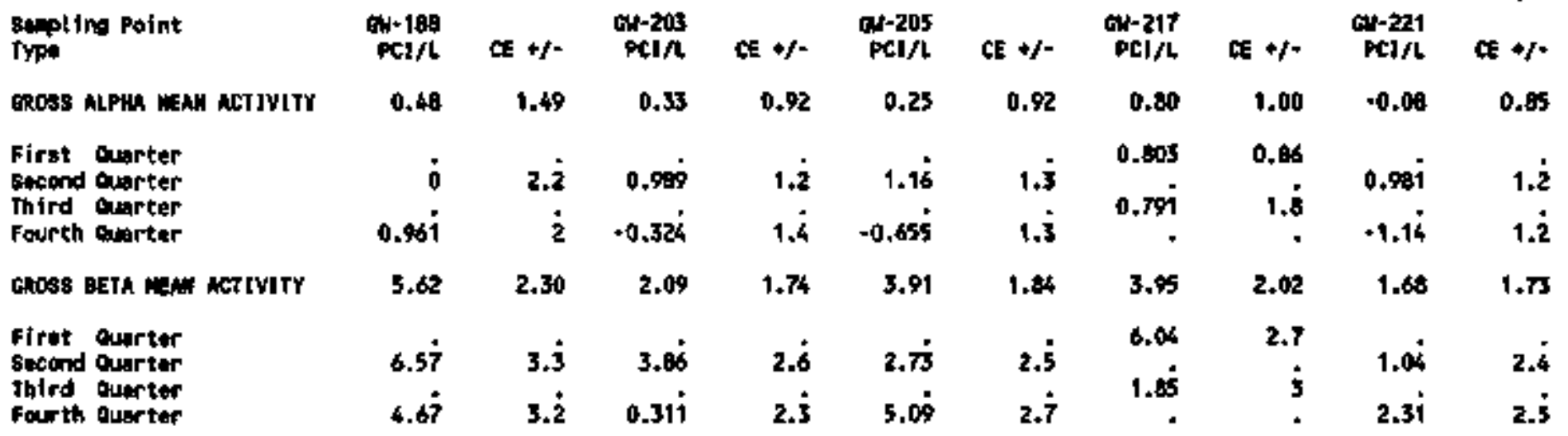


APFELDIX 6

Grose Alpho + groos cote Activity Date subary, 19\%5

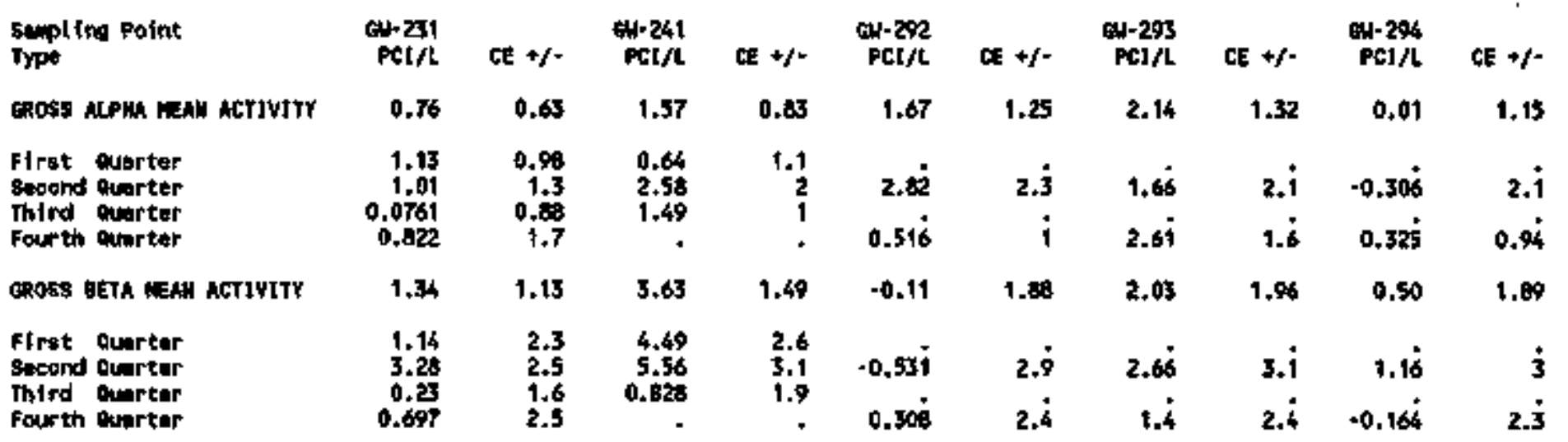


APPEIOIX $\mathrm{E}$

Groos Alphes aroos beta Activity Dote suanry, 1905

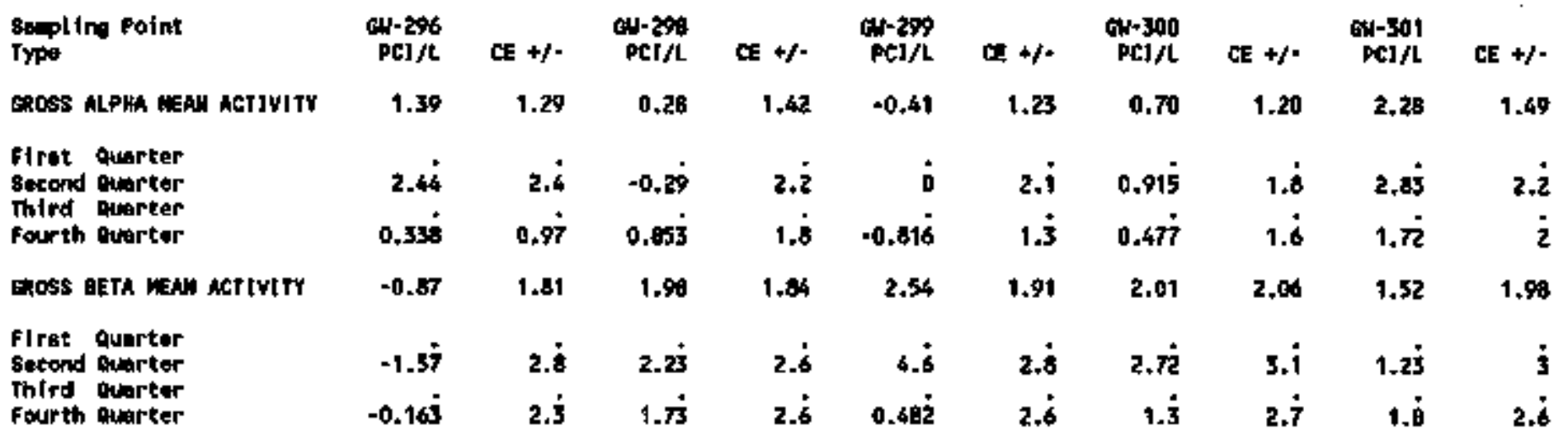


Grosto At pho oreas beta Ativity sata sumbry, 1995

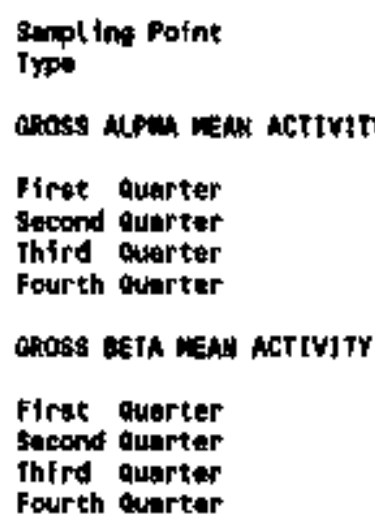

\begin{tabular}{|c|c|c|c|c|c|c|c|c|c|}
\hline $\begin{array}{c}\mathrm{PCI} / \mathrm{t} \\
\mathrm{P}\end{array}$ & $C E \bullet /-$ & $\begin{array}{c}\text { بu-303 } \\
\text { PCI/L }\end{array}$ & CE $+/-$ & $\begin{array}{l}\text { W-304 } \\
\text { PCI/ }\end{array}$ & CE $+/-$ & $\begin{array}{c}\text { ov-305 } \\
\text { PCI/th }\end{array}$ & CE $+/-$ & $\begin{array}{r}\mathrm{CH}-\mathbf{3 2 1} \\
\mathrm{PCI} / \mathrm{L}\end{array}$ & CE +\%- \\
\hline 0,64 & 1.06 & 1.35 & 0.91 & 1,41 & 0,83 & 0.39 & 1,07 & $=0.26$ & 0,85 \\
\hline 1.64 & 1.5 & $\begin{array}{r}2.16 \\
0.199 \\
1.65\end{array}$ & $\begin{array}{r}1.4 \\
2.2 \\
1.2\end{array}$ & $\begin{array}{l}1.33 \\
1.20 \\
1.63\end{array}$ & $\begin{array}{l}1.3 \\
1.4 \\
1.1\end{array}$ & $\begin{array}{l}0.158 \\
0.62 i\end{array}$ & $\begin{array}{r}1.3 \\
1.5\end{array}$ & $\begin{array}{r}0.155 \\
-0.604\end{array}$ & $\begin{array}{l}1.2 \\
1.6\end{array}$ \\
\hline-0.354 & 1.5 & - & . & + & . & . & . & -0.318 & 1.5 \\
\hline 1,37 & 1,66 & 3.70 & 1,45 & 1.71 & 1,42 & 2.27 & 2.02 & 1.23 & 1.57 \\
\hline 2.74 & 2.4 & $\begin{array}{l}4.63 \\
1.34 \\
2.94\end{array}$ & $\begin{array}{r}2.5 \\
1.9 \\
1.9\end{array}$ & $\begin{array}{r}2.52 \\
2.12 \\
0.489\end{array}$ & $\begin{array}{l}2.5 \\
2.9 \\
1.9\end{array}$ & $\begin{array}{r}0.234 \\
4.5\end{array}$ & $\begin{array}{l}2.6 \\
3.1\end{array}$ & $\begin{array}{r}1.99 \\
4.81\end{array}$ & $\begin{array}{l}2.5 \\
3.2\end{array}$ \\
\hline .00506 & 2.3 & - & . & • & . & & . & 0.871 & 4 \\
\hline
\end{tabular}


APPEDIDLX 0

trose Alpha of erose Bete Aetlvity Dote sumery, 1995

\begin{tabular}{|c|c|c|c|c|c|c|c|c|c|c|}
\hline Type & $\begin{array}{c}\text { ond 339 } \\
\text { PC1/L }\end{array}$ & Ex $\bullet /$ & $\begin{array}{r}\text { edt-5t1 } \\
\text { peith }\end{array}$ & CE $4 /$ & $\begin{array}{c}04-512 \\
P \& 1 / L\end{array}$ & CE $+/-$ & $\begin{array}{c}\mathrm{P}-513 \\
\mathrm{PCI} / \mathrm{L}\end{array}$ & $\operatorname{ce}+/-$ & $\begin{array}{c}\text { an+514 } \\
\text { Pet } / 4\end{array}$ & 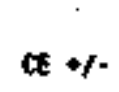 \\
\hline EROSS ALPAH HEA ACTIVITY & 0.47 & 1.06 & 2.08 & 0.90 & 1.13 & 1.17 & 0.24 & 1.02 & 0.93 & 1.10 \\
\hline $\begin{array}{l}\text { I I rst Guacter } \\
\text { Socond Ouarter } \\
\text { Third Ouarter } \\
\text { Fourth ouartor }\end{array}$ & $\begin{array}{r}1 . \dot{3} \\
-0.3 \dot{6}\end{array}$ & $\begin{array}{r}1 . \dot{5} \\
1.5\end{array}$ & $\begin{array}{r}0,631 \\
6.81 \\
-0,326 \\
1.19\end{array}$ & $\begin{array}{l}1.1 \\
2.6 \\
1.2 \\
1.9\end{array}$ & $\begin{array}{l}1.11 \\
1.14\end{array}$ & $\begin{array}{r}i \\
1 . i\end{array}$ & $\begin{array}{r}\dot{0} \\
0.45 i\end{array}$ & $\begin{array}{r}1 . \dot{8} \\
0.9 \dot{8}\end{array}$ & $\begin{array}{r}1.55 \\
0.318\end{array}$ & $\begin{array}{r}2 \\
0.92\end{array}$ \\
\hline GRoss uth ind activtfy & 1,48 & 1.66 & 1.00 & $\uparrow .34$ & 3.40 & 2,06 & 0.99 & 1.88 & 1.29 & 1.91 \\
\hline $\begin{array}{l}\text { Firat warter } \\
\text { scond werter } \\
\text { Third ouarter } \\
\text { Fourth ouarter }\end{array}$ & $\begin{array}{r}2.03 \\
0.0934\end{array}$ & $\begin{array}{l}2.4 \\
2 . j\end{array}$ & $\begin{array}{r}3.92 \\
2.78 \\
0.313 \\
0.599\end{array}$ & $\begin{array}{l}2.6 \\
2.7 \\
2.5 \\
2.7\end{array}$ & $\begin{array}{l}3.0 \dot{3} \\
4.52\end{array}$ & $\begin{array}{r}3.1 \\
2.7\end{array}$ & $\begin{array}{l}0.79 \\
1.19\end{array}$ & $\begin{array}{l}2.9 \\
2.4\end{array}$ & $\begin{array}{r}0.176 \\
2.39\end{array}$ & $2 . \dot{5}$ \\
\hline
\end{tabular}


AppaDIX 6

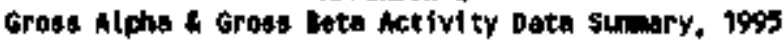

\begin{tabular}{|c|c|c|c|c|c|c|c|c|c|c|}
\hline $\begin{array}{l}\text { sappling Point } \\
\text { Typo }\end{array}$ & $\begin{array}{c}\mathrm{GN}-52 \dagger \\
\mathrm{PCI} / \mathrm{L}\end{array}$ & CE $+/-$ & $\begin{array}{l}a 14-522 \\
\mathrm{PCt} / \mathrm{c}\end{array}$ & CE $+/-$ & $\begin{array}{r}64-539 \\
\mathrm{PCI} / \mathrm{t}\end{array}$ & CE $\%$ & $\begin{array}{l}\mathrm{Cu}-540 \\
\mathrm{PC1} / \mathrm{L}\end{array}$ & CE $4 /^{-}$ & $\begin{array}{r}\text { cut } 541 \\
\text { PCt } / \mathrm{L}\end{array}$ & $\mathrm{CE}+/-$ \\
\hline GROSS ALPHA MEAH ACTtVITY & 0.38 & 0.99 & 0.07 & 1.04 & 0.94 & 0.96 & -1.53 & 2.39 & 3.21 & 2.86 \\
\hline $\begin{array}{l}\text { First quarter } \\
\text { Sircond Gurter } \\
\text { Third quarter } \\
\text { fourth Quster }\end{array}$ & $\begin{array}{r}-0.00578 \\
0.774 \\
\end{array}$ & $\begin{array}{r}1 \\
1.7 \\
.\end{array}$ & $\begin{array}{r}-0.167 \\
0.313 \\
\end{array}$ & $\begin{array}{r}1.2 \\
1.7 \\
.\end{array}$ & $\begin{array}{r}2.86 \\
0.302 \\
-0.334\end{array}$ & $\begin{array}{r}1.5 \\
2 \\
1.4\end{array}$ & $\begin{array}{r}\dot{0} \\
-2.6 \dot{5}\end{array}$ & $\begin{array}{r}1.6 \\
4.5\end{array}$ & $\begin{array}{l}2.61 \\
3.81\end{array}$ & $\begin{array}{r}2 . \dot{4} \\
5 . \dot{2}\end{array}$ \\
\hline EROSS BETA MEAN WCTIVITY & 1.93 & 1,91 & -0.49 & 1.91 & 2.83 & 1.55 & -5.05 & 3.58 & 2.22 & 3.95 \\
\hline $\begin{array}{l}\text { Firse Guarter } \\
\text { second Guarter } \\
\text { Third Guarter } \\
\text { fourth Guarter }\end{array}$ & $\begin{array}{r}2.62 \\
1.28 \\
.\end{array}$ & $\begin{array}{r}2.5 \\
2.9 \\
.\end{array}$ & $\begin{array}{r}-0.986 \\
\dot{0} \\
.\end{array}$ & $\begin{array}{r}2.5 \\
2.9\end{array}$ & $\begin{array}{l}3.54 \\
3.17 \\
1.74\end{array}$ & $\begin{array}{r}2.6 \\
\vdots \\
2.4\end{array}$ & $\begin{array}{r}1 . j \\
-11 . j\end{array}$ & $\begin{array}{r}2.8 \\
6.6\end{array}$ & $\begin{array}{l}1.37 \\
3.07\end{array}$ & $\begin{array}{r}\mathbf{3} \\
7 . j\end{array}$ \\
\hline
\end{tabular}


APEENOIX 0

Grosa Alphe 8 brose Beta Mctivity Data suriwy, 195

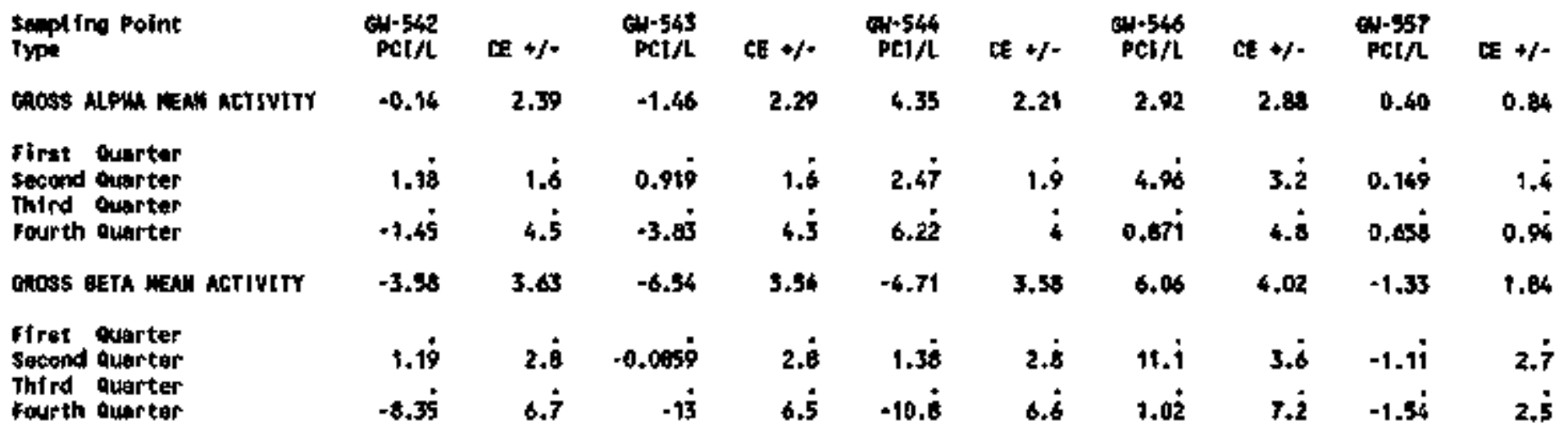


APPETDIX 6

Gross Alphs orowe Bete Aetfyity Osta sumbry, 1995

\begin{tabular}{|c|c|c|c|c|c|c|c|c|c|c|}
\hline $\begin{array}{l}\text { senpling Point } \\
\text { Type }\end{array}$ & $\begin{array}{l}0.560 \\
\text { PCt/t }\end{array}$ & c $\pitchfork / *$ & $\begin{array}{c}01-56 z \\
\text { pct } / 2\end{array}$ & ce $\bullet$ & $\begin{array}{l}\text { an-564 } \\
\text { pct/L }\end{array}$ & $\mathbf{W} \bullet$ & $\begin{array}{l}\text { cy-tos } \\
\text { PCI/L }\end{array}$ & CE + / - & $\begin{array}{l}\text { ced-60) } \\
\text { PCI/L }\end{array}$ & CE $+\%$ \\
\hline seass MLPHA VEN MCTIVITY & 0.80 & 1.19 & 24.97 & 1.00 & 0,54 & 0.88 & 1.02 & 0.83 & 0.47 & 0.70 \\
\hline $\begin{array}{l}\text { flrst cuarter } \\
\text { second ourtor } \\
\text { Third ouster } \\
\text { fourth aurter }\end{array}$ & $\begin{array}{l}0.611 \\
0.9 \% 4\end{array}$ & $\begin{array}{r}2.1 \\
1.1\end{array}$ & $\begin{array}{r}-0.15 i \\
50 . i\end{array}$ & $\begin{array}{r}\dot{z} \\
0.89\end{array}$ & $\begin{array}{l}0.442 \\
0.641\end{array}$ & 1.5 & $\begin{array}{r}-0.51 \\
2.50 \\
1.27 \\
0.329\end{array}$ & $\begin{array}{l}1.3 \\
2.1 \\
1.8 \\
1.5\end{array}$ & $\begin{array}{r}0.505 \\
0.872 \\
0.486 \\
-0.00078\end{array}$ & $\begin{array}{l}1.2 \\
1.5 \\
1.5 \\
1.4\end{array}$ \\
\hline GROSS BETA WEAN METIVITY & 0.37 & 1.98 & -0.84 & 1.91 & -0.19 & $1,8 s$ & 0.75 & 1.29 & 0.48 & $\$ .28$ \\
\hline $\begin{array}{l}\text { FIrst aurter } \\
\text { sucond ouarter } \\
\text { third Gurtor } \\
\text { fourth oubrter }\end{array}$ & $\begin{array}{l}0.258 \dot{8} \\
0.473\end{array}$ & $\begin{array}{r}\dot{3} \\
2.8\end{array}$ & $\begin{array}{l}-1.12 \dot{2} \\
-0.36 \dot{8}\end{array}$ & $\begin{array}{r}2 . \dot{9} \\
2 . \dot{5}\end{array}$ & $\begin{array}{r}0.85 \dot{0} \\
-1.38\end{array}$ & $\begin{array}{r}2 . \dot{8} \\
2 . \dot{5}\end{array}$ & $\begin{array}{r}2.09 \\
1.27 \\
-1.86 \\
1.5\end{array}$ & $\begin{array}{l}2.6 \\
2.6 \\
2.6 \\
2.7\end{array}$ & $\begin{array}{r}-1.67 \\
1.47 \\
0.94 \\
1.16\end{array}$ & $\begin{array}{l}2.5 \\
2.5 \\
7.5 \\
2.7\end{array}$ \\
\hline
\end{tabular}


MPENDIX $G$

Gross Alphe s arost Bote Activity Dote Sumary, 1995

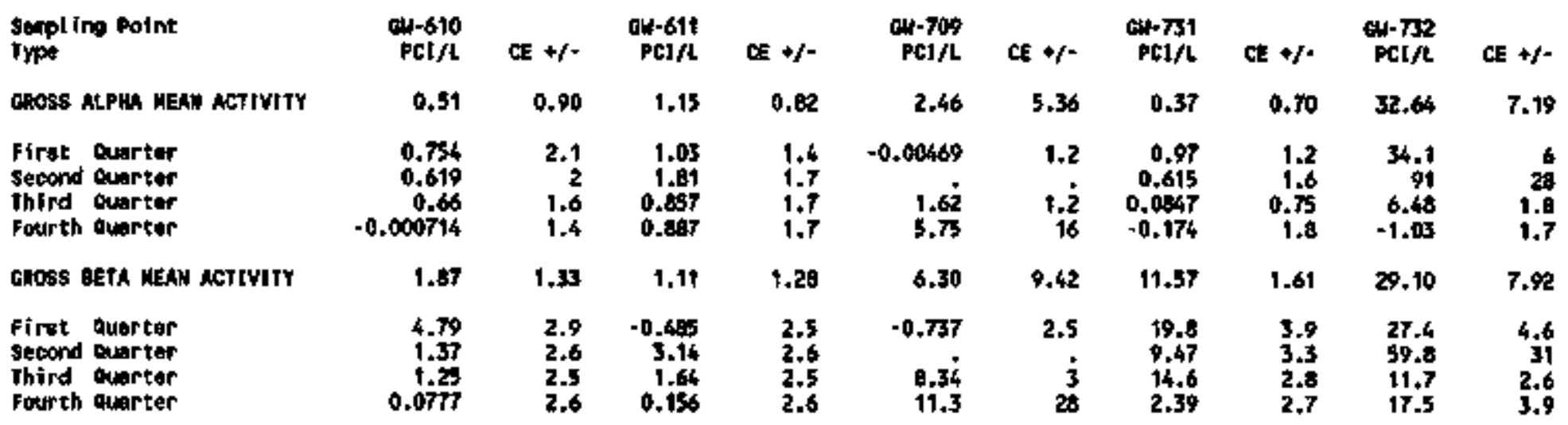


Groes Alphe is Grows bete ictivity bats sumery. 1995

\begin{tabular}{|c|c|c|c|c|c|c|c|c|c|c|}
\hline $\begin{array}{l}\text { Supl ing Doint } \\
\text { Type }\end{array}$ & $\begin{array}{c}0+742 \\
A C 1 / L\end{array}$ & $\operatorname{ces}+\pi$ & $\begin{array}{r}0.743 \\
\mathrm{PCO} / \mathrm{L}\end{array}$ & $\mathrm{CE}+/ \cdot$ & $\begin{array}{l}01-757 \\
\mathrm{PCI} / \mathrm{L}\end{array}$ & $\boldsymbol{\alpha E} \bullet /$ & $\begin{array}{c}\text { CH-7\%6 } \\
\mathrm{PCl} / \mathrm{L}\end{array}$ & CE $+f-$ & $\begin{array}{l}0+797 \\
\operatorname{Pc}[/ 1\end{array}$ & CEE $* / *$ \\
\hline EROSS WOMA WENM NCTIVITY & 5.50 & 1.25 & 1.93 & 0.96 & 4.74 & 5.40 & 0.40 & 0.70 & 0.75 & 0.82 \\
\hline $\begin{array}{l}\text { Flrot quarter } \\
\text { seccind Querter } \\
\text { Third Quarter } \\
\text { Fourth Duterter }\end{array}$ & $\begin{array}{r}4.22 \\
2.15 \\
-0.0412 \\
4.72\end{array}$ & $\begin{array}{l}2.8 \\
2.7 \\
2.5 \\
3.1\end{array}$ & $\begin{array}{r}3.29 \\
4.06 \\
0.0707 \\
13.4\end{array}$ & $\begin{array}{l}2.8 \\
2.8 \\
2.4 \\
3.6\end{array}$ & $\begin{array}{l}1.92 \\
7.98 \\
9.57\end{array}$ & $\begin{array}{r}2.7 \\
3 \\
25\end{array}$ & $\begin{array}{r}1.85 \\
-1.97\end{array}$ & $\begin{array}{l}2 . \dot{6} \\
2 . \dot{5}\end{array}$ & $\begin{array}{l}1.59 \\
0.709\end{array}$ & 2.6 \\
\hline
\end{tabular}


APPENDIX o

Grosa Alpha \& Groes bete Activity Dats \$nomery, 1995

\begin{tabular}{|c|c|c|c|c|c|c|c|c|c|c|}
\hline $\begin{array}{l}\text { 8nppt ing Point } \\
\text { Typo }\end{array}$ & $\begin{array}{l}\mathrm{CW}-7 \% \\
\mathrm{PCI} / \mathrm{L}\end{array}$ & $\boldsymbol{c}+\boldsymbol{*}$ & $\begin{array}{l}\text { GH-759 } \\
\text { PCI/ }\end{array}$ & CE + $/-$ & $\begin{array}{c}\mathrm{W}-801 \\
\mathrm{PC} 1 / \mathrm{L}\end{array}$ & $C E+/=$ & $\begin{array}{l}\mathrm{Er}+827 \\
\mathrm{PCl} / \mathrm{L}\end{array}$ & CE $+t-$ & $\begin{array}{r}\mathrm{sck2}, 2 \mathrm{gP} \\
\mathrm{PCI} / \mathrm{L}\end{array}$ & ce $* f$ \\
\hline GOOSS ALPHA MEM ACTIWIFY & 0.73 & 0.78 & 0.48 & 0.94 & 0.16 & 0.66 & -0.60 & 2.46 & 0.92 & 2.10 \\
\hline $\begin{array}{l}\text { Firat quarter } \\
\text { Second aurter }\end{array}$ & 0.324 & 1.1 & 0,052 & 1.6 & 0.164 & 1.1 & 0.449 & $2 . i$ & 0.924 & 2.1 \\
\hline Fourth tuerter & $1 . t 3$ & $1 . i$ & 0.014 & $0 . \dot{\theta}$ & 0.159 & $0 . \dot{73}$ & $-2, \infty$ & 4.5 & * & $\dot{-}$ \\
\hline CNOSS BETR MEAH MCTIVITY & 1.15 & 1.84 & 0.39 & 1.91 & 0.27 & 1.77 & +6.82 & 3,65 & 1.63 & 2.90 \\
\hline $\begin{array}{l}\text { First Ourter } \\
\text { Second ourarter }\end{array}$ & 1.17 & 2.5 & 1.26 & 2.8 & 1.59 & 2.5 & -0.428 & 2.9 & 1.63 & 2.9 \\
\hline Fourth ouster & 1.13 & 2.7 & $-0.497^{*}$ & $2 . \dot{6}$ & -0.979 & 2.5 & -9.21 & 6.7 & : & * \\
\hline
\end{tabular}



APPENDIX H

GROUNDWATER ELEVATIONS, 1995 



\section{EXPLANATION}

LOCATION:

\begin{tabular}{|c|c|c|}
\hline $\begin{array}{r}\text { ADB } \\
\text { CDLVI } \\
\text { CDE VII } \\
\text { CRBAWP } \\
\text { CRDT } \\
\text { CRSDB } \\
\text { CRSP } \\
\text { ECRWP } \\
\text { KHQ } \\
\text { LII } \\
\text { LIV } \\
\text { LV } \\
\text { ORSF } \\
\text { RQ } \\
\text { SSCR } \\
\text { UNCS }\end{array}$ & 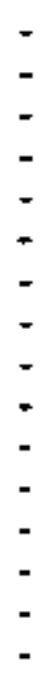 & 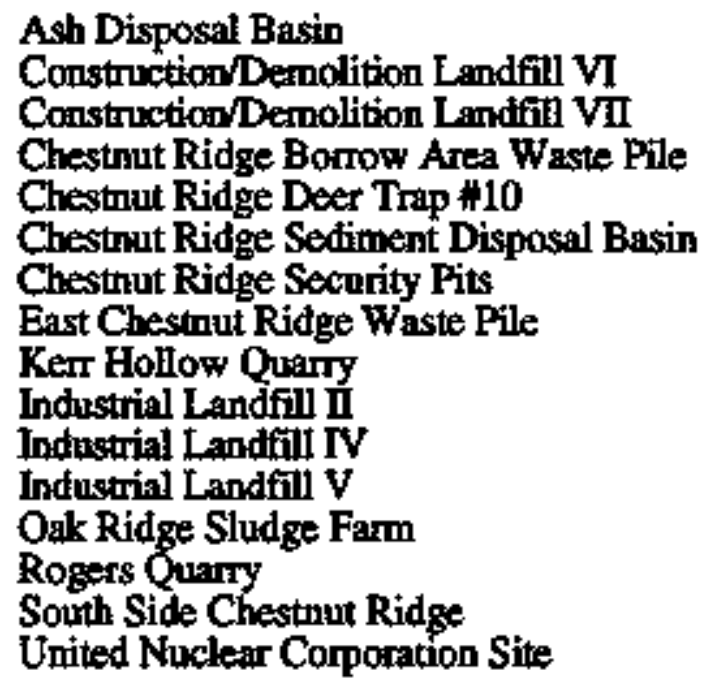 \\
\hline
\end{tabular}

SAMPLEM IN 95;

Y - Well included in the 1995 groundwater monitoring program.

NOTES:
CIP Elev. - The elevation of the centerpoint of the monitored interval. April - Measuring event from April 3 to 7, 1995.
Oxtobet - Measuring event from October 16 to 23, 1995.
Depth - The distance in $\mathrm{ft}$ from the top-of-casing measuring point to the static water level.
Elev. - The groundwater elevation in $\mathrm{ft}$ above mean sea level. H-Ft - The cakculated drop in water-level from the April to the October measuring events. Negative results show a rise in water level.
No information available.




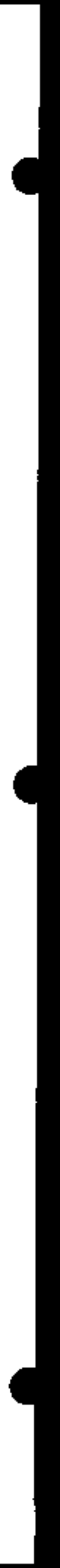


APFENDDXH

Grosindweter Elevations, 1995

\begin{tabular}{|c|c|c|c|c|c|c|c|c|}
\hline \multirow{2}{*}{$\begin{array}{c}\text { Sumpling } \\
\text { P. }\end{array}$} & \multirow[b]{2}{*}{ Locution } & \multirow{2}{*}{$\begin{array}{l}\text { Sampled } \\
\text { in } 95\end{array}$} & \multirow{2}{*}{$\begin{array}{l}\text { CIP } \\
\text { Elevt }\end{array}$} & \multicolumn{2}{|c|}{ Aptil } & \multicolumn{2}{|c|}{$O x=0$ ber } & \multirow{2}{*}{$\begin{array}{l}+* \\
\mathrm{Ft} .\end{array}$} \\
\hline & & & & $D=p h$ & Elev. & Deph & Elev, & \\
\hline
\end{tabular}

Woter Table Interval

\begin{tabular}{|c|c|c|c|c|c|c|c|c|}
\hline 1082 & ORSF & & 789.44 & 25,4 & 811.88 & 25.6 & 811.68 & 02 \\
\hline 1090 & uNCS & $\mathbf{Y}$ & 1101.58 & 50.3 & 1053.58 & 60.6 & 1043.28 & 10.3 \\
\hline 1096 & CRSDB & & 983.44 & 65.7 & 982.04 & 68.7 & $9 n 9.04$ & 3.0 \\
\hline $6 W-145$ & KHQ & $\mathbf{Y}$ & 739.29 & 3.6 & 836.44 & 64 & B33.64 & 28 \\
\hline GW-160 & CRBAWP & $\mathbf{Y}$ & 870.66 & 146.2 & 946.89 & 1533 & 939.79 & 7.1 \\
\hline GW-179 & CRSP & & 101283 & 115.9 & 101210 & 1160 & 1012.00 & 0.1 \\
\hline GW-241 & CRSDB & $\mathbf{Y}$ & 890.30 & 44.0 & 938.64 & 34.1 & 928.54 & 30.1 \\
\hline GW-512 & ADB & $\mathbf{Y}$ & 944.49 & 19.8 & 981.74 & 31.4 & 970.14 & 11.6 \\
\hline CW-542 & CDLV & $\mathbf{Y}$ & 981.28 & 69.4 & 98220 & 70.7 & 980.90 & 1.3 \\
\hline GW-546 & CDLVI & $\mathbf{Y}$ & 994.34 & 79.3 & 99291 & 85.3 & 926.91 & 6.0 \\
\hline GW-55T & LY & $\mathbf{Y}$ & 953.18 & 117.0 & 964.16 & 1225 & 958.66 & 5.5 \\
\hline GDW-558 & SSCR & & 907.31 & 47.4 & 936.57 & 52.9 & 931.07 & 5.5 \\
\hline GW-559 & SSCR & & 927.20 & 134.7 & 968.09 & 141.4 & $\$ 61.39$ & 6.7 \\
\hline GW-560 & CDLVII & $\mathbf{Y}$ & 879.07 & 28.7 & 910.22 & 36.8 & 902.12 & 8.1 \\
\hline GW-562 & CDLVII & $\mathbf{Y}$ & 883.86 & 3.2 & 931.29 & 10.6 & 923.85 & 7.4 \\
\hline GW-564 & CDLVII & $\mathbf{Y}$ & 868.62 & 10.1 & 927.67 & 115 & 926.27 & 1.4 \\
\hline aw-674 & $A D B$ & & 868.48 & 7.2 & 876.59 & 9.7 & 874.05 & 25 \\
\hline GW-676 & ADB & & 832.02 & 3.8 & 84270 & 6.2 & 840.30 & 24 \\
\hline
\end{tabular}

Bectoct Interval

\begin{tabular}{|c|c|c|c|c|c|c|c|c|}
\hline 1084 & ORSF & & 823.74 & 61.4 & 904.00 & 62.3 & 903.10 & 0.9 \\
\hline GW-14] & L.JV & $\mathbf{Y}$ & 1034.95 & 94.4 & 1091.65 & 98.6 & 1967,46 & 4.2 \\
\hline OW.142 & KHQ & $\mathbf{Y}$ & 696.54 & 133.3 & 837.05 & 136.2 & 834.15 & 29 \\
\hline GW-143 & KHQ & $\mathbf{Y}$ & 682.04 & 77.6 & 835.58 & 70.9 & 842.28 & -6.7 \\
\hline GW-144 & KHQ & $\mathbf{Y}$ & 738.98 & 78.2 & 835.14 & 80.4 & 832.94 & 2.2 \\
\hline$G W-146$ & KHQ & $\mathbf{Y}$ & 632.00 & 113.9 & 724.26 & 82.5 & 755.66 & $-31 . A$ \\
\hline OW-147 & KHQ & $\mathbf{Y}$ & 787.91 & 14.8 & 836.82 & 17.8 & 833.82 & 3.0 \\
\hline GW-156 & CRSDE & $\mathbf{Y}$ & 895,44 & 142.8 & 906.33 & 1434 & 905.73 & 0.6 \\
\hline$G W \cdot 158$ & CRSDB & $\mathbf{Y}$ & 58274 & 44.9 & 938.15 & 49.4 & 933.65 & 45 \\
\hline GW-159 & CRSDE & $\mathbf{Y}$ & 897.29 & 1163 & 934.65 & 128.7 & 922.45 & 12.2 \\
\hline $0 w-161$ & CRBAWP & $\mathbf{Y}$ & 715.91 & 159.1 & 934.44 & 162.8 & 930.74 & 3.7 \\
\hline GW-165 & CRDT & & 789.89 & 83.7 & 1007.67 & 105.4 & 985.97 & 21.7 \\
\hline$G W-166$ & CRDT & & 709.51 & 86.2 & 1007.09 & 107.5 & 985.79 & 21.3 \\
\hline Gw:-173 & CRsP & & 953.47 & 1432. & 971.80 & . & . & . \\
\hline GW-174 & CRSP & & 97456 & 111.2 & 1005.32 & 123.3 & 993.22 & 121 \\
\hline GW.175 & CRSP & $\mathbf{Y}$ & 924.39 & 116.3 & 967.70 & 126.8 & 957.20 & 105 \\
\hline GW-136 & CRSP & & 982.63 & 116.1 & 1009.77 & 117.2 & 1008.9 & 1.1 \\
\hline
\end{tabular}


APPENDLX $\mathrm{H}$

Groundwater Evevations, 1995

\begin{tabular}{|c|c|c|c|c|c|c|c|c|}
\hline \multirow{2}{*}{$\begin{array}{c}\text { Sampling } \\
\mathrm{P} \text {. }\end{array}$} & \multirow[b]{2}{*}{ Location } & \multirow{2}{*}{$\begin{array}{l}\text { Sannjiod } \\
\text { in } 95\end{array}$} & \multirow{2}{*}{$\begin{array}{l}\text { CTP } \\
\text { Elev. }\end{array}$} & \multicolumn{2}{|c|}{ Apt } & \multicolumn{2}{|c|}{ Octobst } & \multirow{2}{*}{$\begin{array}{l}+ \\
\mathbf{P}\end{array}$} \\
\hline & & & & Depth & Elev. & Depth & Elev. & \\
\hline ow-17 & CRSP & $\mathbf{Y}$ & 1017.02 & 107,0 & 1050.95 & 119.9 & 1038.05 & 12.9 \\
\hline OW-178 & CRSP & & 1015.81 & 89.9 & 1053.59 & 98.9 & 1044.59 & 9.0 \\
\hline GW-180 & CRSP & & 966.43 & 104.3 & 999.67 & 130.9 & 973.07 & 26.6 \\
\hline$O W-184$ & $\mathbf{R Q}$ & $\mathbf{Y}$ & 808.90 & 130.1 & 817.53 & 110.9 & 816.73 & 0.8 \\
\hline$G W+186$ & $\mathrm{RQ}$ & $\mathrm{Y}$ & 671.81 & 14.6 & 816.72 & 14.9 & 816.42 & 0.3 \\
\hline 6W-293 & UNCS & $\mathbf{Y}$ & 95234 & 74.5 & 1030.76 & 83.9 & 1021,36 & 9,4 \\
\hline GW-205 & UNCS & $\mathbf{Y}$ & 942.46 & 72.3 & 1031.67 & 81.3 & 1022.67 & 9.0 \\
\hline 6w-217 & LV & $\mathbf{Y}$ & $1001.6 \%$ & 108.9 & 1097.96 & 118.7 & 1058.16 & 9.8 \\
\hline$G W \cdot 221$ & UNCS & $\mathbf{Y}$ & 950.86 & 76.2 & 1029.80 & 85.4 & 1020.60 & 9.2 \\
\hline GW-231 & $\mathrm{KHQ}$ & $\mathbf{Y}$ & 818.00 & 12.7 & 836,7 & 15.7 & 833.7 & 3.0 \\
\hline $0 w-292$ & ECRWP & $\mathbf{Y}$ & 891.06 & 112.0 & 961.00 & 116.4 & 956.60 & 4,4 \\
\hline GW-299 & ECRWP & $\mathbf{Y}$ & 856.20 & I14.4 & 949.50 & 118.9 & 945.00 & 4.5 \\
\hline$\sigma w-29$ & CRBAWP & $\mathbf{Y}$ & 865.85 & 107.8 & 941.21 & 110.8 & 938.21 & 3.0 \\
\hline GW/299 & CKBAWP & $\mathbf{Y}$ & 890.83 & 953 & 958.56 & 1032 & 950.66 & 7.9 \\
\hline GW-300 & CRBAWP & $\mathbf{Y}$ & 931.19 & 107.9 & 965.22 & 117.6 & 955.52 & 9.7 \\
\hline $0 w-301$ & CRBAWP & $\mathbf{Y}$ & 927.94 & 129.1 & 957.28 & 135.9 & 950.48 & 6.8 \\
\hline GW-302 & UNCS & $\mathbf{Y}$ & 1011,44 & 98,5 & 1043.17 & 108.1 & 1033.57 & 9.6 \\
\hline 6W-303 & CRSDB & $\mathbf{Y}$ & 693.94 & 86.8 & 920.36 & 89.4 & 917.76 & 26 \\
\hline $6 w-304$ & CRSLB & $\mathbf{Y}$ & 885.07 & 116.7 & 928.79 & 118.6 & 926.89 & $1+9$ \\
\hline GW-305 & LV & $\mathbf{Y}$ & 2008,62 & 120.4 & 1063.15 & 128.4 & 1055.15 & 8.0 \\
\hline GW-321 & ADB & $\mathbf{Y}$ & 831.80 & 18.0 & 907.58 & 22.7 & 90288 & 4.7 \\
\hline GW-322 & CRSP & & 971.31 & 149.7 & 984.55 & 165.9 & 968.35 & 16.2 \\
\hline GW-339 & UNCS & $\mathbf{Y}$ & 1014.68 & 70.9 & 1053.69 & 81.3 & 1043.29 & 10.4 \\
\hline GW-511 & CRSP & $\mathbf{Y}$ & 943.85 & 100.6 & 99261 & 114.1 & 979.11 & 13.5 \\
\hline$G W-514$ & $\mathrm{ADB}$ & $\mathbf{Y}$ & 814.16 & 19.8 & $\$ 1,42$ & 31.9 & 969.32 & 12.1 \\
\hline $0 W-521$ & uv & $\mathbf{Y}$ & 1049.86 & 81.6 & 1101.08 & 88.9 & 1093.78 & 3.3 \\
\hline OW-522 & LIV & $\mathbf{Y}$ & 982.89 & 92.2 & 3083.11 & 102.6 & 1072.71 & 10.4 \\
\hline GW-539 & 뉴 & $\mathbf{Y}$ & 944.19 & 100.4 & $\$ 92.60$ & 109.5 & $983 \leq 0$ & 9.1 \\
\hline OW.\$40 & CDLVI & $\mathbf{Y}$ & 904.38 & 79.3 & 992.82 & 85.2 & 986.92 & 5.9 \\
\hline GW-541 & CDLVI & $y$ & 950.19 & 65.2 & 993.20 & 65.6 & 992.80 & 0.4 \\
\hline GW-\$43 & CDLVI & $\mathbf{Y}$ & 936.29 & 63.2 & 960.60 & 64.1 & 959.70 & 0.9 \\
\hline OW-544 & CDLVI & $\mathbf{Y}$ & $\$ 42.38$ & 63.9 & 981.09 & 68.3 & 976.69 & 4.4 \\
\hline$G W-608$ & CRSP & $\mathbf{Y}$ & 887,00 & 131.8 & 942.15 & 138.4 & 935.55 & 6.6 \\
\hline GW-609 & CRSP & $\mathbf{Y}$ & 847.00 & 168.0 & 944.11 & 173.2 & 938,91 & 5.2 \\
\hline $9 W-610$ & CRsP & $\mathbf{Y}$ & $945 \leq 3$ & 81,4 & 978.04 & 93.7 & 965.74 & 12.3 \\
\hline$G W-611$ & CRSP & $\mathbf{Y}$ & 933.88 & 86.6 & 961.78 & 130.1 & 938.28 & 23.5 \\
\hline$G w-612$ & CRSP & & 884.15 & 121.1 & 1009.93 & 131.3 & 999.73 & 10.2 \\
\hline GW-673 & $A D B$ & & 757,70 & 8.2 & 873.81 & 10.9 & 871.11 & 2.7 \\
\hline$G W-67$ & $A D B$ & & 873.30 & 24.9 & 1005.50 & 32.7 & 997.70 & 7.8 \\
\hline GW-679 & ADB & & 899.70 & 47.8 & 979.10 & $\$ 9.0$ & $\$ 67.90$ & 11.2 \\
\hline
\end{tabular}


APPENDLX H

Groumtwater Eevutiors, 1995

\begin{tabular}{|c|c|c|c|c|c|c|c|c|}
\hline \multirow{2}{*}{$\begin{array}{c}\text { Surpling } \\
\text { Pt. }\end{array}$} & \multirow[b]{2}{*}{ Locoticon } & \multirow{2}{*}{$\begin{array}{l}\text { Sumpled } \\
\text { in } 95 \\
\end{array}$} & \multirow{2}{*}{$\begin{array}{l}\text { CTP } \\
\text { Eivev. }\end{array}$} & \multicolumn{2}{|c|}{ Afril } & \multicolumn{2}{|c|}{ October } & \multirow{2}{*}{$\begin{array}{l}4 \\
\mathrm{~F} .\end{array}$} \\
\hline & & & & Degph & Elev, & Depdr & Eky. & \\
\hline$G W-709$ & LII & $\mathbf{Y}$ & 829.19 & 29.5 & 877.10 & 29.7 & 876.90 & 0.2 \\
\hline GW-73! & CRSDB & $\mathbf{Y}$ & 874.40 & 124.2 & 924.98 & 125.2 & 923.98 & 1.0 \\
\hline $0 w-732$ & CRSDB & $\mathbf{Y}$ & 876.50 & 157.4 & 906.69 & 1578 & 906.29 & 0.4 \\
\hline GW-743 & CRSP & $\mathbf{Y}$ & 943.12 & 120.4 & 979.96 & . & . & . \\
\hline GW.757 & tit & $\mathbf{Y}$ & 808.30 & 84,4 & 877.03 & 84.5 & 876.93 & 0.1 \\
\hline $0 \$-796$ & $2 V$ & $\mathbf{Y}$ & 919.10 & 69.8 & 982.62 & 84.6 & 967.82 & 14.8 \\
\hline GW-797 & LV & $\mathbf{Y}$ & 930.05 & 65.4 & 994.40 & 70.7 & 989.10 & 5.3 \\
\hline CW-798 & CDLVII & $\mathbf{Y}$ & 857.90 & 73.8 & 926.50 & 84.2 & 916.10 & 10.4 \\
\hline GW-799 & CDLVII & $\mathbf{Y}$ & 892.75 & 8.7 & 972.39 & 17,3 & 963.79 & 8.6 \\
\hline GW-801 & $\mathbf{L V}$ & $\mathbf{Y}$ & 911.30 & 101.9 & 995.46 & 112.9 & 984.06 & 11.0 \\
\hline CW-827 & CDLVI & $\mathbf{Y}$ & 919.03 & 39.8 & 1011.59 & 44,4 & 1006,99 & 4.6 \\
\hline
\end{tabular}




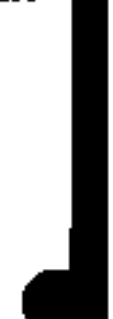


APPENDIX I

ION-CHARGE BALANCE CALCULATIONS 



\section{EXPLANATION}

SAMPLING POINT:

$\begin{array}{rll}\text { GW } & - & \text { Monitoring Well } \\ \text { CBS-1 } & - & \text { Chestnut Branch Spring } 1 \\ \text { SCR2.2SP } & - & \text { South Chestunt Ridge Spring 2.2 } \\ 1090 & - & \text { Historical 1000-Series Monitoring Well }\end{array}$

\section{LOCATION:}

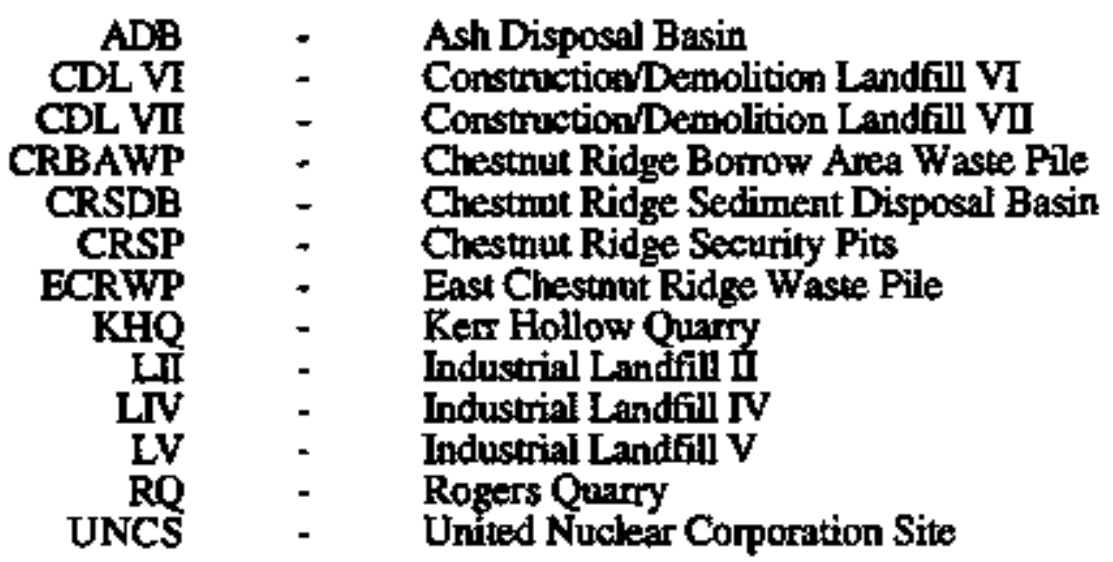

NOTES:

$\begin{array}{ccl}\text { mgll } & - & \text { milligrams per liter } \\ \text { mogl } & - & \text { milliequivalents per liter } \\ \text { NTU } & - & \text { Nephelometric Turbidity Units } \\ \text { TDS } & - & \text { Total Dissolved Solids } \\ \text { TSS } & - & \text { Total Suspended Solids }\end{array}$

Bicarbonate and carbonate alkalizities (reported as $\mathrm{CaCO}_{3}$ ) were converted to $\mathrm{HCO}_{3}$ and $\mathrm{CO}_{3}$.

Charge balances for each sample were evaluated by computing a relative percent difference (RPD) between the summed milliequivalent concentrations of the principal cations and anions using the following formula:

$$
R P D=\left(\frac{\sum \text { cations }-\sum \text { anions }}{\sum \text { cations }+\sum \text { anions }}\right) \times 100
$$

Absolute Value of the RPD calculated for the following samples exceeded $10 \%$

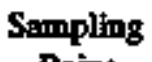

Point

GW-205

GW-732

GW.732

GW-732
Loctico

UNCS

CRSDB

CRSDB

CRSDB
Date

Sompled

$04 / 18495$

$10 \times 2495$

$10 / 25 / 95$

102695
RPD

$-11$

$-57$

$-39$

$-13$ 


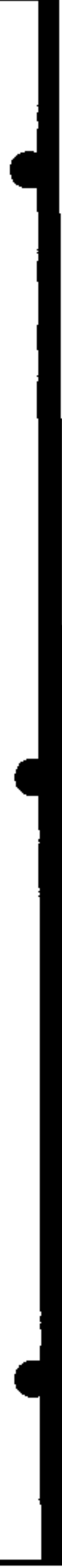


APPENDIX 1

Jon-Charge Balance talculattons, 1995

\begin{tabular}{|c|c|c|c|c|c|c|c|c|c|c|}
\hline $\begin{array}{l}\text { sempling Polnt } \\
\text { Location } \\
\text { Dute supled }\end{array}$ & $\begin{array}{r}1090 \\
\text { vincs } \\
0420 / 95\end{array}$ & $\begin{array}{r}1090 \\
\text { 1Nts } \\
10 / 09 / 95\end{array}$ & $\begin{array}{r}\text { ces:1 } \\
L V \\
0,17 / \%\end{array}$ & $\begin{array}{r}\text { cos+1 } \\
\text { Ly } \\
10 / 11 / 95\end{array}$ & $\begin{array}{r}6+141 \\
114 \\
01 / 0 \% / 55\end{array}$ & $\begin{array}{r}61+141 \\
\text { LIV } \\
07 / 10 / 55\end{array}$ & $\begin{array}{r}6+142 \\
\times 1 \% \\
01 / 10 / 45\end{array}$ & $\begin{array}{r}04+142 \\
\text { KHa } \\
04 / 20 / \% 5\end{array}$ & $\begin{array}{r}c W \cdot 142 \\
k 110 \\
07 / 94 / 45\end{array}$ & $\begin{array}{r}4+142 \\
\times 110 \\
11 / 05 / 95\end{array}$ \\
\hline CATJOAS (WEO/L & & & & & & & & & & \\
\hline 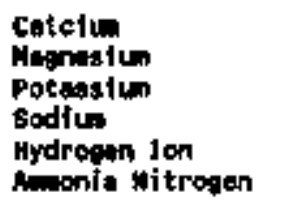 & $\begin{array}{l}2.74 \\
2.55 \\
0.03 \\
0.45 \\
0.00 \\
.\end{array}$ & $\begin{array}{l}2.64 \\
2.63 \\
0.03 \\
0.46 \\
0.00 \\
\end{array}$ & $\begin{array}{l}1.45 \\
0.90 \\
0.05 \\
0.07 \\
0.00 \\
0.00\end{array}$ & $\begin{array}{l}1.80 \\
0.99 \\
0.04 \\
0.1 \% \\
0.00 \\
0.00\end{array}$ & $\begin{array}{l}2.40 \\
2.30 \\
0.02 \\
0.04 \\
0.00\end{array}$ & $\begin{array}{r}2.40 \\
2.55 \\
0.00 \\
0.04 \\
0.00 \\
.\end{array}$ & $\begin{array}{l}2.15 \\
2.39 \\
0.08 \\
0.04 \\
0.09 \\
.\end{array}$ & $\begin{array}{l}2.20 \\
2.55 \\
0.10 \\
0.04 \\
0.00 \\
.\end{array}$ & $\begin{array}{l}2.25 \\
2.55 \\
0.11 \\
0.05 \\
0.00 \\
.\end{array}$ & $\begin{array}{r}2.00 \\
2.47 \\
0.10 \\
0.04 \\
0.00 \\
.\end{array}$ \\
\hline Fotal catione & 5. 的 & 5.78 & 2.53 & 2.94 & 4.76 & 4.99 & 4.66 & 6.89 & 4.76 & 4.61 \\
\hline Ak10us \{mea/t\} & & & & & & & & & & \\
\hline 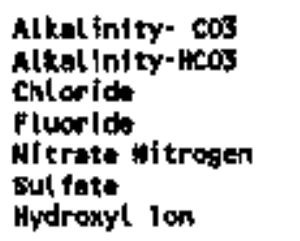 & $\begin{array}{l}0.00 \\
5.34 \\
0.65 \\
0.00 \\
0.07 \\
0.11 \\
0.00\end{array}$ & $\begin{array}{l}0.00 \\
5.08 \\
0.56 \\
0.00 \\
0.06 \\
0.08 \\
0.00\end{array}$ & $\begin{array}{l}0.00 \\
2.48 \\
0.11 \\
0.00 \\
0.05 \\
0.12 \\
0.00\end{array}$ & $\begin{array}{l}0.00 \\
2.52 \\
0.15 \\
0.01 \\
0.05 \\
0.27 \\
0.01\end{array}$ & $\begin{array}{l}0.00 \\
4.68 \\
0.07 \\
0.00 \\
0.06 \\
0.05 \\
0.00\end{array}$ & $\begin{array}{l}0.00 \\
4.64 \\
0.07 \\
0.00 \\
0.05 \\
0.04 \\
0.00\end{array}$ & $\begin{array}{l}0.00 \\
4.70 \\
0.06 \\
0.03 \\
0.09 \\
0.11 \\
0.00\end{array}$ & $\begin{array}{l}0.00 \\
4.84 \\
0.07 \\
0.03 \\
0.00 \\
0.12 \\
0.00\end{array}$ & $\begin{array}{l}0.00 \\
4.72 \\
0.06 \\
0.03 \\
0.04 \\
0.12 \\
0.00\end{array}$ & $\begin{array}{l}0.00 \\
4.68 \\
0.05 \\
0.05 \\
0.00 \\
0.11 \\
0.00\end{array}$ \\
\hline Totat Anions & 6.17 & 5.79 & 2.74 & 2.99 & 4.86 & 4.79 & 4.99 & 5.06 & 4.77 & 6.87 \\
\hline Chane balance $(\mathbf{z})$ & -3 & -0 & -4 & -1 & -1 & 2 & -3 & $\cdot 2$ & -0 & -3 \\
\hline $\begin{array}{ll}\text { Tos } & (\mathrm{ng} / \mathrm{L}) \\
\text { TSS } & \text { (ng/l) } \\
\text { turteldlty } & \text { (nTU) }\end{array}$ & $\begin{array}{r}322 \\
3 \\
3.7\end{array}$ & $\begin{array}{r}2.8 \\
1 \\
0.35\end{array}$ & $\begin{array}{l}152 \\
2.5 \\
2.5\end{array}$ & $\begin{array}{r}160 \\
4 \\
10\end{array}$ & $\begin{array}{r}260 \\
18 \\
55\end{array}$ & $\begin{array}{r}250 \\
4.5 \\
30\end{array}$ & $\begin{array}{r}180 \\
8 \\
27\end{array}$ & $\begin{array}{r}268 \\
3.8 \\
34\end{array}$ & $\begin{array}{r}208 \\
9 \\
40\end{array}$ & $\begin{array}{r}226 \\
5 \\
35\end{array}$ \\
\hline
\end{tabular}


APPEADIX I

Ion-Charge Balance calculatlons, 1995

sempl ing Point
Locatton
odt sempled

CATIONS (MEO/L)

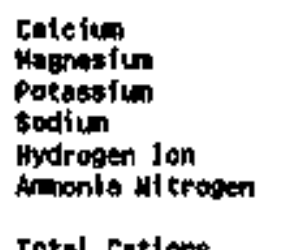

Total Catlens

winows (VEO/L)

Alkal Inity- $\cos$

Alkal Inity-nces

chloride

Witrate Hitrogen

sulfate

Hydronyl Iom

Toted Antone

Ctarke Bunice (x)

Tos (noth)

TSS (me/L)
GN-143

KHe
ou/17/45

$64+143$
KHa
$04 / 24 / 95$

$0 N+143$
$k H 4$
$07 / 16 / 55$

64. 143

11/00/9s

sul 144
K104
$01 /$ totos

$4+144$
$k+19$
$04 / 25 / 55$

KN-144
$07 / 96 / 48$

GW- 144
KMG

$11+4 / 95$

$\begin{array}{rrr}1.50 & 1.45 & 1.40 \\ 2.30 & 1.69 & 2.06 \\ 0.41 & 0.43 & 0.49 \\ 1.04 & 1.17 & 1.26 \\ 0.00 & 0.00 & 0.00 \\ . & . & . \\ 5.25 & 4.95 & 5.40\end{array}$

1.40
1.89
0.45
1.35
0.00
-
4.85

2.10

1.40

0.04

0.05

0.00

4.85

3.58

0.00
4.06

4.05

0.19

0.00

0.71

0.00

0.00

4.14

0.17

0.17

0.00

0.73

0.00

4.09

0.17

0.14

0.00

0.75

5.13

5.20

$-2$

5,12

5

278286

7
22

0.00
4.20
0.15
0.16
0.00
0.65
0.00
5.14
-3
266
7.

0.00
4.20
0.15
0.16
0.00
0.65
0.00
5.14
.3
266
$\times 1$
7.5

0.00

3.02

0.06

0.61

0.11

0.11

0.00

3.32

4

104

0.1

$\begin{array}{rrr}1.95 & 1.95 & 2.05 \\ 1.15 & 1.25 & 1.32 \\ 0.05 & 0.04 & 0.04 \\ 0.06 & 0.06 & 0.05 \\ 0.00 & 0.00 & 0.00 \\ . & . & . \\ 3.20 & 3.27 & 3.45\end{array}$

0.00

3.02

0.00

0.01

0.12

0.00

3.36

$-2$

166

41
0.4
0.00

3,30
0,07

0.00

0.18

0.10

0.01

3.65

$-3$

108
01
0.00

$$
3.10 .
$$

0.07

0.01

0.16

0.11

0.00

3.44

$-2$

16

0.25 
apperdix I

Ion-Charges eslance Calulations, $19 \%$

\begin{tabular}{|c|c|c|c|c|c|c|c|c|}
\hline $\begin{array}{l}\text { sempl ing Foint } \\
\text { Lotention } \\
\text { Date sapled }\end{array}$ & $\begin{array}{r}64.145 \\
k+16 \\
01 / 18 / 95\end{array}$ & $\begin{array}{r}\text { Cw- 145 } \\
\text { KH10 } \\
04 / 26 / 95\end{array}$ & $\begin{array}{r}\text { cw-t45 } \\
k \mathrm{kHa} \\
07 / 17 / 95\end{array}$ & $\begin{array}{r}\text { an-145 } \\
\text { K19 } \\
11 / 15 / 99\end{array}$ & $\begin{array}{r}a N-146 \\
k+0 \\
01 / 17 / 65\end{array}$ & $\begin{array}{r}w-146 \\
K 10 \\
04 / 24 / 95\end{array}$ & $\begin{array}{r}6+164 \\
\text { KHQ } \\
07 / 17 / 95\end{array}$ & $\begin{array}{r}04-146 \\
k 116 \\
11 / 05 / 95\end{array}$ \\
\hline CATIOWS (HEa/L & & & & & & & & \\
\hline 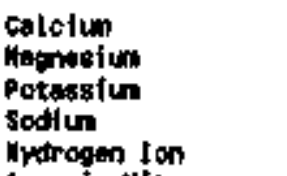 & $\begin{array}{l}2.20 \\
3.65 \\
0.31 \\
0.20 \\
0.00\end{array}$ & $\begin{array}{l}2.10 \\
2.80 \\
0.31 \\
0.26 \\
0.00\end{array}$ & $\begin{array}{l}2.25 \\
2.96 \\
0.35 \\
0.26 \\
0.00\end{array}$ & $\begin{array}{l}2.10 \\
2.86 \\
0.20 \\
0.25 \\
0.00\end{array}$ & $\begin{array}{l}1.90 \\
3.13 \\
0.35 \\
0.48 \\
0.00\end{array}$ & $\begin{array}{l}1.90 \\
2.30 \\
0.31 \\
0.40 \\
0.00\end{array}$ & $\begin{array}{l}2.10 \\
2.89 \\
0.46 \\
0.61 \\
0.00\end{array}$ & $\begin{array}{l}1.60 \\
2.63 \\
0.39 \\
0.52 \\
0.00\end{array}$ \\
\hline mmonts Nttrogen & • & • & - & . & $\cdot$ & . & . & $\cdot$ \\
\hline Totel Cuttona & 6.24 & 5.46 & $5.7 t$ & 5.51 & 5.80 & 4.98 & 6.04 & 5.33 \\
\hline$(0 \mathrm{EO} / \mathrm{L})$ & & & & & & & & \\
\hline 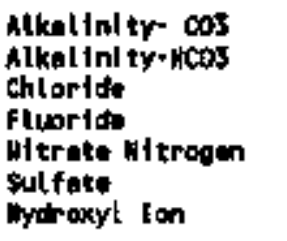 & $\begin{array}{l}0.00 \\
4.38 \\
0.25 \\
0.15 \\
0.03 \\
0.75 \\
0.00\end{array}$ & $\begin{array}{l}0.00 \\
4.64 \\
0.27 \\
0.15 \\
0.02 \\
0.77 \\
0.00\end{array}$ & $\begin{array}{l}0.00 \\
4.34 \\
0.27 \\
0.12 \\
0.07 \\
0.77 \\
0.00\end{array}$ & $\begin{array}{l}0.00 \\
4.70 \\
0.24 \\
0.13 \\
0.03 \\
0.20 \\
0.00\end{array}$ & $\begin{array}{l}0.00 \\
3.94 \\
0.31 \\
0.16 \\
0.05 \\
1.37 \\
0.00\end{array}$ & $\begin{array}{l}0.00 \\
3.96 \\
0.31 \\
0.15 \\
0.04 \\
1.46 \\
0.00\end{array}$ & $\begin{array}{l}0.00 \\
3.92 \\
0.34 \\
0.14 \\
0.07 \\
1.40 \\
0.00\end{array}$ & $\begin{array}{l}0.00 \\
4.18 \\
0.32 \\
0.14 \\
0.00 \\
1.36 \\
0.00\end{array}$ \\
\hline Total Anlone & 5.76 & 5.83 & 5.76 & 5.89 & 5.83 & 5.91 & 5.86 & 5.50 \\
\hline CWARAE OLLWCE (X) & 4 & -3 & 0 & -3 & 0 & $-\theta$ & 2 & -6 \\
\hline $\begin{array}{ll}\text { TDS } & (n, L) \\
\text { TSS } & (n g / k) \\
\text { Turbidity } & \text { (nTU) }\end{array}$ & $\begin{array}{r}226 \\
5.2\end{array}$ & $\begin{array}{r}276 \\
27 \\
4.2\end{array}$ & $\begin{array}{r}310 \\
2.5 \\
6\end{array}$ & $\begin{array}{r}164 \\
17 \\
20\end{array}$ & $\begin{array}{r}269 \\
2 \\
6\end{array}$ & $\begin{array}{r}342 \\
4 \\
6.2\end{array}$ & $\begin{array}{r}344 \\
7.6\end{array}$ & $\begin{array}{l}310 \\
9.1 \\
9.6\end{array}$ \\
\hline
\end{tabular}


ApPEIOIX I

ton-charge belance Coleulatione, 1995

\begin{tabular}{|c|c|c|c|c|c|c|c|c|}
\hline $\begin{array}{l}\text { Senpl ind Point } \\
\text { locat ion } \\
\text { Date sampled }\end{array}$ & $\begin{array}{r}64-147 \\
146 \\
01 / 15 / 95\end{array}$ & $\begin{array}{r}0,-147 \\
04 / 21 / 65\end{array}$ & $\begin{array}{r}04-147 \\
1040 \\
07 / 4455\end{array}$ & $\begin{array}{r}0 w+147 \\
\times 119 \\
11 / 06 / 95\end{array}$ & $\begin{array}{r}\text { Gi-156 } \\
\text { chest5 } \\
01 / 18 / 95\end{array}$ & $\begin{array}{r}\text { CH-154 } \\
\text { Ck\$5D } \\
04 / 47 / 95\end{array}$ & $\begin{array}{r}\text { an-156 } \\
\text { chisti } \\
07 / 13 / 95\end{array}$ & 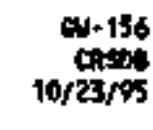 \\
\hline \multicolumn{9}{|l|}{ CATION8 (NEQ/L) } \\
\hline 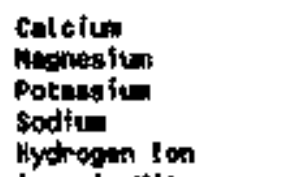 & $\begin{array}{l}1.85 \\
1.65 \\
0.14 \\
0.13 \\
0.00\end{array}$ & $\begin{array}{l}1.65 \\
1.65 \\
0.14 \\
0.17 \\
0.00\end{array}$ & $\begin{array}{l}1.70 \\
1.56 \\
0.16 \\
0.13 \\
0.00\end{array}$ & $\begin{array}{l}1.65 \\
1.56 \\
0.06 \\
0.10 \\
0.00\end{array}$ & $\begin{array}{l}3.64 \\
3.45 \\
0.10 \\
0.16 \\
0.00\end{array}$ & $\begin{array}{l}3.64 \\
3.37 \\
0.19 \\
0.24 \\
0.00\end{array}$ & $\begin{array}{l}3.59 \\
3.54 \\
0.12 \\
0.14 \\
0.00\end{array}$ & $\begin{array}{l}3.49 \\
3.54 \\
0.09 \\
0.11 \\
0.00\end{array}$ \\
\hline mimonie Mitrogen & - & . & , & $\cdot$ & $\cdot$ & - & - & - \\
\hline Total estions & 3.76 & 3.61 & 3.56 & 3.37 & 7.36 & 7.24 & 7.39 & 7.24 \\
\hline \multicolumn{9}{|l|}{ Nintows (meorls) } \\
\hline 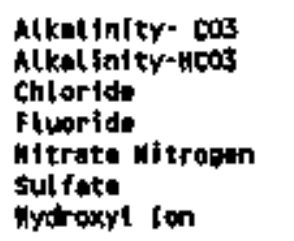 & $\begin{array}{l}0.00 \\
3.44 \\
0.07 \\
0.02 \\
0.09 \\
0.09 \\
0.00\end{array}$ & $\begin{array}{l}0.00 \\
3.38 \\
0.07 \\
0.04 \\
0.08 \\
0.11 \\
0.00\end{array}$ & $\begin{array}{l}0.00 \\
3.16 \\
0.07 \\
0.01 \\
0.11 \\
0.09 \\
0.00\end{array}$ & $\begin{array}{l}0.00 \\
3.28 \\
0.06 \\
0.01 \\
0.10 \\
0.07 \\
0.00\end{array}$ & $\begin{array}{l}0.00 \\
6.03 \\
0.06 \\
0.00 \\
0.04 \\
0.21 \\
0.00\end{array}$ & $\begin{array}{l}0.00 \\
7.05 \\
0.00 \\
0.00 \\
0.05 \\
0.19 \\
0.00\end{array}$ & $\begin{array}{l}0.00 \\
6.03 \\
0.08 \\
0.00 \\
0.04 \\
0.14 \\
0.00\end{array}$ & $\begin{array}{l}0.00 \\
6.85 \\
0.08 \\
0.00 \\
0.05 \\
0.15 \\
0.05\end{array}$ \\
\hline Total Antons & 3.70 & 3.68 & 3.44 & 3.52 & 7.16 & 7.34 & 7.10 & 7.09 \\
\hline CHALGE BALAHCE ( $(H)$ & 1 & +1 & 2 & -2 & 1 & -1 & 2 & 1 \\
\hline $\begin{array}{ll}\text { Fos } & (\mathrm{g} / \mathrm{L}) \\
\text { TSs } & (\mathrm{g} / \mathrm{L}) \\
\text { Turbititity } & \text { (ntu) }\end{array}$ & $\begin{array}{r}164 \\
4 \\
4.5\end{array}$ & $\begin{array}{r}202 \\
19 \\
17\end{array}$ & $\begin{array}{r}192 \\
5 \\
3.9\end{array}$ & $\begin{array}{l}168 \\
1.5 \\
3.2\end{array}$ & $\begin{array}{r}266 \\
10 \\
5.3\end{array}$ & $\begin{array}{r}300 \\
4 \\
3.8\end{array}$ & $\begin{array}{l}358 \\
1.5 \\
1.5\end{array}$ & $\begin{array}{r}304 \\
\uparrow 6 \\
21\end{array}$ \\
\hline
\end{tabular}


APPENOEX :

1em-charse solence Calcutations, 1995

\begin{tabular}{|c|c|c|c|c|c|c|c|c|c|c|}
\hline $\begin{array}{l}\text { sampl in Point } \\
\text { Locettion } \\
\text { Date Senpled }\end{array}$ & $\begin{array}{r}\text { an-156 } \\
\text { chsog } \\
10 / 24 / 95\end{array}$ & $\begin{array}{r}\text { CH-156 } \\
\text { caspB } \\
10 / 25 / \% 5\end{array}$ & $\begin{array}{r}a+156 \\
\text { CRs08 } \\
10 / 26 / 95\end{array}$ & $\begin{array}{r}\text { aH-15日 } \\
\text { Crsps } \\
01 / 20 / 85\end{array}$ & $\begin{array}{r}\text { SH-158 } \\
\text { CRSDB } \\
04 / 13 / 95\end{array}$ & 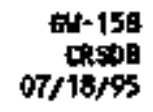 & $\begin{array}{r}\text { cut-159 } \\
\text { tansos } \\
01 / 15 / 95\end{array}$ & $\begin{array}{r}\text { th-159 } \\
\text { ckeps } \\
0,06 / 065\end{array}$ & $\begin{array}{r}\text { Cy-159 } \\
\text { CRSD: } \\
07 / 12 / 95\end{array}$ & $\begin{array}{r}\text { ON-159 } \\
\text { CR848 } \\
10 / 23 / 95\end{array}$ \\
\hline CATIONS (NEO/L) & & & & & & & & & & \\
\hline 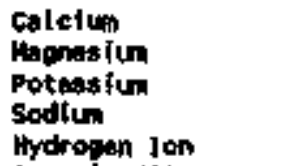 & $\begin{array}{l}3.44 \\
3.45 \\
0.08 \\
0.08 \\
0.00\end{array}$ & $\begin{array}{l}3.29 \\
3.45 \\
0.12 \\
0.09 \\
0.00\end{array}$ & $\begin{array}{l}2.94 \\
3.13 \\
0.15 \\
0.09 \\
0.00\end{array}$ & $\begin{array}{l}2.00 \\
1.81 \\
0.02 \\
0.05 \\
0.00\end{array}$ & $\begin{array}{l}1.00 \\
1.65 \\
0.02 \\
0.02 \\
0.00\end{array}$ & $\begin{array}{l}1.95 \\
1.81 \\
0.02 \\
0.03 \\
0.00\end{array}$ & $\begin{array}{l}2.05 \\
2.22 \\
0.02 \\
0.03 \\
0.00\end{array}$ & $\begin{array}{l}1.05 \\
1.01 \\
0.04 \\
0.03 \\
0.00\end{array}$ & $\begin{array}{l}1.95 \\
1.89 \\
0.67 \\
0.06 \\
0.00\end{array}$ & $\begin{array}{l}2.05 \\
2.14 \\
0.06 \\
0.03 \\
0.00\end{array}$ \\
\hline Nemia Mitrogen & $\cdot$ & $\cdot$ & $\cdot$ & $*$ & $\cdot$ & $\cdot$ & $\cdot$ & $\cdot$ & - & * \\
\hline Totel Cotions & 7.00 & 6.96 & 6.30 & 3.96 & 3.49 & 3.81 & $4+31$ & 3.72 & 3.94 & 4.26 \\
\hline wrows (man) & & & & & & & & & & \\
\hline 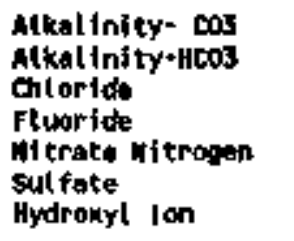 & $\begin{array}{l}0.00 \\
6.47 \\
0.06 \\
0.00 \\
0.03 \\
0.98 \\
0.00\end{array}$ & $\begin{array}{l}0.00 \\
6.71 \\
0.07 \\
0.00 \\
0.08 \\
0.09 \\
0.00\end{array}$ & $\begin{array}{l}0.02 \\
6.77 \\
0.06 \\
0.00 \\
0.05 \\
0.09 \\
0.00\end{array}$ & $\begin{array}{l}0.00 \\
3.50 \\
0.03 \\
0.00 \\
0.00 \\
0.21 \\
0.00\end{array}$ & $\begin{array}{l}0.00 \\
3.50 \\
0.04 \\
0.00 \\
0.00 \\
0.19 \\
0.00\end{array}$ & $\begin{array}{l}0.00 \\
3.44 \\
0.05 \\
0.00 \\
0.00 \\
0.26 \\
0.00\end{array}$ & $\begin{array}{l}0.00 \\
3.60 \\
0.06 \\
0.00 \\
0.05 \\
0.18 \\
0.00\end{array}$ & $\begin{array}{l}0.00 \\
5.90 \\
0.05 \\
0.00 \\
0.05 \\
0.18 \\
0.00\end{array}$ & $\begin{array}{l}0.00 \\
3.76 \\
0.05 \\
0.00 \\
0.03 \\
0.16 \\
0.00\end{array}$ & $\begin{array}{l}0.00 \\
3.68 \\
0.04 \\
0.00 \\
0.05 \\
0.14 \\
0.00\end{array}$ \\
\hline Total unliona & 6.84 & 6.90 & 6.98 & 3.74 & 3.7 & 3.68 & 3.59 & 4.17 & 4.01 & $3,8 t$ \\
\hline chores bulditas (x) & $\mathbf{z}$ & 0 & -5 & 5 & -3 & 2 & $\mathbf{5}$ & -6 & -1 & $\mathbf{s}$ \\
\hline 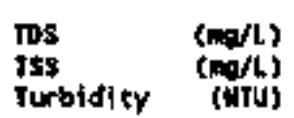 & $\begin{array}{r}386 \\
\times f \\
2.2\end{array}$ & $\begin{array}{r}336 \\
0.95\end{array}$ & $\begin{array}{r}350 \\
5 \\
3.1\end{array}$ & $\begin{array}{r}105 \\
5 \\
5\end{array}$ & $\begin{array}{r}190 \\
41 \\
1.4\end{array}$ & $\begin{array}{r}210 \\
5.7\end{array}$ & $\begin{array}{r}180 \\
6 \\
1.8\end{array}$ & $\begin{array}{r}226 \\
3 \\
7.7\end{array}$ & $\begin{array}{r}222 \\
1 \\
0.45\end{array}$ & $\begin{array}{r}176 \\
13 \\
19\end{array}$ \\
\hline
\end{tabular}


APPELDIX 1

Jon-Charge balence Caleulations, 1995

\begin{tabular}{|c|c|c|c|c|c|c|c|}
\hline $\begin{array}{l}\text { Sempl tnd Point } \\
\text { locetion } \\
\text { Date sempled }\end{array}$ & $\begin{array}{r}\text { C14-159 } \\
\text { CRSOS } \\
10 / 24 / 95\end{array}$ & $\begin{array}{r}c N-159 \\
\text { costos } \\
10 / 25 / 95\end{array}$ & $\begin{array}{r}6 \%-159 \\
\text { CRtspo } \\
10 / 26 / 95\end{array}$ & 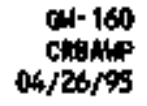 & $\begin{array}{l}\text { GN-160 } \\
\text { Collatp } \\
11 / 02 / 05\end{array}$ & $\begin{array}{r}\text { GN-161 } \\
\text { Chimplip } \\
\text { O4/20/95 }\end{array}$ & $\begin{array}{r}\text { MN-161 } \\
\text { CRANANP } \\
10 / 31 / \% 5\end{array}$ \\
\hline \multicolumn{8}{|l|}{ CATIOWS (NEO/L) } \\
\hline 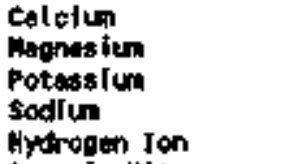 & $\begin{array}{l}2.00 \\
2.06 \\
0.05 \\
0.04 \\
0.00\end{array}$ & $\begin{array}{l}1.00 \\
1.07 \\
0.05 \\
0.03 \\
0.00\end{array}$ & $\begin{array}{l}1.85 \\
2.06 \\
0.04 \\
0.03 \\
0.00\end{array}$ & $\begin{array}{l}2.40 \\
2.30 \\
0.02 \\
0.13 \\
0.00\end{array}$ & $\begin{array}{l}2.25 \\
2.39 \\
0.03 \\
0.11 \\
0.00\end{array}$ & $\begin{array}{l}2.00 \\
1.97 \\
0.02 \\
0.03 \\
0.00\end{array}$ & $\begin{array}{l}1.70 \\
2.06 \\
0.03 \\
0.06 \\
0.00\end{array}$ \\
\hline lapomis Mltrogen & - & - & + & • & - & - & - \\
\hline Totel tations & 4.14 & 3.95 & 3.98 & 4.86 & 4.77 & 4.02 & 3.84 \\
\hline$(N \in Q / L)$ & & & & & & & \\
\hline 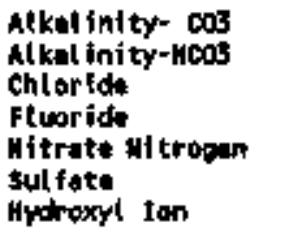 & $\begin{array}{l}0.00 \\
3.78 \\
0.06 \\
0.00 \\
0.03 \\
0.18 \\
0.00\end{array}$ & $\begin{array}{l}0.02 \\
3.84 \\
0.04 \\
0.00 \\
0.05 \\
0.17 \\
0.00\end{array}$ & $\begin{array}{l}0.00 \\
3.00 \\
0.04 \\
0.00 \\
0.03 \\
0.18 \\
0.00\end{array}$ & $\begin{array}{l}0.00 \\
4.04 \\
0.20 \\
0.00 \\
0.07 \\
0.06 \\
0.00\end{array}$ & $\begin{array}{l}0.00 \\
4.34 \\
0.15 \\
0.00 \\
0.06 \\
0.00 \\
0.00\end{array}$ & $\begin{array}{l}0.00 \\
6.12 \\
0.08 \\
0.00 \\
0.04 \\
0.04 \\
0.01\end{array}$ & $\begin{array}{l}0.00 \\
3.82 \\
0.07 \\
0.00 \\
0.02 \\
0.03 \\
0.00\end{array}$ \\
\hline Total inions & 4.04 & 4.10 & 4.05 & 4.99 & 4.64 & 4.27 & 3.94 \\
\hline CHARGE BALAMES $(\mathbf{X})$ & $\$$ & -2 & -1 & -1 & 1 & -3 & -1 \\
\hline 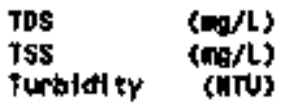 & $\begin{array}{r}224 \\
1696 \\
41\end{array}$ & $\begin{array}{l}216 \\
2.5 \\
2.9\end{array}$ & $\begin{array}{r}186 \\
1 \\
3\end{array}$ & $\begin{array}{l}402 \\
117 \\
260\end{array}$ & $\begin{array}{r}750 \\
5365 \\
7600\end{array}$ & $\begin{array}{r}226 \\
<1 \\
0.7\end{array}$ & $\begin{array}{r}199 \\
41 \\
5.3\end{array}$ \\
\hline
\end{tabular}


APFENDIX I

Ion-Chargye talunce Colculations, 1995

\begin{tabular}{|c|c|c|c|c|c|c|c|c|}
\hline $\begin{array}{l}\text { soipl ing Polm } \\
\text { Cocet tion } \\
\text { Date sapted }\end{array}$ & $\begin{array}{r}0.175 \\
\text { chsp } \\
02 / 14 / 95\end{array}$ & $\begin{array}{r}0+175 \\
\text { chss } \\
05 / 15 / 95\end{array}$ & $\begin{array}{r}\text { 64-175 } \\
\text { CRsp } \\
\text { 09rotios }\end{array}$ & 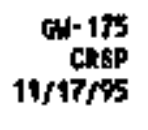 & $\begin{array}{r}04-177 \\
\text { chisp } \\
02 / 13 / 95\end{array}$ & $\begin{array}{r}0 \times 177 \\
0 / 11 / 45\end{array}$ & $\begin{array}{r}0+177 \\
\text { cksp } \\
\text { arot/ss }\end{array}$ & $\begin{array}{r}\text { au-177 } \\
\text { ctsp } \\
11 / 4 / 95\end{array}$ \\
\hline (HEO/L) & & & & & & & & \\
\hline 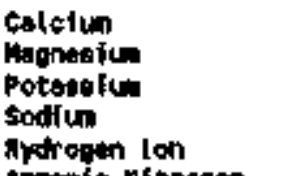 & $\begin{array}{l}2.40 \\
2.39 \\
0.02 \\
0.04 \\
0.06\end{array}$ & $\begin{array}{l}2.45 \\
2.47 \\
0.05 \\
0.04 \\
0.40\end{array}$ & $\begin{array}{l}2.40 \\
2.39 \\
0.02 \\
0.07 \\
0.00\end{array}$ & $\begin{array}{l}2.15 \\
2.22 \\
0.00 \\
0.10 \\
0.04\end{array}$ & $\begin{array}{l}2.25 \\
2.22 \\
0.08 \\
0.07 \\
0.00\end{array}$ & $\begin{array}{l}2.25 \\
2.14 \\
0.07 \\
0.05 \\
0.00\end{array}$ & $\begin{array}{l}2.20 \\
2.30 \\
0.05 \\
0.06 \\
0.00\end{array}$ & $\begin{array}{l}2.25 \\
2.39 \\
0.07 \\
0.07 \\
0.00\end{array}$ \\
\hline niming nitrogen & ' & $\cdot$ & $\cdot$ & $\cdot$ & $\cdot$ & $\cdot$ & $\cdot$ & - \\
\hline Total cationa & 4.84 & $4 . \%$ & 4.86 & 4.47 & 4.62 & 4,50 & 4.61 & 4.78 \\
\hline Antows (NEO/L) & & & & & & & & \\
\hline 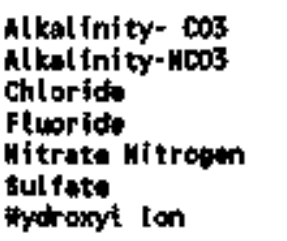 & $\begin{array}{l}0.00 \\
4.70 \\
0.06 \\
0.00 \\
0.05 \\
0.03 \\
0.04\end{array}$ & $\begin{array}{l}0.00 \\
4.52 \\
0.06 \\
0.00 \\
0.02 \\
0.00 \\
0.00\end{array}$ & $\begin{array}{l}0.00 \\
4.60 \\
0.07 \\
0.00 \\
0.04 \\
0.02 \\
0.00\end{array}$ & $\begin{array}{l}0.00 \\
4.34 \\
0.07 \\
0.00 \\
0.05 \\
0.00 \\
0.00\end{array}$ & $\begin{array}{l}0.00 \\
4.30 \\
0.08 \\
0.00 \\
0.05 \\
0.10 \\
0.00\end{array}$ & $\begin{array}{l}0.00 \\
4.32 \\
0.05 \\
0.00 \\
0.00 \\
0.21 \\
0.00\end{array}$ & $\begin{array}{l}0.00 \\
4.32 \\
0.08 \\
0.00 \\
0.03 \\
0.17 \\
0.00\end{array}$ & $\begin{array}{l}0.00 \\
4.58 \\
0.00 \\
0.00 \\
0.06 \\
0.14 \\
0.00\end{array}$ \\
\hline Totel intons: & 4.84 & 4.60 & 4.76 & 4.46 & 4.61 & 4.50 & 4.61 & 4.86 \\
\hline CHAKCE BALAMCE $(x)$ & 0 & 4 & 1 & 0 & 0 & -1 & 0 & -1 \\
\hline $\begin{array}{ll}\text { TDS } & (m g / \mathrm{C}) \\
\text { TS\$ } & \text { (mg/L) } \\
\text { Turbldtity } & \text { (NTU) }\end{array}$ & $\begin{array}{r}214 \\
1 \\
2\end{array}$ & $\begin{array}{r}278 \\
1.4\end{array}$ & $\begin{array}{l}236 \\
3.4\end{array}$ & $\begin{array}{r}190 \\
2 \\
4.1\end{array}$ & $\begin{array}{r}260 \\
2 \\
4\end{array}$ & $\begin{array}{r}234 \\
24 \\
4.1\end{array}$ & $\begin{array}{r}272 \\
4.5\end{array}$ & $\begin{array}{r}120 \\
12 \\
5.2\end{array}$ \\
\hline
\end{tabular}


Afpeimp I

Ion-cherge Belence Celeuletiong, 1995

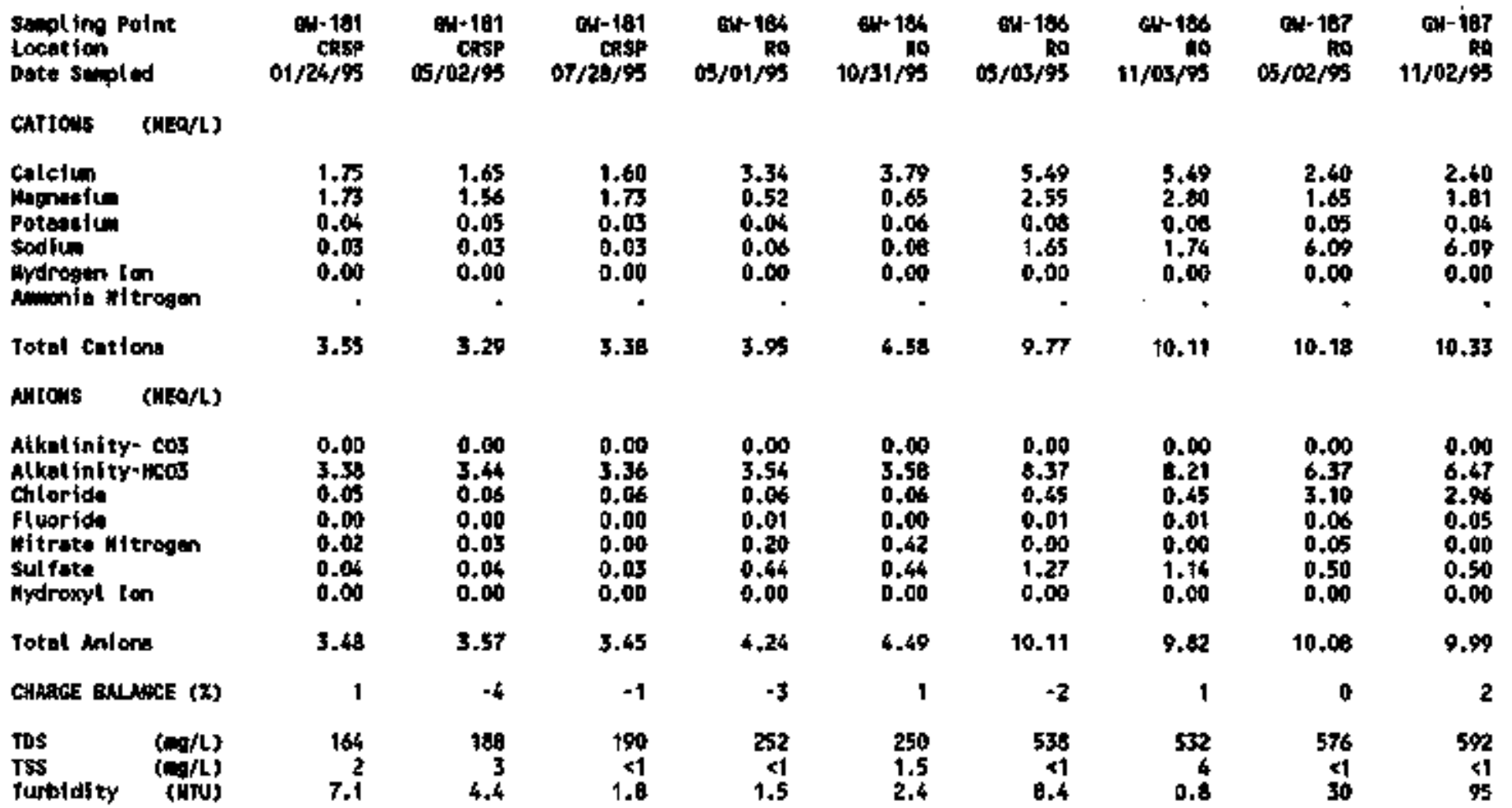


APPEWIX I

Jon-charge dalance Coleulekions, 1955

\begin{tabular}{|c|c|c|c|c|c|c|c|c|c|c|}
\hline $\begin{array}{l}\text { Sonpling Point } \\
\text { tocotion } \\
\text { Date 8tepled }\end{array}$ & 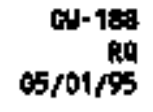 & $\begin{array}{r}04 \cdot 185 \\
10 / 31 / 95\end{array}$ & $\begin{array}{r}\text { ru+203 } \\
\text { UnC5 } \\
04 / 18 / 93\end{array}$ & $\begin{array}{r}60-203 \\
\text { thacs } \\
10 / 07 / 05\end{array}$ & $\begin{array}{r}\text { cu-205 } \\
\text { Uwes } \\
0 \times / 18 / 95\end{array}$ & $\begin{array}{r}\text { ON-20S } \\
\text { UNCS } \\
10 / 07 / \% S\end{array}$ & $\begin{array}{r}\text { OW-217 } \\
\text { LIV } \\
01 / 06 / 95\end{array}$ & $\begin{array}{r}\text { cu-217 } \\
\text { L1V } \\
07 / 10 / 95\end{array}$ & $\begin{array}{r}\text { a } \\
\text { uncs } \\
04 / 19 / \% 5\end{array}$ & $\begin{array}{r}0,-221 \\
\text { Lincs } \\
10 / 08 / 95\end{array}$ \\
\hline CArLows (NEO/L) & & & & & & & & & & \\
\hline 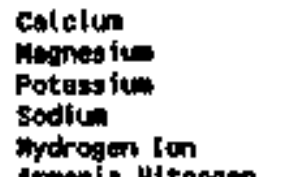 & $\begin{array}{l}1.70 \\
1.07 \\
0.07 \\
0.83 \\
0.00\end{array}$ & $\begin{array}{l}1.75 \\
2.14 \\
0.07 \\
0.86 \\
0.00\end{array}$ & $\begin{array}{l}1.50 \\
1.32 \\
0.03 \\
0.02 \\
0.00\end{array}$ & $\begin{array}{l}1.35 \\
1.32 \\
0.02 \\
0.02 \\
0.00\end{array}$ & $\begin{array}{l}1.55 \\
1.45 \\
0.05 \\
0.05 \\
0.00\end{array}$ & $\begin{array}{l}1.15 \\
1.56 \\
0.25 \\
0.09 \\
0.00\end{array}$ & $\begin{array}{l}1.40 \\
1.48 \\
0.11 \\
0.24 \\
0.00\end{array}$ & $\begin{array}{l}1.50 \\
1.56 \\
0.09 \\
0.27 \\
0.00\end{array}$ & $\begin{array}{l}1.45 \\
1.40 \\
0.03 \\
0.03 \\
0.00\end{array}$ & $\begin{array}{l}1.45 \\
1.48 \\
0.04 \\
0.05 \\
0.00\end{array}$ \\
\hline Amonia Hitrogen & $\cdot$ & - & $\bullet$ & + & • & , & - & * & $\cdot$ & - \\
\hline Totel tations & 4.56 & 4.91 & 2.86 & $2.7 \dagger$ & 3.09 & 3.05 & 3.22 & 3,42 & 2.00 & 2.97 \\
\hline Antons (NLO/L) & & & & & & & & & & \\
\hline 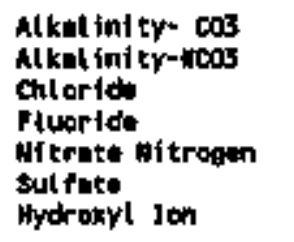 & $\begin{array}{l}0.00 \\
4.14 \\
0.14 \\
0.04 \\
0.05 \\
0.56 \\
0.00\end{array}$ & $\begin{array}{l}0.00 \\
4.16 \\
0.14 \\
0.04 \\
0.02 \\
0.55 \\
0.00\end{array}$ & $\begin{array}{l}0.00 \\
2.76 \\
0.05 \\
0.00 \\
0.10 \\
0.08 \\
0.00\end{array}$ & $\begin{array}{l}0.00 \\
2.74 \\
0.05 \\
0.00 \\
0.09 \\
0.00 \\
0.00\end{array}$ & $\begin{array}{l}0.00 \\
3.20 \\
0.08 \\
0.00 \\
0.45 \\
0.13 \\
0.00\end{array}$ & $\begin{array}{l}0.00 \\
2.90 \\
0.07 \\
0.00 \\
0.03 \\
0.06 \\
0.00\end{array}$ & $\begin{array}{l}0.00 \\
3.02 \\
0.06 \\
0.00 \\
0.08 \\
0.11 \\
0.00\end{array}$ & $\begin{array}{l}0.00 \\
3.12 \\
0.07 \\
0.00 \\
0.04 \\
0.14 \\
0.00\end{array}$ & $\begin{array}{l}0.00 \\
3.02 \\
0.05 \\
0.00 \\
0.06 \\
0.03 \\
0.00\end{array}$ & $\begin{array}{l}0.00 \\
3.04 \\
0.05 \\
0.00 \\
0.05 \\
0.03 \\
0.00\end{array}$ \\
\hline Total intons & 4.81 & 4.90 & 2.96 & 2.87 & 3.65 & 3.06 & 3.27 & 3.37 & 3.17 & 3.16 \\
\hline CHARTE BaLAHCE (X) & -4 & 0 & +1 & +3 & .11 & -0 & -1 & 1 & .4 & -3 \\
\hline $\begin{array}{ll}\text { Ios } & (\mathrm{G} / \mathrm{L}) \\
\text { TSs } & (\mathrm{D} / \mathrm{l}) \\
\text { Turbidity } & \text { (NTU) }\end{array}$ & $\begin{array}{r}276 \\
<1 \\
2\end{array}$ & $\begin{array}{r}324 \\
74 \\
74\end{array}$ & $\begin{array}{l}154 \\
5.5 \\
7.1\end{array}$ & $\begin{array}{r}140 \\
3 \\
3.6\end{array}$ & $\begin{array}{r}150 \\
41 \\
2.8\end{array}$ & $\begin{array}{r}162 \\
4 \\
2\end{array}$ & $\begin{array}{r}109 \\
5 \\
8.9\end{array}$ & $\begin{array}{r}176 \\
27 \\
5.1\end{array}$ & $\begin{array}{r}162 \\
41 \\
1\end{array}$ & $\begin{array}{r}142 \\
<1 \\
1.1\end{array}$ \\
\hline
\end{tabular}


APPEIDIX I

Ion-Chnrge Bolanes Celcutatlons, 1995

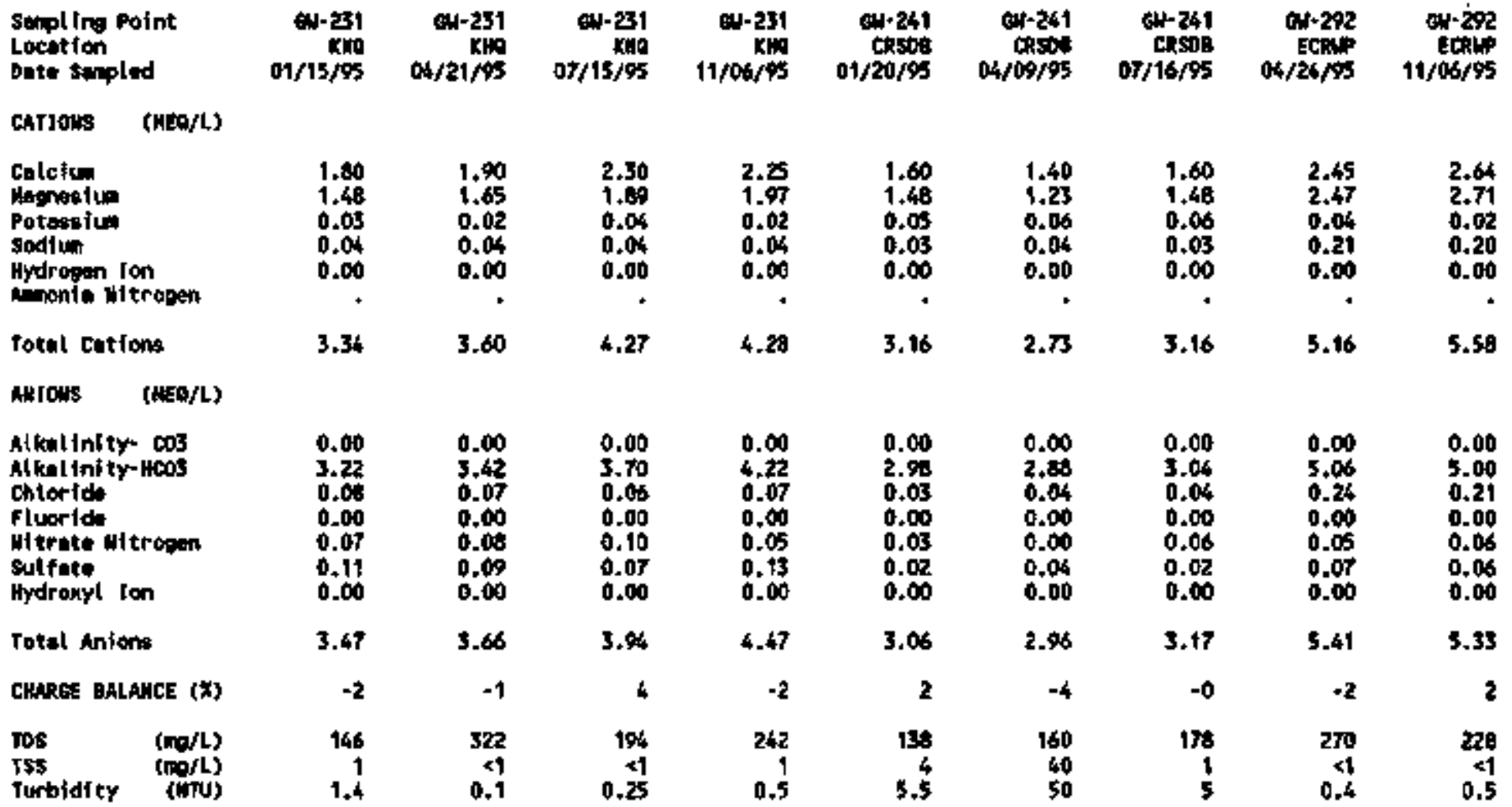


APPEND [K J

Ion-Cherge anlence Col cukations, 1995

\begin{tabular}{|c|c|c|c|c|c|c|c|c|c|c|}
\hline $\begin{array}{l}\text { Sempling Polint } \\
\text { Lacetion } \\
\text { Dote sanpted }\end{array}$ & $\begin{array}{r}61-293 \\
\text { ECGAP } \\
04 / 23 / 95\end{array}$ & $\begin{array}{r}\text { EN-293 } \\
\text { ECRHP } \\
11 / 06 / 95\end{array}$ & $\begin{array}{r}\text { CN-204 } \\
\text { Ectis } \\
04 / 22 / 95\end{array}$ & $\begin{array}{r}\text { SN-296 } \\
\text { ECRTP } \\
11 / \cos / 95\end{array}$ & $\begin{array}{r}0+296 \\
\text { EcR1P } \\
04 / 22 / 45\end{array}$ & $\begin{array}{r}\text { CW-296 } \\
\text { ECRLP } \\
11 / 05 / 65\end{array}$ & $\begin{array}{r}\text { GN-259 } \\
\text { Crastip } \\
\text { O5/02/95 }\end{array}$ & $\begin{array}{r}\text { 6h-2s: } \\
\text { Chians } \\
11 / 06 / 95\end{array}$ & $\begin{array}{r}0.299 \\
05 \text { cholpos }\end{array}$ & $\begin{array}{r}\text { chr299 } \\
\text { CR:ALP } \\
11 / 03 / 95\end{array}$ \\
\hline CATJOHS (nea/L) & & & & & & & & & & \\
\hline 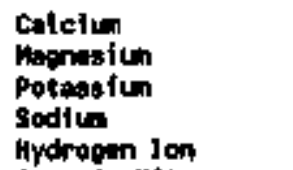 & $\begin{array}{l}2.40 \\
2.47 \\
0.05 \\
0.21 \\
0.00\end{array}$ & $\begin{array}{l}2.79 \\
2.00 \\
0.03 \\
0.25 \\
0.00\end{array}$ & $\begin{array}{l}2.40 \\
2.39 \\
0.04 \\
0.18 \\
0.00\end{array}$ & $\begin{array}{l}2.45 \\
2.55 \\
0.02 \\
0.13 \\
0.00\end{array}$ & $\begin{array}{l}2.15 \\
2.14 \\
0.02 \\
0.07 \\
0.00\end{array}$ & $\begin{array}{l}2.25 \\
2.30 \\
0.02 \\
0.06 \\
0.00\end{array}$ & $\begin{array}{l}1.70 \\
1.73 \\
0.02 \\
0.07 \\
0.00\end{array}$ & $\begin{array}{l}1.65 \\
1.81 \\
0.03 \\
0.08 \\
0.00\end{array}$ & $\begin{array}{l}1.45 \\
t .40 \\
0.05 \\
0.05 \\
0.00\end{array}$ & $\begin{array}{l}1.40 \\
1.40 \\
0.02 \\
0.03 \\
0.00\end{array}$ \\
\hline Nisonit Hitrogen & - & $\cdot$ & $\cdot$ & $\cdot$ & - & $\cdot$ & - & • & * & 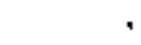 \\
\hline Total Cations & 5.13 & 5.87 & 5.01 & 5.14 & 4.36 & 4.62 & 3.51 & 3.57 & 2.82 & 2.85 \\
\hline huons (wea/t) & & & & & & & & & & \\
\hline 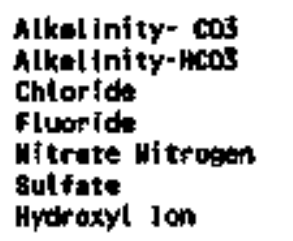 & $\begin{array}{l}0.00 \\
4.92 \\
0.25 \\
0.00 \\
0.04 \\
0.07 \\
0.00\end{array}$ & $\begin{array}{l}0.00 \\
5.14 \\
0.31 \\
0.00 \\
0.05 \\
0.07 \\
0.00\end{array}$ & $\begin{array}{l}0.00 \\
4.42 \\
0.25 \\
0.00 \\
0.19 \\
0.07 \\
0.00\end{array}$ & $\begin{array}{l}0.00 \\
4.52 \\
0.29 \\
0.00 \\
0.19 \\
0.07 \\
0.00\end{array}$ & $\begin{array}{l}0.00 \\
4.40 \\
0.10 \\
0.00 \\
0.05 \\
0.02 \\
0.09\end{array}$ & $\begin{array}{l}0.00 \\
4.44 \\
0.08 \\
0.00 \\
0.06 \\
0.03 \\
0.00\end{array}$ & $\begin{array}{l}0.00 \\
3.40 \\
0.05 \\
0.01 \\
0.05 \\
0.14 \\
0.00\end{array}$ & $\begin{array}{l}0.00 \\
3.40 \\
0.03 \\
0.00 \\
0.00 \\
0.17 \\
0.00\end{array}$ & $\begin{array}{l}0.00 \\
2.82 \\
0.05 \\
0.00 \\
0.03 \\
0.08 \\
0.00\end{array}$ & $\begin{array}{l}0.00 \\
2.82 \\
0.04 \\
0.00 \\
0.02 \\
0.06 \\
0.00\end{array}$ \\
\hline Total Antons & 5.31 & 5.57 & 4.86 & 5.07 & 4.56 & 4.60 & 3.62 & 3,61 & 2.87 & 2.94 \\
\hline CHaree baLnCE (x) & $\cdot 2$ & $\mathbf{3}$ & 0 & 1 & -2 & 0 & -1 & -1 & +1 & -2 \\
\hline $\begin{array}{ll}\text { Tos } & \text { (ns/L) } \\
\text { Tss } & \text { (forlt) } \\
\text { Turbldier } & \text { (HTU) }\end{array}$ & $\begin{array}{r}276 \\
\times 1 \\
8,4\end{array}$ & $\begin{array}{l}166 \\
3.5 \\
8.8\end{array}$ & $\begin{array}{r}254 \\
0.1 \\
0.2\end{array}$ & $\begin{array}{r}2 \$ 2 \\
0.7\end{array}$ & $\begin{array}{r}226 \\
1 \\
0.6\end{array}$ & $\begin{array}{r}184 \\
1 \\
2\end{array}$ & $\begin{array}{r}180 \\
19 \\
90\end{array}$ & $\begin{array}{r}172 \\
13 \\
16\end{array}$ & $\begin{array}{r}270 \\
<1 \\
0.6\end{array}$ & $\begin{array}{r}88 \\
<1 \\
1.1\end{array}$ \\
\hline
\end{tabular}


APPEDIX $t$

Ion-Cherge Bulence Celcutationa, 1995

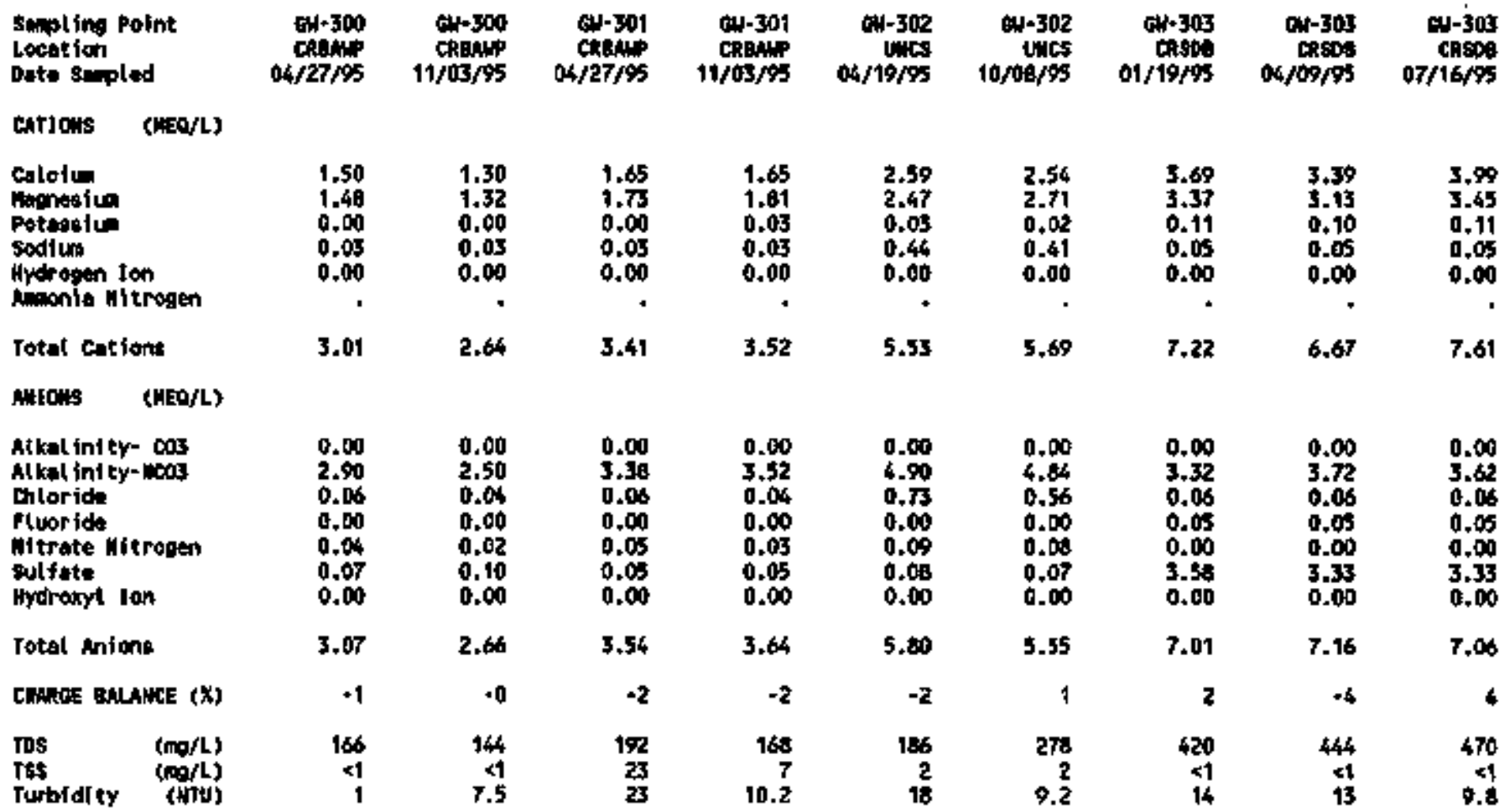


APPENoIX I

Jon-Charge folence taleulations, 199\%

\begin{tabular}{|c|c|c|c|c|c|c|c|c|c|}
\hline $\begin{array}{l}\text { sempl Ing Foint } \\
\text { tocation } \\
\text { Dete stipled }\end{array}$ & $\begin{array}{r}04-304 \\
\operatorname{coses} \\
0.175 / 95\end{array}$ & $\begin{array}{r}64-304 \\
04 / 06 / 95\end{array}$ & $\begin{array}{r}\text { cw-304 } \\
\text { crspa } \\
07 / 12 / 45\end{array}$ & $\begin{array}{r}01-305 \\
\operatorname{Lry} \\
01 / 10 / 05\end{array}$ & $\begin{array}{r}\text { cu-3os } \\
\text { LIV } \\
\text { or/12/95 }\end{array}$ & $\begin{array}{r}64-321 \\
108 \\
04 / 2+3 / 95\end{array}$ & $\begin{array}{r}0+321 \\
10 / 26 / 95\end{array}$ & $\begin{array}{r}6 N-339 \\
\text { thes } \\
04 / 19 / 95\end{array}$ & 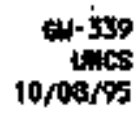 \\
\hline \multicolumn{10}{|l|}{ CATJOHS (NEQ/L) } \\
\hline 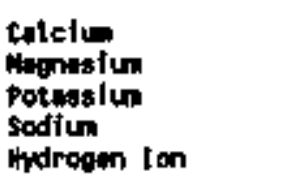 & $\begin{array}{l}1.90 \\
1.97 \\
0.05 \\
0.20 \\
0.00\end{array}$ & $\begin{array}{l}1.00 \\
1.89 \\
0.03 \\
0.20 \\
0.00\end{array}$ & $\begin{array}{l}1.95 \\
2.06 \\
0.06 \\
0.22 \\
0.00\end{array}$ & $\begin{array}{l}1.40 \\
1.40 \\
0.00 \\
0.06 \\
0.00\end{array}$ & $\begin{array}{l}1.35 \\
\$ .48 \\
0.02 \\
0.09 \\
0.00\end{array}$ & $\begin{array}{l}1.25 \\
1 . \$ 5 \\
0.02 \\
0.02 \\
0.00\end{array}$ & $\begin{array}{l}1.30 \\
1.25 \\
0.02 \\
0.03 \\
0.00\end{array}$ & $\begin{array}{l}2.84 \\
2.63 \\
0.04 \\
0.48 \\
0.00\end{array}$ & $\begin{array}{l}2.70 \\
2.80 \\
0.03 \\
0.52 \\
0.00\end{array}$ \\
\hline Totent cations & 4.12 & 3.94 & 4.20 & 2.86 & 2.94 & 2.44 & 2.58 & 5.89 & 6.16 \\
\hline \multicolumn{10}{|l|}{ Nhrows (kE⿰亻) } \\
\hline 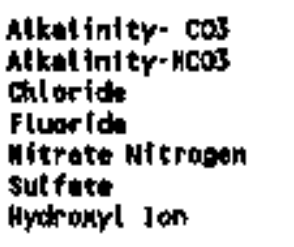 & $\begin{array}{l}0.00 \\
3.70 \\
0.06 \\
0.00 \\
0.04 \\
0.37 \\
0.00\end{array}$ & $\begin{array}{l}0.00 \\
3.82 \\
0.09 \\
0.00 \\
0.04 \\
0.40 \\
0.00\end{array}$ & $\begin{array}{l}0.00 \\
3.74 \\
0.08 \\
0.00 \\
0.05 \\
0.47 \\
0.00\end{array}$ & $\begin{array}{l}0.00 \\
2.66 \\
0.07 \\
0.00 \\
0.05 \\
0.09 \\
0.00\end{array}$ & $\begin{array}{l}0.00 \\
2.74 \\
0.00 \\
0.00 \\
0.03 \\
0.03 \\
0.00\end{array}$ & $\begin{array}{l}0.00 \\
2.52 \\
0.05 \\
0.00 \\
0.05 \\
0.04 \\
0.00\end{array}$ & $\begin{array}{l}0.00 \\
2.54 \\
0.04 \\
0.00 \\
0.05 \\
0.05 \\
0.00\end{array}$ & $\begin{array}{l}0.00 \\
5.30 \\
0.76 \\
0.00 \\
0.08 \\
0.11 \\
0.00\end{array}$ & $\begin{array}{l}0.00 \\
5.26 \\
0.65 \\
0.00 \\
0.06 \\
0.09 \\
0.00\end{array}$ \\
\hline Fotal Antors & 4.20 & 4.33 & 4.27 & 2.86 & 2.80 & 2.63 & 2.66 & 6.25 & 6.06 \\
\hline CHAROE BALACE $(X)$ & .1 & -5 & 0 & .0 & 1 & .4 & -2 & -2 & $t$ \\
\hline $\begin{array}{ll}\text { Tos } & (m a / L) \\
\text { Tss } & \text { (ng/L) } \\
\text { Turbidity } & \text { (nTU) }\end{array}$ & $\begin{array}{r}222 \\
24 \\
2+6\end{array}$ & $\begin{array}{l}22.2 \\
6.5 \\
2.8\end{array}$ & $\begin{array}{r}226 \\
10 \\
4.7\end{array}$ & $\begin{array}{r}128 \\
5 \\
3.5\end{array}$ & $\begin{array}{r}139 \\
2 \\
3.9\end{array}$ & $\begin{array}{r}140 \\
<1 \\
0.1\end{array}$ & $\begin{array}{r}166 \\
\times 1 \\
0.25\end{array}$ & $\begin{array}{r}326 \\
<1 \\
0.4\end{array}$ & $\begin{array}{l}142 \\
2.5 \\
7.2\end{array}$ \\
\hline
\end{tabular}


APPEW IX 1

Ion-Cherge Balence calculat lono, \$995

\begin{tabular}{|c|c|c|c|c|c|c|c|c|c|c|}
\hline $\begin{array}{l}\text { semplting Point } \\
\text { Loction } \\
\text { Bate sinpled }\end{array}$ & $\begin{array}{r}\text { ar-511 } \\
\text { ERsp } \\
01 / 24 / 95\end{array}$ & $\begin{array}{r}\text { ev-51t } \\
\text { ansp } \\
05 / 05 / 9 \%\end{array}$ & $\begin{array}{r}\text { en-511 } \\
\text { G15p } \\
07 / 29 / 95\end{array}$ & $\begin{array}{r}\text { EN-511 } \\
\text { Ckesp } \\
11 / 07 / 98\end{array}$ & $\begin{array}{r}64-512 \\
\text { ind } \\
04 / 26 / 95\end{array}$ & $\begin{array}{r}64-512 \\
\text { 10B } \\
10 / 31 / 95\end{array}$ & $\begin{array}{r}\text { u-51s } \\
\text { 10698 }\end{array}$ & $\begin{array}{r}5+513 \\
10 / 31 / 95\end{array}$ & $\begin{array}{r}01+514 \\
04 / 27 / \% 5\end{array}$ & $\begin{array}{r}04-514 \\
101 / 02 / 95\end{array}$ \\
\hline \multicolumn{11}{|l|}{ CATLOHS (NEA/L) } \\
\hline 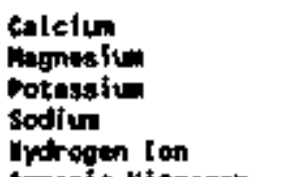 & $\begin{array}{l}1.30 \\
1.32 \\
0.11 \\
0.08 \\
0.00\end{array}$ & $\begin{array}{l}1.45 \\
1.40 \\
0.07 \\
0.05 \\
0.00\end{array}$ & $\begin{array}{l}1.35 \\
1.48 \\
0.08 \\
0.06 \\
0.00\end{array}$ & $\begin{array}{l}1.40 \\
1.40 \\
0.08 \\
0.05 \\
0.00\end{array}$ & $\begin{array}{l}1.60 \\
1.48 \\
0.10 \\
0.03 \\
0.00\end{array}$ & $\begin{array}{l}1.55 \\
1.56 \\
0.11 \\
0.05 \\
0.00\end{array}$ & $\begin{array}{l}1.75 \\
1.75 \\
0.05 \\
0.05 \\
0.00\end{array}$ & $\begin{array}{l}1.00 \\
1.81 \\
0.05 \\
0.04 \\
0.06\end{array}$ & $\begin{array}{l}1.75 \\
1.65 \\
0.03 \\
0.02 \\
0.00\end{array}$ & $\begin{array}{l}1.13 \\
1.81 \\
0.04 \\
0.02 \\
0.00\end{array}$ \\
\hline monit Nitrogen & . & , & - & ? & • & + & $\bullet$ & - & $\cdot$ & - \\
\hline Total Cotions: & 2.81 & 2.87 & 2.95 & 2.95 & 3.21 & 3.27 & 3.55 & 3.68 & 3.44 & 3.61 \\
\hline \multicolumn{11}{|l|}{ wlons (NEa/L) } \\
\hline 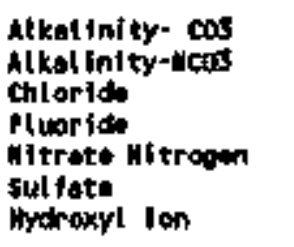 & $\begin{array}{l}0.00 \\
2.70 \\
0.09 \\
0.00 \\
0.02 \\
0.04 \\
0.00\end{array}$ & $\begin{array}{l}0.00 \\
3.00 \\
0.07 \\
0.00 \\
0.02 \\
0.04 \\
0.00\end{array}$ & $\begin{array}{l}0.00 \\
2.06 \\
0.00 \\
0.00 \\
0.02 \\
0.04 \\
0.00\end{array}$ & $\begin{array}{l}0.00 \\
2.64 \\
0.07 \\
0.00 \\
0.02 \\
0.03 \\
0.00\end{array}$ & $\begin{array}{l}0.00 \\
3.26 \\
0.05 \\
0.00 \\
0.00 \\
0.06 \\
0.00\end{array}$ & $\begin{array}{l}0.00 \\
3.14 \\
0.04 \\
0.09 \\
0.00 \\
0.08 \\
0.00\end{array}$ & $\begin{array}{l}0.00 \\
3.60 \\
0.06 \\
0.00 \\
0.02 \\
0.06 \\
0.00\end{array}$ & $\begin{array}{l}0.00 \\
3.60 \\
0.06 \\
0.00 \\
0.02 \\
0.03 \\
0.00\end{array}$ & $\begin{array}{l}0.00 \\
3.66 \\
0.06 \\
0.00 \\
0.05 \\
0.09 \\
0.00\end{array}$ & $\begin{array}{l}0.00 \\
3.50 \\
0.04 \\
0.00 \\
0.00 \\
0.00 \\
0.00\end{array}$ \\
\hline Total intons & 2.84 & 3.12 & 2.99 & 2.96 & 3.57 & 3.25 & 3.74 & 3.69 & 3.24 & 3.71 \\
\hline CWARGE BHANCE $(x)$ & -1 & -3 & -1 & -1 & -3 & a & -3 & 0 & -5 & $-\uparrow$ \\
\hline 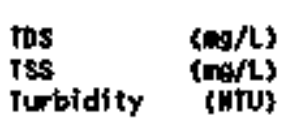 & $\begin{array}{r}132 \\
1 \\
2.5\end{array}$ & $\begin{array}{l}168 \\
2.5 \\
4.7\end{array}$ & $\begin{array}{r}166 \\
1 \\
0.7\end{array}$ & $\begin{array}{r}162 \\
41 \\
0.5\end{array}$ & $\begin{array}{r}180 \\
\times 1 \\
4.9\end{array}$ & $\begin{array}{r}214 \\
3.7\end{array}$ & $\begin{array}{r}176 \\
<1 \\
1.3\end{array}$ & $\begin{array}{l}20.4 \\
3.5 \\
1.9\end{array}$ & $\begin{array}{r}190 \\
\times 1 \\
3.5\end{array}$ & $\begin{array}{r}142 \\
2 \\
8.6\end{array}$ \\
\hline
\end{tabular}


ippgintix I

ton-charge betance Colculationa, 1995

\begin{tabular}{|c|c|c|c|c|c|c|c|c|c|}
\hline $\begin{array}{l}\text { sepling Polnt } \\
\text { Locetion } \\
\text { pot tipled }\end{array}$ & $\begin{array}{r}0.521 \\
41 v \\
01 / 09 / 95\end{array}$ & $\begin{array}{r}\text { GN-sz1 } \\
\text { LIV } \\
0 t / 11 / 9 s\end{array}$ & $\begin{array}{r}\text { GH-522 } \\
\text { LJV } \\
01 / 10 / 95\end{array}$ & $\begin{array}{r}\text { a+s22 } \\
\text { LIV } \\
07 / 12 / 95\end{array}$ & $\begin{array}{r}0+539 \\
\text { Ltl } \\
01 / 04 / 45\end{array}$ & $\begin{array}{r}04-539 \\
\text { LtI } \\
07 / 10 / 95\end{array}$ & $\begin{array}{r}0,539 \\
\text { LI] } \\
10 / 06 / 95\end{array}$ & $\begin{array}{r}a+540 \\
6 L 11 \\
04 / 04 / 95\end{array}$ & $\begin{array}{r}\text { eN-540 } \\
\text { colvI } \\
10 / 11 / 9 S\end{array}$ \\
\hline EATIONS (KESO/L) & & & & & & & & & \\
\hline 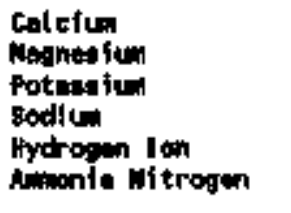 & $\begin{array}{r}1.50 \\
1.56 \\
0.00 \\
0.03 \\
0.00 \\
.\end{array}$ & $\begin{array}{r}1.50 \\
1.65 \\
0.00 \\
0.04 \\
0.00 \\
.\end{array}$ & $\begin{array}{r}1.45 \\
1.40 \\
0.02 \\
0.03 \\
0.00 \\
.\end{array}$ & $\begin{array}{r}1.30 \\
1.32 \\
0.05 \\
0.05 \\
0.00 \\
.\end{array}$ & $\begin{array}{l}1.90 \\
1.73 \\
0.03 \\
0.11 \\
0.00 \\
0.00\end{array}$ & $\begin{array}{l}1.60 \\
1.81 \\
0.04 \\
0.17 \\
0.00 \\
0.00\end{array}$ & $\begin{array}{l}1.00 \\
1.01 \\
0.05 \\
0.10 \\
0.00 \\
0.00\end{array}$ & $\begin{array}{l}2.05 \\
1.09 \\
0.02 \\
0.10 \\
0.00 \\
0.00\end{array}$ & $\begin{array}{l}1.95 \\
1.97 \\
0.04 \\
0.13 \\
0.00 \\
0.00\end{array}$ \\
\hline Tatal Cations & 3.09 & 3.18 & 2.69 & 2.67 & 3.77 & 3.81 & 3.76 & 4.06 & 4.00 \\
\hline Nustors (MES/L) & & & & & & & & & \\
\hline 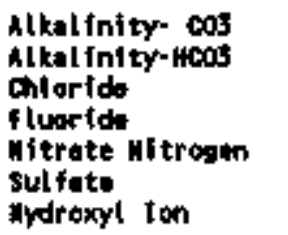 & $\begin{array}{l}0.00 \\
3.04 \\
0.05 \\
0.00 \\
0.04 \\
0.03 \\
0.00\end{array}$ & $\begin{array}{l}0.00 \\
3.02 \\
0.05 \\
0.00 \\
0.04 \\
0.00 \\
0.00\end{array}$ & $\begin{array}{l}0.00 \\
2.00 \\
0.06 \\
0.00 \\
0.06 \\
0.00 \\
0.00\end{array}$ & $\begin{array}{l}0.00 \\
2.52 \\
0.06 \\
0.00 \\
0.04 \\
0.00 \\
0.00\end{array}$ & $\begin{array}{l}0.00 \\
2.98 \\
0.59 \\
0.00 \\
0.07 \\
0.16 \\
0.00\end{array}$ & $\begin{array}{l}0.00 \\
3.00 \\
0.56 \\
0.00 \\
0.05 \\
0.23 \\
0.00\end{array}$ & $\begin{array}{l}0.00 \\
3.04 \\
0.51 \\
0.00 \\
0.03 \\
0.15 \\
0.00\end{array}$ & $\begin{array}{l}0.00 \\
3.96 \\
0.07 \\
0.00 \\
0.03 \\
0.09 \\
0.00\end{array}$ & $\begin{array}{l}0.00 \\
3.92 \\
0.05 \\
0.00 \\
0.03 \\
0.09 \\
0.00\end{array}$ \\
\hline Iottil intons & 3.18 & 3.11 & 2.92 & 2.62 & 3.60 & 3.84 & 3.72 & 4.16 & 4.09 \\
\hline CHARGE BALAMCE ( $\boldsymbol{\hbar}$ ) & -1 & 1 & -1 & 1 & $\bullet 0$ & -0 & 0 & -1 & .0 \\
\hline $\begin{array}{ll}\text { Tos } & (m / L) \\
\text { T\$S } & (m / L) \\
\text { Turbidity } & \text { (WTt) }\end{array}$ & $\begin{array}{r}118 \\
2 \\
6.7\end{array}$ & $\begin{array}{r}176 \\
41 \\
2,9\end{array}$ & $\begin{array}{r}100 \\
5 \\
3.5\end{array}$ & $\begin{array}{r}146 \\
17 \\
9.2\end{array}$ & $\begin{array}{l}40 \\
76 \\
34\end{array}$ & $\begin{array}{r}229 \\
3.6\end{array}$ & $\begin{array}{r}202 \\
9 \\
12\end{array}$ & $\begin{array}{r}204 \\
1 \\
0.2\end{array}$ & $\begin{array}{r}232 \\
41 \\
0.15\end{array}$ \\
\hline
\end{tabular}


APEIDIX 1

Ion+cherge tolence caleulatlons, 1995

\begin{tabular}{|c|c|c|c|c|c|c|c|c|c|c|}
\hline $\begin{array}{l}\text { pompt ing point } \\
\text { Locat ion } \\
\text { Oute sepled }\end{array}$ & $\begin{array}{r}0 \%-541 \\
\cot w] \\
04 / 05 / 95\end{array}$ & $\begin{array}{r}\text { (0N-54) } \\
\text { Dotyt } \\
10 / 12 / 95\end{array}$ & $\begin{array}{r}0 N-542 \\
\cos 142 \\
04 / 06 / 95\end{array}$ & $\begin{array}{r}\text { Oal-542 } \\
\text { CDLUI } \\
10 / 16 / 95\end{array}$ & $\begin{array}{r}c y-543 \\
\operatorname{col} 1 / \\
06 / 06 / 95\end{array}$ & $\begin{array}{r}04-543 \\
\operatorname{coL} 41 \\
10 / 17 / \% 5\end{array}$ & $\begin{array}{r}64-544 \\
\text { CoLv] } \\
04 / 06 / 95\end{array}$ & $\begin{array}{r}64-546 \\
\text { coLv] } \\
10 / 77 / 95\end{array}$ & $\begin{array}{r}\text { an-546 } \\
\text { colvi } \\
04 / 05 / 95\end{array}$ & $\begin{array}{r}\text { o.546 } \\
\text { chevt } \\
10 / 11 / s\end{array}$ \\
\hline \multicolumn{11}{|l|}{ ChTJous (IEQ/L) } \\
\hline 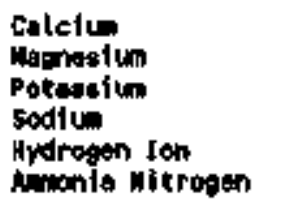 & $\begin{array}{l}1.00 \\
1.65 \\
0.02 \\
0.02 \\
0.00 \\
0.00\end{array}$ & $\begin{array}{l}1.05 \\
1.73 \\
0.02 \\
0.03 \\
0.00 \\
0.00\end{array}$ & $\begin{array}{l}2.00 \\
+.81 \\
0.04 \\
0.04 \\
0.00 \\
0.00\end{array}$ & $\begin{array}{l}1.40 \\
1.32 \\
0.04 \\
0.04 \\
0.00 \\
0.00\end{array}$ & $\begin{array}{l}2.56 \\
2.35 \\
0.02 \\
0.04 \\
0.00 \\
0.00\end{array}$ & $\begin{array}{l}2.35 \\
2.39 \\
0.03 \\
0.05 \\
0.00 \\
0.00\end{array}$ & $\begin{array}{l}2.40 \\
2.22 \\
0.04 \\
0.22 \\
0.00 \\
0.00\end{array}$ & $\begin{array}{l}2.25 \\
2.30 \\
0.03 \\
0.22 \\
0.00 \\
0.00\end{array}$ & $\begin{array}{l}1.65 \\
1.56 \\
0.05 \\
0.10 \\
0.00 \\
0.00\end{array}$ & $\begin{array}{l}1.40 \\
1.58 \\
0.05 \\
0.09 \\
0.00 \\
0.00\end{array}$ \\
\hline Total cations & 3.59 & 3.62 & 3.89 & 2.80 & 4.99 & 4.80 & 6,67 & 4,80 & 3.35 & 3.10 \\
\hline \multicolumn{11}{|l|}{ MIOHs (NEQ/L) } \\
\hline 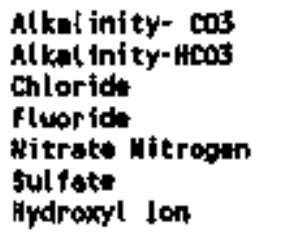 & $\begin{array}{l}0.00 \\
3.76 \\
0.05 \\
0.09 \\
0.00 \\
0.25 \\
0.00\end{array}$ & $\begin{array}{l}0.00 \\
3.30 \\
0.04 \\
0.00 \\
0.00 \\
0.75 \\
0.00\end{array}$ & $\begin{array}{l}0.00 \\
3.70 \\
0.07 \\
0.00 \\
0.05 \\
0.15 \\
0.00\end{array}$ & $\begin{array}{l}0.09 \\
2.70 \\
0.05 \\
0.00 \\
0.05 \\
0.07 \\
0.00\end{array}$ & $\begin{array}{l}0.00 \\
4.60 \\
0.00 \\
0.00 \\
0.06 \\
0.07 \\
0.00\end{array}$ & $\begin{array}{l}0.00 \\
4.72 \\
0.09 \\
0.00 \\
0.03 \\
0.06 \\
0.00\end{array}$ & $\begin{array}{l}0.00 \\
4.34 \\
0.28 \\
0.00 \\
0.06 \\
0.14 \\
0.00\end{array}$ & $\begin{array}{l}0.00 \\
4.20 \\
0.25 \\
0.00 \\
0.06 \\
0.16 \\
0.00\end{array}$ & $\begin{array}{l}0.00 \\
2.00 \\
0.07 \\
0.00 \\
0.03 \\
0.09 \\
0.00\end{array}$ & $\begin{array}{l}0.00 \\
2.50 \\
0.05 \\
0.00 \\
0.05 \\
0.05 \\
0.00\end{array}$ \\
\hline Totel mions & 4.06 & 3.67 & $3 . \%$ & 2.86 & 5.00 & 4.49 & 4.64 & 6.75 & 3.00 & 2.63 \\
\hline CHARGE BALAIKCE (X) & -6 & -1 & +4 & +1 & -0 & -1 & 0 & 1 & 4 & 8 \\
\hline $\begin{array}{lc}\text { Tes } & (m / L) \\
\text { TSS } & (m / L) \\
\text { Turbldity } & (W T U)\end{array}$ & $\begin{array}{r}2000 \\
3 \\
0.5\end{array}$ & $\begin{array}{r}240 \\
1.3 \\
1.3\end{array}$ & $\begin{array}{r}209 \\
1 \\
1.3\end{array}$ & $\begin{array}{r}36 \\
4 \\
2.6\end{array}$ & $\begin{array}{r}2 \$ 2 \\
1 \\
2\end{array}$ & $\begin{array}{l}256 \\
3.1\end{array}$ & $\begin{array}{l}262 \\
1.5 \\
2\end{array}$ & $\begin{array}{r}178 \\
1.7\end{array}$ & $\begin{array}{r}172 \\
62 \\
152\end{array}$ & $\begin{array}{l}176 \\
194 \\
160\end{array}$ \\
\hline
\end{tabular}


APEDEIXI

[on-Cherge volence Colculationa, 1995

\begin{tabular}{|c|c|c|c|c|c|c|c|c|}
\hline $\begin{array}{l}\text { sopl ing Point } \\
\text { cocetion } \\
\text { Dats simplet }\end{array}$ & $\begin{array}{r}0.557 \\
t y \\
04 / 06195\end{array}$ & $\begin{array}{r}61.557 \\
t v \\
10 / 07 / 95\end{array}$ & $\begin{array}{r}0+560 \\
041 v 11 \\
0405 / \% 5\end{array}$ & $\begin{array}{r}0 \mathrm{H}-560 \\
\text { DLw] } \\
10 / 06 / 95\end{array}$ & 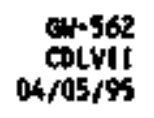 & $\begin{array}{r}61-562 \\
10 L W 11 \\
10 / 06 / 45\end{array}$ & $\begin{array}{r}\text { cu-564 } \\
\text { colvil } \\
04 / 06 / 95\end{array}$ & $\begin{array}{r}\text { ch-s4t } \\
\text { colvit } \\
10 / 07 / 93\end{array}$ \\
\hline carlows (NEO/L) & & & & & & & & \\
\hline 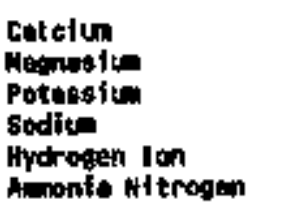 & $\begin{array}{l}1.05 \\
1.01 \\
0.04 \\
0.03 \\
0.00 \\
0.00\end{array}$ & $\begin{array}{l}1.80 \\
1.65 \\
0.02 \\
0.03 \\
0.00 \\
0.00\end{array}$ & $\begin{array}{l}1.00 \\
1.23 \\
0.04 \\
0.03 \\
0.00 \\
0.00\end{array}$ & $\begin{array}{l}1.00 \\
1.23 \\
0.05 \\
0.03 \\
0.00 \\
0.00\end{array}$ & $\begin{array}{l}2.00 \\
1.97 \\
0.00 \\
0.06 \\
0.00 \\
0.09\end{array}$ & $\begin{array}{l}1.90 \\
1.01 \\
0.05 \\
0.04 \\
0.00 \\
0.00\end{array}$ & $\begin{array}{l}1.35 \\
1.23 \\
0.05 \\
0.02 \\
0.00 \\
0.00\end{array}$ & $\begin{array}{l}1.40 \\
0.99 \\
0.04 \\
0.05 \\
0.00 \\
0.00\end{array}$ \\
\hline Total Cationo & 3.73 & 3.49 & 3.21 & 3.08 & 4.01 & $3 . \pi$ & 2,24 & 2.46 \\
\hline (MEOA) & & & & & & & & \\
\hline 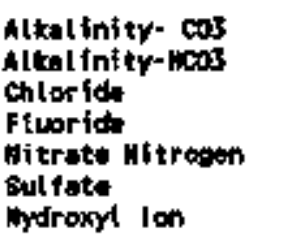 & $\begin{array}{l}0.00 \\
3.30 \\
0.08 \\
0.00 \\
0.08 \\
0.04 \\
0.00\end{array}$ & $\begin{array}{l}0.00 \\
3.36 \\
0.05 \\
0.00 \\
0.07 \\
0.03 \\
0.00\end{array}$ & $\begin{array}{l}0.00 \\
2.94 \\
0.05 \\
0.00 \\
0.03 \\
0.04 \\
0.00\end{array}$ & $\begin{array}{l}0.00 \\
3.00 \\
0.04 \\
0.00 \\
0.02 \\
0.04 \\
0.00\end{array}$ & $\begin{array}{l}0.00 \\
3.68 \\
0.06 \\
0.00 \\
0.04 \\
0.04 \\
0.00\end{array}$ & $\begin{array}{l}0.00 \\
3.62 \\
0.05 \\
0.00 \\
0.04 \\
0.04 \\
0.00\end{array}$ & $\begin{array}{l}0.00 \\
2.09 \\
0.05 \\
0.00 \\
0.05 \\
0.03 \\
0.00\end{array}$ & $\begin{array}{l}0.00 \\
2.32 \\
0.07 \\
0.00 \\
0.06 \\
0.10 \\
0.00\end{array}$ \\
\hline Totel intions & 3.55 & 3.50 & 3.06 & 3.10 & 3.65 & 3.75 & 2.93 & 2.55 \\
\hline CHAHEE aALAHCE (x) & $z$ & -0 & 2 & -0 & 2 & 0 & -2 & -1 \\
\hline 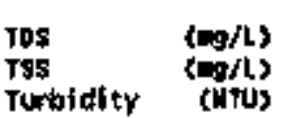 & $\begin{array}{r}190 \\
5 \\
4.4\end{array}$ & $\begin{array}{r}158 \\
1.5\end{array}$ & $\begin{array}{r}140 \\
\times 1 \\
0.3\end{array}$ & $\begin{array}{r}152 \\
41 \\
1.3\end{array}$ & $\begin{array}{r}19.4 \\
9.5\end{array}$ & $\begin{array}{r}194 \\
5 \\
0.9\end{array}$ & $\begin{array}{r}174 \\
\times 1 \\
1\end{array}$ & $\begin{array}{l}128 \\
1,5 \\
1.4\end{array}$ \\
\hline
\end{tabular}


APPENDIX I

Ion-Charge Balenes Colculationa, 1995

\begin{tabular}{|c|c|c|c|c|c|c|c|c|}
\hline $\begin{array}{l}\text { samtind polnt } \\
\text { Locution } \\
\text { Dot: sompled }\end{array}$ & $\begin{array}{r}\mathrm{cu}+608 \\
\text { cRsp } \\
02 / 02 / \% 5\end{array}$ & $\begin{array}{r}\text { GN-cos } \\
\text { CRsp } \\
\text { os/11/25 }\end{array}$ & $\begin{array}{r}01-605 \\
\text { CRsp } \\
07 / 31 / 95\end{array}$ & $\begin{array}{l}\text { ar.tops } \\
\text { chsp } \\
11 / 16 / 95\end{array}$ & $\begin{array}{r}6-609 \\
\text { CRSP } \\
02 / 15 / 95\end{array}$ & $\begin{array}{r}c+609 \\
\cos s p \\
05 / t 6 / 95\end{array}$ & $\begin{array}{r}40-60 \% \\
\text { cosp } \\
00 / 01 / 95\end{array}$ & $\begin{array}{r}64-609 \\
\text { Chsp } \\
11 / 20 / 95\end{array}$ \\
\hline \multicolumn{9}{|l|}{ chrtons (weo/h) } \\
\hline $\begin{array}{l}\text { Calcium } \\
\text { Mognestion } \\
\text { Potessitem } \\
\text { sodiun } \\
\text { aydrowion ion }\end{array}$ & $\begin{array}{l}1.55 \\
1.48 \\
0.02 \\
0.03 \\
0.00\end{array}$ & $\begin{array}{l}4.50 \\
1.40 \\
0.04 \\
0.05 \\
0.00\end{array}$ & $\begin{array}{l}1.50 \\
1.48 \\
0.06 \\
0.04 \\
0.00\end{array}$ & $\begin{array}{l}1.65 \\
1.65 \\
0.02 \\
0.05 \\
0.00\end{array}$ & $\begin{array}{l}1.95 \\
1.59 \\
0.04 \\
0.04 \\
0.00\end{array}$ & $\begin{array}{l}2.05 \\
1.09 \\
0.03 \\
0.03 \\
0.00\end{array}$ & $\begin{array}{l}2.15 \\
2.16 \\
0.02 \\
0.03 \\
0.00\end{array}$ & $\begin{array}{l}1.90 \\
1.97 \\
0.03 \\
0.03 \\
0.00\end{array}$ \\
\hline Animila 너 troosen & $\cdot$ & • & $\bullet$ & - & • & $\cdot$ & $\bullet$ & - \\
\hline Total totions & 3.08 & 2.96 & 3.07 & 3.34 & 3.92 & 4.00 & 4.34 & 3.94 \\
\hline \multicolumn{9}{|l|}{ Awlous (NEQ/L) } \\
\hline 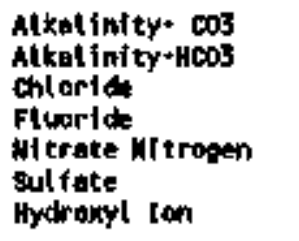 & $\begin{array}{l}0.00 \\
3.06 \\
0.07 \\
0.00 \\
0.05 \\
0.05 \\
0.00\end{array}$ & $\begin{array}{l}0.00 \\
2.04 \\
0.06 \\
0.00 \\
0.03 \\
0.05 \\
0.00\end{array}$ & $\begin{array}{l}0.00 \\
2.90 \\
0.07 \\
0.00 \\
0.05 \\
0.06 \\
0.00\end{array}$ & $\begin{array}{l}0.00 \\
3.10 \\
0.07 \\
0.00 \\
0.00 \\
0.05 \\
0.00\end{array}$ & $\begin{array}{l}0.00 \\
3.92 \\
0.05 \\
0.00 \\
0.09 \\
0.05 \\
0.00\end{array}$ & $\begin{array}{l}0.00 \\
4.05 \\
0.04 \\
0.00 \\
0.08 \\
0.05 \\
0.00\end{array}$ & $\begin{array}{l}0.00 \\
3.94 \\
0.06 \\
0.00 \\
0.09 \\
0.05 \\
0.00\end{array}$ & $\begin{array}{l}0.00 \\
4.04 \\
0.06 \\
0.00 \\
0.08 \\
0.04 \\
0.00\end{array}$ \\
\hline Totul Anions & 3.24 & 3.12 & 3.07 & 3.30 & 4.12 & 4.22 & 4.14 & 4.22 \\
\hline COARES HLAACE $(z)$ & -3 & -3 & -0 & 1 & -2 & -3 & 2 & -3 \\
\hline $\begin{array}{ll}\text { TDS } & (\omega, / L) \\
\text { TSs } & \left(\omega_{0} / L\right) \\
\text { Turbidity } & \text { (WTU) }\end{array}$ & $\begin{array}{l}178 \\
1 \\
1.7\end{array}$ & $\begin{array}{r}176 \\
3 \\
5.5\end{array}$ & $\begin{array}{r}132 \\
\times 3 \\
2,2\end{array}$ & $\begin{array}{r}149 \\
5 \\
5.1\end{array}$ & $\begin{array}{r}212 \\
1 \\
0.3\end{array}$ & $\begin{array}{r}216 \\
\times 1 \\
0.6\end{array}$ & $\begin{array}{r}212 \\
0.1\end{array}$ & $\begin{array}{r}202 \\
0.3\end{array}$ \\
\hline
\end{tabular}


Ion-Cherot tellence Caleulations, $19 \% 5$

\begin{tabular}{|c|c|c|c|c|c|c|c|c|}
\hline $\begin{array}{l}\text { stanting Foint } \\
\text { tocation } \\
\text { Dote sapled }\end{array}$ & $\begin{array}{r}6 N-610 \\
\text { CASP } \\
0 t / 31 / \%\end{array}$ & $\begin{array}{r}\text { U-615 } \\
\text { CRes } \\
\text { 05/07/O5 }\end{array}$ & $\begin{array}{r}04-610 \\
\text { casp } \\
07 / 31 / 95\end{array}$ & $\begin{array}{r}0 x+610 \\
t+\$ p \\
11 / 13 / \% 5\end{array}$ & $\begin{array}{r}0.611 \\
\text { chis } \\
02 / 14 / 95\end{array}$ & $\begin{array}{r}\text { w-6\$1 } \\
\text { atsp } \\
05 / 15 / 95\end{array}$ & $\begin{array}{r}\text { en-611 } \\
\text { cisp } \\
0901 / 95\end{array}$ & $\begin{array}{r}\text { ciN-611 } \\
\text { CR5P } \\
11 / 17 / \% s\end{array}$ \\
\hline \multicolumn{9}{|l|}{ SATIONS (MEO/L) } \\
\hline 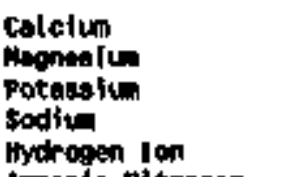 & $\begin{array}{l}2.15 \\
2.14 \\
0.02 \\
0.04 \\
0.00\end{array}$ & $\begin{array}{l}2.40 \\
2.30 \\
0.02 \\
0.05 \\
0.00\end{array}$ & $\begin{array}{l}2.15 \\
2.22 \\
0.00 \\
0.04 \\
0.00\end{array}$ & $\begin{array}{l}2.25 \\
2.30 \\
0.03 \\
0.06 \\
0.00\end{array}$ & $\begin{array}{l}2.84 \\
2.71 \\
0.03 \\
0.10 \\
0.00\end{array}$ & $\begin{array}{l}2.04 \\
2.63 \\
0.07 \\
0.09 \\
0.00\end{array}$ & $\begin{array}{l}2.76 \\
2.80 \\
0.00 \\
0.05 \\
0.00\end{array}$ & $\begin{array}{l}2.69 \\
2.71 \\
0.03 \\
0.27 \\
0.00\end{array}$ \\
\hline Anonta Aitrogen & $\cdot$ & $\cdot$ & $\cdot$ & $\cdot$ & - & $\cdot$ & ' & - \\
\hline Total Cetions & 4.54 & 4.77 & 4,40 & 4.62 & 5.68 & 5.63 & 5.62 & S.71 \\
\hline \multicolumn{9}{|l|}{ nintons (1;0/h) } \\
\hline 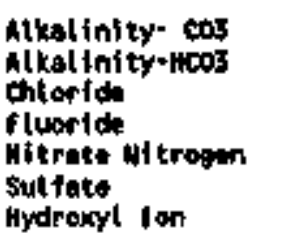 & $\begin{array}{l}0.00 \\
4.54 \\
0.00 \\
0.00 \\
0.07 \\
0.03 \\
0.00\end{array}$ & $\begin{array}{l}0.00 \\
4.54 \\
0.08 \\
0.00 \\
0.06 \\
0.00 \\
0.00\end{array}$ & $\begin{array}{l}0.00 \\
4.42 \\
0.08 \\
0.00 \\
0.06 \\
0.00 \\
0.00\end{array}$ & $\begin{array}{l}0.00 \\
4.54 \\
0.04 \\
0.00 \\
0.06 \\
0.00 \\
0.00\end{array}$ & $\begin{array}{l}9.00 \\
5.44 \\
0.05 \\
0.00 \\
0.24 \\
0.04 \\
0.00\end{array}$ & $\begin{array}{l}0.00 \\
5.64 \\
0.04 \\
0.00 \\
0.24 \\
0.05 \\
0.00\end{array}$ & $\begin{array}{l}0.00 \\
5.32 \\
0.06 \\
0.00 \\
0.75 \\
0.05 \\
0.00\end{array}$ & $\begin{array}{l}0.00 \\
5.26 \\
0.09 \\
0.00 \\
0.25 \\
0.12 \\
0.00\end{array}$ \\
\hline Totel Antiont & 4.72 & 4.68 & 4.58 & 4,6 & 5.77 & 5.44 & 5.66 & 3.70 \\
\hline CHARGE BALAMCE (X) & -4 & 1 & -2 & -1 & -1 & -3 & -0 & 0 \\
\hline $\begin{array}{ll}\text { Tos } & \text { (ng/L) } \\
\text { TSS } & \text { (ng/l) } \\
\text { Turblall or } & \text { (fitu) }\end{array}$ & $\begin{array}{r}210 \\
1 \\
0.8\end{array}$ & $\begin{array}{r}242 \\
1 \\
1.6\end{array}$ & $\begin{array}{r}225 \\
1 \\
0.5\end{array}$ & $\begin{array}{r}92 \\
0.3\end{array}$ & $\begin{array}{r}274 \\
\times 1 \\
0.8\end{array}$ & $\begin{array}{r}332 \\
11 \\
1.2\end{array}$ & $\begin{array}{r}226 \\
1.5 \\
15\end{array}$ & $\begin{array}{r}206 \\
6 \\
6.9\end{array}$ \\
\hline
\end{tabular}


Jon-charge Belance Colculations, $19 \% 5$

\begin{tabular}{|c|c|c|c|c|c|c|c|c|c|c|}
\hline $\begin{array}{l}\text { seppling Potnt } \\
\text { coction } \\
\text { Dote stipled }\end{array}$ & $\begin{array}{r}\text { 6u-70s } \\
\text { LII } \\
01 / 00 / 95\end{array}$ & $\begin{array}{r}4-70 \% \\
\text { t11 } \\
07 / 10 / 9 \%\end{array}$ & $\begin{array}{r}0 w-709 \\
411 \\
10 / 06 / 95\end{array}$ & $\begin{array}{r}n+73 t \\
01 / 7100\end{array}$ & $\begin{array}{r}5 \cdot 751 \\
\text { cospd } \\
04 / 07 / \% 5\end{array}$ & $\begin{array}{r}4 N-731 \\
\text { CRston } \\
07 / 13 / 95\end{array}$ & $\begin{array}{r}\text { c1-731 } \\
\text { chios } \\
10 / 23 / 95\end{array}$ & $\begin{array}{r}04+731 \\
0 \neq 508 \\
10 / 24 / 95\end{array}$ & $\begin{array}{r}\text { ches } \\
\text { t0/25/\% }\end{array}$ & $\begin{array}{r}n-731 \\
0+2604 \\
70 / 26 / 85\end{array}$ \\
\hline \multicolumn{11}{|l|}{ cattoin (IEo/) } \\
\hline 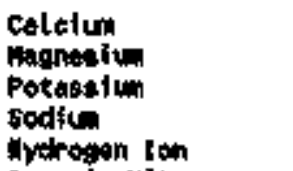 & $\begin{array}{l}1.90 \\
1.73 \\
0.03 \\
0.02 \\
0.00\end{array}$ & $\begin{array}{l}1.75 \\
1.75 \\
0.00 \\
0.03 \\
0.00\end{array}$ & $\begin{array}{l}1.45 \\
1.89 \\
0.03 \\
0.03 \\
0.00\end{array}$ & $\begin{array}{l}0.65 \\
0.19 \\
0.99 \\
0.29 \\
0.00\end{array}$ & $\begin{array}{l}0.85 \\
1.07 \\
0.25 \\
0.16 \\
0.00\end{array}$ & $\begin{array}{l}0.75 \\
1.56 \\
0.59 \\
0.22 \\
0.00\end{array}$ & $\begin{array}{l}1.05 \\
1.23 \\
0.14 \\
0.12 \\
0.00\end{array}$ & $\begin{array}{l}0.70 \\
1.07 \\
0.31 \\
0.17 \\
0.00\end{array}$ & $\begin{array}{l}0.60 \\
1.07 \\
0.36 \\
0.19 \\
0.00\end{array}$ & $\begin{array}{l}0.47 \\
1.07 \\
0.41 \\
0.20 \\
0.00\end{array}$ \\
\hline Anonia witregen & - & 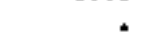 & - & . & . & 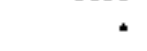 & - & - & + & , \\
\hline Iotek cottons & 3.68 & 3.50 & 3.60 & 2.71 & 2.36 & 3.12 & 2.56 & 2.25 & 2.22 & 2.14 \\
\hline nitows (wed/) & & & & & & & & & & \\
\hline 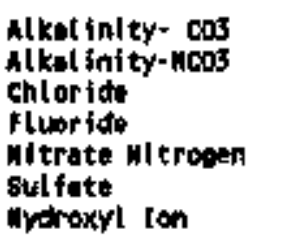 & $\begin{array}{l}0.00 \\
3.54 \\
0.06 \\
0.00 \\
0.04 \\
0.04 \\
0.00\end{array}$ & $\begin{array}{l}0.00 \\
3.42 \\
0.05 \\
0.00 \\
0.03 \\
0.03 \\
0.00\end{array}$ & $\begin{array}{l}0.00 \\
3.52 \\
0.05 \\
0.00 \\
0.02 \\
0.06 \\
0.00\end{array}$ & $\begin{array}{l}0.12 \\
1.94 \\
0.00 \\
0.00 \\
0.05 \\
0.15 \\
0.01\end{array}$ & $\begin{array}{l}0.36 \\
2.02 \\
0.05 \\
0.01 \\
0.05 \\
0.12 \\
0.01\end{array}$ & $\begin{array}{l}0.76 \\
2.04 \\
0.03 \\
0.00 \\
0.04 \\
0.10 \\
0.03\end{array}$ & $\begin{array}{l}0.00 \\
2.24 \\
0.08 \\
0.00 \\
0.05 \\
0.00 \\
0.01\end{array}$ & $\begin{array}{l}0.20 \\
1.24 \\
0.01 \\
0.00 \\
0.03 \\
0.11 \\
0.01\end{array}$ & $\begin{array}{l}0.40 \\
1.69 \\
0.04 \\
0.00 \\
0.03 \\
0.11 \\
0.01\end{array}$ & $\begin{array}{l}0.32 \\
1.60 \\
0.04 \\
0.00 \\
0.03 \\
0.12 \\
0.02\end{array}$ \\
\hline Total Anino & 3.68 & 3.54 & 3.64 & 2.50 & 2.62 & 3.02 & 2.41 & 2.22 & 2.21 & 2.20 \\
\hline CHARGE GALAHCE $(x)$ & -0 & -1 & -3 & 4 & -5 & 2 & 3 & $\dagger$ & 0 & -1 \\
\hline $\begin{array}{ll}\text { Tos } & (\operatorname{mg} / L) \\
\text { TS8 } & (\operatorname{mg} / L) \\
\text { Turbtdt tr } & \text { (HIU) }\end{array}$ & $\begin{array}{r}+50 \\
\times 1 \\
0.7\end{array}$ & $\begin{array}{r}200 \\
0.4\end{array}$ & $\begin{array}{r}172 \\
48 \\
25\end{array}$ & $\begin{array}{l}94 \\
26 \\
26\end{array}$ & $\begin{array}{r}148 \\
41 \\
2.7\end{array}$ & $\begin{array}{r}182 \\
2 \\
0.35\end{array}$ & $\begin{array}{r}150 \\
4.5 \\
13\end{array}$ & $\begin{array}{r}146 \\
3 \\
7\end{array}$ & $\begin{array}{r}142 \\
9 \\
33\end{array}$ & $\begin{array}{r}136 \\
51 \\
5.2\end{array}$ \\
\hline
\end{tabular}


Appeno IX I

Jon-Chorge oetence Gelculations, 1995

\begin{tabular}{|c|c|c|c|c|c|c|c|c|}
\hline $\begin{array}{l}\text { sapling Point } \\
\text { location } \\
\text { bate sicpled }\end{array}$ & $\begin{array}{r}\text { EN-737 } \\
\text { CRSTi } \\
01 / 48 / 45\end{array}$ & $\begin{array}{r}\text { an-732 } \\
\text { costis } \\
04 / 07 / 65\end{array}$ & $\begin{array}{r}6+732 \\
07+13 / 95\end{array}$ & $\begin{array}{r}\text { ru-732 } \\
\text { ctstes } \\
08 / 14 / 95\end{array}$ & $\begin{array}{r}\text { 6N-732 } \\
\text { GR:P5 } \\
10 / 23 / 95\end{array}$ & $\begin{array}{r}\text { ad-732 } \\
\text { CRsol } \\
10 / 24 / 95\end{array}$ & $\begin{array}{r}61732 \\
\text { ch508 } \\
10 / 25 / 95\end{array}$ & $\begin{array}{r}\text { an-732 } \\
\text { calspB } \\
10 / 26 / 45\end{array}$ \\
\hline rattows (MEO/L) & & & & & & & & \\
\hline $\begin{array}{l}\text { Coletiun } \\
\text { Mophestum } \\
\text { Poteosilum } \\
\text { sodiun } \\
\text { Hydropen jon }\end{array}$ & $\begin{array}{l}1.45 \\
1.23 \\
0.25 \\
0.48 \\
0.00\end{array}$ & $\begin{array}{l}1.25 \\
1.07 \\
0.23 \\
0.52 \\
0.00\end{array}$ & $\begin{array}{l}1.05 \\
1.15 \\
0.25 \\
0.34 \\
0.00\end{array}$ & $\begin{array}{l}1.45 \\
1.48 \\
0.05 \\
0.04 \\
0.00\end{array}$ & $\begin{array}{l}1.55 \\
1.56 \\
0.06 \\
0.06 \\
0.00\end{array}$ & $\begin{array}{l}0.37 \\
0.10 \\
0.84 \\
0.37 \\
0.00\end{array}$ & $\begin{array}{l}0.61 \\
0.75 \\
0.61 \\
0.30 \\
0.00\end{array}$ & $\begin{array}{l}0.35 \\
0.90 \\
0.56 \\
0.26 \\
0.00\end{array}$ \\
\hline Mmonte Witrogen & $\star$ & • & $\cdot$ & • & $*$ & $\star$ & $\cdot$ & $\cdot$ \\
\hline Totel Cotions & 3.41 & 3.06 & 2.82 & 3.01 & 3.10 & 1,69 & 2.08 & 2.07 \\
\hline MHows (EEO/L) & & & & & & & & \\
\hline 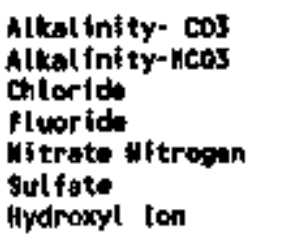 & $\begin{array}{l}0.02 \\
3.24 \\
0.04 \\
0.00 \\
0.04 \\
0.04 \\
0.00\end{array}$ & $\begin{array}{l}0.00 \\
3.14 \\
0.05 \\
0.00 \\
0.05 \\
0.06 \\
0.00\end{array}$ & $\begin{array}{l}1.52 \\
0.55 \\
0.05 \\
0.00 \\
0.05 \\
0.10 \\
0.10\end{array}$ & $\begin{array}{l}0.00 \\
3.12 \\
0.04 \\
0.00 \\
0.07 \\
0.04 \\
0.06\end{array}$ & $\begin{array}{l}0.00 \\
2.99 \\
0.05 \\
0.00 \\
0.02 \\
0.03 \\
0.00\end{array}$ & $\begin{array}{l}2.07 \\
0.00 \\
0.05 \\
0.00 \\
0.03 \\
0.07 \\
3.16\end{array}$ & $\begin{array}{l}2.94 \\
0.00 \\
0.06 \\
0.00 \\
0.04 \\
0.08 \\
1.58\end{array}$ & $\begin{array}{l}2.40 \\
0.00 \\
0.04 \\
0.00 \\
0.03 \\
0.07 \\
0.16\end{array}$ \\
\hline Fotal infien: & 3.38 & 3.29 & 2.40 & 3.27 & 3.07 & 6.22 & 4.71 & 2.70 \\
\hline CHARGE DALAMCE $(X)$ & 0 & +4 & 4 & +4 & 2 & -57 & -39 & -13 \\
\hline $\begin{array}{ll}\text { tos } & (\mathrm{m} / \mathrm{t}) \\
\text { T8s } & (\mathrm{mg} / \mathrm{t}) \\
\text { Turbidt ty } & \text { (utu) }\end{array}$ & $\begin{array}{l}166 \\
020 \\
180\end{array}$ & $\begin{array}{r}200 \\
1152 \\
300\end{array}$ & $\begin{array}{r}330 \\
1045 \\
200\end{array}$ & $\begin{array}{r}154 \\
21 \\
2.8\end{array}$ & $\begin{array}{l}164 \\
2.5 \\
1.4\end{array}$ & $\begin{array}{r}160 \\
70 \\
19\end{array}$ & $\begin{array}{r}130 \\
32 \\
28\end{array}$ & $\begin{array}{r}139 \\
41 \\
38\end{array}$ \\
\hline
\end{tabular}


Jom-charge Balance talculetlans, 1995

\begin{tabular}{|c|c|c|c|c|c|c|c|c|}
\hline $\begin{array}{l}\text { sempling polnt } \\
\text { Locsetion } \\
\text { Date 8spled }\end{array}$ & $\begin{array}{r}04-742 \\
\operatorname{crsp} \\
0 t / 27 / 95\end{array}$ & $\begin{array}{r}0 \mathrm{TH}+742 \\
\operatorname{crsp} \\
05 / 07 / 05\end{array}$ & $\begin{array}{r}40-742 \\
\operatorname{Cr} 89 \\
07 / 30 / 95\end{array}$ & $\begin{array}{r}6-742 \\
\operatorname{cosp} \\
11 / 09 / 95\end{array}$ & $\begin{array}{r}\text { w-743 } \\
\text { coss } \\
01 / 51 / 05\end{array}$ & $\begin{array}{r}0 w-743 \\
\text { Chsp } \\
05 / 07 / 95\end{array}$ & $\begin{array}{r}4.745 \\
\text { cRtsp } \\
07 / 51 / \% 3\end{array}$ & $\begin{array}{r}\text { ol-743 } \\
\text { CRsp } \\
11 / 13 / 95\end{array}$ \\
\hline \multicolumn{9}{|l|}{ Cattows (neo/t) } \\
\hline 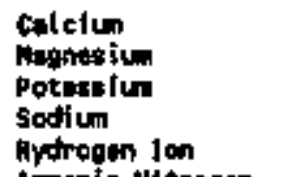 & $\begin{array}{l}1.90 \\
1.81 \\
0.03 \\
0.03 \\
0.00\end{array}$ & $\begin{array}{l}1.90 \\
1.75 \\
0.03 \\
0.03 \\
0.01\end{array}$ & $\begin{array}{l}1.73 \\
1.73 \\
0.03 \\
0.03 \\
0.00\end{array}$ & $\begin{array}{l}1.00 \\
1.09 \\
0.05 \\
0.03 \\
0.00\end{array}$ & $\begin{array}{l}1.55 \\
1.46 \\
0.03 \\
0.04 \\
0.00\end{array}$ & $\begin{array}{l}1.55 \\
1.48 \\
0.04 \\
0.05 \\
0.00\end{array}$ & $\begin{array}{l}1.50 \\
1.56 \\
0.02 \\
0.06 \\
0.00\end{array}$ & $\begin{array}{l}1.55 \\
1.56 \\
0.06 \\
0.05 \\
0.00\end{array}$ \\
\hline monfe Witrogen & - & • & - & - & . & ? & • & • \\
\hline Total cations & 3.77 & 3.69 & 3.53 & 3.67 & $\$ .10$ & 3.11 & 3.13 & 3.21 \\
\hline \multicolumn{9}{|l|}{ Antows (WEQVL) } \\
\hline 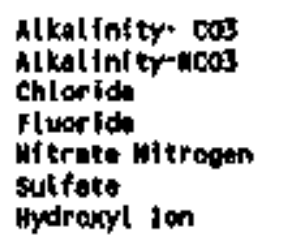 & $\begin{array}{l}0.00 \\
3.34 \\
0.05 \\
0.00 \\
0.00 \\
0.25 \\
0.00\end{array}$ & $\begin{array}{l}0.00 \\
3.50 \\
0.00 \\
0.00 \\
0.00 \\
0.27 \\
0.00\end{array}$ & $\begin{array}{l}0.00 \\
3.30 \\
0.05 \\
0.00 \\
0.00 \\
0.27 \\
0.00\end{array}$ & $\begin{array}{l}0.00 \\
3.40 \\
0.04 \\
0.00 \\
0.00 \\
0.26 \\
0.00\end{array}$ & $\begin{array}{l}0.00 \\
2.86 \\
0.08 \\
0.00 \\
0.00 \\
0.10 \\
0.00\end{array}$ & $\begin{array}{l}0.00 \\
2.90 \\
0.06 \\
0.00 \\
0.06 \\
0.07 \\
0.00\end{array}$ & $\begin{array}{l}0.00 \\
2.98 \\
0.06 \\
0.00 \\
0.04 \\
0.07 \\
0.00\end{array}$ & $\begin{array}{l}0.00 \\
2.92 \\
0.06 \\
0.00 \\
0.07 \\
0.08 \\
0.00\end{array}$ \\
\hline Total Antone & 3.64 & 3.81 & 3.70 & 3.70 & 3.12 & 3.09 & 3.17 & 3.14 \\
\hline CBARE BALAHCE ( $\mathrm{H}$ ) & 2 & -2 & -2 & -0 & -0 & 0 & -1 & 1 \\
\hline $\begin{array}{ll}\text { tos } & (m / L) \\
\text { TSs } & (\operatorname{mo/L}) \\
\text { Turbidity } & \text { (tTu) }\end{array}$ & $\begin{array}{r}188 \\
2 \\
15\end{array}$ & $\begin{array}{r}990 \\
21 \\
2.4\end{array}$ & $\begin{array}{r}196 \\
1 \\
13\end{array}$ & $\begin{array}{r}190 \\
11 \\
10\end{array}$ & $\begin{array}{r}186 \\
41 \\
1.4\end{array}$ & $\begin{array}{r}168 \\
1 \\
1\end{array}$ & $\begin{array}{r}152 \\
41 \\
1\end{array}$ & $\begin{array}{r}106 \\
41 \\
0.65\end{array}$ \\
\hline
\end{tabular}


APPE:DIX 1

ton-cheres bilence Colculations, 1995

\begin{tabular}{|c|c|c|c|c|c|c|c|c|c|}
\hline $\begin{array}{l}\text { sempling Point } \\
\text { Locetion } \\
\text { Ootte 8mpled }\end{array}$ & $\begin{array}{r}04-757 \\
\text { LII } \\
09100 / 95\end{array}$ & $\begin{array}{r}\text { ah- } 757 \\
\text { LII } \\
07 / 11 / 95\end{array}$ & $\begin{array}{r}04-757 \\
411 \\
10 / 06 / 95\end{array}$ & $\begin{array}{r}4-796 \\
L V \\
0,12 / 45\end{array}$ & $\begin{array}{r}0.7 \% 6 \\
10 / 00 / \% 5\end{array}$ & $\begin{array}{r}\text { Gh-797 } \\
\text { Ly }\end{array}$ & $\begin{array}{r}\text { LU } 7 y \\
10 / 00 / \% s\end{array}$ & $\begin{array}{r}\text { ert-7sa } \\
\text { CoLvi } \\
04 / 11 / 45\end{array}$ & $\begin{array}{r}\text { c14-798 } \\
\text { cDLVII } \\
10 / 0 \text { - } / 95\end{array}$ \\
\hline CATIOWS (NEO/L) & & & & & & & & & \\
\hline 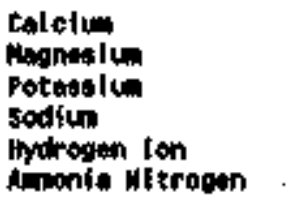 & $\begin{array}{l}1.65 \\
1.40 \\
0.04 \\
0.06 \\
0.00 \\
.\end{array}$ & $\begin{array}{l}1.50 \\
1.40 \\
0.07 \\
0.11 \\
0.00 \\
.\end{array}$ & $\begin{array}{l}1.65 \\
1.65 \\
0.05 \\
0.22 \\
0.00\end{array}$ & $\begin{array}{l}1.00 \\
1.15 \\
0.07 \\
0.05 \\
0.00 \\
0.00\end{array}$ & $\begin{array}{l}1.10 \\
1.15 \\
0.07 \\
0.04 \\
0.00 \\
0.00\end{array}$ & $\begin{array}{l}1.70 \\
1.65 \\
0.05 \\
0.05 \\
0.00 \\
0.00\end{array}$ & $\begin{array}{l}1.55 \\
1.60 \\
0.02 \\
0.05 \\
0.00 \\
0.00\end{array}$ & $\begin{array}{l}1.40 \\
1.40 \\
0.04 \\
0.03 \\
0.00 \\
0.00\end{array}$ & $\begin{array}{l}1.35 \\
1.25 \\
0.04 \\
0.05 \\
0.00 \\
0.00\end{array}$ \\
\hline Totel cotions & 3.14 & 3.08 & 3.56 & 2.27 & 2.37 & 3.41 & 3.10 & 2.86 & 2.66 \\
\hline Antows (NES/L) & & & & & & & & & \\
\hline 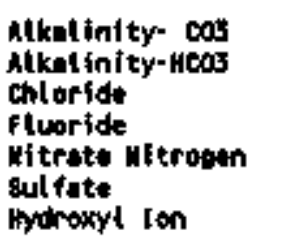 & $\begin{array}{l}0.00 \\
2.82 \\
0.07 \\
0.11 \\
0.00 \\
0.21 \\
0.00\end{array}$ & $\begin{array}{l}0.00 \\
2.02 \\
0.07 \\
0.09 \\
0.02 \\
0.23 \\
0.00\end{array}$ & $\begin{array}{l}0.00 \\
3.26 \\
0.06 \\
0.00 \\
0.02 \\
0.20 \\
0.00\end{array}$ & $\begin{array}{l}0.00 \\
2.25 \\
0.06 \\
0.00 \\
0.04 \\
0.04 \\
0.00\end{array}$ & $\begin{array}{l}0.00 \\
2.36 \\
0.05 \\
0.00 \\
0.02 \\
0.05 \\
0.00\end{array}$ & $\begin{array}{l}0.00 \\
3.18 \\
0.05 \\
0.09 \\
0.04 \\
0.04 \\
0.00\end{array}$ & $\begin{array}{l}0.00 \\
3.02 \\
0.04 \\
0.00 \\
0.03 \\
0.05 \\
0.00\end{array}$ & $\begin{array}{l}0.00 \\
2.56 \\
0.09 \\
0.00 \\
0.07 \\
0.06 \\
0.00\end{array}$ & $\begin{array}{l}0.00 \\
2.66 \\
0.06 \\
0.00 \\
0.06 \\
0.05 \\
0.00\end{array}$ \\
\hline Totel mions & 3.21 & 3.23 & 3.62 & 2.42 & 2.46 & 3.30 & 3.16 & 2.77 & 2.60 \\
\hline CHAR日e Baldince $(x)$ & .1 & -3 & -9 & -3 & -2 & 2 & -1 & 2 & -3 \\
\hline $\begin{array}{ll}\text { IOS } & (m / L) \\
\text { TSs } & \text { (WT/L) } \\
\text { Turbidity } & \text { (WTU) }\end{array}$ & $\begin{array}{r}140 \\
4 \\
2.3\end{array}$ & $\begin{array}{r}164 \\
1.7 \\
1.7\end{array}$ & 200 & $\begin{array}{r}144 \\
1 \\
1.5\end{array}$ & $\begin{array}{r}140 \\
4.3\end{array}$ & $\begin{array}{l}104 \\
9.5 \\
2.5\end{array}$ & $\begin{array}{r}300 \\
1 \\
1.7\end{array}$ & $\begin{array}{r}164 \\
11 \\
0.8\end{array}$ & $\begin{array}{r}124 \\
<1 \\
0.65\end{array}$ \\
\hline
\end{tabular}


APFinix 1

Jor-Chors balence Colculations, ips

\begin{tabular}{|c|c|c|c|c|c|c|c|}
\hline $\begin{array}{l}\text { smal ing polnt } \\
\text { Lotat im } \\
\text { onte stimpled }\end{array}$ & $\begin{array}{r}104-799 \\
04 / 06 / 95\end{array}$ & $\begin{array}{r}14-79 ? \\
10 / 07 / 95\end{array}$ & $\begin{array}{r}04-801 \\
2 v \\
04 / 13 / 95\end{array}$ & $\begin{array}{r}\text { 6n- } 0101 \\
\text { Ly } \\
10 / 09 / 45\end{array}$ & 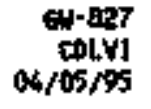 & $\begin{array}{r}a+827 \\
\cos v 1 \\
10 / 16 / 45\end{array}$ & $\begin{array}{r}\text { selve.2sp } \\
\text { cesp } \\
01 / 15 / 95\end{array}$ \\
\hline \multicolumn{8}{|l|}{ CAIJWMS (nEO/L) } \\
\hline 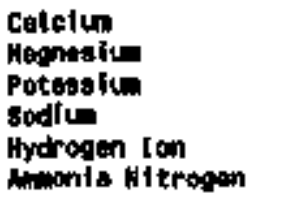 & $\begin{array}{l}1.53 \\
1.32 \\
0.02 \\
0.08 \\
0.09 \\
0.00\end{array}$ & $\begin{array}{l}1.53 \\
1.25 \\
0.02 \\
0.04 \\
0.00 \\
0.00\end{array}$ & $\begin{array}{l}1.45 \\
1.40 \\
0.03 \\
0.03 \\
0.00 \\
0.00\end{array}$ & $\begin{array}{l}1.45 \\
1.40 \\
0.02 \\
0.03 \\
0.00 \\
0.00\end{array}$ & $\begin{array}{l}1.50 \\
1.40 \\
0.03 \\
0.03 \\
0.00 \\
0.00\end{array}$ & $\begin{array}{l}1.45 \\
1.40 \\
0.03 \\
0.04 \\
0.00 \\
0.00\end{array}$ & $\begin{array}{l}2.40 \\
0.58 \\
0.02 \\
0.11 \\
0.00 \\
.\end{array}$ \\
\hline Totet Cotions & 2.72 & 2.66 & 2,99 & 2, & $2 . \$ 8$ & 2.91 & 3.10 \\
\hline \multicolumn{8}{|l|}{ Anlous (nto/h) } \\
\hline 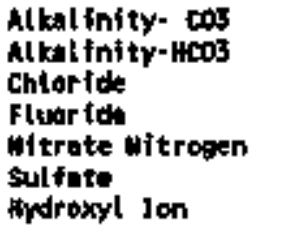 & $\begin{array}{l}0.00 \\
2.58 \\
0.05 \\
0.00 \\
0.09 \\
0.03 \\
0.00\end{array}$ & $\begin{array}{l}0.00 \\
2.64 \\
0.04 \\
0.01 \\
0.07 \\
0.03 \\
0.00\end{array}$ & $\begin{array}{l}0.00 \\
2.06 \\
0.08 \\
0.00 \\
0.02 \\
0.06 \\
0.00\end{array}$ & $\begin{array}{l}0.00 \\
2.90 \\
0.05 \\
0.00 \\
0.02 \\
0.05 \\
0.00\end{array}$ & $\begin{array}{l}0.00 \\
2.04 \\
0.05 \\
0.00 \\
0.02 \\
0.04 \\
0.00\end{array}$ & $\begin{array}{l}0.00 \\
2.80 \\
0.04 \\
0.00 \\
0.08 \\
0.04 \\
0.00\end{array}$ & $\begin{array}{l}0.00 \\
2.86 \\
0.13 \\
0.00 \\
0.15 \\
0.17 \\
0.00\end{array}$ \\
\hline Total Antent & 2.74 & 2.78 & 3.03 & 3.03 & 3.05 & 2.99 & 3.30 \\
\hline CAREC EALAMCE (x) & .0 & -3 & -2 & -2 & -2 & $\cdot 1$ & -3 \\
\hline 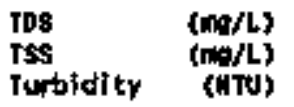 & $\begin{array}{r}164 \\
41 \\
3.2\end{array}$ & $\begin{array}{r}134 \\
11 \\
14\end{array}$ & $\begin{array}{r}160 \\
41 \\
2.5\end{array}$ & $\begin{array}{r}190 \\
41 \\
3.7\end{array}$ & $\begin{array}{r}160 \\
<1 \\
0.8\end{array}$ & $\begin{array}{r}160 \\
41 \\
0.8\end{array}$ & $\begin{array}{r}224 \\
3.4\end{array}$ \\
\hline
\end{tabular}




\section{APPENDIX J}

TRACE METAL CONCENTRATIONS THAT EXCEED MAXIMUM CONTAMINANT LEVELS 



\section{EXPI.ANATION}

\section{SAMPLING POINT:}

$\begin{array}{rll}\text { GW } & - & \text { Monitaring Well } \\ \text { CBS-1 } & - & \text { Chestnut Branch Spring 1 } \\ \text { SCR2.2SP } & - & \text { South Chestmut Ridge Spring 2.2 } \\ 1090 & - & \text { Historical 1000-Series Monitoring Well }\end{array}$

\section{LOCATION:}

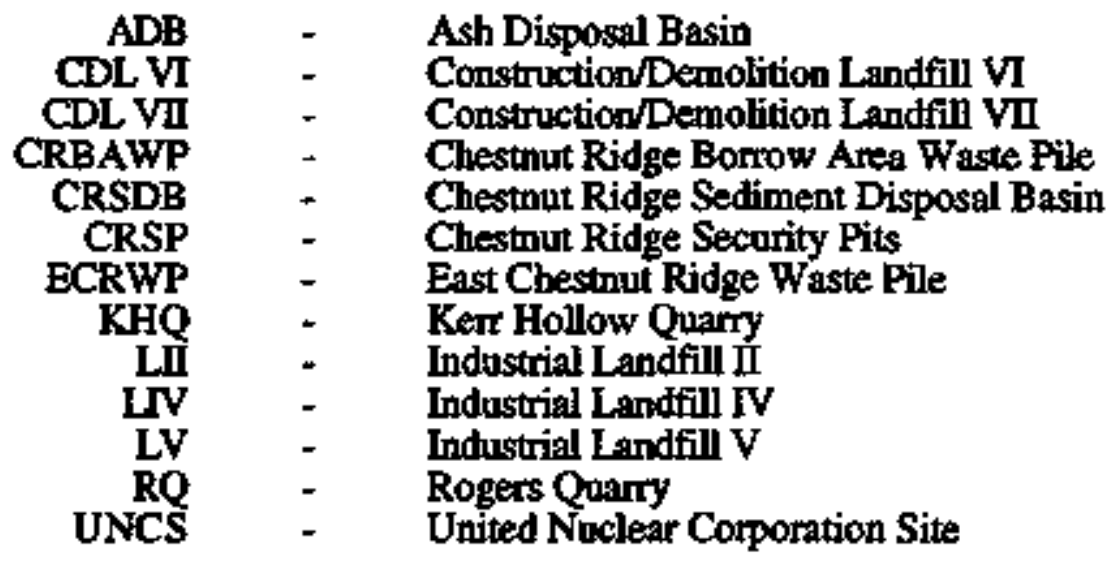

\section{NOTES:}

\begin{tabular}{|c|c|c|}
\hline $\mathrm{mg} / \mathbf{L}$ & & $\begin{array}{l}\text { Compound less than detection limit } \\
\text { milligrams per liter } \\
\text { Total Motal Concentration less than the } \\
\text { Maximum Contaminant Level (MCL) }\end{array}$ \\
\hline TO & & Total Concentration (Unfiltered Sample) \\
\hline & & Dissolved Concentration (Filtered Sample) \\
\hline & & Total Suspended Solids \\
\hline & & $\begin{array}{l}\text { Nephelometric Tutbidity Urats } \\
\text { Atomic Absorption Spectroscon }\end{array}$ \\
\hline & & vely Coupled Arc Plasma s \\
\hline & & Absomtion Soections \\
\hline
\end{tabular}

MCLS:

\section{Metst}

Barium (ICP)

Cadmitum (AAS)

Chromium (AAS)

Lead (AAS)

Menciry (CVAA)

\section{Maximum Conmminart.Level. $(\mathrm{mg} / \mathrm{L}$ )}

\section{0}

0.005

0.10

0.05

0.002

Because of inherent analytical interferences, ICP results for arsenic, selenium, and silver were not evalnated with respect to MCLs. Historical AAS results for these metals have shown only isolated and sporadic occurrences where concentrations exceeded MCls. 

APPENIX J

Trese botel Concentrations Thet Excead

Max inum Conte-inunt Levele, 1995

WEIL 1DENTITY

mate os shoshe site wate

DATE SATHED

SATPLE

GU-145 GN-1

145

cin-150

Gir- 759
CRsas

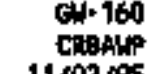

$03 / 24 / 95$

$03 / 24 / 95$

$40 / 25 / 95$

G. 160

CRENTP

uncs

CN-302

ey-soz

vincs

$1 / 02 / 05$
015

$04 / 19 / 95$

this

to/ 0 is $/ 95$

SNPLE TYPE

\section{Butrit:}

Crowlun

cronilu

Chrowtin

chontu

LEND

ICAP (NG/L)

ASS (NOS/L)

IEAP ( $\mathrm{N} / \mathrm{H} / \mathrm{L}$ )

MAS (NG/L)

ICAP (NG/L)

Ans (NO/L)

ICtP (WIG/A

MERCURY

cvas (Me/R)

$\begin{array}{rr}0.007 & 40,003 \\ : & : \\ : & :\end{array}$

TOTAL EUSPENDEO SOLIOS

TERAJoITY

\begin{tabular}{|c|c|c|}
\hline - & , & \\
\hline ' & * & $<0.002$ \\
\hline & • & $\begin{array}{r}0.0082 \\
0.31\end{array}$ \\
\hline & • & $\begin{array}{l}0.31 \\
0.14\end{array}$ \\
\hline 0.12 & $<0.004$ & 0.55 \\
\hline 0.16 & 40,05 & 0.52 \\
\hline • & - & * \\
\hline $\begin{array}{l}2.5 \\
2.9\end{array}$ & 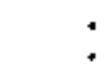 & $\begin{array}{l}5365 \\
7600\end{array}$ \\
\hline
\end{tabular}

0.002
0.003
0.01
0.01
0.004
0.05

0.3

\begin{tabular}{|c|c|c|}
\hline - & - & - \\
\hline ; & " & * \\
\hline $\begin{array}{l}\infty .01 \\
\infty 0.01\end{array}$ & $\begin{array}{l}0.23 \\
0.26\end{array}$ & $\begin{array}{l}0.01 \\
0.01\end{array}$ \\
\hline • & . & ' \\
\hline . & " & * \\
\hline - & - & • \\
\hline : & 9.2 & ; \\
\hline
\end{tabular}




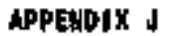

Irace intel concentration Thet Exced

Haxina containinant levels, iss

WELL DOETTIY

WhATE OJSPOSAL STTE WHE

OATE SAMPLE

SAPLE

SHYLE TYPE

Banjuy

cenpres

canntum

Comanjun

chifkowitu

LEAO

ENo

EERCURY

$64-511$
CRsp
$07 / 29 / 95$

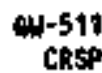

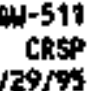

$\begin{array}{rr}0+539 & 6 w-539 \\ \text { LII } & \text { CII }\end{array}$

54-757 64-757

$07+2 r^{\circ}$

$07 / 29 / 95$

$07 / 10 / \% 507 / 10 / \% 5$

LIt tII

(2)

ICAP (NG/)

MS (NB/L)

JCAP (NG/L)

MAs ( $\mathrm{NOL} / \mathrm{l}$ )

ICN $(\mathrm{mo/L})$

Nots (more)

icap (mons?

Cwh (ing/l)

:

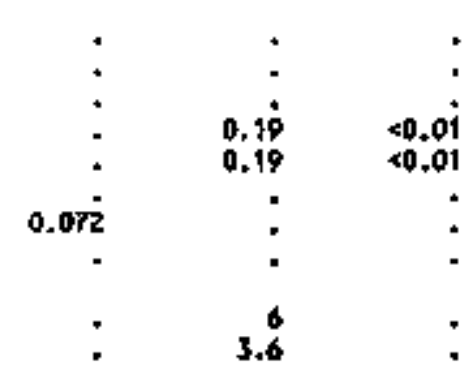

SOLID

TUREJdTr

(Niv)

0.7

Iot o1s


APPENDIX $K$

NITRATE CONCENTRATIONS 



\section{EXPLANATION}

SAMPLING POINT:

$\begin{array}{rll}\text { OW } & - & \text { Monitoring Well } \\ \text { CBS-1 } & - & \text { Chestmut Branch Spring } 1 \\ \text { SCR2.2SP } & - & \text { South Chestnut Ridge Spring 2.2 } \\ 1090 & - & \text { Historical } 1000 \text {-Series Monitoring Well }\end{array}$

\section{LOCATION:}

$\begin{array}{rcl}\text { ADH } & - & \text { Ash Disposal Basin } \\ \text { CDL VI } & - & \text { Construction/Demolition Landfill VI } \\ \text { CDL VII } & - & \text { Construction/Demolition Landfill VII } \\ \text { CRBAWP } & - & \text { Chesmut Ridge Bortow Area Waste Pile } \\ \text { CRSDB } & - & \text { Chestnut Ridge Sediment Disposal Basin } \\ \text { CRSP } & - & \text { Chestmut Ridge Security Pits } \\ \text { ECRWP } & - & \text { East Chestuut Ridge Waste Pile } \\ \text { KHQ } & - & \text { Kert Hollow Quary } \\ \text { LII } & - & \text { Industrial Lamdfill II } \\ \text { LIV } & - & \text { Industrial Landfill IV } \\ \text { LV } & - & \text { Industrial Landfill V } \\ \text { RQ } & - & \text { Rogers Quarty } \\ \text { UNCS } & - & \text { United Nuclear Corporation Site }\end{array}$

NOTES:

All concentrations reported as nitrate (as $\mathrm{N}$ ) in milligrams per liter (mg/L).

Results reported as less than the detection limit were not used to calculate mean concentrations.

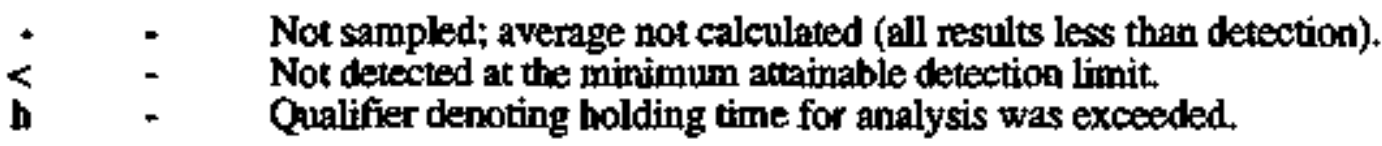



Appendix $\mathrm{K}$

Average Annual Detected Nitrate Concentrations (mg/L), 1995

Sampling

Point

\begin{tabular}{|c|c|}
\hline $\begin{array}{l}1090 \\
C B S-1 \\
G W-141 \\
G W-142 \\
G W-143 \\
G W-144 \\
G W-145 \\
G W-146 \\
G W-147 \\
G W-156 \\
G W-158 \\
G W-159 \\
G W-160 \\
G W-161 \\
G W-175 \\
G W-177 \\
G W-181 \\
G W-184 \\
G W-186 \\
G W-187 \\
G W-188 \\
G W-203 \\
G W-205 \\
G W-217 \\
G W-221 \\
G W-231 \\
G W-241 \\
G W-292 \\
G W-293 \\
G W-294 \\
G W-296 \\
G W-298 \\
G W-299 \\
G W-300 \\
G W-301 \\
G W-302 \\
G W-303 \\
G W-304 \\
G W-305 \\
G W-321 \\
G W-339 \\
G W-511 \\
G W-512 \\
G W-513\end{array}$ & $\begin{array}{l}\text { UNCS } \\
\text { LV } \\
\text { LIV } \\
\text { KHQ } \\
\text { KHQ } \\
\text { KHQ } \\
\text { KHQ } \\
\text { KHQ } \\
\text { KHQ } \\
\text { CRSDB } \\
\text { CRSDB } \\
\text { CRSDB } \\
\text { CRBAWP } \\
\text { CRBAWP } \\
\text { CRSP } \\
\text { CRSP } \\
\text { CRSP } \\
\text { RQ } \\
\text { RQ } \\
\text { RQ } \\
\text { RQ } \\
\text { UNCS } \\
\text { UNCS } \\
\text { LIV } \\
\text { UNCS } \\
\text { KHQ } \\
\text { CRSDB } \\
\text { ECRWP } \\
\text { ECRWP } \\
\text { ECRWP } \\
\text { ECRWP } \\
\text { CRBAWP } \\
\text { CRBAWP } \\
\text { CRBAWP } \\
\text { CRBAWP } \\
\text { UNCS } \\
\text { CRSDB } \\
\text { CRSDB } \\
\text { LIV } \\
\text { ADB } \\
\text { UNCS } \\
\text { CRSP } \\
\text { ADB } \\
\text { ADB }\end{array}$ \\
\hline
\end{tabular}

No. Location Detected

2

$\mathbf{2}$

2

2

0

4

4

3

4

4

0

4

2

2

4

3

3

2

0

1

2

2

2

2

2

4

2

2

2

2

1

2

2

2

2

0

3

2

2

2

4

o

2
1995 Average

10

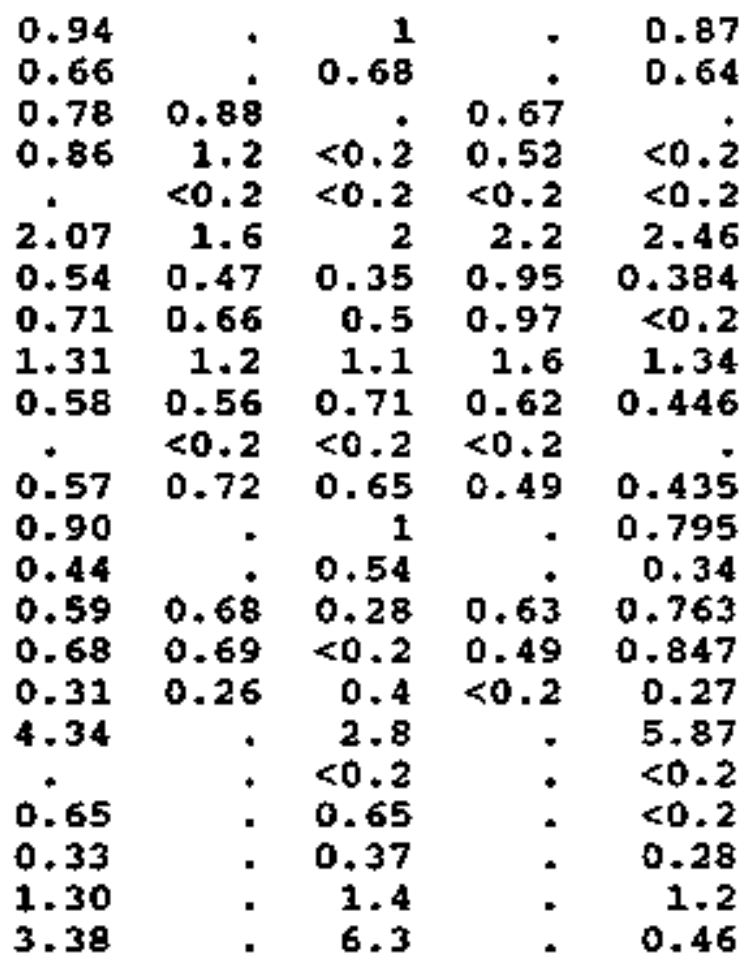

0.85

0.83

1.07

0.62

0.79

0.72

2.70

0.64

0.42

0.30

0.38

0.52

1.15

0.53

0.56

0.44

1.00

0.26

0.30

2nd

$3 r d$

4th

1. 1

0.9

1. 1

$0.36<0.2$

0.67

0.58

2.7

0.47

0.42

0.37

0.5

0.64

1. 2

$<0.2$

$<0.2$

0.58

0.54

0.68

0.45

1.1

0.26

0.22

$<0.2$

0.33
0.59

0.75

1. 4

0.88

0.76

0.9

0.86

2.7

0.8

$<0.2$

0.227

0. 404

1.1

$<0.2$

0.46

0.44

0.424

$\begin{array}{rr}0.28 & 0.89 \\ 0.28\end{array}$

$<0.2$

0.26
0.254 


\section{Appendix $\mathrm{K}$}

Average Annual Detected Nitrate Concentrations (mg/L), 1995

Sampling

Point

$\begin{array}{ll}G W-514 & \text { ADB } \\ G W-521 & \text { LIV } \\ G W-522 & \text { LIV } \\ G W-539 & \text { LII } \\ G W-540 & \text { CDLVI } \\ G W-541 & \text { CDLVI } \\ G W-542 & \text { CDLVI } \\ G W-543 & \text { CDLVI } \\ G W-544 & \text { CDLVI } \\ G W-546 & \text { CDLVI } \\ G W-557 & \text { LV } \\ G W-560 & \text { CDLVII } \\ G W-562 & \text { CDLVII } \\ G W-564 & \text { CDLVII } \\ G W-608 & \text { CRSP } \\ G W-609 & \text { CRSP } \\ G W-610 & \text { CRSP } \\ G W-621 & \text { CRSP } \\ G W-709 & \text { LII } \\ G W-731 & \text { CRSDB } \\ G W-732 & \text { CRSDB } \\ G W-742 & \text { CRSP } \\ G W-743 & \text { CRSP } \\ G W-757 & \text { LII } \\ G W-796 & \text { LV } \\ G W-797 & \text { LV } \\ G W-798 & \text { CDLVII } \\ G W=799 & \text { LV } \\ G W-801 & \text { LV } \\ G W-827 & \text { CDLVI } \\ \text { GCR2.2SP } & \text { CRSP } \\ & \end{array}$

No. Location Detected

1

2

2

3

2

o

2

2

2

2

2

2

2

2

4

4

4

3

4

4

0

4

2

2

2

2

2

2

2

1
1995 Average

0.41

0.60

0.70

0.70

0.40

.

0.67

0.47

0.82

0.39

1.05

0.36

0.55

0.75

0.75

1.22

0.89

3.31

0.45

0.58

0.60

0.99

0.28

0.42

0.48

0.90

1.03

0.33

0.37

2.10 1s Q

$\begin{array}{rrr}0.63 & 0.41 & 0.56 \\ 0.81 & : & 0.58 \\ 1 & : & 0.69\end{array}$

0.39

$<0.2$

0.68

0.57

0.84

0.39

1.1

0.39

0.58

0.68

0.77

0.48

1.3

1

3,3

0.58

0.67

0.54

$<0.2$

1.3

$<0.2$

1.1

0.81

3.3

$0.6 \dot{5}$

0.68

$<0.2$

0.81

0.52

.0 .56

- 1

- 1.1

- 0.32

2.1

0.33
3 rd 4 th

Q $\quad$ Q

$<0.2$ $\begin{array}{ll}0.69 & 0.42\end{array}$

0.41

$<0.2 \mathrm{~h}$

0.65

0.373

0.797

0.39

0.32

0.52

0.81

0.67

1.3

0.85

3.2

0.43

0.57

0.69

$<0.2$

0.8

0.32

1.08

1.17

0.91

3.44

0.33

0.418

0.485

$<0.2$

1.05

0.24

0.32

0.39

0.8

0.96

0.34

0.4 
APPENDIX L

QA/QC DATA SUMMARIES 



\section{EXPLANATION}

SAMPLING POINT:

$\begin{array}{rll}\text { GW } & - & \text { Monitoring Well } \\ \text { CBS-1 } & - & \text { Chestrut Branch Spring 1 } \\ \text { SCR2.2SP } & - & \text { South Chestnut Ridge Spring 2.2 } \\ 1090 & - & \text { Historical 1000-Series Monitoring Well }\end{array}$

\section{LOCATION:}

$\begin{array}{rll}\text { ADB } & - & \text { Ash Disposal Basin } \\ \text { CDLVI } & - & \text { ConstructionDemolition Landfill vI } \\ \text { CDL VII } & - & \text { ConstructionDernolition Landfill VII } \\ \text { CRBAWP } & - & \text { Chestnut Ridge Borrow Area Waste Pile } \\ \text { CRSDB } & - & \text { Chestnut Ridge Sediment Disposal Basin } \\ \text { CRSP } & - & \text { Chestnut Ridge Security Pits } \\ \text { ECRWP } & - & \text { East Chestnut Ridge Waste Pile } \\ \text { EXP-SW } & - & \text { Exit Pathway, Surface Water Sampling Location } \\ \text { KHQ } & - & \text { Kerr Hollow Quary } \\ \text { LII } & - & \text { Industrial Landfill II } \\ \text { LVV } & - & \text { Industrial Landfill IV } \\ \text { LV } & - & \text { Industrial Landfill V } \\ \text { RQ } & - & \text { Rogers Quatry } \\ \text { UNCS } & - & \text { United Nuclear Corporation Site }\end{array}$

NOTES:
Not analyzed
U - Compound not detected at the reported minimum attainable detection linit

\section{UNITS:}

ugl - micrograms per liter

mglL - milligrams per liter

pCi/L - picoCuries per liter

BqL - Becquerels per liter

$\mathrm{CE}+$ - $_{-} \quad$ Counting Error (two standard deviations) 


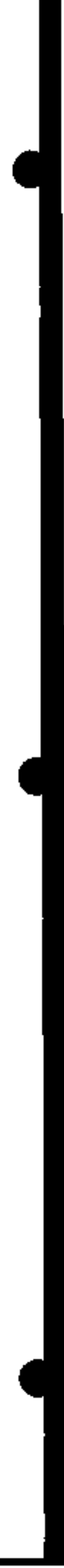


Appendices L. 2 through L. 4 show only results for the target compound list of VOCs that were detected in each type of QAQC sample. Ethanol was the only organic compound not in the target compound list that was detected, and was detected only in the following first and second quarter trip and laboratory blank samples:

\begin{tabular}{|c|c|c|c|c|c|c|}
\hline $\begin{array}{c}\text { Trip Blank } \\
\text { Namber }\end{array}$ & $\begin{array}{c}\text { Date } \\
\text { Sumpted }\end{array}$ & $\begin{array}{l}\text { Ethanol } \\
\text { (ug/L) }\end{array}$ & : & $\begin{array}{c}\text { Laboratory } \\
\text { Blant Number }\end{array}$ & $\begin{array}{c}\text { Date } \\
\text { Sapupted }\end{array}$ & $\begin{array}{l}\text { Ethanol } \\
\text { (ug/L) }\end{array}$ \\
\hline $950104 \cdot 284$ & $1 / 4 / 95$ & 620 & 策 & $950109-046$ & $1 / 4 / 99$ & 570 \\
\hline $950105-054$ & $1 / 4 / 95$ & 800 & & 950111.001 & $1 / 9 / 95$ & 560 \\
\hline 950110.044 & $1 / 9 / 95$ & 630 & & $950112-001$ & $1 / 10 / 95$ & 1100 \\
\hline $950110 \cdot \mathrm{A} 12$ & $1 / 9 / 95$ & 810 & 4 & $950407-001$ & $4 / 5 / 95$ & 780 \\
\hline $950111-089$ & $1 / 10 / 95$ & 800 & 管 & $950411-001$ & $4 / 6 / 95$ & 750 \\
\hline $950406-016$ & $4 / 5 / 95$ & 450 & w & $9 \$ 0421-001$ & $4 / 20 / 95$ & 920 \\
\hline $950407-013$ & $4 / 6 / 95$ & 710 & 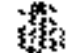 & $950501-002$ & $4 / 26 / 95$ & 870 \\
\hline $950420-131$ & $4 / 20 / 95$ & 770 & 舆 & $950502-001$ & $5 / 1 / 95$ & 1000 \\
\hline $950427-126$ & $4 / 26 / 95$ & 530 & 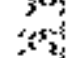 & $950504-001$ & $5 / 3 / 95$ & 860 \\
\hline $950427-199$ & $4 / 27 / 95$ & 420 & 8 & & & \\
\hline $950501-131$ & $5 / 1 / 95$ & 610 & $\therefore$ & & & \\
\hline $950502-093$ & $5 / 2 / 95$ & 700 & $\because$ & & & \\
\hline $950503-139$ & $5 / 3 / 95$ & 490 & 糙 & & & \\
\hline
\end{tabular}


Compounds Detected to QNQC Samples, CY 1995

\begin{tabular}{|c|c|c|c|c|c|}
\hline \multirow[t]{2}{*}{ Trip Blank Samples } & \multicolumn{4}{|c|}{ Querter of CY 1995} & \multirow{2}{*}{$\begin{array}{c}\text { Anndal } \\
\text { Total }\end{array}$} \\
\hline & First & Second & Thland & Pourth & \\
\hline Totst Number of Sanples & 23 & 42 & 20 & 38 & 123 \\
\hline Saraptes Wuth VOCg & 19 & 24 & 14 & $3-4$ & 91 \\
\hline Pextent Contwmlanted & 83 & 57 & 70 & 89 & 75 \\
\hline Acetton: & 4 & 4 & 1 & 4 & 13 \\
\hline 2-Butaninn: & 4 & I & 0 & 7 & 12 \\
\hline 12-Dichloroethane & 0 & $\mathbf{l}$ & 0 & 0 & l \\
\hline Ethyibenzene & 0 & 0 & 0 & 2 & 2 \\
\hline 2-Hesanone & 0 & 0 & 0 & 1 & 1 \\
\hline Methylene chloride & 3 & 2 & 0 & 2 & 7 \\
\hline Tohuene & 5 & 0 & 0 & 0 & 5 \\
\hline 1,1,1-Ttuctioroethane & 19 & 22 & 14 & 31 & 86 \\
\hline Xylenes & 0 & 0 & 0 & 2 & 2 \\
\hline Total Organic Halide & $\mathrm{l}$ & $\underline{0}$ & 0 & 0 & 1 \\
\hline \multirow[t]{2}{*}{ Loboratory Bänle Sumples } & \multicolumn{4}{|c|}{ Quarter of CY 1995} & Agnual \\
\hline & Fryt & Seoond & Third & Ponrth & Tatal \\
\hline Total Nunber of Stapples & 15 & 24 & 10 & 21 & 70 \\
\hline Sumples With VOCs & 3 & 6 & $\mathbf{]}$ & 5 & 15 \\
\hline Percent Costeminated & 20 & 25 & 10 & 24 & 21 \\
\hline Acetone & 2 & 4 & 0 & 1 & 7 \\
\hline 2-Butanione & 3 & 1 & $\mathbf{]}$ & 4 & 9 \\
\hline Ethylbenzene & 0 & 0 & 0 & 1 & $\mathbf{l}$ \\
\hline Methylene chlorids & 0 & 2 & 0 & 0 & 2 \\
\hline Toluene & 2 & 0 & 0 & 0 & 2 \\
\hline Xylenes & 0 & 0 & 0 & 1 & 1 \\
\hline
\end{tabular}

\begin{tabular}{|c|c|c|c|c|c|}
\hline \multirow[t]{2}{*}{ Rinsnte Sanples } & \multicolumn{4}{|c|}{ Querter of CY 1995} & \multirow{2}{*}{$\begin{array}{l}\text { Annutal } \\
\text { Totall }\end{array}$} \\
\hline & Ftst & Second & Thind & Foarls & \\
\hline Total Nunber of Somples & 6 & 10 & 6 & 11 & 32 \\
\hline Soraples With VOCS & 3 & 2 & 4 & $\tau$ & 1s \\
\hline Parcent Contaminated & 50 & 20 & 67 & 64 & 47 \\
\hline Acetont & 2 & 0 & 0 & 3 & 5 \\
\hline 2-Butingone & 1 & 0 & 0 & 1 & 2 \\
\hline Chloroform & 0 & 0 & 0 & 1 & $\mathbf{l}$ \\
\hline Ethylbenzene & 0 & 0 & 0 & 2 & 2 \\
\hline Matrylene chioride & 2 & 0 & 0 & 2 & 4 \\
\hline Toluene & $\mathbf{1}$ & 0 & 0 & I & 2 \\
\hline 1.I.I-T jishkscothane & 3 & 2 & 4 & d & 13 \\
\hline Xylenes & $\theta$ & 0 & 0 & 2 & 2 \\
\hline
\end{tabular}

\begin{tabular}{|c|c|c|c|c|c|}
\hline \multirow[t]{2}{*}{ Fïeld Binnk Semnples } & \multicolumn{4}{|c|}{ Qusirter of CY 1998} & \multirow{2}{*}{$\begin{array}{l}\text { Annual } \\
\text { Total }\end{array}$} \\
\hline & First & Second & Thind & Fowth & \\
\hline Total Noubler of Sanptes & $\mathbf{1}$ & 1 & 1 & 1 & 4 \\
\hline Sanples With VOCs & $\mathbf{l}$ & 1 & 0 & 1 & 3 \\
\hline Percent Contumiagted & 100 & 100 & 0 & 100 & 75 \\
\hline 1.1.1-Trichkxoethane & $\mathbf{I}$ & ] & 0 & 1 & 3 \\
\hline
\end{tabular}





\section{APPENDIX $\mathrm{L} .1$}

CORRELATION OF GROUNDWATER AND SURFACE WATER SAMPLES TO ASSOCIATED BLANKS 


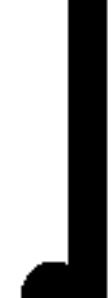


APPERIX L. 1

Corrolation of Sampleg to Ageociated Blanks

First puarter 1995

\begin{tabular}{|c|c|c|c|c|c|}
\hline $\begin{array}{l}\text { Sampling } \\
\text { Point }\end{array}$ & Location & $\begin{array}{l}\text { Data } \\
\text { sampled }\end{array}$ & Sample & $\begin{array}{c}\text { Trip Blank } \\
\text { No. }\end{array}$ & $\begin{array}{c}\text { Lab Blank } \\
\text { No. }\end{array}$ \\
\hline $\begin{array}{l}G W-141 \\
G W-141 \\
G W-141 \\
G W-142 \\
G W-142 \\
G W-143 \\
G W-144 \\
G W-144 \\
G W-345 \\
G W-145 \\
G W-146 \\
G W-146 \\
G W=147 \\
G W-156 \\
G W-158 \\
G W-159 \\
G W-175 \\
G W-177 \\
G W-181 \\
G W-237 \\
G W-217 \\
G W-217 \\
G W-231 \\
G W-241 \\
G W=303 \\
G W-304 \\
G W-305 \\
G W-305 \\
G W-305 \\
G W-321 \\
G W-511 \\
G W-521 \\
G W-521 \\
G W-521 \\
G H-522 \\
G W-522 \\
G W-522 \\
G W=539 \\
G W-539 \\
G W-539 \\
G W-608 \\
G W-609 \\
G W-610 \\
G W-611 \\
G W-709 \\
G W-709 \\
G W-709 \\
G W-731 \\
G W-732 \\
G W-742 \\
G W-743 \\
G W-757 \\
G W-757 \\
G W-757 \\
G C R 2.259\end{array}$ & $\begin{array}{l}\text { LIV } \\
\text { LIV } \\
\text { LIV } \\
\text { KHQ } \\
\text { RHQ } \\
\text { KHQ } \\
\text { RHQ } \\
\text { KHQ } \\
\text { KHO } \\
\text { KHO } \\
\text { KHO } \\
\text { RHQ } \\
\text { KHD } \\
\text { CRSDB } \\
\text { GRSDB } \\
\text { CRSDB } \\
\text { CRSP } \\
\text { CRSP } \\
\text { CRSP } \\
\text { LIV } \\
\text { LIV } \\
\text { LIV } \\
\text { KHQ } \\
\text { CRSDB } \\
\text { CRSDB } \\
\text { CRSDB } \\
\text { LIV } \\
\text { LIV } \\
\text { LIV } \\
\text { LDB } \\
\text { CRSP } \\
\text { LIV } \\
\text { LIV } \\
\text { LIV } \\
\text { LIV } \\
\text { LIV } \\
\text { LIV } \\
\text { LII } \\
\text { LII } \\
\text { III } \\
\text { CRSP } \\
\text { CRSP } \\
\text { CRSP } \\
\text { CRSP } \\
\text { LII } \\
\text { LII } \\
\text { LII } \\
\text { CRSDB } \\
\text { CRSDB } \\
\text { CRSP } \\
\text { CRSP } \\
\text { LII } \\
\text { LII } \\
\text { LII } \\
\text { EXP-St }\end{array}$ & $\begin{array}{l}01 / 09 / 95 \\
01 / 09 / 95 \\
01 / 09 / 95 \\
01 / 10 / 95 \\
03 / 20 / 95 \\
01 / 17 / 95 \\
01 / 18 / 95 \\
03 / 22 / 95 \\
01 / 18 / 95 \\
03 / 24 / 95 \\
01 / 17 / 95 \\
03 / 23 / 95 \\
01 / 15 / 95 \\
01 / 18 / 95 \\
01 / 20 / 95 \\
01 / 15 / 95 \\
02 / 14 / 95 \\
02 / 13 / 95 \\
01 / 24 / 95 \\
01 / 04 / 95 \\
01 / 04 / 95 \\
01 / 04 / 95 \\
01 / 15 / 95 \\
01 / 20 / 95 \\
01 / 19 / 95 \\
01 / 15 / 95 \\
01 / 10 / 95 \\
01 / 10 / 95 \\
01 / 10 / 95 \\
01 / 10 / 95 \\
01 / 24 / 95 \\
01 / 09 / 95 \\
01 / 09 / 95 \\
01 / 09 / 95 \\
01 / 10 / 95 \\
01 / 10 / 95 \\
01 / 10 / 95 \\
01 / 04 / 95 \\
01 / 04 / 95 \\
01 / 04 / 95 \\
02 / 02 / 95 \\
02 / 15 / 95 \\
01 / 31 / 95 \\
02 / 14 / 95 \\
01 / 09 / 95 \\
01 / 09 / 95 \\
01 / 09 / 95 \\
01 / 17 / 95 \\
01 / 18 / 95 \\
01 / 27 / 95 \\
01 / 31 / 95 \\
01 / 09 / 95 \\
01 / 09 / 95 \\
01 / 09 / 95 \\
03 / 15 / 95\end{array}$ & $\begin{array}{l}950110-035 \\
950110-037 \\
950110-038 \\
950111-100 \\
950320-173 \\
950118-012 \\
950118-053 \\
950322-085 \\
950118-051 \\
950324-048 \\
950118-010 \\
950324-008 \\
950117-115 \\
950119-022 \\
950120-125 \\
950117-076 \\
950214-094 \\
950213-105 \\
950125-073 \\
950104-279 \\
950104-281 \\
950104-282 \\
950117-117 \\
950120-129 \\
950119-093 \\
950117-078 \\
950111-077 \\
950111-079 \\
950111-080 \\
950111-103 \\
950125-075 \\
950110-031 \\
950110-033 \\
950110-034 \\
950111-081 \\
950111-093 \\
950111-094 \\
950105-049 \\
950105-051 \\
950105-052 \\
950203-049 \\
950216-005 \\
950201-003 \\
950214-096 \\
950110-996 \\
950110-998 \\
950110-999 \\
950118-007 \\
950119-015 \\
950130-040 \\
950201-001 \\
950110-804\end{array}$ & $\begin{array}{l}950110-043 \\
950110-044 \\
950110-045 \\
950111-102 \\
950118-016 \\
950118-055 \\
950118-055 \\
950118-016 \\
950117-119 \\
950119-017 \\
950120-127 \\
950117-080 \\
950214-098 \\
950213-107 \\
950125=079 \\
950104-283 \\
950104-284 \\
950104-285 \\
950117-119 \\
950120-131 \\
950119-095 \\
950117-080 \\
950111-088 \\
950111-089 \\
950111-090 \\
950111-102 \\
950125-079 \\
950110-043 \\
950110-044 \\
950110-045 \\
950111-088 \\
950111-089 \\
950111-090 \\
950105-053 \\
950105-054 \\
950105-055 \\
950203-051 \\
950216-009 \\
950201-095 \\
950214-098 \\
950110-A 11 \\
950110-412 \\
950110-713 \\
950118-009 \\
950119-017 \\
950130-042 \\
950201-005 \\
950110-311\end{array}$ & 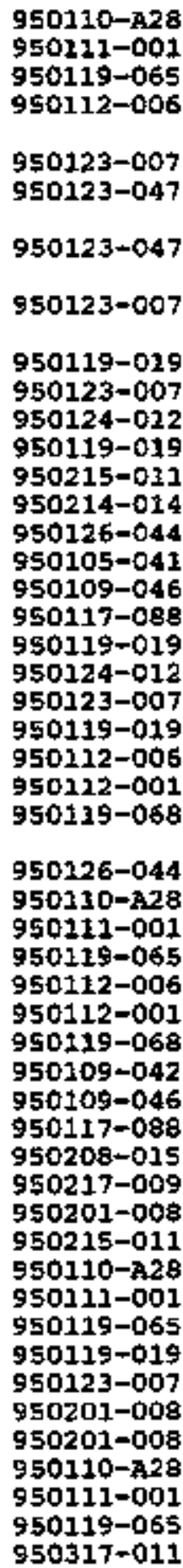 \\
\hline
\end{tabular}


APPENDIX L. 1

Correlation of Samples to Atsociated Blanks serona oueter 3995

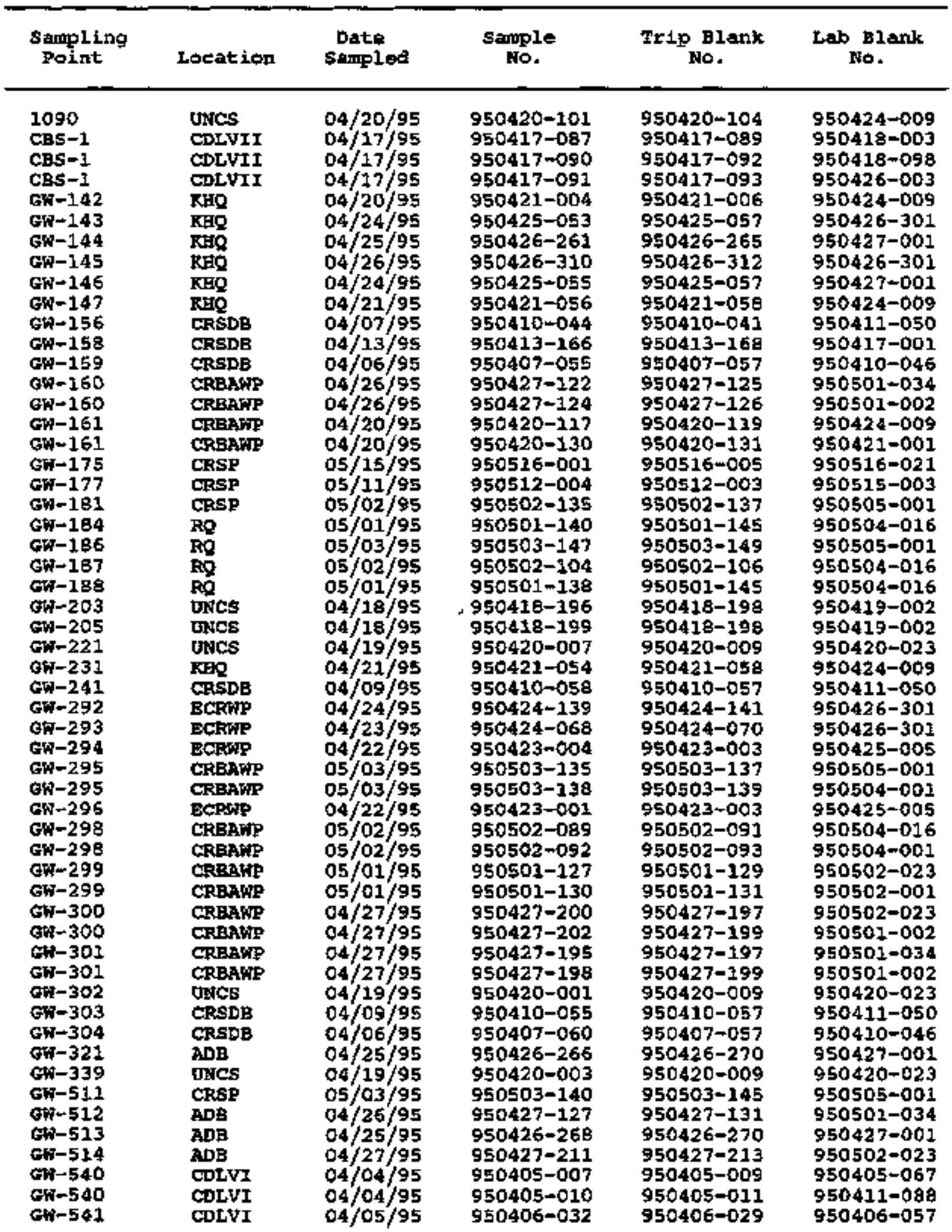


APPERTII L 1

Correlation of Samplet to Atrociated Blanks second Quarter 1995

\begin{tabular}{|c|c|c|c|c|c|}
\hline $\begin{array}{l}\text { Sampling } \\
\text { Point }\end{array}$ & Location & $\begin{array}{l}\text { Date } \\
\text { Saspled }\end{array}$ & $\begin{array}{l}\text { Sample } \\
\text { No. }\end{array}$ & Trip Blank & $\begin{array}{c}\text { Lab Blank } \\
\text { No. }\end{array}$ \\
\hline 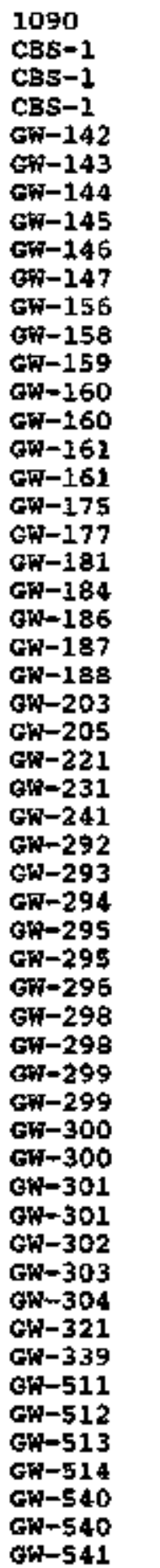 & 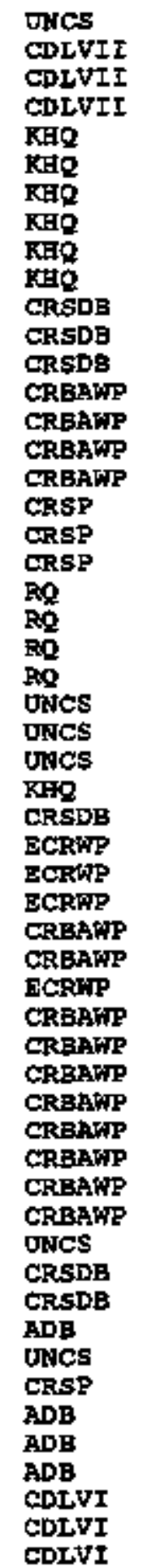 & $\begin{array}{l}04 / 20 / 95 \\
04 / 17 / 95 \\
04 / 17 / 95 \\
04 / 17 / 95 \\
04 / 20 / 95 \\
04 / 24 / 95 \\
04 / 25 / 95 \\
04 / 26 / 95 \\
04 / 24 / 95 \\
04 / 21 / 95 \\
04 / 07 / 95 \\
04 / 13 / 95 \\
04 / 06 / 95 \\
04 / 25 / 95 \\
04 / 26 / 95 \\
04 / 20 / 95 \\
04 / 20 / 95 \\
05 / 15 / 95 \\
05 / 11 / 95 \\
05 / 02 / 95 \\
05 / 01 / 95 \\
05 / 03 / 95 \\
05 / 02 / 95 \\
05 / 01 / 95 \\
04 / 18 / 95 \\
04 / 18 / 95 \\
04 / 19 / 95 \\
04 / 21 / 95 \\
04 / 09 / 95 \\
04 / 24 / 95 \\
04 / 23 / 95 \\
04 / 22 / 95 \\
05 / 03 / 95 \\
05 / 03 / 95 \\
04 / 22 / 95 \\
05 / 02 / 95 \\
05 / 02 / 95 \\
05 / 01 / 95 \\
05 / 01 / 95 \\
04 / 27 / 95 \\
04 / 27 / 95 \\
04 / 27 / 95 \\
04 / 27 / 95 \\
04 / 19 / 95 \\
04 / 09 / 95 \\
04 / 06 / 95 \\
04 / 25 / 95 \\
04 / 19 / 95 \\
05 / 03 / 95 \\
04 / 26 / 95 \\
04 / 25 / 95 \\
04 / 27 / 95 \\
04 / 04 / 95 \\
04 / 04 / 95 \\
04 / 05 / 95\end{array}$ & $\begin{array}{l}950420-101 \\
950417-087 \\
950417-090 \\
950417-091 \\
950421-004 \\
950425-053 \\
950426-261 \\
950426-310 \\
950425-055 \\
950421-056 \\
950410-044 \\
950413-166 \\
950407-055 \\
950427-122 \\
950427-124 \\
950420-117 \\
950420-130 \\
950515-001 \\
950512-004 \\
950502-135 \\
950501-140 \\
950503-147 \\
950502-104 \\
950501-138 \\
950418-196 \\
950418-199 \\
950420-007 \\
950421-054 \\
950410-058 \\
950424-139 \\
950424-068 \\
950423-004 \\
950503-135 \\
950503-138 \\
950423-001 \\
950502-099 \\
950502-092 \\
950501-127 \\
950501-130 \\
950427-200 \\
950427-202 \\
950427-195 \\
950427-198 \\
950420-001 \\
950410-055 \\
950407-060 \\
950426-266 \\
950420-003 \\
950503-140 \\
950427-127\end{array}$ & $\begin{array}{l}950420-104 \\
950417-089 \\
950417-092 \\
950417-093 \\
950421-006 \\
950425-057 \\
950426-265 \\
950426-312 \\
950425-057 \\
950421-058 \\
950410-041 \\
950413-168 \\
950407-057 \\
950427-125 \\
950427-126 \\
950420-119 \\
950420-131 \\
950516-005 \\
950512-003 \\
950502-137 \\
950501-145 \\
950503-149 \\
950502-106 \\
950501-145 \\
950416=198 \\
950418-198 \\
950420-009 \\
950421-058 \\
950410-057 \\
950424-141 \\
950424-070 \\
950423-003 \\
950503-137 \\
950503-139 \\
95040405-011 \\
950423-003 \\
950502-091 \\
950502-093 \\
950501-129 \\
950501-131 \\
950427-197 \\
950427-199 \\
950427-197 \\
950427-199 \\
950420-009 \\
950410-057 \\
950407-057 \\
950426-270 \\
950420-009\end{array}$ & 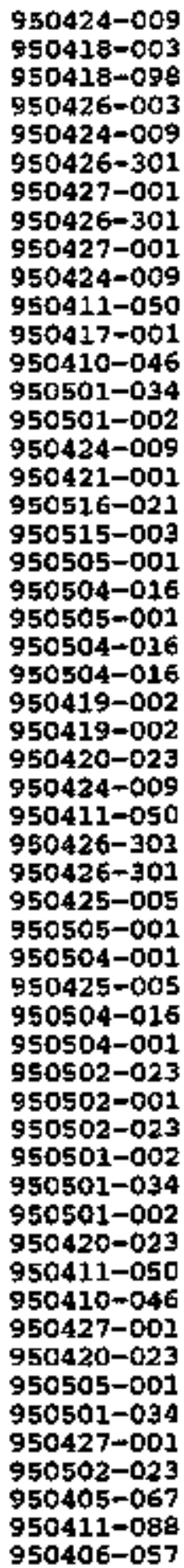 \\
\hline
\end{tabular}


APPEFDIX I 1

Correlation of Samplea to Amsociated Blanks Second Quarter 1995

\begin{tabular}{|c|c|c|c|c|c|}
\hline $\begin{array}{l}\text { Sampling } \\
\text { Point }\end{array}$ & Location & $\begin{array}{l}\text { Date } \\
\text { Sampled }\end{array}$ & $\begin{array}{c}\text { Samplo } \\
\text { no. }\end{array}$ & $\begin{array}{c}\text { Txip Blank } \\
\text { wo- }\end{array}$ & $\begin{array}{c}\text { Iab Blank } \\
\text { Mg. }\end{array}$ \\
\hline 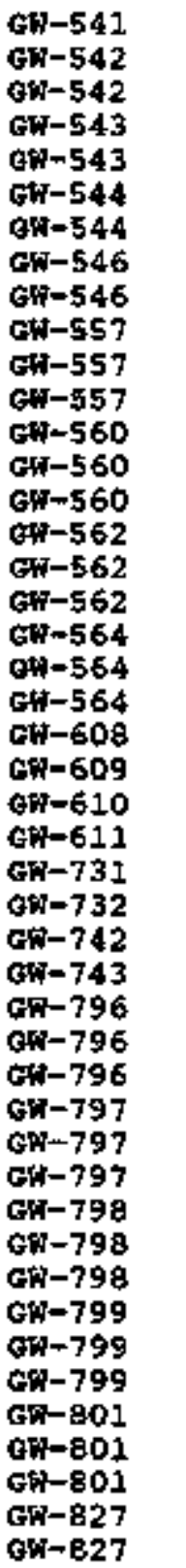 & $\begin{array}{l}\text { CDIVI } \\
\text { CDLVI } \\
\text { CDLVI } \\
\text { CDIVI } \\
\text { CDLVI } \\
\text { CDLVI } \\
\text { CDLVI } \\
\text { CDLVI } \\
\text { CDLVI } \\
\text { LV } \\
\text { LV } \\
\text { LV } \\
\text { CDLVII } \\
\text { CDLVII } \\
\text { CDLVII } \\
\text { CDLVII } \\
\text { CDIVII } \\
\text { CDIVII } \\
\text { CDLVII } \\
\text { CDIVII } \\
\text { CDIVII } \\
\text { CRSP } \\
\text { CRSP } \\
\text { CRSP } \\
\text { CRSP } \\
\text { CRSDB } \\
\text { CRSDB } \\
\text { CRSP } \\
\text { CRSP } \\
\text { LV } \\
\text { IV } \\
\text { LV } \\
\text { LV } \\
\text { LV } \\
\text { LV } \\
\text { CDLVII } \\
\text { CDLVII } \\
\text { CDLVII } \\
\text { CDLVII } \\
\text { CDLVII } \\
\text { CDLVII } \\
\text { LV } \\
\text { LV } \\
\text { CV } \\
\text { CDLVII } \\
\text { CDIVI }\end{array}$ & $\begin{array}{l}04 / 05 / 95 \\
04 / 06 / 95 \\
04 / 06 / 95 \\
04 / 06 / 95 \\
04 / 06 / 95 \\
04 / 06 / 95 \\
04 / 06 / 95 \\
04 / 05 / 95 \\
04 / 05 / 95 \\
04 / 05 / 95 \\
04 / 05 / 95 \\
04 / 06 / 95 \\
04 / 05 / 95 \\
04 / 05 / 95 \\
04 / 05 / 95 \\
04 / 05 / 95 \\
04 / 05 / 95 \\
04 / 05 / 95 \\
04 / 06 / 95 \\
04 / 05 / 95 \\
04 / 06 / 95 \\
05 / 11 / 95 \\
05 / 15 / 95 \\
05 / 07 / 95 \\
05 / 15 / 95 \\
04 / 07 / 95 \\
04 / 07 / 95 \\
05 / 07 / 95 \\
05 / 07 / 95 \\
04 / 12 / 95 \\
04 / 12 / 95 \\
04 / 12 / 95 \\
04 / 11 / 95 \\
04 / 11 / 95 \\
04 / 11 / 95 \\
04 / 11 / 95 \\
04 / 11 / 95 \\
04 / 11 / 95 \\
04 / 06 / 95 \\
04 / 05 / 95 \\
04 / 06 / 95 \\
04 / 13 / 95 \\
04 / 13 / 95 \\
04 / 13 / 95 \\
04 / 05 / 95 \\
04 / 05 / 95\end{array}$ & $\begin{array}{l}950406-034 \\
950407-026 \\
950407-028 \\
950407-029 \\
950407-031 \\
950407-032 \\
950407-034 \\
950406-027 \\
950406-030 \\
950407-020 \\
950407-022 \\
950407-023 \\
950406-019 \\
950406-021 \\
950405-022 \\
950406-012 \\
950406-015 \\
950406-017 \\
950407-016 \\
950407-018 \\
950407-019 \\
950512-001 \\
950516-122 \\
950508-032 \\
950516-003 \\
950410-039 \\
950410-042 \\
950 \$ 08-029 \\
950508-034 \\
950413-113 \\
950413-115 \\
950413-116 \\
950412-041 \\
950412-043 \\
950412-044 \\
950412-034 \\
950412-037 \\
950412-039 \\
950407-009 \\
950407-012 \\
950407-014 \\
950413-139 \\
950413-142 \\
950413-144 \\
950406=035 \\
950406-037\end{array}$ & $\begin{array}{l}950406-031 \\
950407-037 \\
950407-038 \\
950407-037 \\
950407-038 \\
950407-037 \\
950407-038 \\
950406-029 \\
950406-031 \\
950407-011 \\
950407-013 \\
950407-015 \\
950406-014 \\
950406-016 \\
950406-018 \\
950406-014 \\
950406-016 \\
950406-018 \\
950407-011 \\
950407-013 \\
950407-015 \\
950512-003 \\
950516-127 \\
950508-031 \\
950516-005 \\
950410-041 \\
950410-041 \\
950508-031 \\
950508-031 \\
950413-117 \\
950413-118 \\
950413-119 \\
950412-036 \\
950412-038 \\
950412-040 \\
950412-036 \\
950412-038 \\
950412-040 \\
950407-011 \\
950407-013 \\
950407-015 \\
950413-141 \\
950413-143 \\
950413-145 \\
950406-029 \\
950406-031\end{array}$ & $\begin{array}{l}950411-088 \\
950410-046 \\
950413-016 \\
950410-046 \\
950413-016 \\
950410-046 \\
950413-016 \\
950406-057 \\
950411-088 \\
950407-062 \\
950411-001 \\
950413-016 \\
950405-054 \\
950407-001 \\
950411-089 \\
950405-054 \\
950407-001 \\
950411-098 \\
950407-062 \\
950411-001 \\
950413-016 \\
950512-028 \\
950518-022 \\
950508-027 \\
950516-021 \\
950411-050 \\
950411-050 \\
950508-027 \\
950508-027 \\
950417-001 \\
950419-084 \\
950426-003 \\
950413-001 \\
950412-052 \\
950413-016 \\
950413-001 \\
950412-052 \\
950413-016 \\
950407-062 \\
950411-001 \\
950413-016 \\
950417-001 \\
950418-084 \\
950426-003 \\
950406-057 \\
950412-123\end{array}$ \\
\hline
\end{tabular}


APPENDIX L. 1

Correlation of Samples to Rssociated Blanks Third Quarter 1995

\begin{tabular}{|c|c|c|c|c|c|}
\hline $\begin{array}{l}\text { Sampling } \\
\text { Point }\end{array}$ & Location & $\begin{array}{l}\text { Date } \\
\text { sampled }\end{array}$ & $\begin{array}{c}\text { sample } \\
\text { No. }\end{array}$ & $\begin{array}{c}\text { Trip } \operatorname{Blank} \\
\text { to. }\end{array}$ & $\begin{array}{c}\text { Lab Blank } \\
\text { No. }\end{array}$ \\
\hline 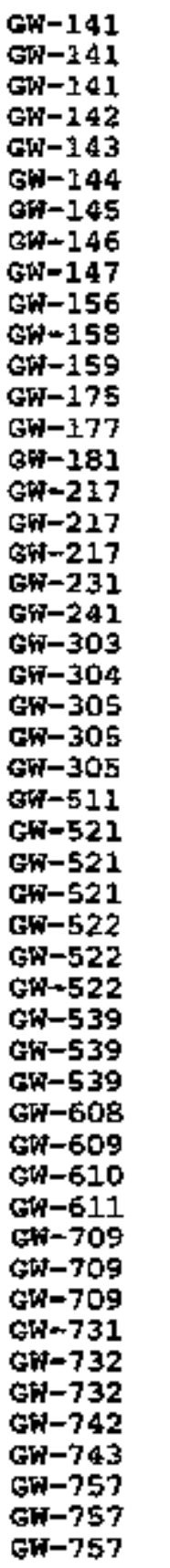 & $\begin{array}{l}\text { LIV } \\
\text { LIV } \\
\text { IIV } \\
\text { KHQ } \\
\text { RHO } \\
\text { KHQ } \\
\text { KEO } \\
\text { KHO } \\
\text { KHO } \\
\text { CRSDB } \\
\text { CRSDB } \\
\text { CRSDB } \\
\text { CRSP } \\
\text { CRSP } \\
\text { CRSP } \\
\text { LIV } \\
\text { LIV } \\
\text { LIV } \\
\text { KHO } \\
\text { CRSDB } \\
\text { CRSDB } \\
\text { CRSDB } \\
\text { LIV } \\
\text { LIV } \\
\text { LIV } \\
\text { CRSP } \\
\text { LIV } \\
\text { LIV } \\
\text { LIV } \\
\text { LIV } \\
\text { LIV } \\
\text { LIV } \\
\text { LII } \\
\text { LII } \\
\text { LII } \\
\text { CRSP } \\
\text { CRSP } \\
\text { CRSP } \\
\text { CRSP } \\
\text { LII } \\
\text { LII } \\
\text { LII } \\
\text { CRSDB } \\
\text { CRSDB } \\
\text { CRSDB } \\
\text { CRSP } \\
\text { CRSP } \\
\text { LII } \\
\text { LII } \\
\text { LII }\end{array}$ & $\begin{array}{l}07 / 10 / 95 \\
07 / 10 / 95 \\
07 / 10 / 95 \\
07 / 14 / 95 \\
07 / 16 / 95 \\
07 / 15 / 95 \\
07 / 17 / 95 \\
07 / 17 / 95 \\
07 / 14 / 95 \\
07 / 13 / 95 \\
07 / 18 / 95 \\
07 / 12 / 95 \\
08 / 01 / 95 \\
08 / 01 / 95 \\
07 / 28 / 95 \\
07 / 10 / 95 \\
07 / 10 / 95 \\
07 / 10 / 95 \\
07 / 15 / 95 \\
07 / 15 / 95 \\
07 / 16 / 95 \\
07 / 12 / 95 \\
07 / 12 / 95 \\
07 / 12 / 95 \\
07 / 12 / 95 \\
07 / 29 / 95 \\
07 / 11 / 95 \\
07 / 11 / 95 \\
07 / 11 / 95 \\
07 / 12 / 95 \\
07 / 12 / 95 \\
07 / 12 / 95 \\
07 / 10 / 95 \\
07 / 10 / 95 \\
07 / 10 / 95 \\
07 / 31 / 95 \\
08 / 01 / 95 \\
07 / 31 / 95 \\
08 / 01 / 95 \\
07 / 10 / 95 \\
07 / 10 / 95 \\
07 / 20 / 95 \\
07 / 13 / 95 \\
07 / 13 / 95 \\
08 / 14 / 95 \\
07 / 30 / 95 \\
07 / 31 / 95 \\
07 / 11 / 95 \\
07 / 11 / 95 \\
07 / 11 / 95\end{array}$ & $\begin{array}{l}950711-057 \\
950711-059 \\
950711-060 \\
950714-054 \\
950717-106 \\
950717-109 \\
950718-004 \\
950718-007 \\
950714-057 \\
950713-120 \\
950719-290 \\
950713-068 \\
950802-031 \\
950801-069 \\
950731-002 \\
950711-061 \\
950711-063 \\
950711-064 \\
950717-103 \\
950717-113 \\
950717-113 \\
950713-071 \\
950713-026 \\
950713-028 \\
950713-029 \\
950731-022 \\
950712-001 \\
950712-004 \\
950712-006 \\
950713-014 \\
950713-017 \\
950713-019 \\
950711-001 \\
950711-004 \\
950711-006 \\
950801-010 \\
950802-028 \\
950801-030 \\
950807-066 \\
950711-008 \\
9507 \pm 1-010 \\
950711-011 \\
950713-124 \\
950713-122 \\
950815-079 \\
950731-051 \\
950801-032 \\
950712-009 \\
950712-012 \\
950712-014\end{array}$ & $\begin{array}{l}950711-065 \\
950711-072 \\
950711-066 \\
950714-056 \\
950717-108 \\
950717-108 \\
950718-006 \\
950718-006 \\
950714-056 \\
950713-126 \\
950719-292 \\
950713-070 \\
950902-033 \\
950801-071 \\
950731-084 \\
950711-065 \\
950711-072 \\
950711-066 \\
950717-105 \\
950717-115 \\
950717-115 \\
950713-070 \\
950713-016 \\
950713-018 \\
950713=020 \\
950731-024 \\
950712-003 \\
950712-005 \\
950712-007 \\
950713-016 \\
950713-018 \\
950713-020 \\
950711-003 \\
950711-005 \\
950711-007 \\
950801-012 \\
950802-033 \\
950801-034 \\
950901-071 \\
950711-003 \\
950711-005 \\
950711-007 \\
950713-126 \\
950713-126 \\
950731-053 \\
950801-034 \\
950712-011 \\
950712-013 \\
950712-015\end{array}$ & $\begin{array}{l}950713-021 \\
950717-062 \\
950716-015 \\
950712-048 \\
950718-003 \\
950718-003 \\
950718-003 \\
950718-003 \\
950712-048 \\
950712-048 \\
950726-030 \\
950717-003 \\
950804-040 \\
950804-040 \\
950803-044 \\
950713-021 \\
950717-062 \\
950716-015 \\
950718-035 \\
950718-003 \\
950718-035 \\
950717-003 \\
950717-003 \\
950714-001 \\
950721-006 \\
950731-106 \\
950713-021 \\
950717-062 \\
950716-015 \\
950713-021 \\
950714-001 \\
950715-015 \\
950711-016 \\
950712-049 \\
950716-015 \\
950803-044 \\
950804-040 \\
950803-044 \\
950804-040 \\
950711-016 \\
950712-049 \\
950716-015 \\
950712-048 \\
950712-048 \\
950803-044 \\
950803-044 \\
950713-021 \\
950714-001 \\
950716-015\end{array}$ \\
\hline
\end{tabular}


APPEIDIX L.1

correlation of Samplos to Aapociated Blanko

Fourth Quarter 1995

\begin{tabular}{|c|c|c|c|c|c|}
\hline $\begin{array}{l}\text { Sampling } \\
\text { Point }\end{array}$ & Iocation & $\begin{array}{l}\text { Dater } \\
\text { sampled }\end{array}$ & $\begin{array}{c}\text { Sample } \\
\text { To. }\end{array}$ & $\begin{array}{c}\text { Trip Blank } \\
\text { No. }\end{array}$ & $\begin{array}{c}\text { I ab B1 ant } \\
\text { No. }\end{array}$ \\
\hline 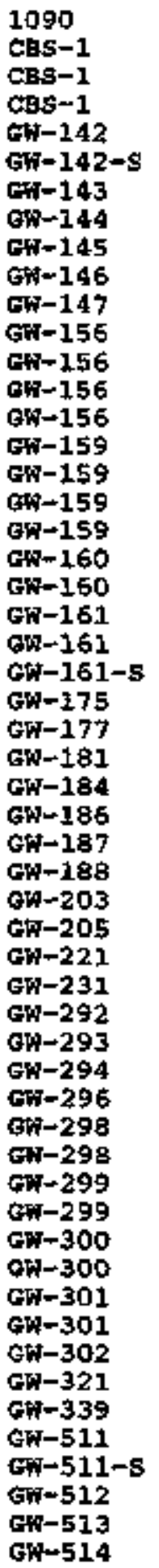 & 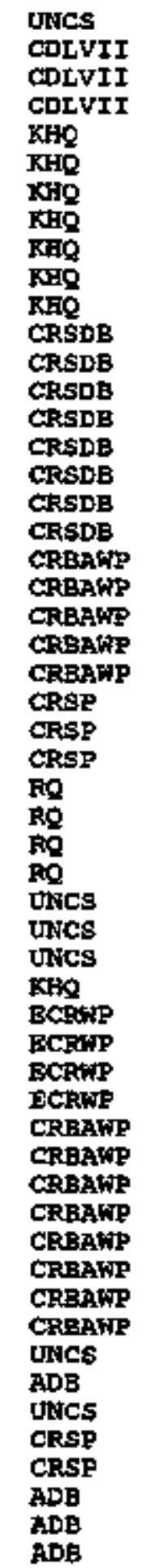 & $\begin{array}{l}10 / 09 / 95 \\
10 / 11 / 95 \\
10 / 11 / 95 \\
10 / 11 / 95 \\
11 / 05 / 95 \\
11 / 05 / 95 \\
11 / 09 / 95 \\
11 / 14 / 95 \\
11 / 15 / 95 \\
11 / 08 / 95 \\
11 / 06 / 95 \\
10 / 26 / 95 \\
10 / 23 / 95 \\
10 / 24 / 95 \\
10 / 25 / 95 \\
10 / 26 / 95 \\
10 / 23 / 95 \\
10 / 24 / 95 \\
10 / 25 / 95 \\
11 / 02 / 95 \\
11 / 02 / 95 \\
10 / 31 / 95 \\
10 / 31 / 95 \\
10 / 31 / 95 \\
11 / 17 / 95 \\
11 / 16 / 95 \\
11 / 05 / 95 \\
10 / 31 / 95 \\
11 / 03 / 95 \\
11 / 02 / 95 \\
10 / 31 / 95 \\
10 / 07 / 95 \\
10 / 07 / 95 \\
10 / 08 / 95 \\
11 / 05 / 95 \\
11 / 05 / 95 \\
11 / 06 / 95 \\
11 / 05 / 95 \\
11 / 05 / 95 \\
11 / 04 / 95 \\
11 / 04 / 95 \\
11 / 03 / 95 \\
11 / 03 / 95 \\
11 / 03 / 95 \\
11 / 03 / 95 \\
11 / 03 / 95 \\
11 / 03 / 95 \\
10 / 08 / 95 \\
10 / 26 / 95 \\
10 / 08 / 95 \\
11 / 07 / 95 \\
11 / 07 / 95 \\
10 / 31 / 95 \\
10 / 31 / 95 \\
11 / 02 / 95\end{array}$ & $\begin{array}{l}951010-011 \\
951011-058 \\
951011-065 \\
951011-066 \\
951106-061 \\
951107-068 \\
951110-004 \\
951114-093 \\
951115-056 \\
951108-170 \\
951105-095 \\
951027-071 \\
951024-046 \\
951025-001 \\
951025-110 \\
951027-073 \\
951025-011 \\
951025-003 \\
951025-122 \\
951103-030 \\
951103-033 \\
951102-042 \\
951102-045 \\
951102-065 \\
951120-003 \\
951117-001 \\
951107-014 \\
951101-050 \\
951103-050 \\
951103-041 \\
951103-048 \\
951009-073 \\
951009-076 \\
951010-005 \\
951106-093 \\
951107-001 \\
951107-005 \\
951106-030 \\
951106-035 \\
951106-002 \\
951106-005 \\
951104-009 \\
951104-011 \\
953104-006 \\
951104-008 \\
951104-001 \\
951104-004 \\
951010-003 \\
951027-053 \\
951010-001 \\
951107-005 \\
951109-025 \\
951101-027 \\
951101-029 \\
951103-060\end{array}$ & $\begin{array}{l}951103-032 \\
951103-034 \\
951102-044 \\
951102-046 \\
951120-007 \\
951117-003 \\
951107-056 \\
951101-052 \\
951103-052 \\
951103-043 \\
951101-052 \\
951009-075 \\
951009-075 \\
951010-007 \\
951106-097 \\
951107-007 \\
951107-007 \\
951106-037 \\
951106-037 \\
951106-004 \\
951106-006 \\
951104-003 \\
951104-005 \\
951104-003 \\
951104-005 \\
951104-003 \\
951104-005 \\
951010-007 \\
951027-055 \\
951010-007 \\
951107-087 \\
951101-031 \\
951101-031 \\
951103-062\end{array}$ & $\begin{array}{l}951106-041 \\
951107-071 \\
951102-001 \\
951102-014 \\
951102-014 \\
951120-002 \\
951120-082 \\
951109-004 \\
951102-001 \\
951106-041 \\
951106-041 \\
951102-001 \\
951023-009 \\
951019-076 \\
951012-008 \\
951109-004 \\
951110-048 \\
951109-024 \\
951107-009 \\
951107-009 \\
951107-020 \\
951107-071 \\
951107-020 \\
951107-071 \\
951107-020 \\
951107-071 \\
951107-020 \\
951107-071 \\
951012-008 \\
951031-007 \\
951012-008 \\
951109-024 \\
951109-024 \\
951102-001 \\
951102-001 \\
951107-020\end{array}$ \\
\hline
\end{tabular}


APPENDIX L. 1

Correlation of Bamples to Associated Blanke

Fourth ouster 1995

\begin{tabular}{|c|c|c|c|c|c|}
\hline $\begin{array}{l}\text { Sampling } \\
\text { point }\end{array}$ & Location & $\begin{array}{l}\text { Date } \\
\text { Sampled }\end{array}$ & $\begin{array}{c}\text { Sample } \\
\text { to. }\end{array}$ & Trip Blank & $\begin{array}{c}\text { Lab Blenk } \\
\text { No. }\end{array}$ \\
\hline 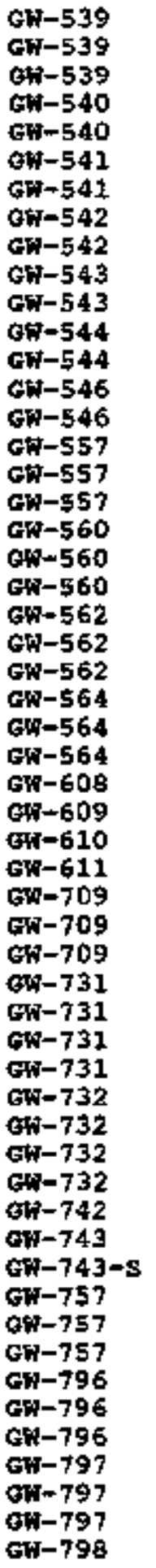 & 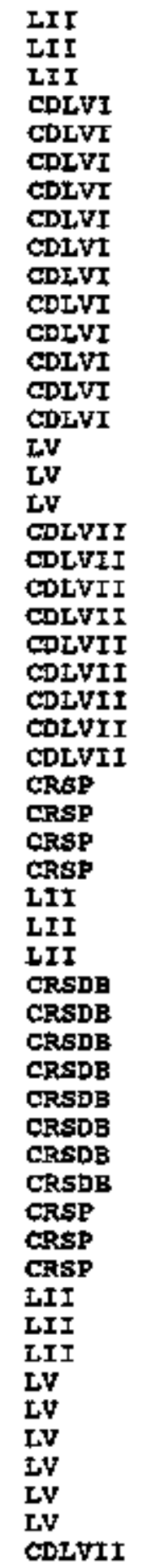 & $\begin{array}{l}10 / 06 / 95 \\
10 / 06 / 95 \\
10 / 06 / 95 \\
10 / 11 / 95 \\
10 / 11 / 95 \\
10 / 12 / 95 \\
10 / 12 / 95 \\
10 / 16 / 95 \\
10 / 16 / 95 \\
10 / 17 / 95 \\
10 / 17 / 95 \\
10 / 17 / 95 \\
10 / 17 / 95 \\
10 / 11 / 95 \\
10 / 11 / 95 \\
10 / 07 / 95 \\
10 / 07 / 95 \\
10 / 07 / 95 \\
10 / 06 / 95 \\
10 / 06 / 95 \\
10 / 06 / 95 \\
10 / 06 / 95 \\
10 / 06 / 95 \\
10 / 06 / 95 \\
10 / 07 / 95 \\
10 / 07 / 95 \\
10 / 07 / 95 \\
11 / 16 / 95 \\
11 / 20 / 95 \\
11 / 13 / 95 \\
11 / 17 / 95 \\
10 / 06 / 95 \\
10 / 06 / 95 \\
10 / 06 / 95 \\
10 / 26 / 95 \\
10 / 23 / 95 \\
10 / 24 / 95 \\
10 / 25 / 95 \\
10 / 26 / 95 \\
10 / 23 / 95 \\
10 / 24 / 95 \\
10 / 25 / 95 \\
11 / 09 / 95 \\
11 / 13 / 95 \\
11 / 13 / 95 \\
10 / 06 / 95 \\
10 / 06 / 95 \\
10 / 06 / 95 \\
10 / 08 / 95 \\
10 / 08 / 95 \\
10 / 08 / 95 \\
10 / 08 / 95 \\
10 / 08 / 95 \\
10 / 08 / 95 \\
10 / 08 / 95\end{array}$ & $\begin{array}{l}951009-041 \\
951009-043 \\
951009-044 \\
951012-026 \\
951012-031 \\
951013-008 \\
951013-011 \\
951017-034 \\
951017-036 \\
951018-014 \\
951018-017 \\
951018-011 \\
951019-013 \\
951013-005 \\
951013-007 \\
951009-143 \\
951009-145 \\
951009-146 \\
951009-005 \\
951009-008 \\
951009-010 \\
951009-016 \\
951009-018 \\
951009-019 \\
951009-119 \\
951009-122 \\
951009-137 \\
951117-003 \\
951121-001 \\
951214-009 \\
951320-005 \\
951009-029 \\
951009-031 \\
951009-032 \\
951027-075 \\
951024-044 \\
951025-005 \\
951025-104 \\
951027-077 \\
951025-013 \\
951025-007 \\
951025-106 \\
951110-001 \\
951114-006 \\
951115-053 \\
951009-033 \\
951009-035 \\
951009-036 \\
951009-170 \\
951009-172 \\
951009-173 \\
951009-166 \\
951009-169 \\
951009-169 \\
951009-159\end{array}$ & $\begin{array}{l}951009-045 \\
951009-134 \\
951009-135 \\
951012-030 \\
951012-032 \\
951013-010 \\
951013-012 \\
951017-031 \\
951017-033 \\
951019-016 \\
951018-028 \\
951018-016 \\
951018-018 \\
951012-030 \\
951012-032 \\
951009-121 \\
951009-136 \\
951009-139 \\
951009-007 \\
951009-009 \\
951009-011 \\
951009-007 \\
951009-009 \\
951009-011 \\
951009-121 \\
951009-136 \\
951009-138 \\
951117-005 \\
951121-003 \\
951114-008 \\
951120-007 \\
951009-045 \\
951009-134 \\
951009-135\end{array}$ & $\begin{array}{l}951019-076 \\
951010-015 \\
951023-045 \\
951012-036 \\
951024-041 \\
951016-028 \\
951024-041 \\
951019-002 \\
951025-023 \\
951023-003 \\
951025-023 \\
951019-002 \\
951025-023 \\
951012-036 \\
951025-023 \\
951019=076 \\
951017-057 \\
951024-139 \\
951011-008 \\
951010-015 \\
951023-045 \\
951011-008 \\
951010-015 \\
951023-045 \\
951019-076 \\
951017-057 \\
951023-045 \\
951120-092 \\
951122-005 \\
951115-052 \\
951120-092 \\
951011-008 \\
951010-015 \\
951023-045\end{array}$ \\
\hline
\end{tabular}


APFEHDIX L.1

Correlation of Bamples to Associated Binkt Fourth Duarter 1995

\begin{tabular}{|c|c|c|c|c|c|}
\hline $\begin{array}{l}\text { Sampling } \\
\text { Point }\end{array}$ & Location & $\begin{array}{l}\text { Date } \\
\text { samplad }\end{array}$ & $\begin{array}{l}\text { sample } \\
\text { tio. }\end{array}$ & $\begin{array}{c}\text { Trip Blank } \\
\text { No. }\end{array}$ & $\begin{array}{c}\text { Iab Blanik } \\
\text { No. }\end{array}$ \\
\hline 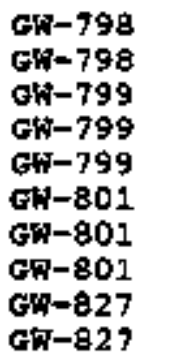 & $\begin{array}{l}\text { CDLVII } \\
\text { CDLVII } \\
\text { CDLVII } \\
\text { CDLUII } \\
\text { CDLVII } \\
\text { LV } \\
\text { LV } \\
\text { LV } \\
\text { CDLVI } \\
\text { CDLVI }\end{array}$ & $\begin{array}{l}30 / 08 / 95 \\
10 / 08 / 95 \\
10 / 07 / 95 \\
10 / 07 / 95 \\
10 / 07 / 95 \\
10 / 09 / 95 \\
10 / 09 / 95 \\
10 / 09 / 95 \\
10 / 16 / 95 \\
10 / 16 / 95\end{array}$ & $\begin{array}{l}951009-162 \\
951009-164 \\
951009-339 \\
951009-141 \\
951009-142 \\
951010-026 \\
951010-029 \\
951010-033 \\
951017-029 \\
951017-032\end{array}$ & $\begin{array}{l}951009-163 \\
951009-165 \\
951009-121 \\
951009-136 \\
951009-138 \\
951010-028 \\
951010-032 \\
951010-034 \\
951017-031 \\
951017-033\end{array}$ & $\begin{array}{l}951011-001 \\
951024-041 \\
951019-076 \\
951017-057 \\
951024-041 \\
951023-009 \\
951011-001 \\
951024-041 \\
951019-002 \\
951025-023\end{array}$ \\
\hline
\end{tabular}




\section{APPENDIX L.2}

TRIP BLANK DATA SUMMARY 

APPEXIOL L.2

vots potuated in Trip Elank somples, First cuarter 1995

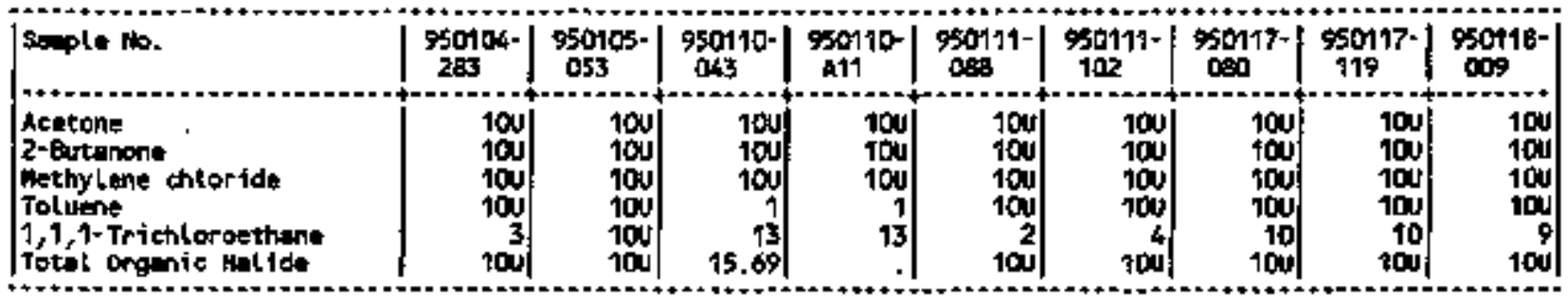

(CONTIMUED)

\begin{tabular}{|c|c|c|c|c|c|c|c|c|c|}
\hline sarapte to. & $\begin{array}{c}950118- \\
016\end{array}$ & osto118- & $\begin{array}{c}950149- \\
017\end{array}$ & $\underset{0 \% 5}{950119-}$ & $\frac{950120-}{127}$ & $\begin{array}{c}950120- \\
131\end{array}$ & $\begin{array}{c}950125- \\
0 \% 9\end{array}$ & $\underset{042}{950130}$ & $\begin{array}{l}950001- \\
006\end{array}$ \\
\hline 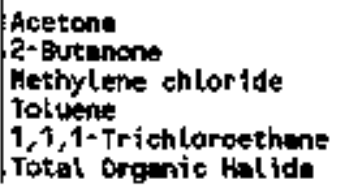 & $\begin{array}{r}15 \\
13 \\
100 \\
7 \\
9 \\
100\end{array}$ & $\begin{array}{r}17 \\
11 \\
100 \\
7 \\
2 \\
1001\end{array}$ & $\begin{array}{c}100 \\
100 \\
100 \\
100 \\
2 \\
100\end{array}$ & $\begin{array}{r}100 \\
100 \\
100 \\
100 \\
3 \\
100\end{array}$ & $\begin{array}{l}100 \\
100 \\
100 \\
100 \\
\text { tou }\end{array}$ & $\begin{array}{l}200 \\
200 \\
100 \\
100 \\
100 \\
100\end{array}$ & $\begin{array}{r}9 \\
8 \\
1001 \\
8 \\
6\end{array}$ & $\begin{array}{r}100 \\
100 \\
100 \\
100 \\
10\end{array}$ & $\begin{array}{l}100 \\
100 \\
100 \\
100\end{array}$ \\
\hline
\end{tabular}

(COHIIMUED)

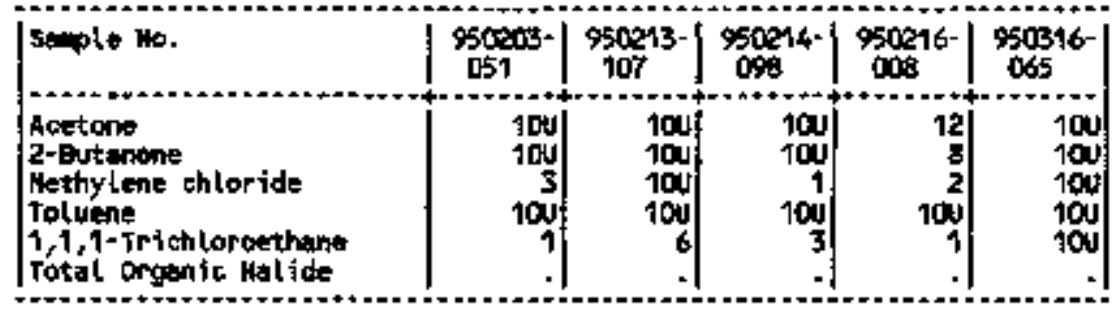


APpandx L.2

Yoce Datected In Trip Blank saleles, second ourter $19 \%$

\begin{tabular}{|c|c|c|c|c|c|c|c|c|c|}
\hline sonple No. & $\begin{array}{c}950405- \\
009\end{array}$ & $\begin{array}{c}950406- \\
044\end{array}$ & $\begin{array}{c}950406- \\
029\end{array}$ & $\begin{array}{l}\text { 950407- } \\
091\end{array}$ & $\begin{array}{c}950507- \\
037\end{array}$ & $\begin{array}{c}950407^{-} \\
057\end{array}$ & $\begin{array}{c}50410 \\
041\end{array}$ & $\begin{array}{c}950410-1 \\
\text { o57 }\end{array}$ & $\begin{array}{c}950412- \\
036\end{array}$ \\
\hline $\begin{array}{l}\text { Acetone } \\
2 \text {-eutenone } \\
1,2-\text { - biehloroethene } \\
\text { Mothylene chloride } \\
1,1,1 \text {-Trichloroethene }\end{array}$ & $\begin{array}{r}100 \\
100 \\
100 \\
100 \\
3\end{array}$ & $\begin{array}{l}100 \\
100 \\
100 \\
100 \\
3\end{array}$ & $\begin{array}{r}100 \\
100 \\
100 \\
3 \\
3\end{array}$ & $\begin{array}{c}100 \\
100 \\
100 \\
100 \\
100\end{array}$ & $\begin{array}{c}100 \\
100 \\
700 \\
104 \\
3\end{array}$ & $\begin{array}{l}100 \\
100 \\
100 \\
100 \\
100\end{array}$ & $\begin{array}{l}100 \\
100 \\
100 \\
100 \\
100\end{array}$ & $\begin{array}{l}100 \\
100 \\
100 \\
100 \\
2\end{array}$ & \\
\hline
\end{tabular}

(CONTINNED)

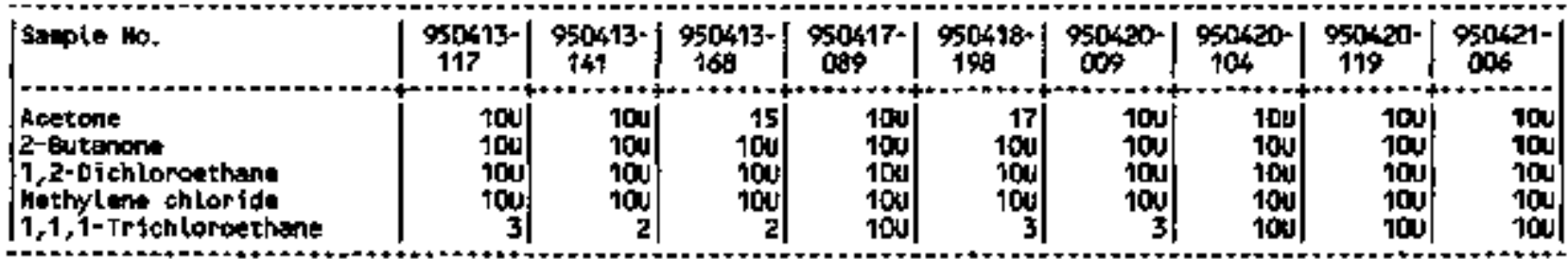

(CONTIMUED)

\begin{tabular}{|c|c|c|c|c|c|c|c|c|c|}
\hline apte w & $\begin{array}{c}930421- \\
05 B\end{array}$ & $\begin{array}{c}950423- \\
003\end{array}$ & $\begin{array}{c}90424- \\
076\end{array}$ & $\begin{array}{c}950426- \\
141\end{array}$ & $\begin{array}{c}95025- \\
057\end{array}$ & $\begin{array}{c}70426- \\
265\end{array}$ & $\begin{array}{c}950426- \\
270\end{array}$ & $\begin{array}{l}950426- \\
312\end{array}$ & $\begin{array}{l}970427 \\
125\end{array}$ \\
\hline $\begin{array}{l}\text { Acetone } \\
\text { 2-Butanche } \\
1,2 \text {-0ichlorathetere } \\
\text { wethylene ehloride } \\
1,1,1 \text {-Trichioroethene }\end{array}$ & $\begin{array}{l}100 \\
100 \\
100 \\
100\end{array}$ & $\begin{array}{l}100 \\
100 \\
100 \\
104\end{array}$ & $\begin{array}{l}100 \\
100 \\
100 \\
700 \\
2\end{array}$ & $\begin{array}{r}100 \\
100 \\
100 \\
100 \\
2\end{array}$ & $\begin{array}{l}100 \\
100 \\
\text { tou } \\
10 \mathrm{n} \\
2\end{array}$ & $\begin{array}{l}100 \\
100 \\
100 \\
100 \\
100\end{array}$ & $\begin{array}{r}100 \\
11 \\
100 \\
100 \\
2\end{array}$ & $\begin{array}{l}100 \\
100 \\
100 \\
100 \\
100\end{array}$ & \\
\hline
\end{tabular}

(CONTIMUED)

\begin{tabular}{|c|c|c|c|c|c|c|c|c|c|}
\hline spimple to. & $\begin{array}{c}930427- \\
131\end{array}$ & $\begin{array}{c}950427- \\
197\end{array}$ & $\begin{array}{c}950427-1 \\
213\end{array}$ & $\begin{array}{c}950501- \\
129\end{array}$ & $\begin{array}{c}950501- \\
145\end{array}$ & $\begin{array}{c}950502- \\
091\end{array}$ & $\begin{array}{l}\$ 50502- \\
106\end{array}$ & $\frac{950502-}{137}$ & $\begin{array}{l}950505- \\
737\end{array}$ \\
\hline 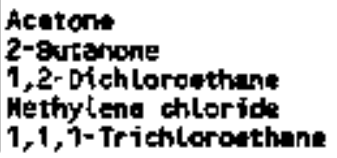 & $\begin{array}{l}100 \\
\text { TDLt } \\
100 \\
100 \\
100\end{array}$ & $\begin{array}{l}100 \\
100 \\
100 \\
100 \\
100\end{array}$ & $\begin{array}{l}100 \\
100 \\
100 \\
100 \\
100\end{array}$ & $\begin{array}{l}100 \\
100 \\
100 \\
100 \\
100\end{array}$ & $\begin{array}{r}700 \\
100 \\
100 \\
100 \\
2\end{array}$ & $\begin{array}{l}100 \\
100 \\
100 \\
100 \\
100\end{array}$ & $\begin{array}{l}10 \mathrm{w} \\
10 \mathrm{w} \\
100 \\
100 \\
10 \mathrm{~N}\end{array}$ & 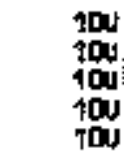 & $\begin{array}{c}100 \\
1004 \\
1001 \\
100\end{array}$ \\
\hline
\end{tabular}

(CONTIRUED)

\begin{tabular}{|c|c|c|c|c|c|c|}
\hline seple Mo. & $\operatorname{sis}_{145}$ & $\begin{array}{l}9505+3- \\
149\end{array}$ & $\begin{array}{l}950505- \\
031\end{array}$ & $\begin{array}{l}95012- \\
\text { ous }\end{array}$ & $\begin{array}{c}950516- \\
\cos \end{array}$ & $\begin{array}{l}\cos 16= \\
127\end{array}$ \\
\hline 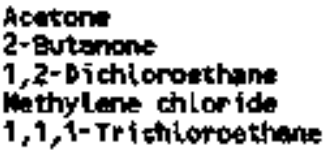 & $\begin{aligned} 10 \mathrm{~J} \\
100 \\
10 \mathrm{i} \\
100 \\
1\end{aligned}$ & $\begin{array}{l}10 \mathrm{y} \\
10 \mathrm{v} \\
10 \mathrm{~N}\end{array}$ & $\begin{array}{l}27 \\
1005 \\
100 \\
100 \\
3\end{array}$ & $\begin{array}{l}100 \\
100 \\
100 \\
100 \\
3\end{array}$ & $\begin{array}{r}100 \\
1004 \\
100 \\
100 \\
2\end{array}$ & $\begin{array}{l}200 \\
100 \\
100 \\
100 \\
100\end{array}$ \\
\hline
\end{tabular}


APPEND1X L.2

VDCs becected in Trip olank syples, Thind ourter 1995

\begin{tabular}{|c|c|c|c|c|c|c|c|c|c|}
\hline I Sample to. & 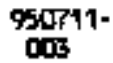 & $\underset{0 \leq 5}{950711-}$ & $\begin{array}{c}950712- \\
003\end{array}$ & $\begin{array}{l}950712- \\
011\end{array}$ & $\underset{\substack{950713 \\
016}}{\cos }$ & $\begin{array}{c}950713- \\
070\end{array}$ & $\begin{array}{c}950713- \\
126\end{array}$ & $\begin{array}{c}950714- \\
056\end{array}$ & $\begin{array}{l}\text { 950717- } \\
105\end{array}$ \\
\hline $\begin{array}{l}\text { Acetone } \\
1,1,1-\text { Frichioroethane }\end{array}$ & 1 & t & 1 & 1 & 1 & 100 & $\begin{array}{l}100 \\
100\end{array}$ & 100 & 10u \\
\hline
\end{tabular}

(CONTINUED)

\begin{tabular}{|c|c|c|c|c|c|c|c|c|}
\hline Sample No. & $\begin{array}{c}950717- \\
100\end{array}$ & $\begin{array}{c}950717- \\
115\end{array}$ & $\begin{array}{c}950718-1 \\
604\end{array}$ & $\begin{array}{l}\text { 950719- } \\
292\end{array}$ & $\underset{014}{950731-}$ & $\begin{array}{c}950731- \\
053\end{array}$ & $\begin{array}{c}950734- \\
084\end{array}$ & \begin{tabular}{c|c}
$950501-$ & $950001-$ \\
012 & $\mathrm{CH}_{4}$
\end{tabular} \\
\hline & & 10 & & 10 & & & & \\
\hline
\end{tabular}

( CONTINLEO)

\begin{tabular}{|c|c|c|}
\hline Salpole to. & $\begin{array}{c}950001-1 \\
071\end{array}$ & $\begin{array}{c}9509072 \\
033\end{array}$ \\
\hline $\begin{array}{l}\text { Acerone } \\
1,1,1-\text { Trithlorothane }\end{array}$ & 10네 & 10 \\
\hline
\end{tabular}


APPEDIX L.2

wocs oetected in Trip Blonk seples, Fourth Gurter $19 \%$

\begin{tabular}{|c|c|c|c|c|c|c|c|c|c|}
\hline serple No. & $\begin{array}{c}9100 \%- \\
007\end{array}$ & $\begin{array}{c}\$ 51009 \\
045\end{array}$ & $\underset{075}{951709-}$ & $\begin{array}{c}951009- \\
121\end{array}$ & $\underset{461}{951009-}$ & $\begin{array}{l}951010- \\
007\end{array}$ & $\begin{array}{c}\text { \%10 } \\
\text { 013 }\end{array}$ & $\begin{array}{c}91010 \\
028\end{array}$ & $\begin{array}{c}\text { cton1- } \\
064\end{array}$ \\
\hline 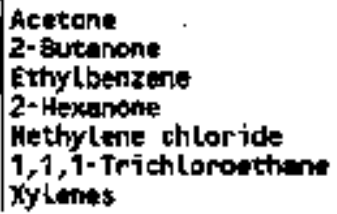 & $\begin{array}{r}100 \\
7 \\
100 \\
100 \\
100 \\
7 \\
100\end{array}$ & $\begin{array}{l}100 \\
100 \\
3000 \\
100 \\
100 \\
8 \\
100\end{array}$ & $\begin{array}{r}100 \\
100 \\
700 \\
100 \\
100 \\
2 \\
104\end{array}$ & $\begin{array}{r}10: 0 \\
100 \\
1001 \\
100 \\
100 \\
2 \\
100\end{array}$ & $\begin{array}{l}100 \\
100 \\
100 \\
100 \\
100 \\
2 \\
100\end{array}$ & $\begin{array}{r}100 \\
10 \\
3 \\
10,1 \\
1 \\
9 \\
12\end{array}$ & $\begin{array}{r}100 \\
9 \\
3 \\
100 \\
1 \\
2 \\
12\end{array}$ & $\begin{array}{r}100 \\
100 \\
100 \\
100 \\
100 \\
2 \\
100\end{array}$ & $\begin{array}{l}100 \\
100 \\
1+2 \\
100 \\
100 \\
2\end{array}$ \\
\hline
\end{tabular}

(CONTIMUED)

\begin{tabular}{|c|c|c|c|c|c|c|c|c|c|}
\hline Sanple to. & $\underset{030}{\$ 51012-}$ & 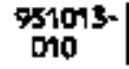 & $\begin{array}{c}\text { \%1017- } \\
\text { 니 }\end{array}$ & 91618- & $\begin{array}{c}95+027-1 \\
055\end{array}$ & $\begin{array}{c}\$ 51101- \\
\mathbf{a 1}\end{array}$ & $\begin{array}{c}951101- \\
0: 2\end{array}$ & $\underset{044}{9+162-}$ & $\begin{array}{c}51,103- \\
102\end{array}$ \\
\hline 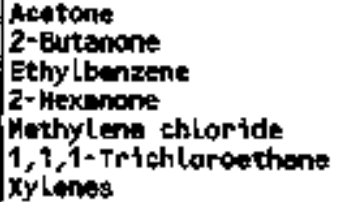 & $\begin{array}{r}100 \\
100 \\
100 \\
10 u \\
2 \\
100\end{array}$ & $\begin{array}{r}10 \\
100 \\
100 \\
904 \\
2 \\
100\end{array}$ & $\begin{array}{r}34 \\
100 \\
8 \\
100 \\
700 \\
100\end{array}$ & $\begin{array}{l}100 \\
100 \\
100 \\
100 \\
100 \\
1001\end{array}$ & $\begin{array}{r}100 \\
100 \\
100 \\
10 \\
4 \\
100\end{array}$ & $\begin{array}{l}100 \\
100 \\
100 \\
100 \\
100\end{array}$ & $\begin{array}{r}100 \\
100 \\
100 \\
10 \mathrm{~N} \\
4 \\
10 \mathrm{v}\end{array}$ & $\begin{array}{r}100 \\
100 \\
100 \\
100 \\
4 \\
100\end{array}$ & 录 \\
\hline
\end{tabular}

\section{(CONTIKNUED)}

\begin{tabular}{|c|c|c|c|c|c|c|c|c|c|}
\hline secple wo. & $\begin{array}{c}951105- \\
043\end{array}$ & $\begin{array}{c}951103- \\
052\end{array}$ & $\begin{array}{c}91100- \\
0<2\end{array}$ & $\underset{\mathrm{DC3}}{951104-1}$ & $\begin{array}{c}95 t 10 s-1 \\
004\end{array}$ & $\begin{array}{c}951105- \\
037\end{array}$ & $\begin{array}{c}951106 \\
0 \leq 3\end{array}$ & $\begin{array}{c}951106- \\
097\end{array}$ & $\begin{array}{c}951107- \\
007\end{array}$ \\
\hline 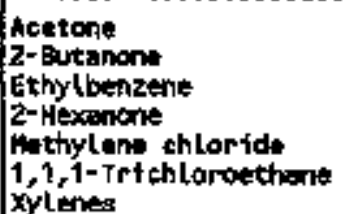 & $\begin{array}{c}100 \\
100 \\
100 \\
100 \\
100 \\
4 \\
104\end{array}$ & $\begin{array}{r}100 \\
104 \\
100 \\
104 \\
100 \\
3 \\
100\end{array}$ & $\begin{array}{l}100 \\
100 \\
100 \\
100 \\
100 \\
100\end{array}$ & $\begin{array}{r}100 \\
100 \\
100 \\
100 \\
100 \\
3 \\
100\end{array}$ & $\begin{array}{r}100 \\
10 \\
100 \\
100 \\
100 \\
10 \\
100\end{array}$ & $\begin{array}{c}100 \\
100 \\
100 \\
100 \\
100 \\
100 \\
100\end{array}$ & $\begin{array}{l}100 \\
100 \\
100 \\
100 \\
100 \\
100 \\
100\end{array}$ & $\begin{array}{l}100 \\
100 \\
100 \\
100 \\
100 \\
100 \\
100\end{array}$ & $\begin{array}{l}100 \\
100 \\
100 \\
\text { tou } \\
100 \\
100 \\
100\end{array}$ \\
\hline
\end{tabular}

(CONTIKNED)

\begin{tabular}{|c|c|c|c|c|c|c|c|c|c|}
\hline Sunpte No. & $\begin{array}{c}951307- \\
056\end{array}$ & $\begin{array}{c}951107- \\
087\end{array}$ & $\begin{array}{c}951100 * \\
17 R\end{array}$ & $\begin{array}{c}951110- \\
003\end{array}$ & $\begin{array}{c}957110- \\
006\end{array}$ & $\begin{array}{c}951114 \\
008\end{array}$ & $\begin{array}{c}951174- \\
095\end{array}$ & $\begin{array}{c}951115 \\
056\end{array}$ & $\begin{array}{c}\text { 951117- } \\
\text { cos }\end{array}$ \\
\hline 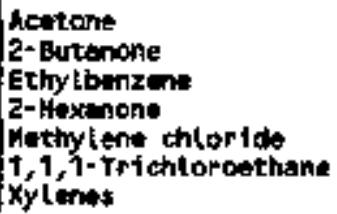 & $\begin{array}{c}100 \\
100 \\
100 \\
100 \\
100 \\
1 \\
100\end{array}$ & $\begin{array}{l}100 \\
100 \\
100 \\
1004 \\
100 \\
104 \\
100\end{array}$ & $\begin{array}{l}100 \\
100 \\
100 \\
100 \\
100 \\
100 \\
100\end{array}$ & $\begin{array}{l}100 \\
100 \\
100 \\
100 \\
100 \\
2 \\
100\end{array}$ & \begin{tabular}{r|}
100 \\
100 \\
100 \\
100 \\
100 \\
2 \\
100
\end{tabular} & $\begin{array}{l}100 \\
100 \\
100 \\
100 \\
100 \\
3 \\
100\end{array}$ & $\begin{array}{l}100 \\
100 \\
100 \\
1004 \\
300 \\
3 \\
1004\end{array}$ & $\begin{array}{c}100 \\
100 \\
100 \\
100 \\
100 \\
3 \\
10 \mathrm{w}\end{array}$ & i \\
\hline
\end{tabular}

(CONTHNED)

\begin{tabular}{|c|c|c|}
\hline saple to. & $\begin{array}{c}\text { 91120- } \\
007\end{array}$ & $\underset{\cos }{\mathrm{S} 11121+}$ \\
\hline 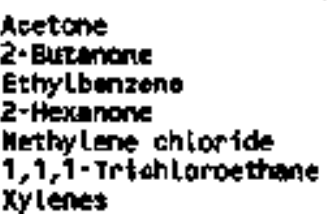 & $\begin{array}{c}12 \\
100 \\
100 \\
100 \\
100 \\
1004 \\
100\end{array}$ & $\begin{array}{c}100 \\
15 \\
200 \\
100 \\
700 \\
2\end{array}$ \\
\hline
\end{tabular}




\section{APPENDIX L.3}

LABORATORY BLANK DATA SUMMARY 

APPENDIX L.3

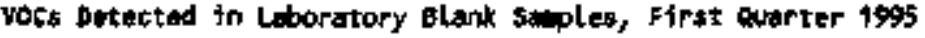

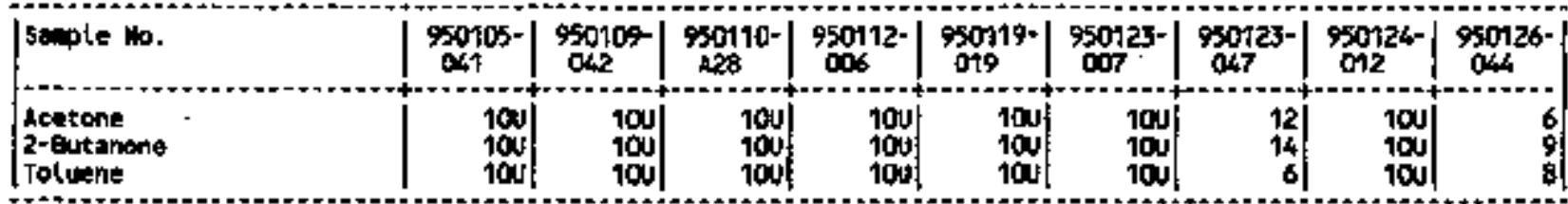

(CONIIHUD)

\begin{tabular}{|c|c|c|c|c|c|c|}
\hline sapale Ho. & 950001- & $\begin{array}{c}950208- \\
0 \leqslant 5\end{array}$ & $\begin{array}{c}950214- \\
\text { of } 4\end{array}$ & $\begin{array}{c}950015-1 \\
011\end{array}$ & $\cos _{\cos 17-}$ & $\begin{array}{l}950317- \\
0+1\end{array}$ \\
\hline one & 100 & $\begin{array}{l}100 \\
100 \\
100\end{array}$ & 100 & 100 & $\begin{array}{l}100 \\
100\end{array}$ & \\
\hline
\end{tabular}


APPENO1X L+3

woch Detected in Leboratory Blank saples, Sacond Quorter 1995

\begin{tabular}{|c|c|c|c|c|c|c|c|c|c|}
\hline Sacple to. & $\begin{array}{l}950405- \\
067\end{array}$ & $\begin{array}{c}950406-1 \\
054\end{array}$ & $\begin{array}{c}950406-1 \\
657\end{array}$ & $\begin{array}{c}950407- \\
062\end{array}$ & $\underset{046}{950410-}$ & $\underset{050}{95411-1}$ & $\underset{60413-}{950413}$ & ${ }_{004}^{950417-1}$ & $\begin{array}{c}950418-1 \\
0,53\end{array}$ \\
\hline onane & 100 & $\begin{array}{l}100 \\
1001\end{array}$ & 40 & 100 & $\begin{array}{l}100 \\
100\end{array}$ & rou & 100 & $\begin{array}{l}10 \mathrm{u} \\
10 \mathrm{v}\end{array}$ & 70 \\
\hline
\end{tabular}

(CONTIU⿴囗十)

\begin{tabular}{|c|c|c|c|c|c|c|c|c|c|}
\hline sanple to. & $\begin{array}{c}950419- \\
002\end{array}$ & $\underset{0,3}{950420}$ & $\underset{\operatorname{Dos}^{2}}{950424}$ & $\begin{array}{c}950425- \\
005\end{array}$ & $\begin{array}{c}950426- \\
301\end{array}$ & $\begin{array}{c}950427 \\
001\end{array}$ & $\underset{034}{950501-1}$ & $\underset{023}{950502-1}$ & $\begin{array}{c}900504-1 \\
016\end{array}$ \\
\hline ore chlortde & $\begin{array}{l}105 \\
100\end{array}$ & 100 & rout & 13 & 10. & 100 & $\begin{array}{l}10 t h \\
100\end{array}$ & $\begin{array}{l}20 \mathrm{y} \\
10 \mathrm{v}\end{array}$ & \\
\hline
\end{tabular}

(CONIINUCD)

\begin{tabular}{|c|c|c|c|c|c|c|}
\hline simple No. & $\begin{array}{c}950505^{\circ} \\
001\end{array}$ & $\begin{array}{c}\text { \%ostos- } \\
\text { ctz }\end{array}$ & $\underset{028}{\cos 12}$ & $\underset{\cos 3}{\cos 15-}$ & $\underset{021}{950516-}$ & $\cos _{0.2}+8-$ \\
\hline $\begin{array}{l}\text { Acetone } \\
\text { 2-Butanone } \\
\text { Wethylene ehloride }\end{array}$ & $\begin{array}{l}100 \\
100 \\
100\end{array}$ & $\begin{array}{l}100 \\
100 \\
100\end{array}$ & $\begin{array}{l}100 \\
1000 \\
1001\end{array}$ & $\begin{array}{l}100 \\
100 \\
100\end{array}$ & $\begin{array}{l}100 \\
100 \\
100\end{array}$ & 10 \\
\hline
\end{tabular}


APPET:DX L.3

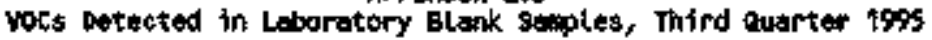

\begin{tabular}{|c|c|c|c|c|c|c|c|c|c|}
\hline Sople Ho. & $\mathrm{OOF}_{6}$ & PS0712- & ${ }_{+21}^{90713-}$ & $\underset{003}{950717}$ & ${ }^{950718-}$ & ${ }_{035}^{20712-}$ & ${ }_{030}^{90726-}$ & $\frac{950731-}{106}$ & ${ }_{044}^{90803-}$ \\
\hline
\end{tabular}

(CONTIMUED)

\begin{tabular}{|c|c|}
\hline sanpite tho. & $950804-1$ \\
\hline 2-Butanon & toul \\
\hline
\end{tabular}


APPEDIX $L+3$

Yocs detected in Laboratory Blank sopies, Fourth quarter T995

\begin{tabular}{|c|c|c|c|c|c|c|c|c|c|}
\hline |sanple No. & $\begin{array}{c}\text { \%to11- } \\
\cos \end{array}$ & $\begin{array}{c}951012- \\
008\end{array}$ & $\begin{array}{c}951012- \\
036\end{array}$ & $\begin{array}{l}951016- \\
028\end{array}$ & $\begin{array}{l}951019- \\
\text { onz }\end{array}$ & $\begin{array}{c}\text { s5f019- } \\
076\end{array}$ & $\begin{array}{c}951023- \\
043\end{array}$ & $\begin{array}{c}951003- \\
009\end{array}$ & $\begin{array}{c}\$ 1031 \\
007\end{array}$ \\
\hline 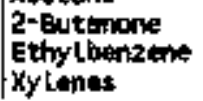 & $\begin{array}{l}10 \\
100 \\
100\end{array}$ & $\begin{array}{l}9 \\
2 \\
7\end{array}$ & $\begin{array}{l}1004 \\
100\end{array}$ & $\begin{array}{l}10 \\
100 \\
100\end{array}$ & $\begin{array}{l}100 \\
100 \\
100\end{array}$ & $\begin{array}{l}100 \\
100 \\
100\end{array}$ & $\begin{array}{l}100 \\
100 \\
100\end{array}$ & $\begin{array}{l}100 \\
100 \\
100\end{array}$ & \\
\hline
\end{tabular}

(COMT IMUED)

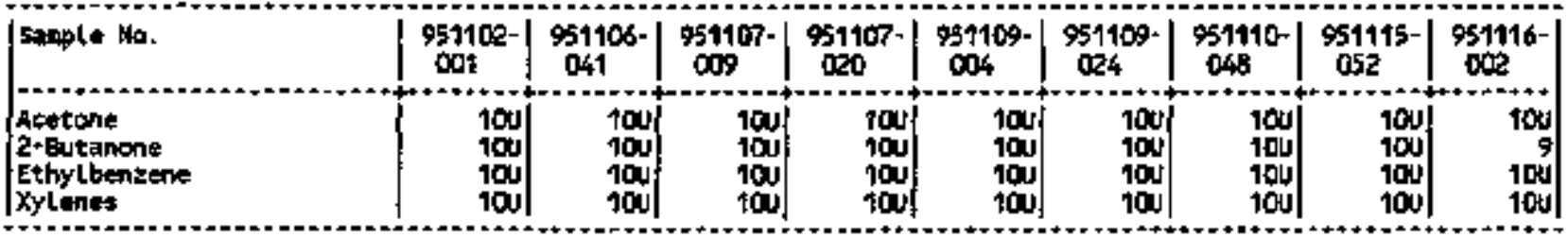

(CONTINUED)

\begin{tabular}{|c|c|c|c|}
\hline saiple Mo. & $\begin{array}{c}951118 \\
\text { od3 }\end{array}$ & $\begin{array}{c}981120- \\
002\end{array}$ & $\begin{array}{c}951122-1 \\
005\end{array}$ \\
\hline $\begin{array}{l}\text { Acetone } \\
2 \text {-furtanome } \\
\text { Ethytbenzene } \\
\text { Xyleng: }\end{array}$ & $\begin{array}{l}100 \\
100 \\
100 \\
100\end{array}$ & $\begin{array}{l}100 \\
100 \\
100 \\
100\end{array}$ & $\begin{array}{l}100 \\
100 \\
100 \\
100\end{array}$ \\
\hline
\end{tabular}


APPENDIX $\mathrm{L} .4$

EQUIPMENT RINSATE DATA SUMMARY 

APPENDIX L.4

Equipment Rinsote buta somary, first Ouster 196s

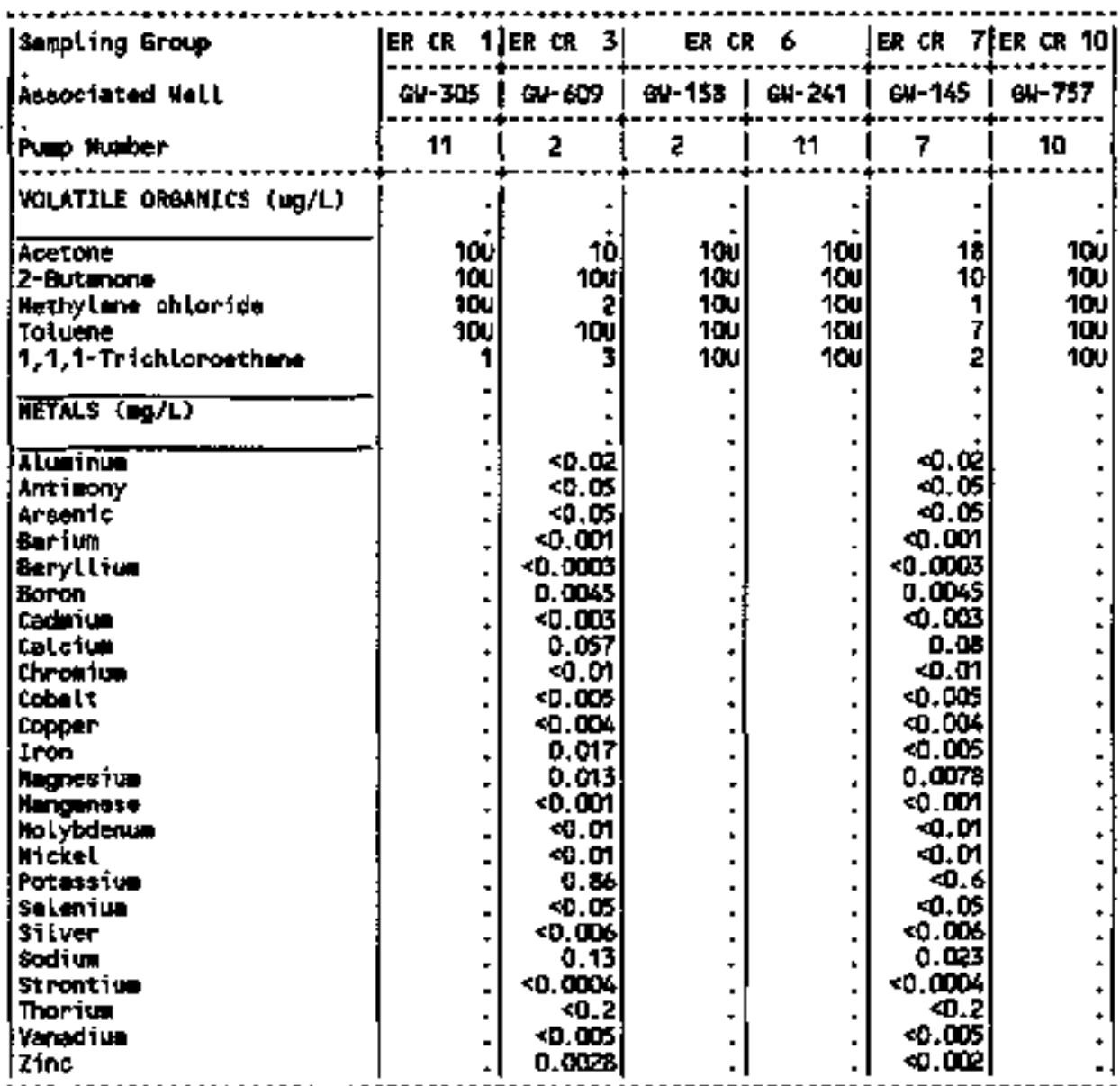


APPEiptx t.4

Equipant Rinsote oate sumbory, second Ourter $19 \%$

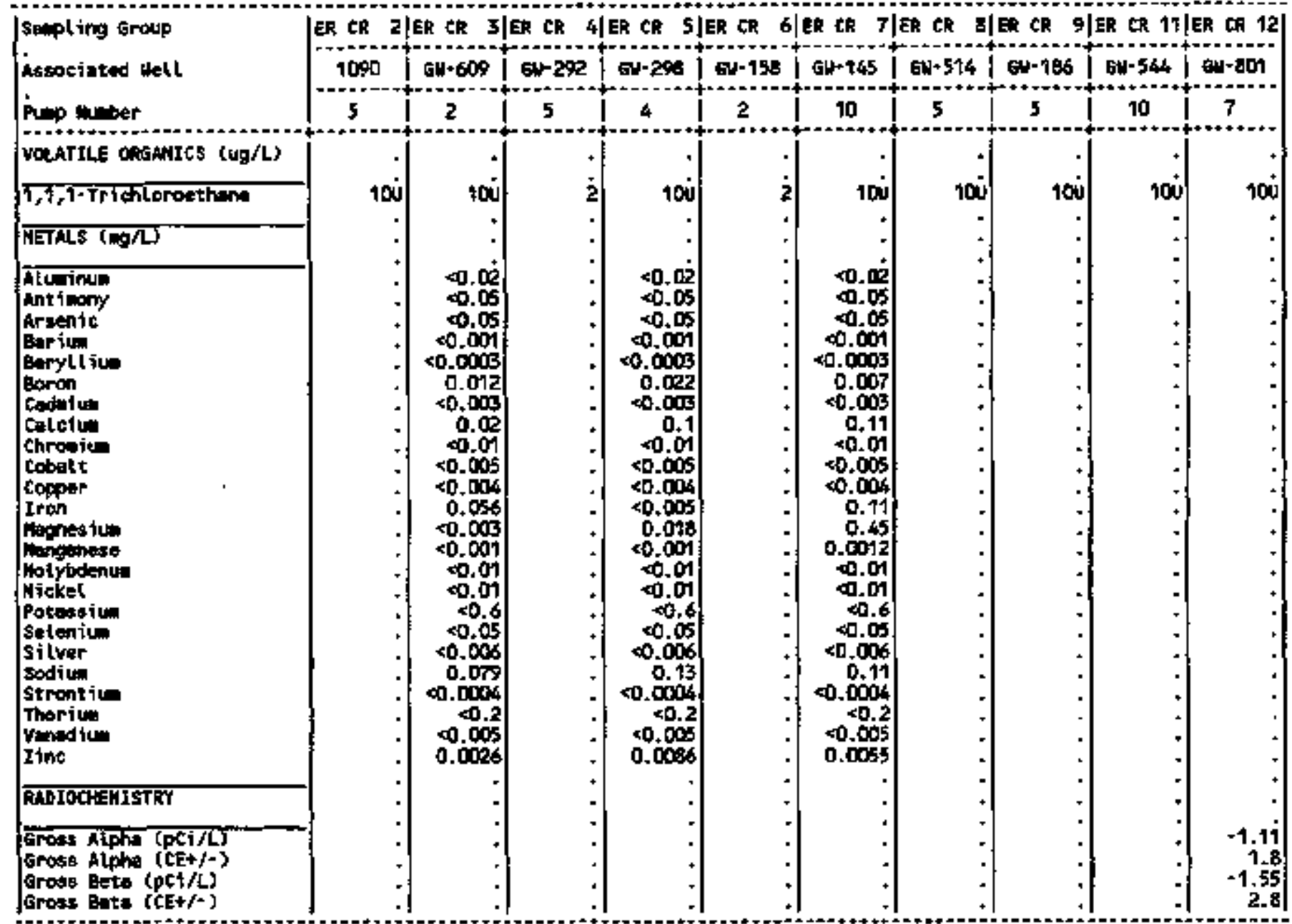


APFEHDIX L.4

Equipant Rinsete Date smary, Third curter 1995

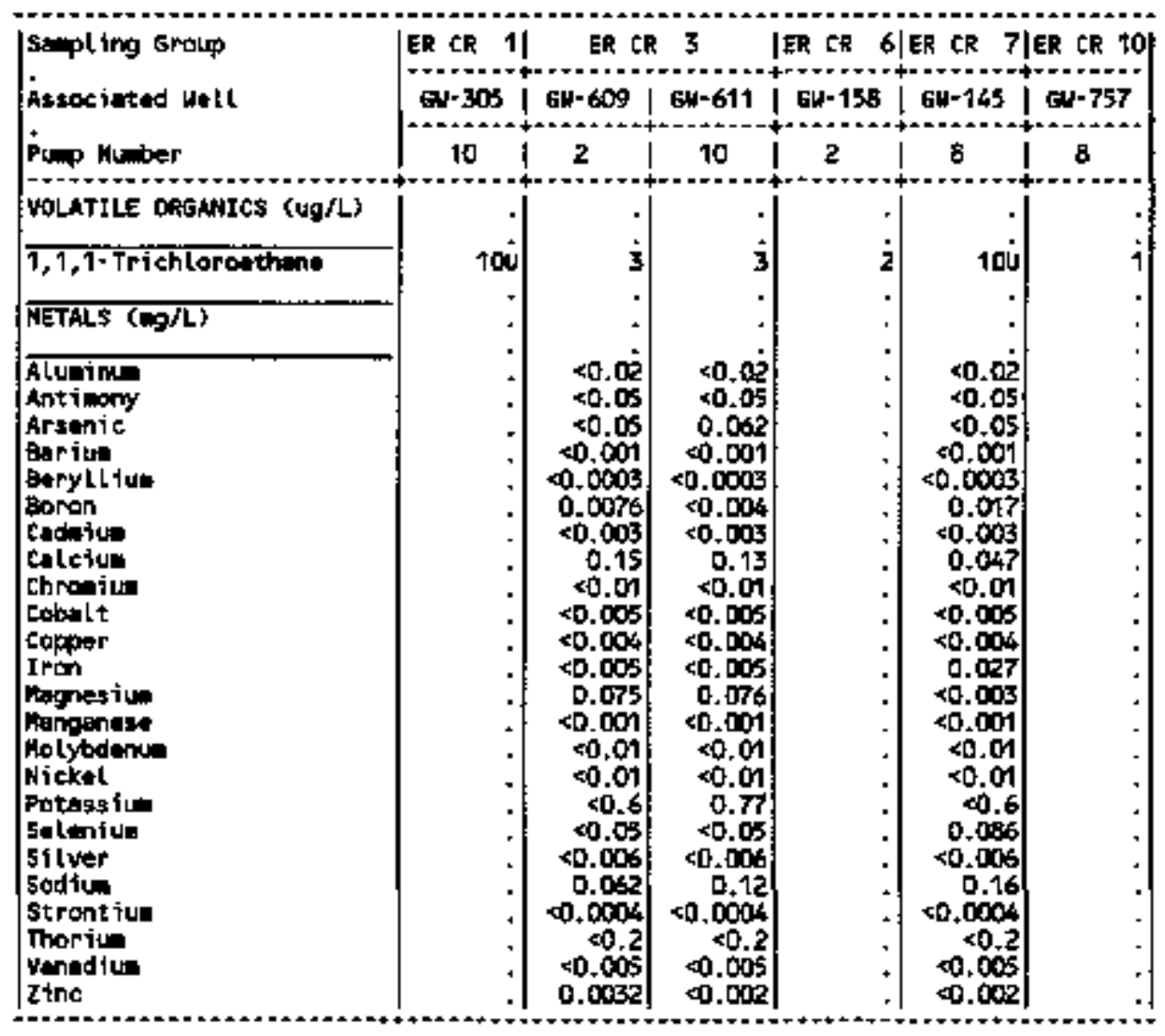


APPENDIX L.4

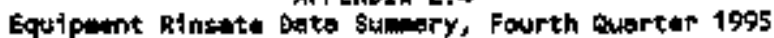

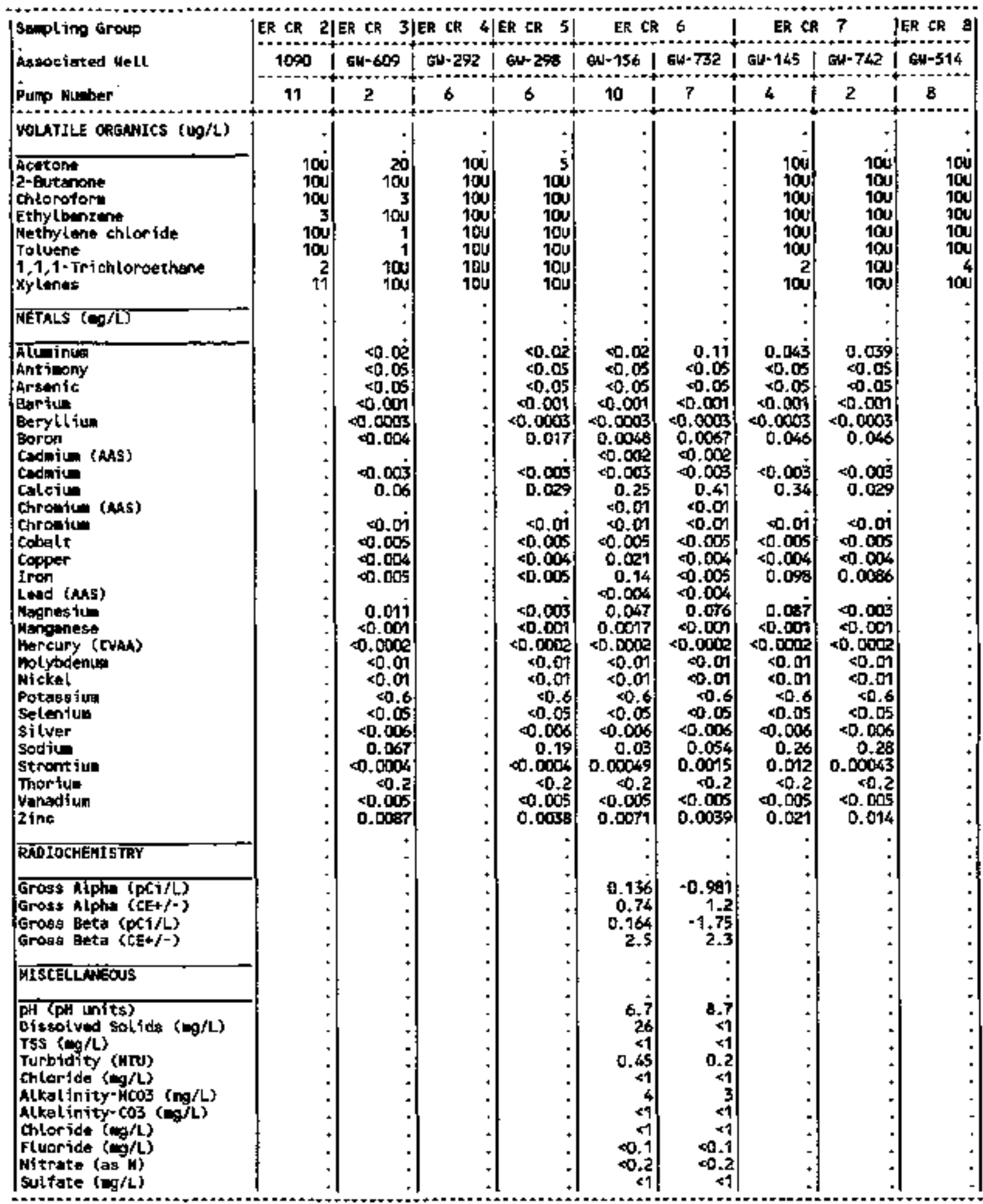

(CDTINEED) 
APPENDIX L.4

Equipanent Rinsate buta Sumisry, fourth ouarter 1995

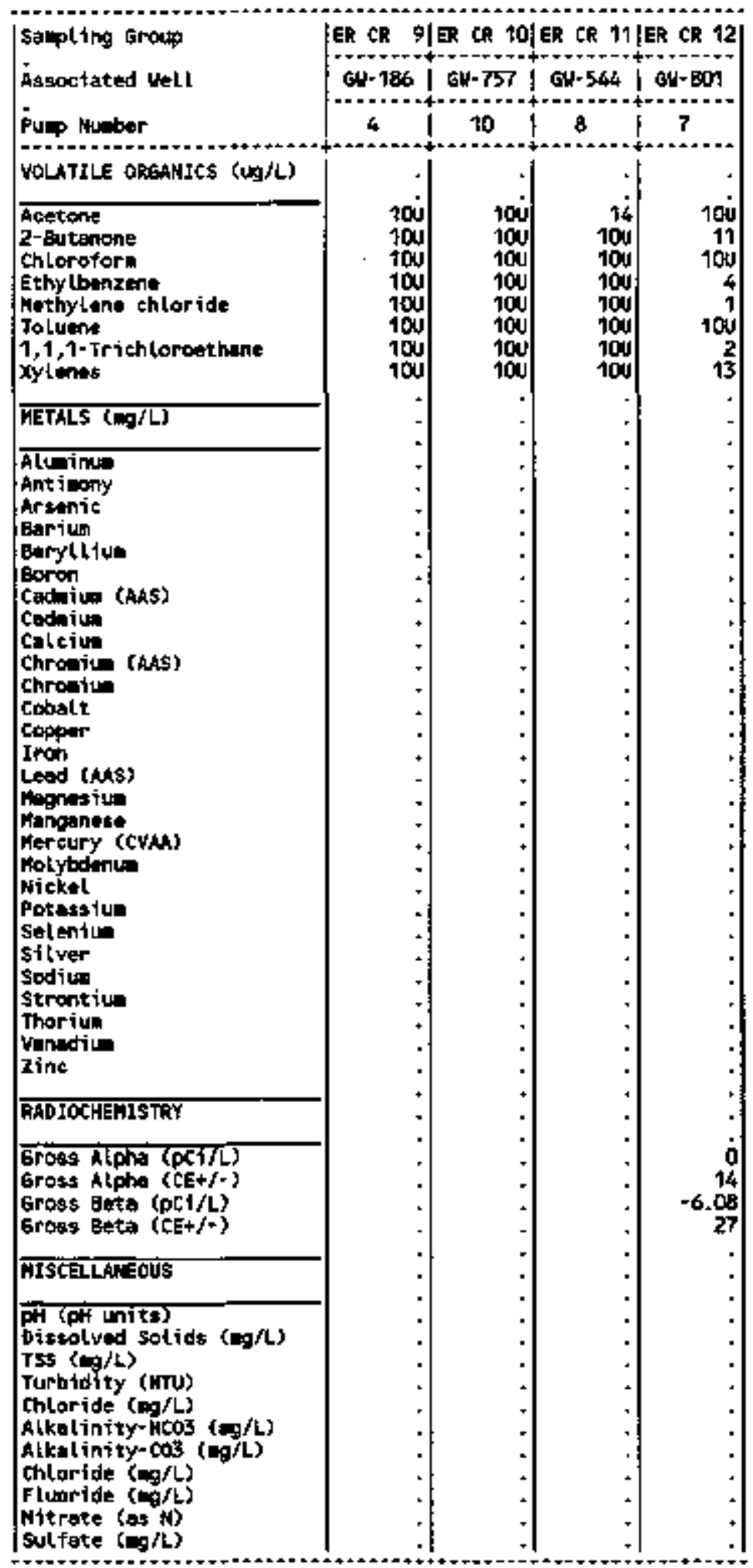




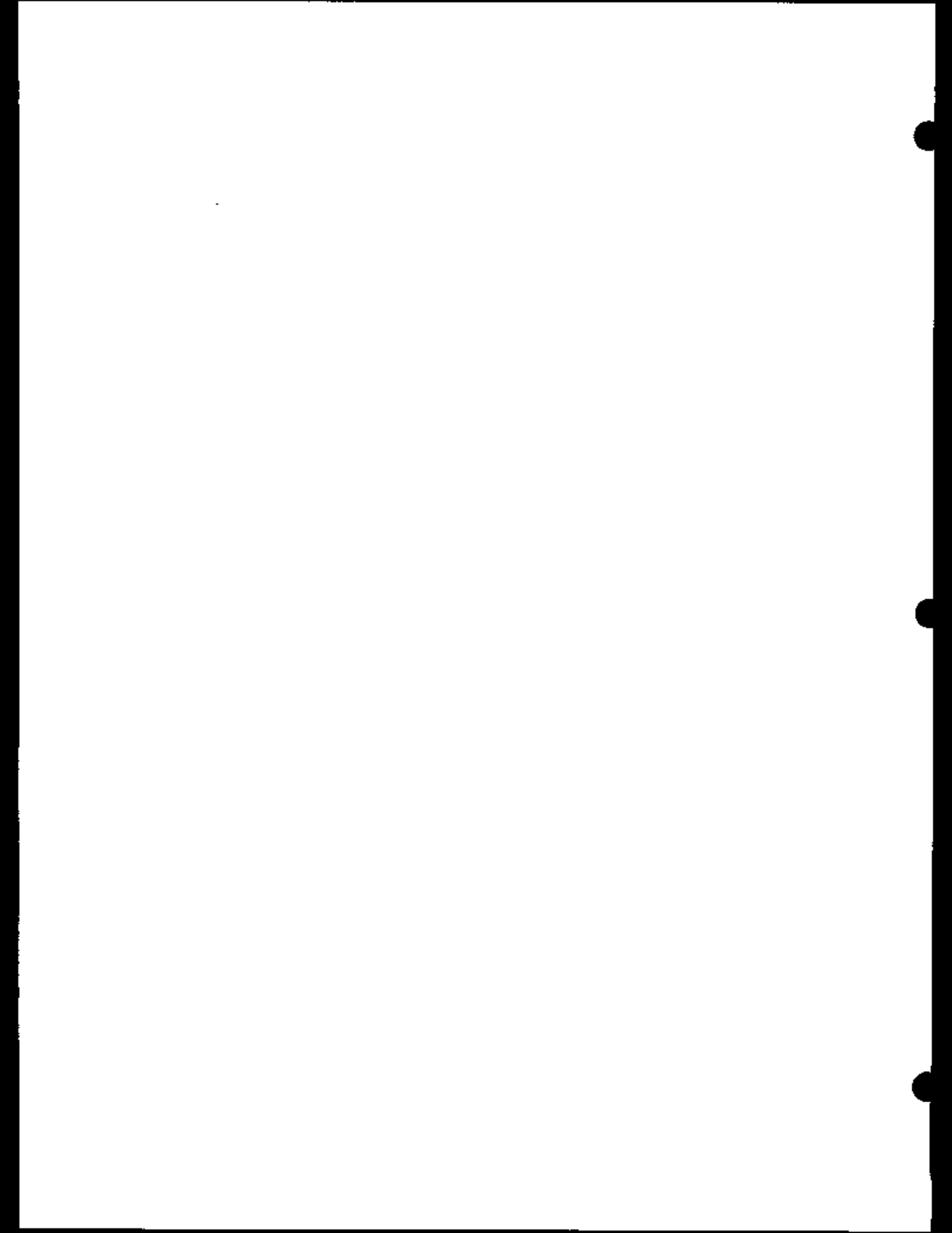




\section{APPENDIX $\mathbf{L . 5}$}

FIELD BLANK DATA SUMMARY 

APFENDIX L+ 5

field Blank Date sienery, 1995

\begin{tabular}{|c|c|c|}
\hline $\begin{array}{l}\text { 5ipling Group } \\
\text { onte sepled }\end{array}$ & $\mid \begin{array}{r}\text { FB CR 6 } 6 \\
\hdashline 01 / 19 / 95 \mid 04 / 09 / 95\end{array}$ & $\begin{array}{l}\quad \text { |FB CR } 7 \\
5 \mid 07 / 18 / 95[11 / 75 / \%\end{array}$ \\
\hline VOLATIEE OREAMLES (Hg/L) & & \\
\hline $7,1,1-\mathrm{Tr}$ & 31 & 10i] \\
\hline
\end{tabular}


APPENDIX M

VALIDATED RESULTS OF

VOLATILE ORGANIC COMPOUND ANALYSES 


\section{EXPLANATION}

SAMPLING POINT:

$\begin{array}{rll}\text { GW } & - & \text { Monitoring Well } \\ \text { CBS-1 } & - & \text { Chestinut Branch Spring 1 } \\ \text { SCR2.2SP } & - & \text { South Chestnut Ridge Spring 2.2 } \\ 1090 & - & \text { Historical 1000-Series Monitoring Well }\end{array}$

LOCATION:

$\begin{array}{rll}\text { ADB } & - & \text { Ash Disposal Basin } \\ \text { CDLVI } & - & \text { Construction/Demolition Landfil VI } \\ \text { CDLVII } & - & \text { Construction/Demolition Landfil VI } \\ \text { CRBAWP } & - & \text { Chestrut Ridge Borrow Area Waste Pile } \\ \text { CRSDB } & - & \text { Chestrut Ridge Sediment Disposal Basin } \\ \text { CRSP } & - & \text { Chestnut Ridge Security Pits } \\ \text { ECRWP } & - & \text { East Chestnut Ridge Waste Pile } \\ \text { EXP-SW } & - & \text { Exit Pathway, Surface Water Sampling Location } \\ \text { KHQ } & - & \text { Ker Hollow Quarry } \\ \text { LII } & - & \text { Industrial Landfill II } \\ \text { LV } & - & \text { Industrial Landfill IV } \\ \text { LV } & - & \text { Industrial Landfill V } \\ \text { RQ } & - & \text { Rogers Quarry } \\ \text { UNCS } & - & \text { United Noclear Corporation Site }\end{array}$

NOTES:

All results in micrograms per liter (ug/L).

FP1 - $\quad$ Folse Positive result, screened by laboratory blank

FP2 - False Positive result, screened by trip blank

Compound not detected at the reported minimum attainable detection limit.

Results of VOC analyses reported for each groundwater sample were evaluated using results for each associated labotatory and trip blank sample to identify false positives (i.e., results that are probable artifacts of the groundwater sampling or analysis procedures). To identify the false positives, the maximum concentration of each VOC detecied in either blank sample (Appendix L.2 and L.3) is multiplied by a qualification factor to determine the blank qualification nesult (BQR) for the compound. A factor of 5 is ussed for most compounds and a factor of 10 is used for the common laboratory reagents methylene chlotide, acetone, 2-butanone, and toluene (U.S. Environmental Protection Agency 1988). If a compound detected in the blank samples is also detected in an associated groundwater sample, the concentration reported for the groundwater sample (Appendix E) is compared to the corresponding $\mathrm{BQR}$. If the concentration of the compound in the groundwater sample is at or below the BQR, the result is considered a false positive. 
APPEMDIX .

Validated VoC Results, 1995

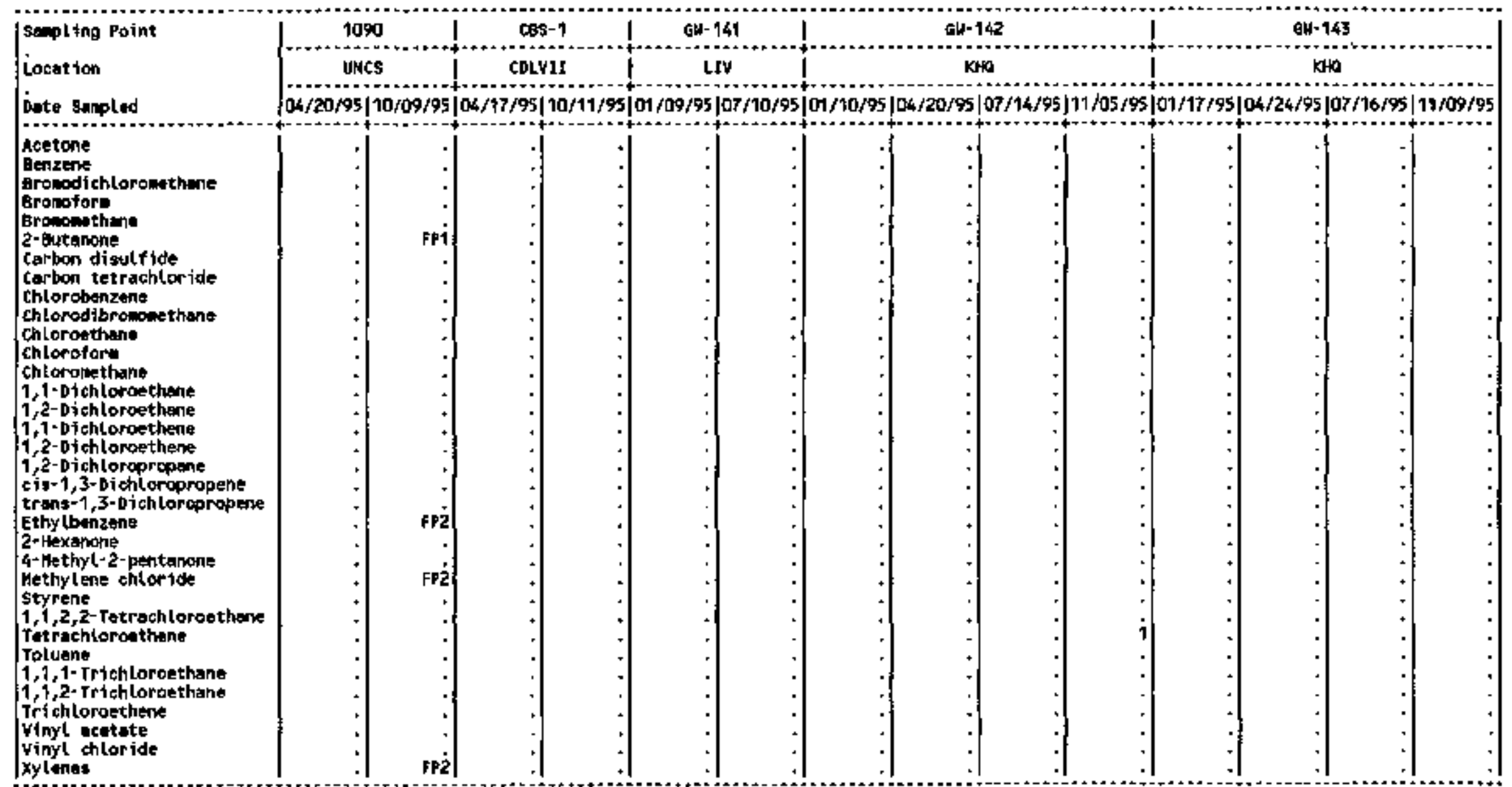

(CONTINUED) 
Validated woC Results, 1895

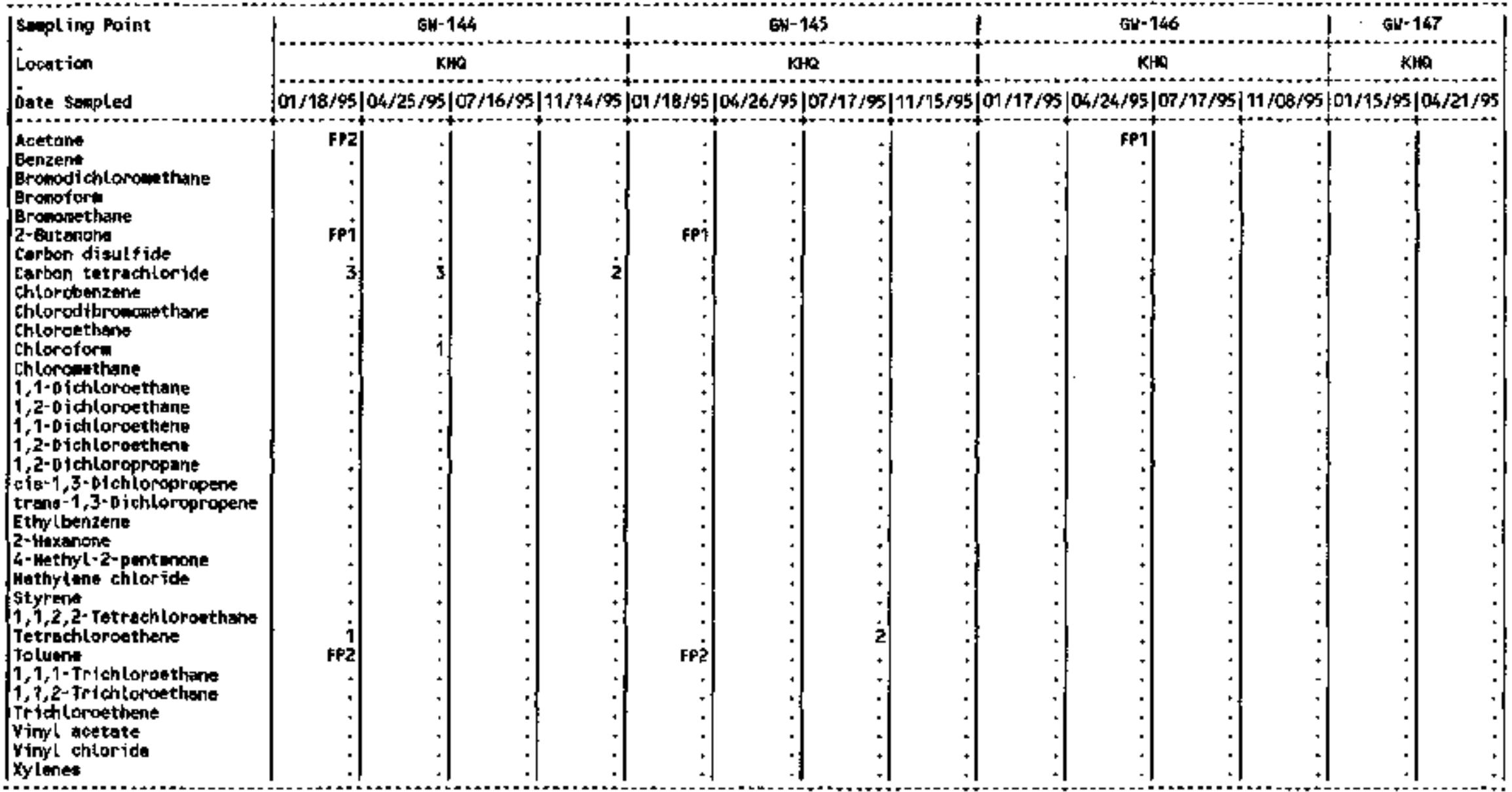

\section{(CONTINUED)}


APPEHPIX

Validated woC Rasslts, 1995

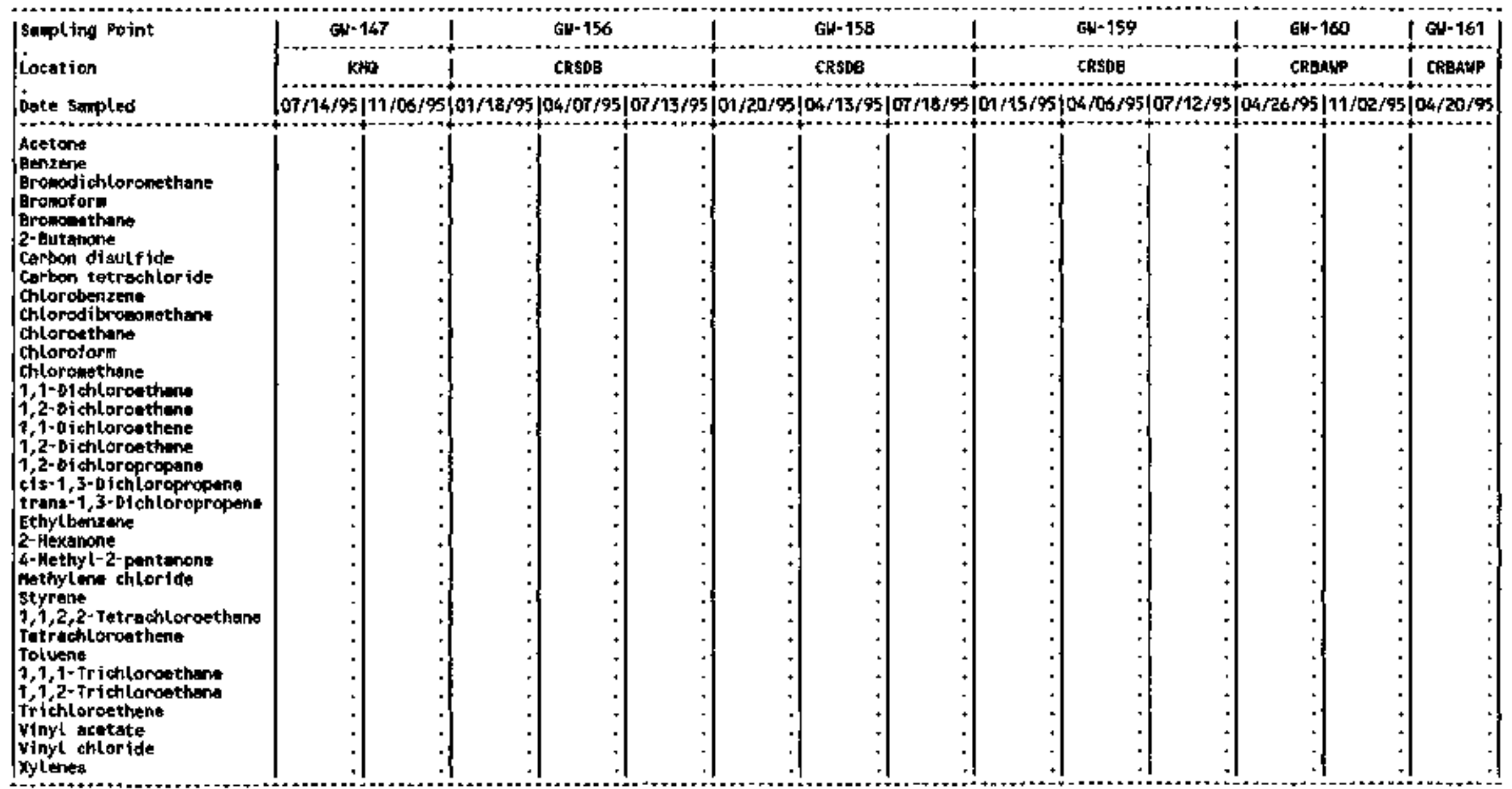

(CONTImueO) 
APPEKDIX H

vol idated yoc Results, 1995

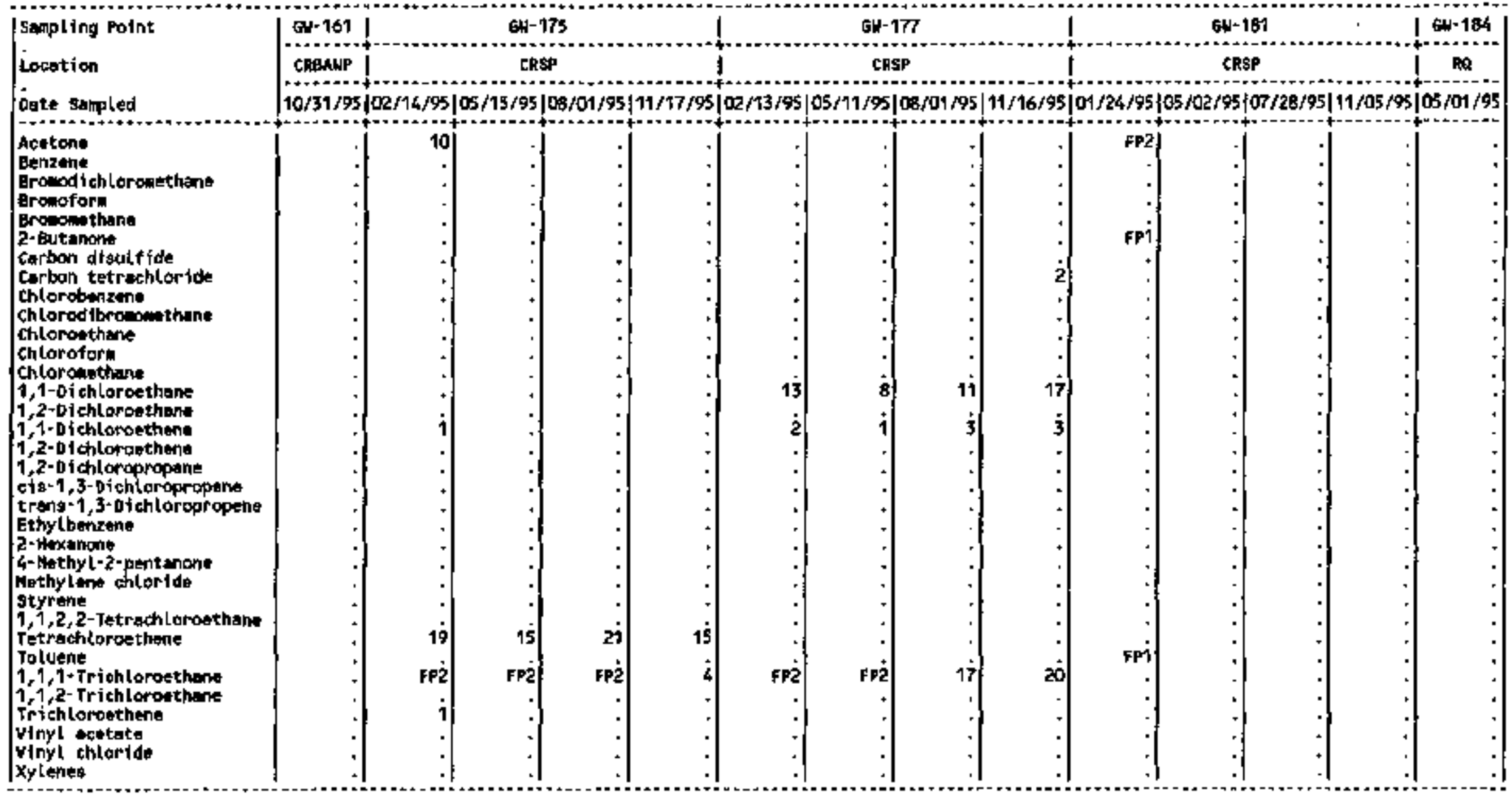

(COATI INJJED) 
APPENDIX

Yalideted Yoc Results, $19 \%$

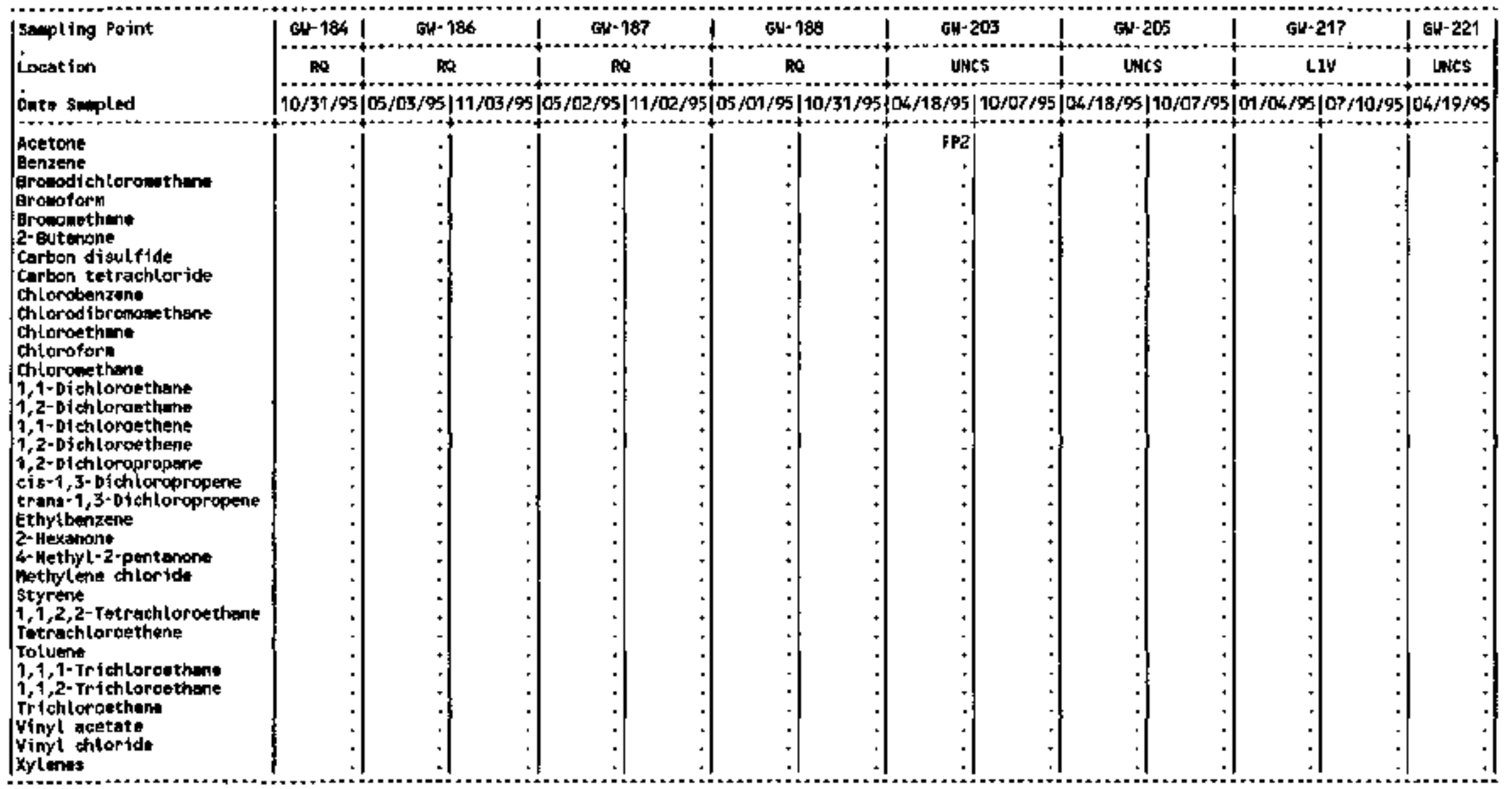

(COHTIKAED) 
Validoted yoc Results, 1995

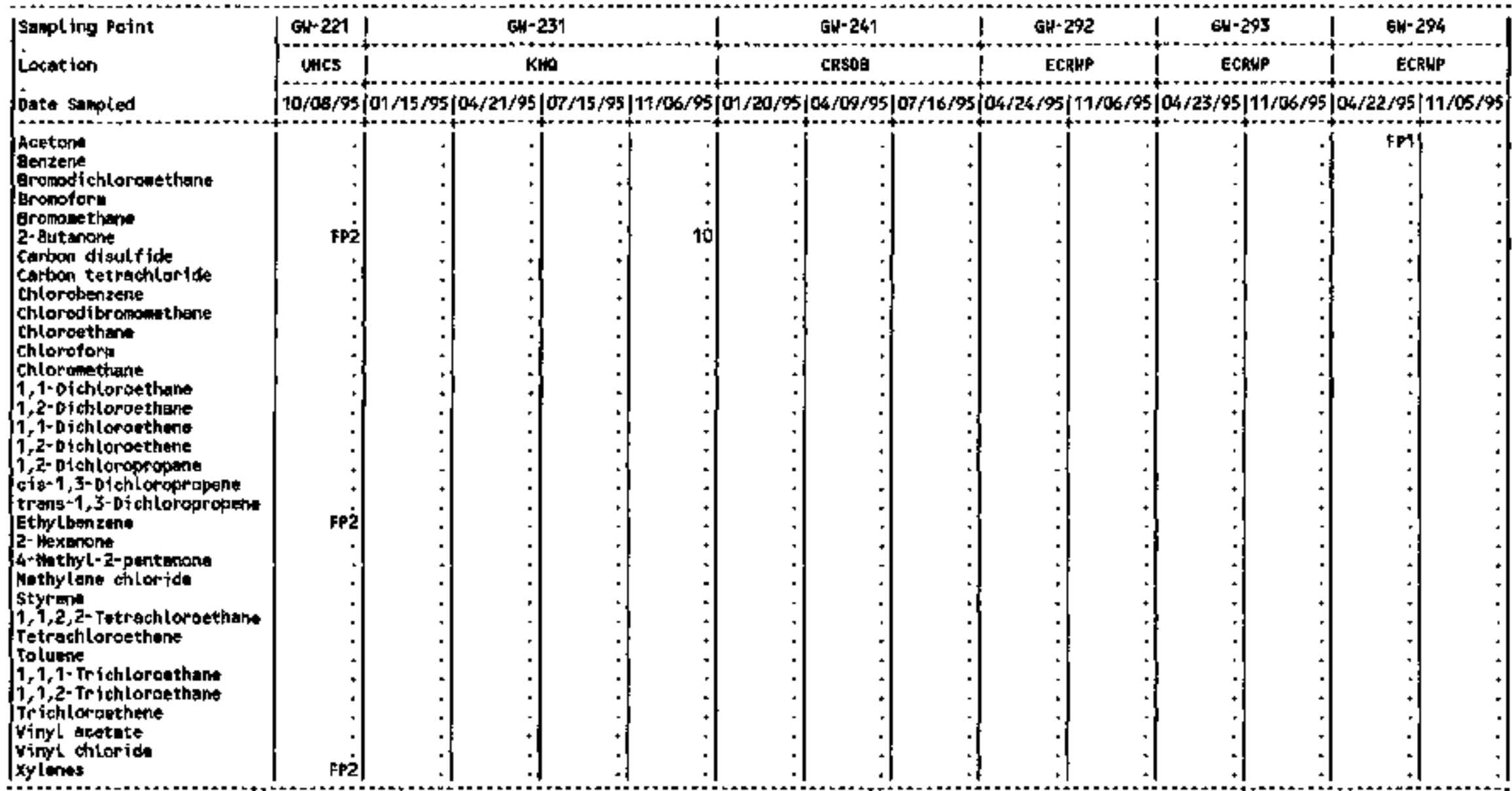

(CONTIn:uEb) 
Validated Voc Regulta, 1995

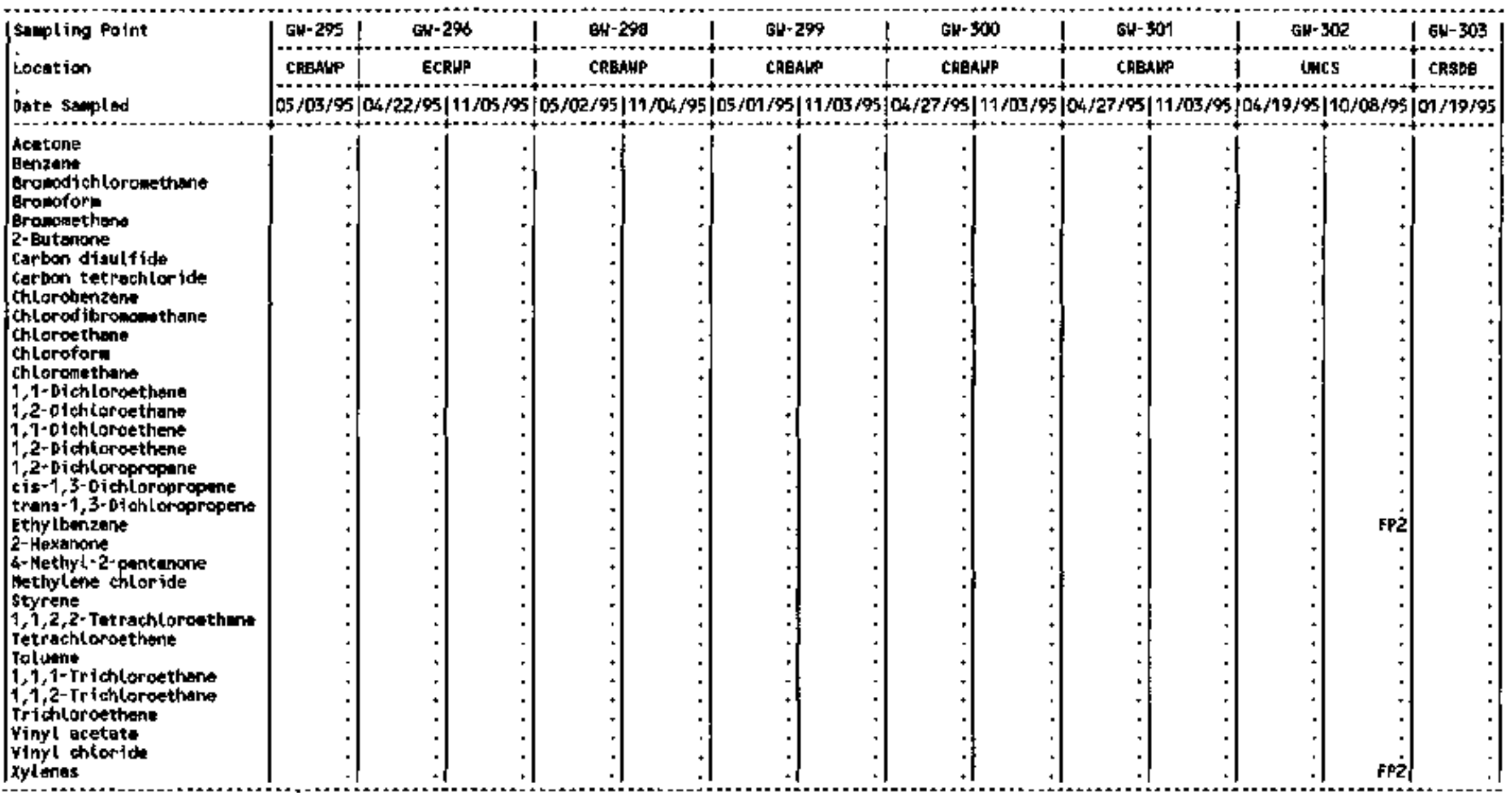

(CONTINUED) 
validoted woc Reoulte, 1955

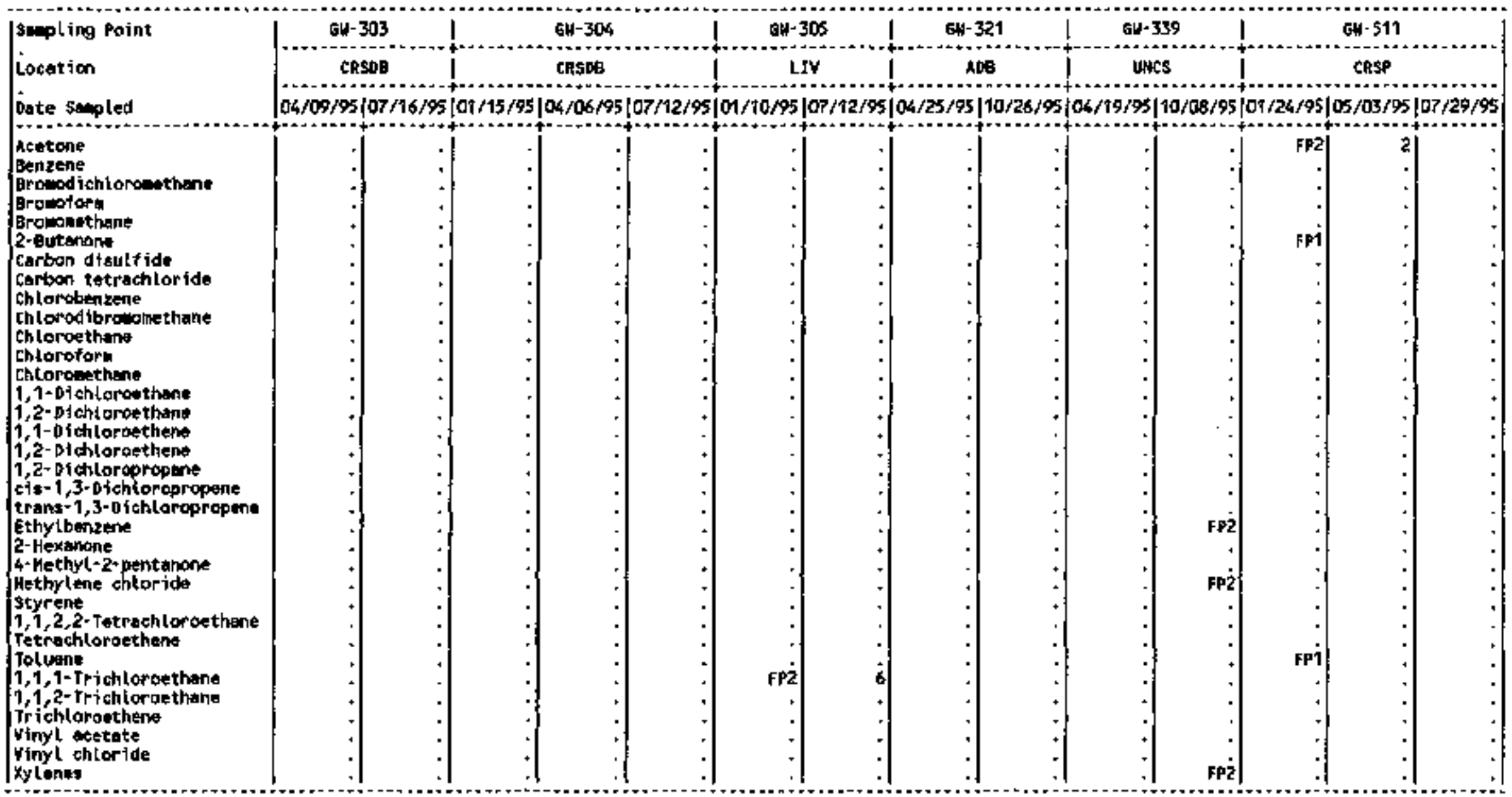

(CowTiMas) 
APPENDIX

Val idated voc iesults, 1995

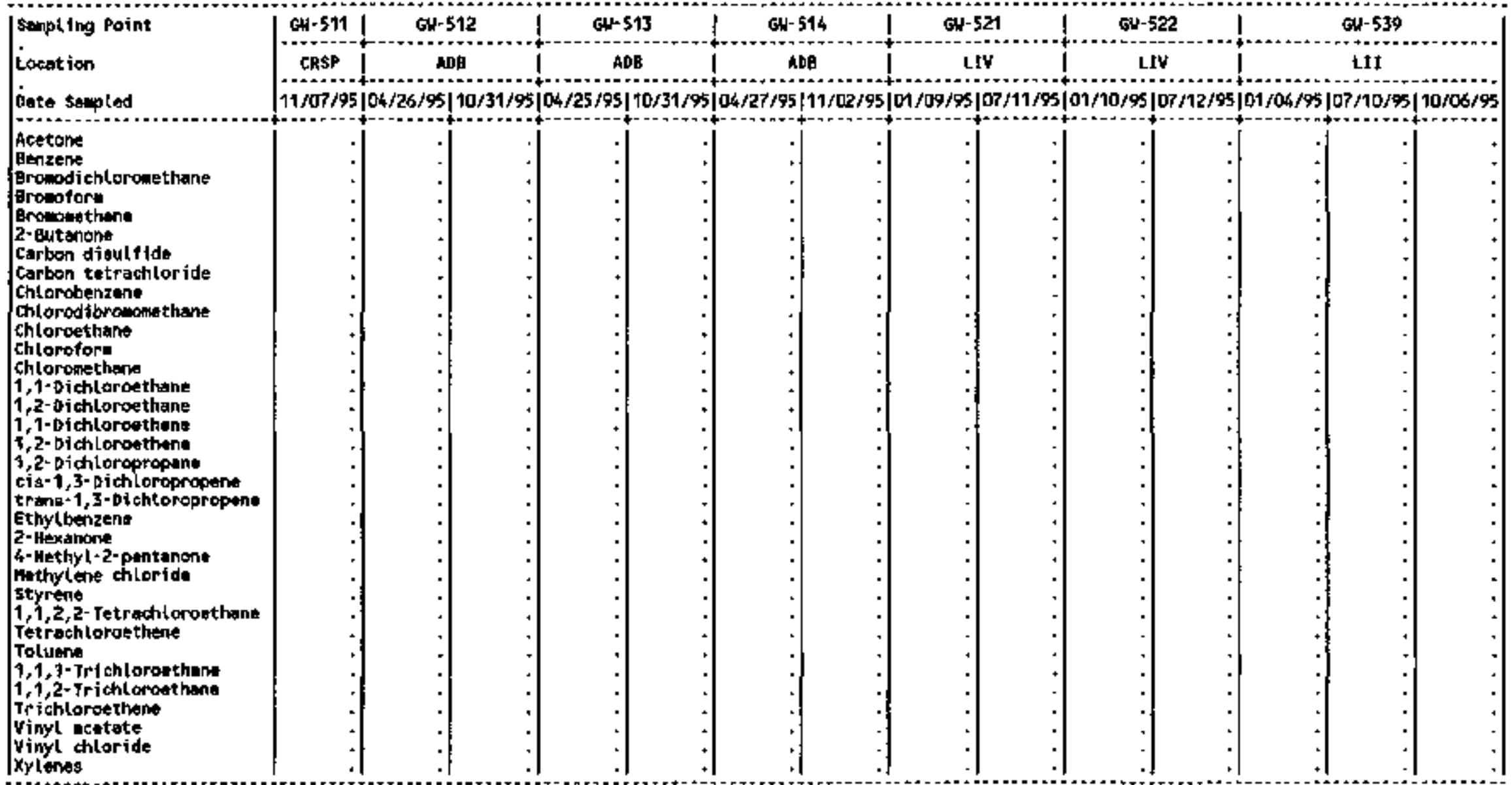

\footnotetext{
(COMTIHUEO)
} 
APPENOIX n

Volideted hoC Regults, 1995

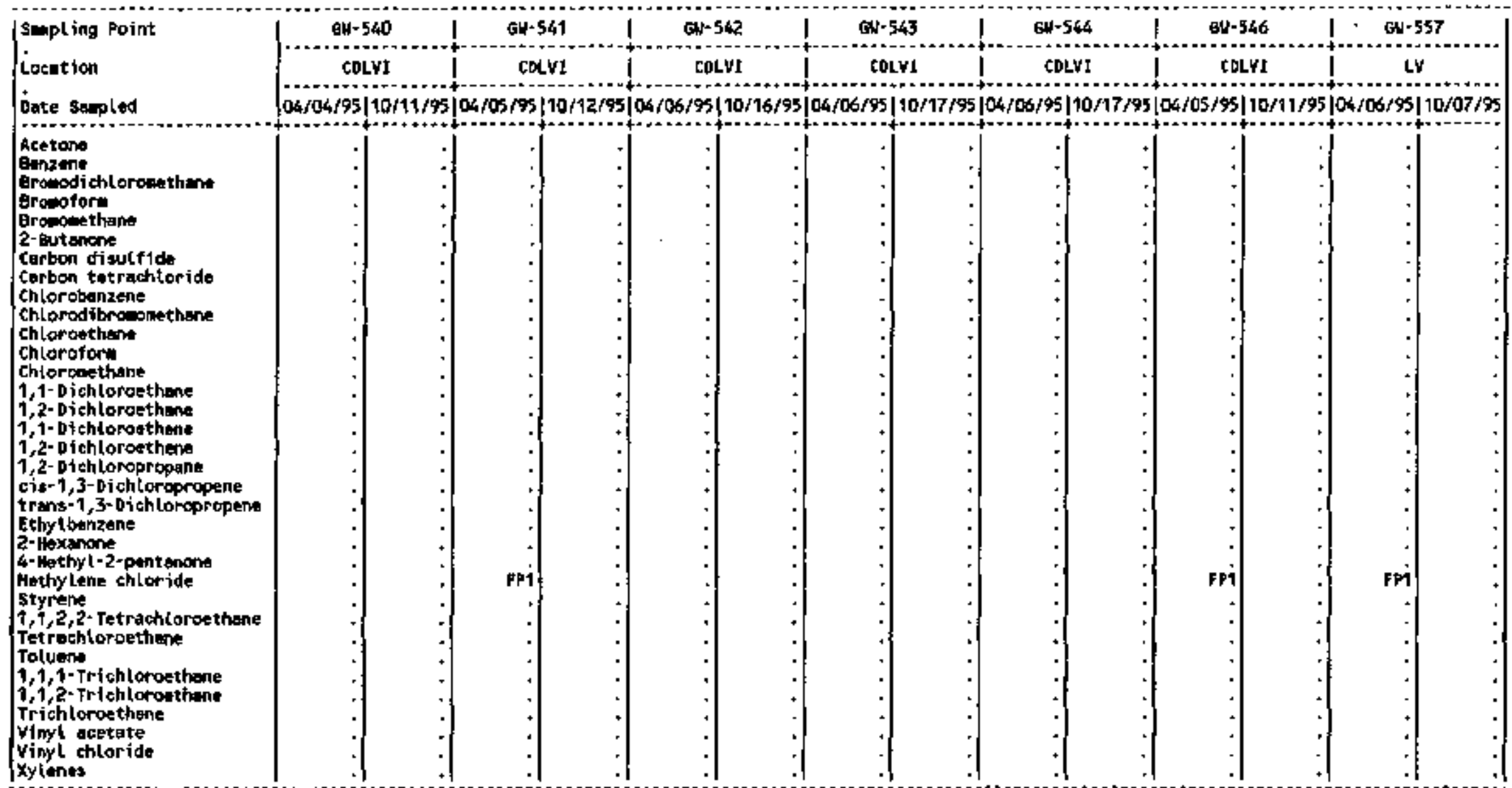

(CONTINUEB) 
Volideted VOC Re⿱宀

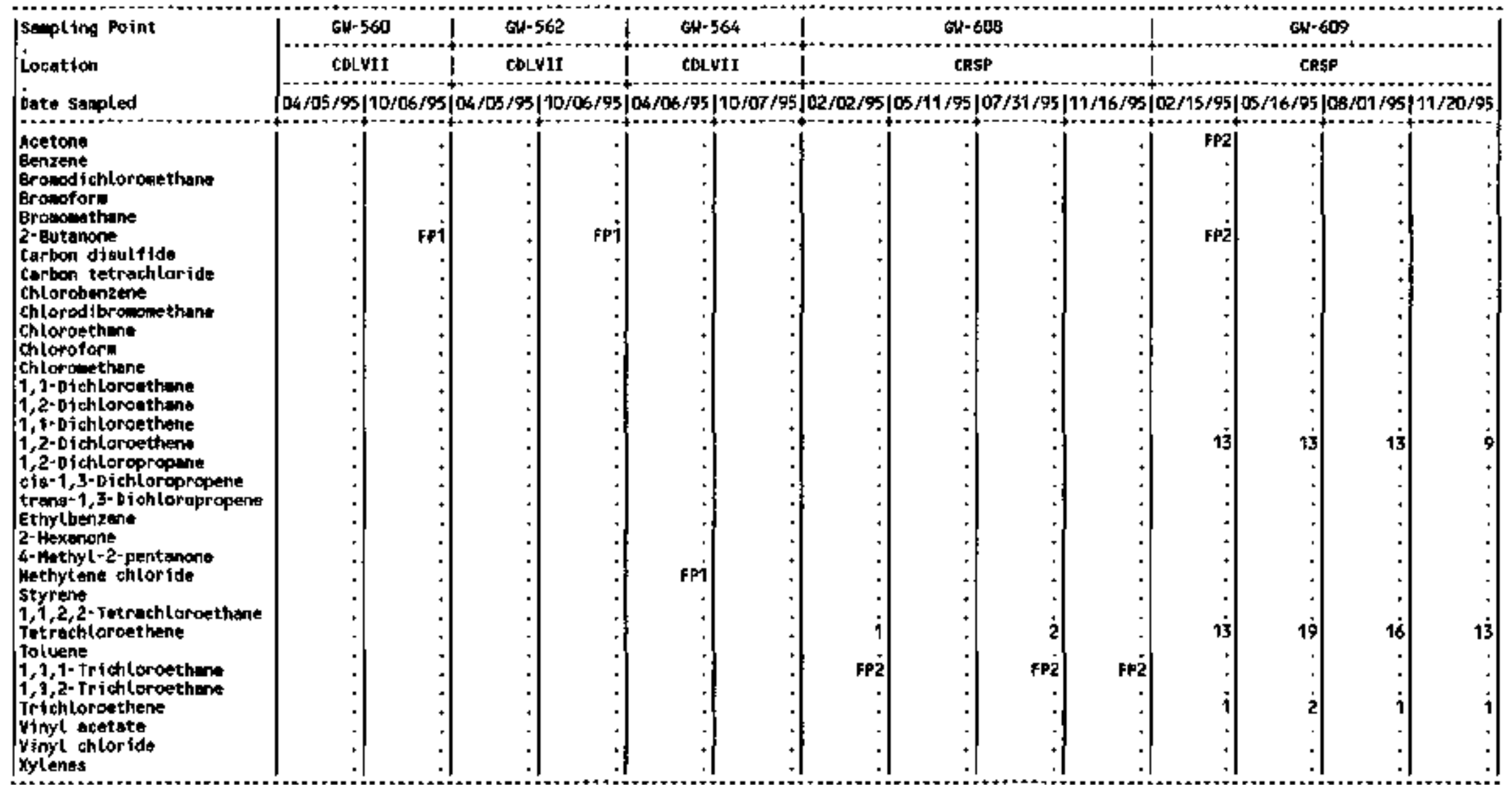

\section{(CONT ZNWED)}


Vol fanted voC Resulte, 1995

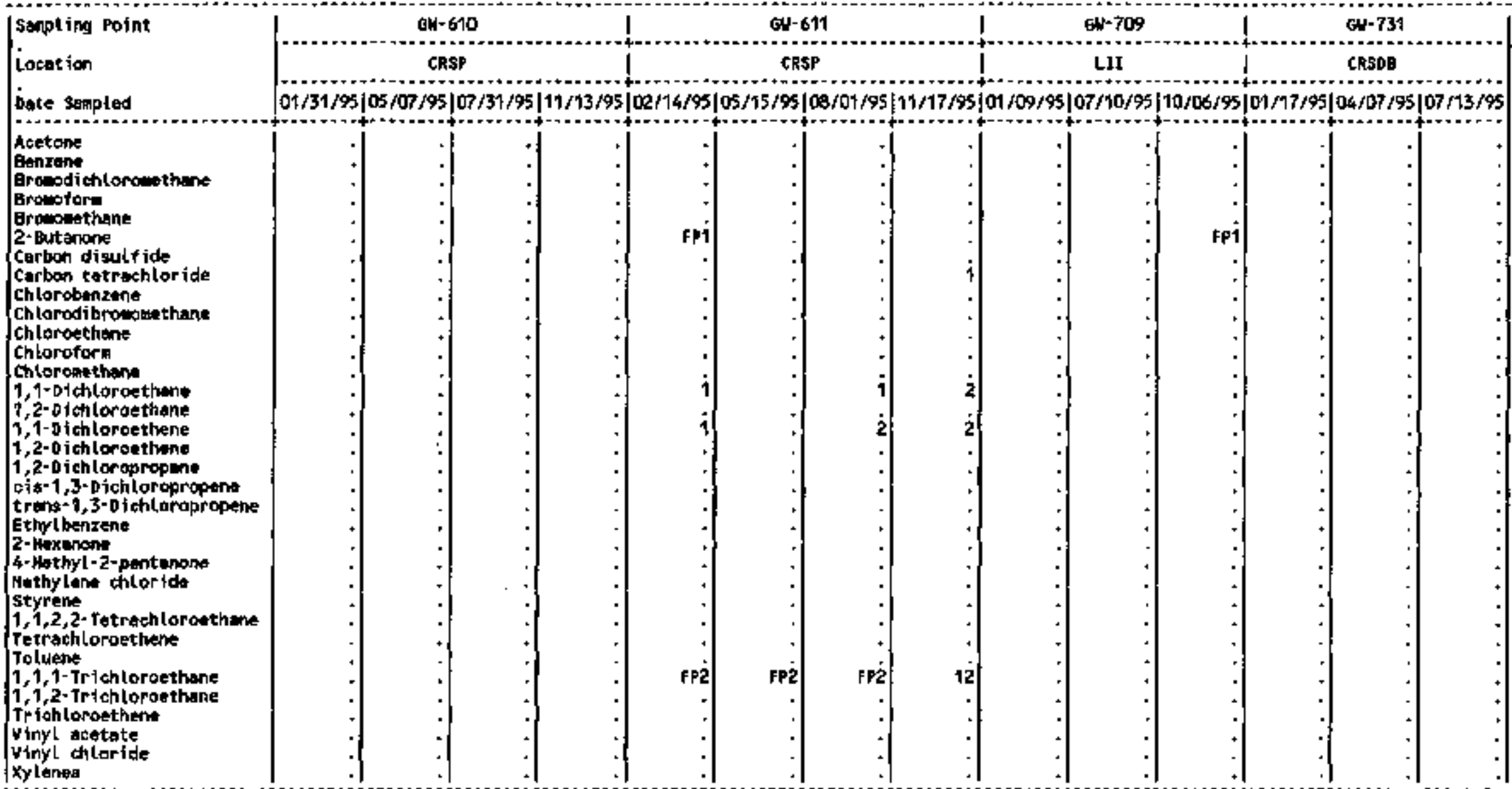

\section{(COMTIRALE)}


APPENOX $\mathrm{H}$

Wulidated Voc Easults, 1995

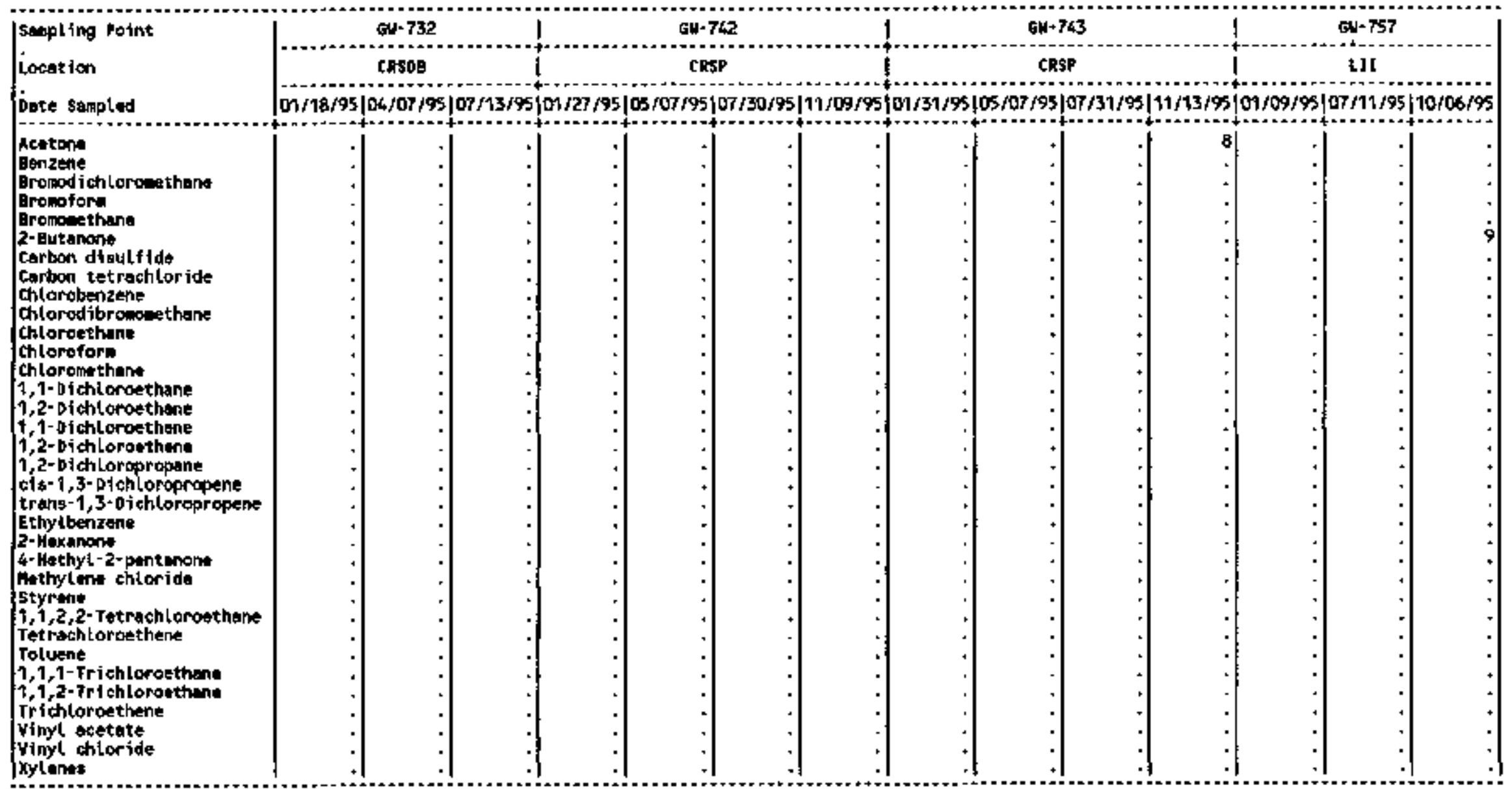

(CONTIMUED) 
Volldoted Yoc Results, 1995

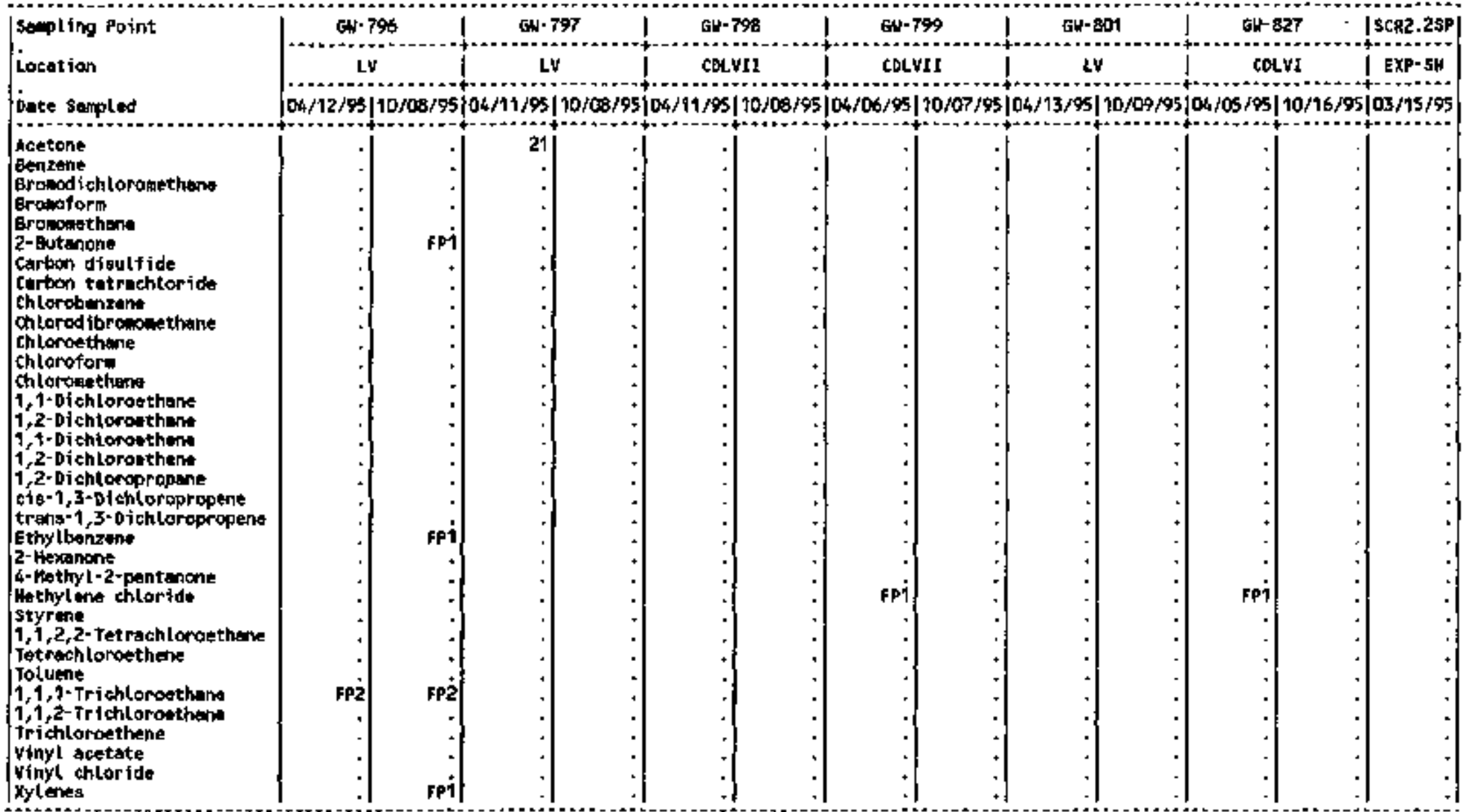




\section{DISIRIFUTION}

DEPARTMMNT OF ENERGY

P.A. Hofiman

P.J. Gtoss

L.M. Sparks

L.L. Radcliffe

W. Mancel

HEALTH. SAEETY ENVIRONMENT.AND

ACCOUNTABILITY ORGANIZATION

W.P. Carlton

C.C. Hill

W.K. Jago

S.B. Jones

J.E. Powelt

E.B. Rundle

L.O. Vaughan

GWPP-File-RC (4)

ENVRONMENTAL RESTORATION PROGRAM

H.J. King

C.S. Walker

Fle-ER DMC-RC

UNIVERSITY OF TENNNESSEE

B.W. McMaster

ORISE

S.M. Field

TENNESSEE DEPARTMIFNT OF

ENVIRONMIENT AND CONSERVATION -

DOE OVERSIGHTDVISION

R.C. Benafietd (3)
ENVIRONMIENTAL SCIENCES DTVISION

R.B. Dreter

D.D. Huff

C.T. Rightmire

R.R. Turner

D.B. Watson

WASTEMANAGEMENT ORGANIZATION

K.D. Delius

C.W. Hutzles

I,W. Jeter

J.E Stone

M.L. Willoughby

ENGINEERING ORGANIZATION

W.E. Manrod

KU25ANALYTICAL SERVICES

ORGANIZATION

J.M. LaBauve

HRR TECHNICAI ASSOCIATES

P.W. Fzell

SCIENCE APPIICATIONS

INTERNATIONAL CORPORATION

S. Selecman/W.P. Kegley

CDM EEDERAL PROGRAMS

M. Leslie/C. Lutz

Y.12 Central Files

A.K. Lee/DOE-OST (2) 